ANL-5914

Reactors - General

AEC Research and

Development Report

\author{
ARGONNE NATIONAL LABORATORY \\ P. O. Box 299 \\ Lemont, Illinois
}

\title{
SELECTED VALUES OF THE PHYSICAL PROPERTIES \\ OF VARIOUS MATERIALS
}

\author{
F. J. Tebo
}

September, 1958

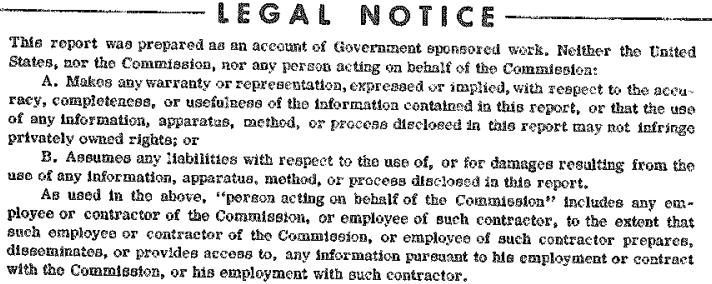

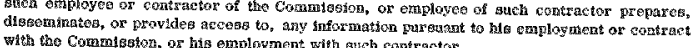

Operated by The University of Chicago

under

Contract $W-31-109-$ eng -38 


\section{DISCLAIMER}

This report was prepared as an account of work sponsored by an agency of the United States Government. Neither the United States Government nor any agency Thereof, nor any of their employees, makes any warranty, express or implied, or assumes any legal liability or responsibility for the accuracy, completeness, or usefulness of any information, apparatus, product, or process disclosed, or represents that its use would not infringe privately owned rights. Reference herein to any specific commercial product, process, or service by trade name, trademark, manufacturer, or otherwise does not necessarily constitute or imply its endorsement, recommendation, or favoring by the United States Government or any agency thereof. The views and opinions of authors expressed herein do not necessarily state or reflect those of the United States Government or any agency thereof. 


\section{DISCLAIMER}

Portions of this document may be illegible in electronic image products. Images are produced from the best available original document. 


\section{SELECTED VALUES OF THE PHYSICAL PROPERTIES OF VARIOUS MATERIALS}

\section{by}

F. J. Tebo

Selected physical properties of various solids, liquids and gases of interest in nuclear reactor heat transfer and fluid flow analysis are presented. In most cases, values for thermal conductivity, specific heat, density, viscosity and PrandtI numbers are given as functions of temperature. A table of room temperatures values of some properties have been compiled on materials for which data as a function of temperature were not found.

A short list of references to additional physical property data on many materials is provided at the end of the compilation.

The values presented here were compiled after a brief survey of the classified and unclassified literature, and no guarantee is given that this is the best existing information. Much of the data came from sources most frequently used for reference, such as the Reactor Handbook and the Liquid Metals Handbook. 
Page Number

\begin{tabular}{|c|c|c|c|}
\hline Solids & $\mathrm{k}^{*}$ & $\mathrm{C}_{p^{*}}$ & $\rho^{*}$ \\
\hline Alumel & 1 & $\infty$ & - \\
\hline Alumina & 2 & - & $=$ \\
\hline Alumi num & 3 & 4 & 5 \\
\hline Aluminum A11oy 25 & - & 6 & - \\
\hline Aluminum AIIOY $24 \mathrm{~S}=\mathrm{TH}$ & 7 & - & 8 \\
\hline Aluminum Alloy 75s-16 & - & - & 9 \\
\hline Beryllia & 10 & 11 & - \\
\hline Beryllium & 12 & 13 & I4s \\
\hline Bismuth & 15 & 16 & 17 \\
\hline Boron Carbide & 18 & - & - \\
\hline Cadmi un & 19 & 20 & - \\
\hline Cadmium-Silver Alloy & 21 & $\mathrm{X}$ & $x$ \\
\hline Concrete, High Density & 22 & $\infty$ & $\infty$ \\
\hline Copper & 23 & 24 & 25 \\
\hline Graphite & $26 \& 27$ & 28 & 29 \\
\hline Incone1 & 30 & - & 31 \\
\hline Inconel "Mx" & 32 & - & 33 \\
\hline Iron, Arnco & 34 & 35 & - \\
\hline Iron, Cast & 36 & - & $=$ \\
\hline Iron, Pure & 37 & $=$ & $\Rightarrow$ \\
\hline Lead & 38 & 39 & - \\
\hline
\end{tabular}


Page Number

Solids

Magnesium

Magnesium AIIoy AN-M-29

Magnesium Oxide

Molybdenum

Monel

MTRP MoneI

Nichrome V

Nicke1

"A" Nicke1

Nickel Oxide

Nicke1-Zi rconium A11oy

Silver

Spine 1

Stainless Steel 301

Stainless Stee1 303

Stainless Steel 304

Stainless Steel 310

Stainless steel 316

Stainless Stee1 321

Stainless SteeI 347

Stainless stee 130

Stainless steel 446

SteeI, SAE 1010

\begin{tabular}{|c|c|c|}
\hline $\mathbf{k}^{*}$ & $\mathrm{C}_{\mathrm{p}}$ & $p^{*}$ \\
\hline 40 & 41 & 42 \\
\hline 43 & - & - \\
\hline 44 & - & $=$ \\
\hline 45 & 46 & - \\
\hline 47 & 48 & $=$ \\
\hline 49 & $\infty$ & 50 \\
\hline- & 51 & - \\
\hline 52 & 53 & - \\
\hline 54 & $\infty$ & - \\
\hline 55 & $\infty$ & - \\
\hline 56 & W & $x$ \\
\hline 57 & 58 & 59 \\
\hline 60 & - & - \\
\hline 61 & - & 62 \\
\hline 63 & - & $=$ \\
\hline 64 & - & - \\
\hline 65 & $=$ & - \\
\hline 66 & - & 67 \\
\hline 68 & 69 & $=$ \\
\hline 70 & 71 & 72 \\
\hline 73 & - & $\infty$ \\
\hline 74 & 75 & - \\
\hline 76 & 77 & 78 \\
\hline
\end{tabular}


TABLE OF CONTENTS (Con't)

Page Number

\begin{tabular}{|c|c|c|c|}
\hline Solids & $k^{*}$ & $\mathrm{C}^{*}$ & $\rho^{*}$ \\
\hline Stre1. I,ow Carbon & 79 & - & - \\
\hline Steel, $\mathrm{diId}$ & 80 & - & - \\
\hline Steel, Tungsten & 81 & - & - \\
\hline Tantalum & 82 & - & - \\
\hline Thorium & 83 & 84 & 85 \\
\hline Thoxium Bioxide & 86 & - & - \\
\hline Titanium & - & 87 & 88 \\
\hline Titanium Dioxide & 89 & - & - \\
\hline Tungsten & 90 & 91 & - \\
\hline Urañum & 92 & 93 & - \\
\hline Uraniunne-Chronium Alloy & 94 & $x$ & $\mathrm{X}$ \\
\hline Uraniun-7i reonium Alloys & 95 & $\mathbb{X}$ & 96 \\
\hline U-2x Alloy (Irradiated) & $97 \& 98$ & - & - \\
\hline Uranium Dioxide & 99 & 200 & - \\
\hline Vanadium & 101 & 102 & $=$ \\
\hline zinc & 103 & 104 & 105 \\
\hline Zinc Oxide & 106 & - & $=$ \\
\hline Zi nconi un & 107 & 108 & 109 \\
\hline Zirconium-Tantalun Alloy & 110 & $\mathrm{x}$ & $x$ \\
\hline Zirconium-Tin Alloy & 111 & $x$ & $\mathbb{x}$ \\
\hline Zireon & 112 & - & - \\
\hline Zixconium DIoxide & 113 & - & - \\
\hline
\end{tabular}


TABLE OF CONTENTS ( $\left.\operatorname{Con}^{\circ} t\right)$

Page Number

\begin{tabular}{|c|c|c|c|c|c|}
\hline Liquids & $k^{*}$ & $C_{0}{ }^{*}$ & $e^{*}$ & $\underline{u}^{*}$ & $\mathrm{Pr}^{*}$ \\
\hline Aluminum & 114 & 115 & 116 & 117 & $x$ \\
\hline Bismuth & 118 & 129 & 120 & 121 & 122 \\
\hline Cadmi un & 123 & 124 & 125 & 126 & $x$ \\
\hline Diphenyl & 127 & $\%$ & 128 & 129 & x \\
\hline Dowtherm & 130 & 131 & 132 & 133 & 羊 \\
\hline Gallium & $\mathrm{x}$ & 134 & 235 & 136 & x \\
\hline Lead & 137 & 138 & 139 & 140 & 14 \\
\hline Lead-Bismuth Alloy & 142 & 143 & Ithe & 145 & 146 \\
\hline Iithium & 147 & 148 & 149 & 150 & 151 \\
\hline Mercury & 152 & 153 & 254 & 155 & $x$ \\
\hline Potassium & 156 & 157 & 158 & 159 & 160 \\
\hline Sodium & 161 & 162 & 163 & 164 & 165 \\
\hline $56 \% \mathrm{Na}, 44 \% \mathrm{~K}$ AIIoy & 166 & 167 & 168 & 169 & 170 \\
\hline $22 \% \mathrm{Na}, 78 \% \mathrm{~K}$ Alloy & 171 & 172 & 173 & 174 & 175 \\
\hline Tin & 176 & 177 & 178 & 179 & $x$ \\
\hline Watex, Heavy & $\mathrm{x}$ & 180 & 181 & 182 & $*$ \\
\hline Water, Iight & 183 & 184 & 185 & 186 & 187 \\
\hline Zinc & 188 & 189 & 190 & 191 & $x$ \\
\hline
\end{tabular}


TABLE OF CONTENTS (COn't)

\begin{tabular}{l} 
Gases \\
\hline Air \\
Argon \\
Carbon Dioxide \\
Carbon Monoxide \\
Hellum \\
Hydrogen \\
Nitric Oxide \\
Nitrogen \\
Oxygen \\
Steam
\end{tabular}

\begin{tabular}{llllll}
\hline$k^{*}$ & $C_{p}^{*}$ & $p^{*}$ & $u^{*}$ & $P^{*}$ \\
192 & 193 & 194 & 195 & 196 \\
197 & 198 & 199 & 200 & 201 \\
202 & 203 & 204 & 205 & 206 \\
207 & 208 & 209 & 210 & 211 \\
212 & 213 & 214 & 215 & 216 \\
217 & 218 & 219 & 220 & 221 \\
222 & 223 & 224 & 225 & 226 \\
227 & 228 & 229 & 230 & 231 \\
232 & 233 & 234 & 235 & 236 \\
237 & 238 & 239 & 240 & 241
\end{tabular}

k - Thermal Conductivity

$C_{p}$ - Heat Capacity

P - Density

u - Dynamic Viscosity

Pr - PrandtI Number

- Single point values

X - No data presented 
TABLE OF CONIENTS (Con't)

Table Number and Title

1. Composition of Some Metals

2. Single Point Value of Some Properties

3. PrandtI Numbers of Gases

4. Thermal Conductivities of Gases

5. Dymamic Viscosities of Gases

6. VIscosities of Argon Under Pressure

7. Viscosities of Carbon Diodide Under Pressure

8. Viscosities of Hydrogen Under Pressure

9. Viscosities of Nitrogen Under Pressure

10. Viscosities of Oxygen Under Pressure

1. Viscosities of Steam Under Pressure
Page Number

242

243

246

247

248

249

250

251

252

253

254

255 


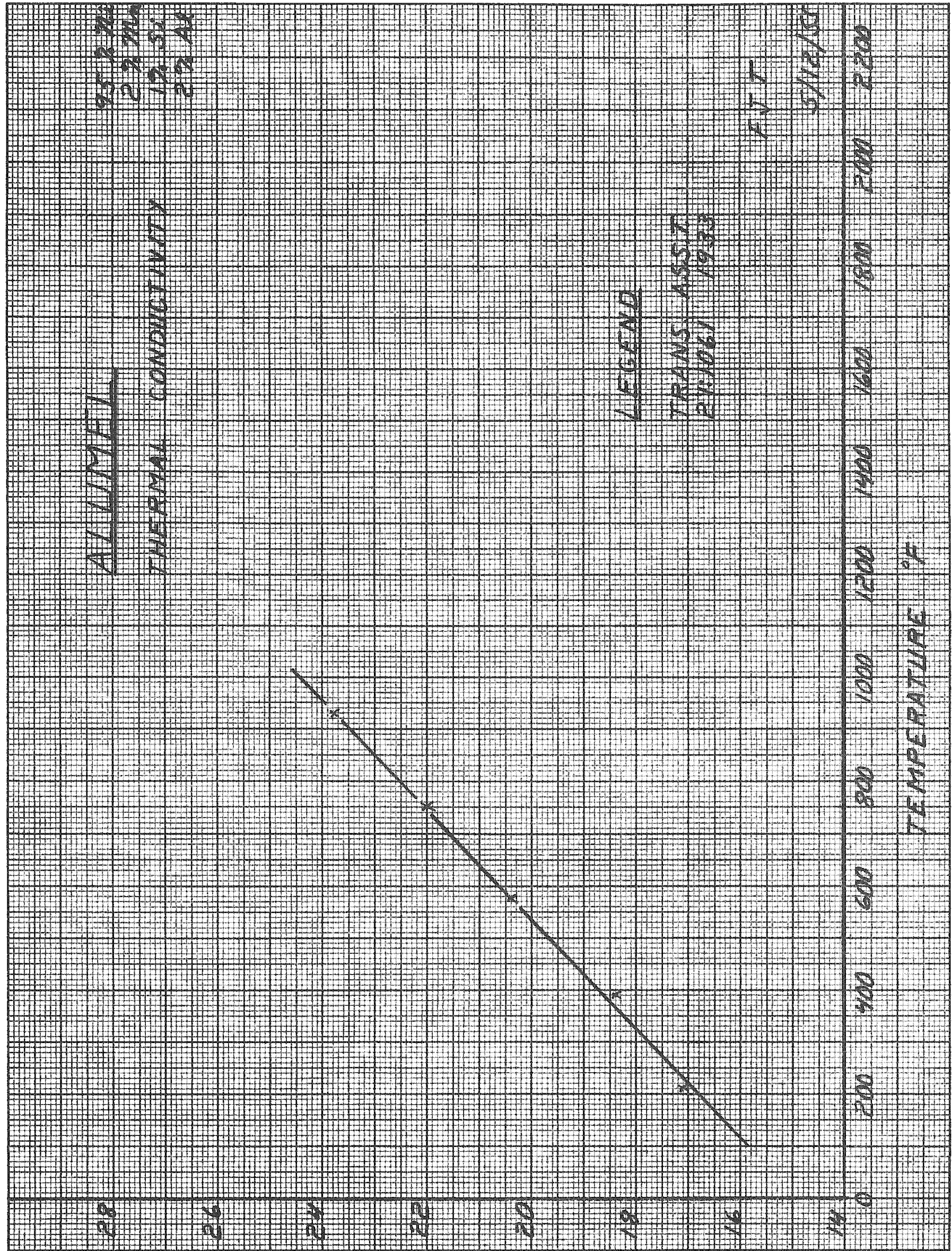

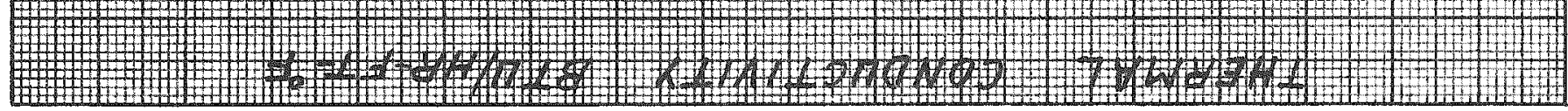




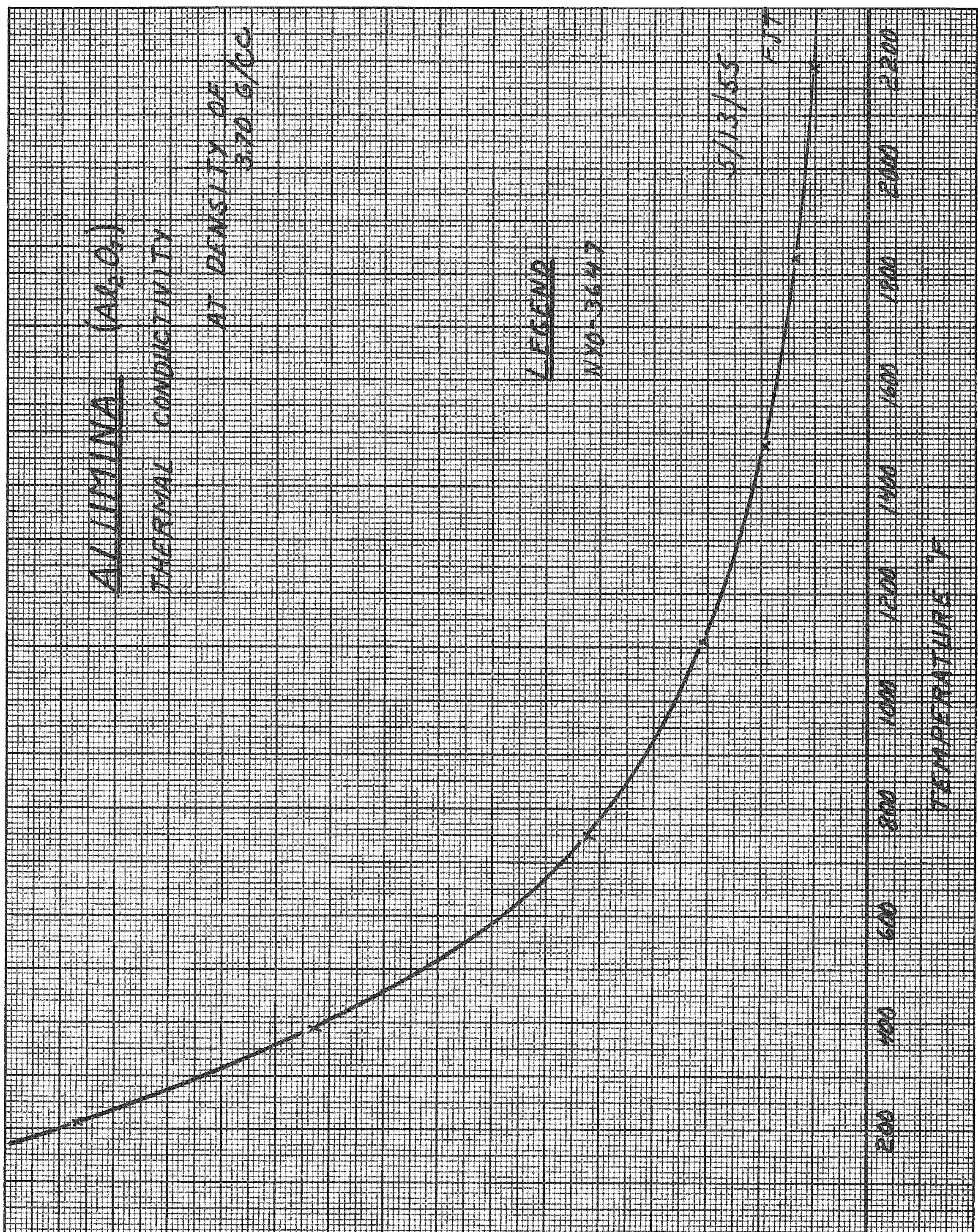




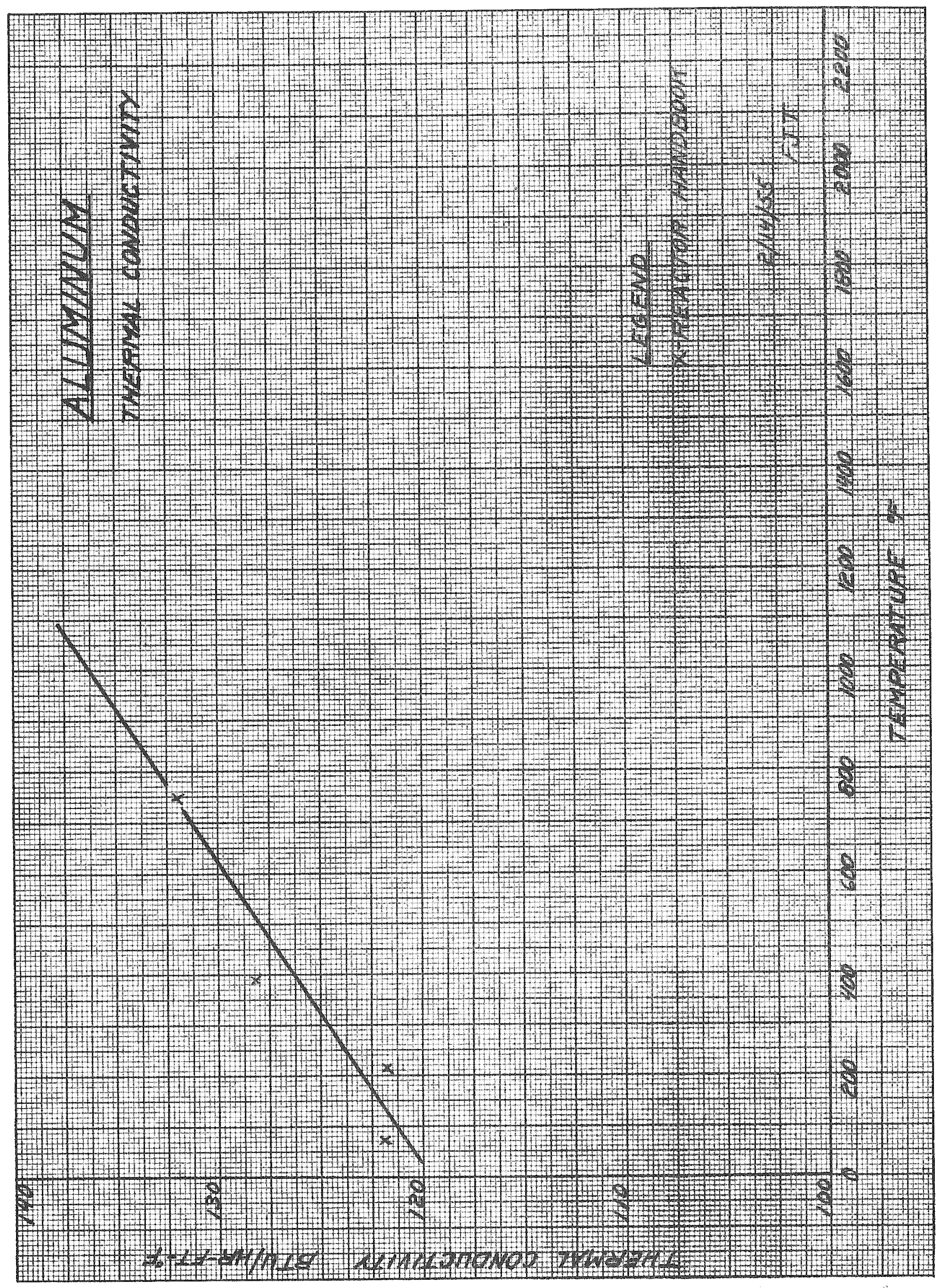




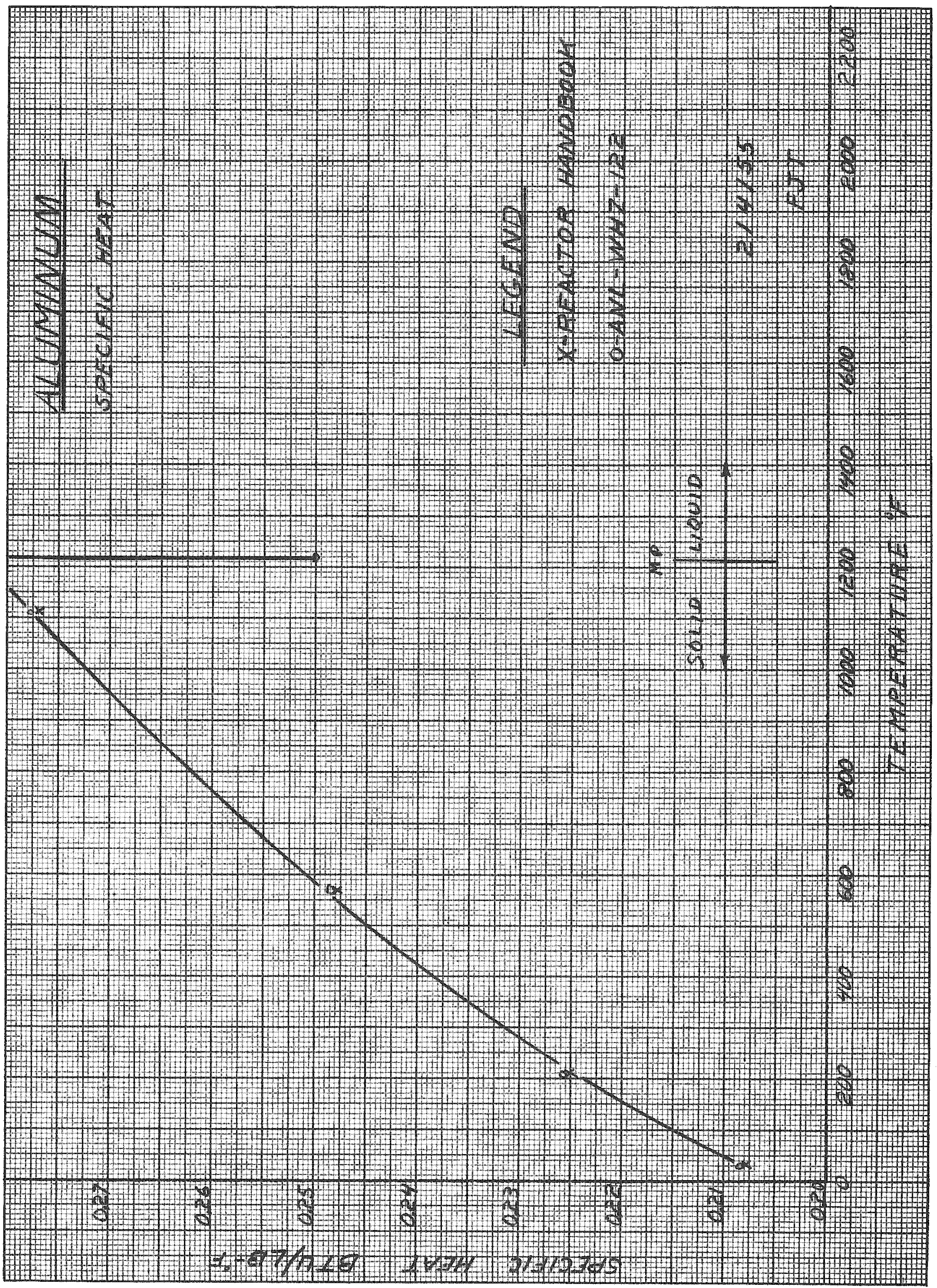




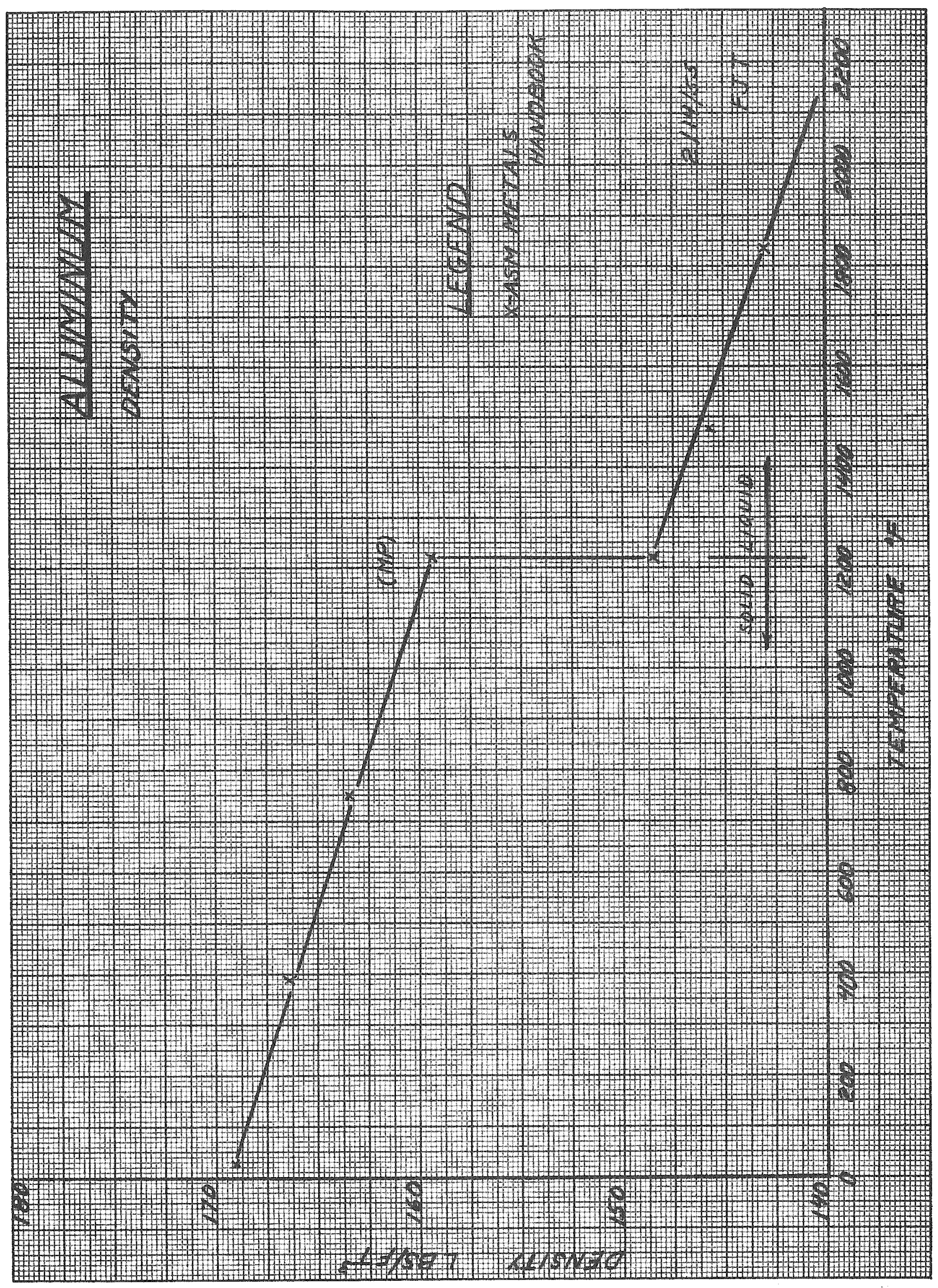




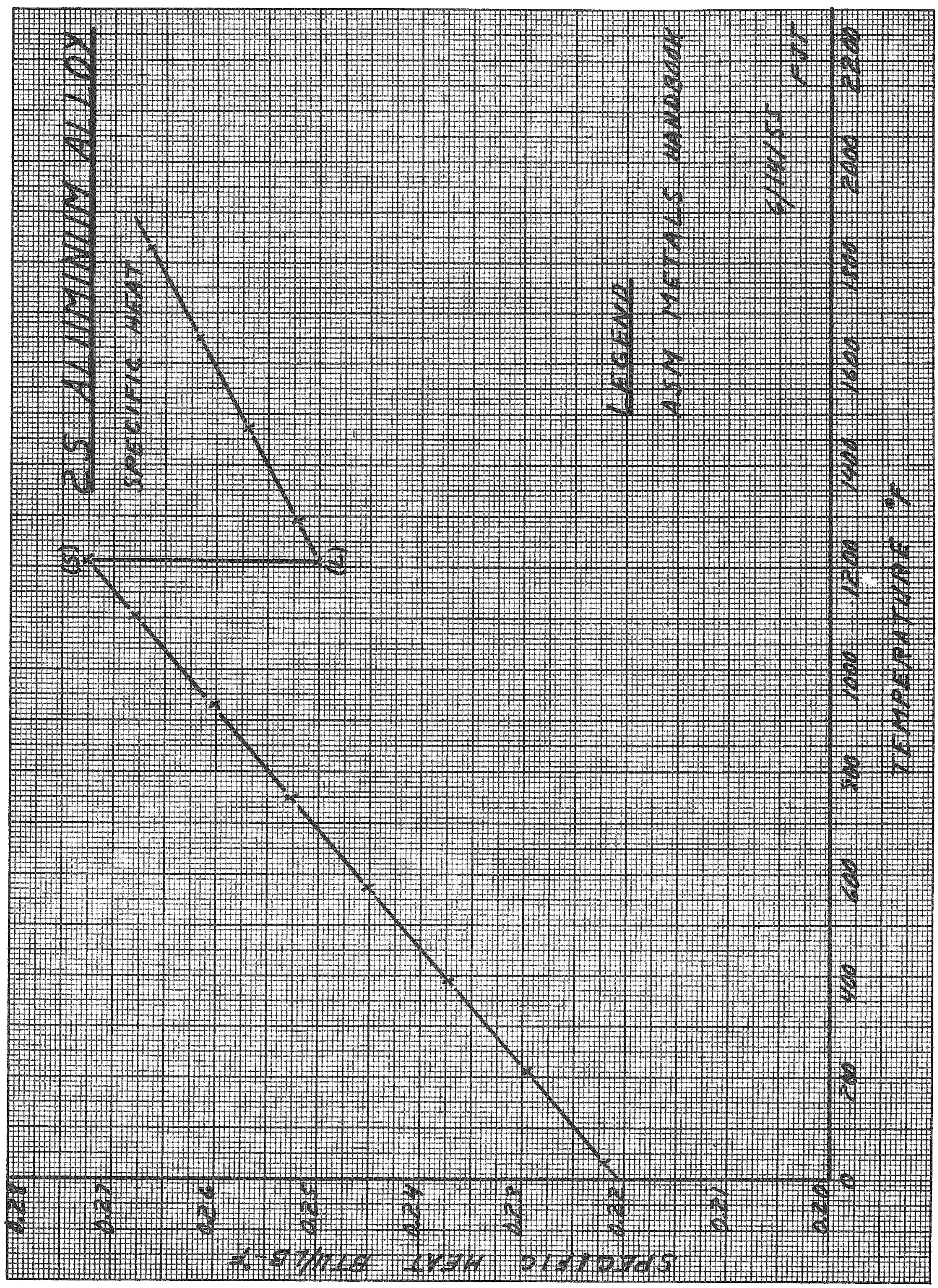


(.

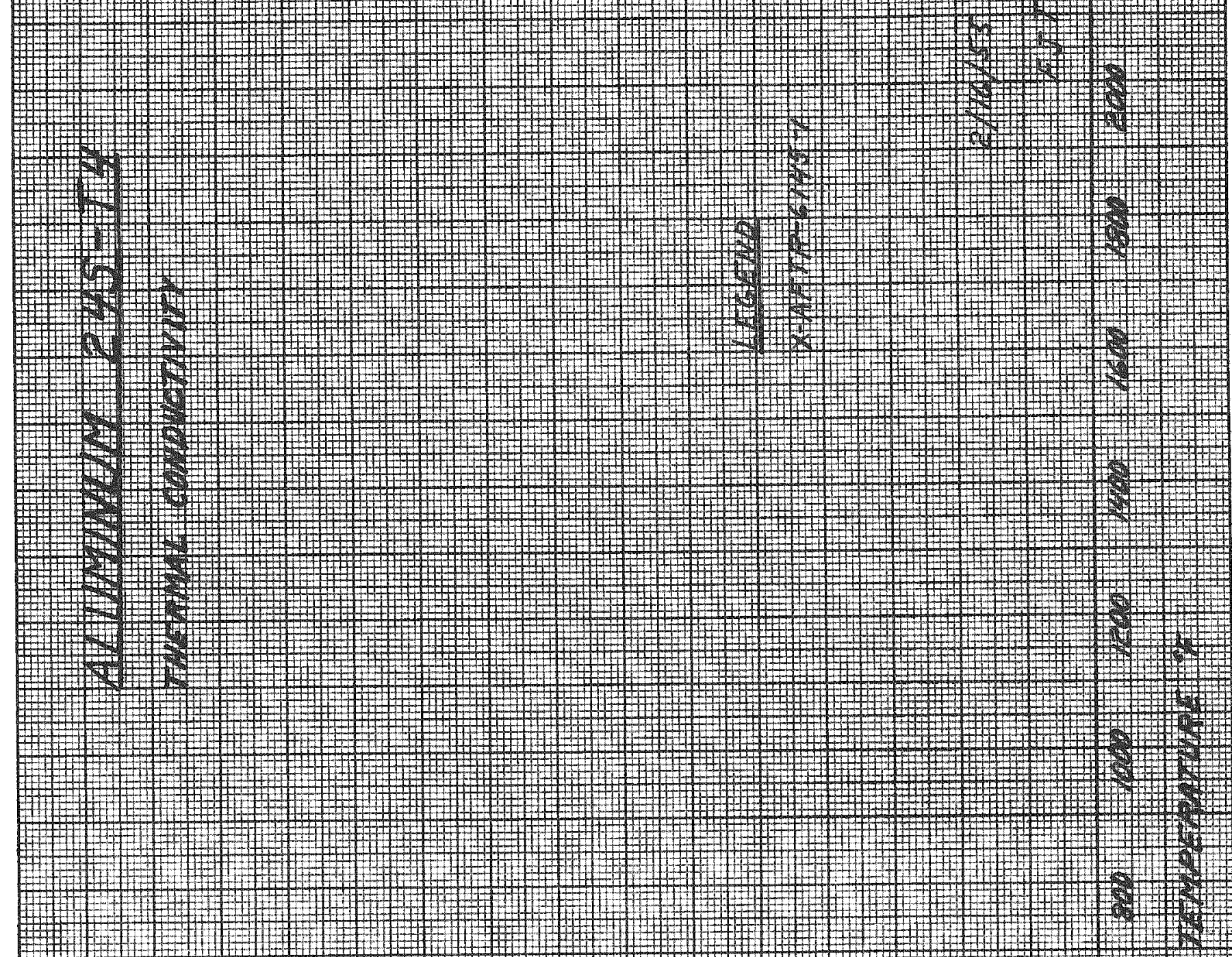

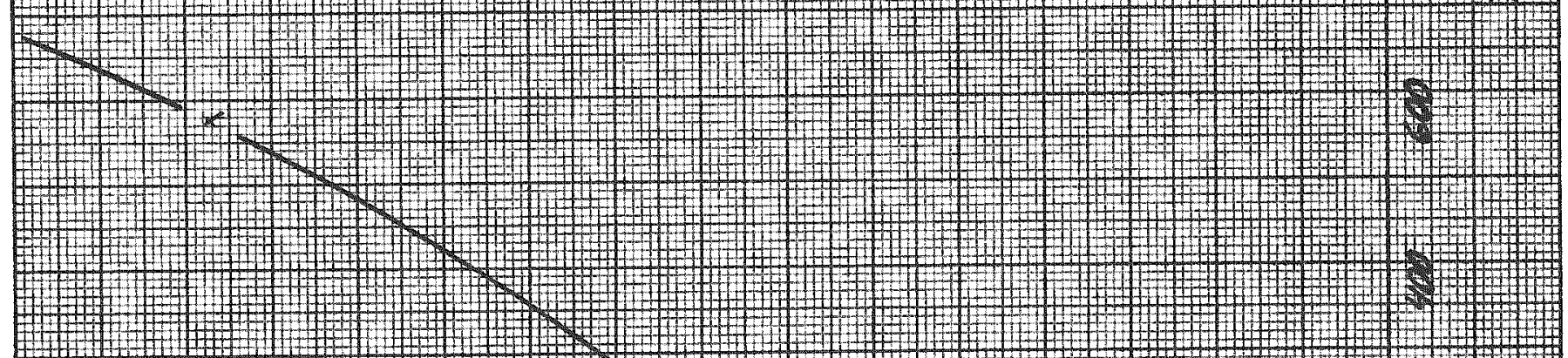
(1) -

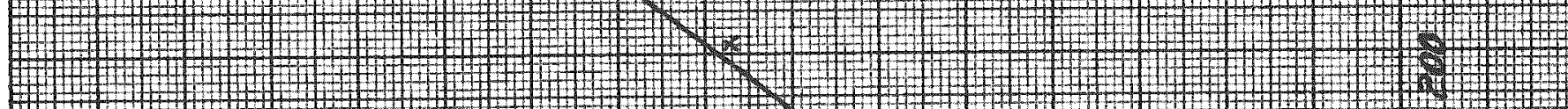
-

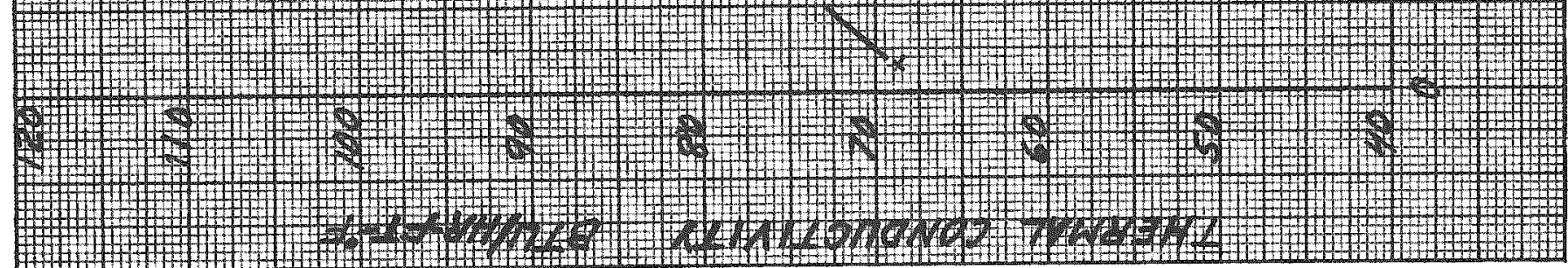




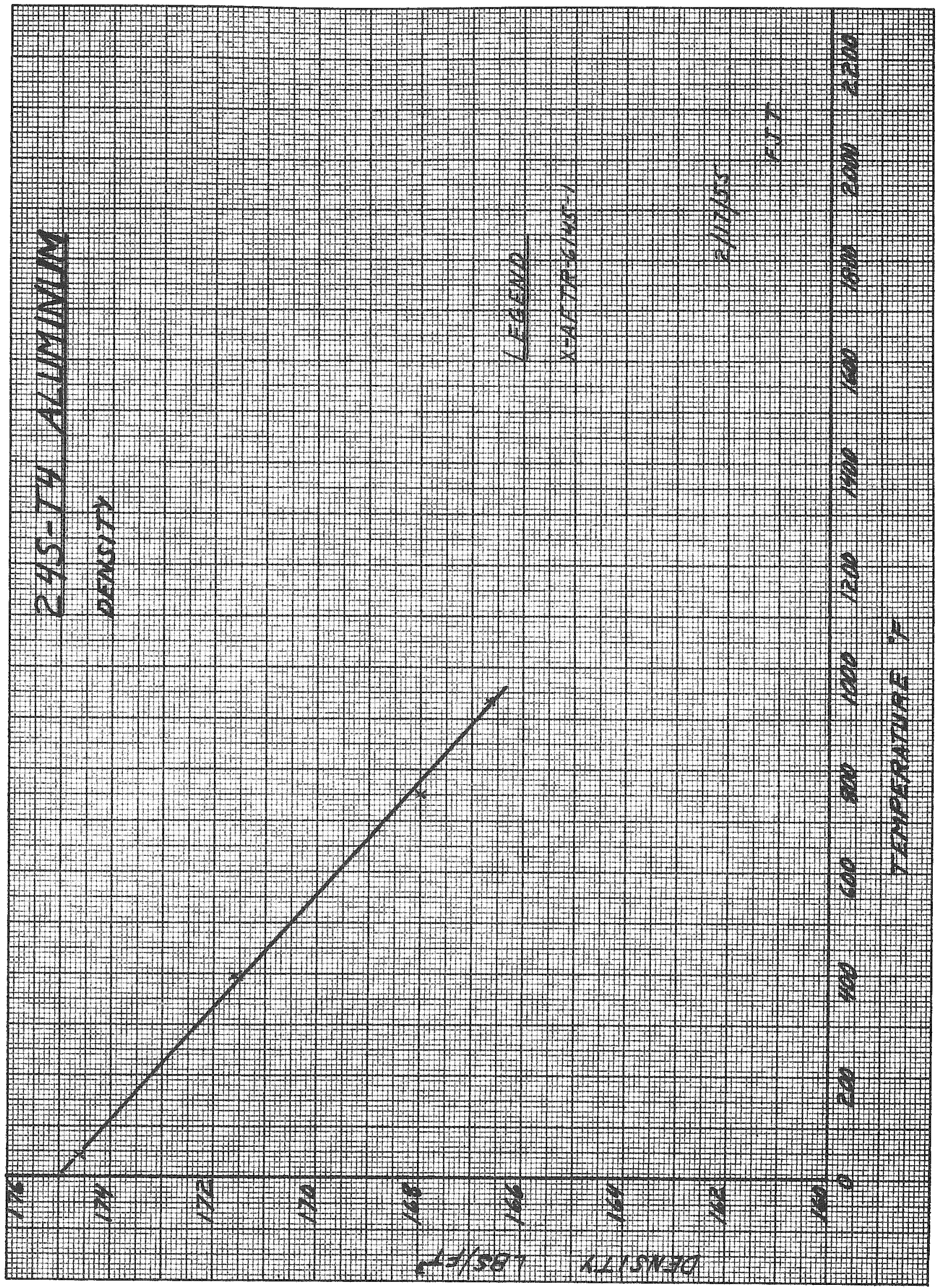




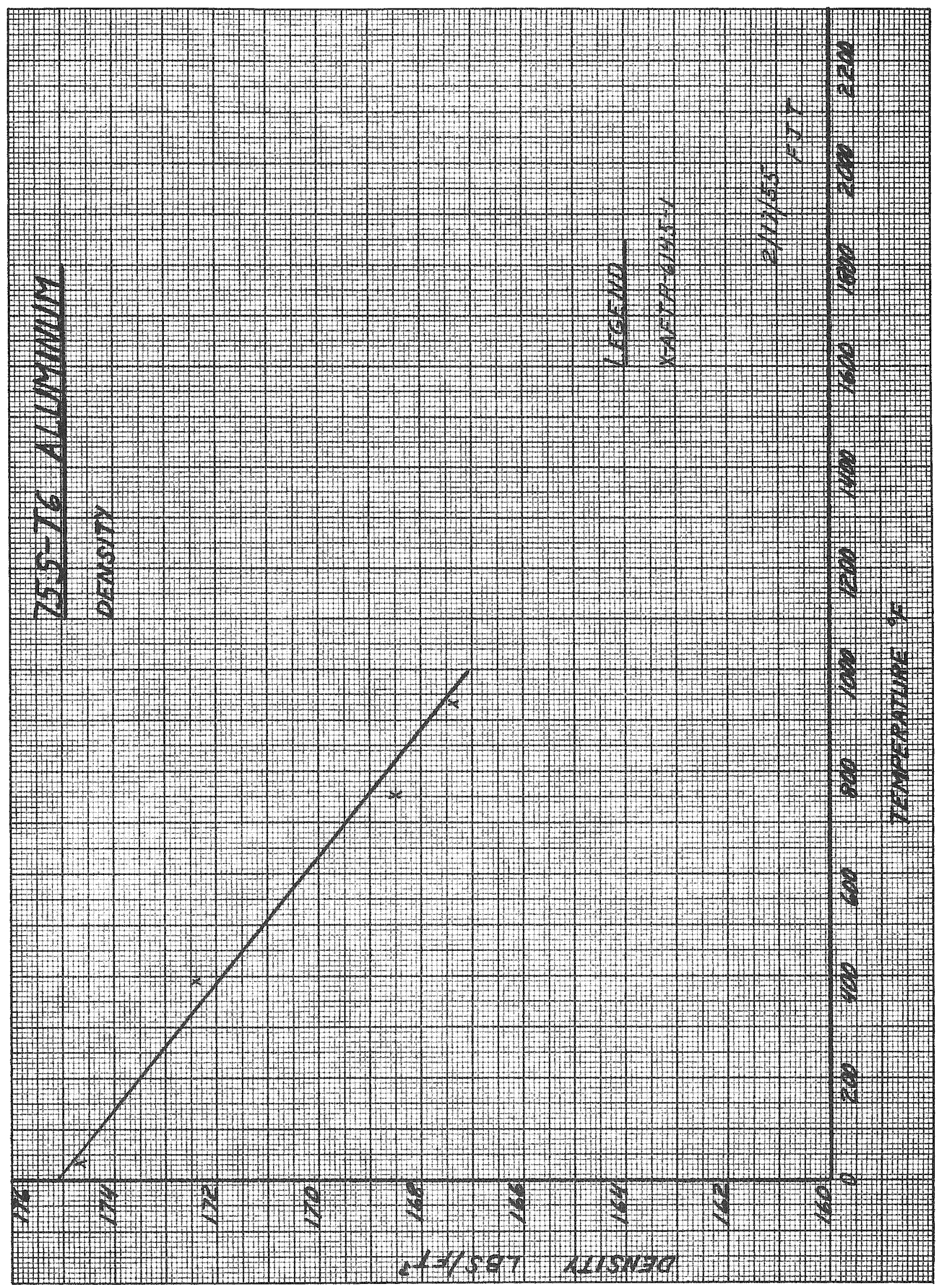




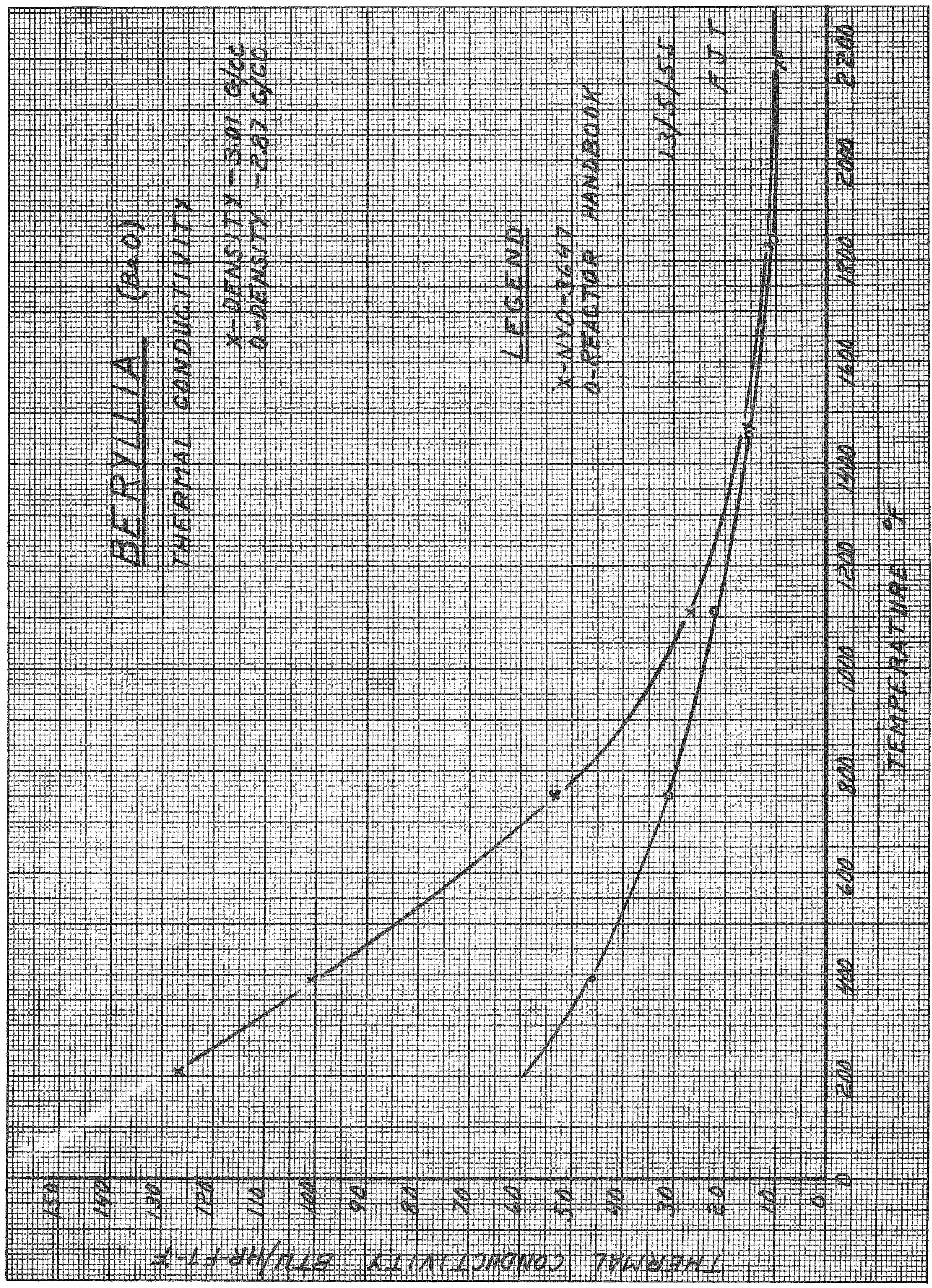




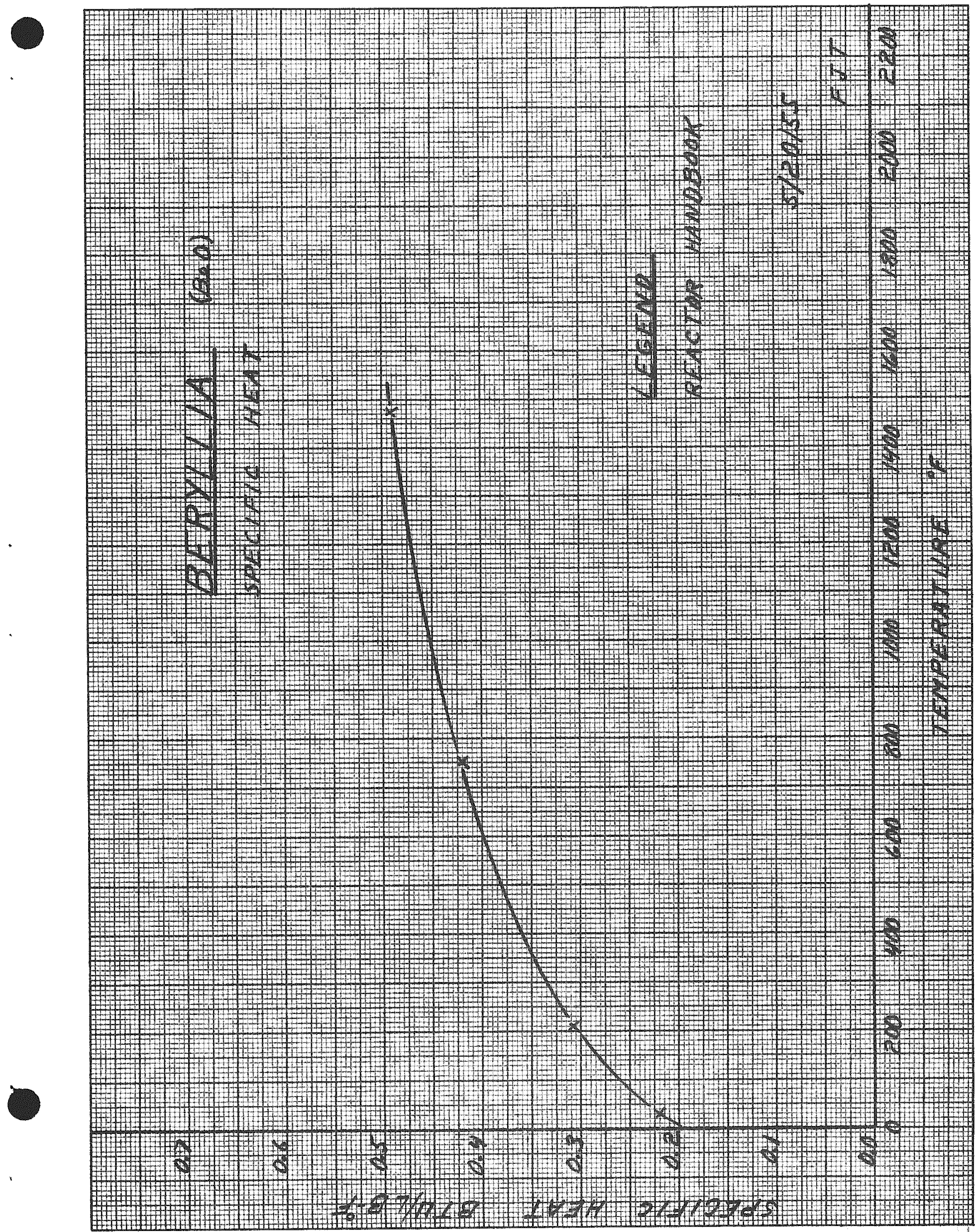




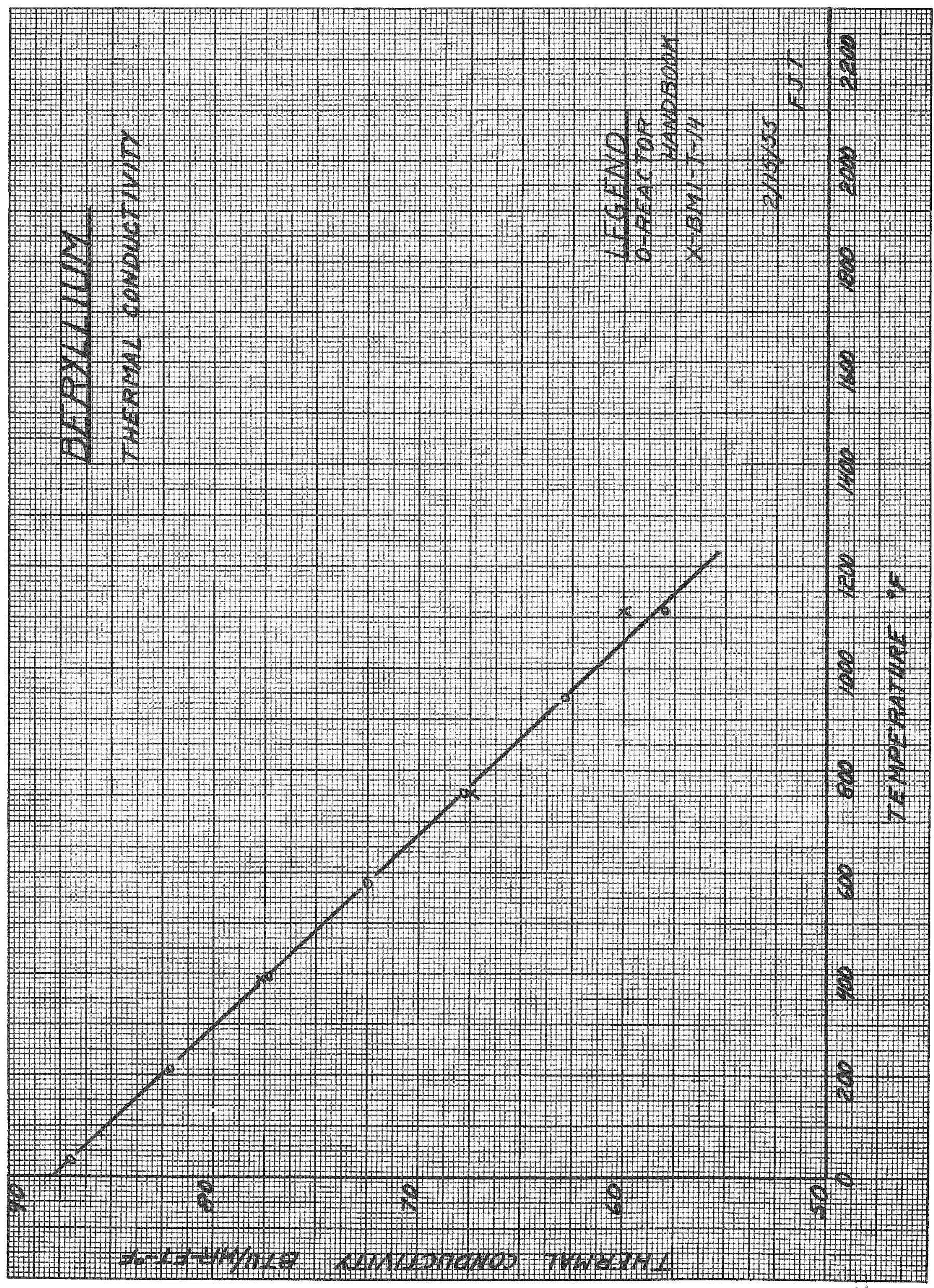




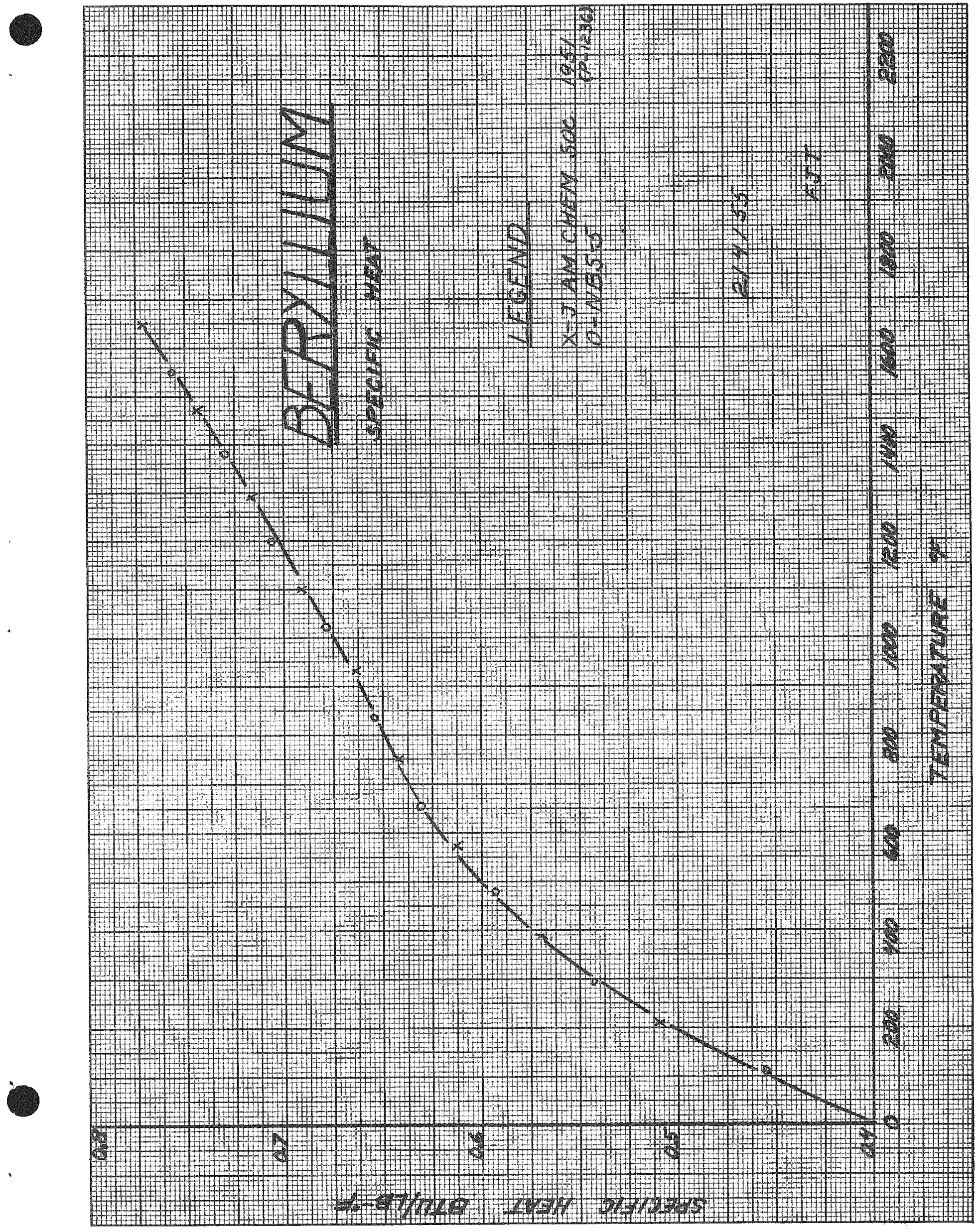




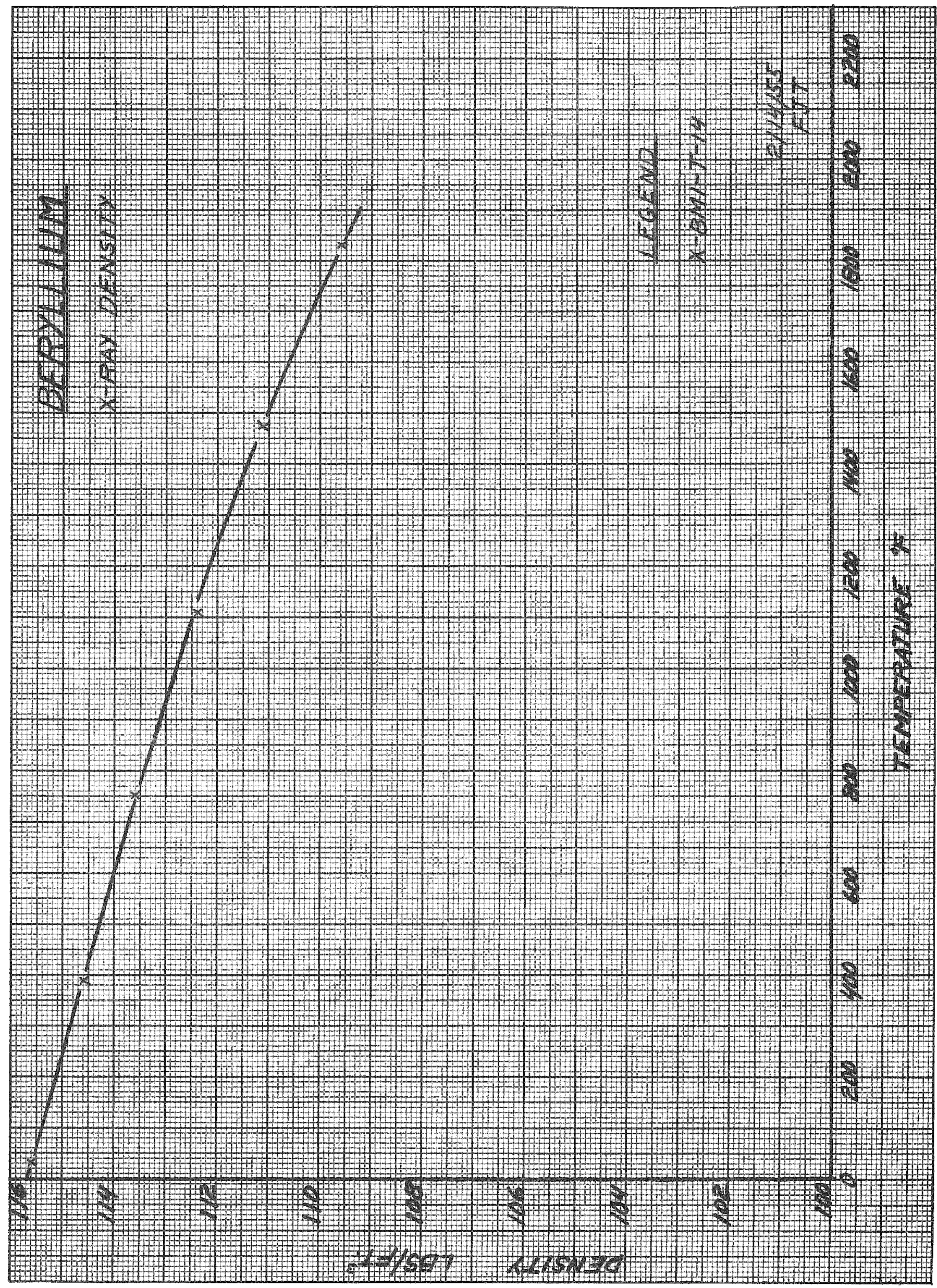




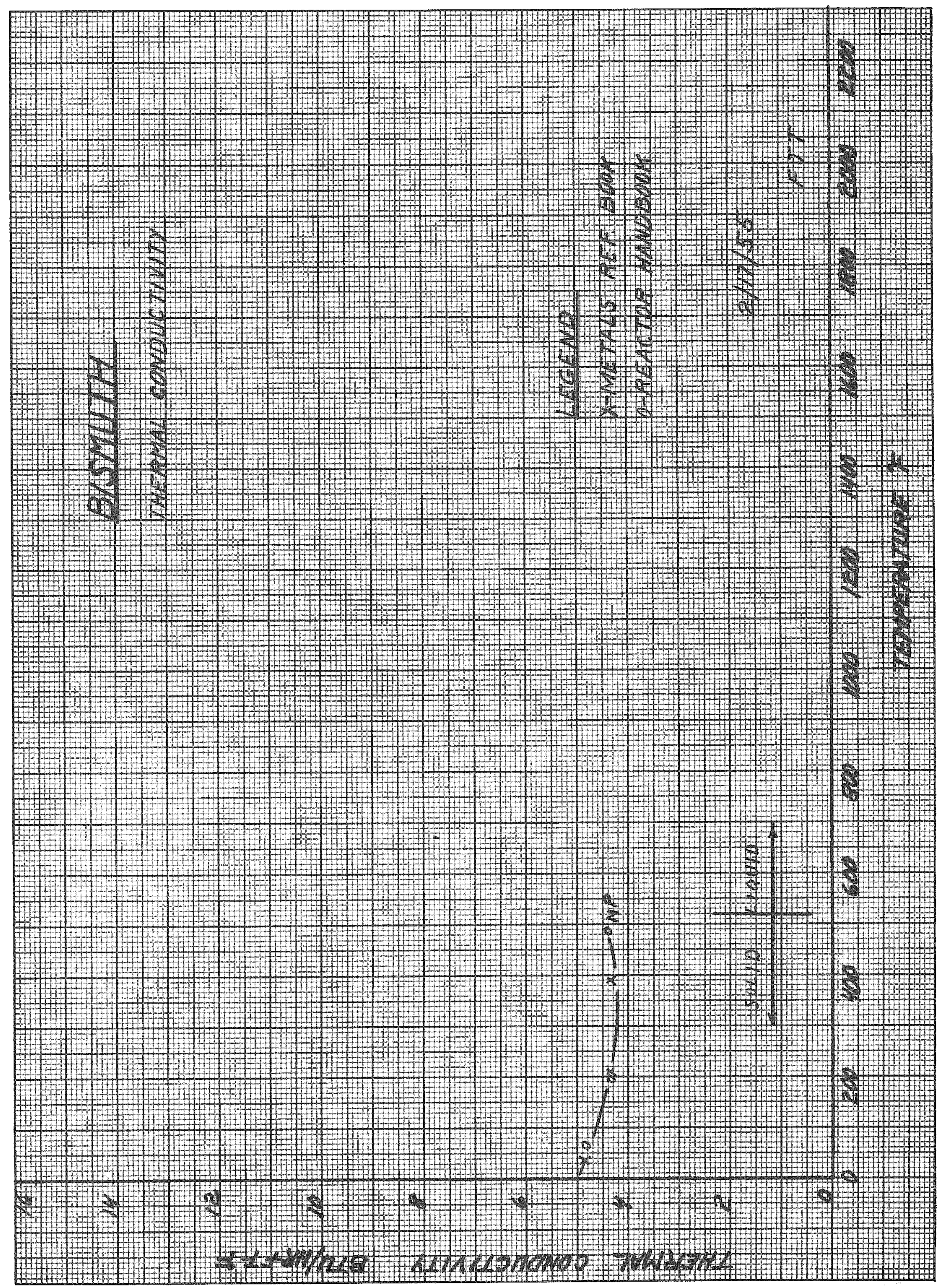




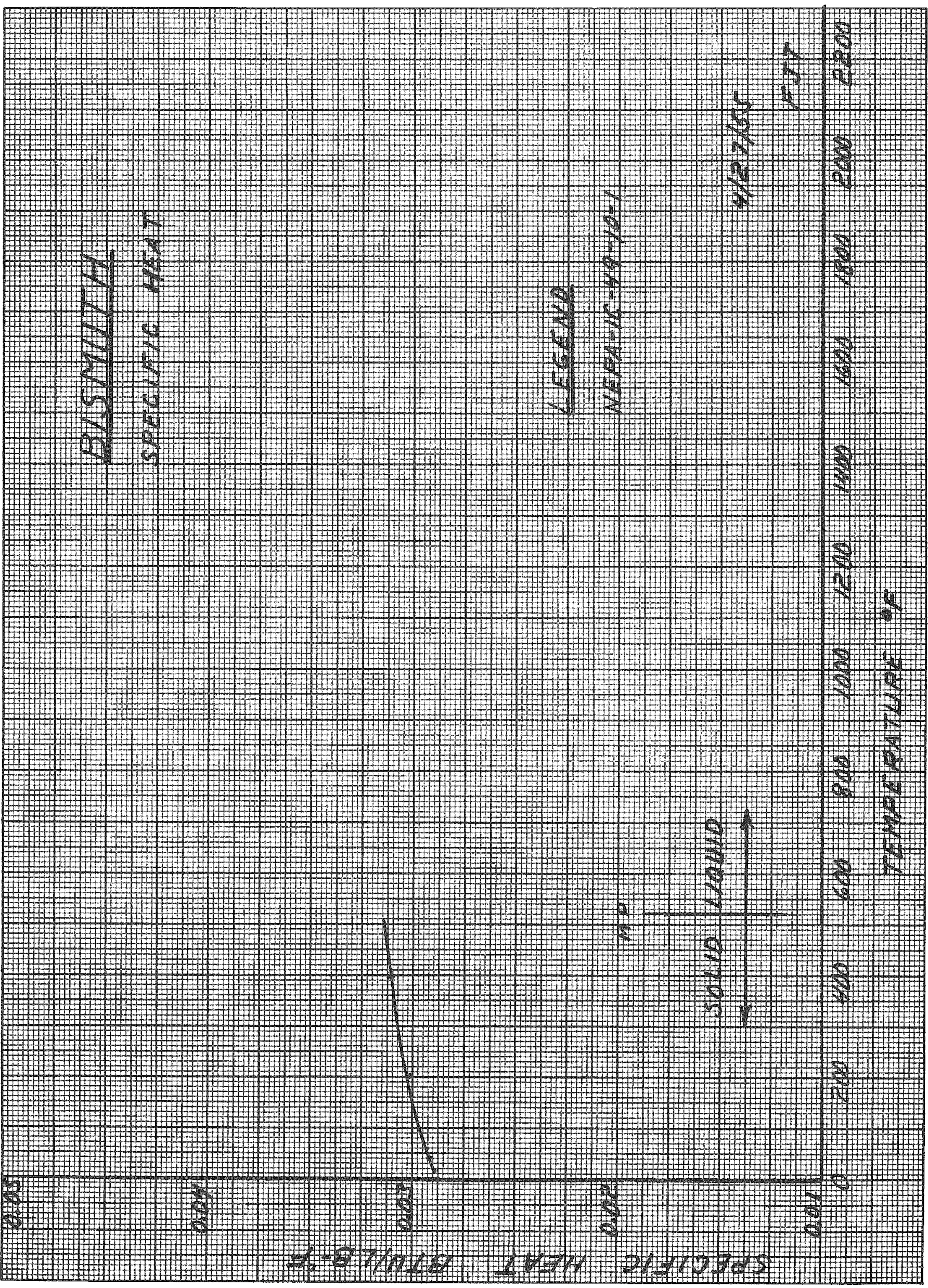




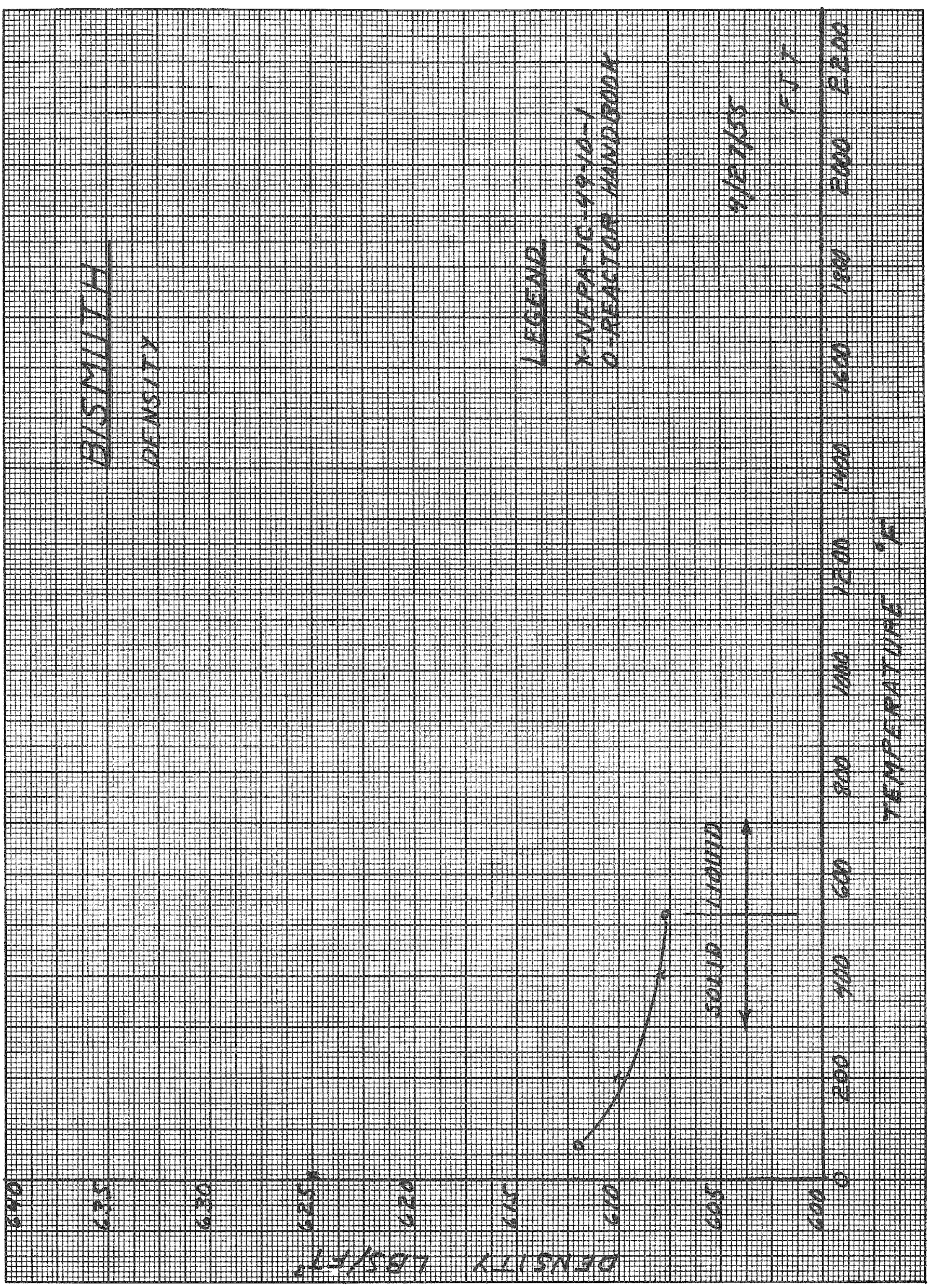




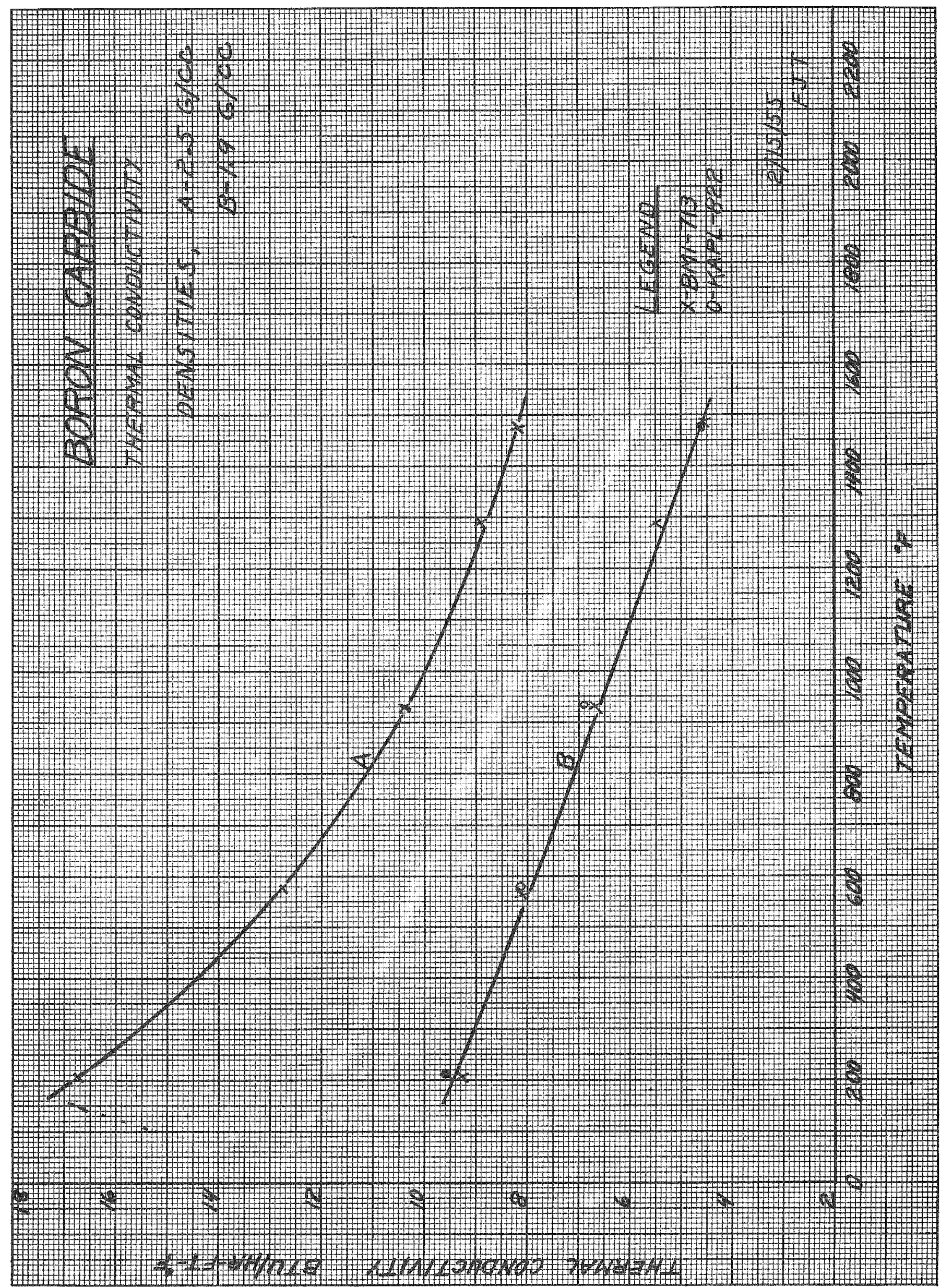




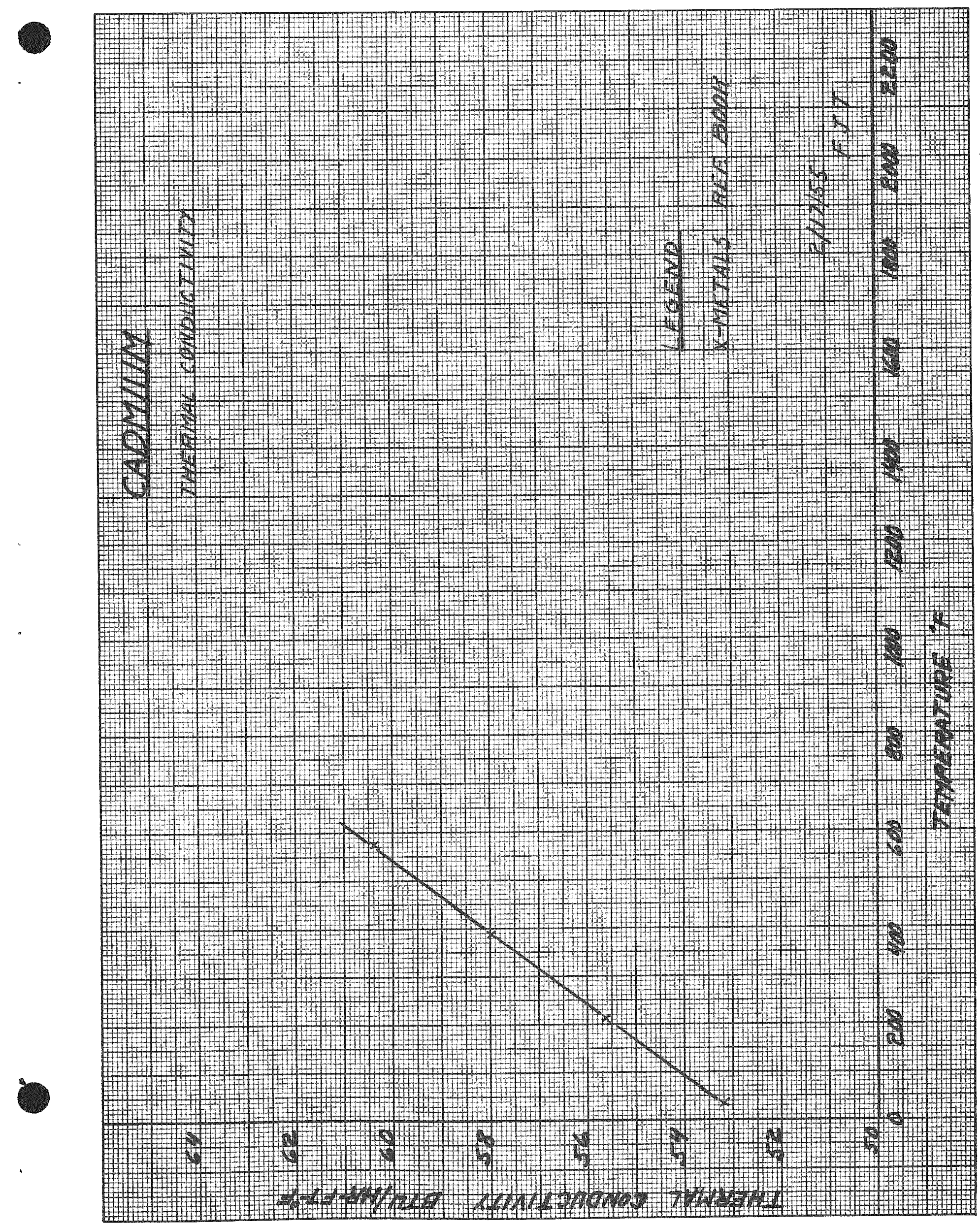




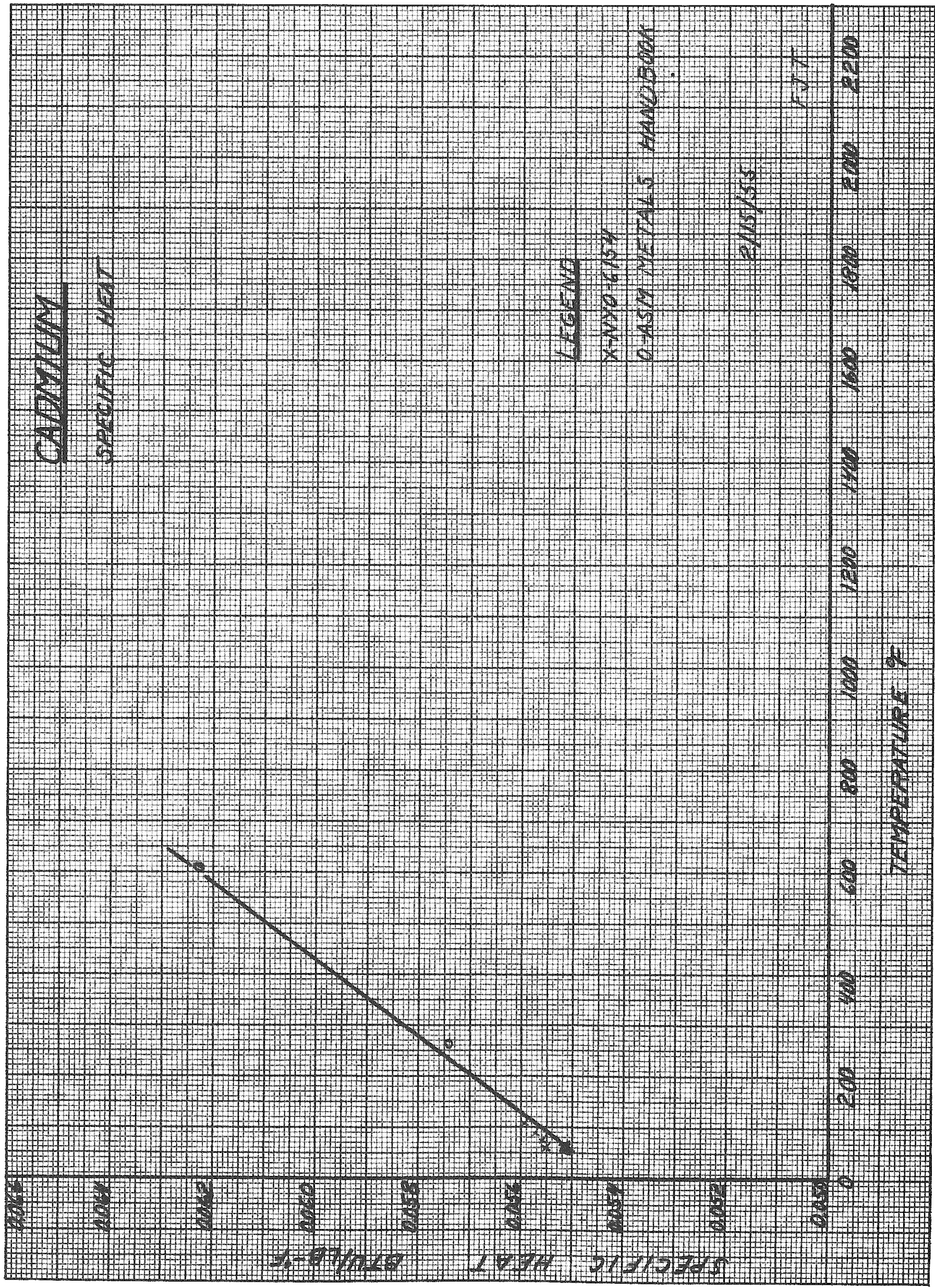




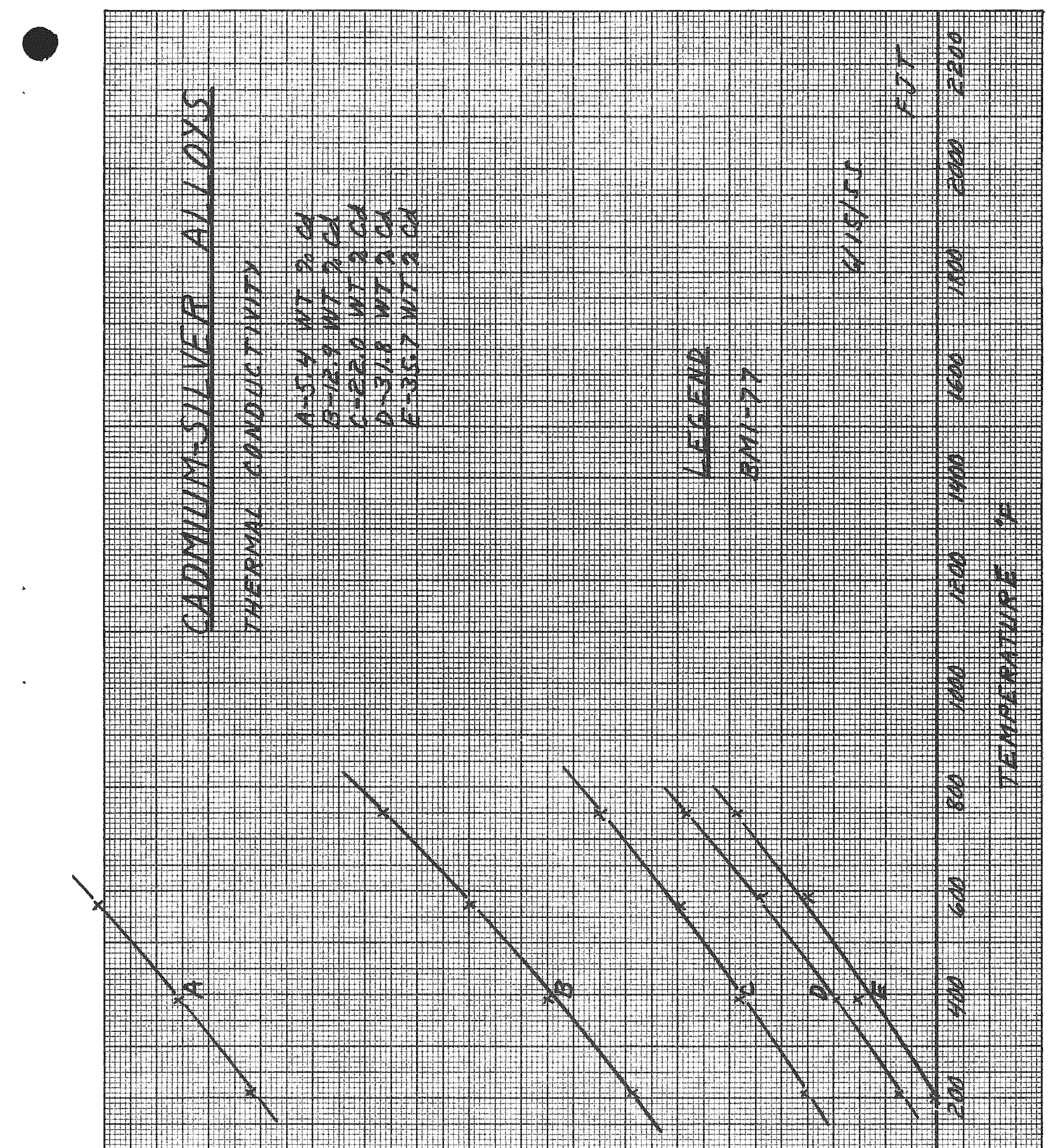

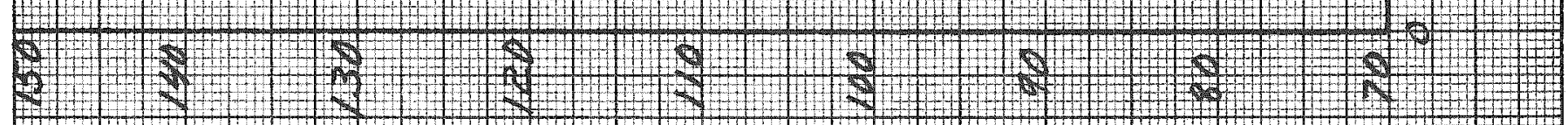

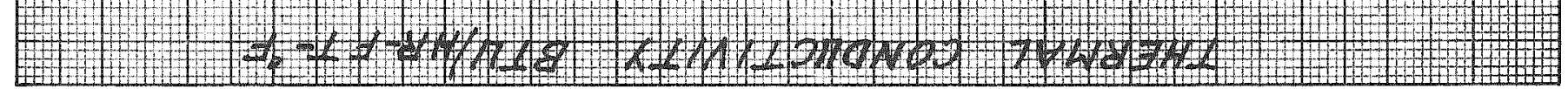




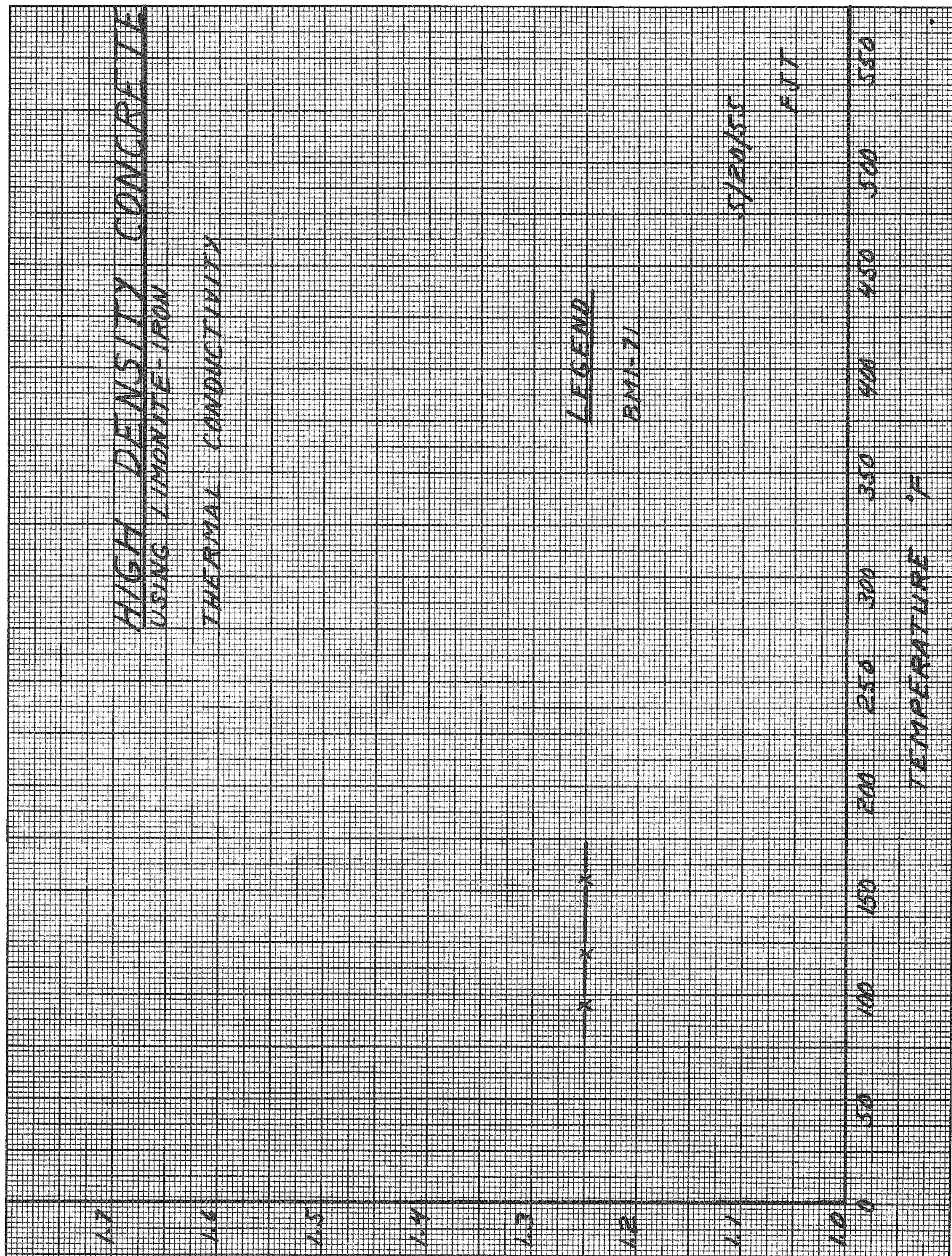




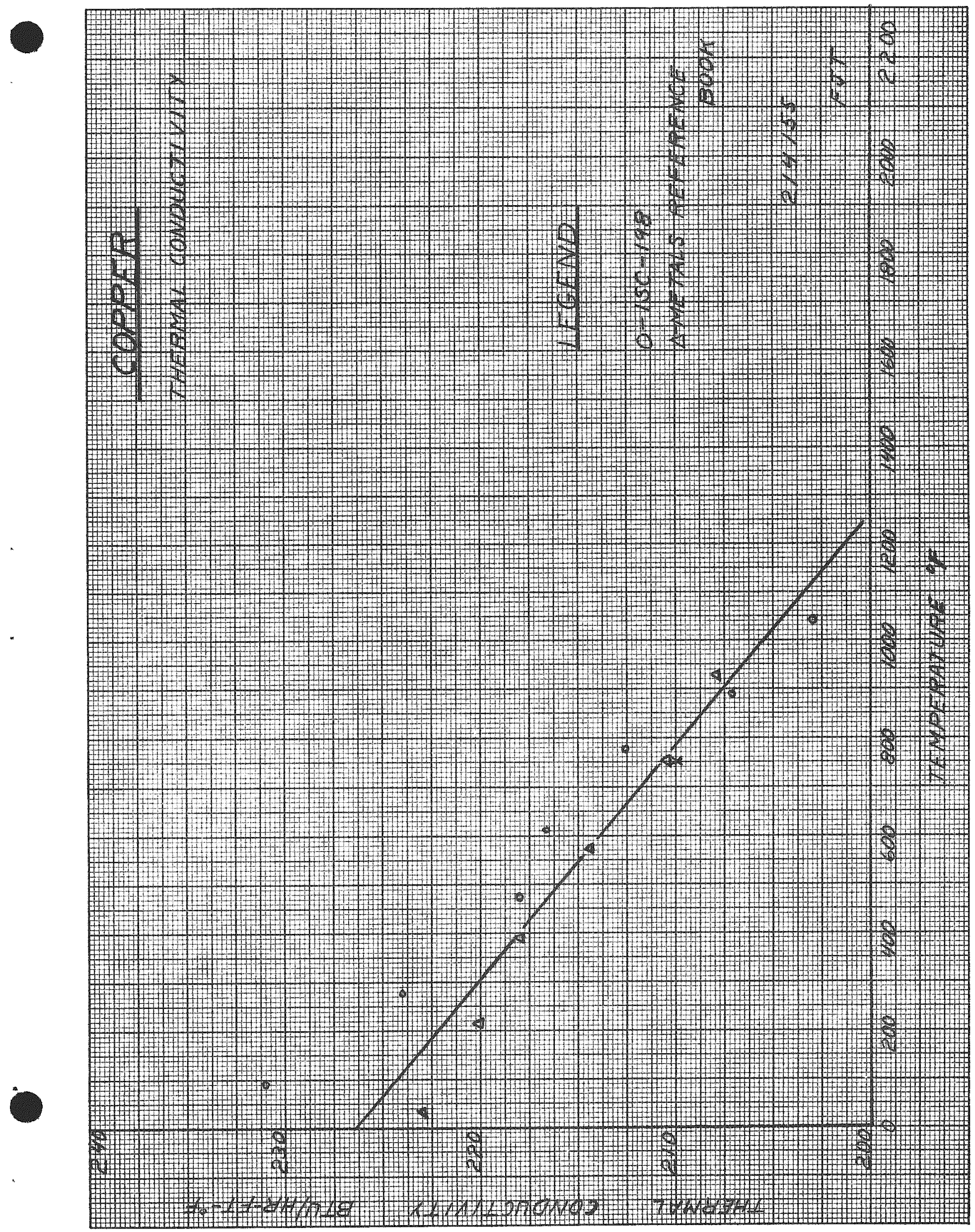




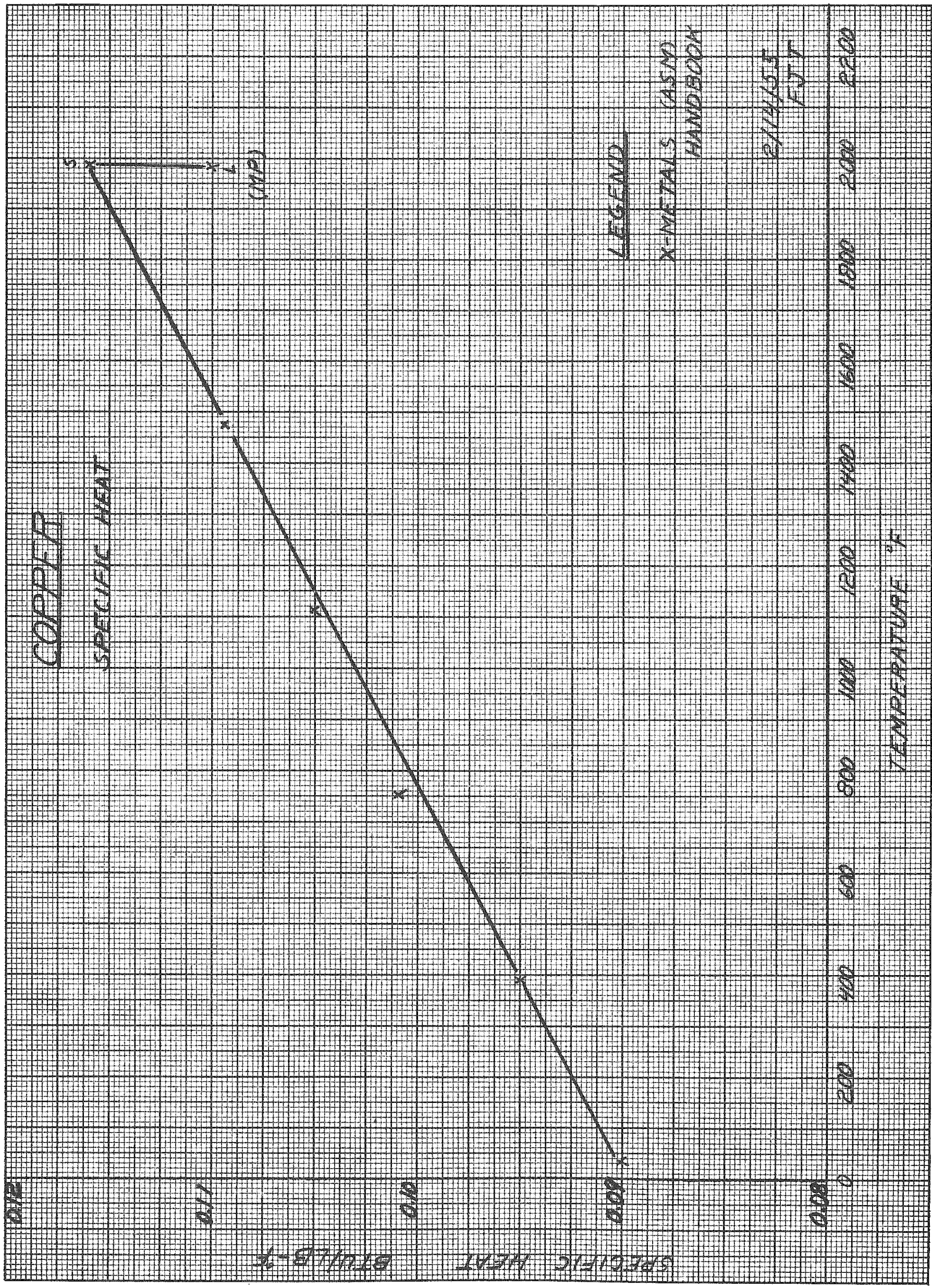




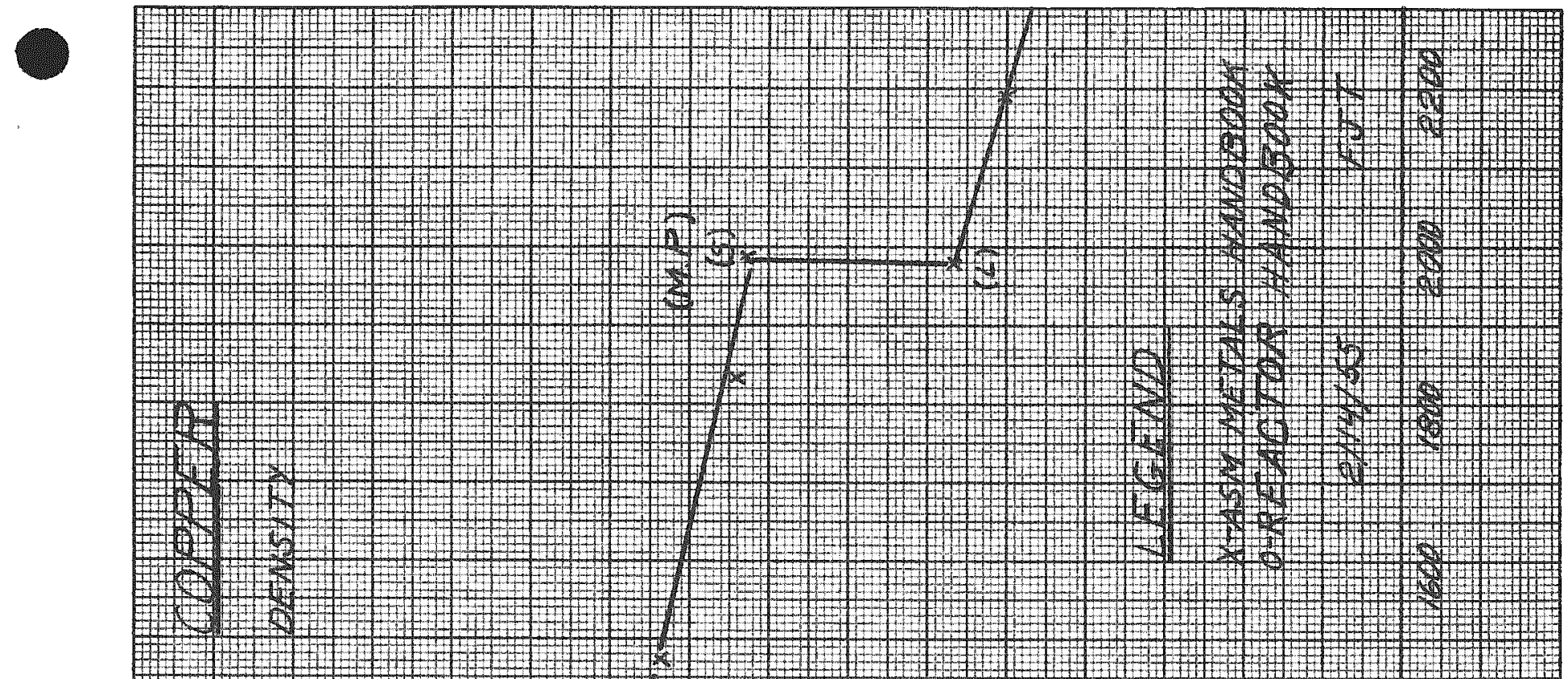

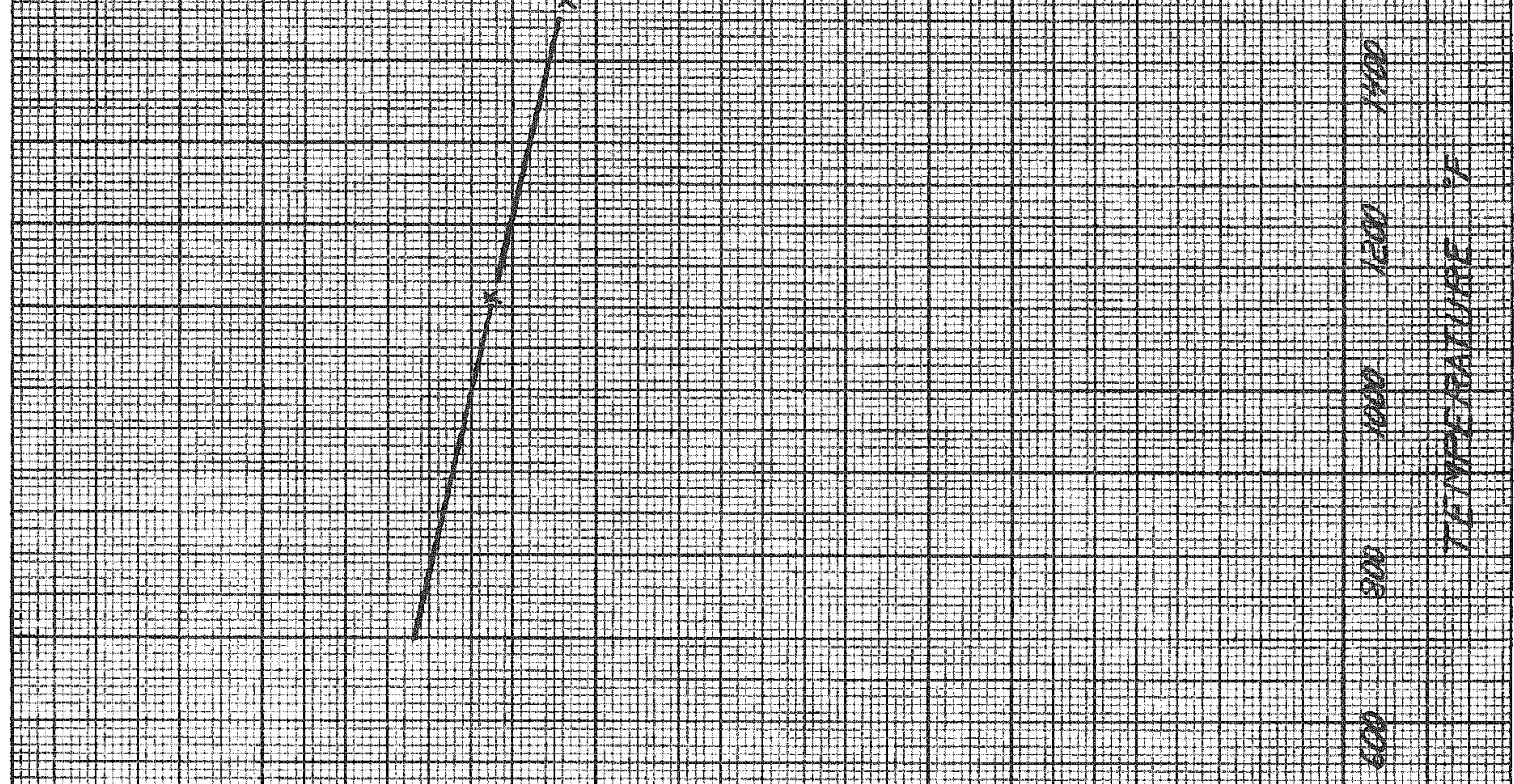

1.7.

\begin{tabular}{lll}
\hline 1 \\
\hline
\end{tabular}

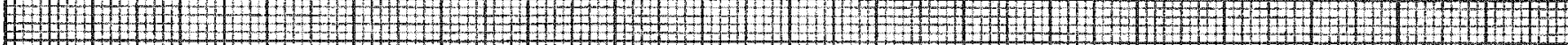
\begin{tabular}{lll}
\hline \\
\hline
\end{tabular}

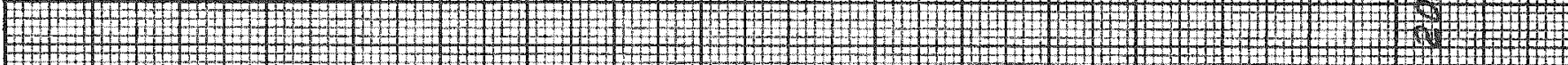

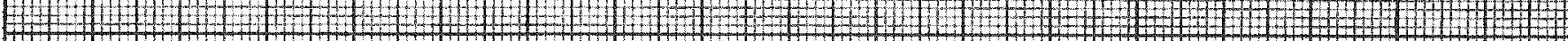

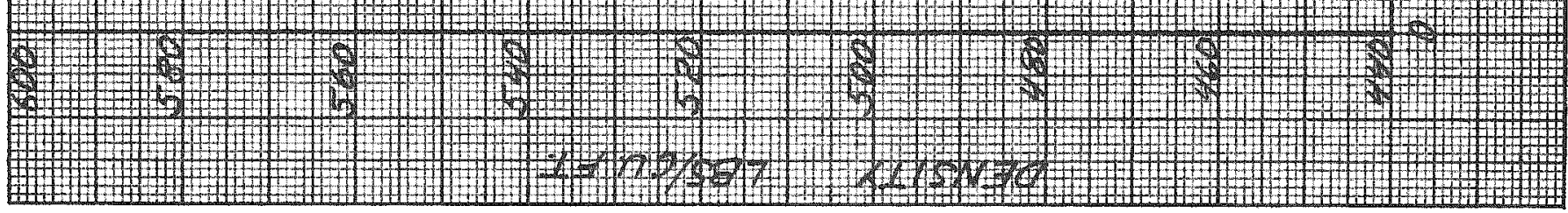




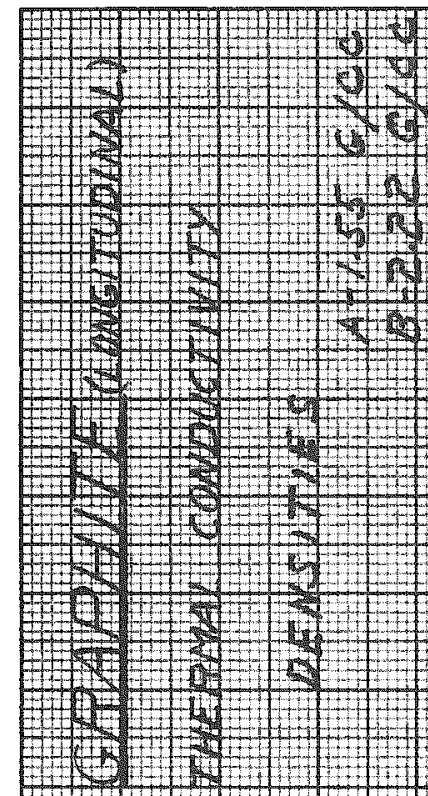

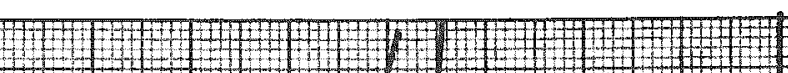

$+4+1+1+1$
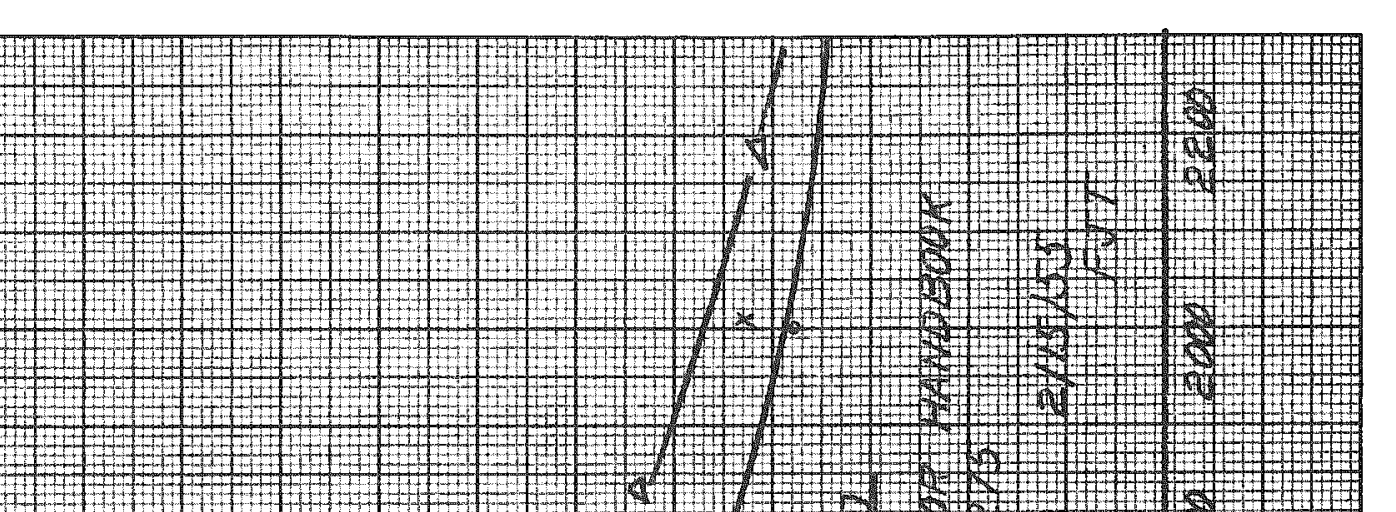

\begin{tabular}{l|l|l|}
\hline$+1+1+\infty$ & +
\end{tabular}

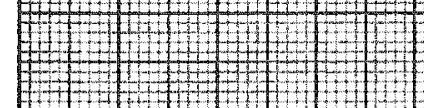

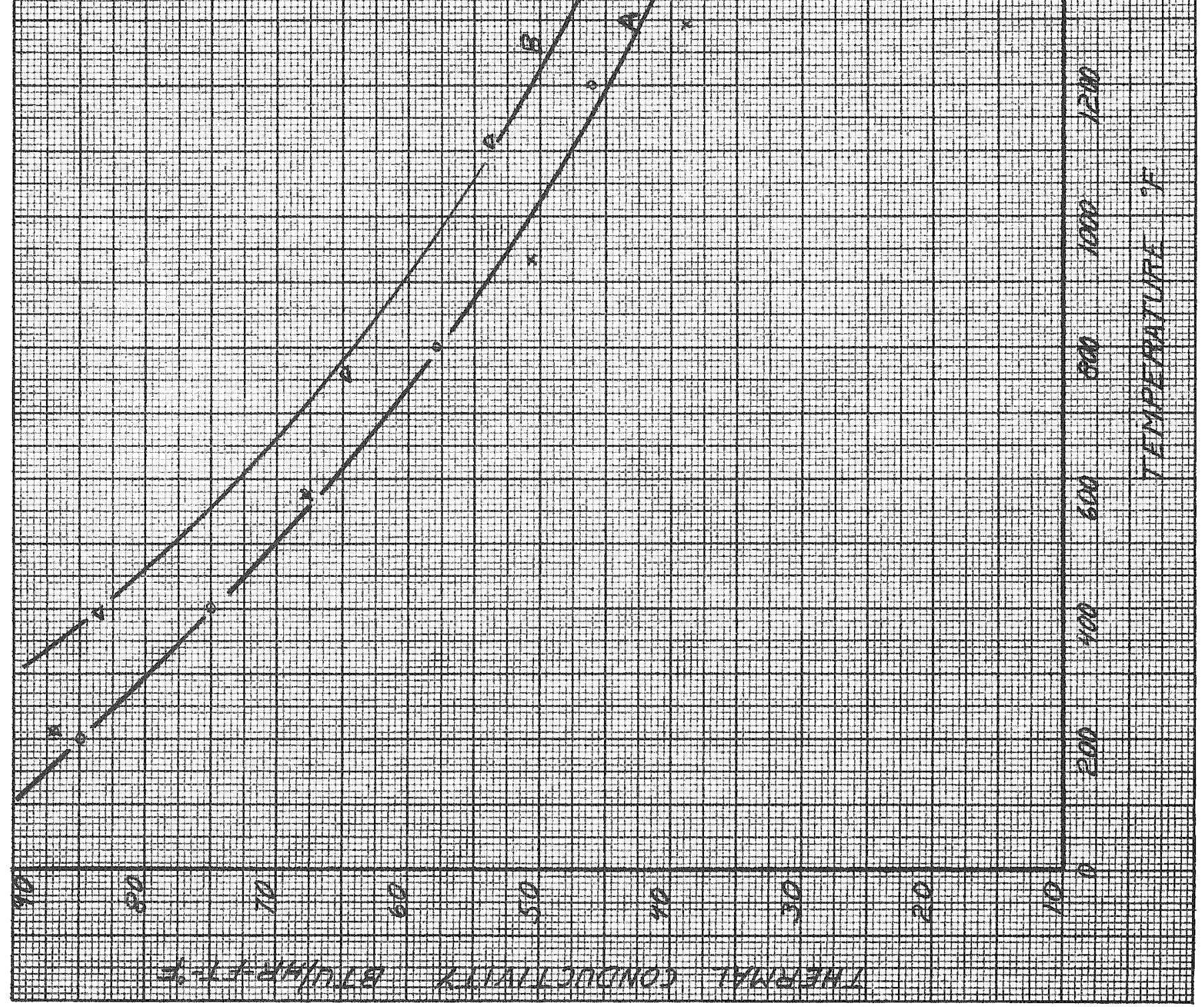




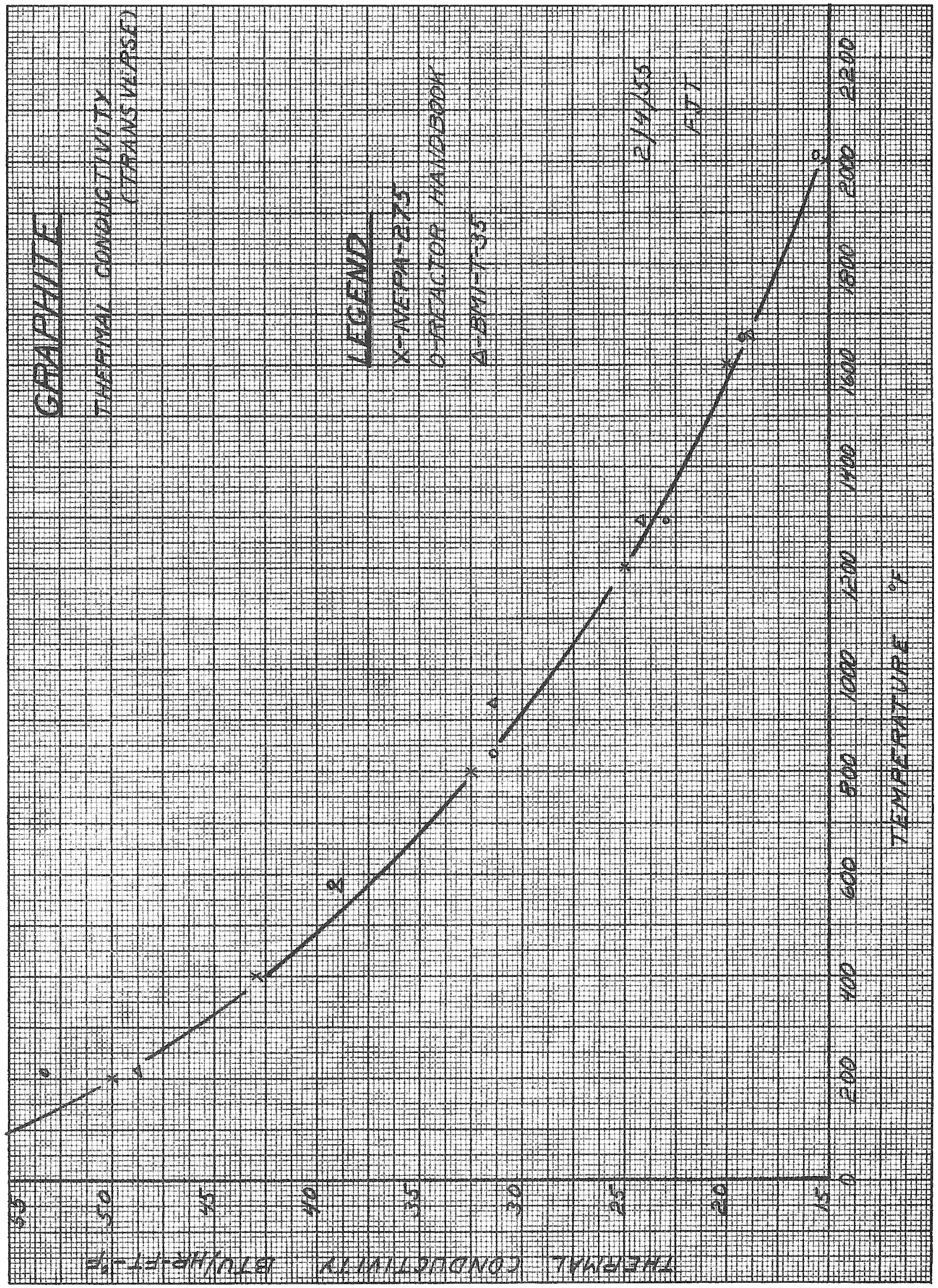




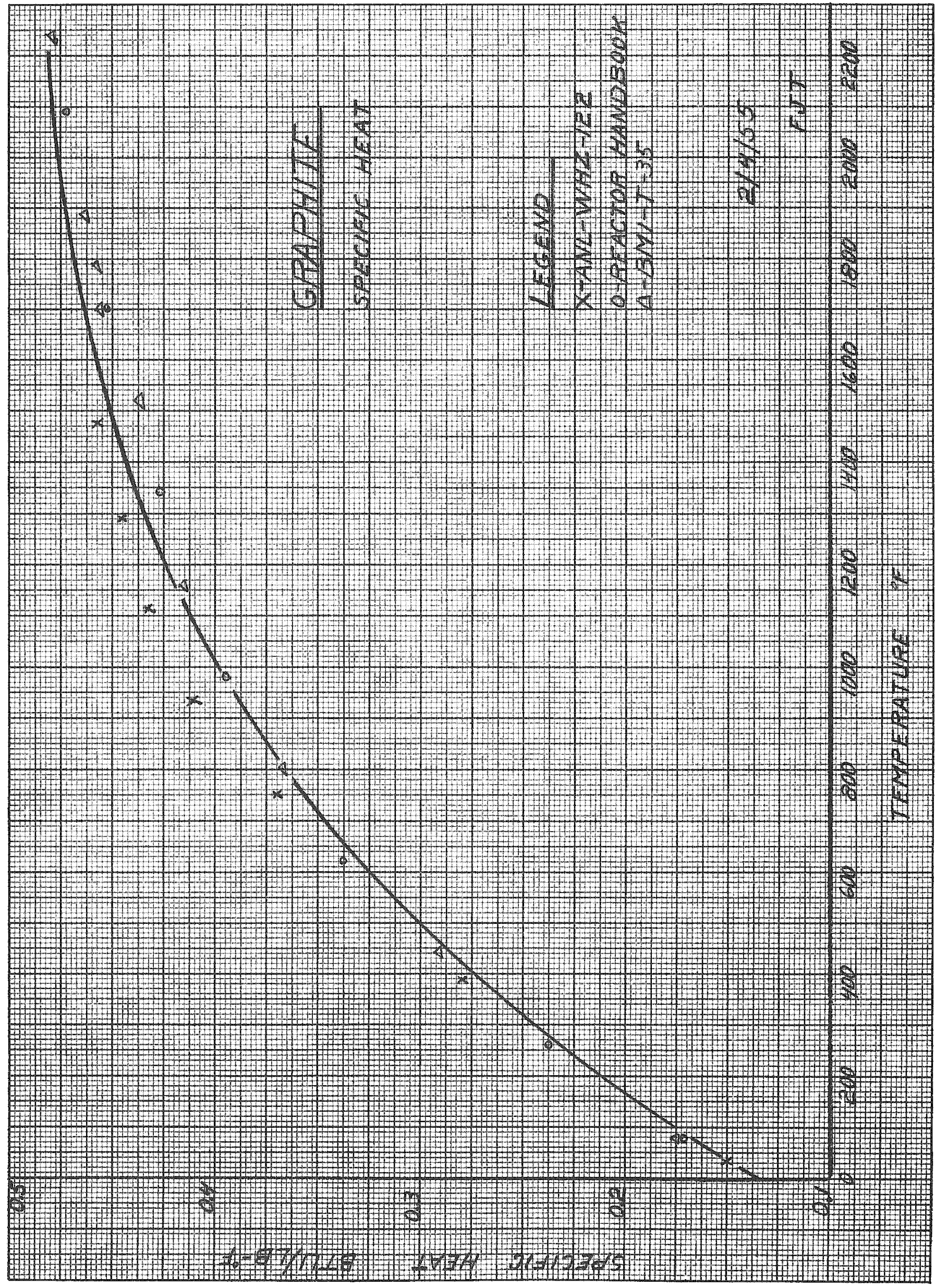




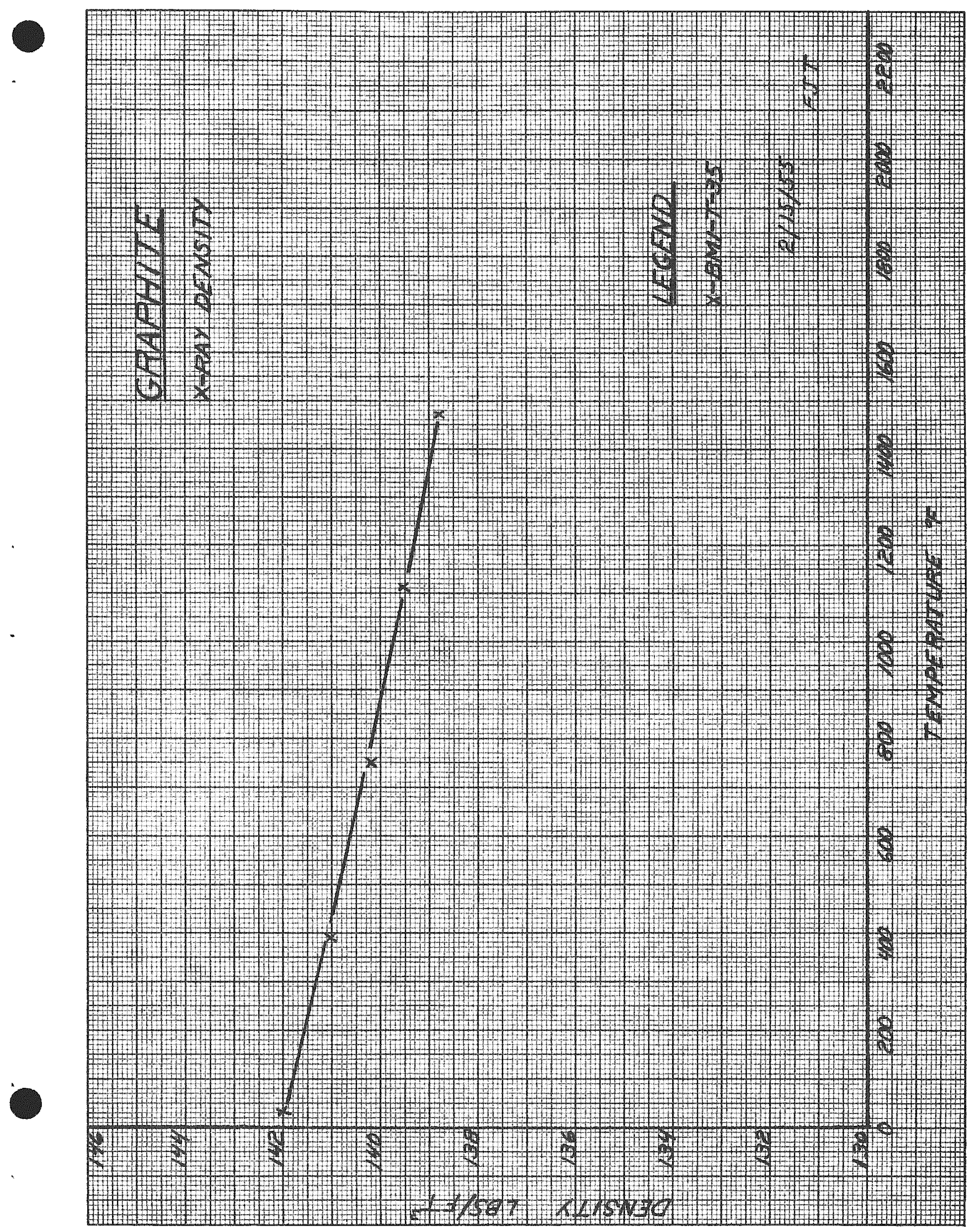




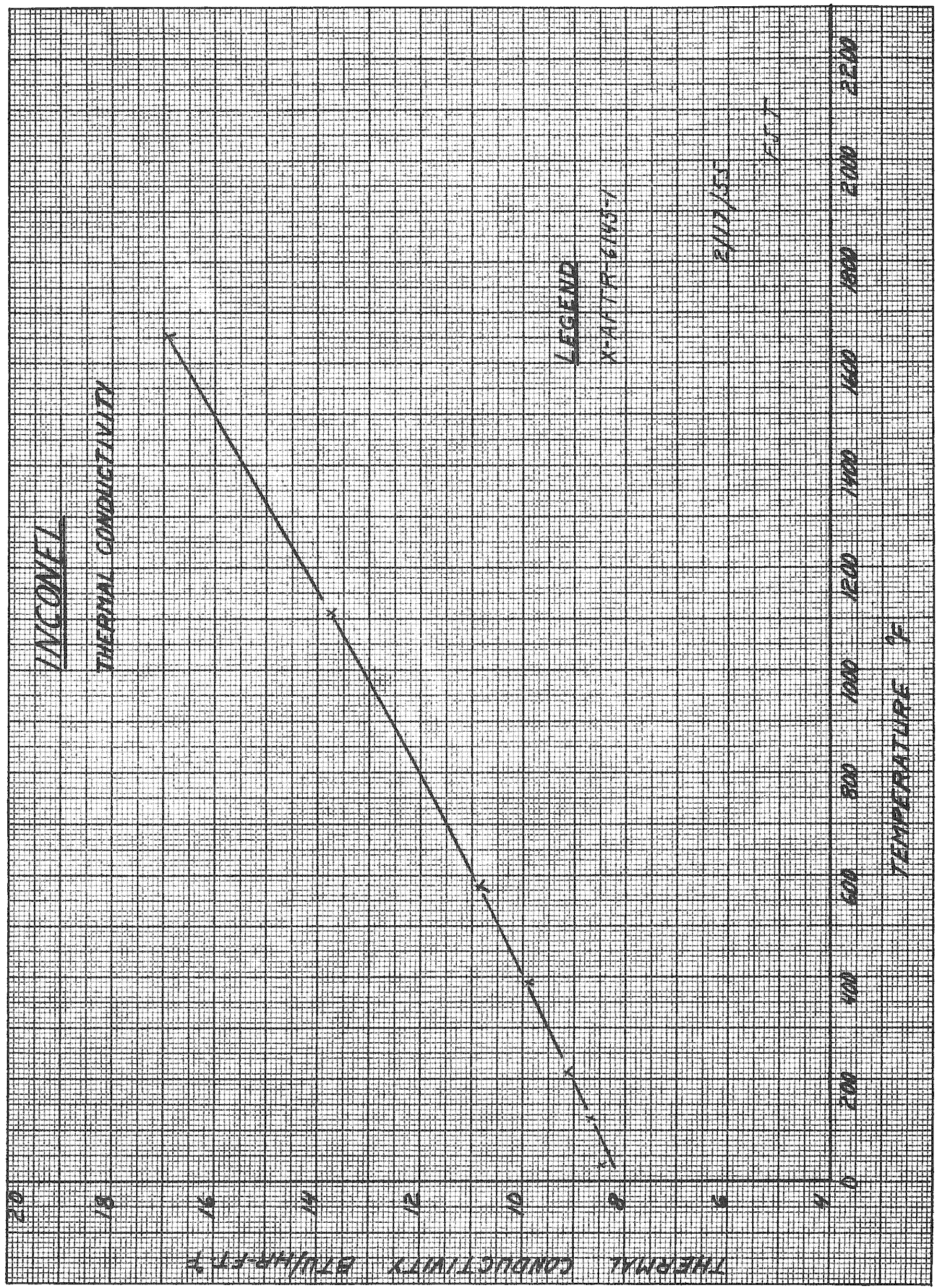




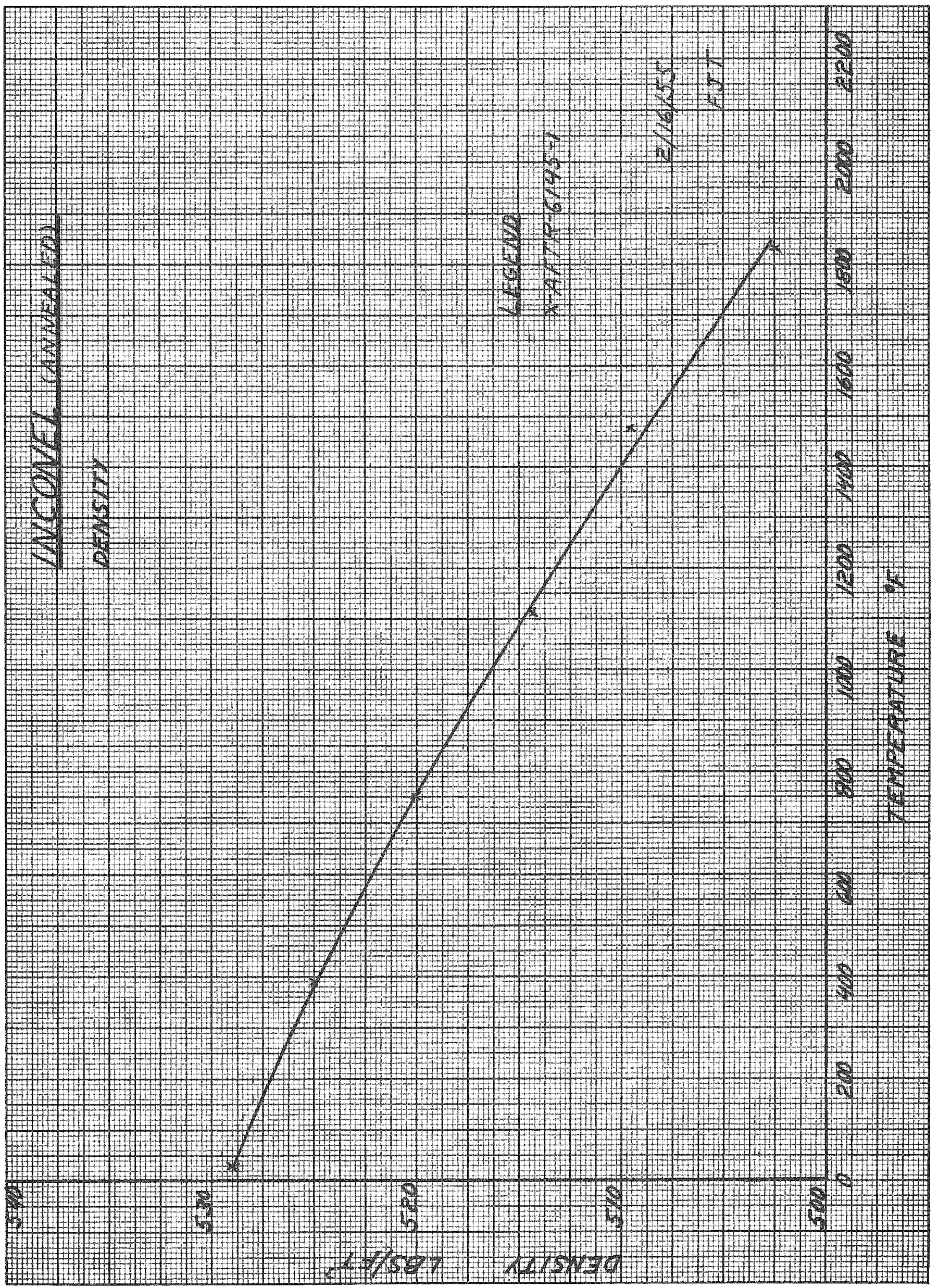




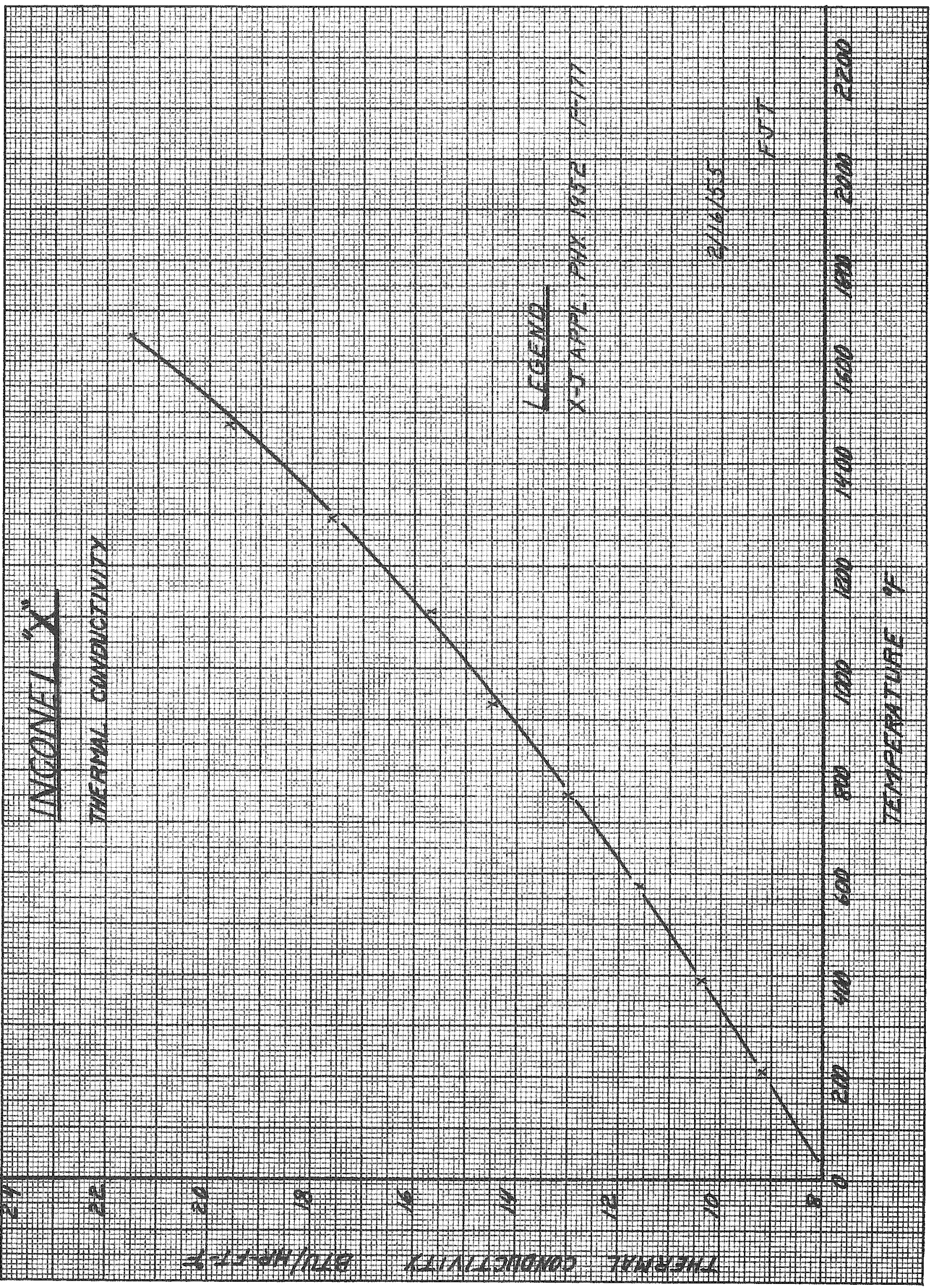




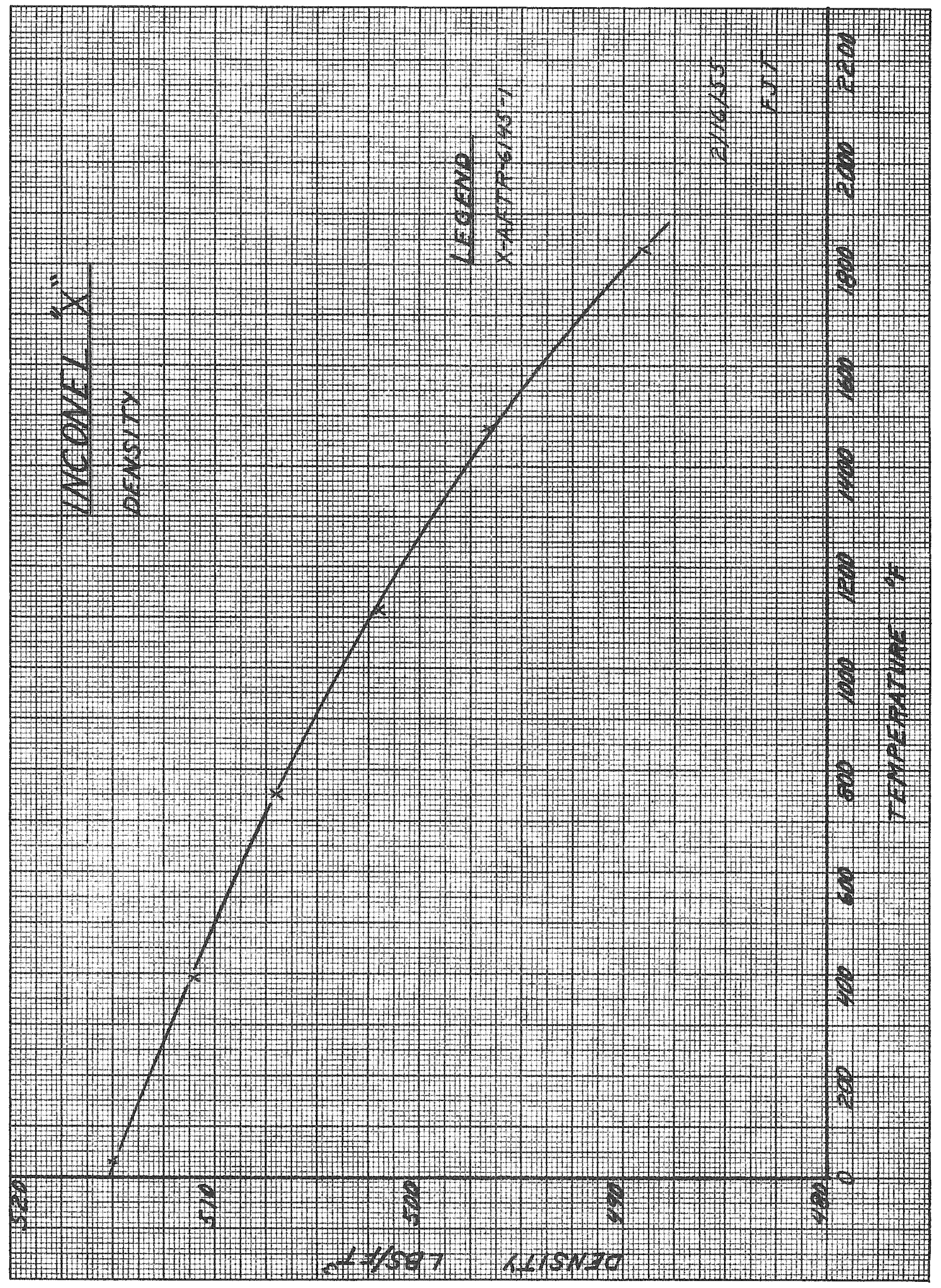




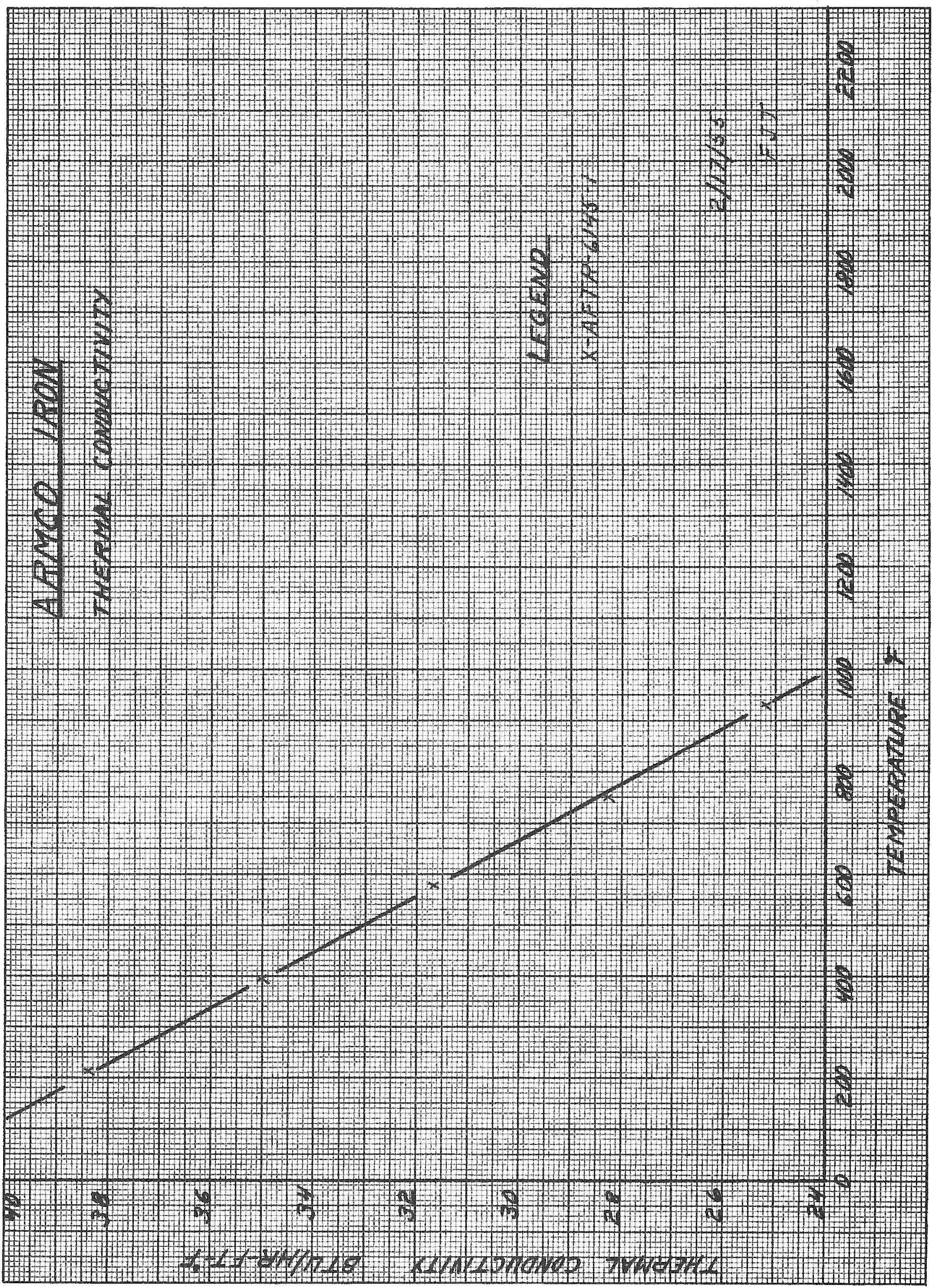




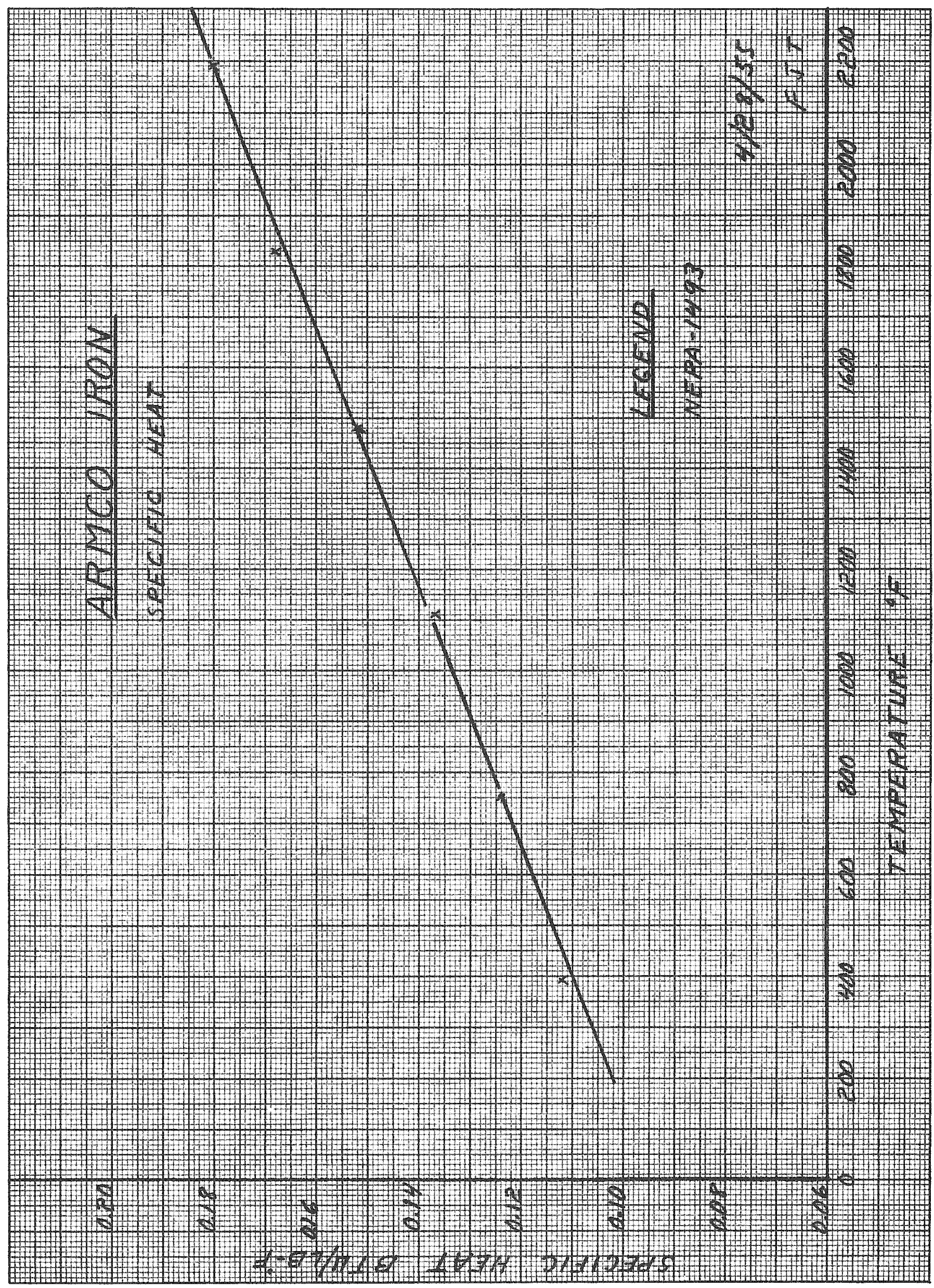




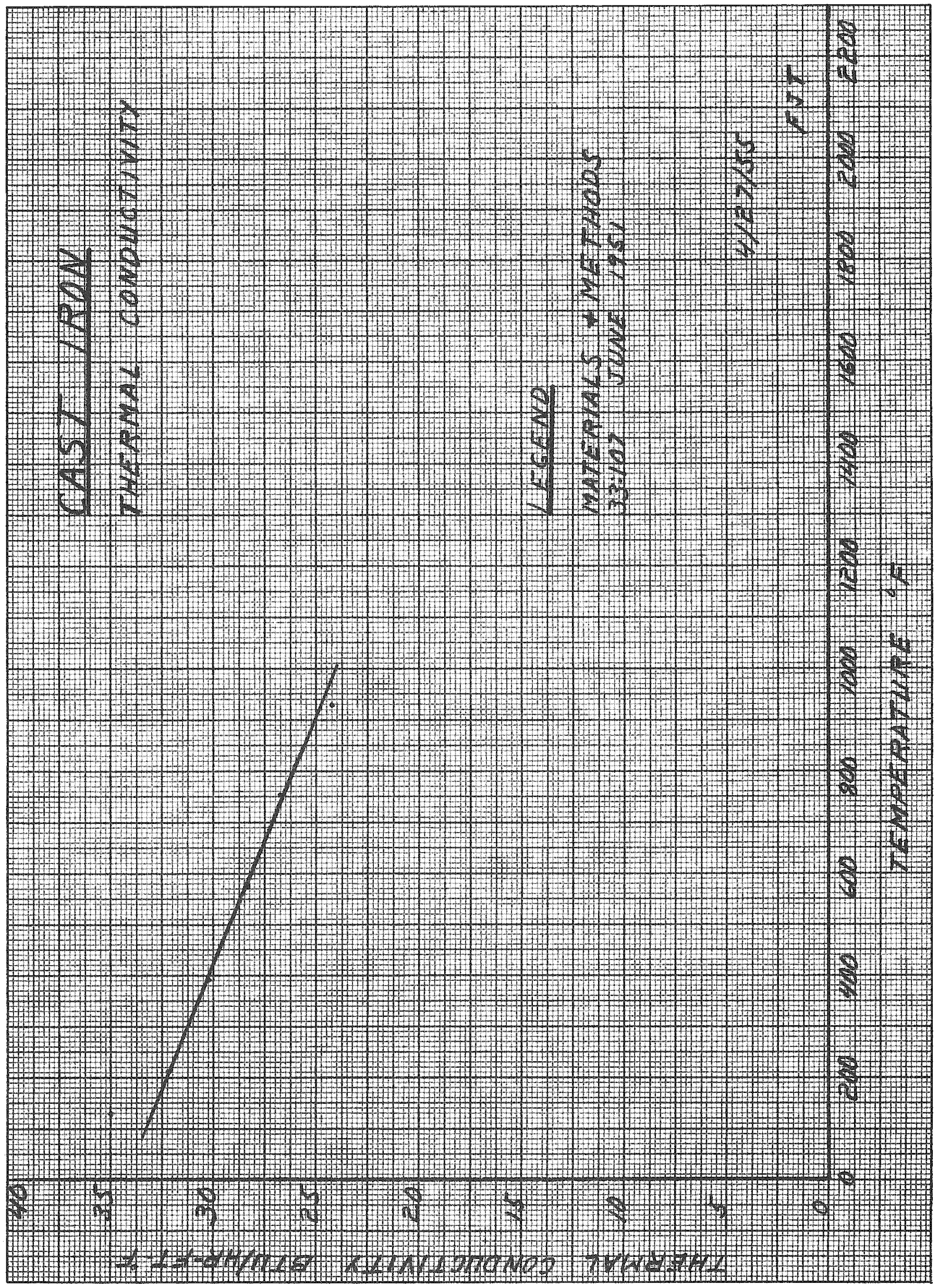




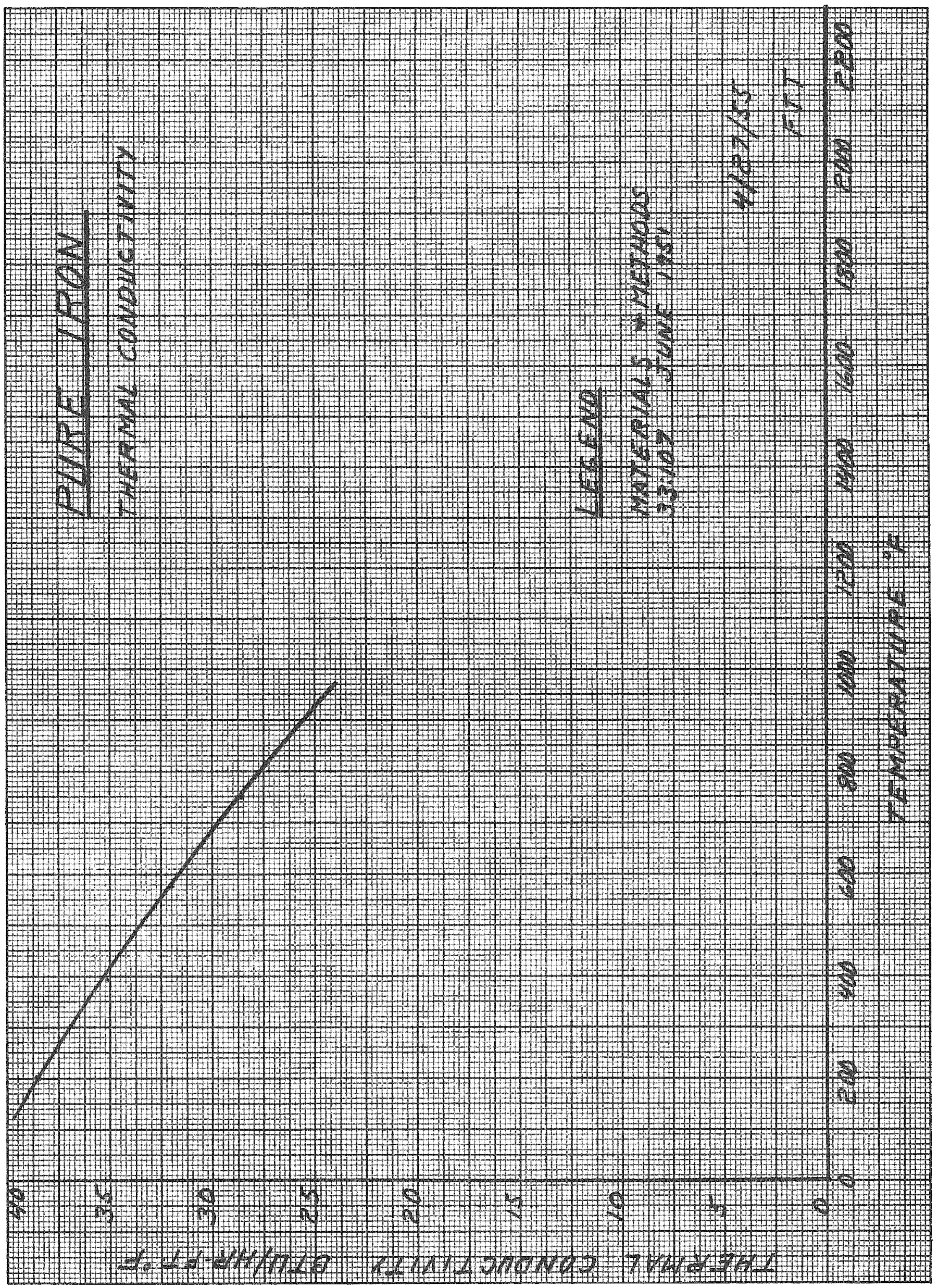




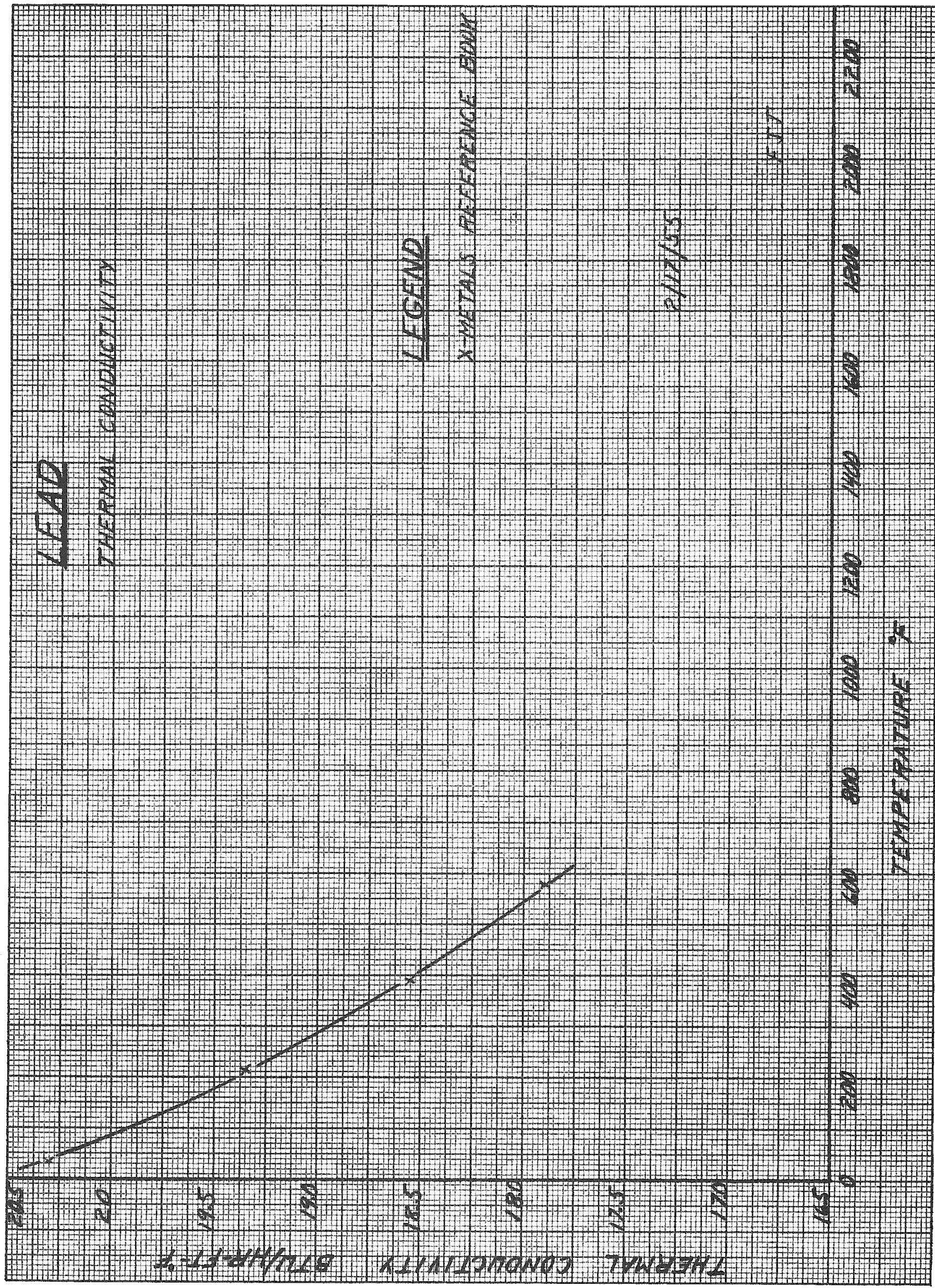




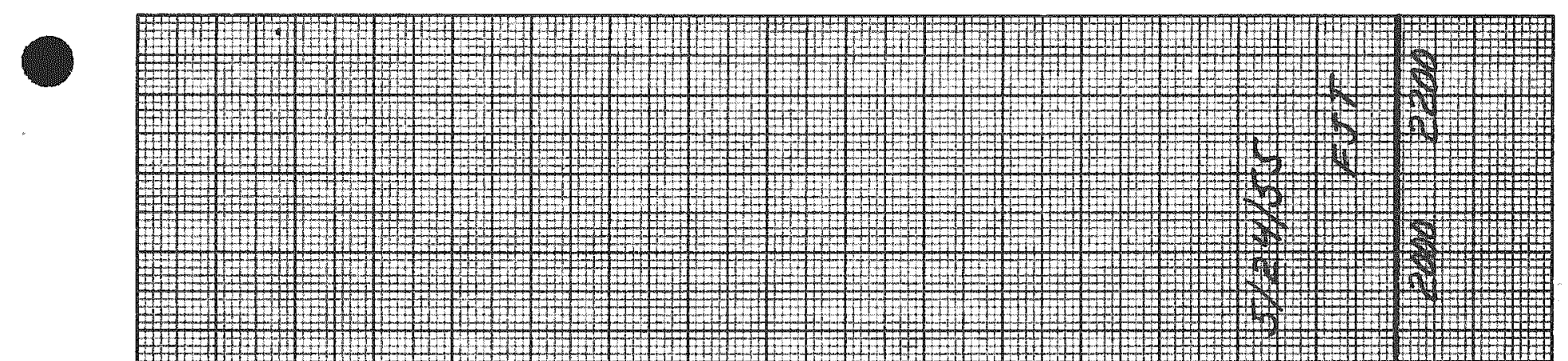
\begin{tabular}{llll}
\hline $1+1$ \\
\hline
\end{tabular}

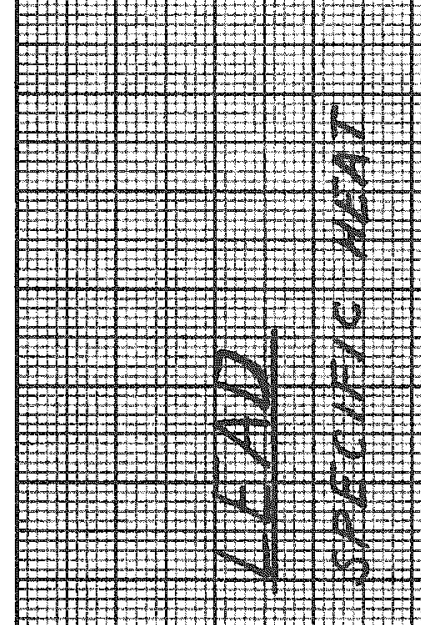

$+2+1+7+1+7+2$

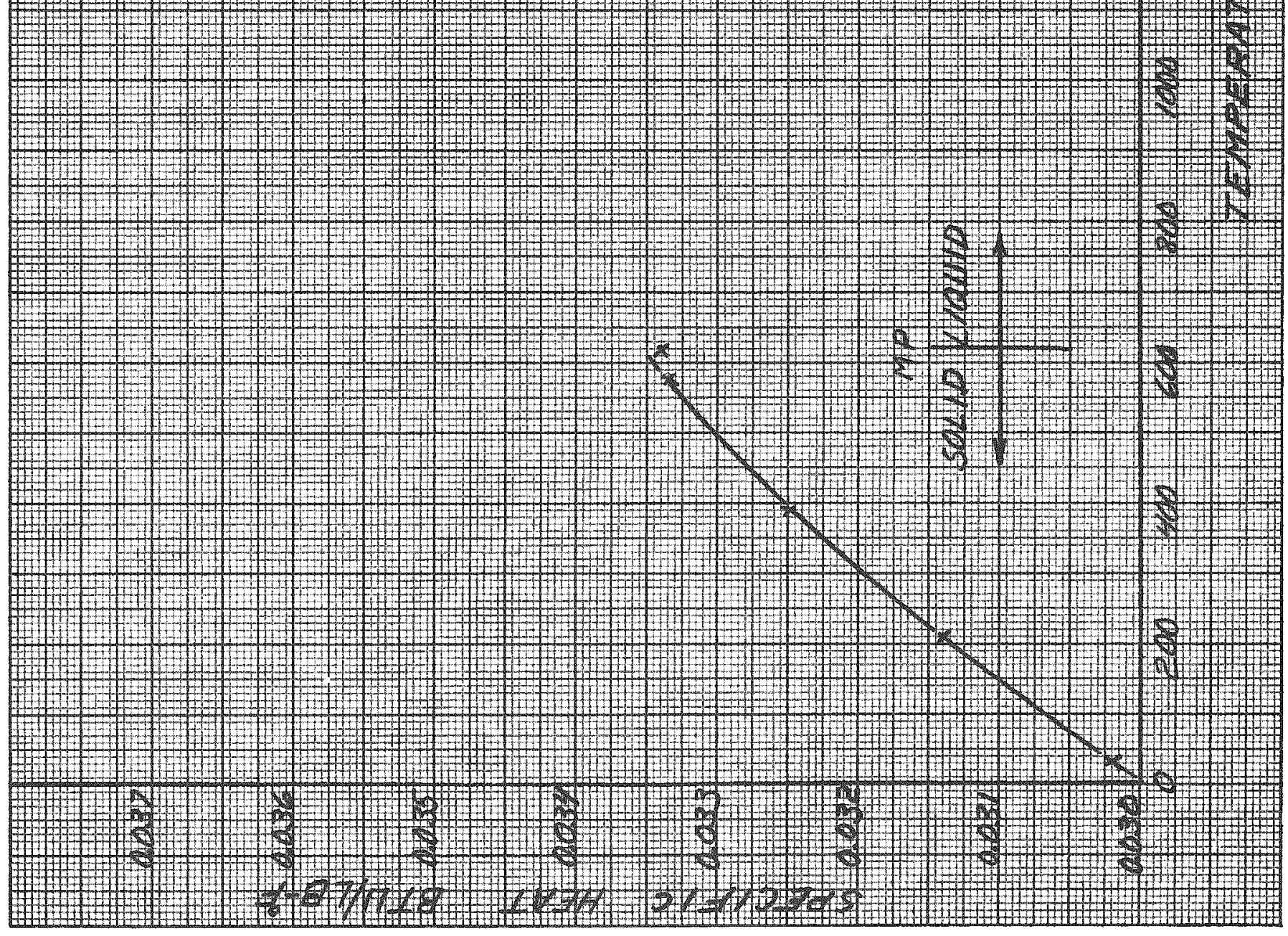




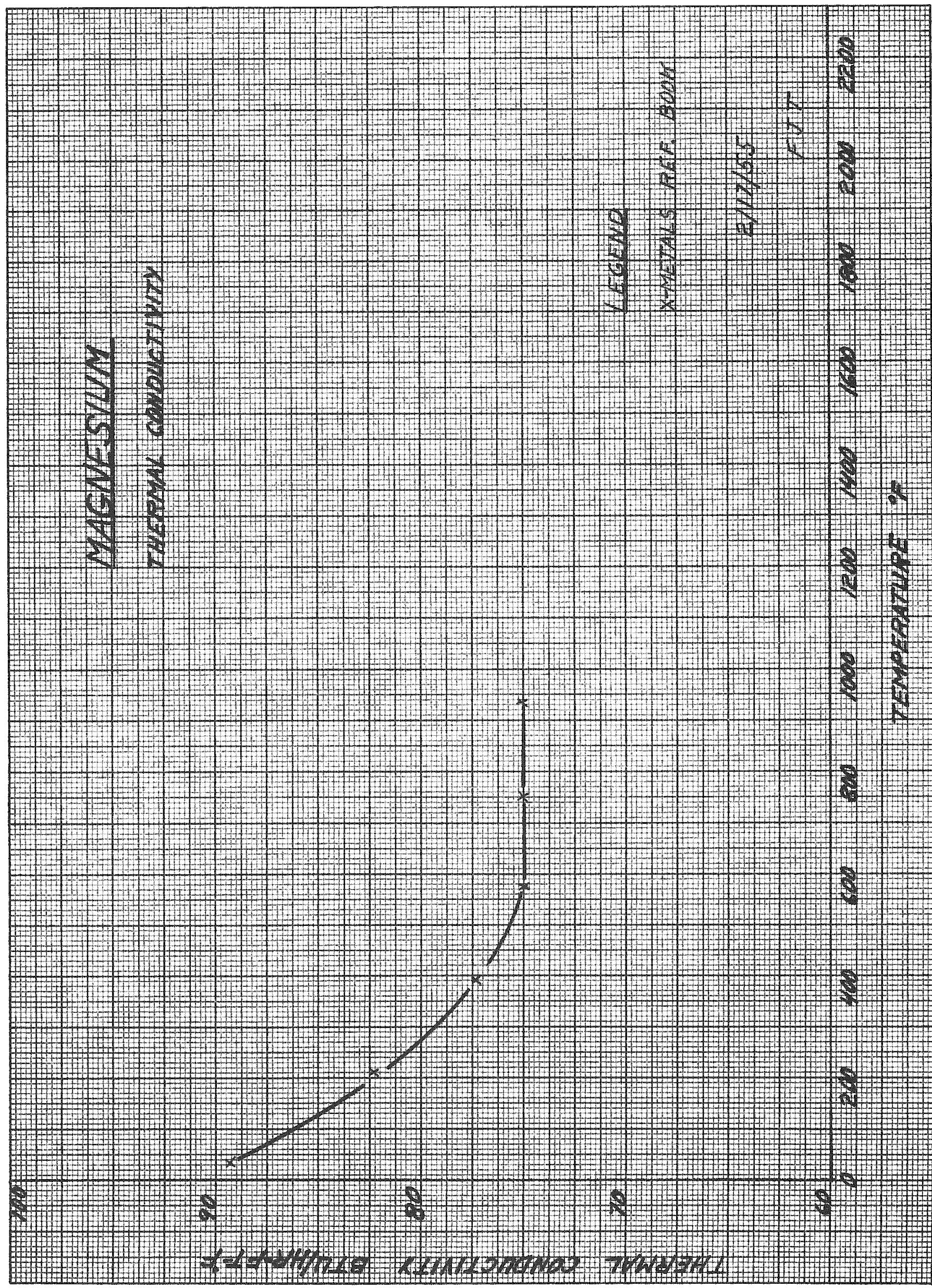




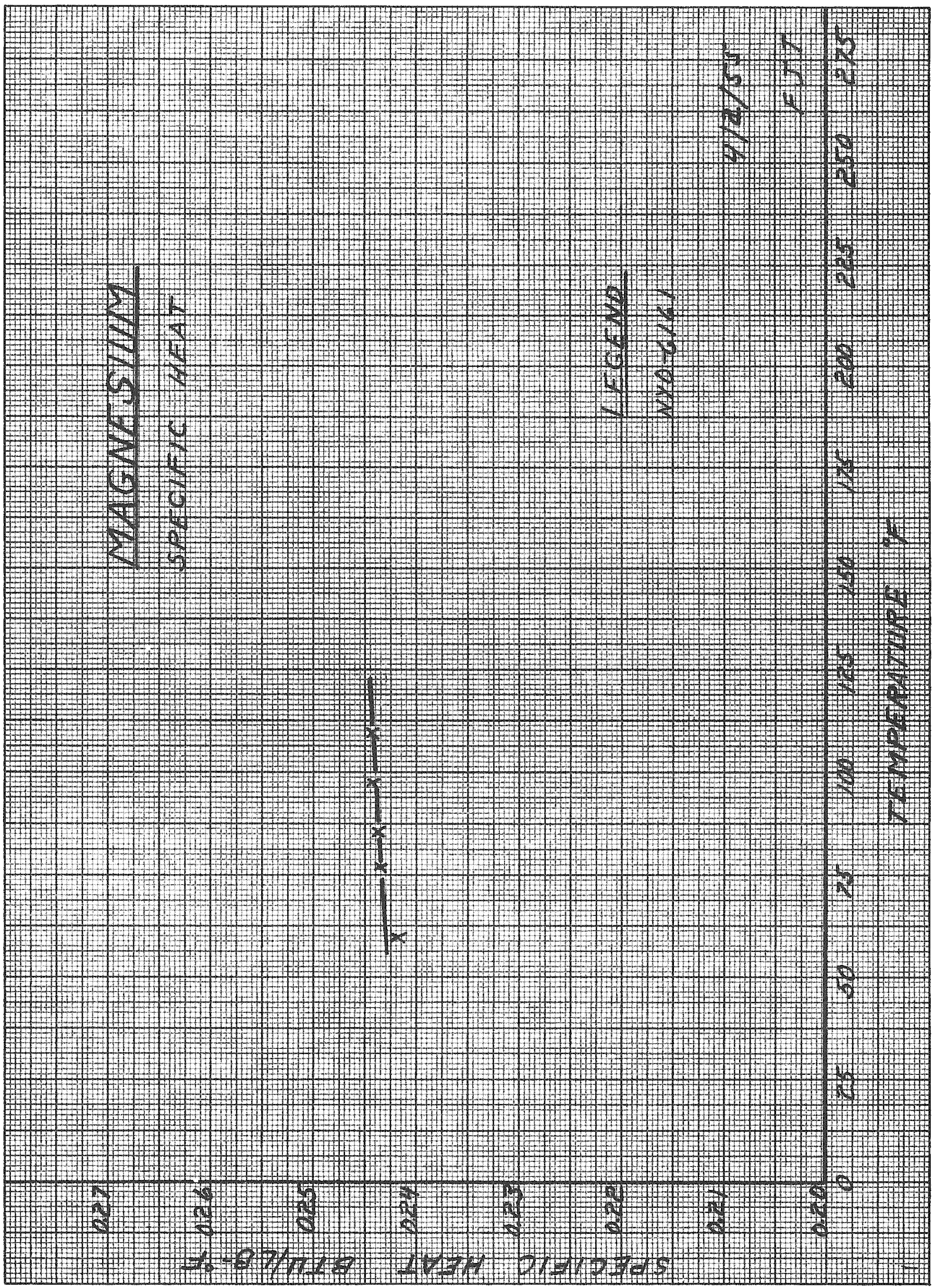




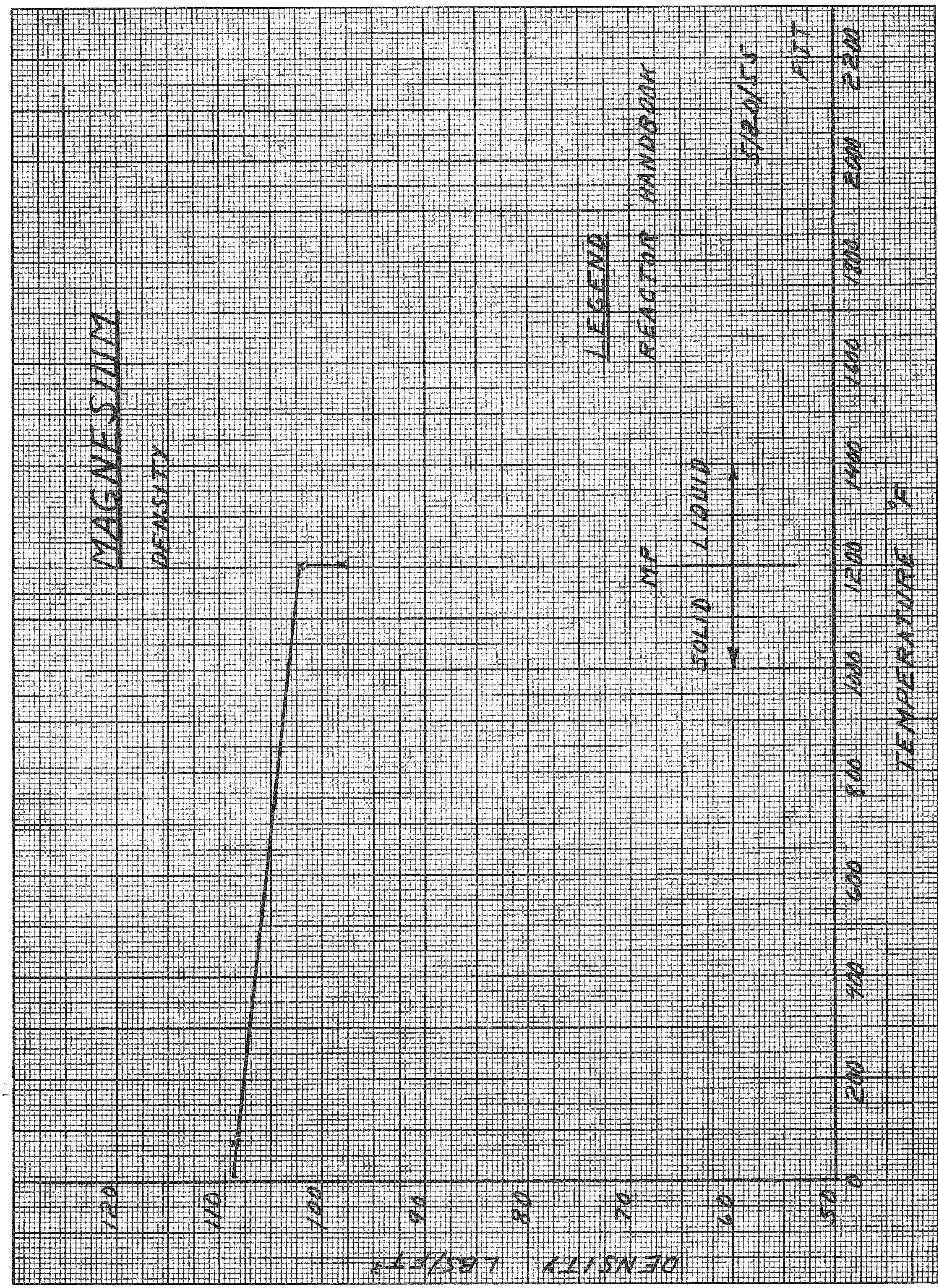




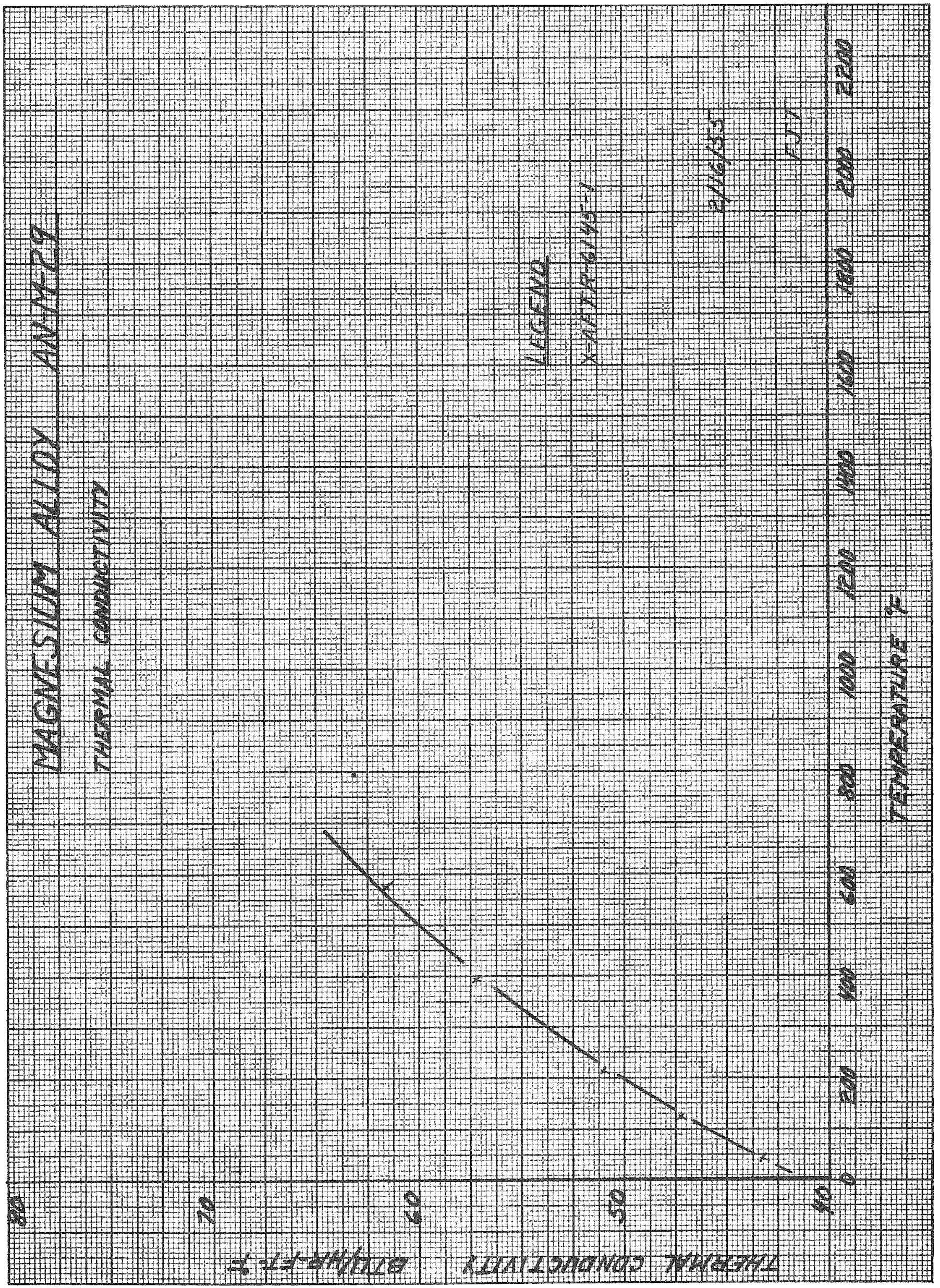




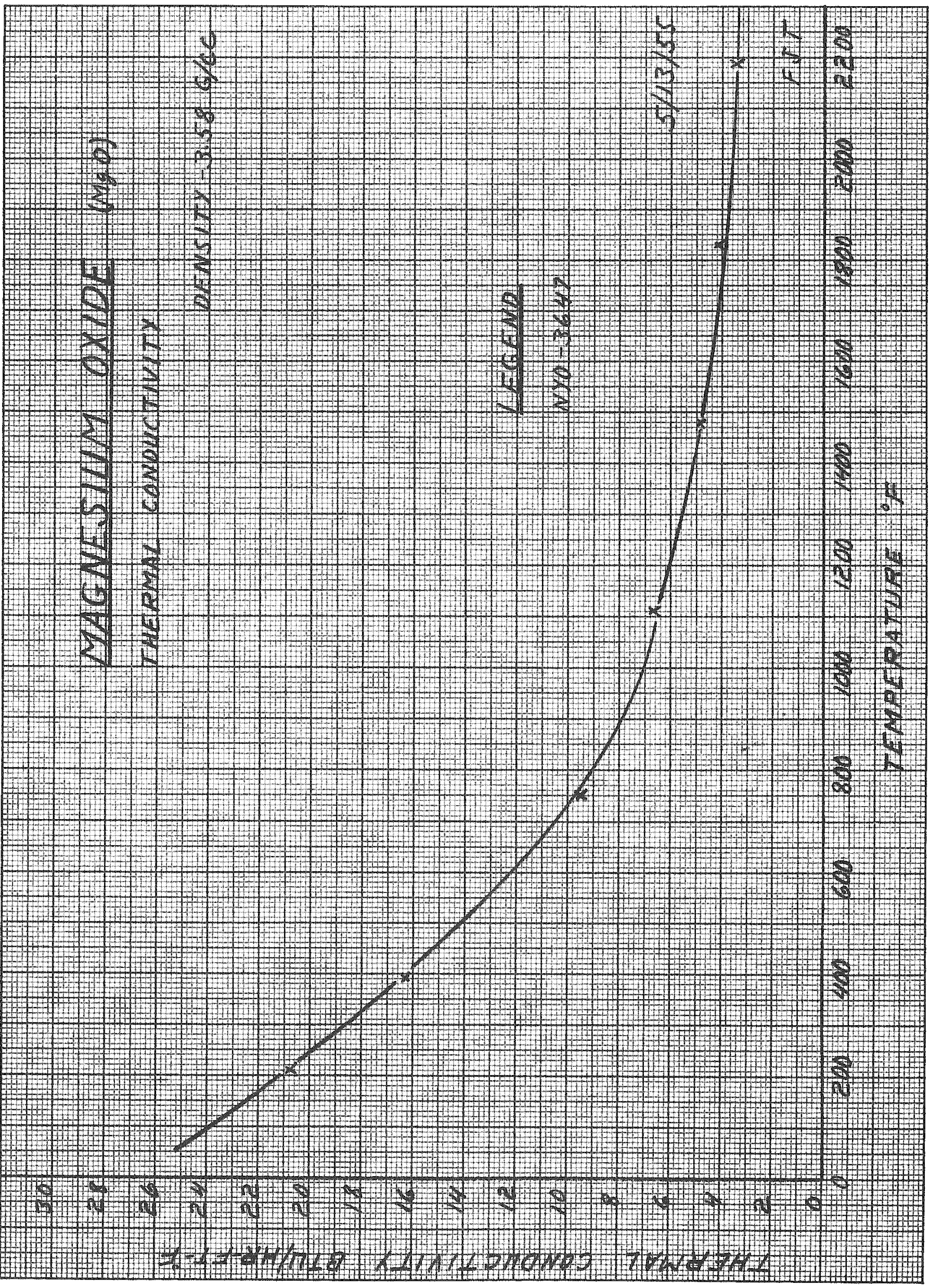




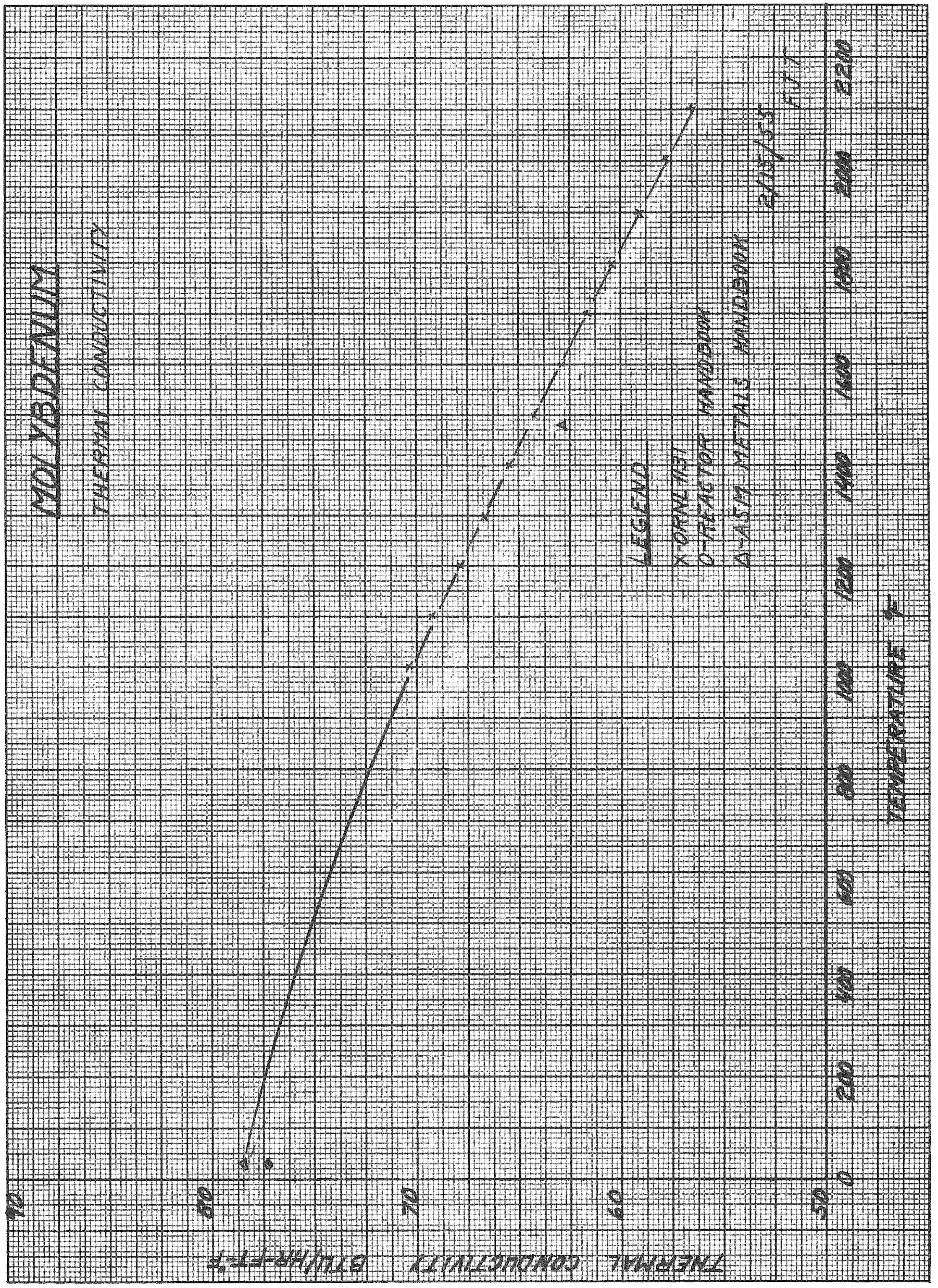




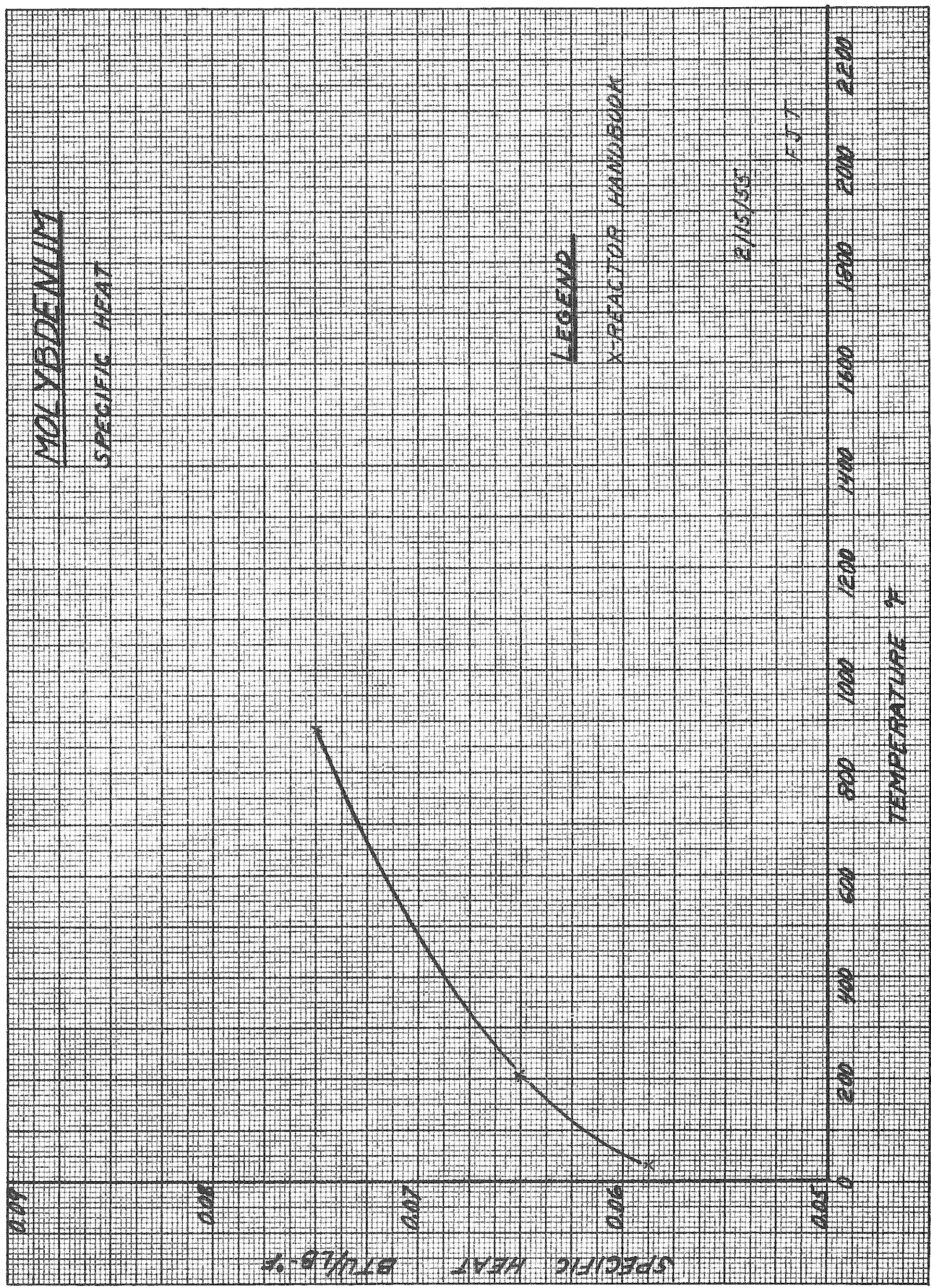




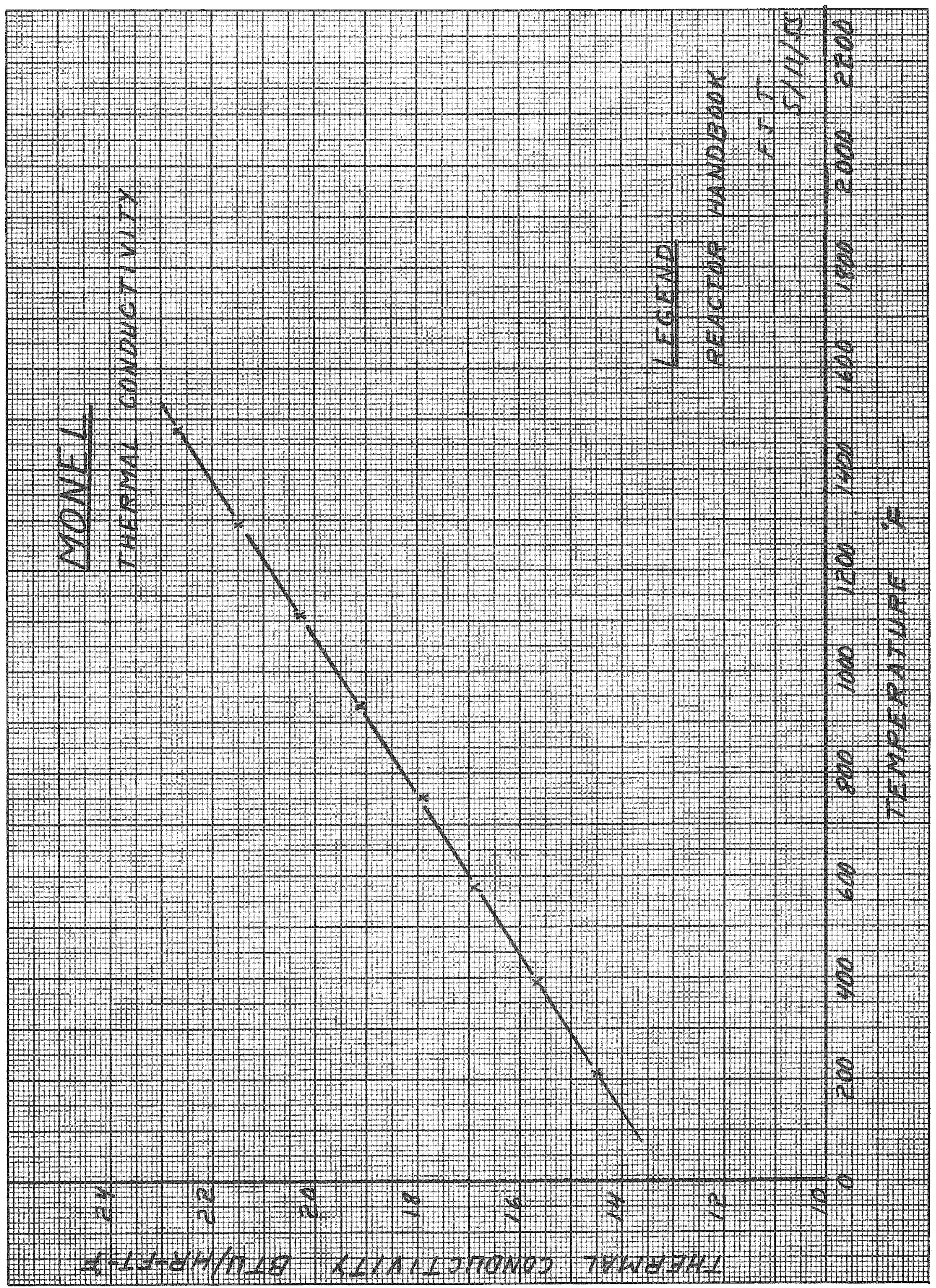




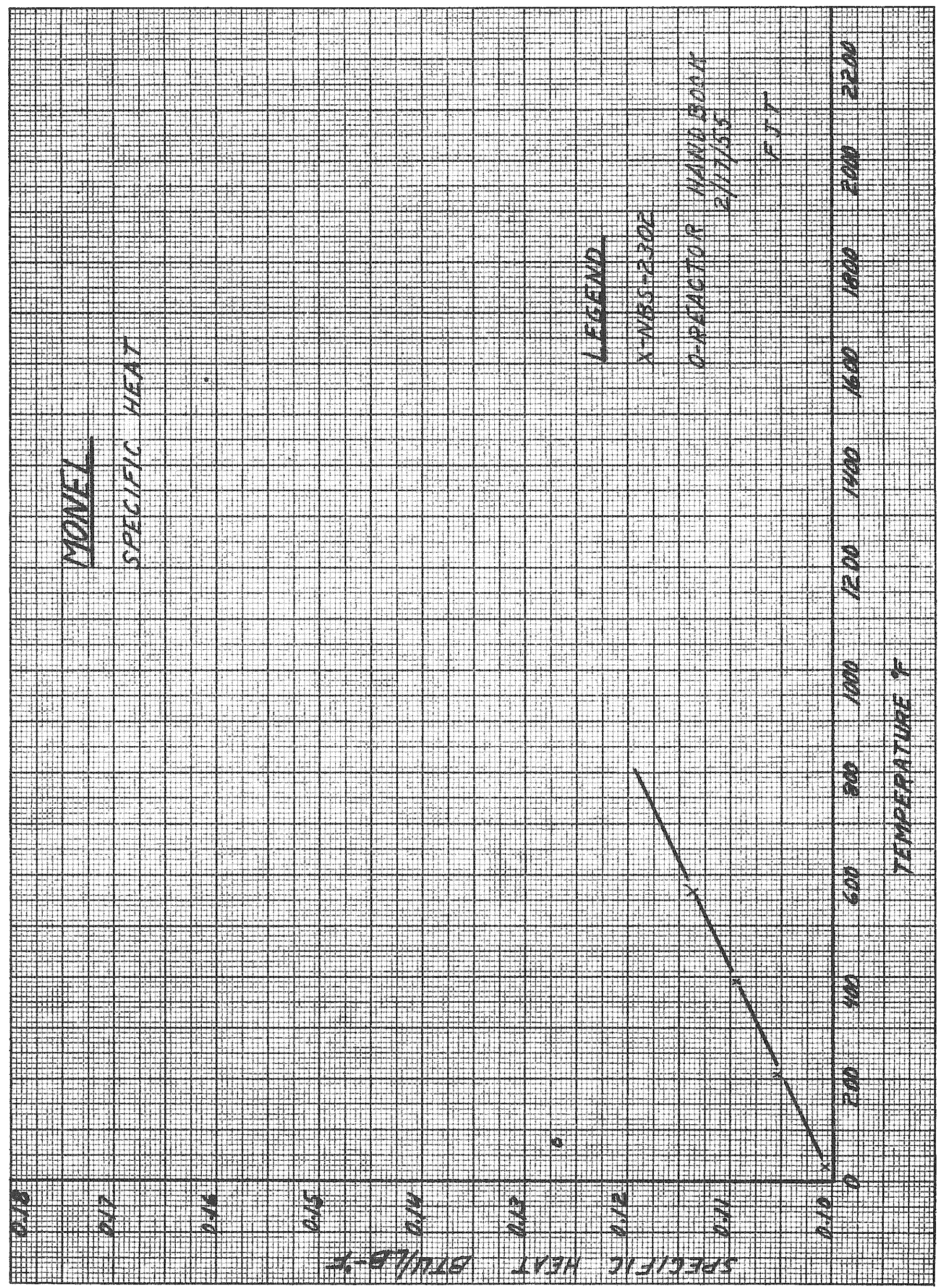




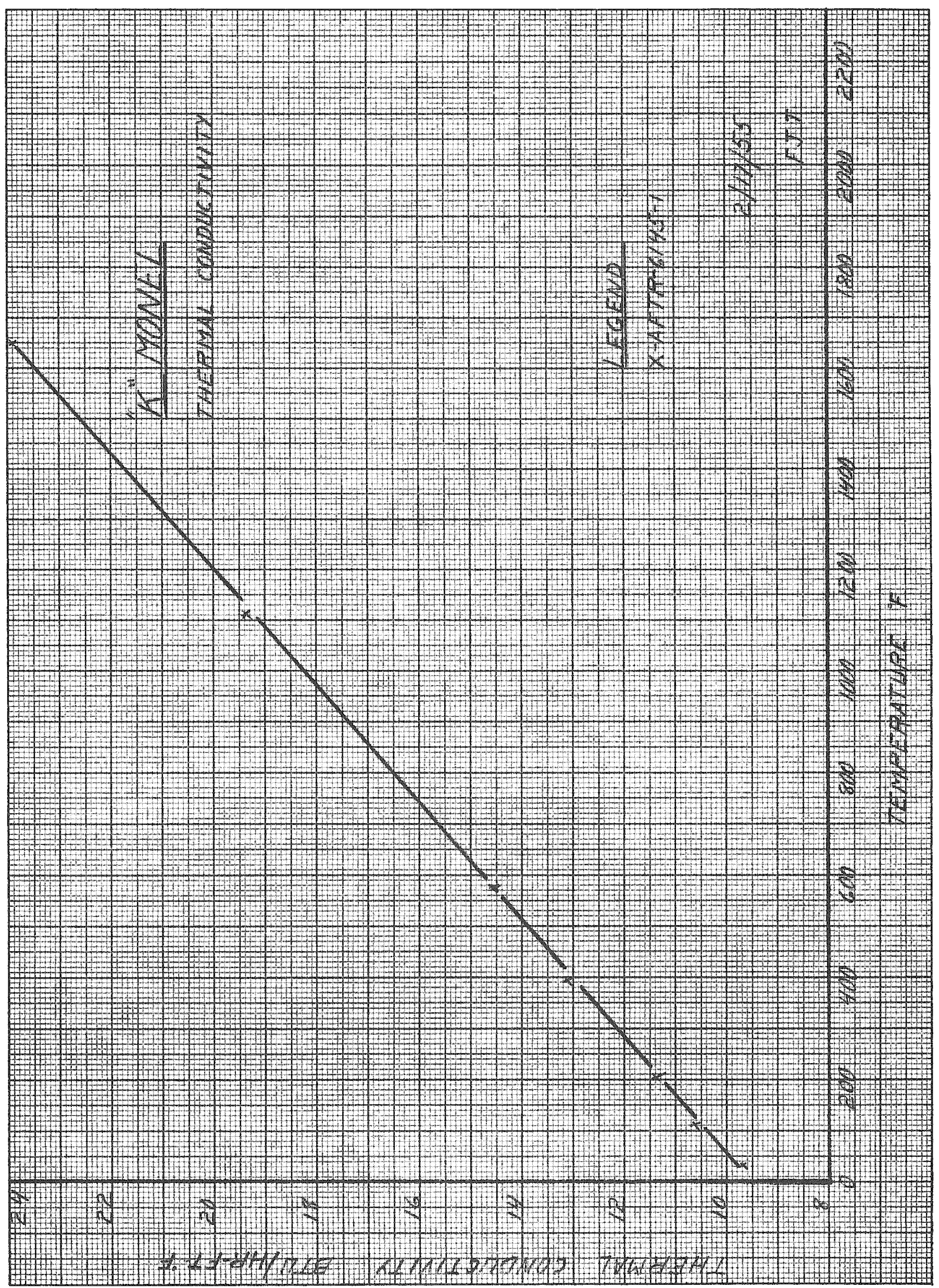




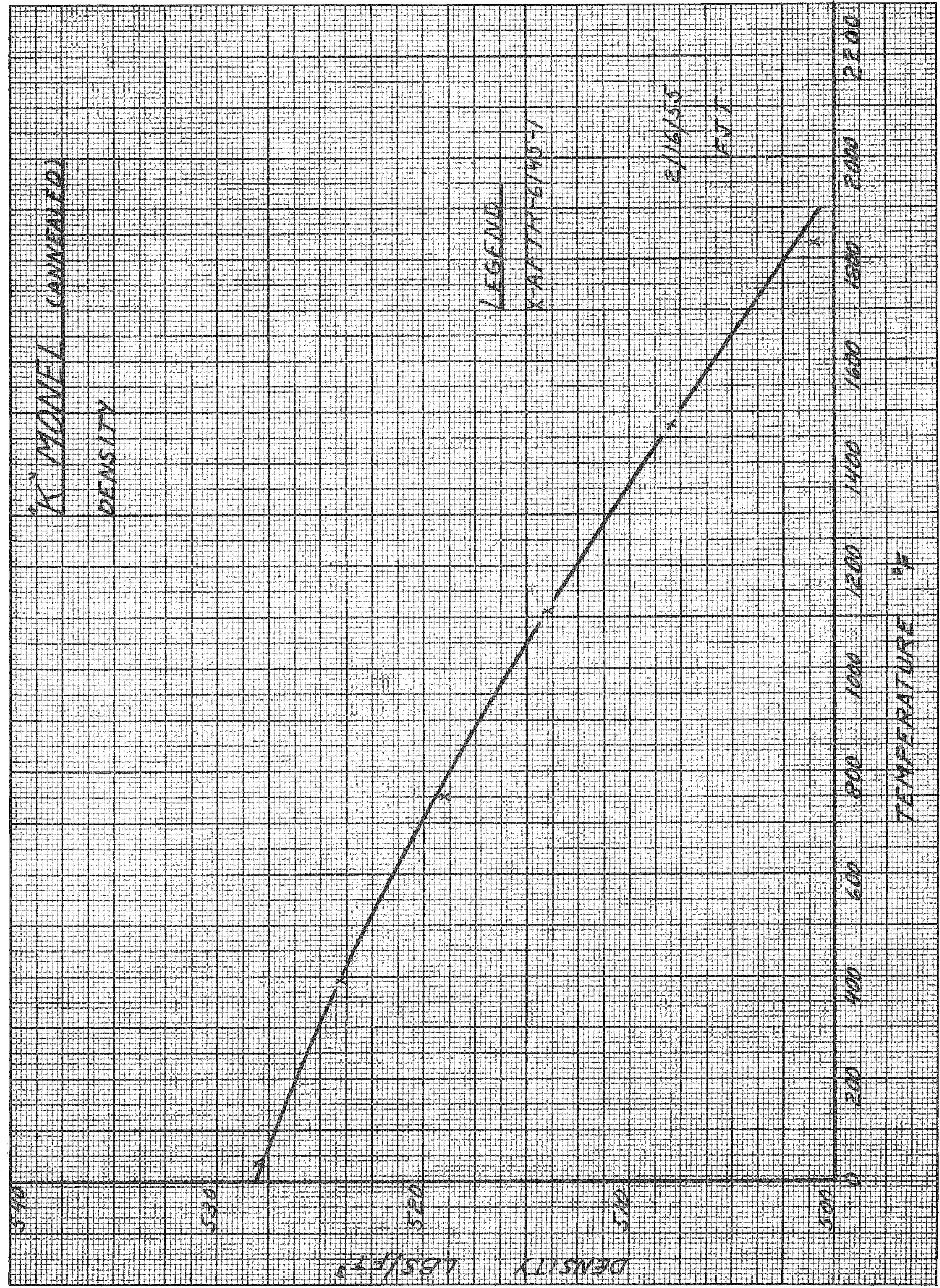




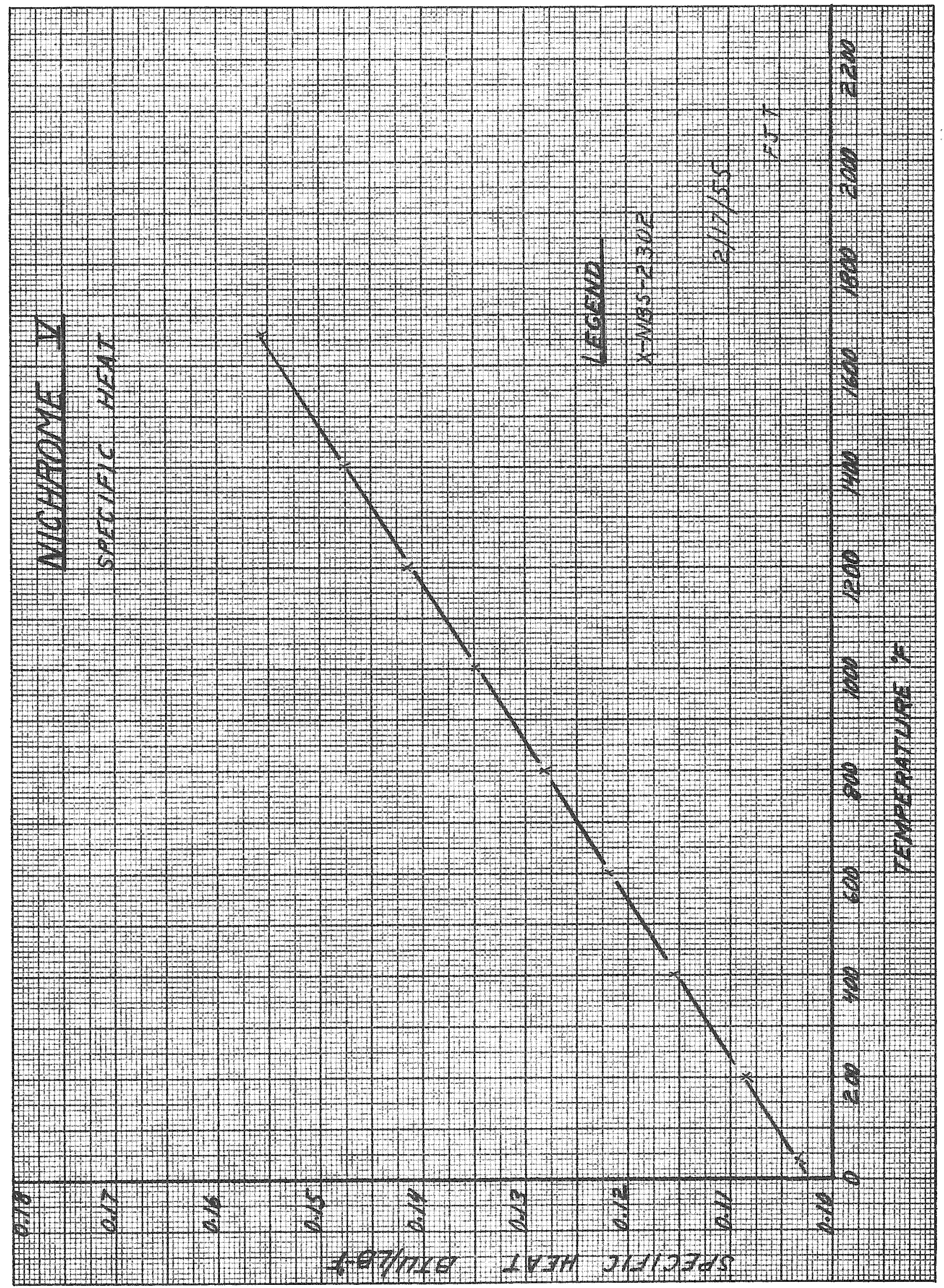




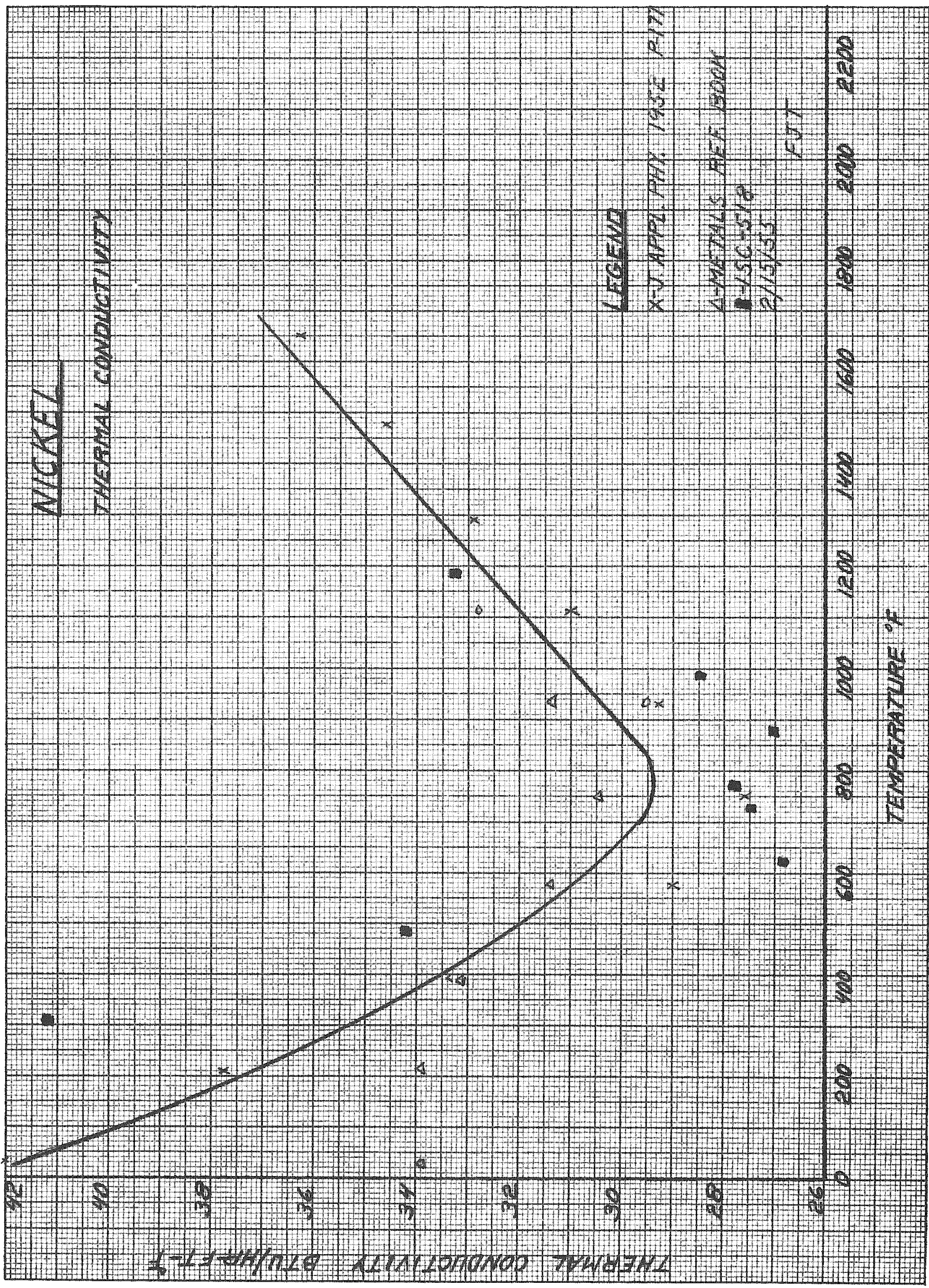




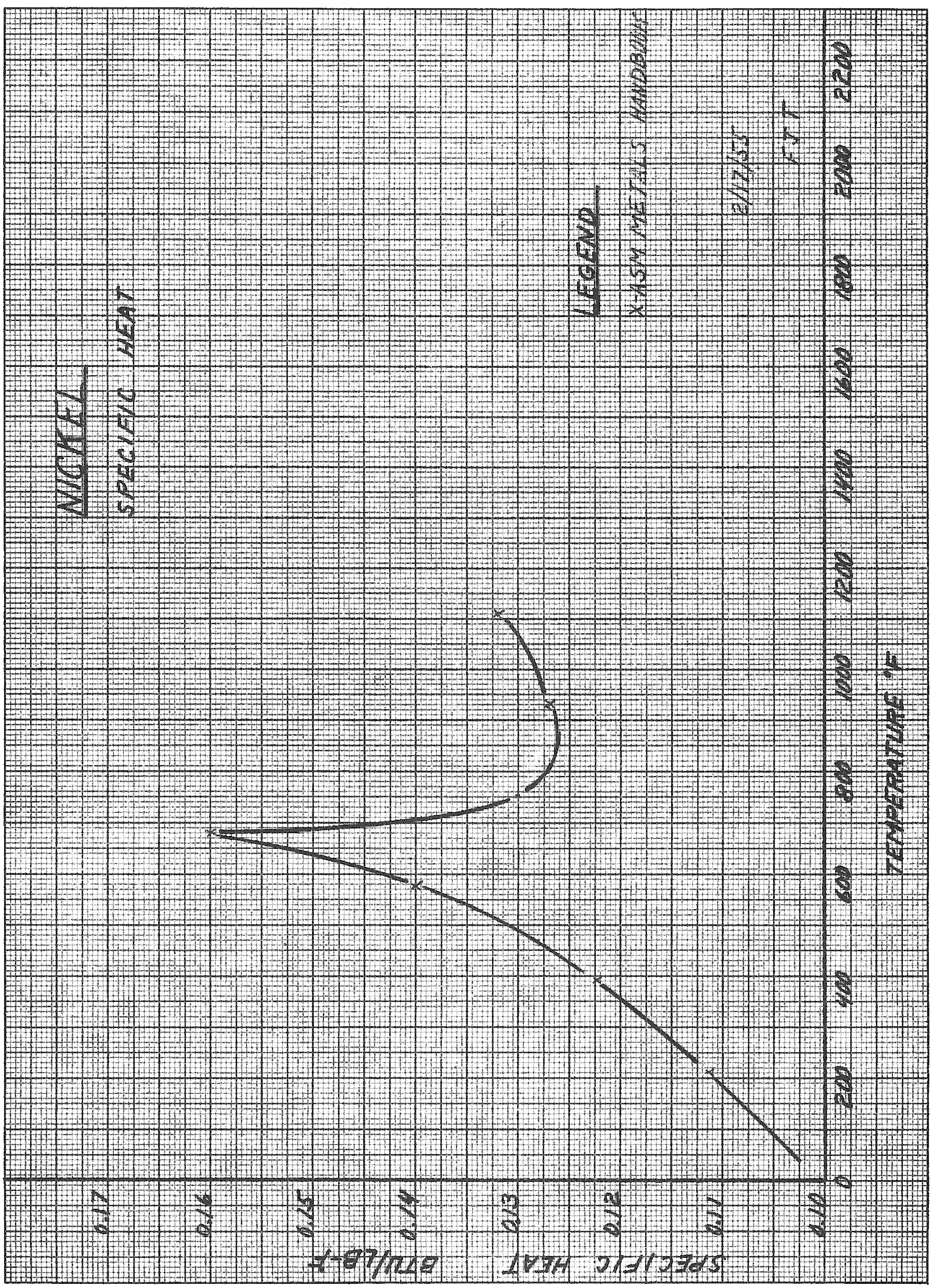




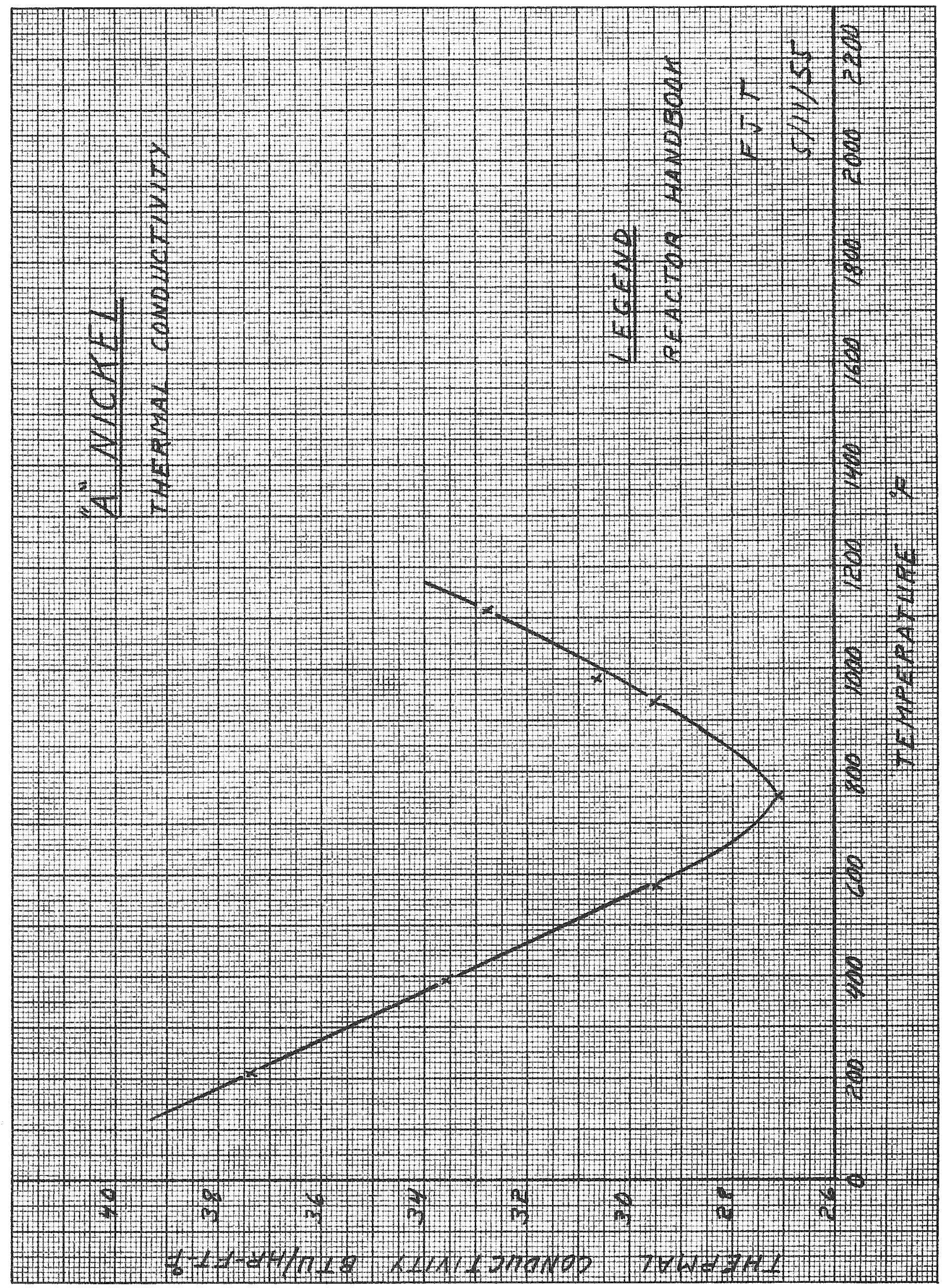




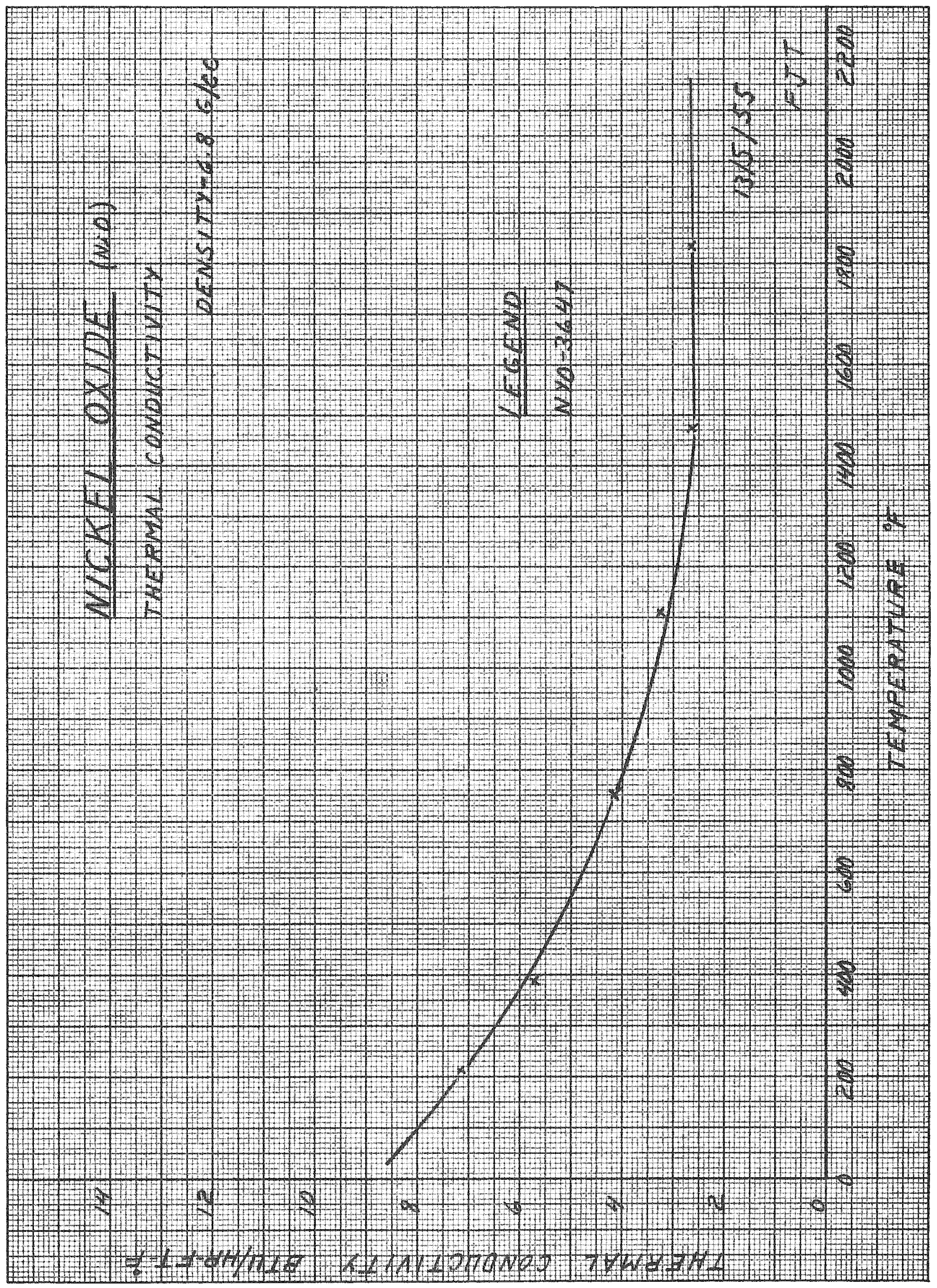




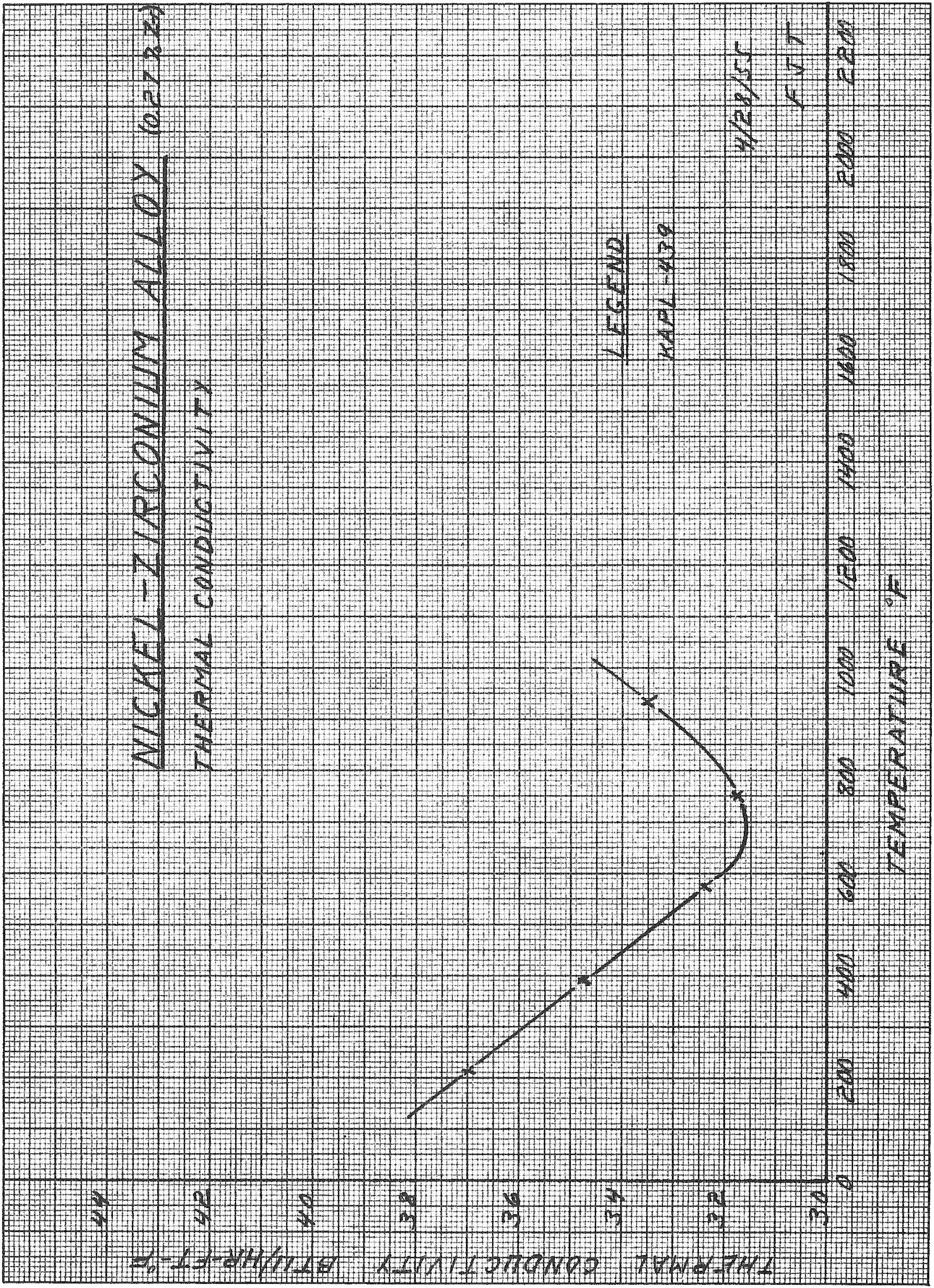




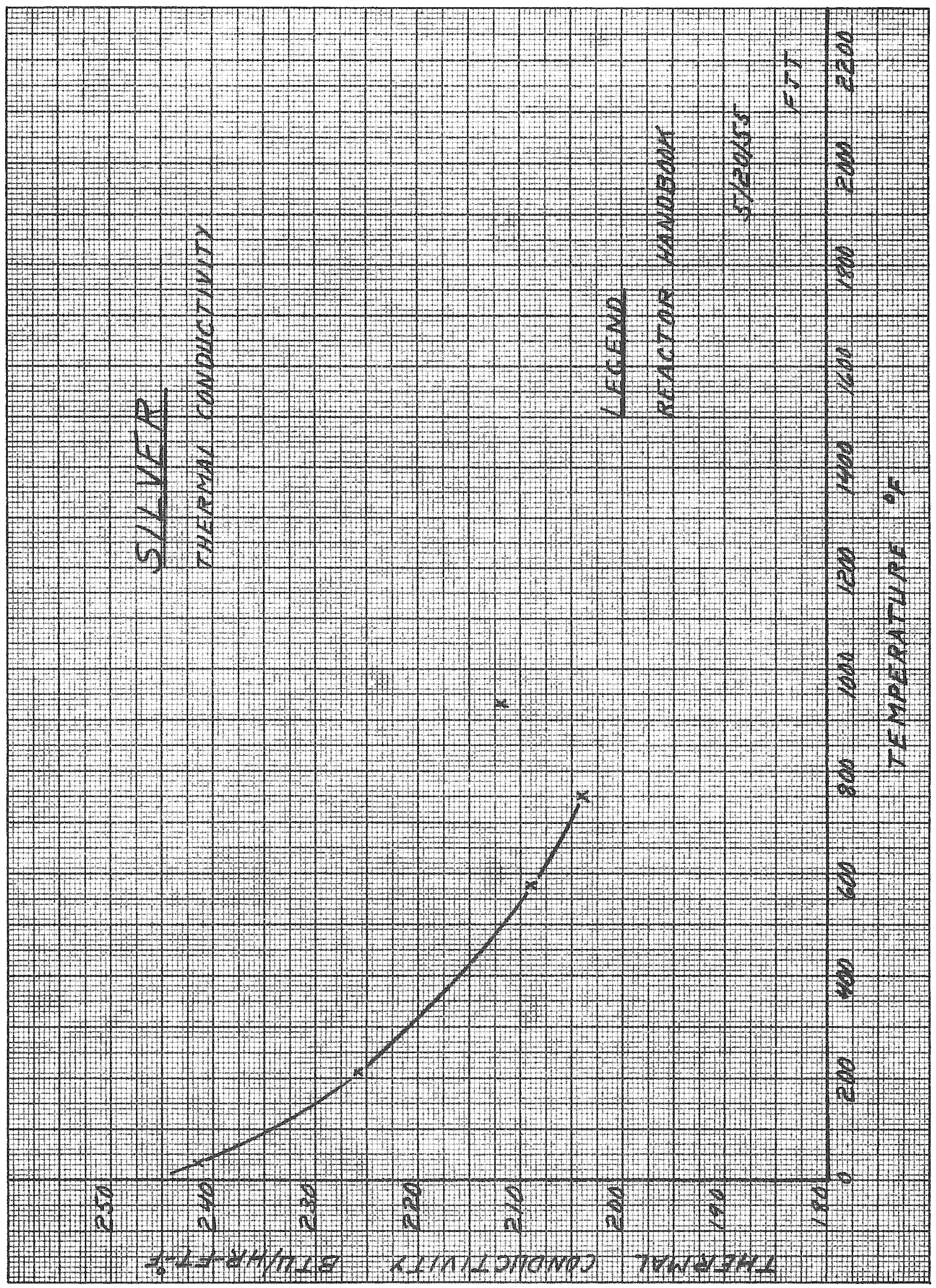




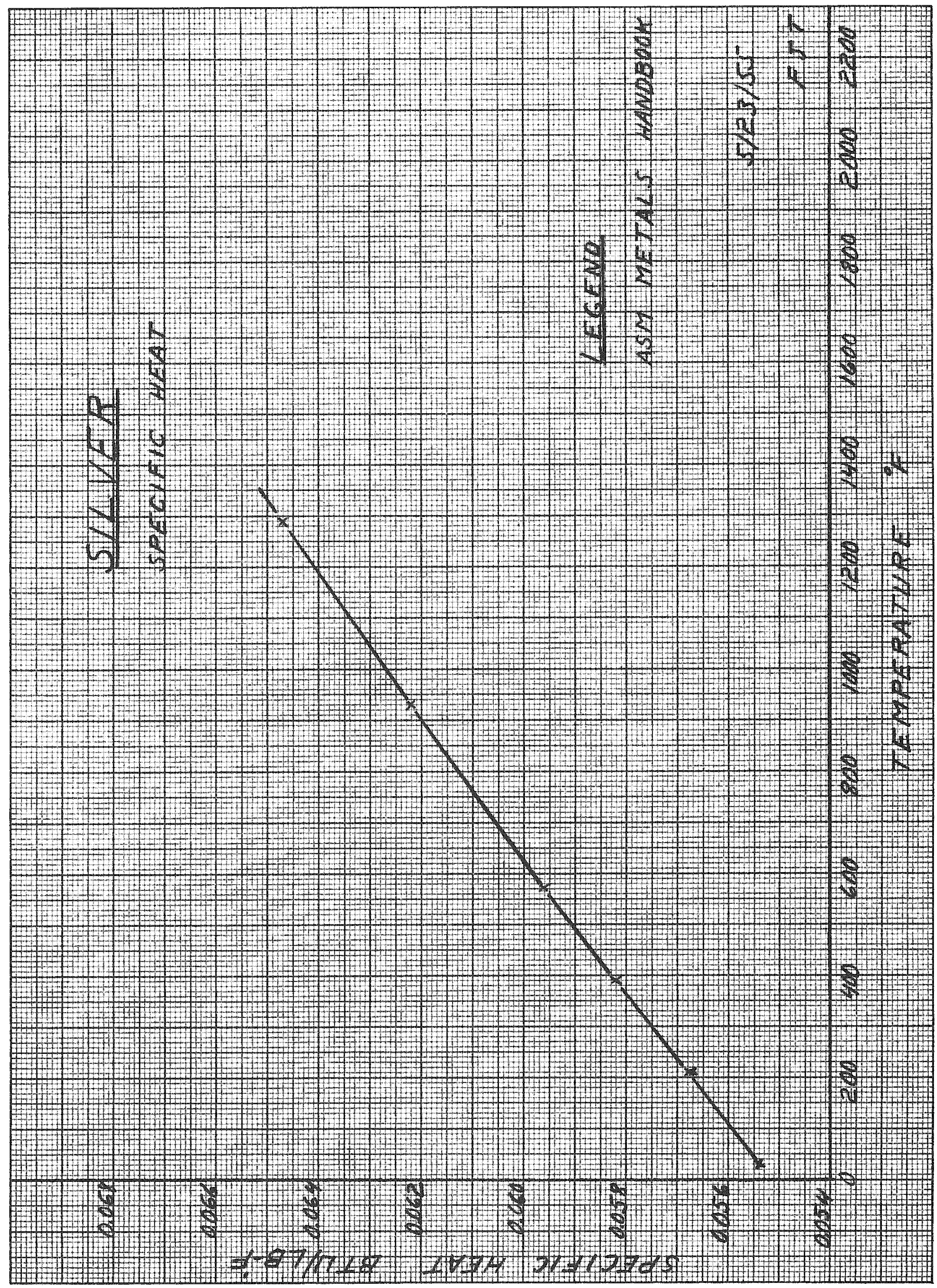




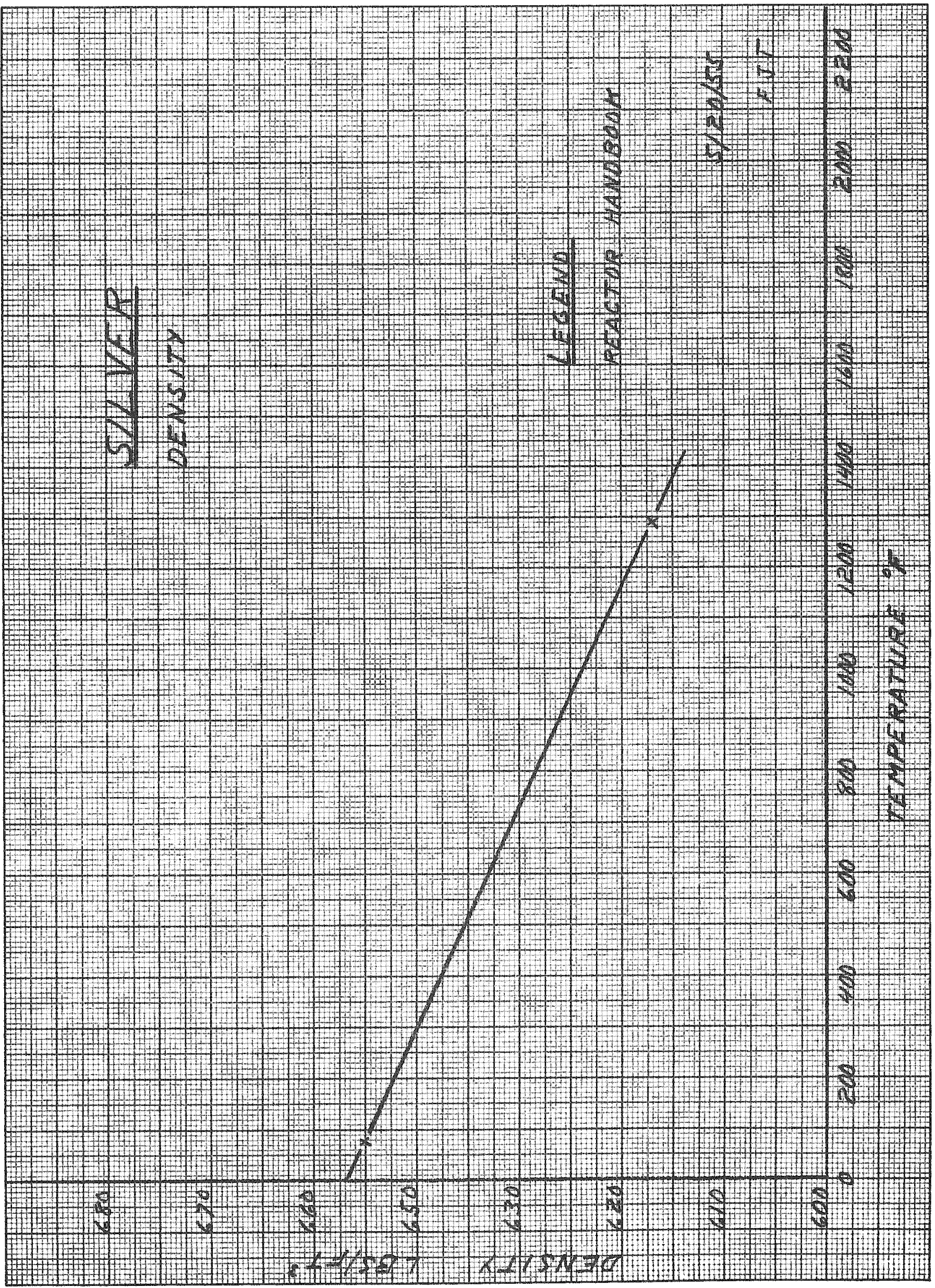




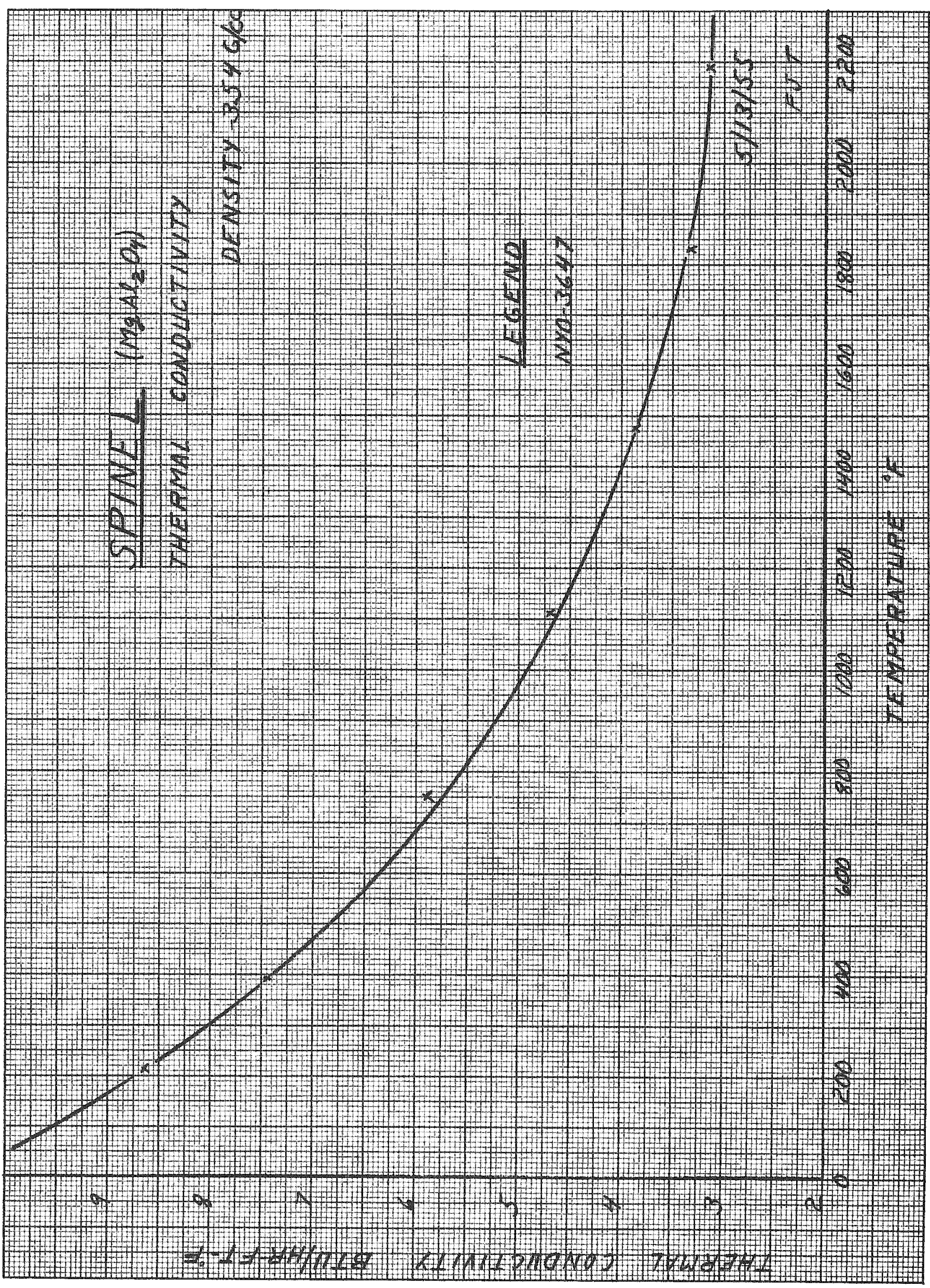




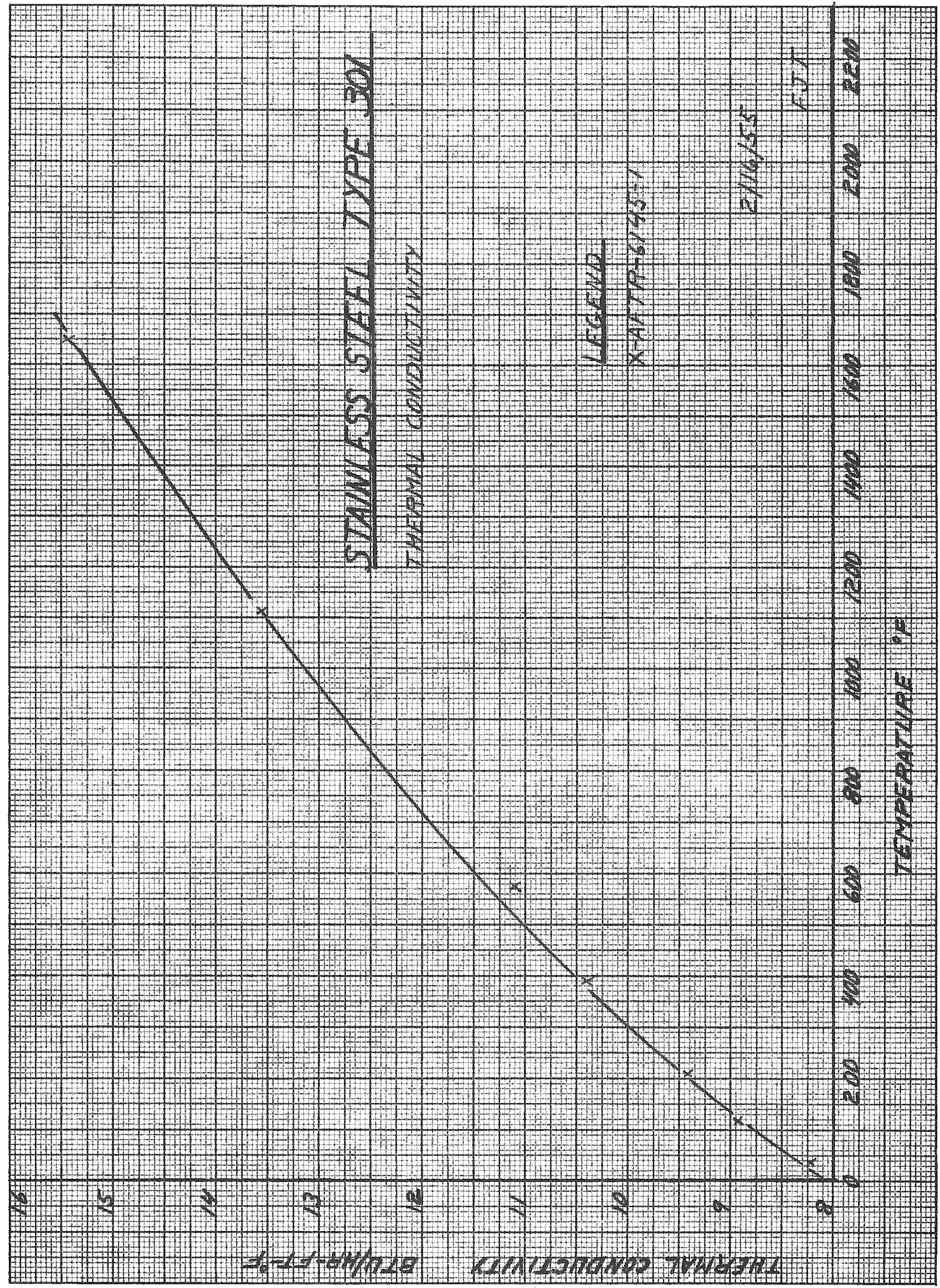




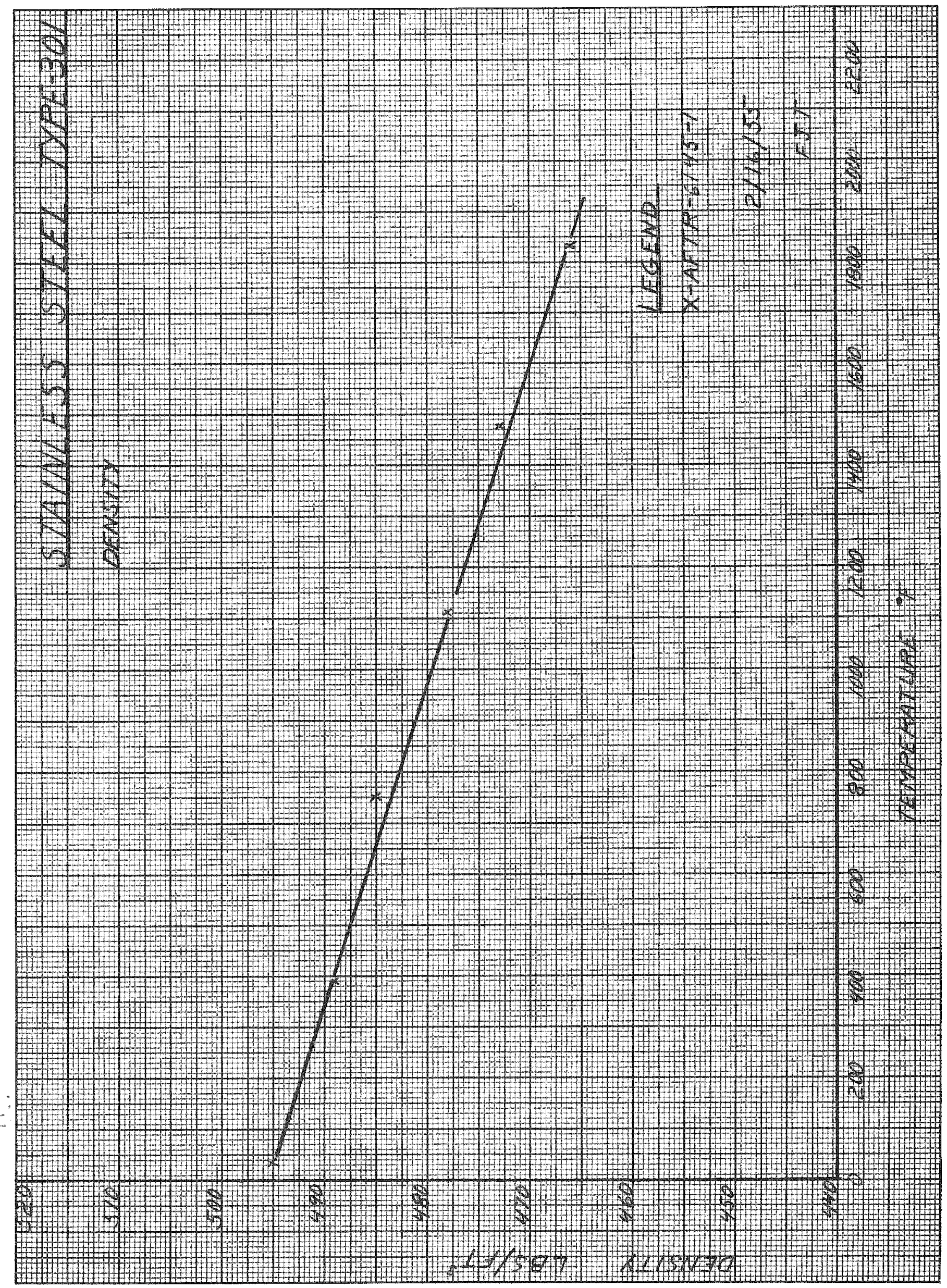




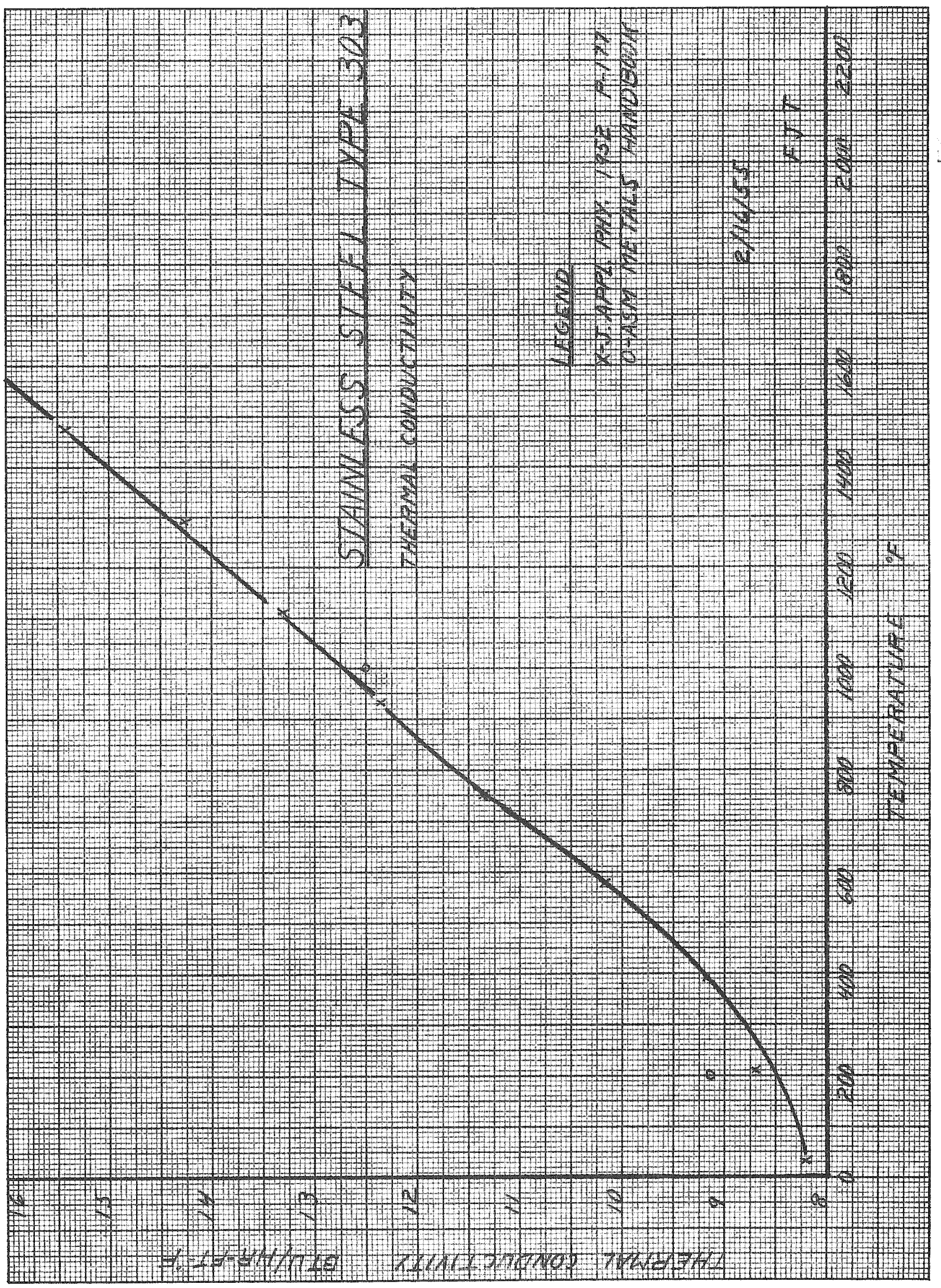




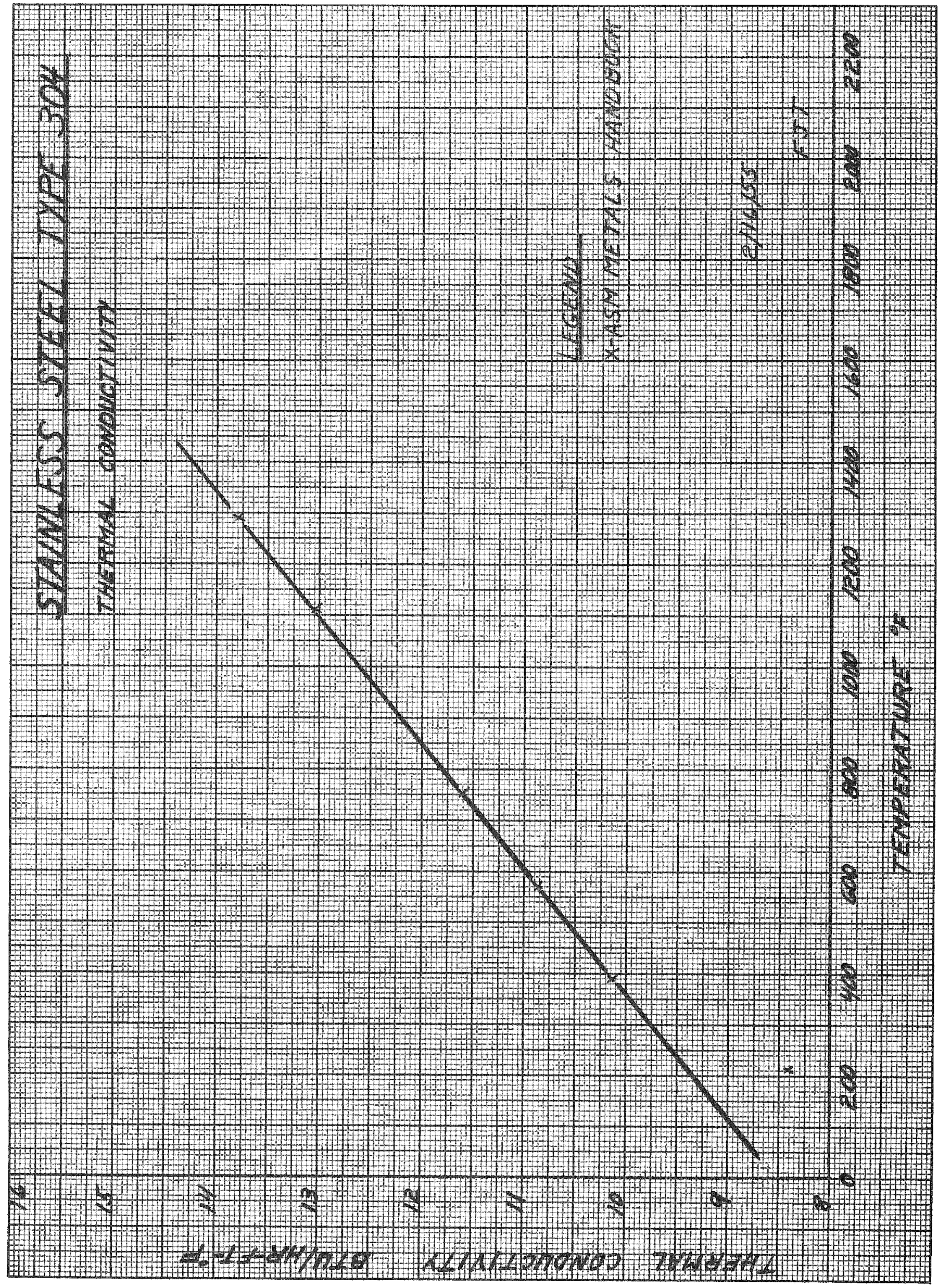




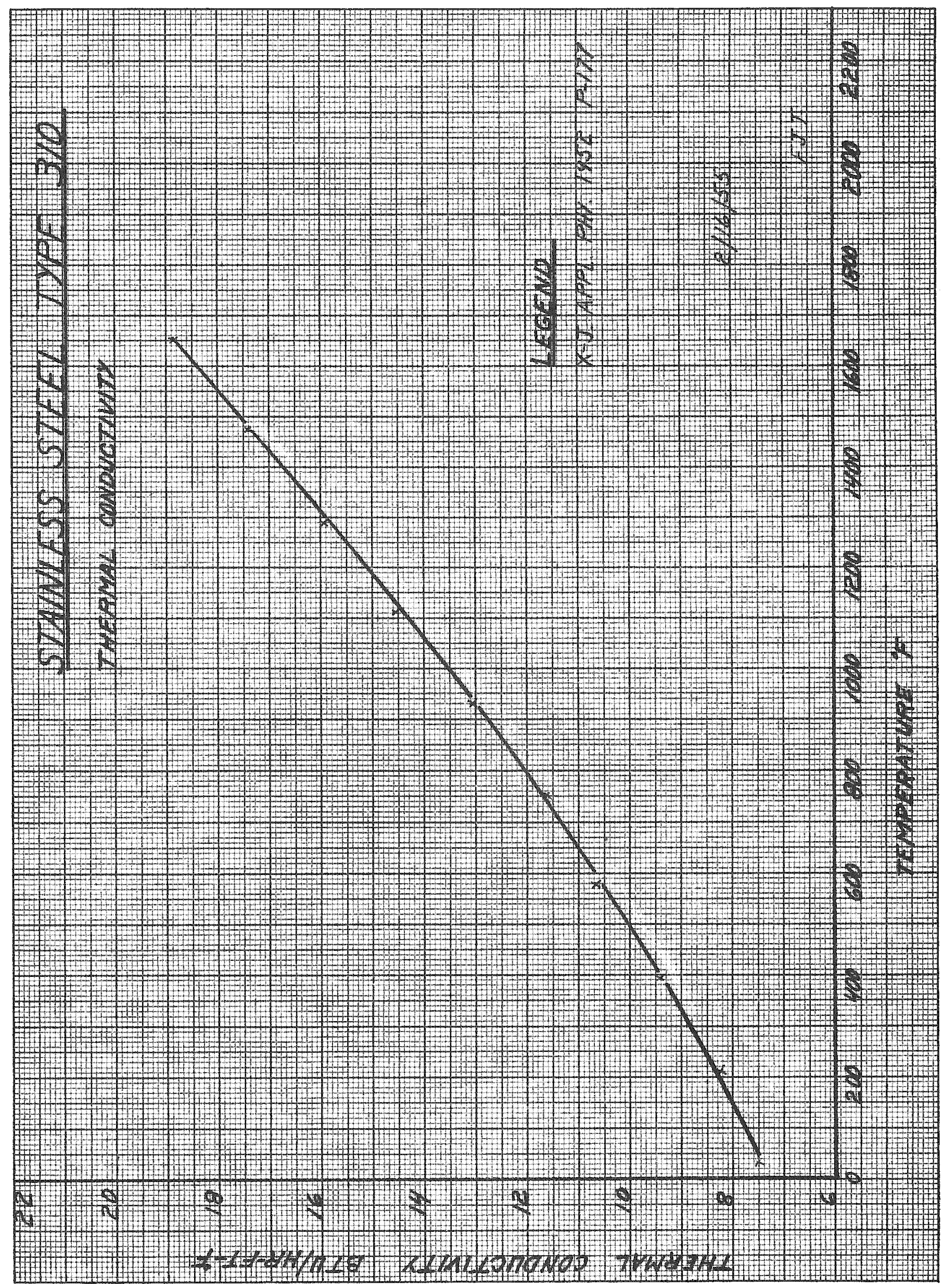




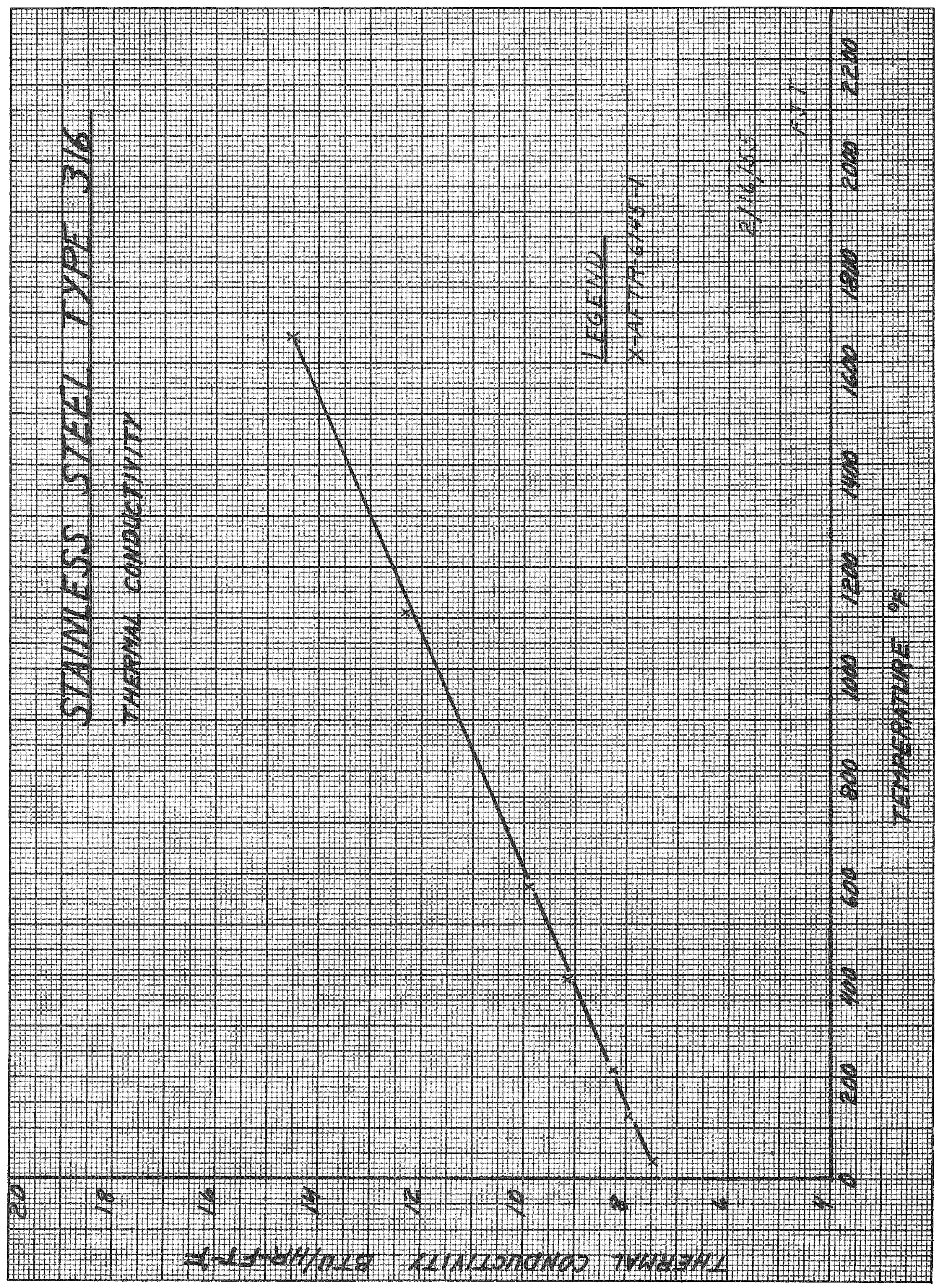




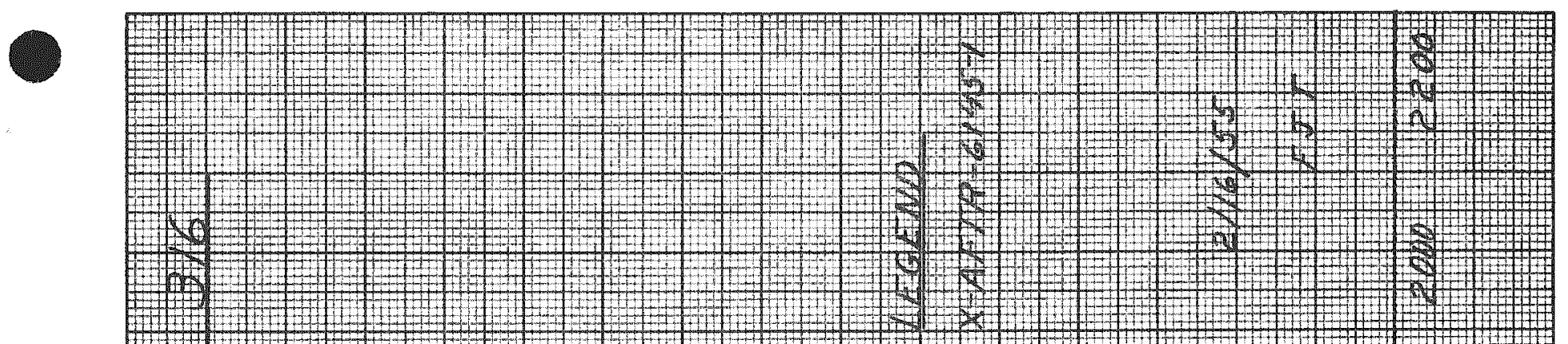

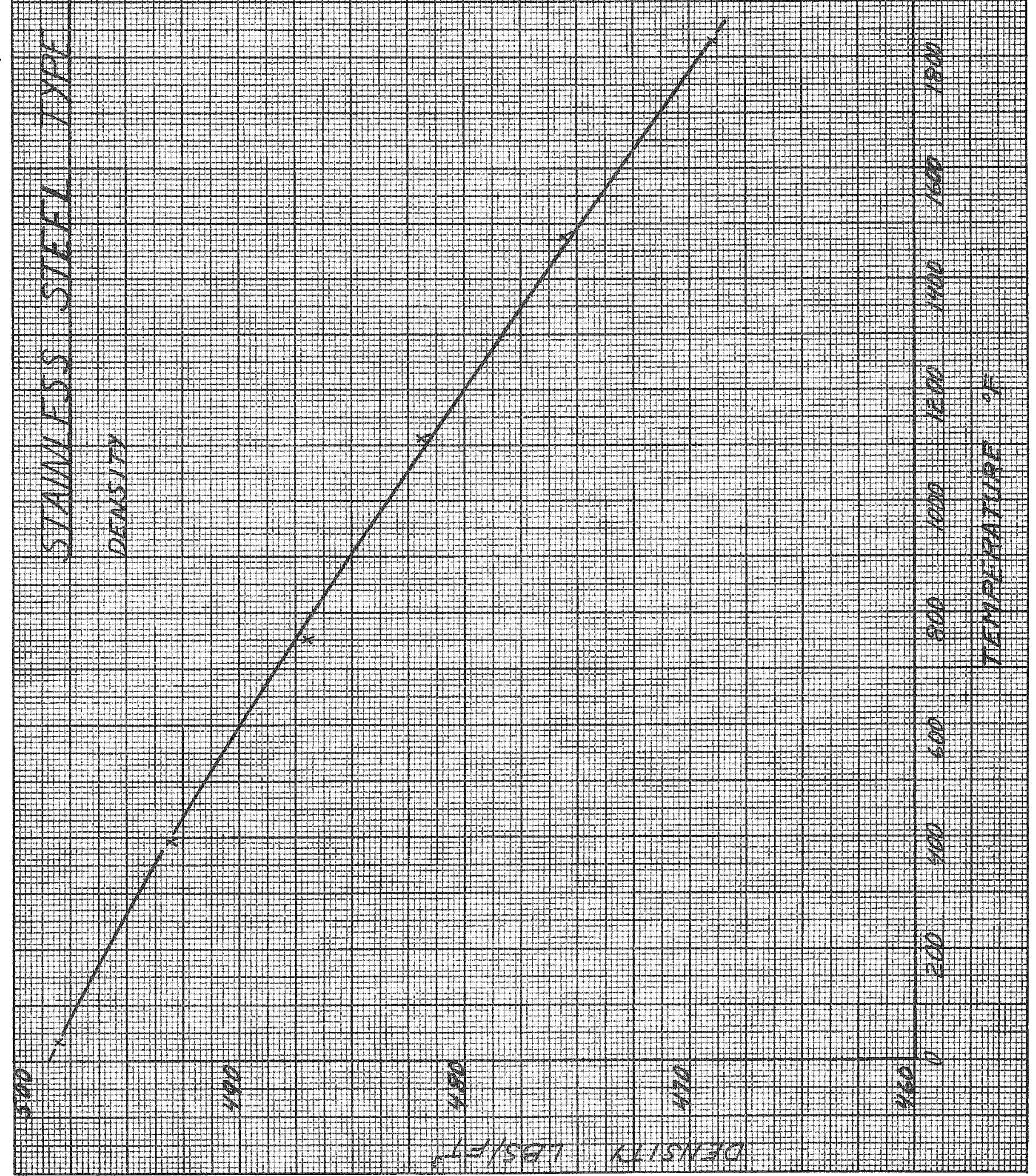




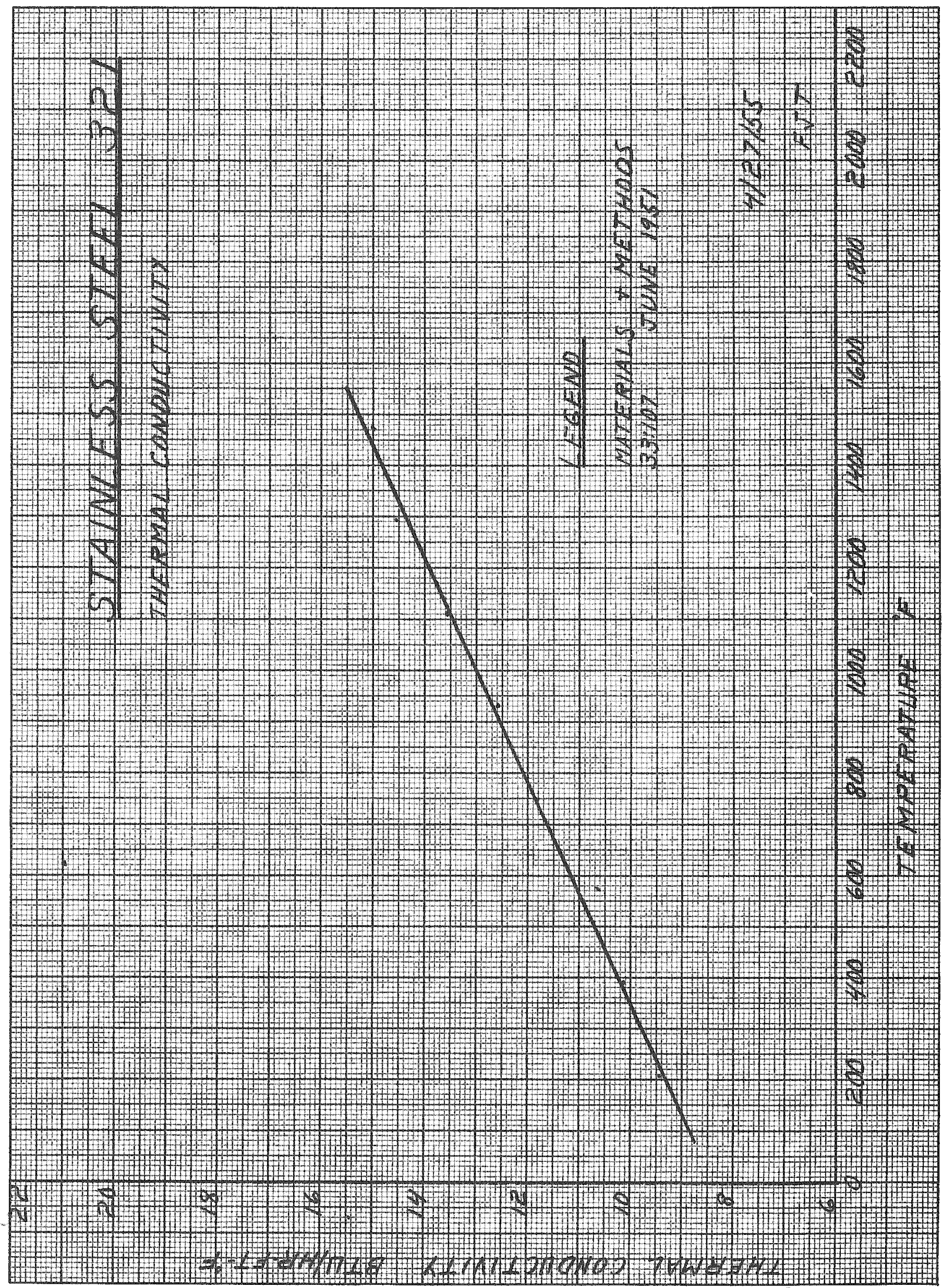




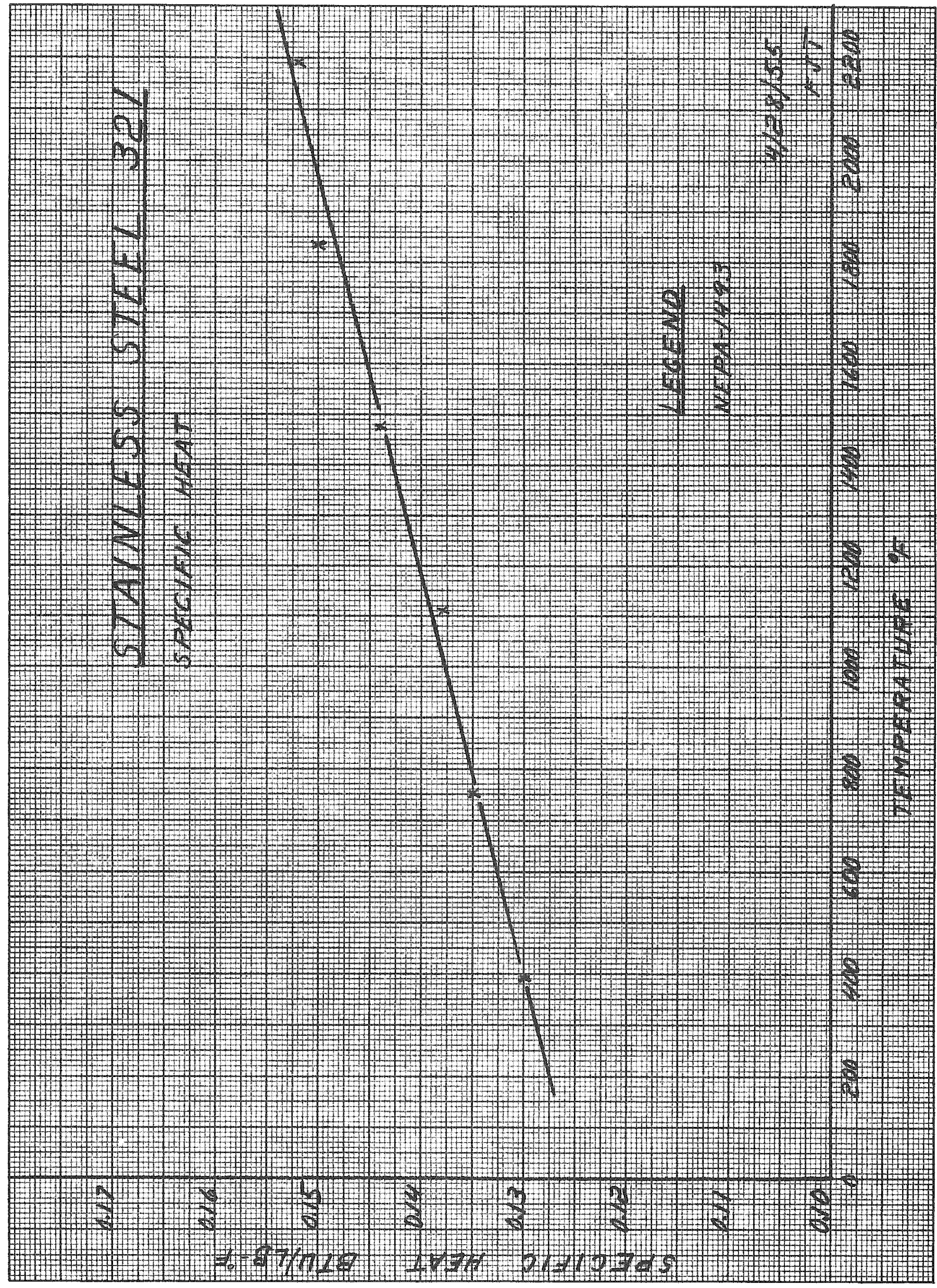




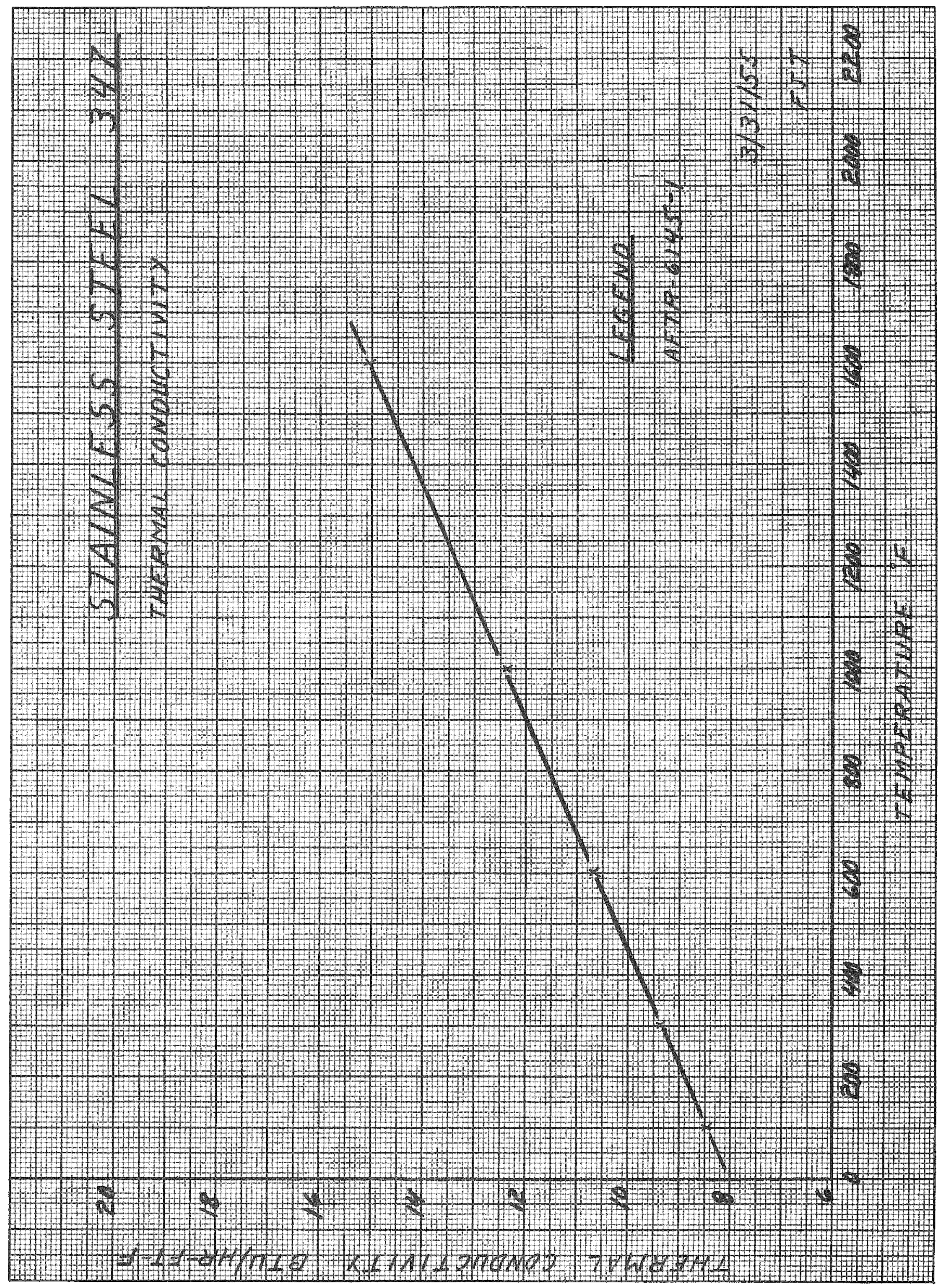




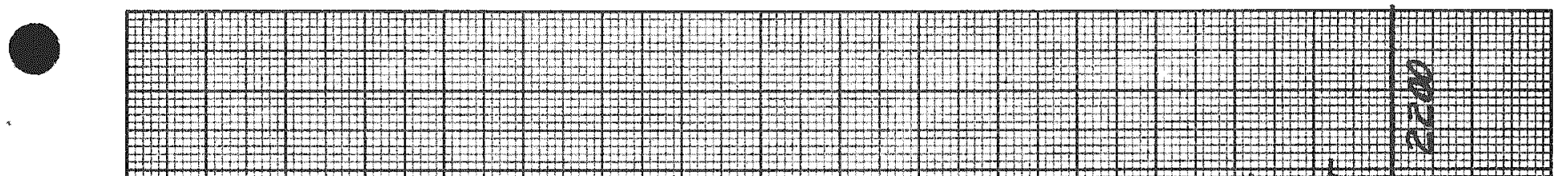

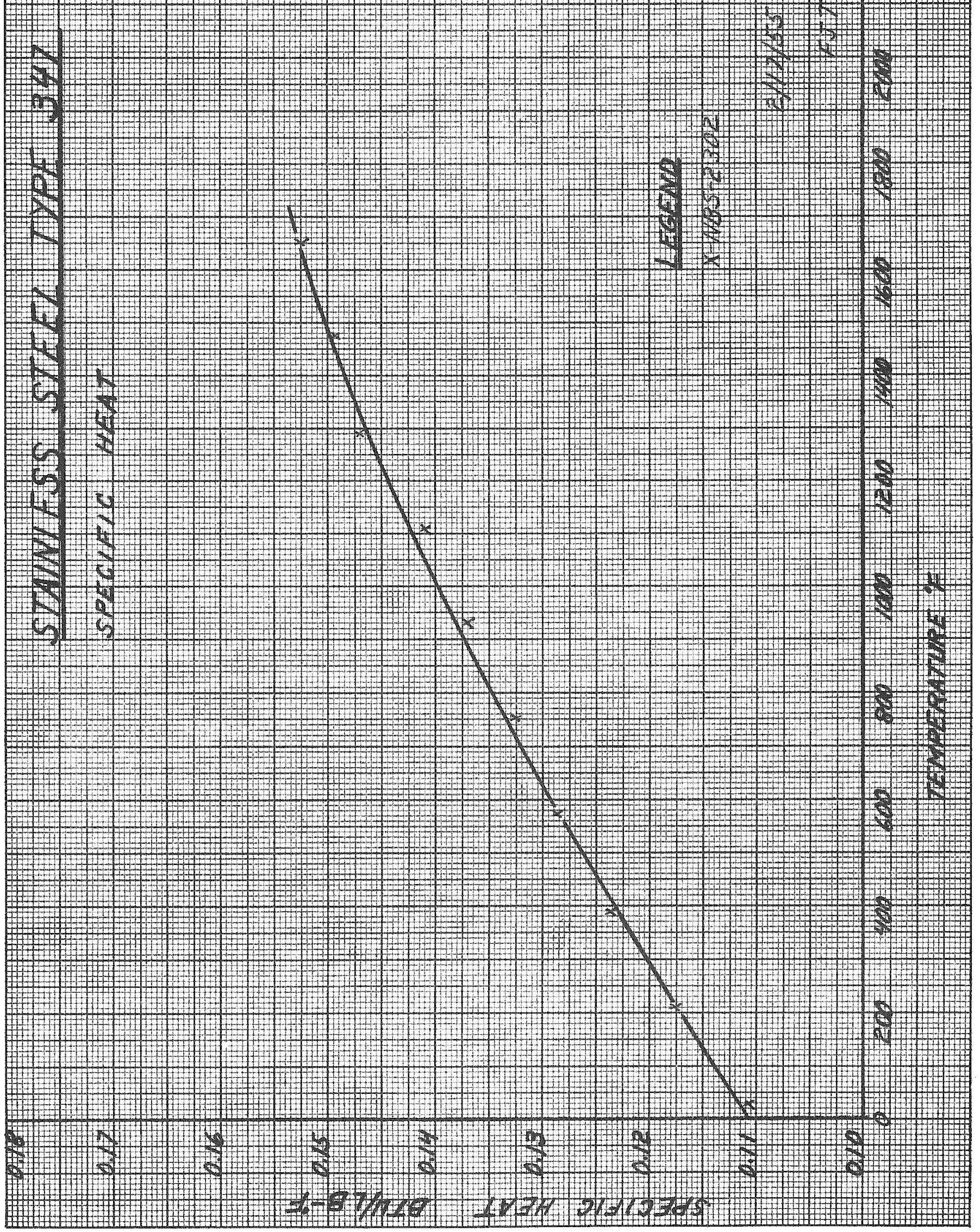




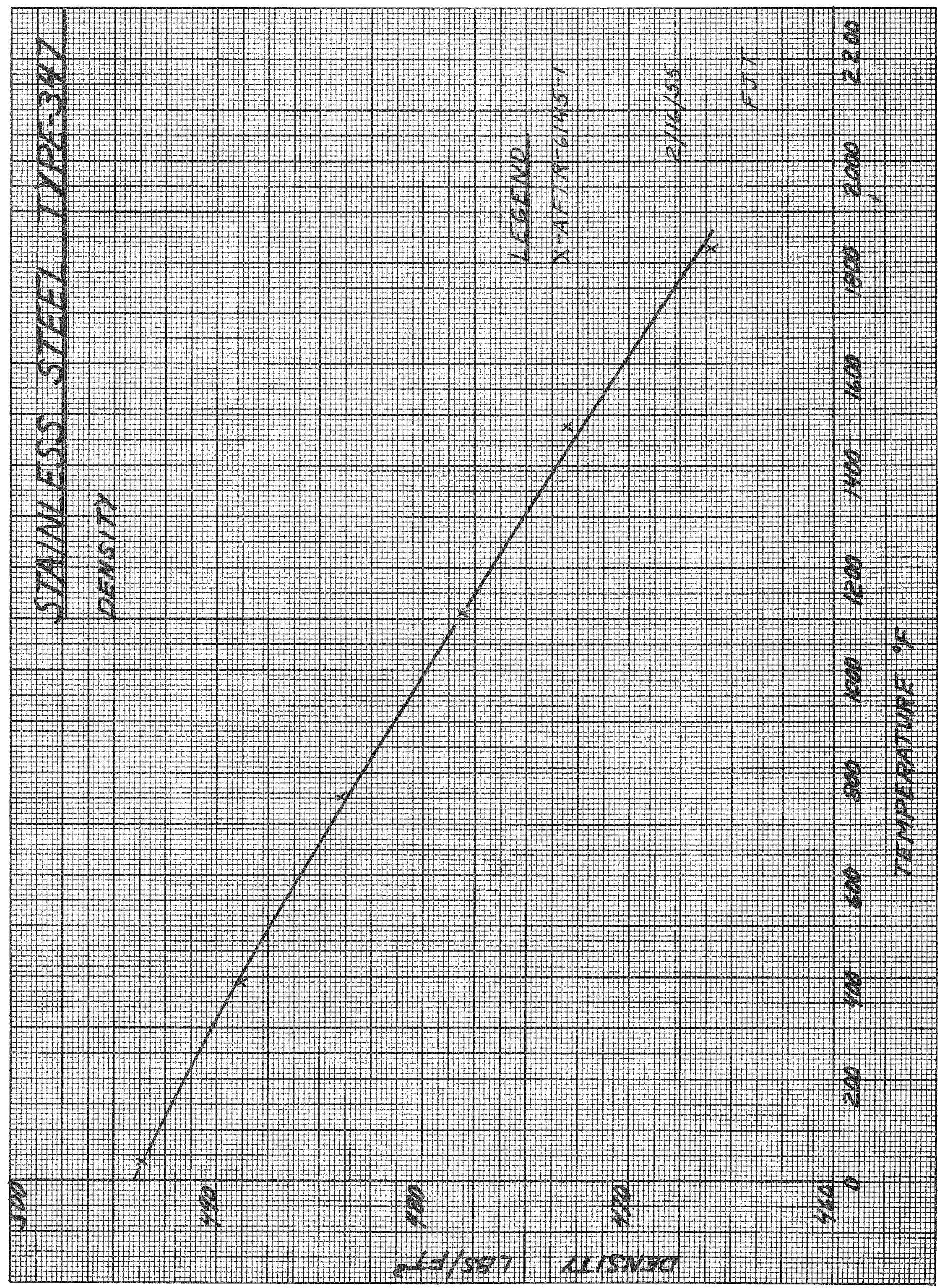




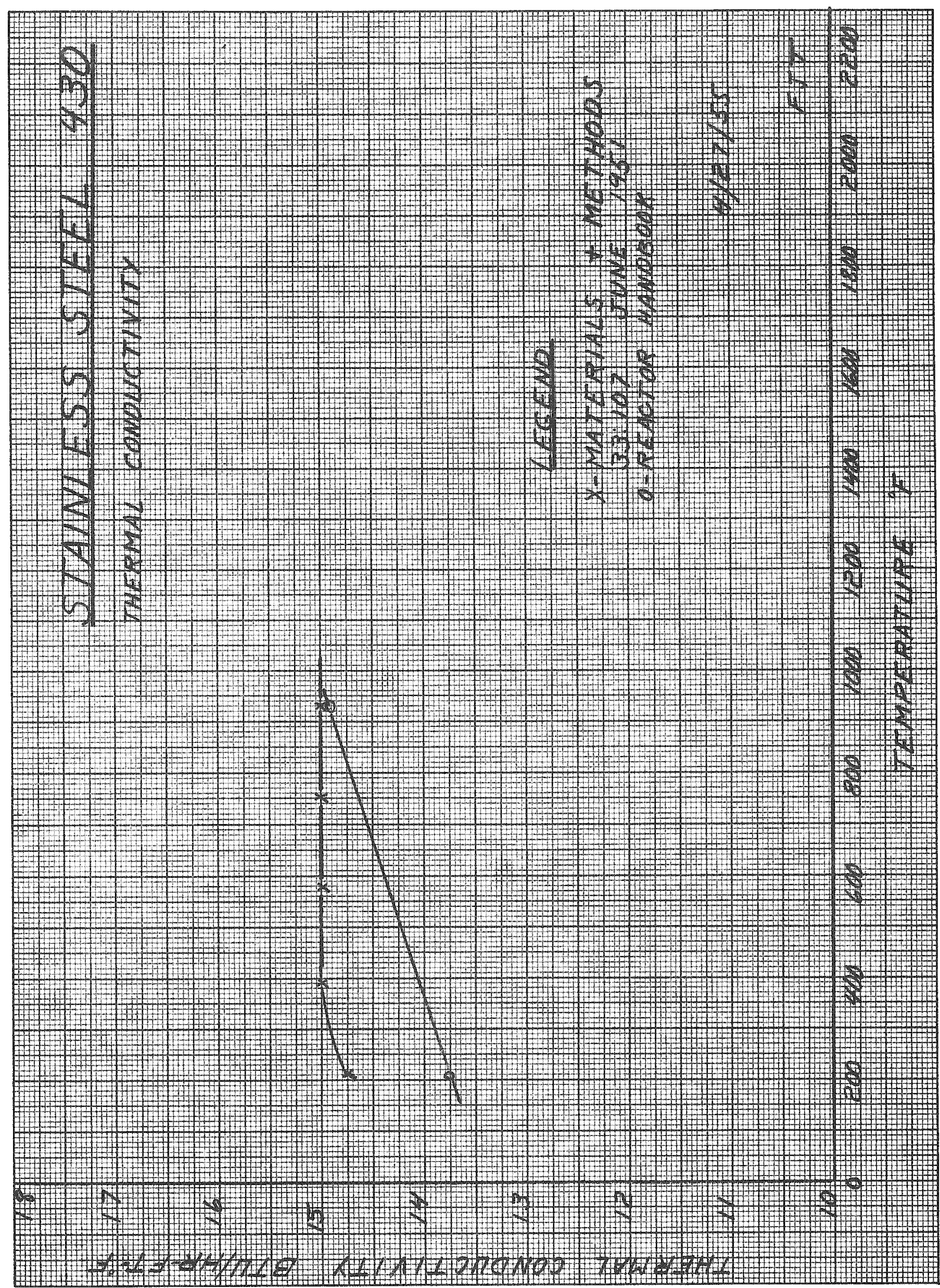




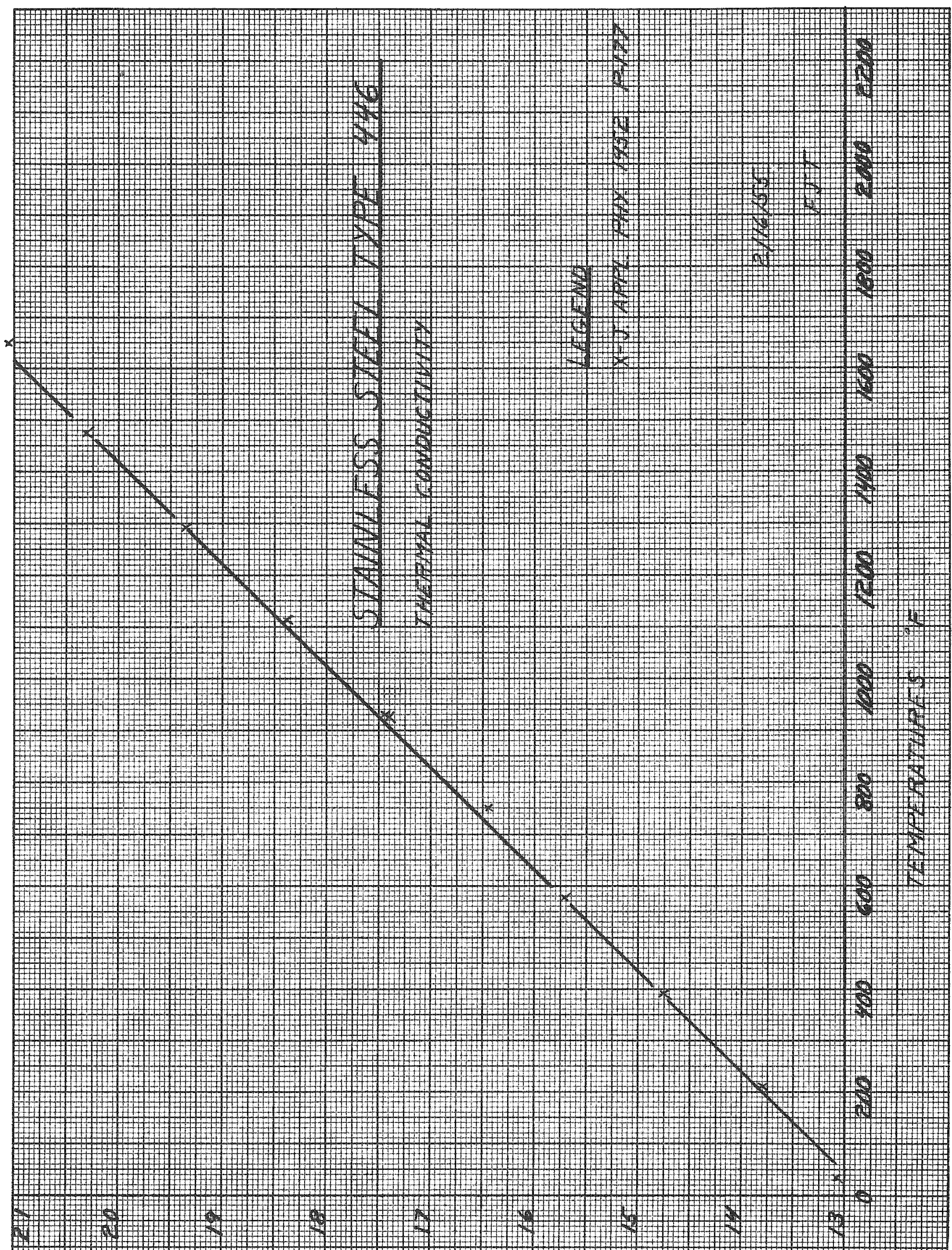

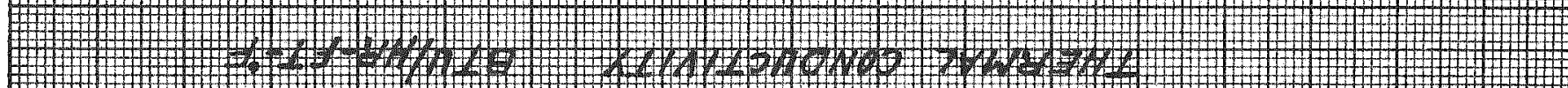




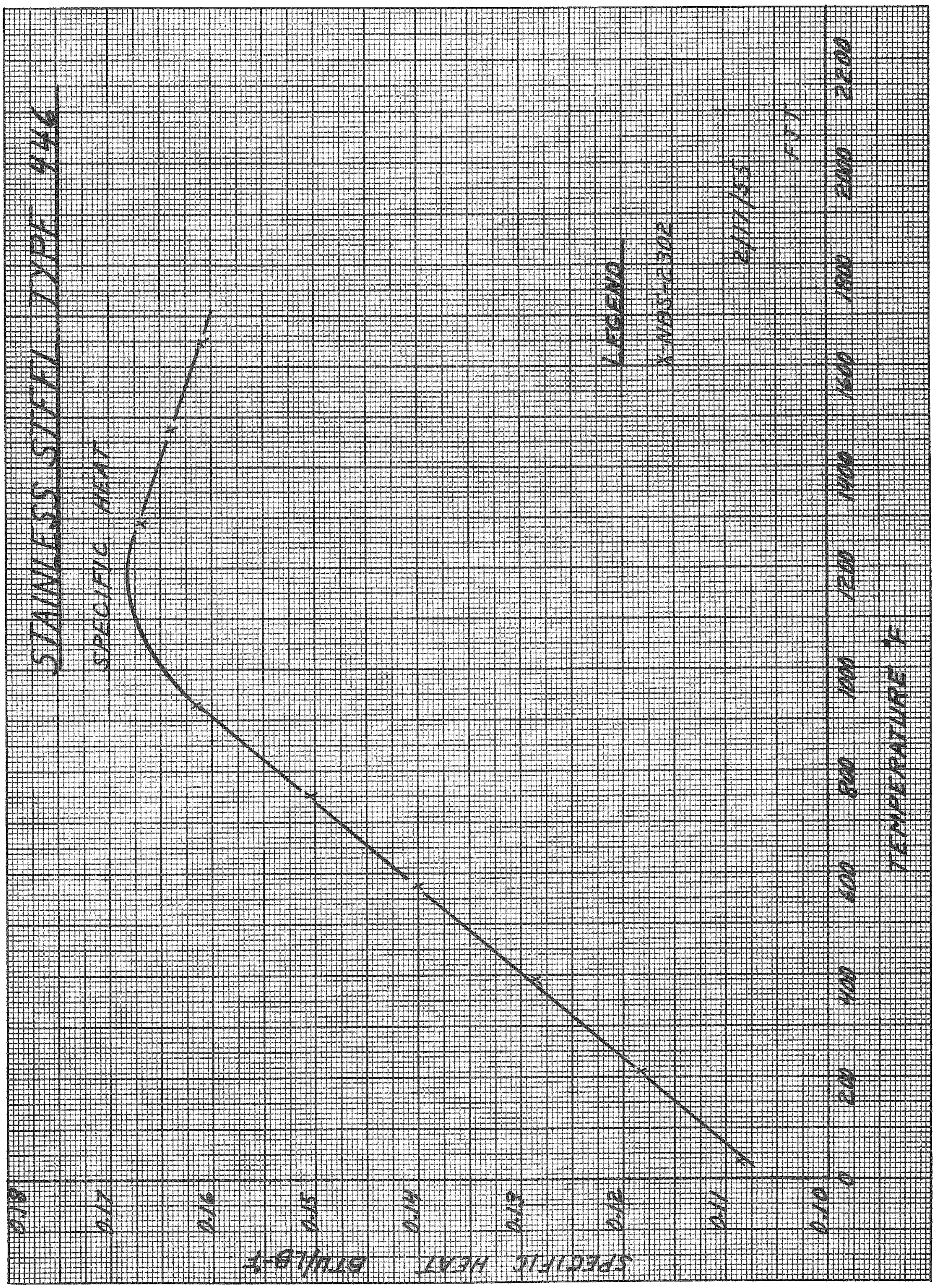




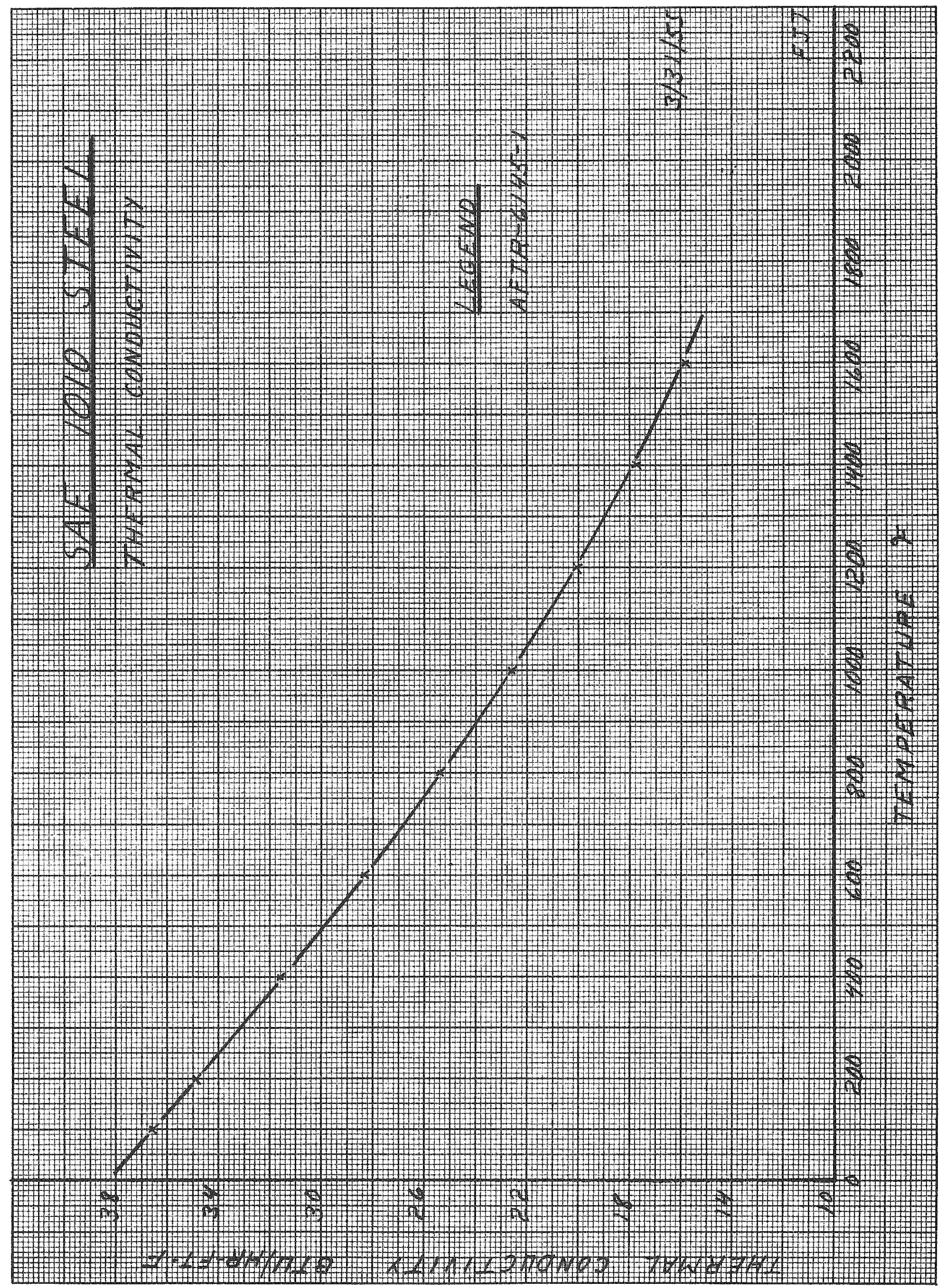




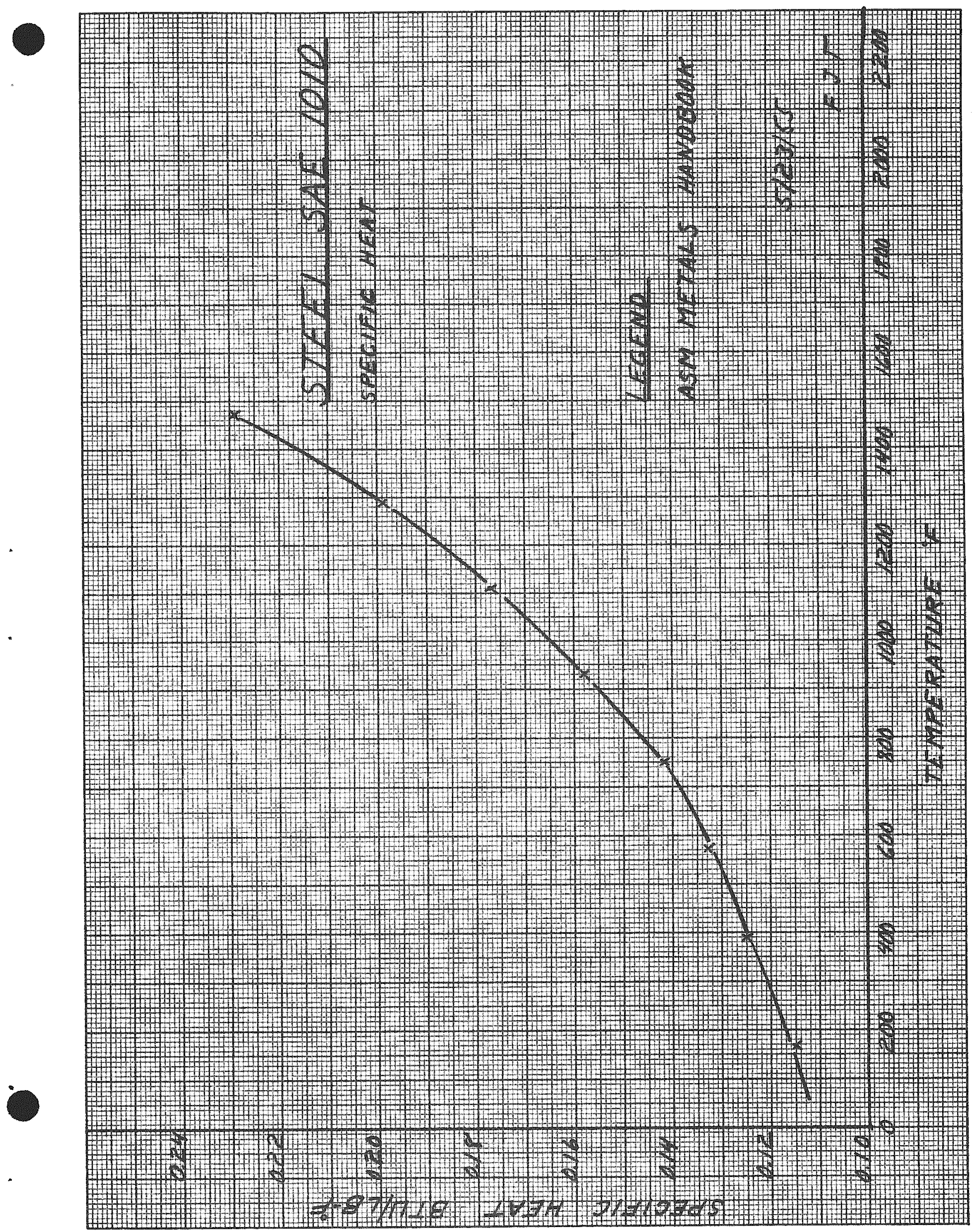




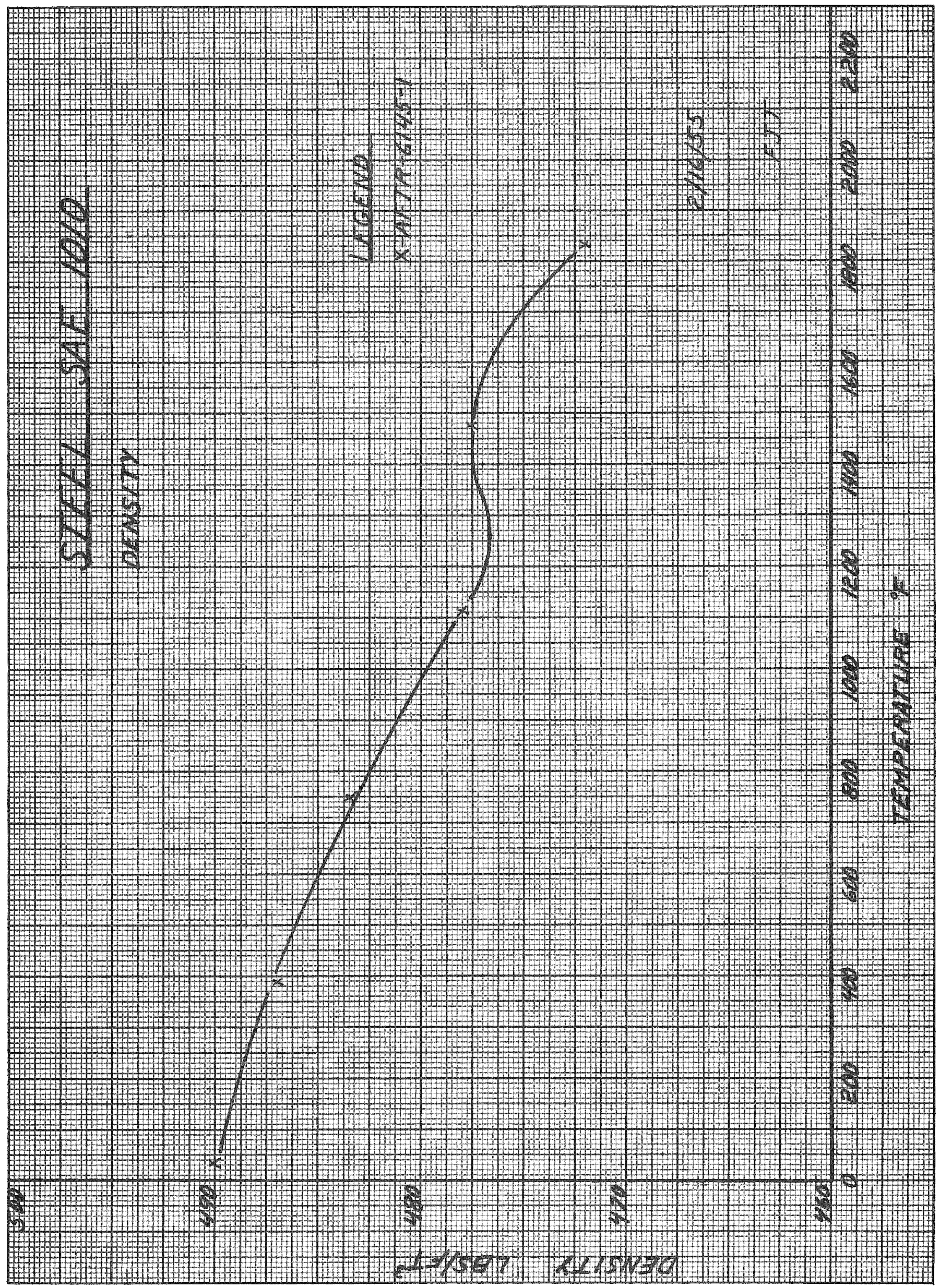




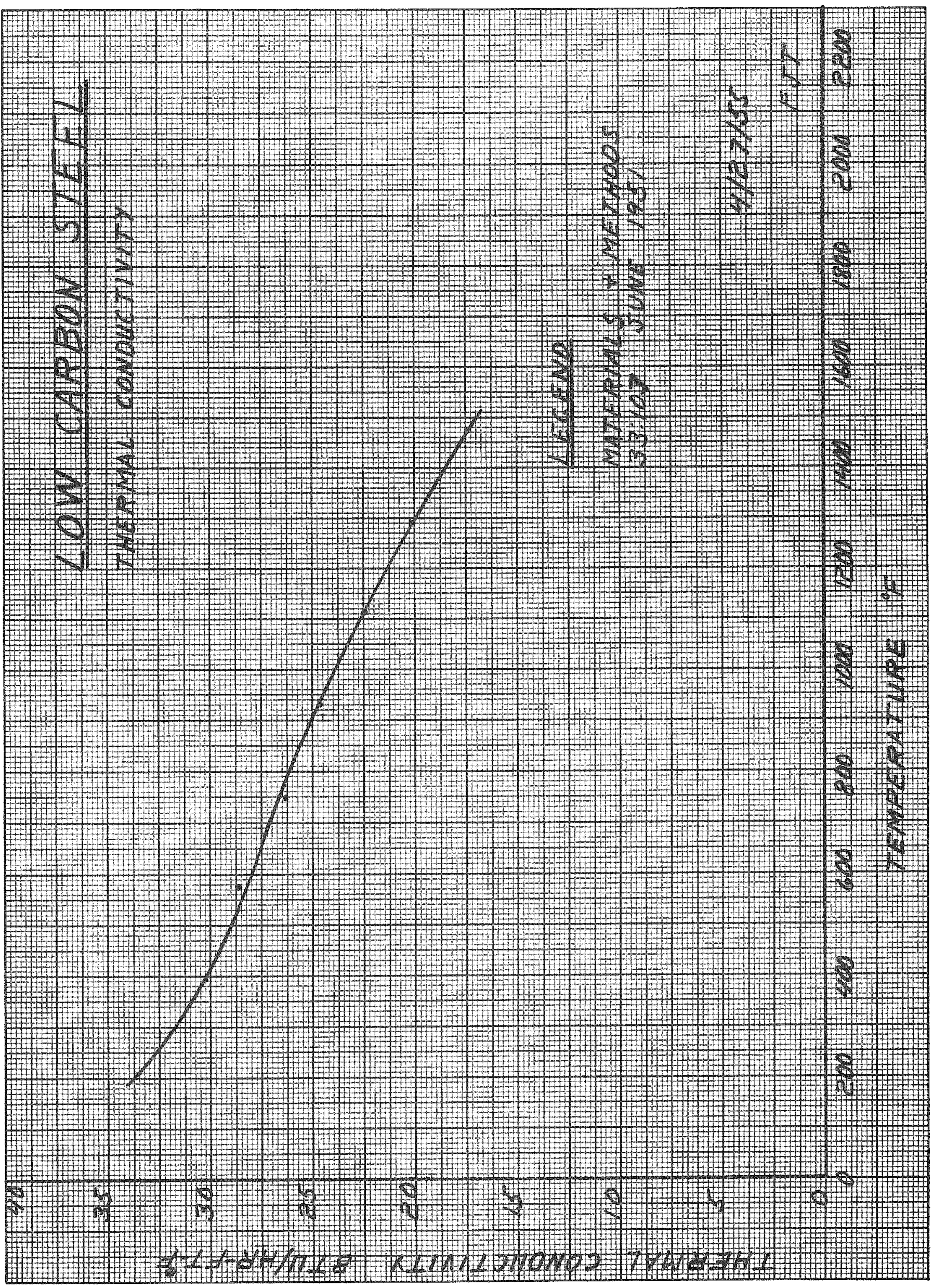




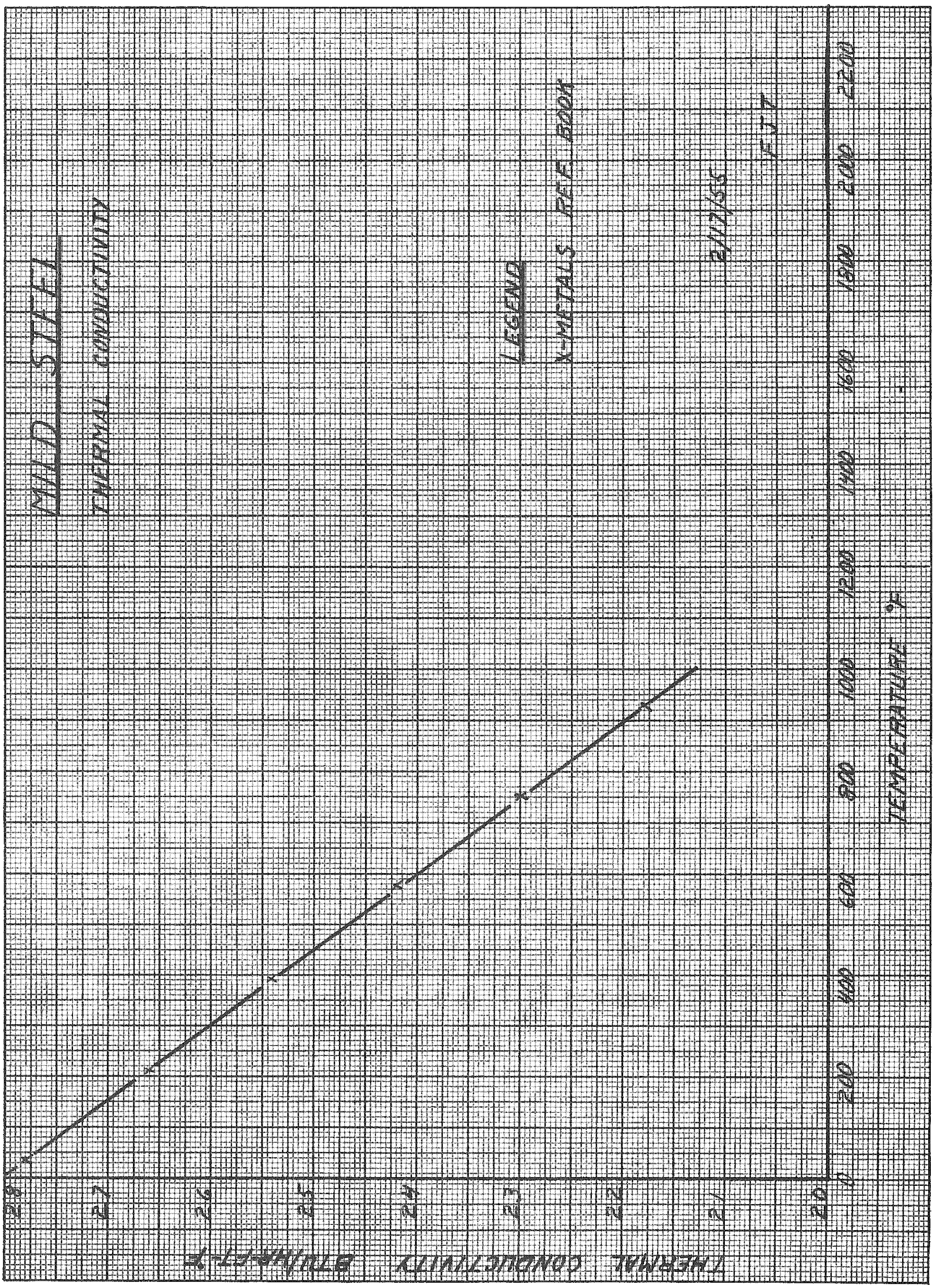



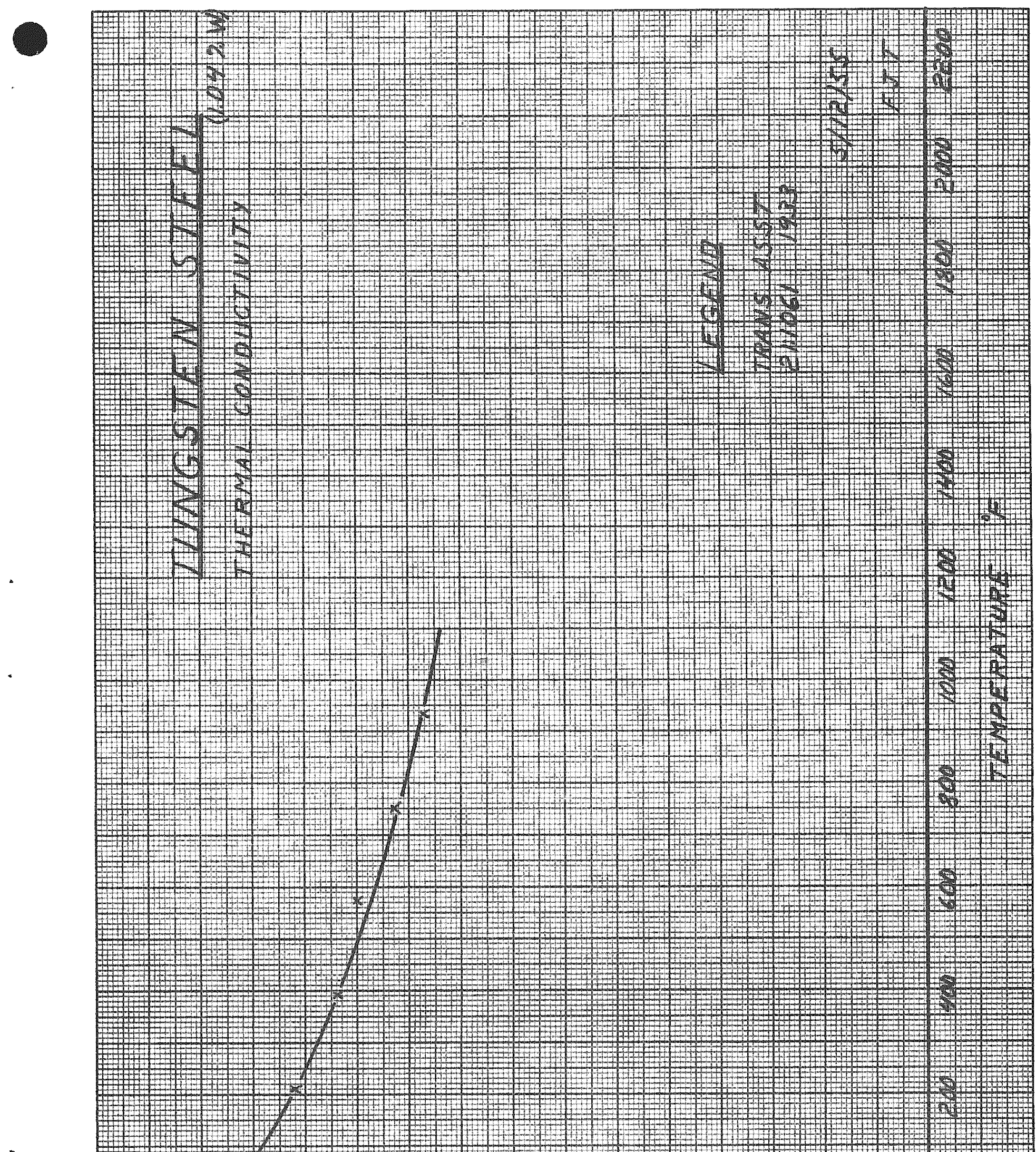

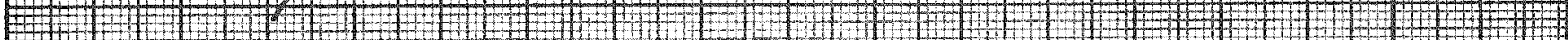

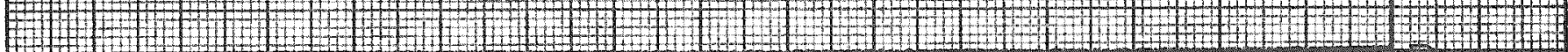

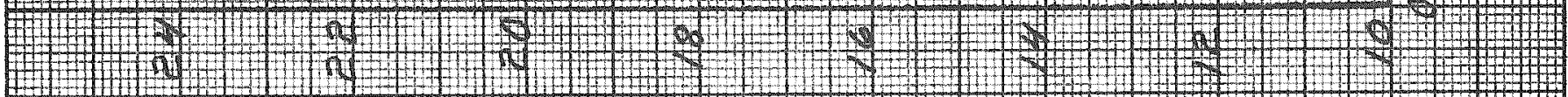

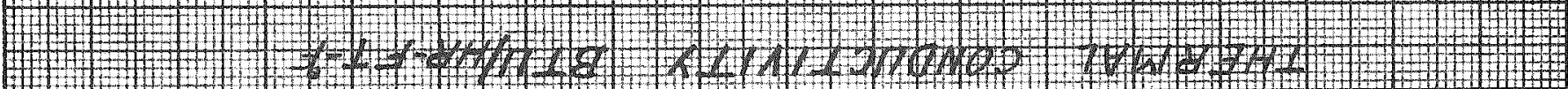




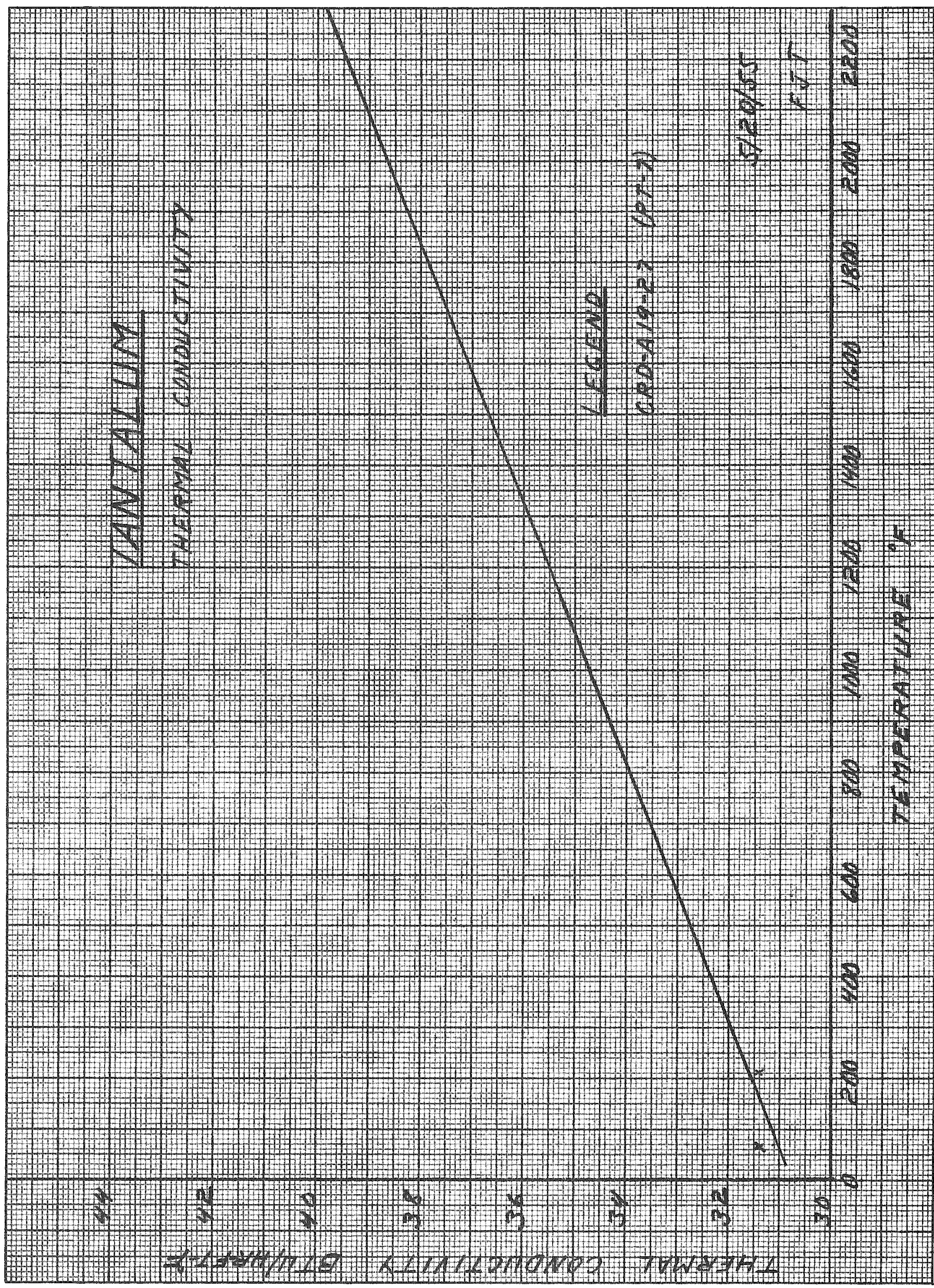




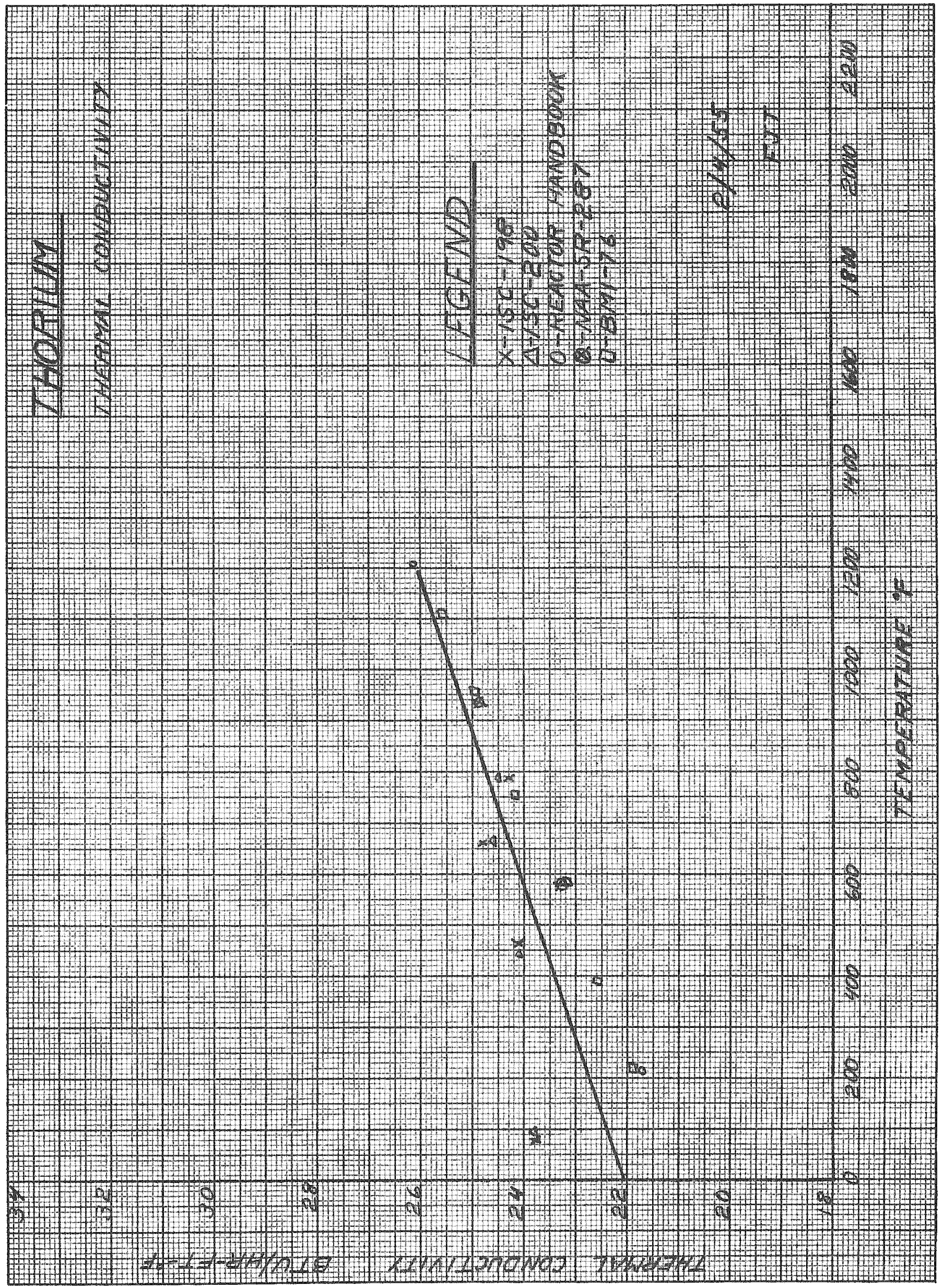




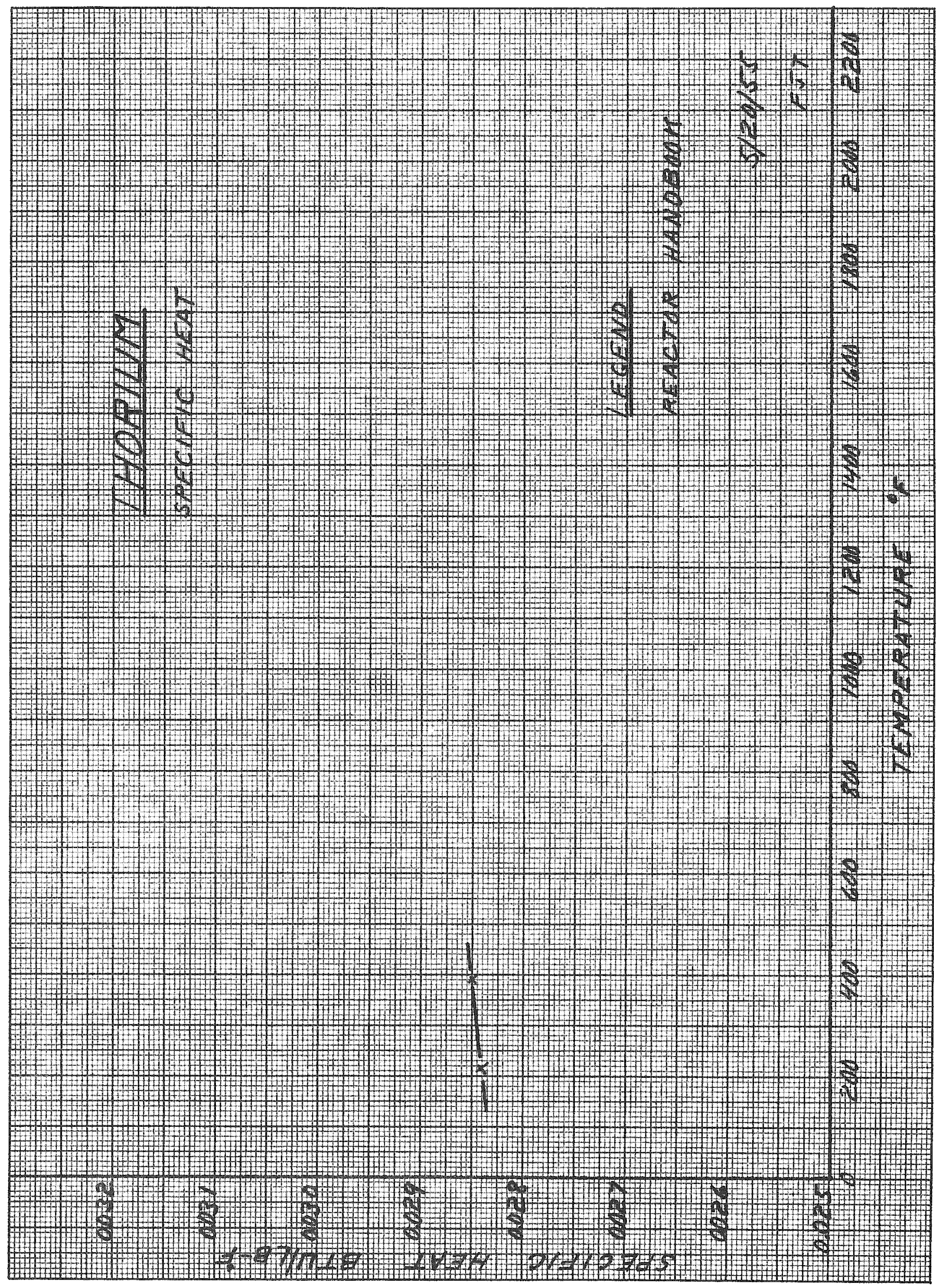




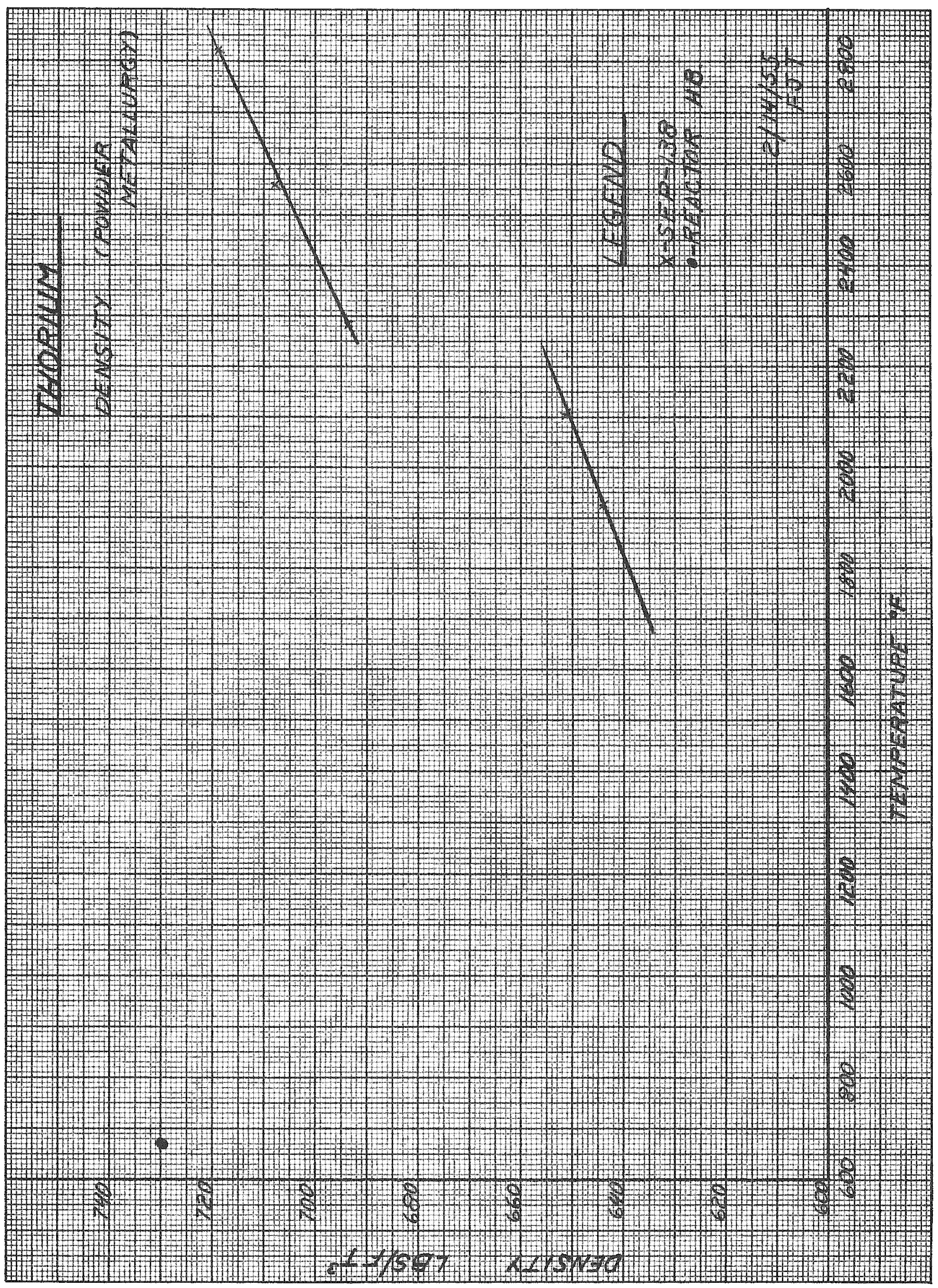




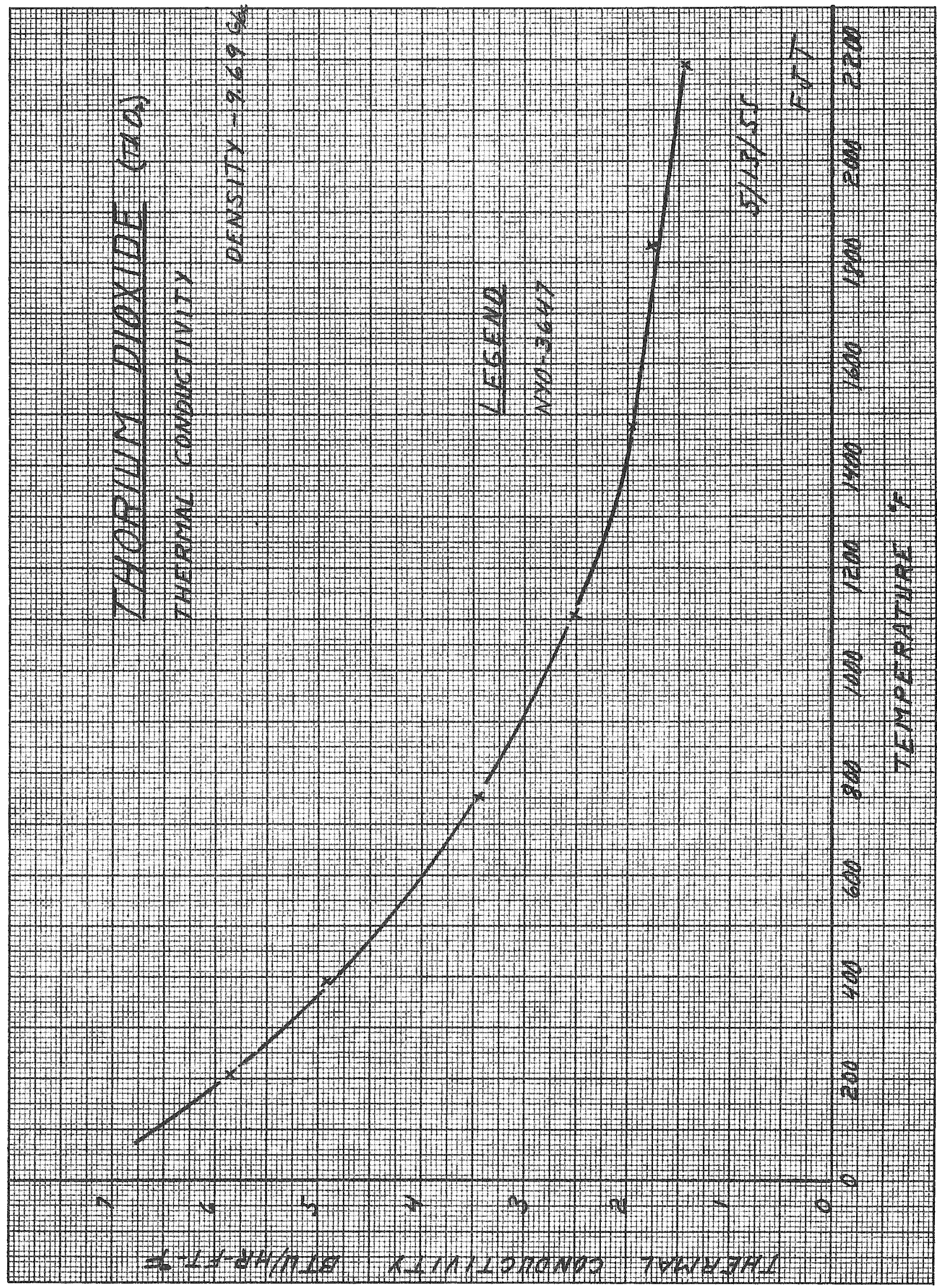




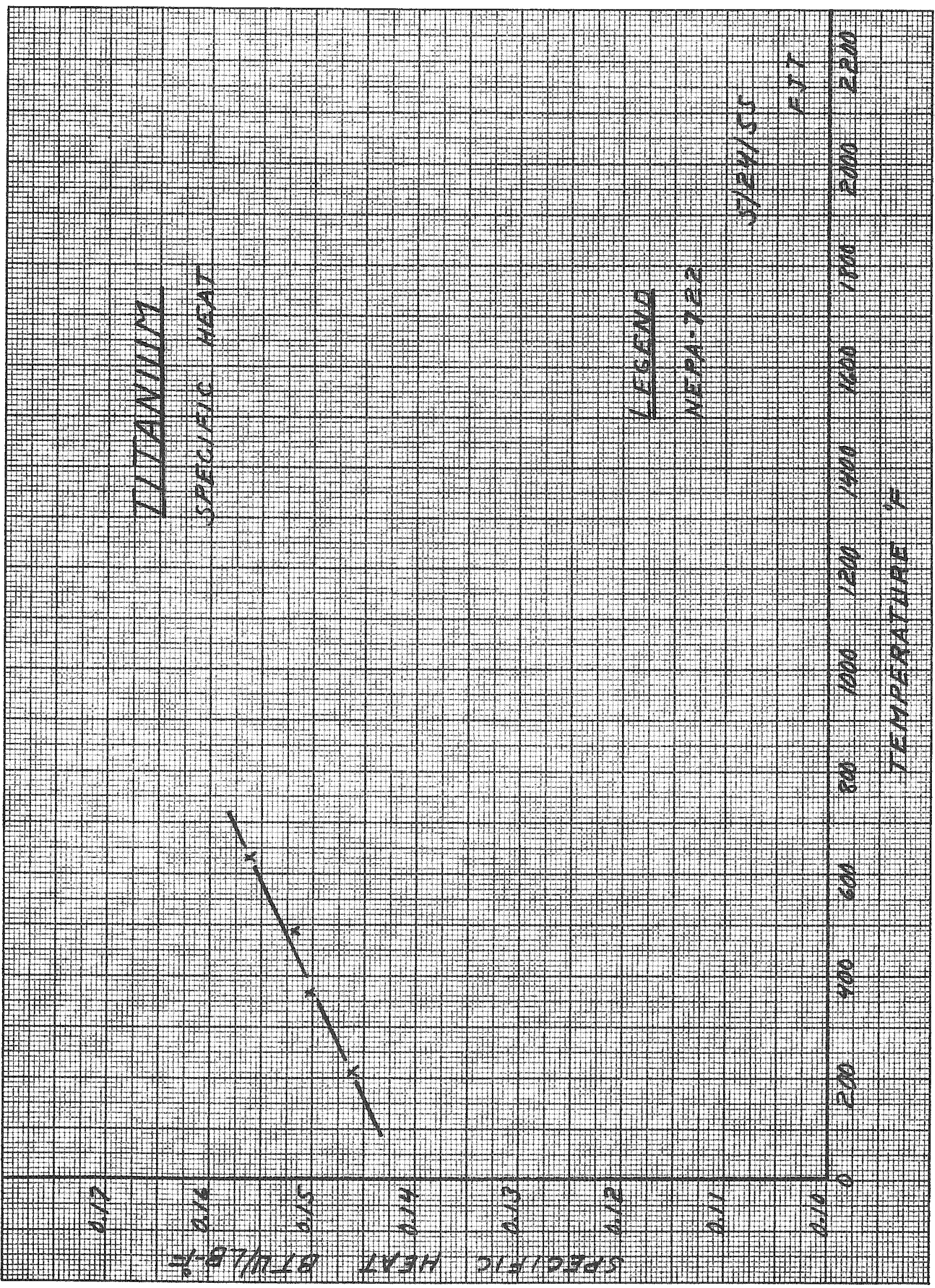




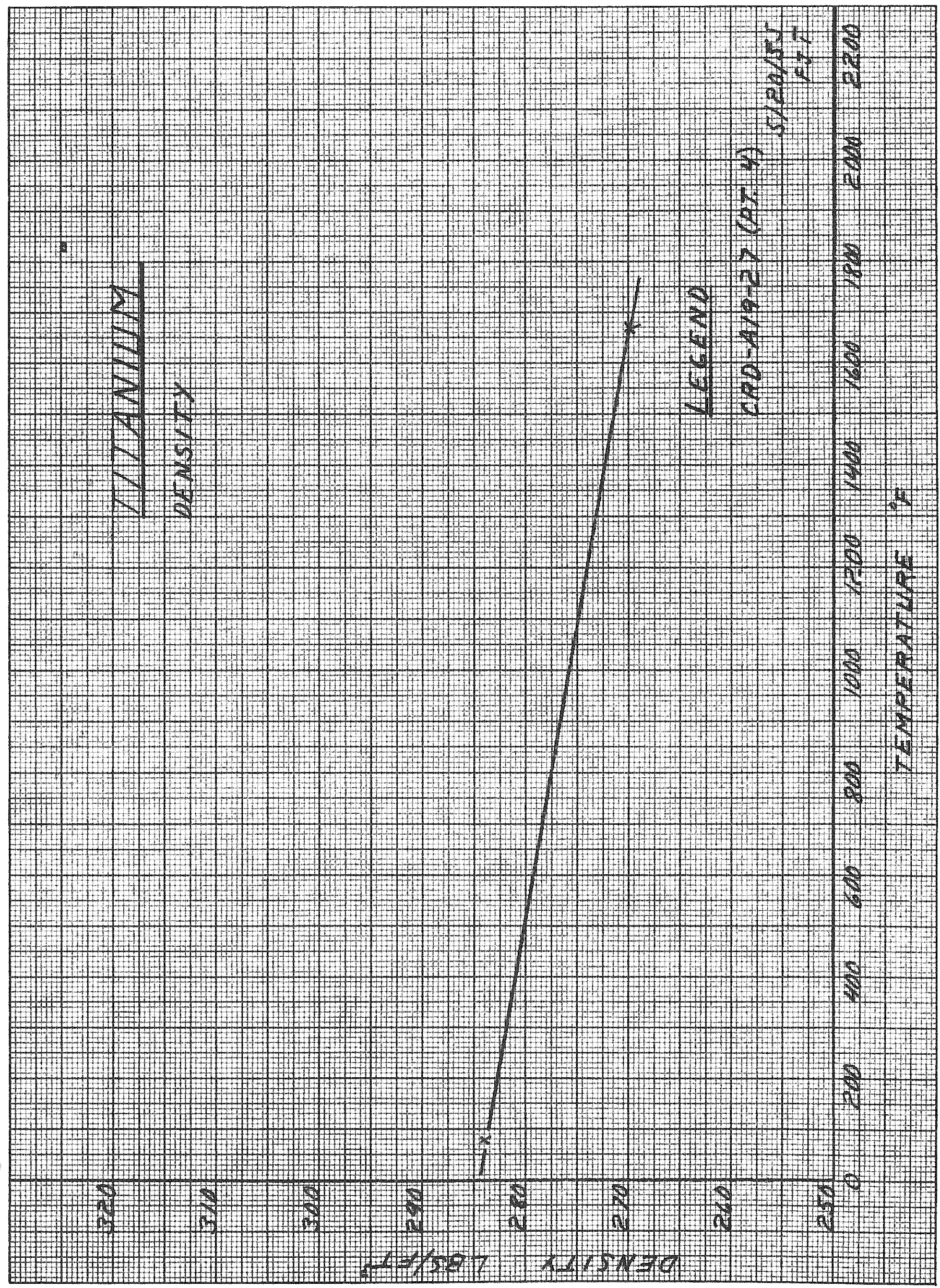




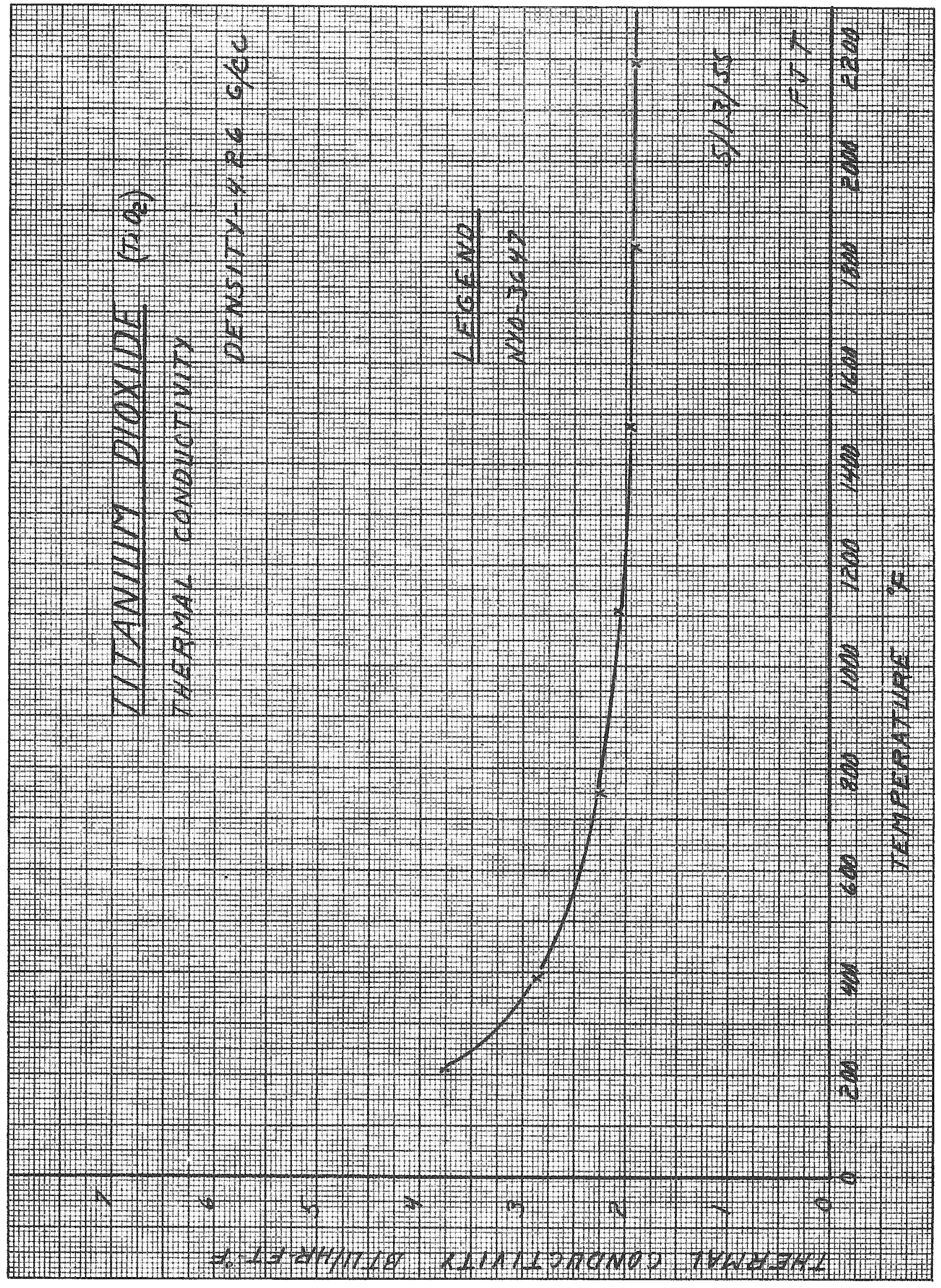




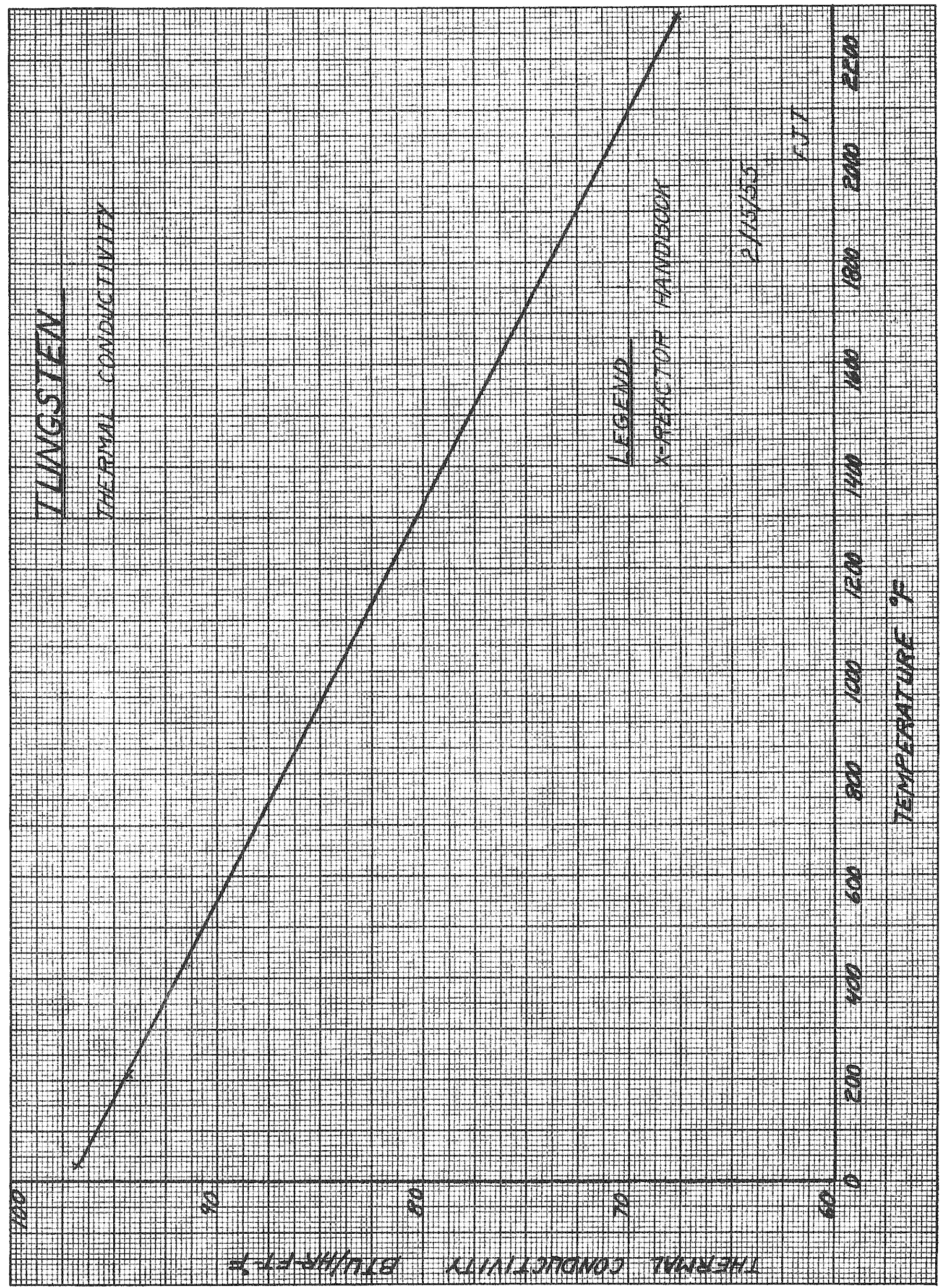




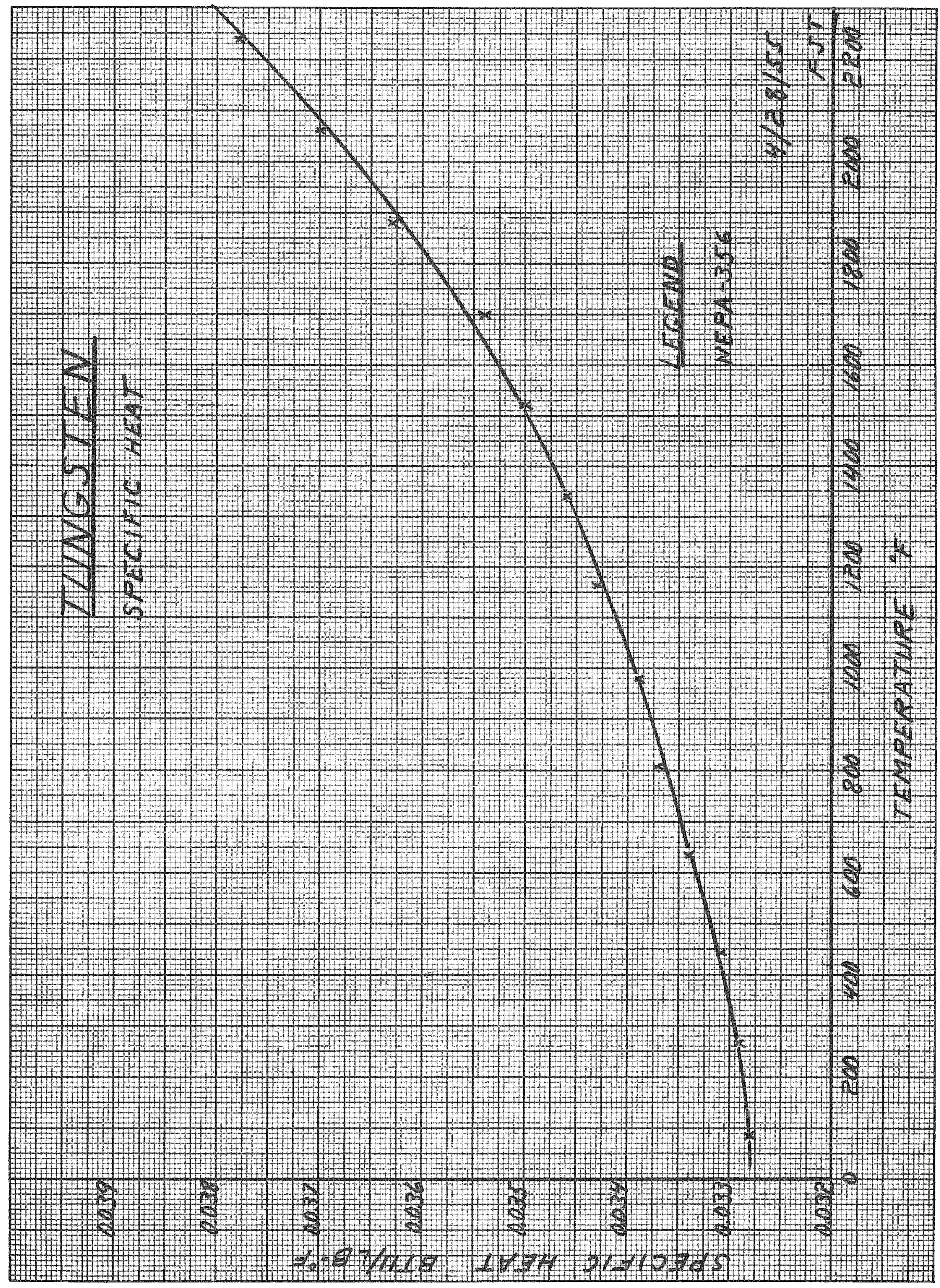




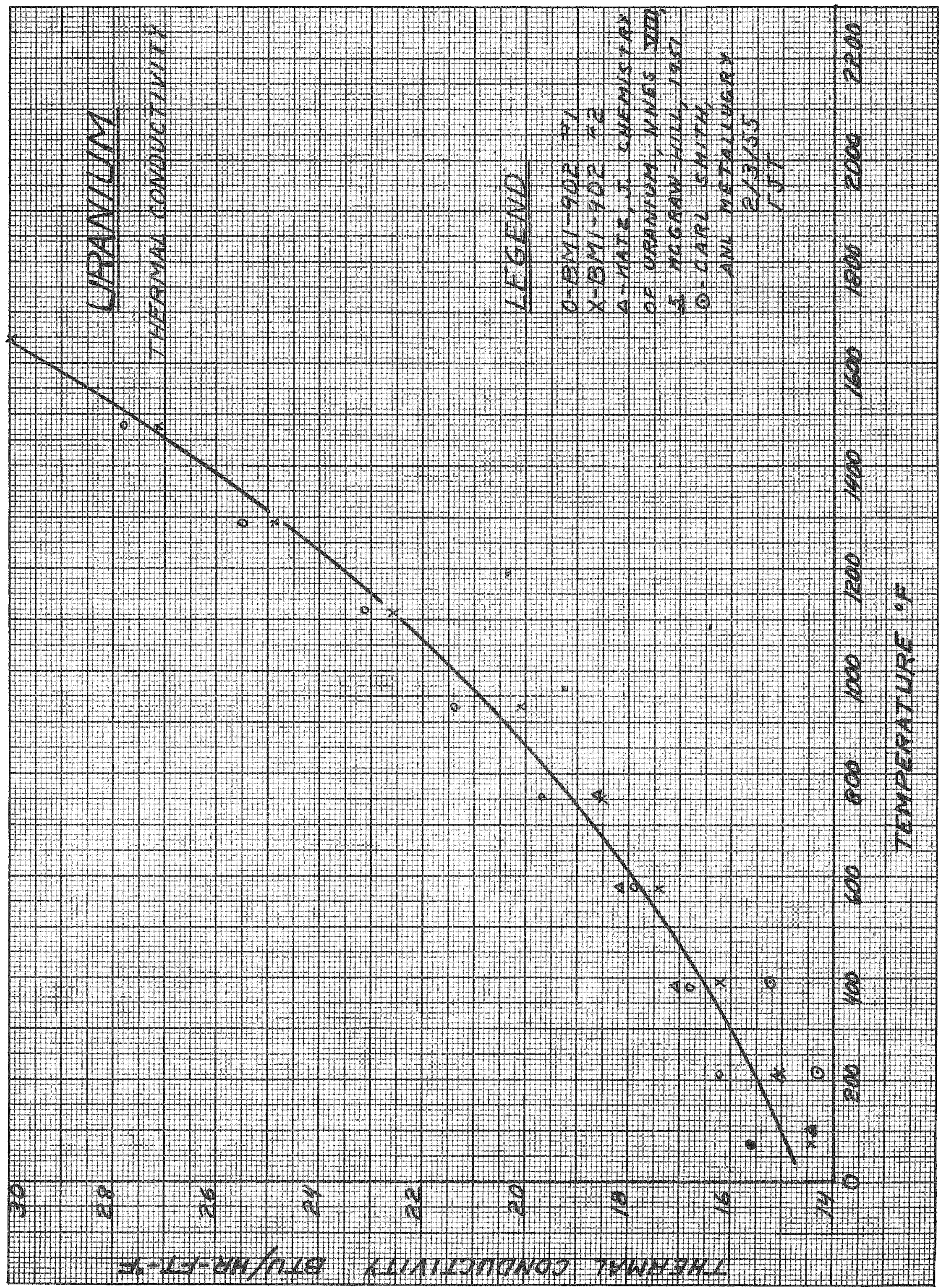




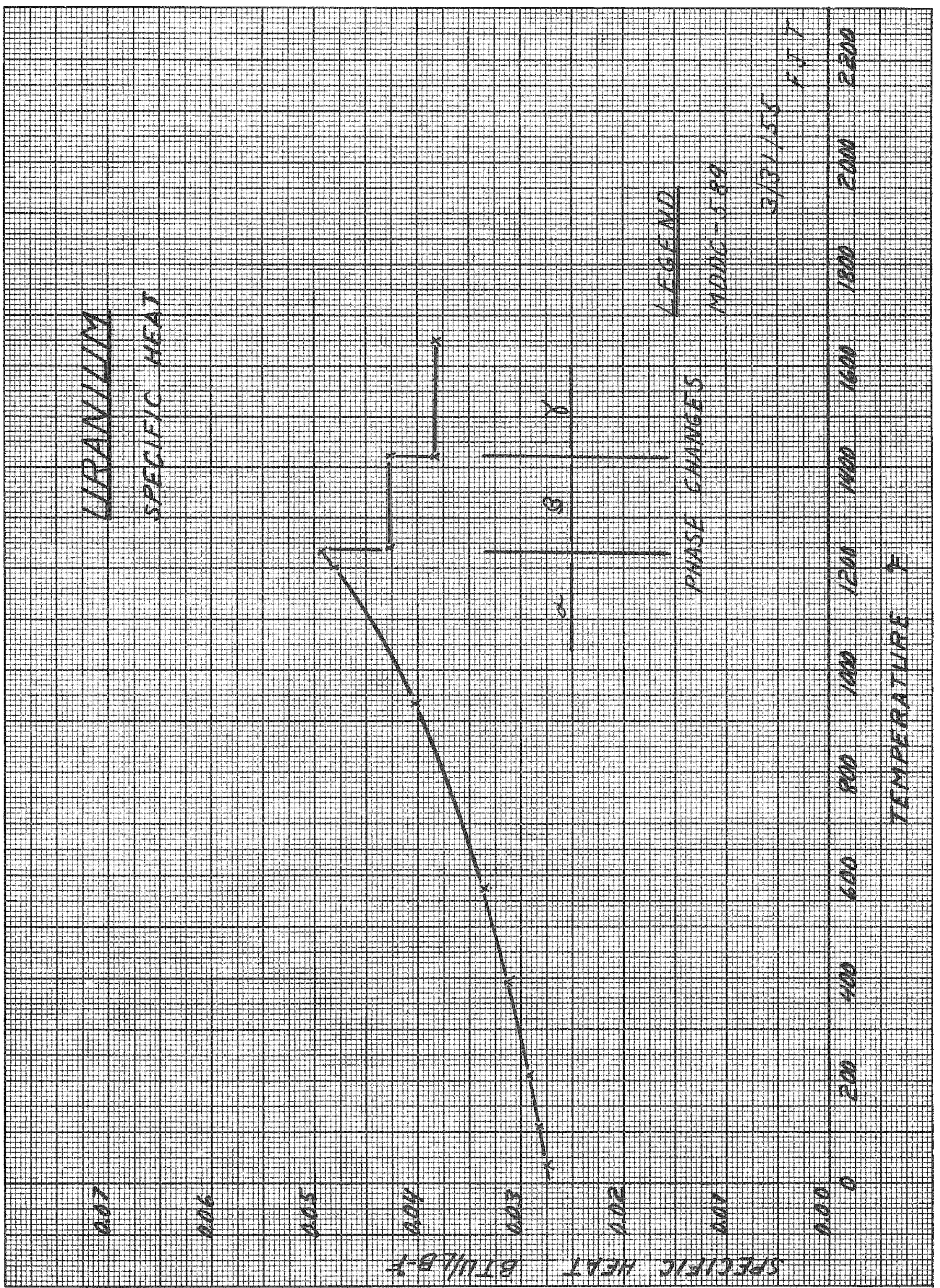




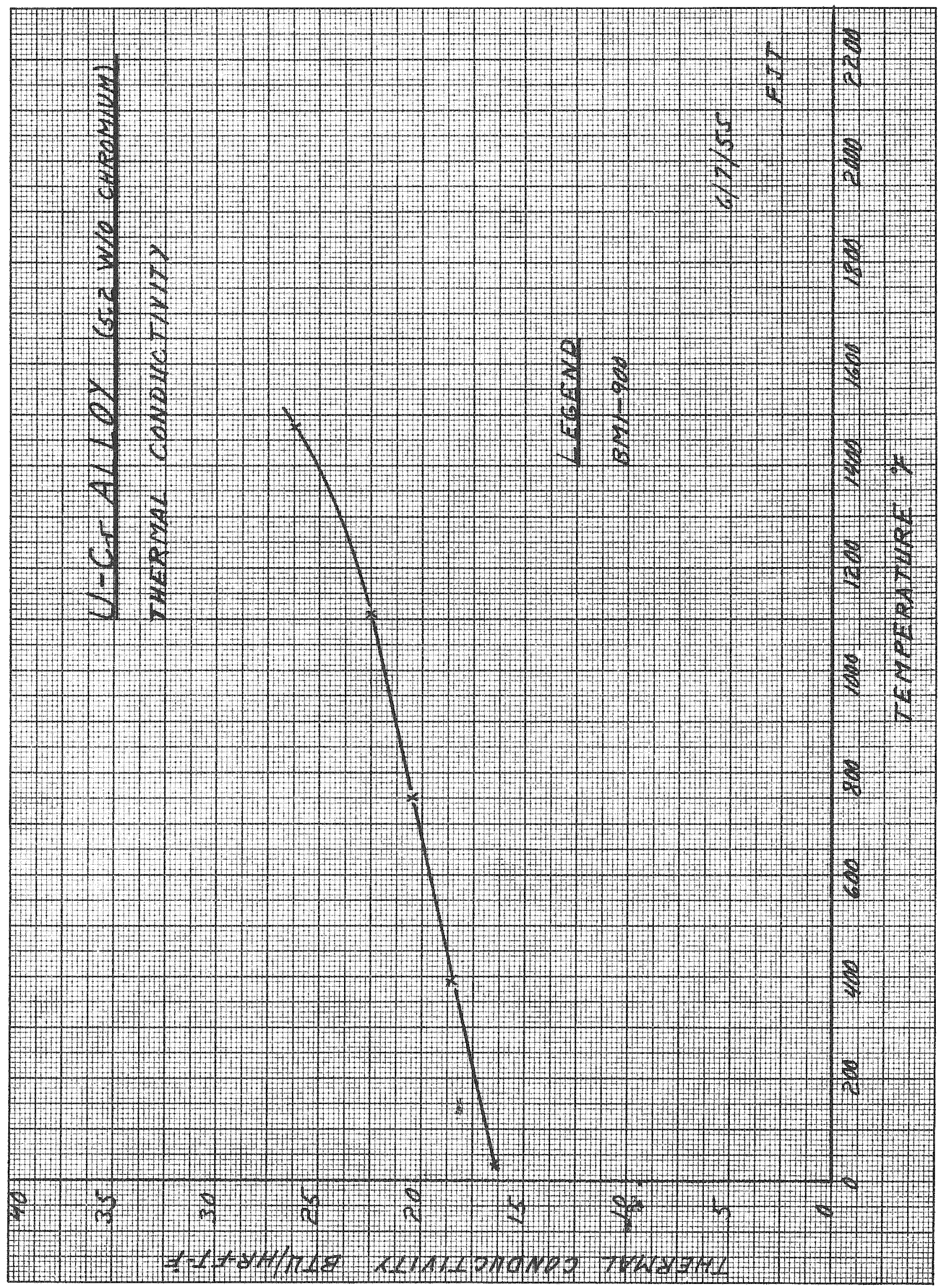




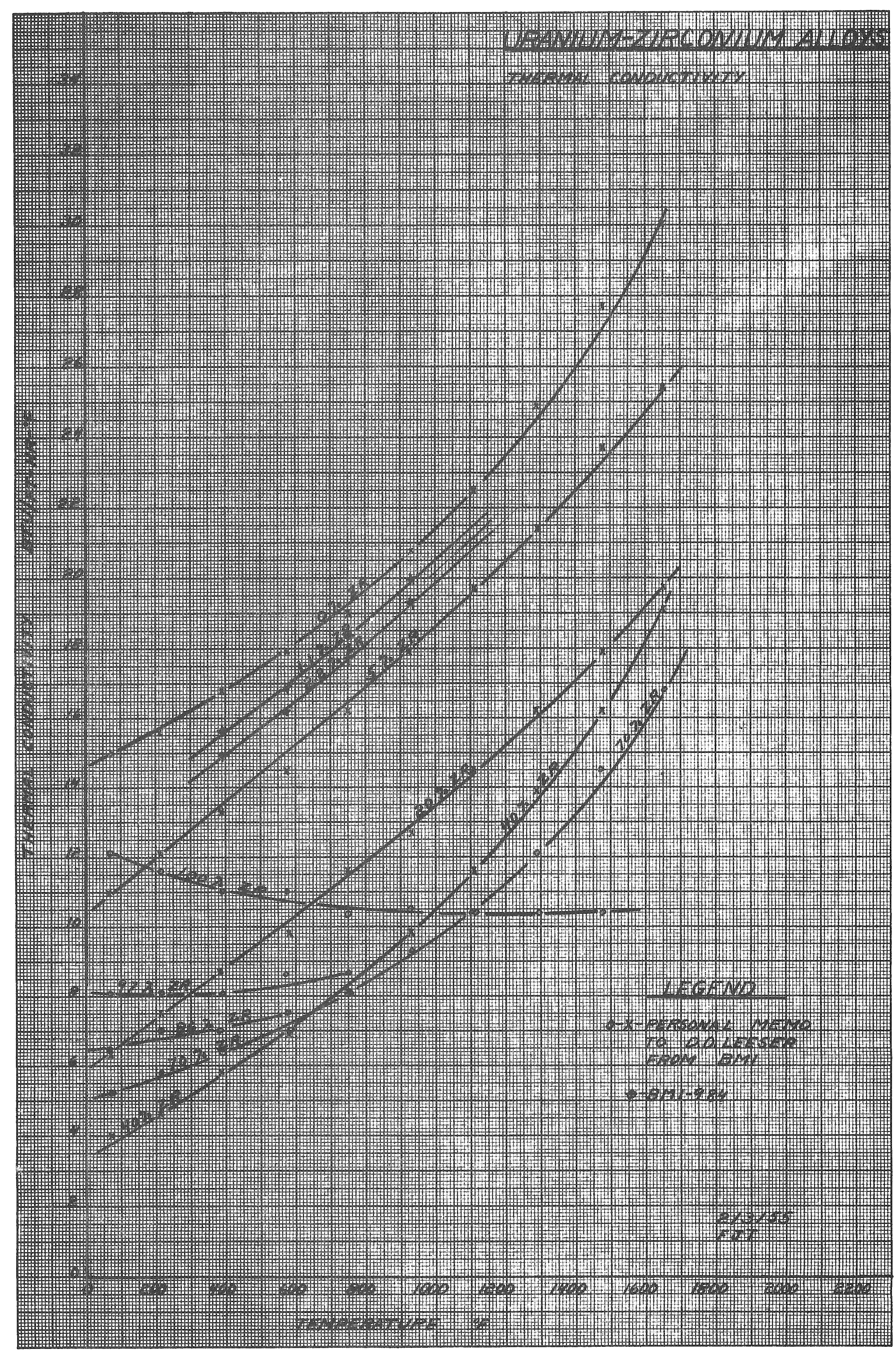




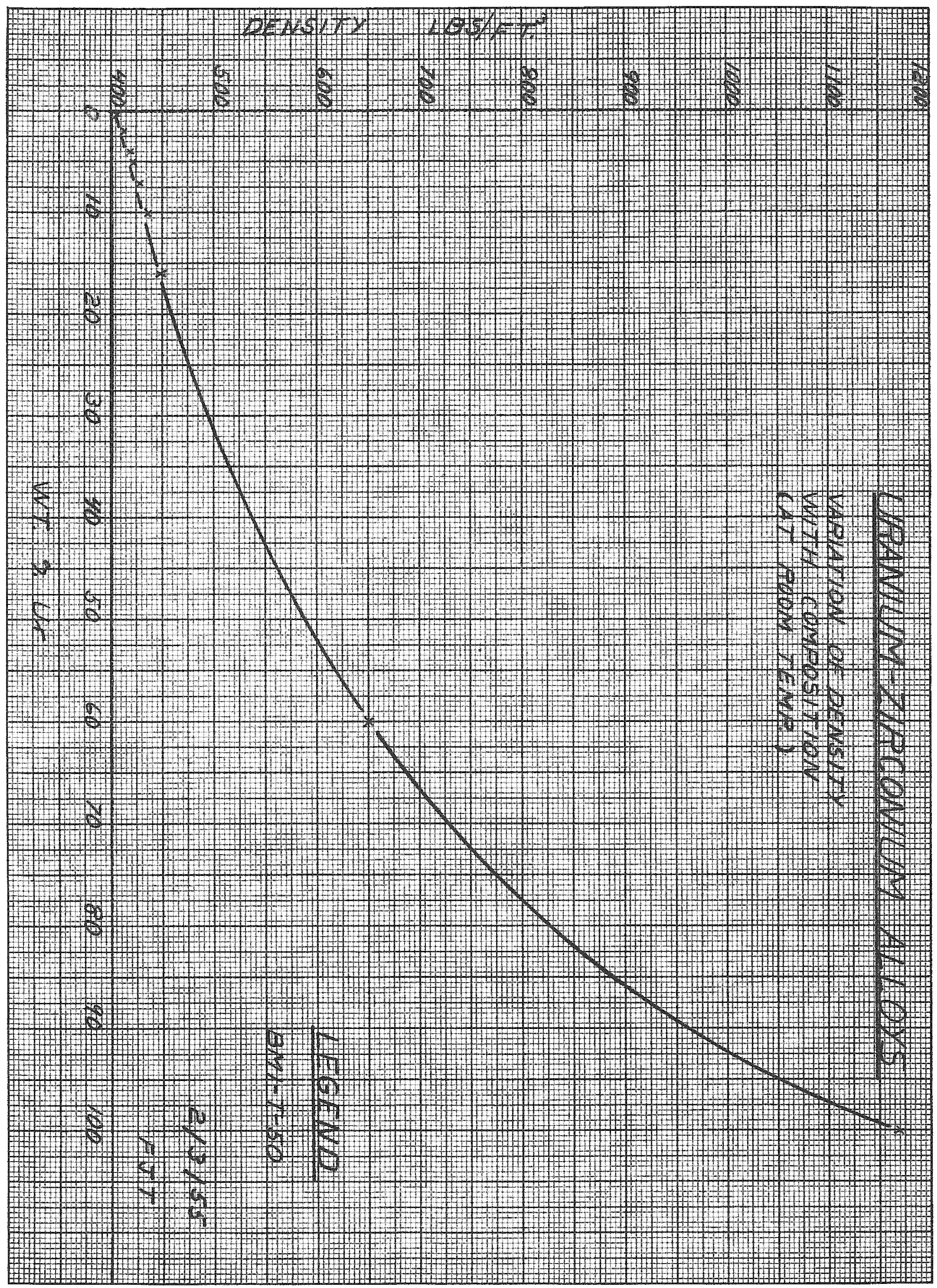




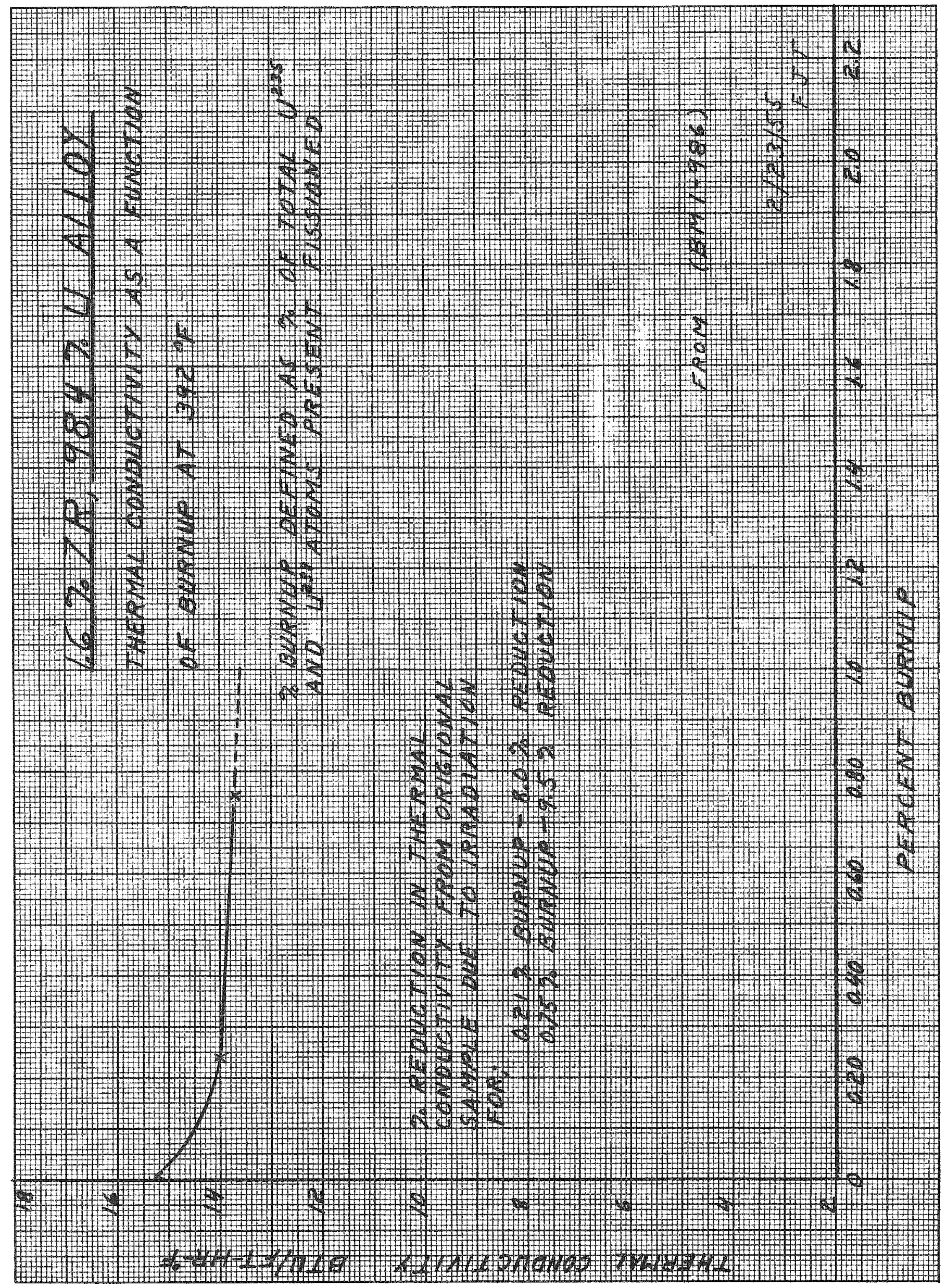




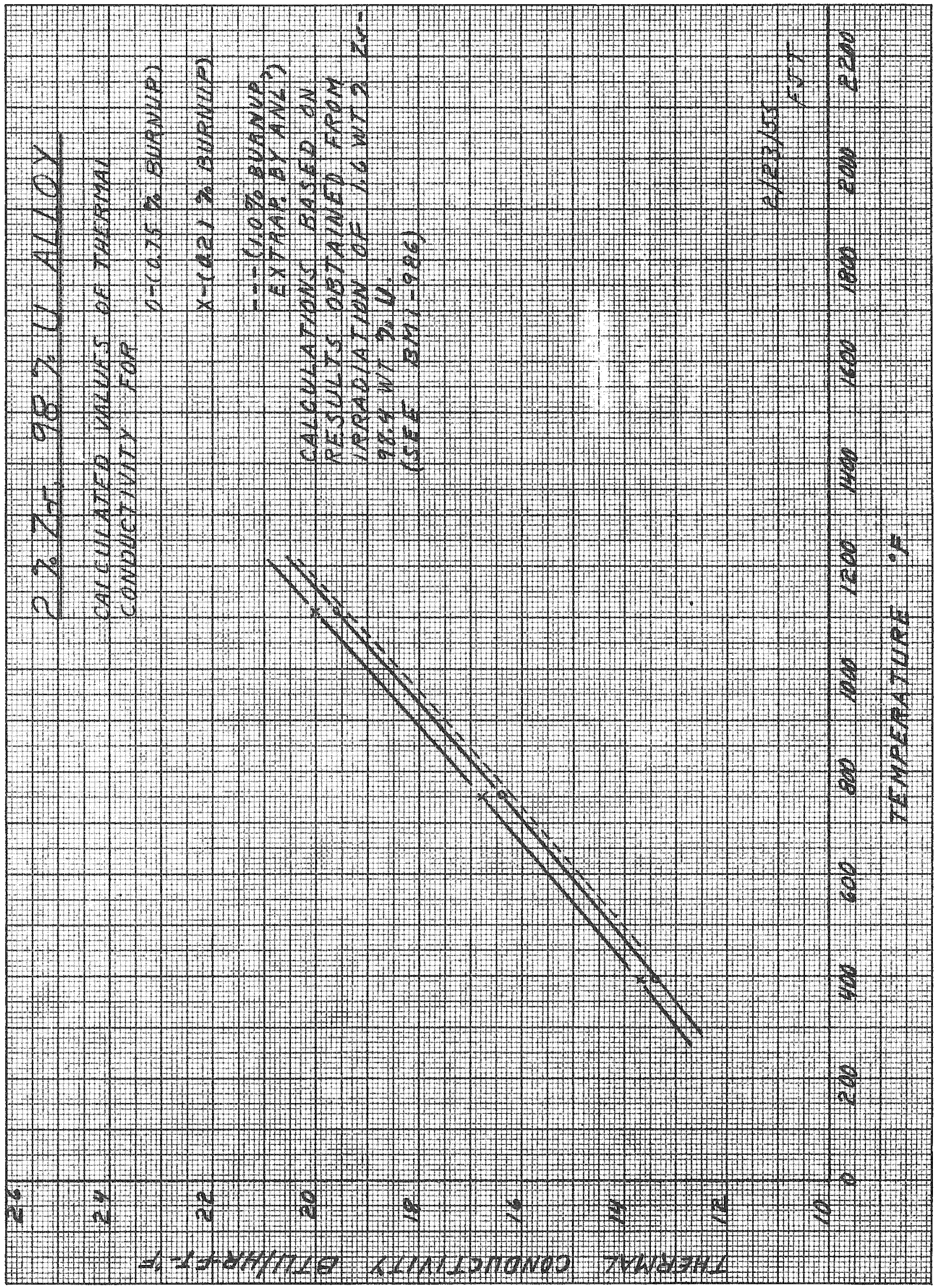




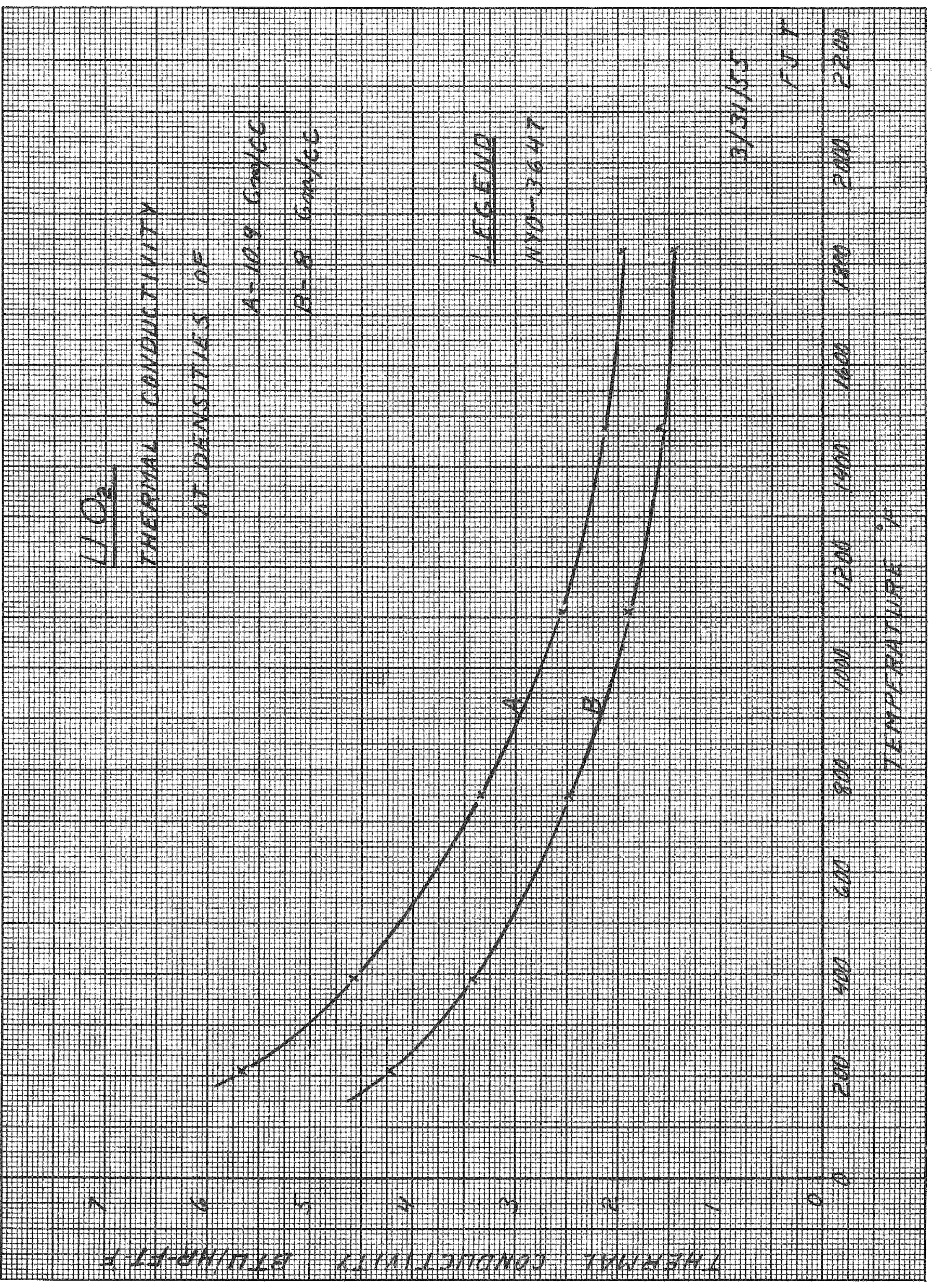




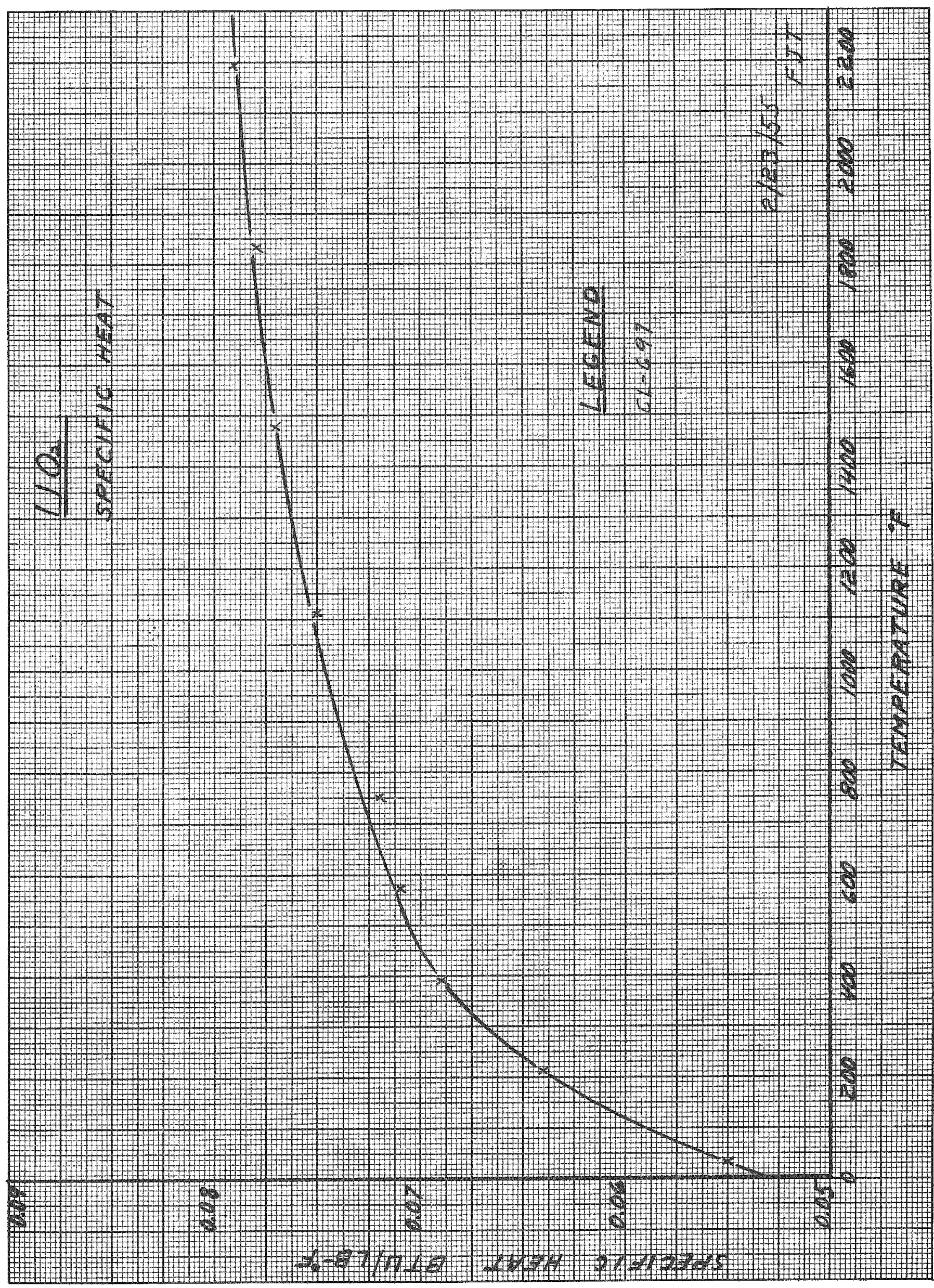




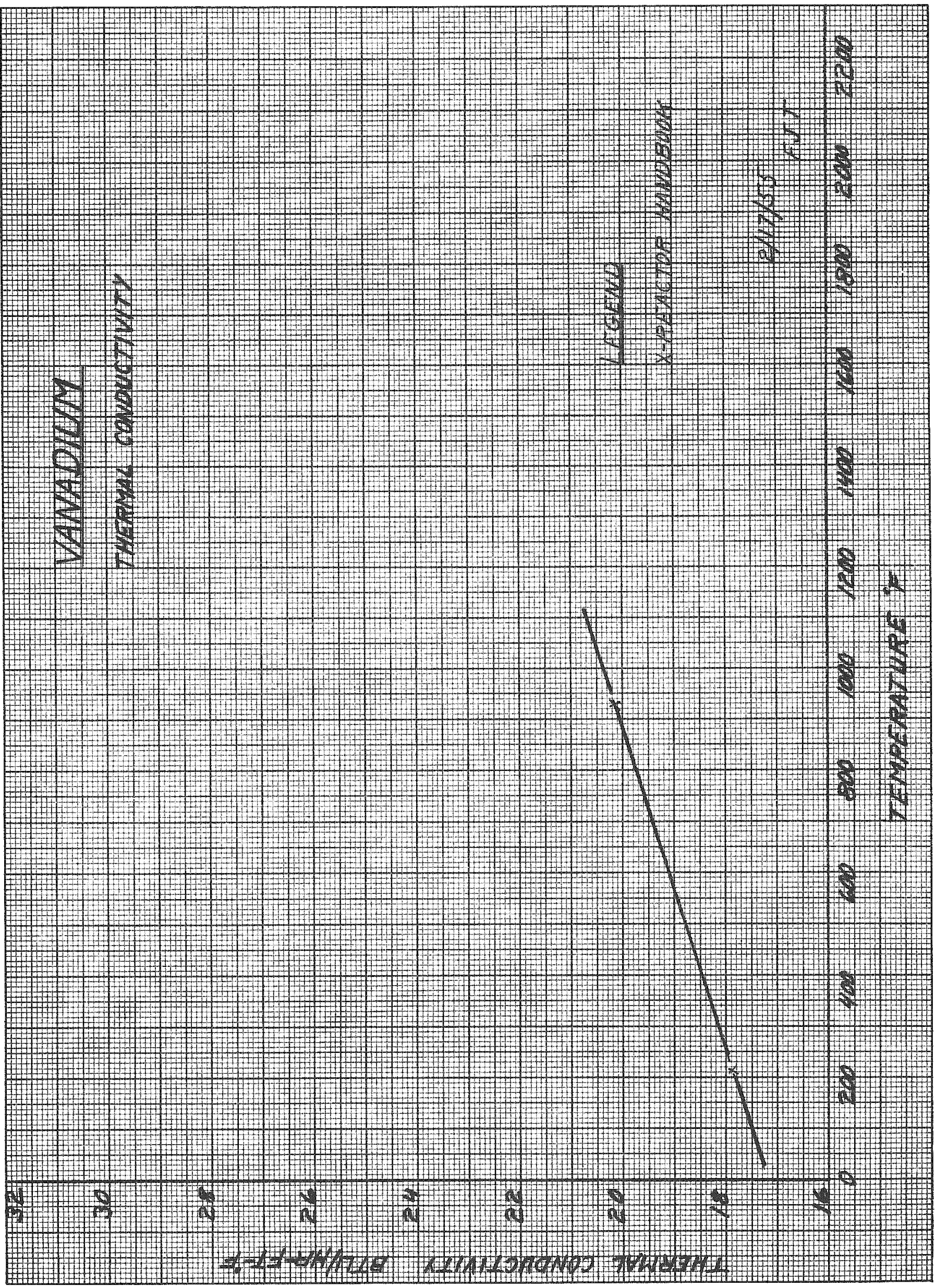




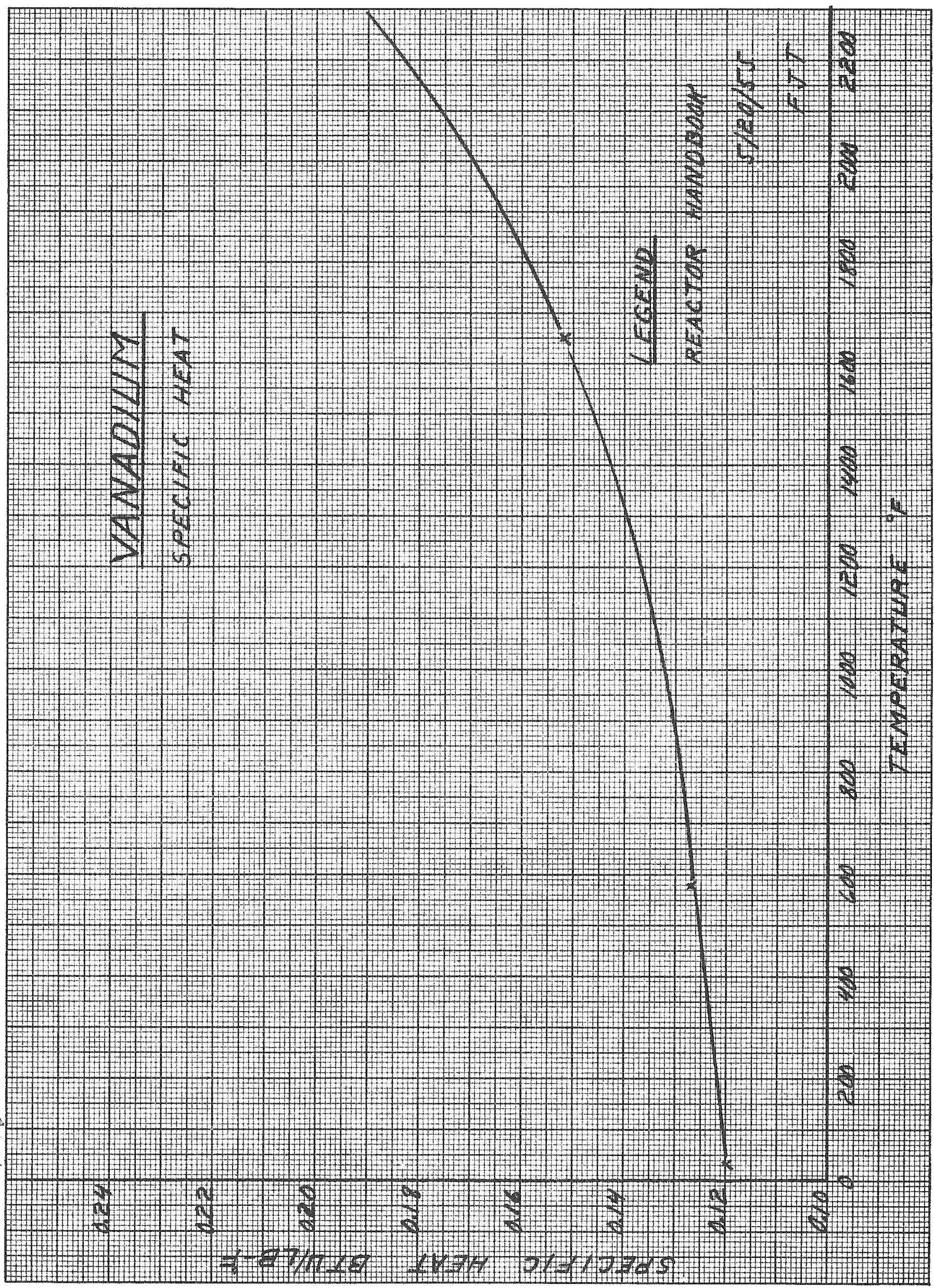




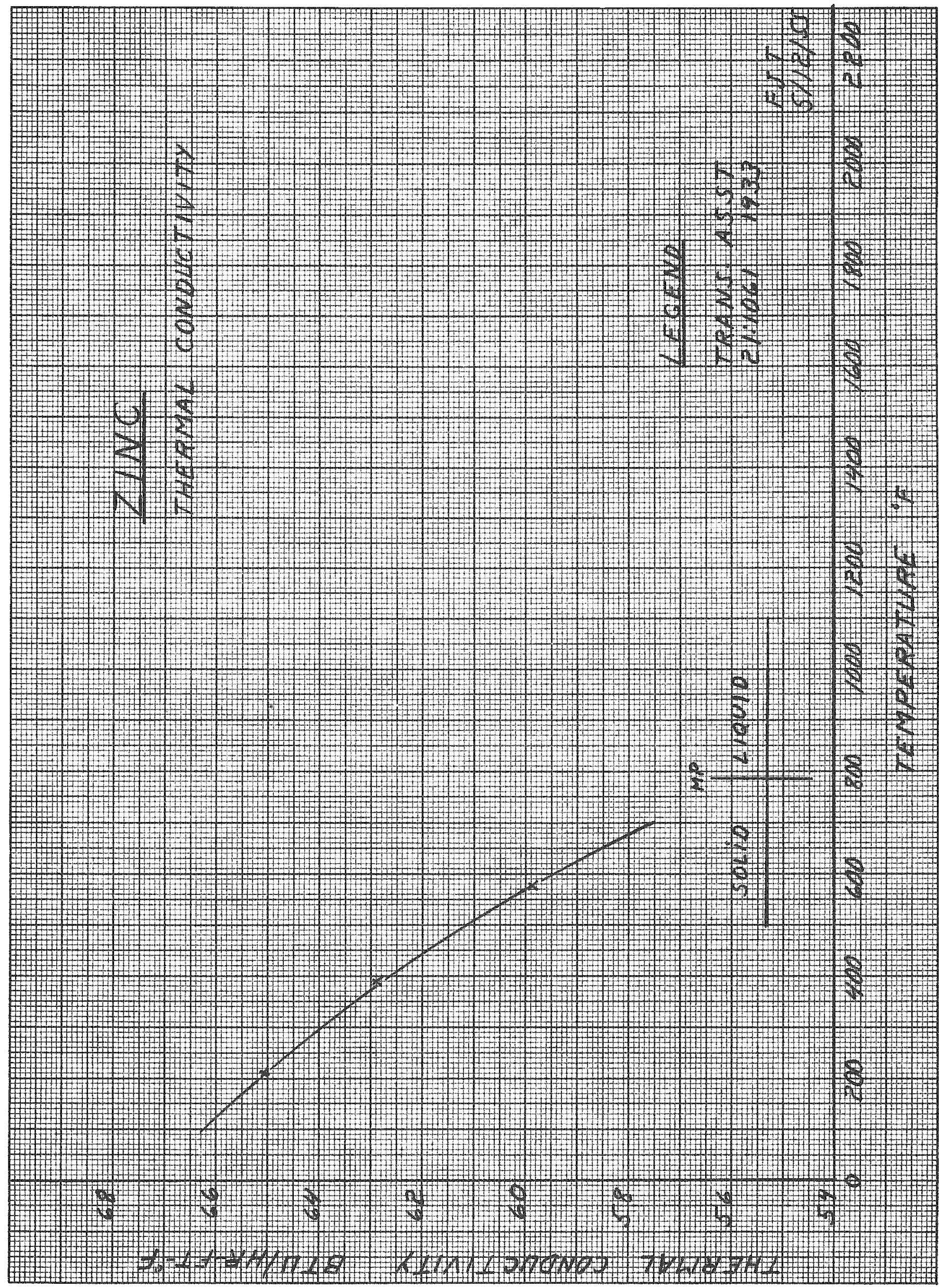




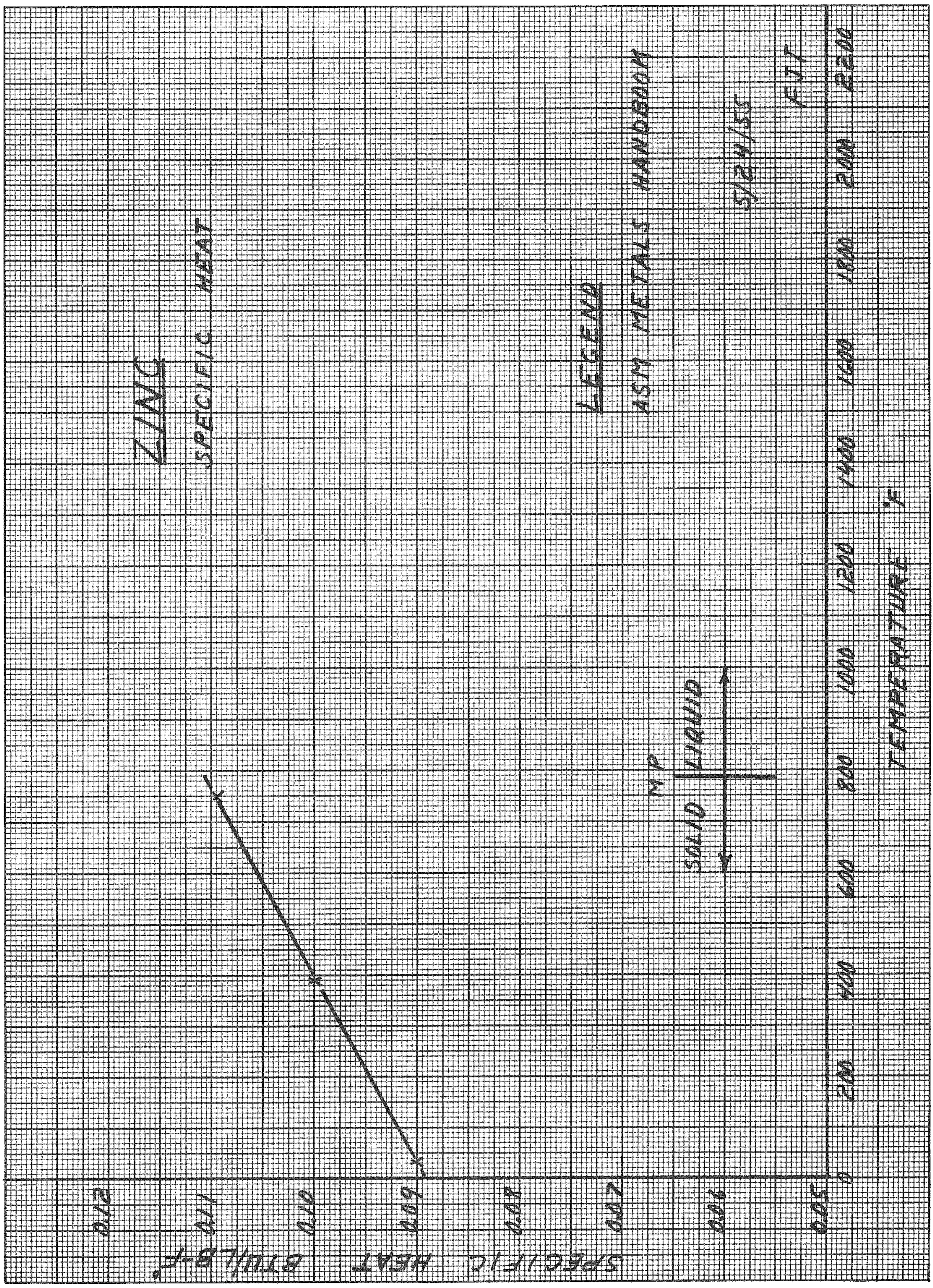




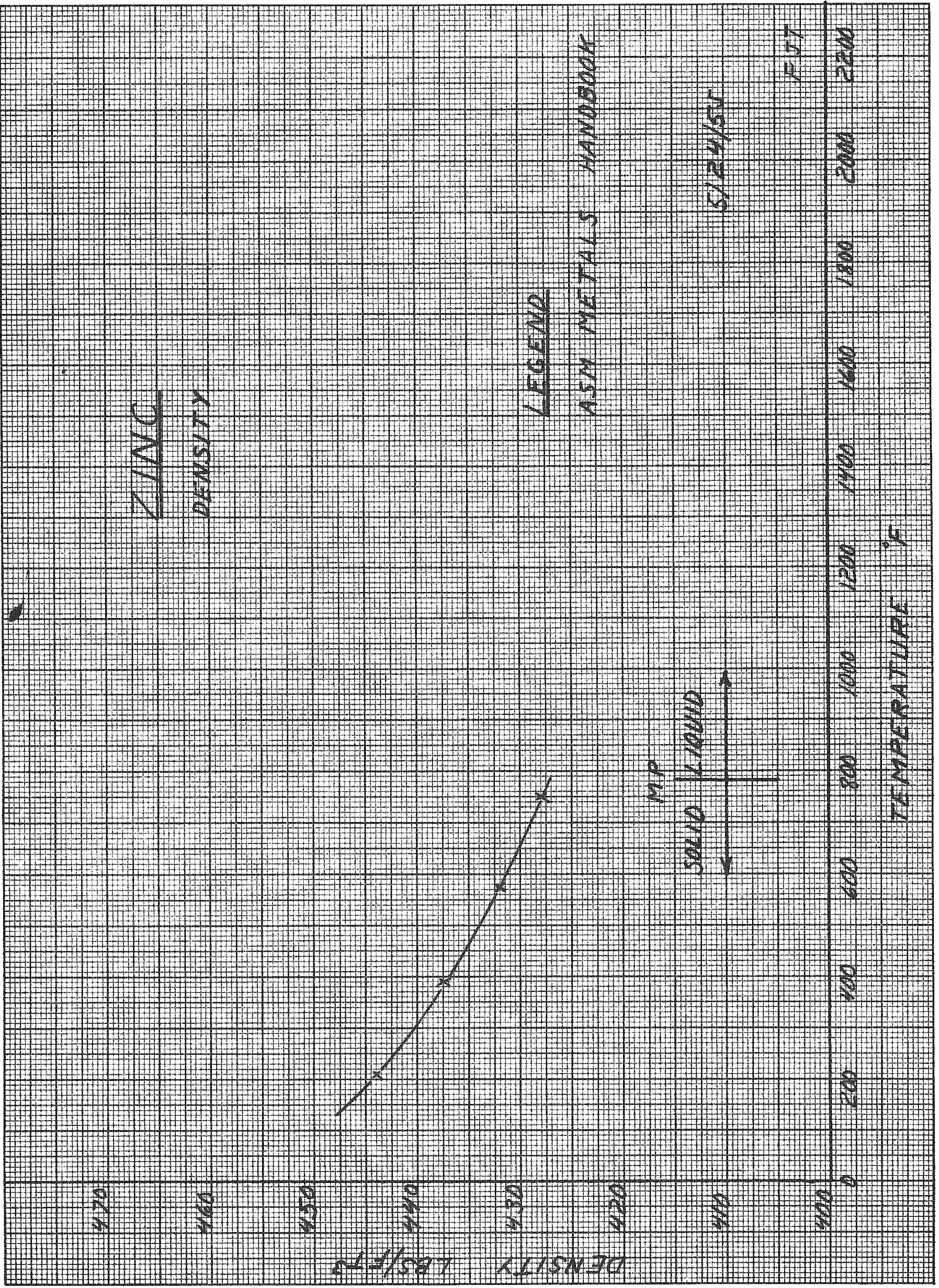




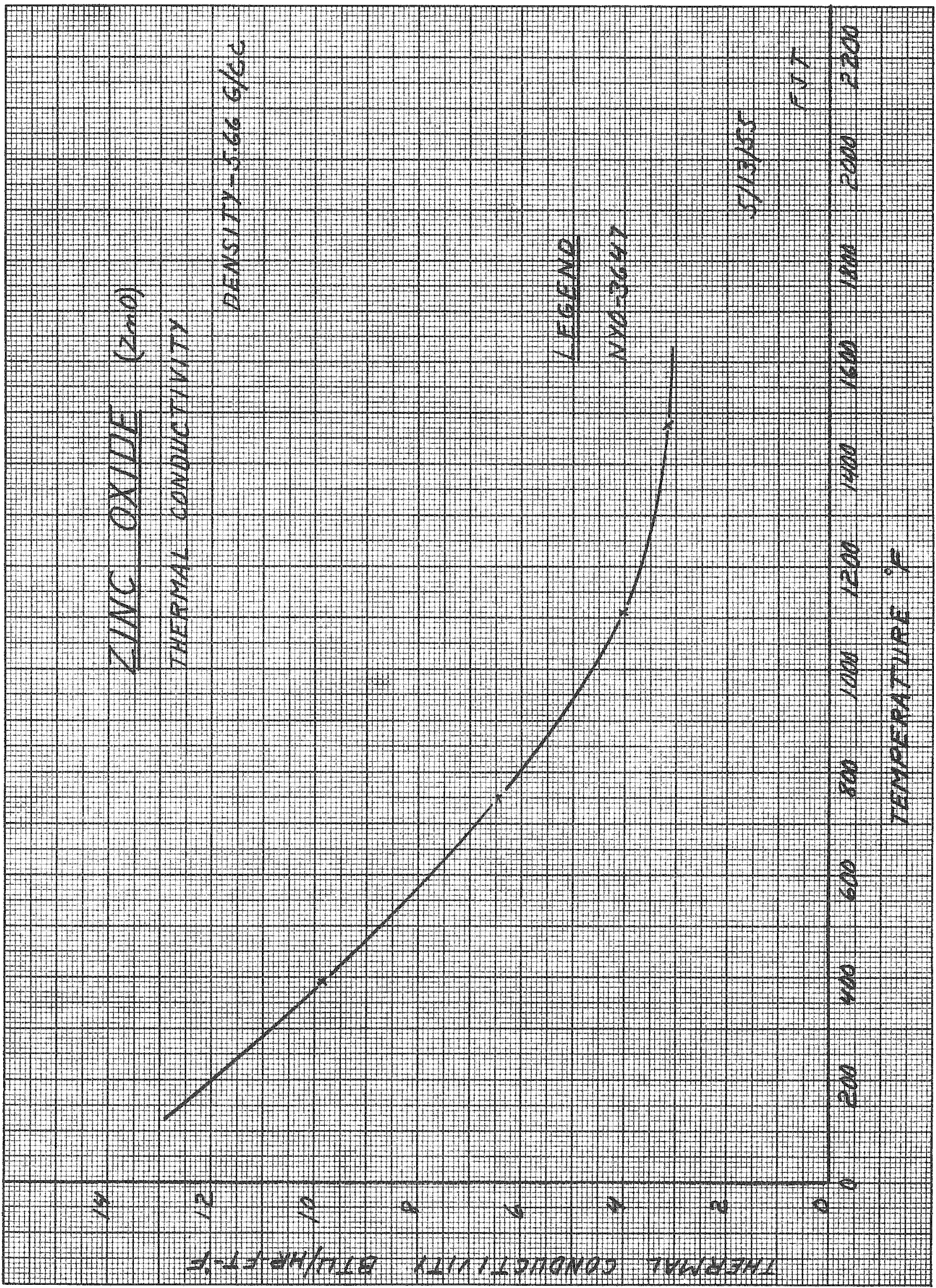




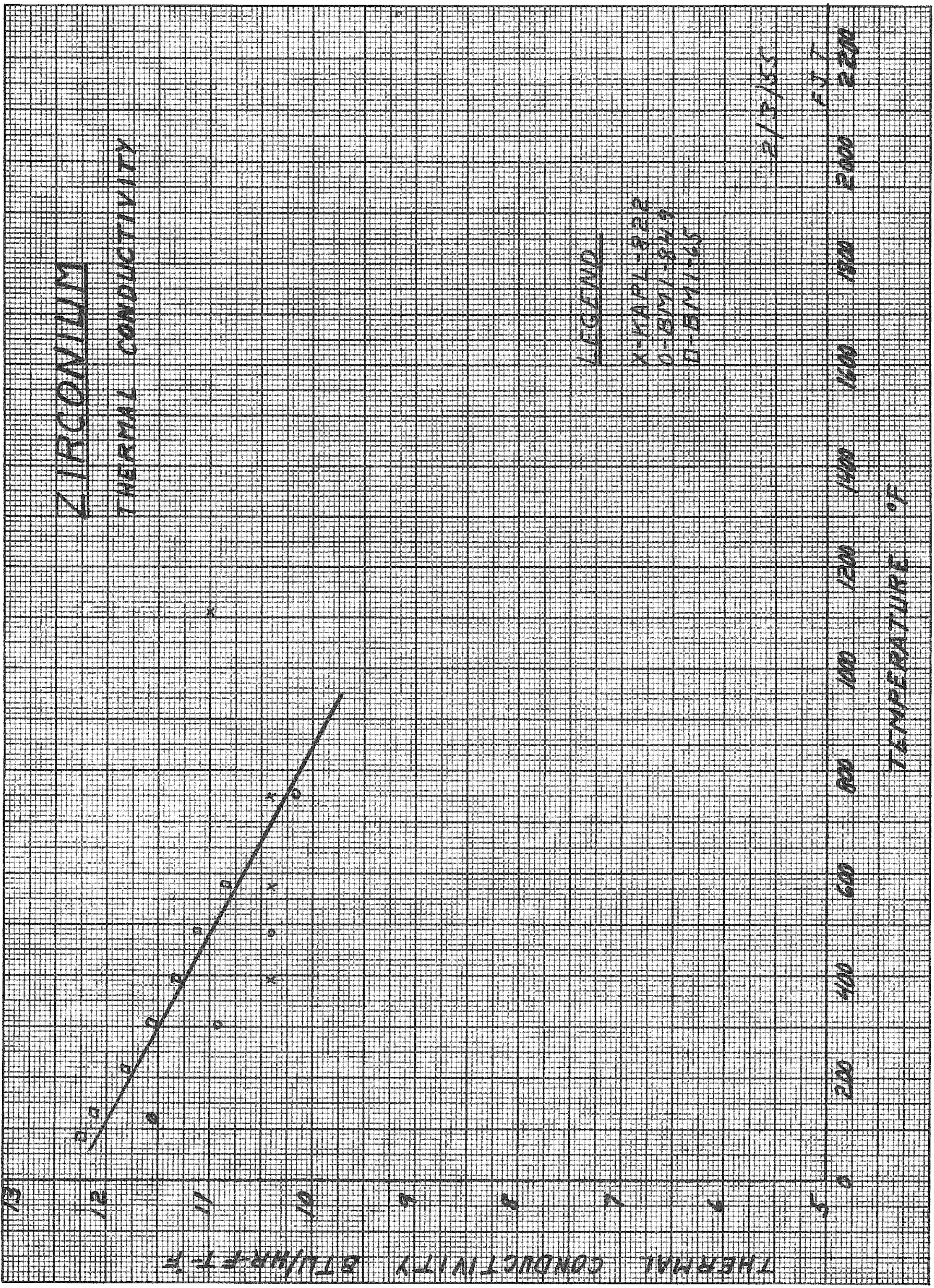




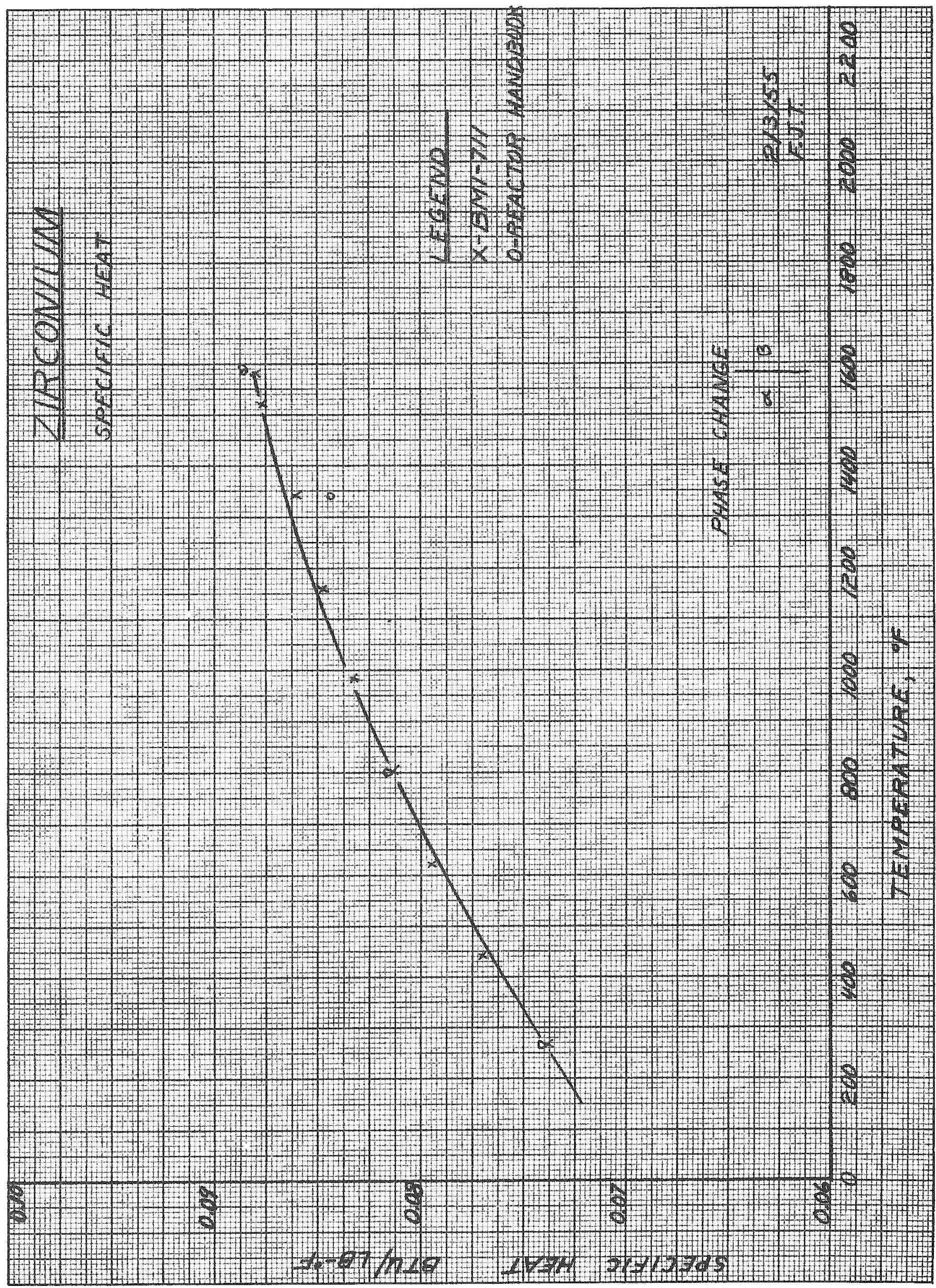




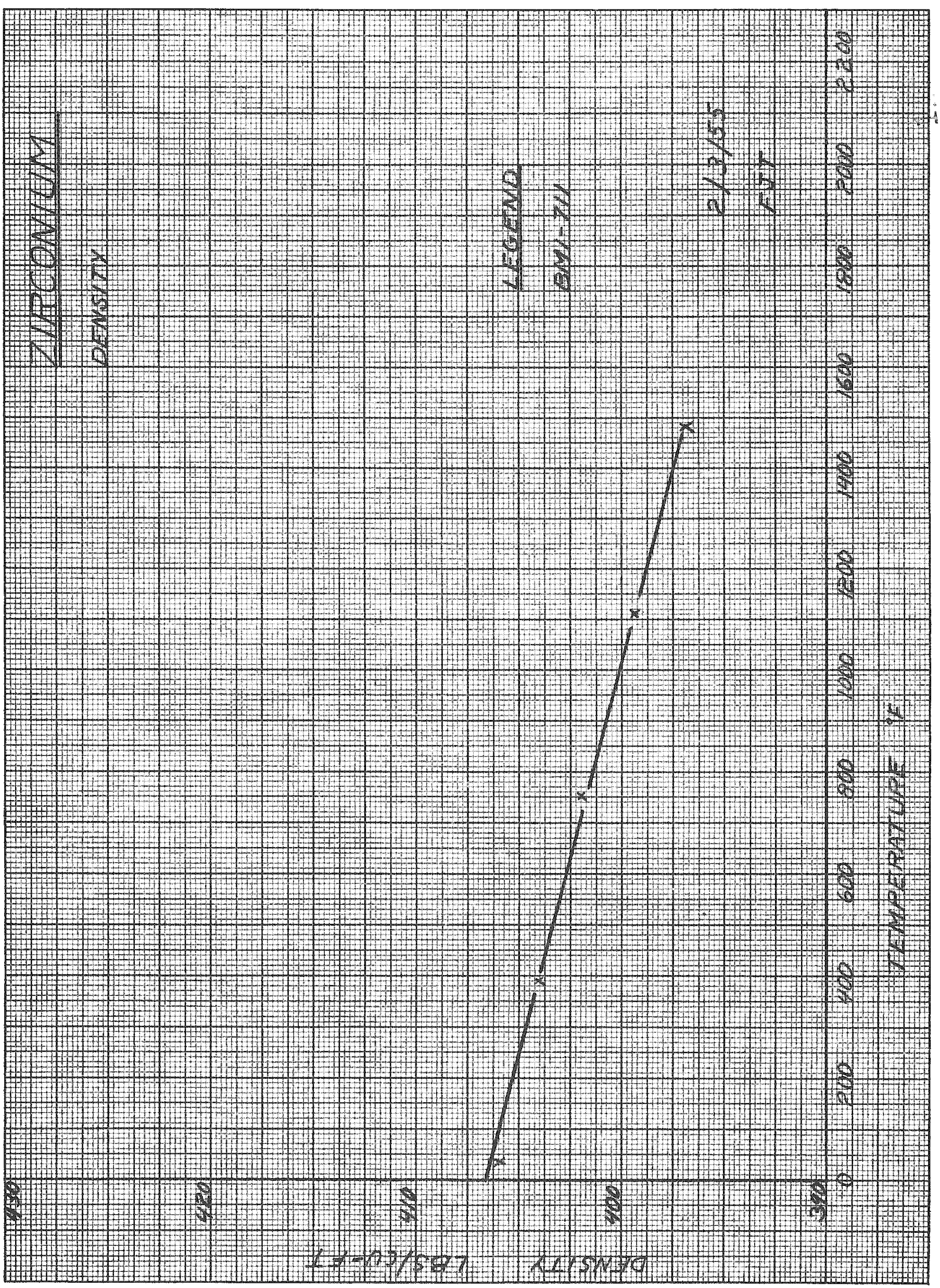




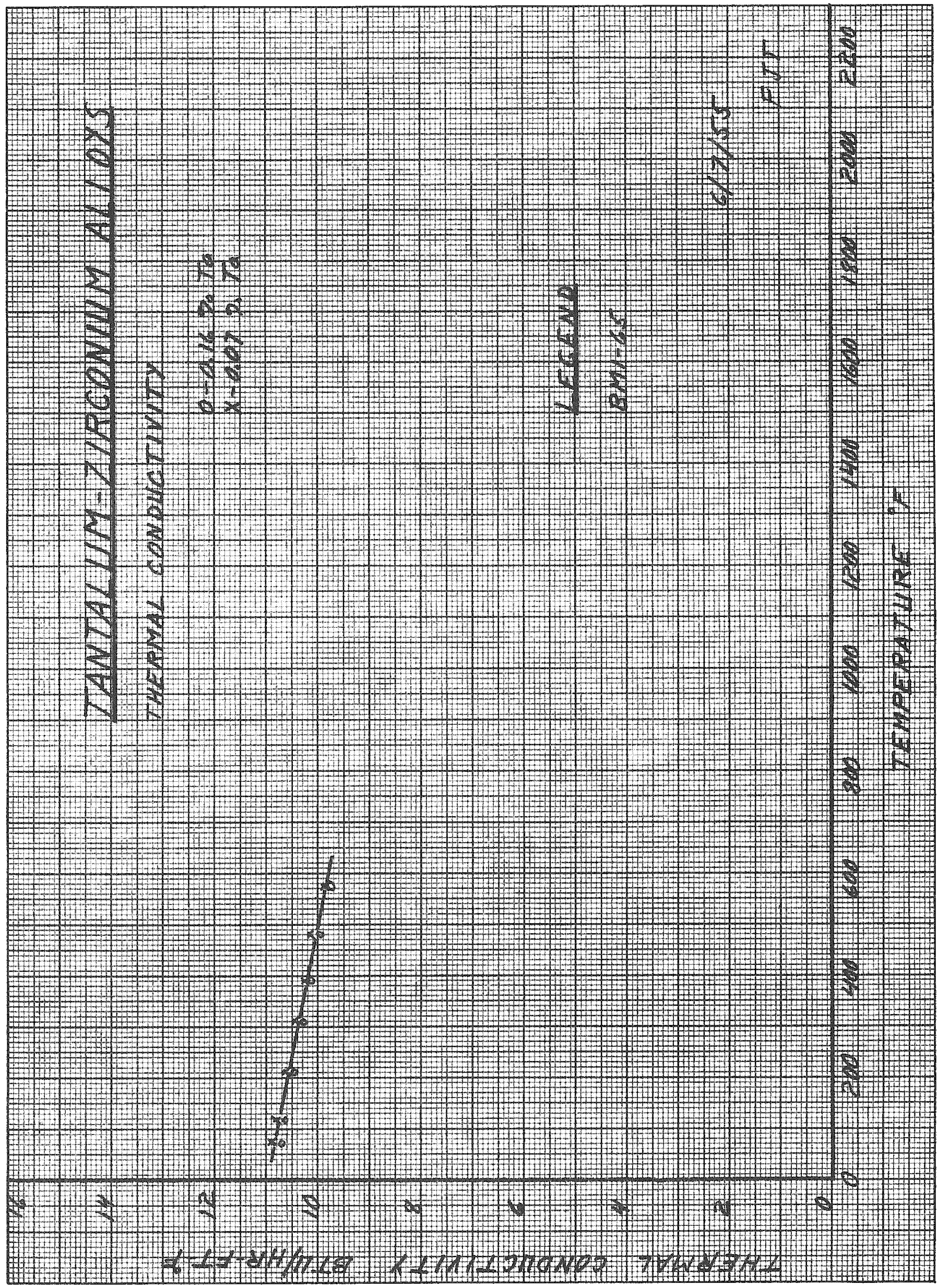




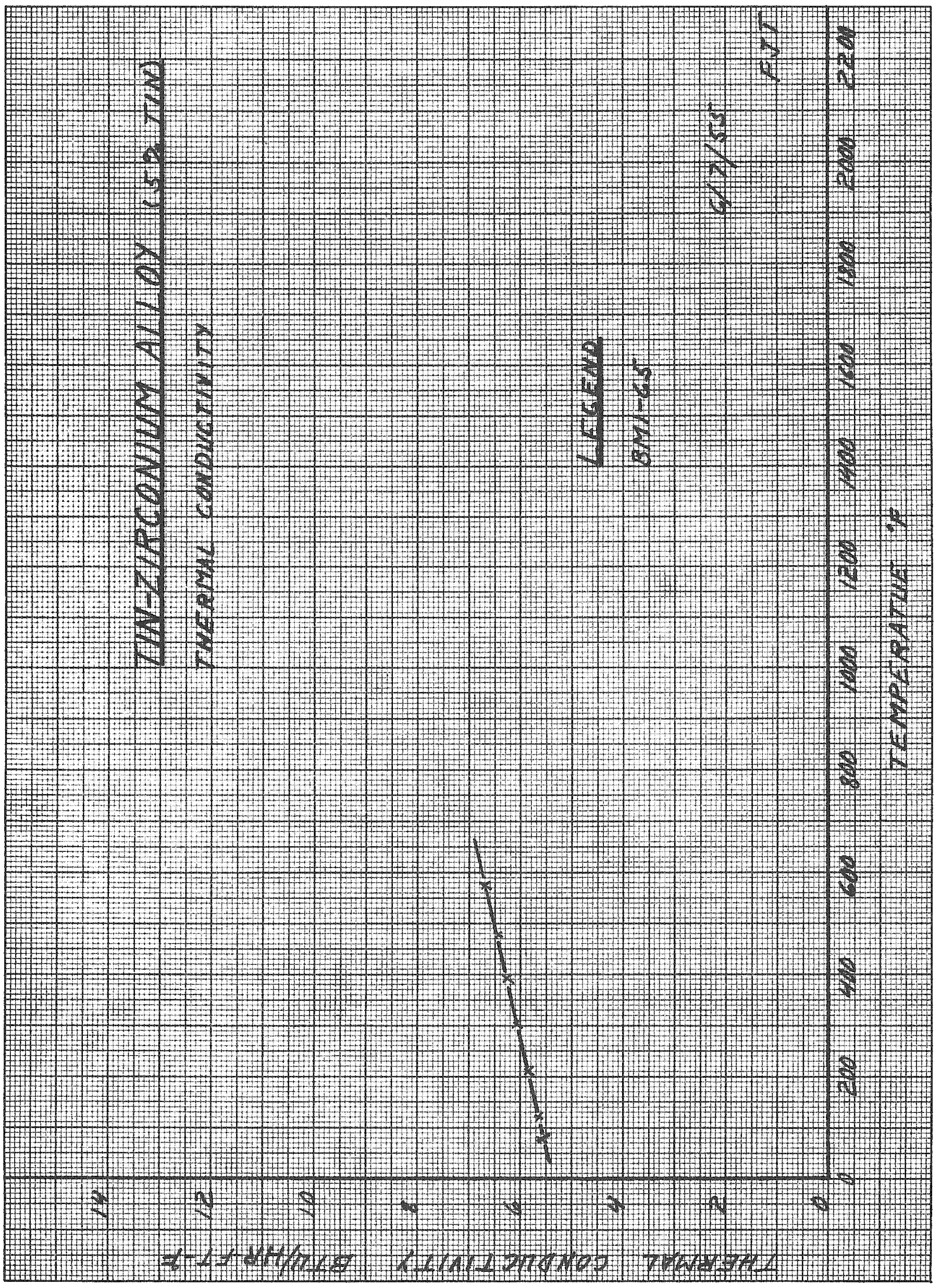




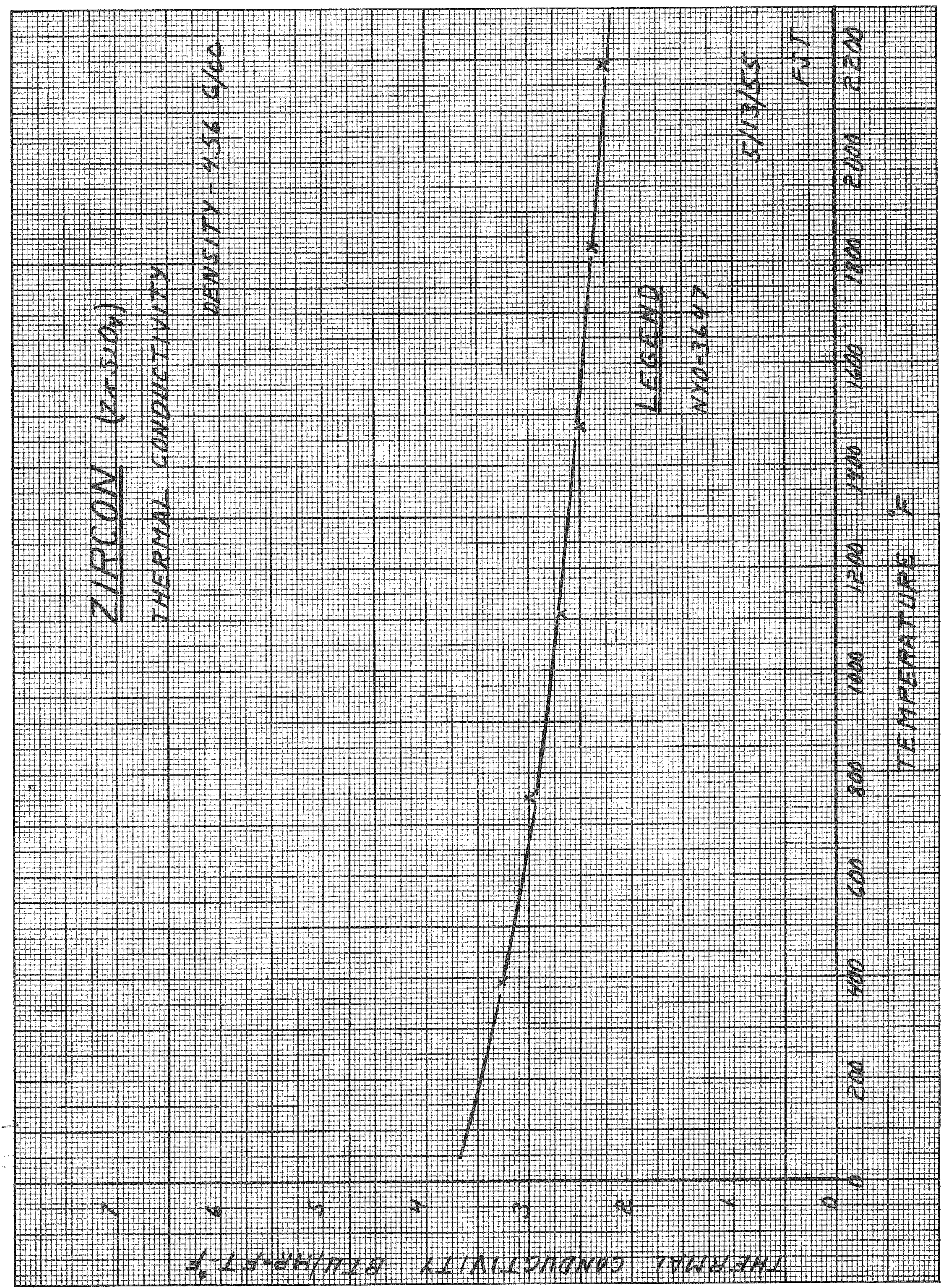




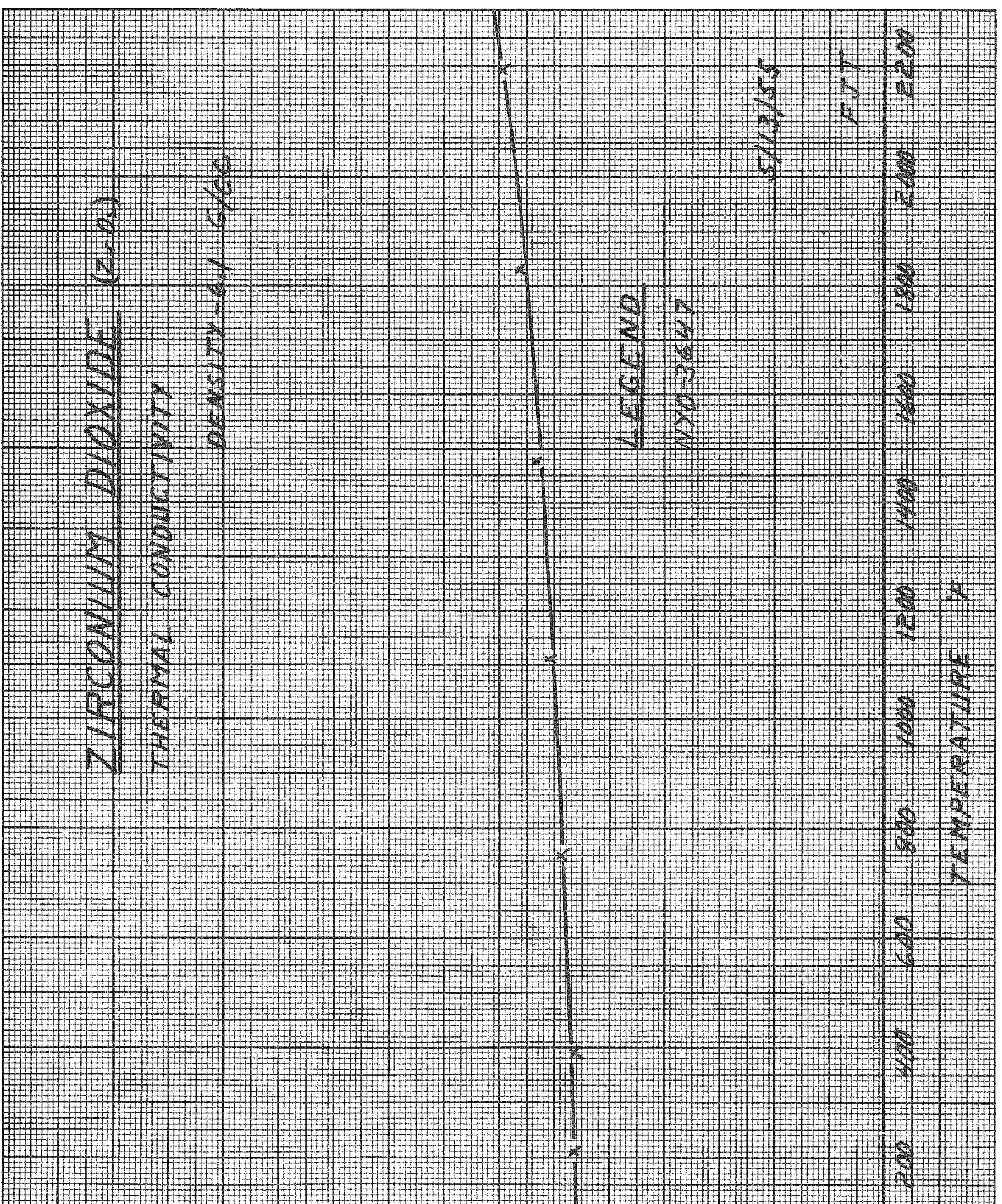
L

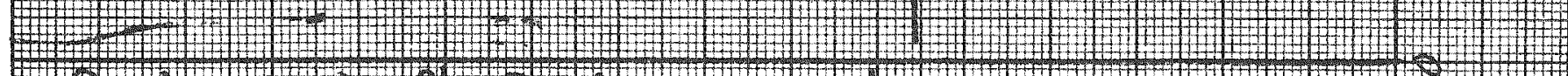

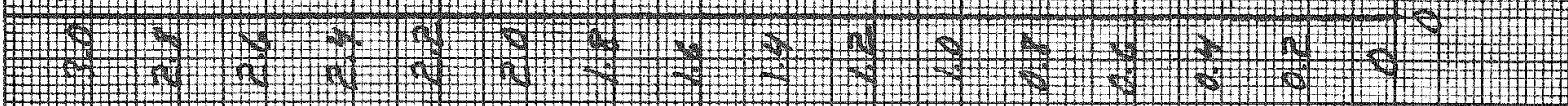

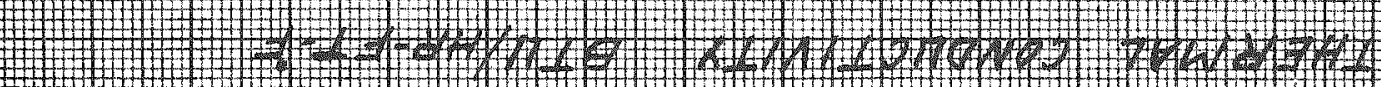




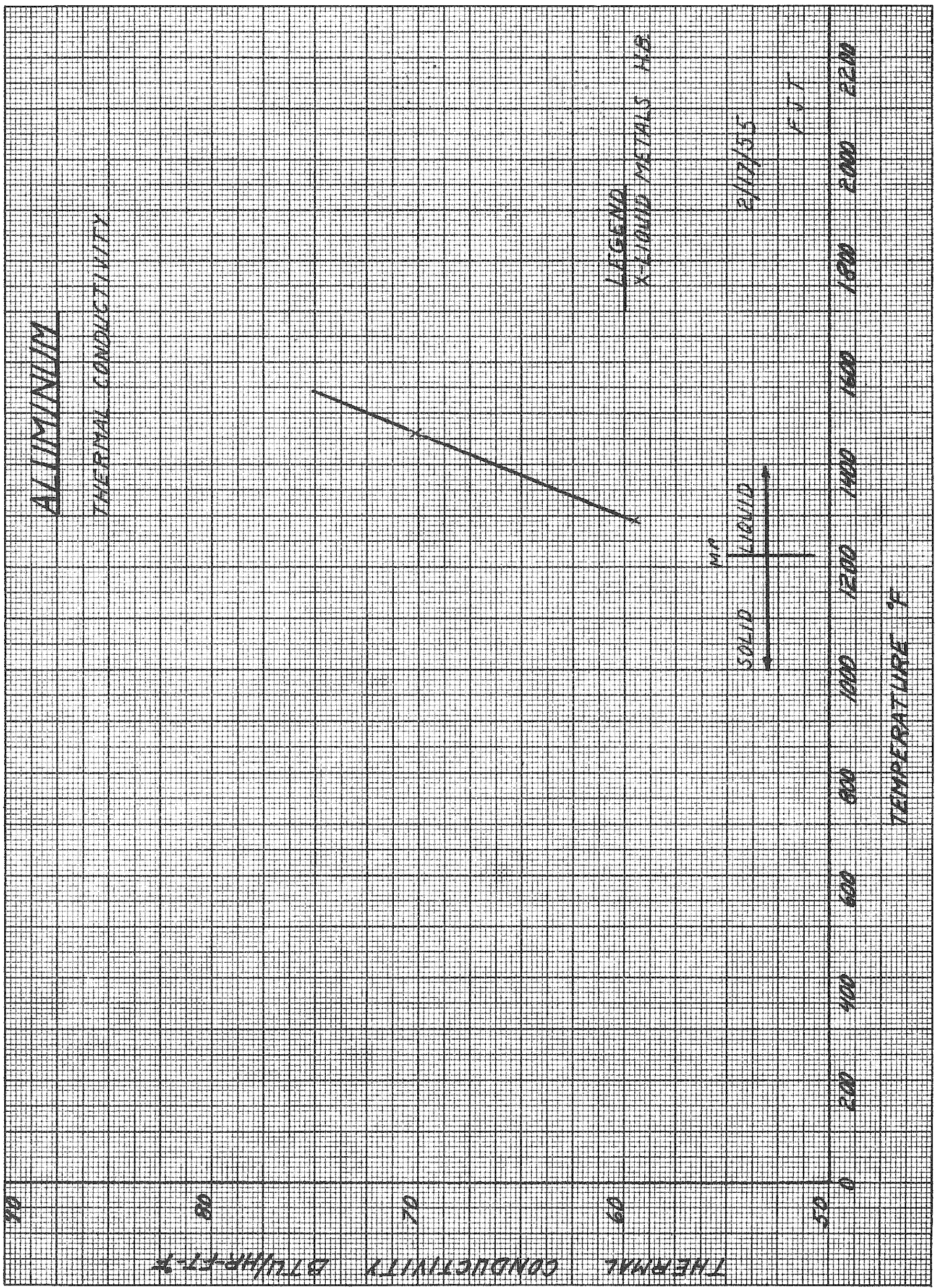




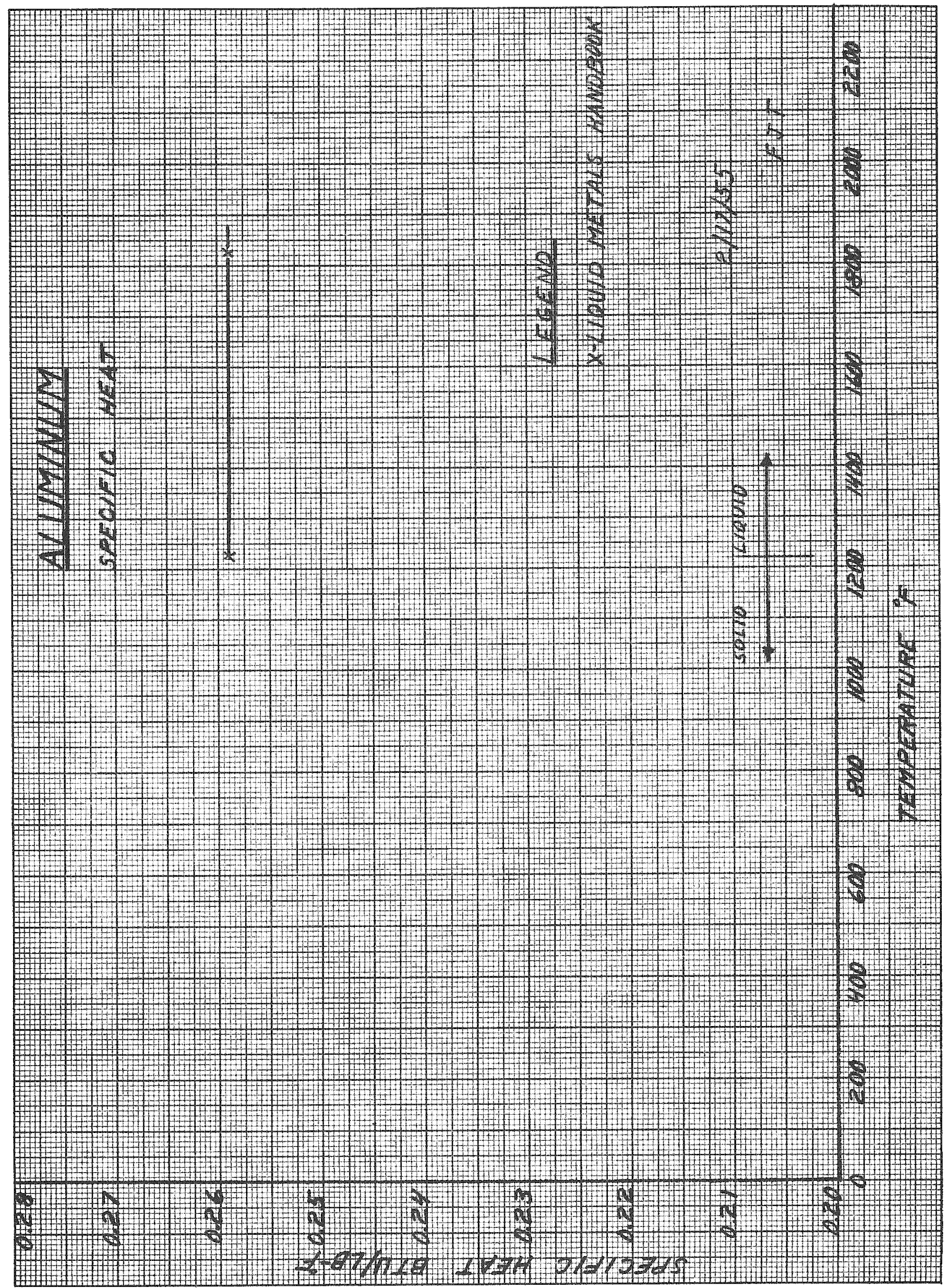




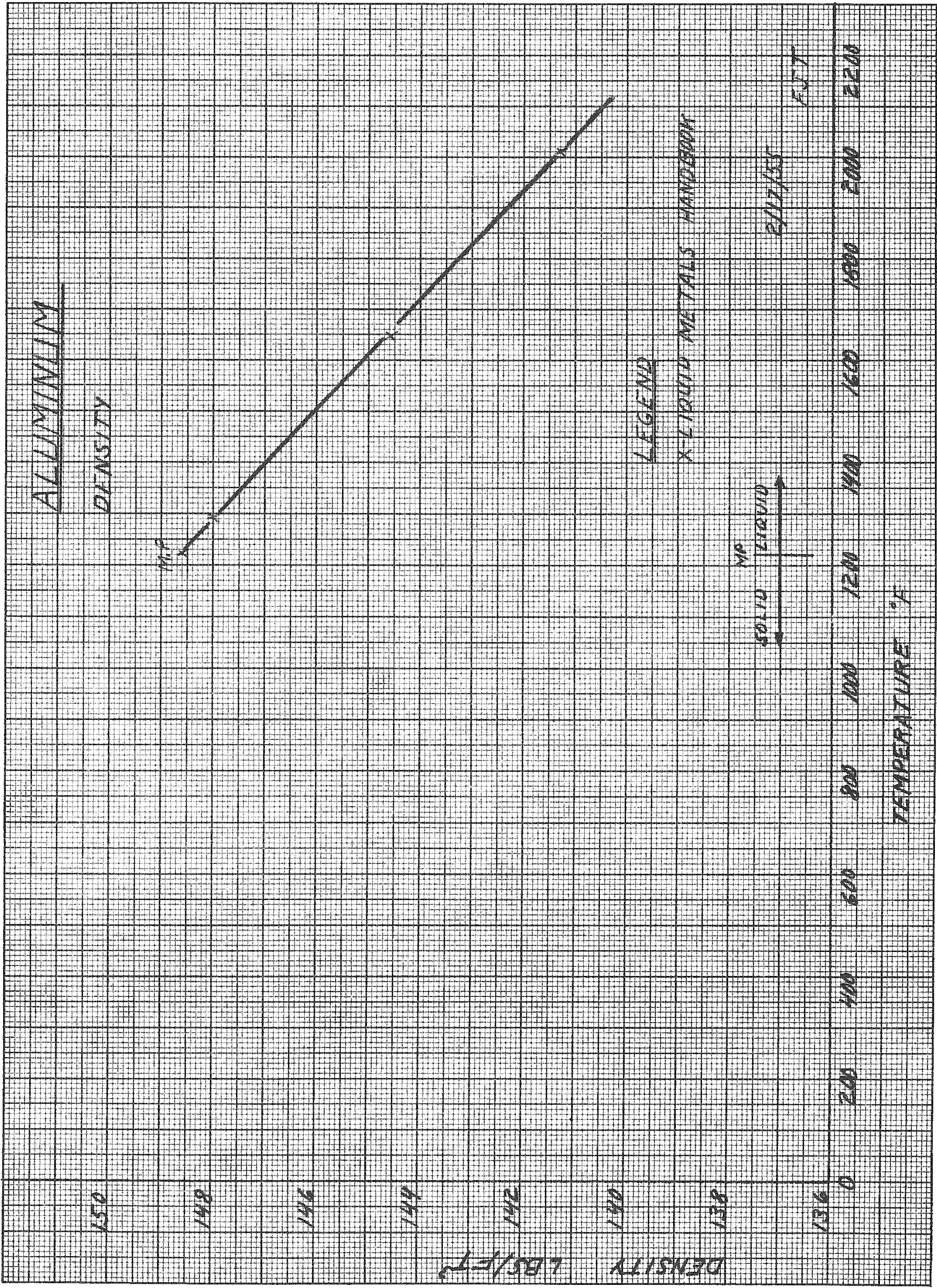




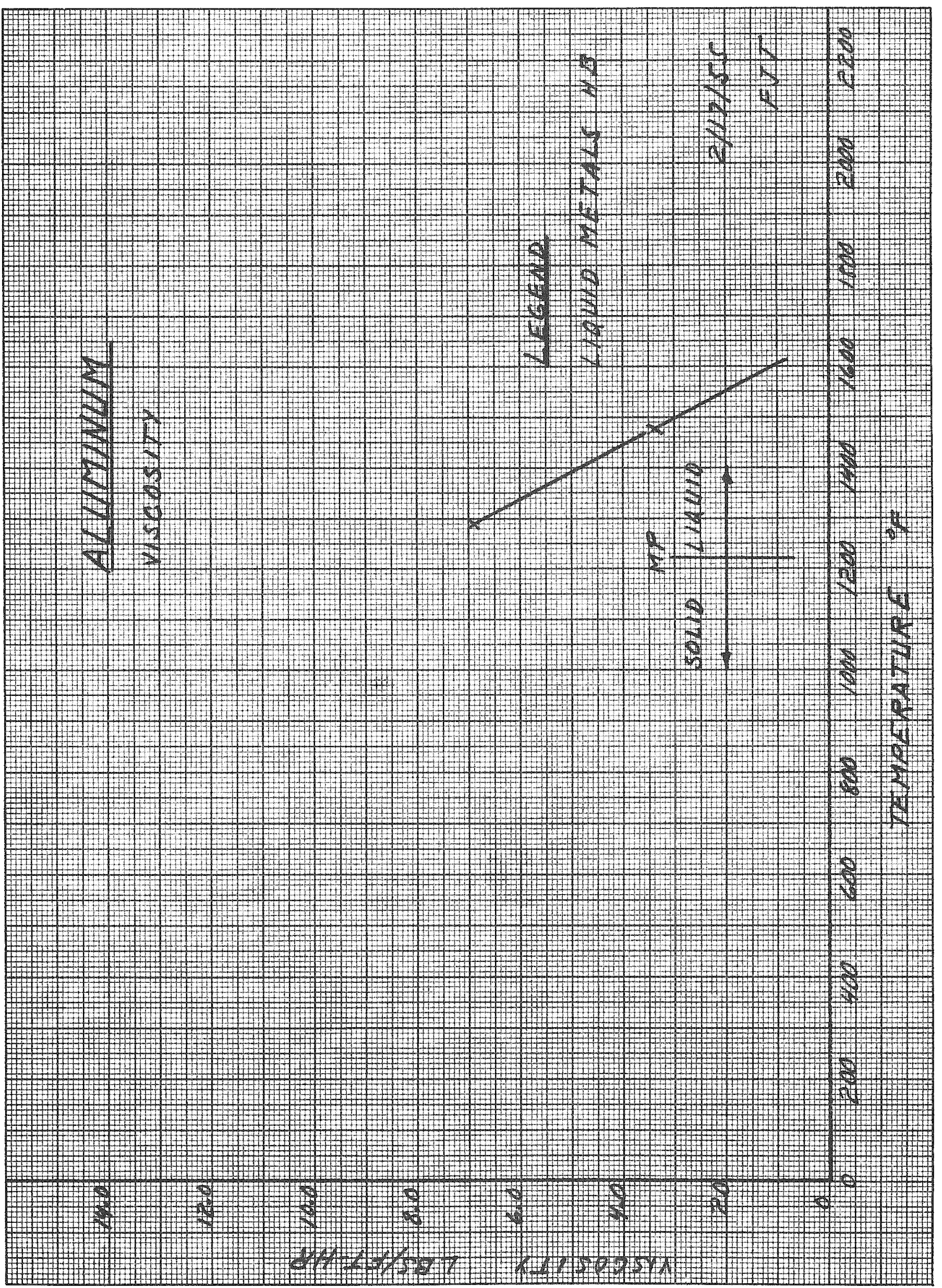




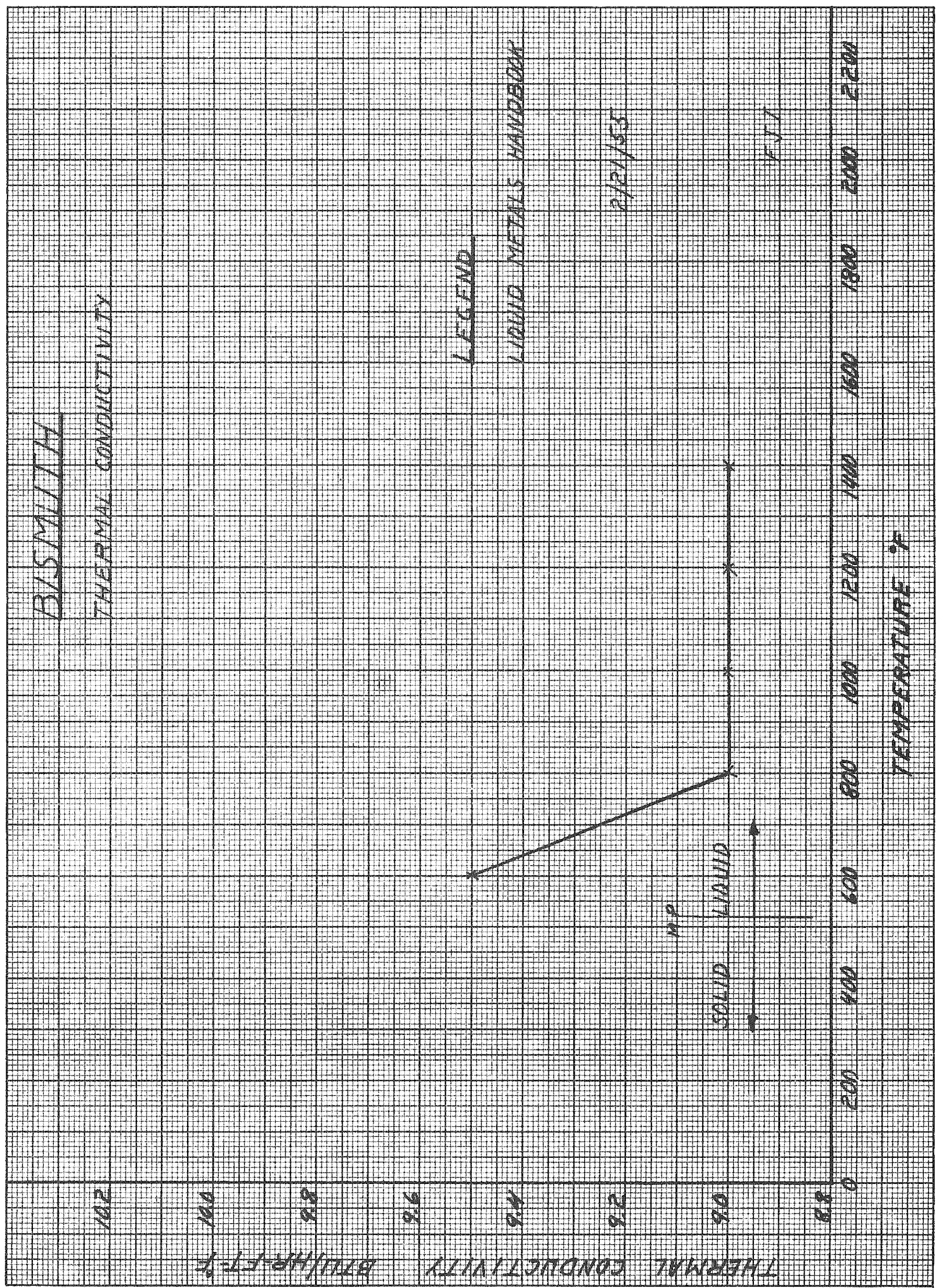




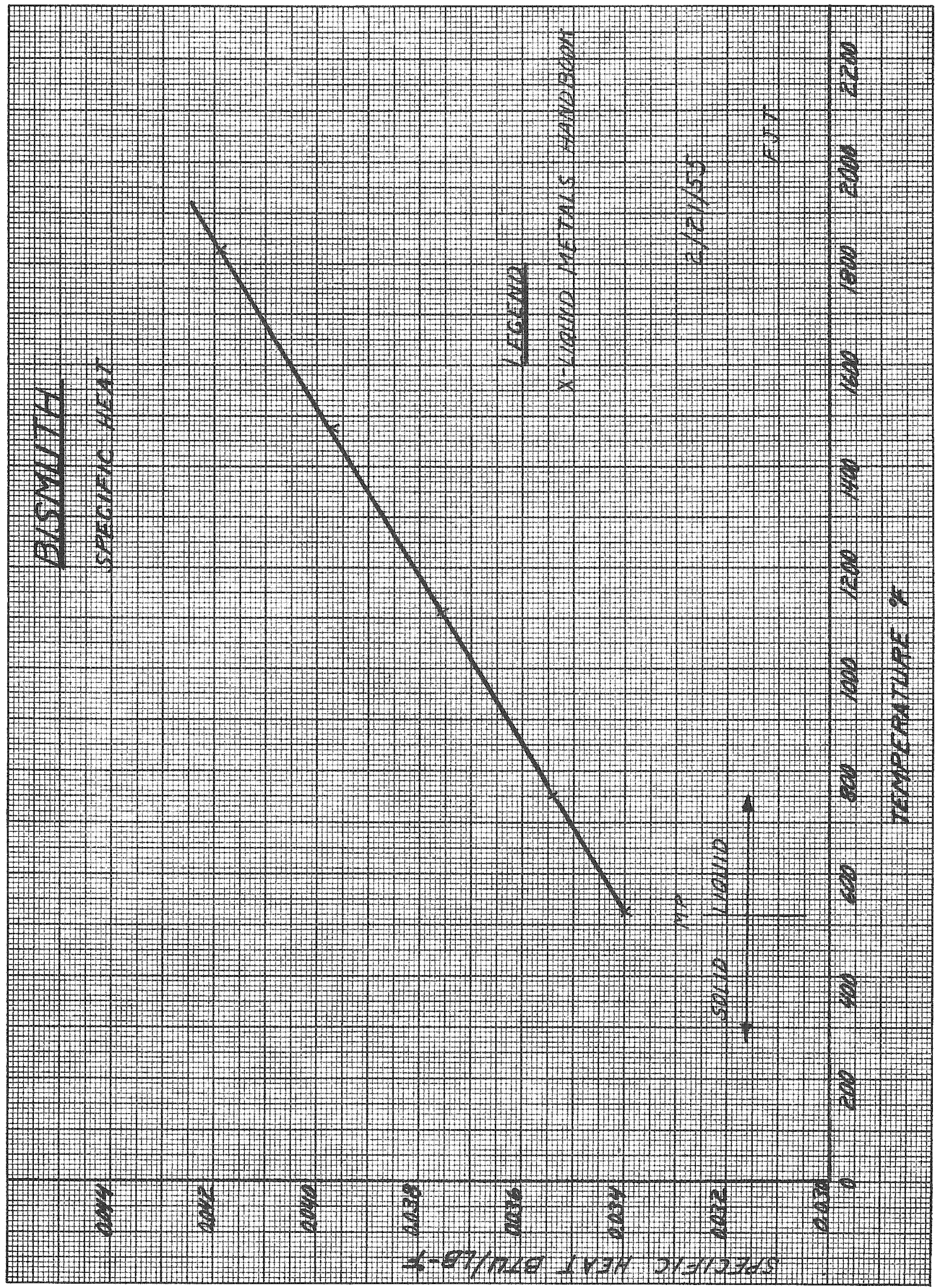




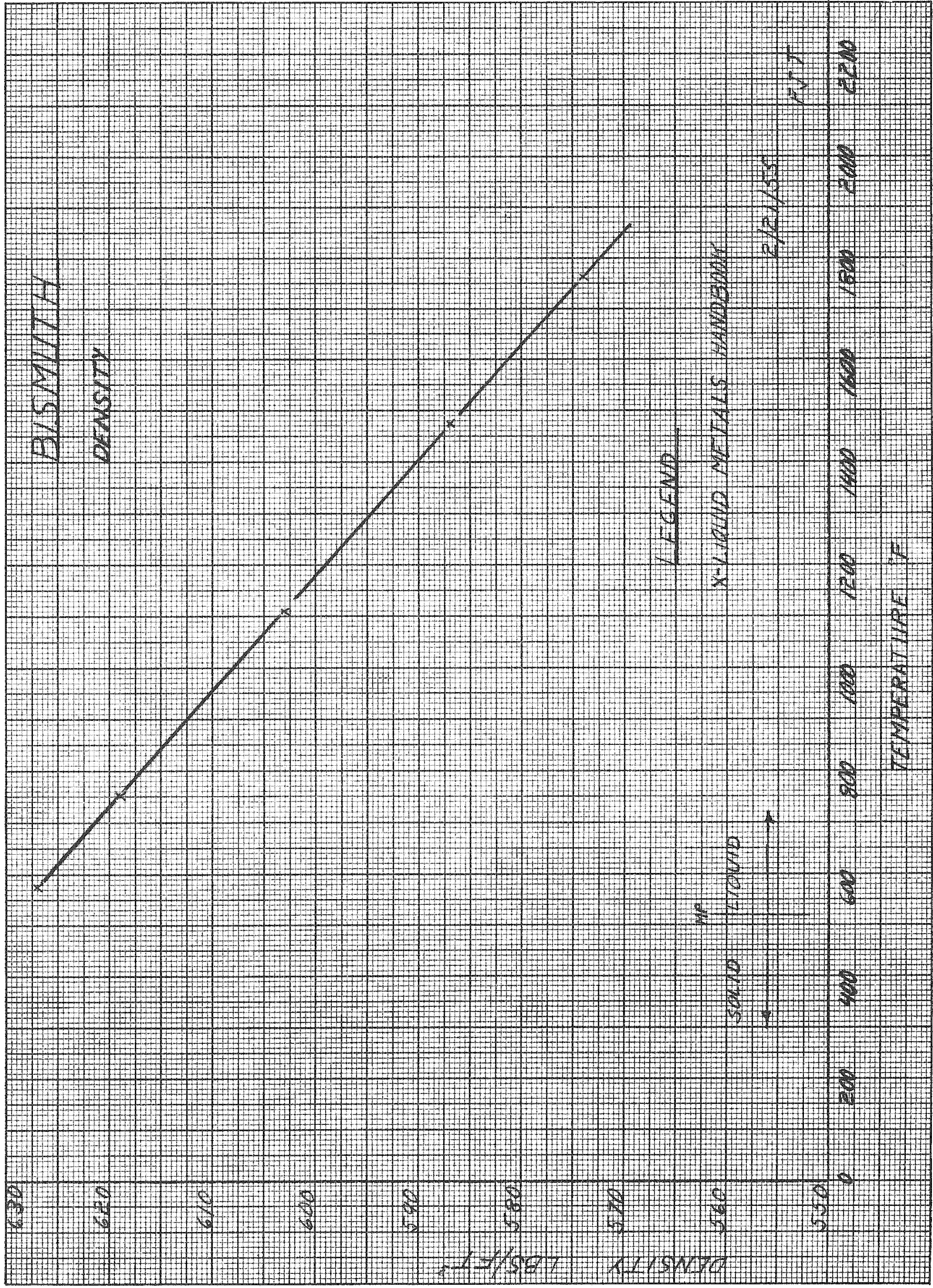




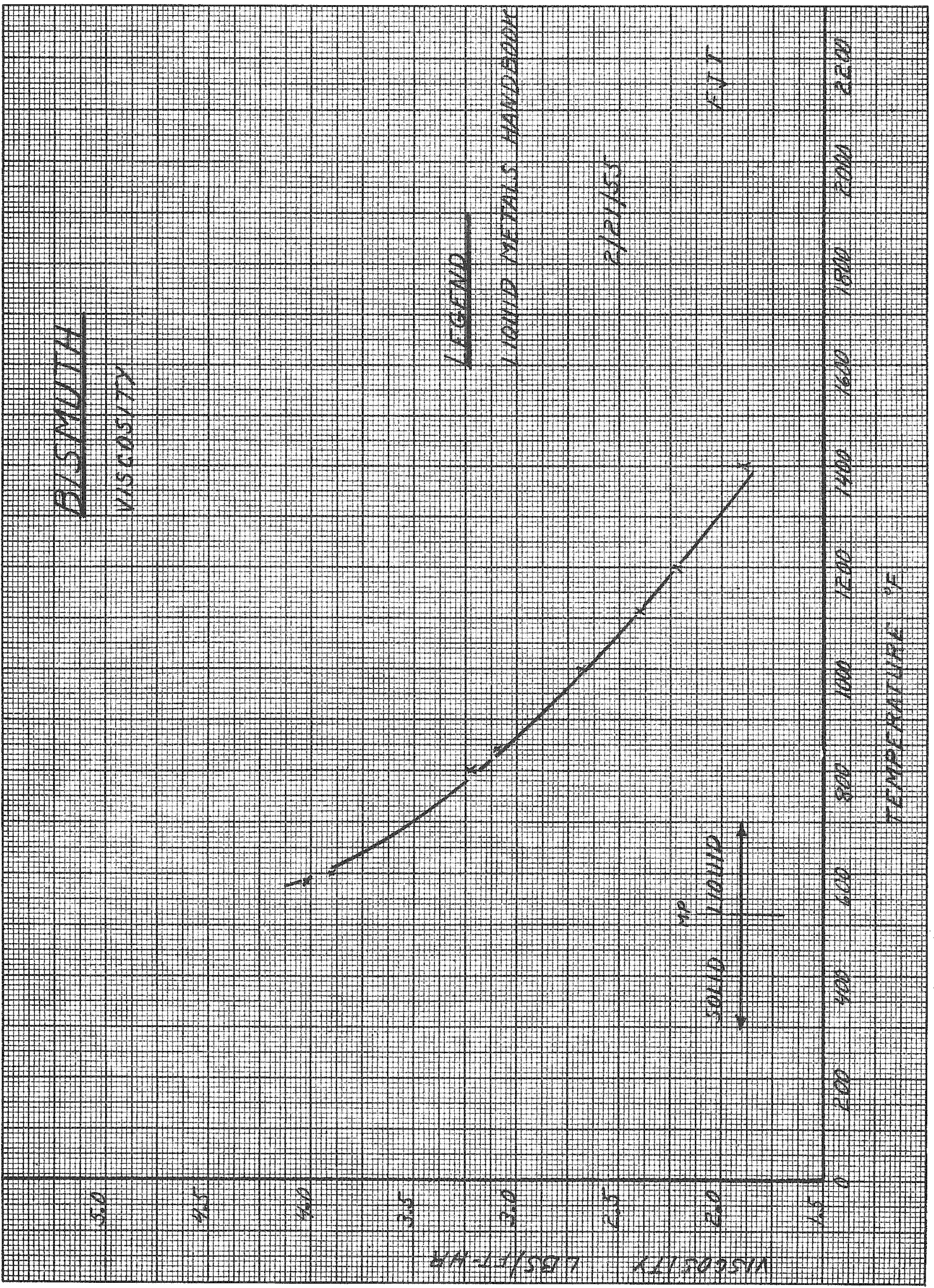




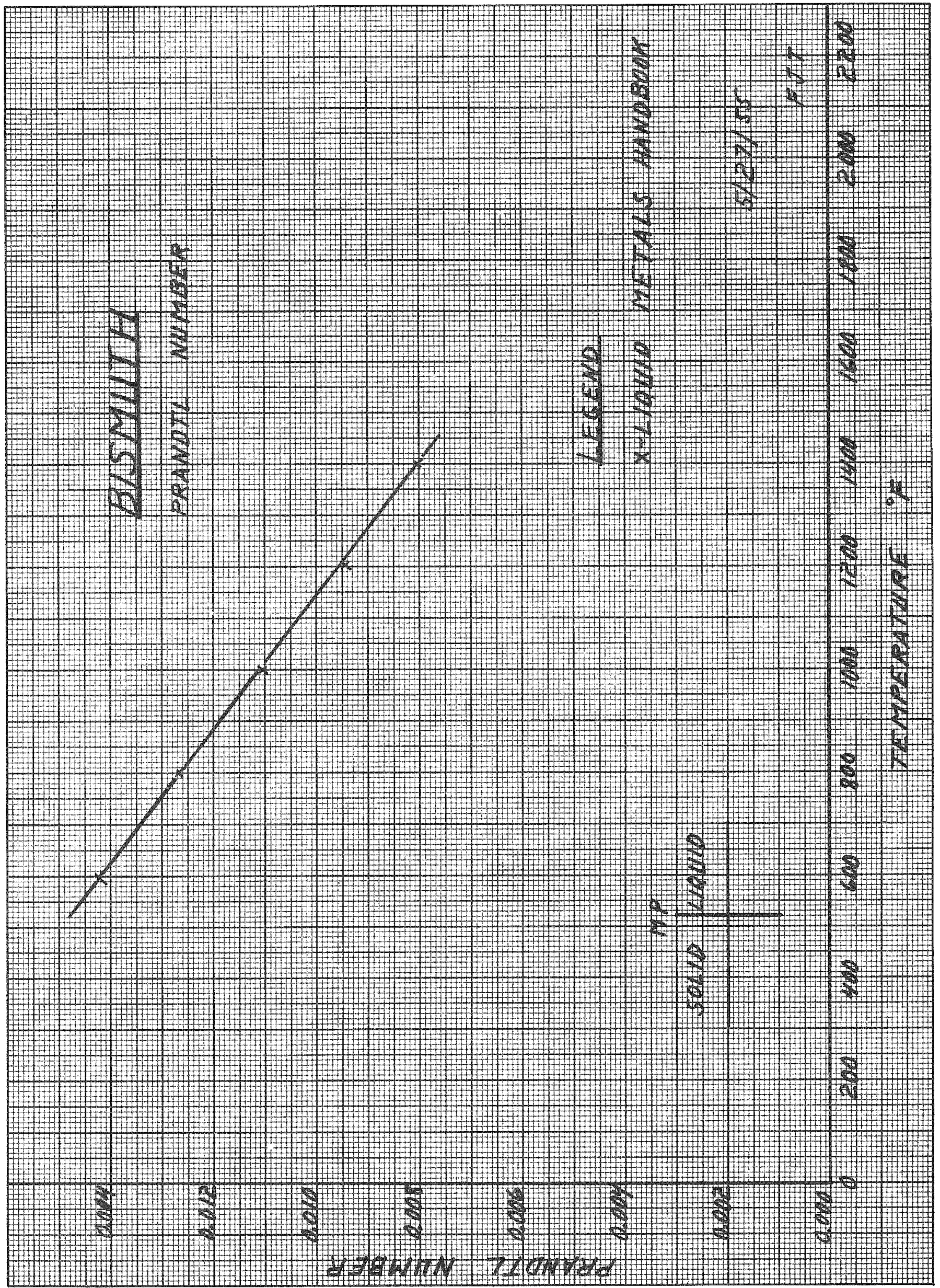




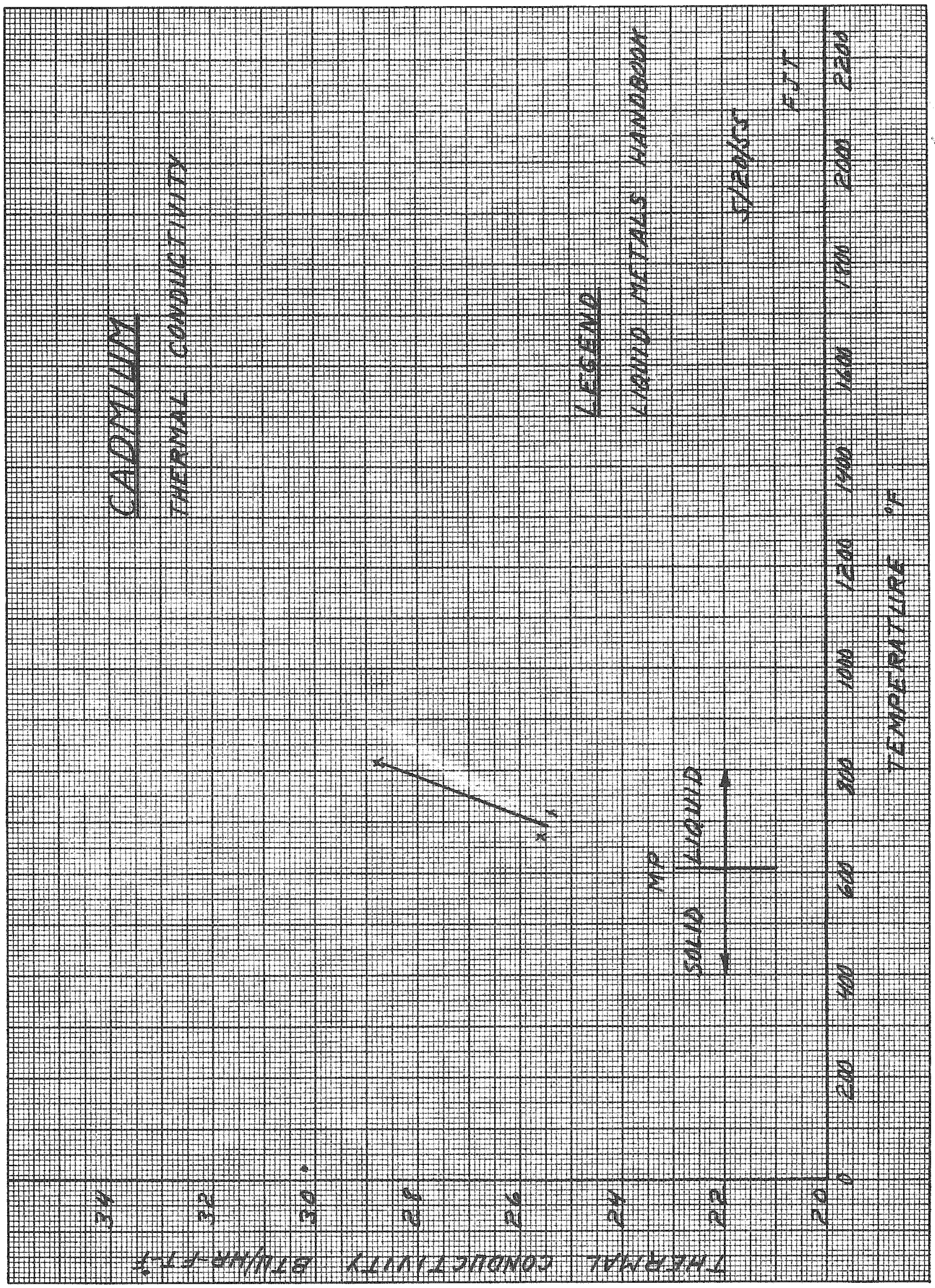




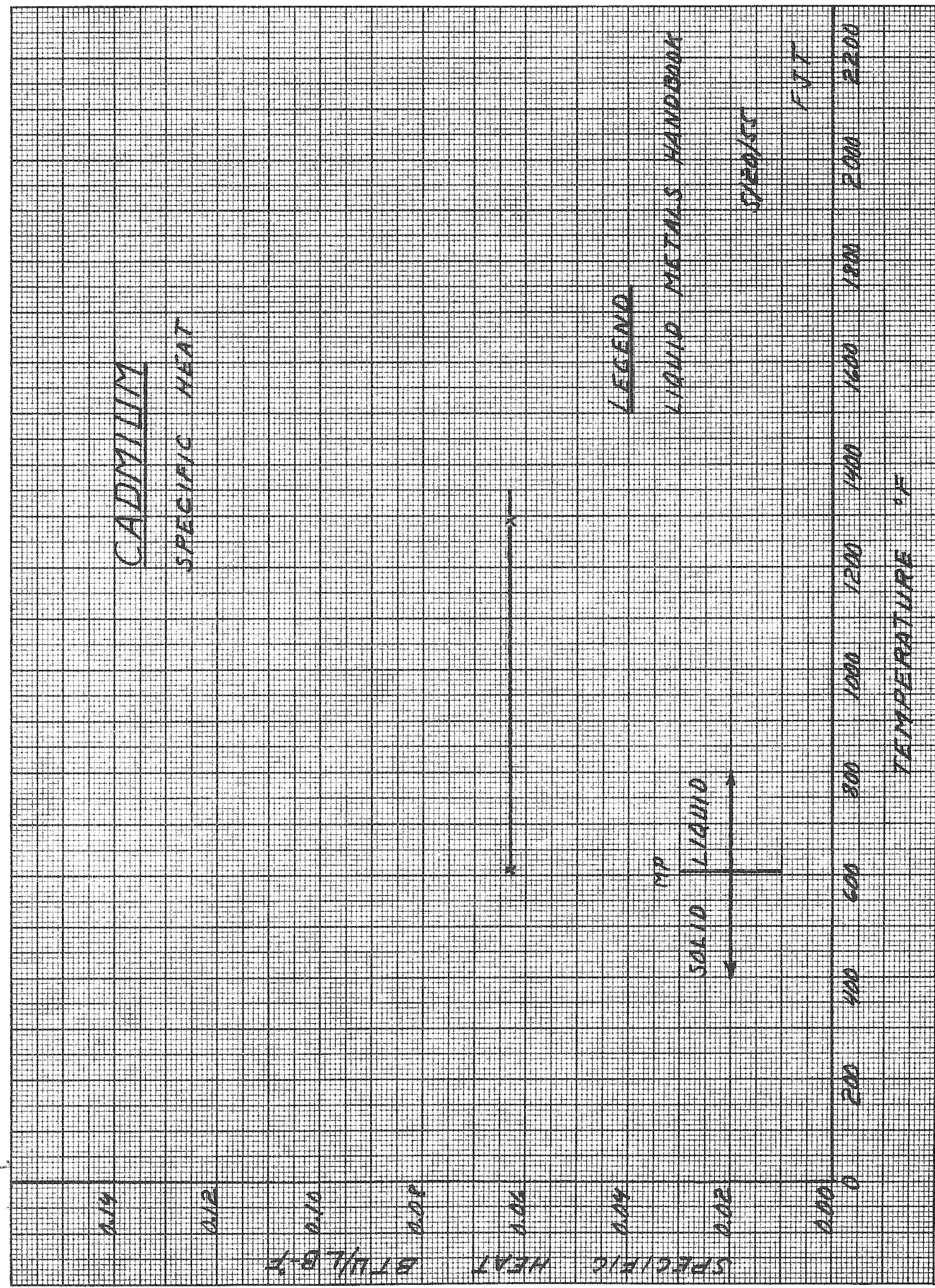




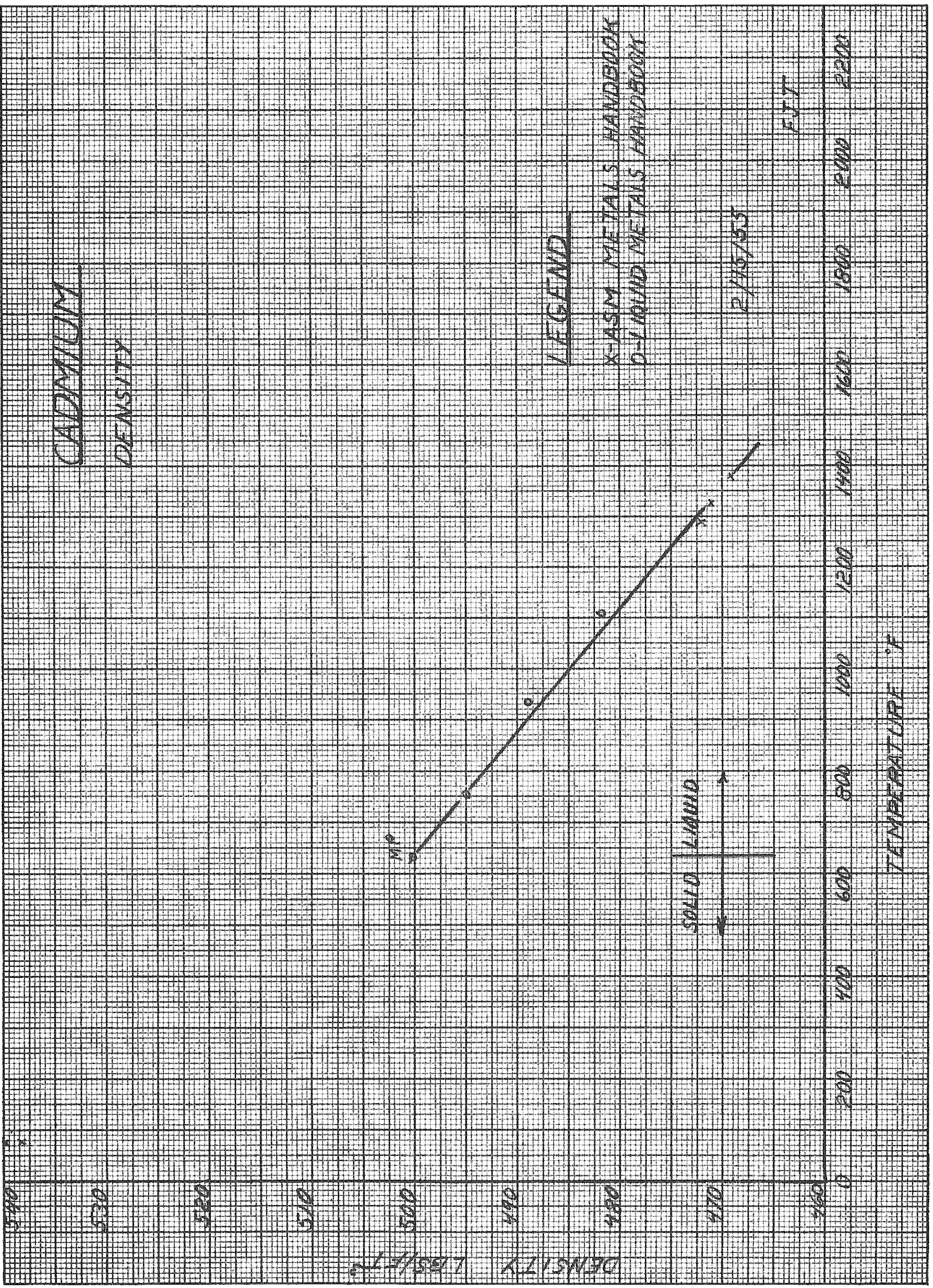




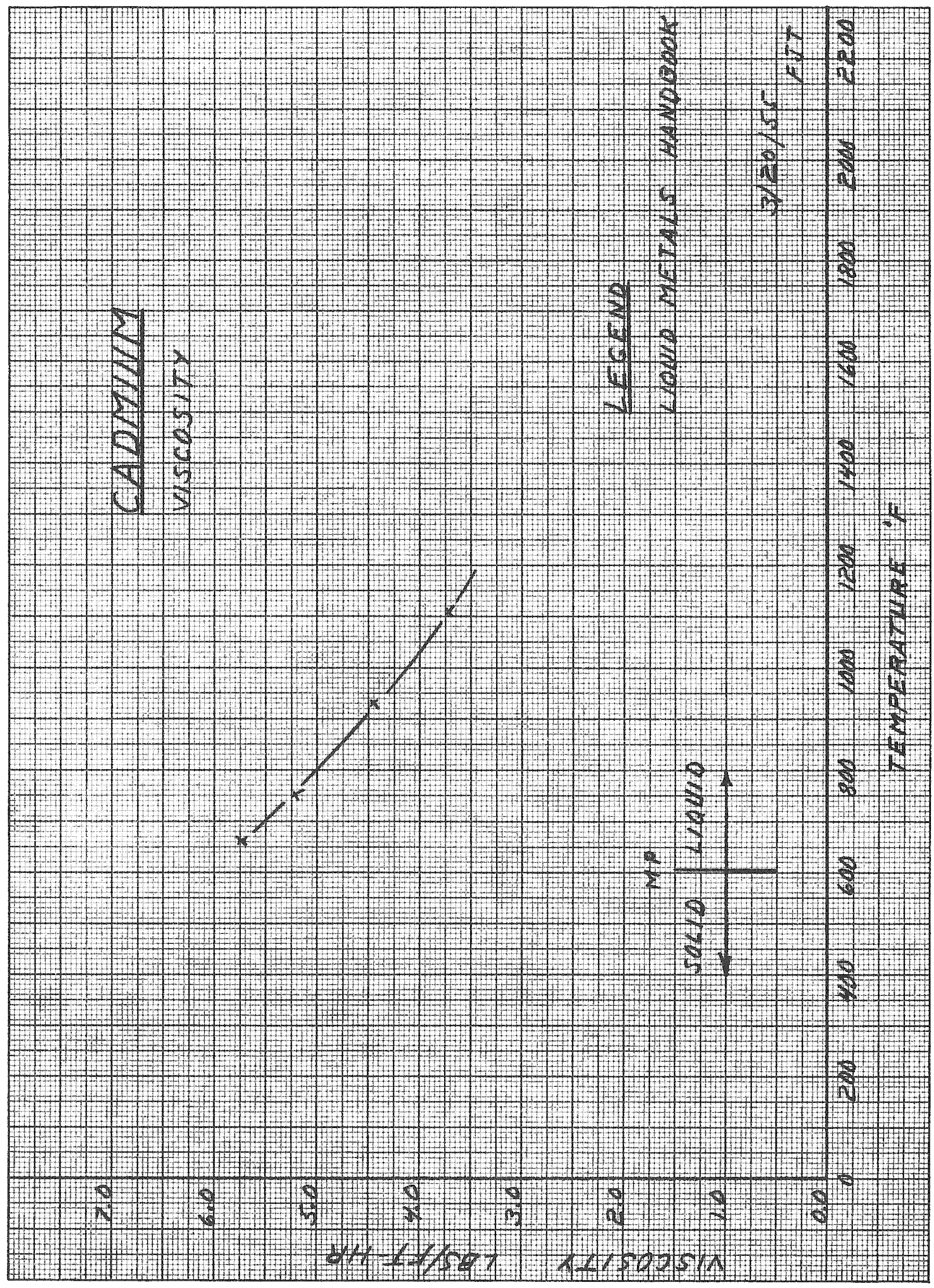




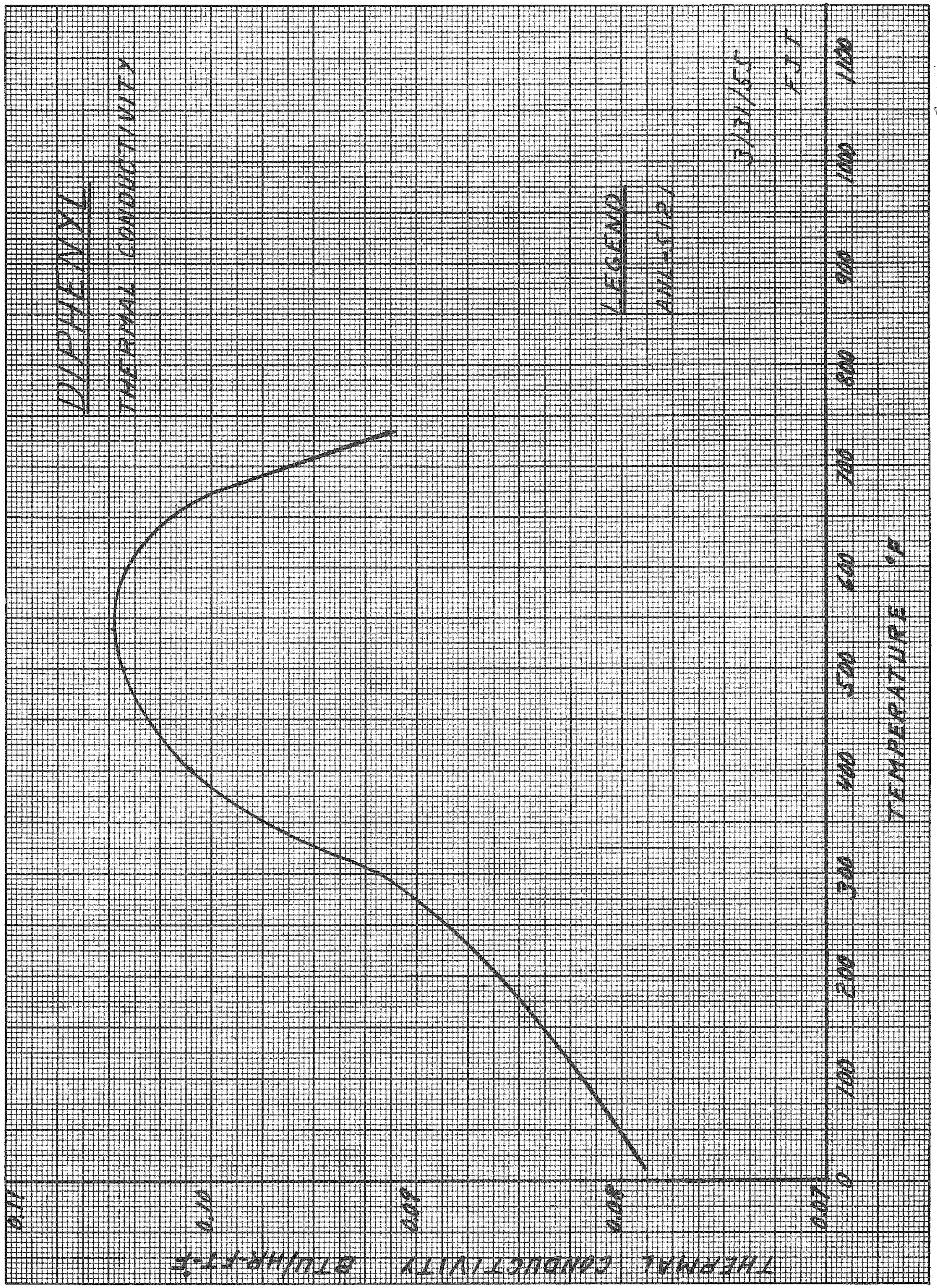




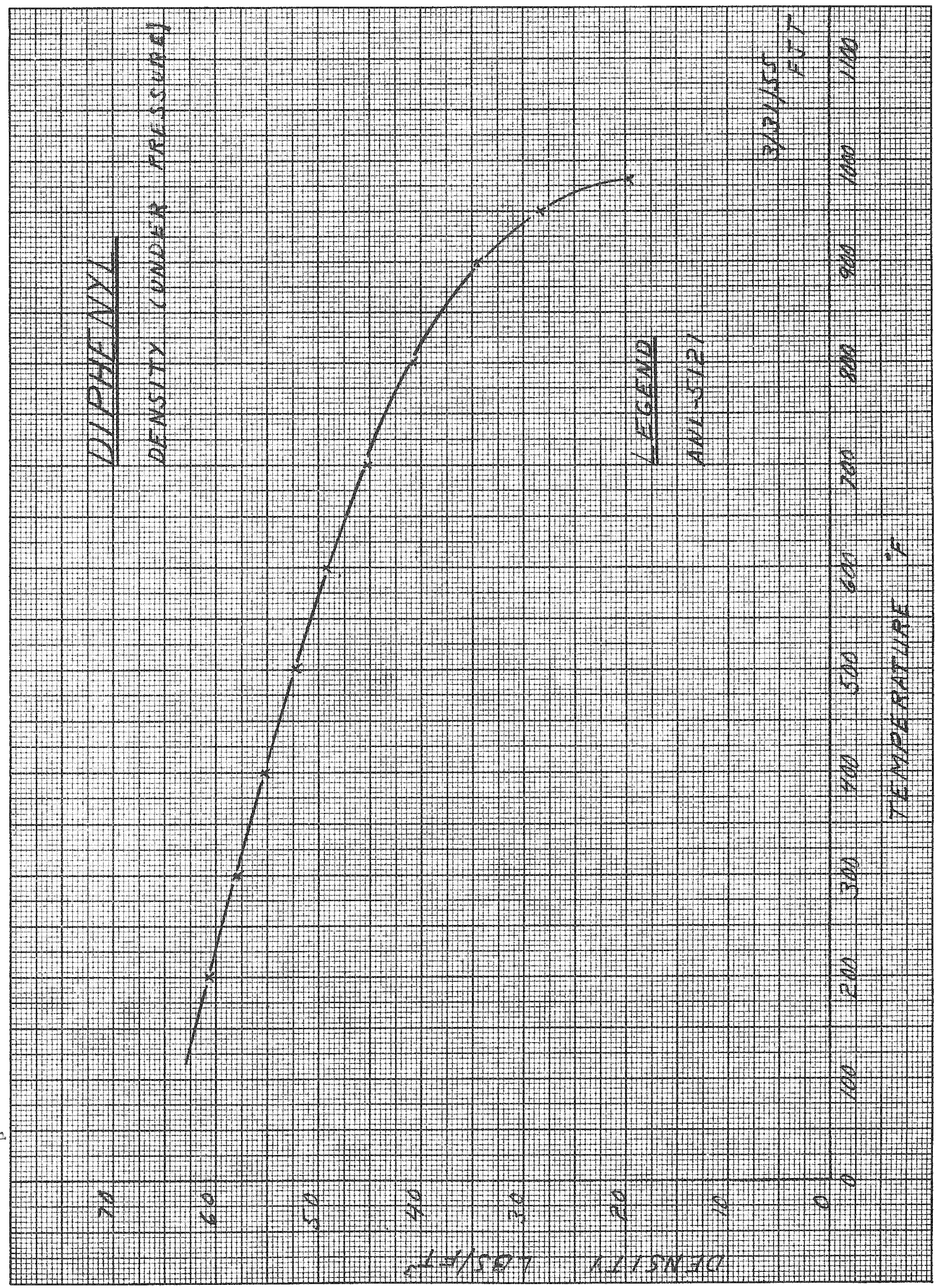




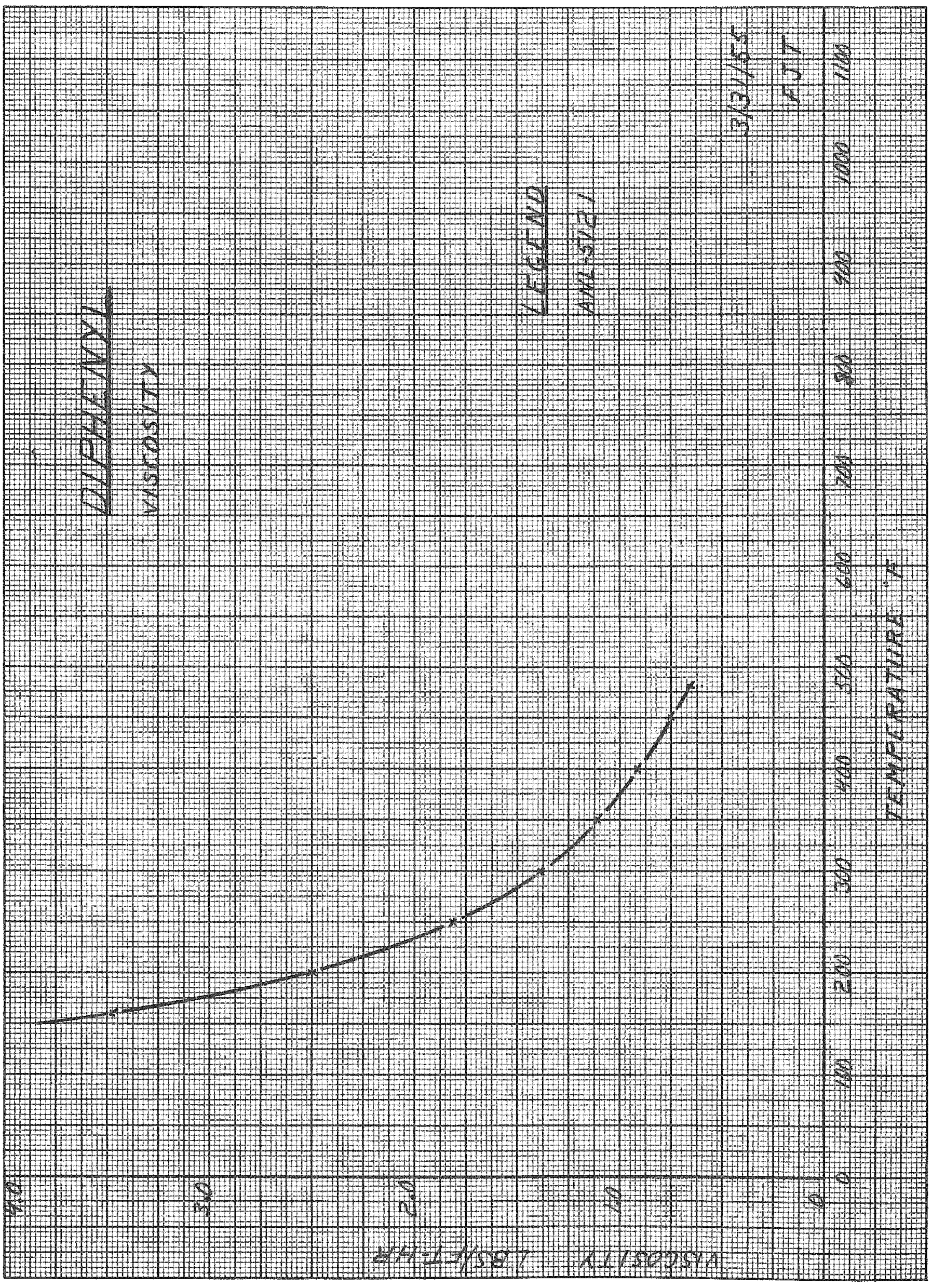




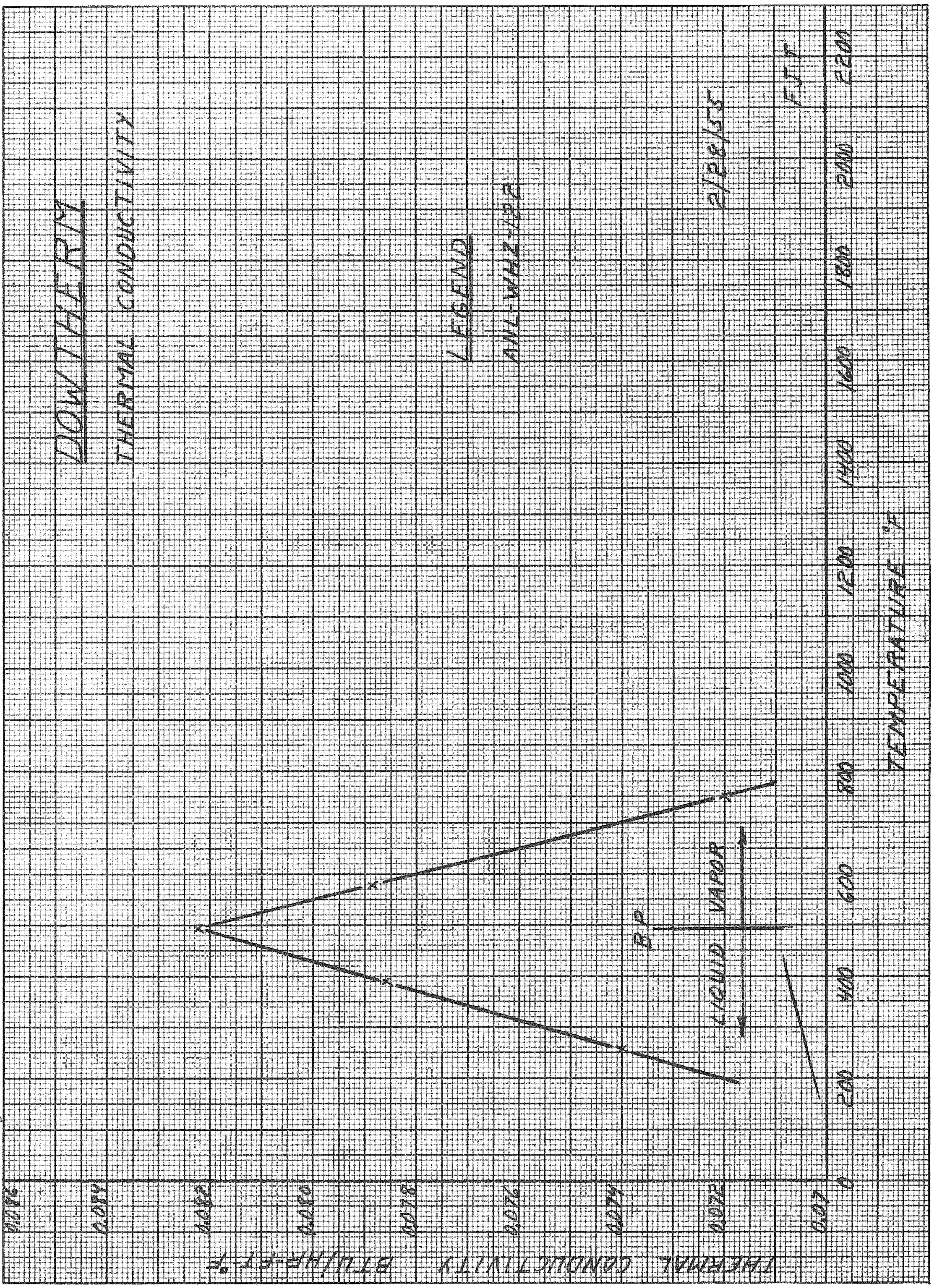




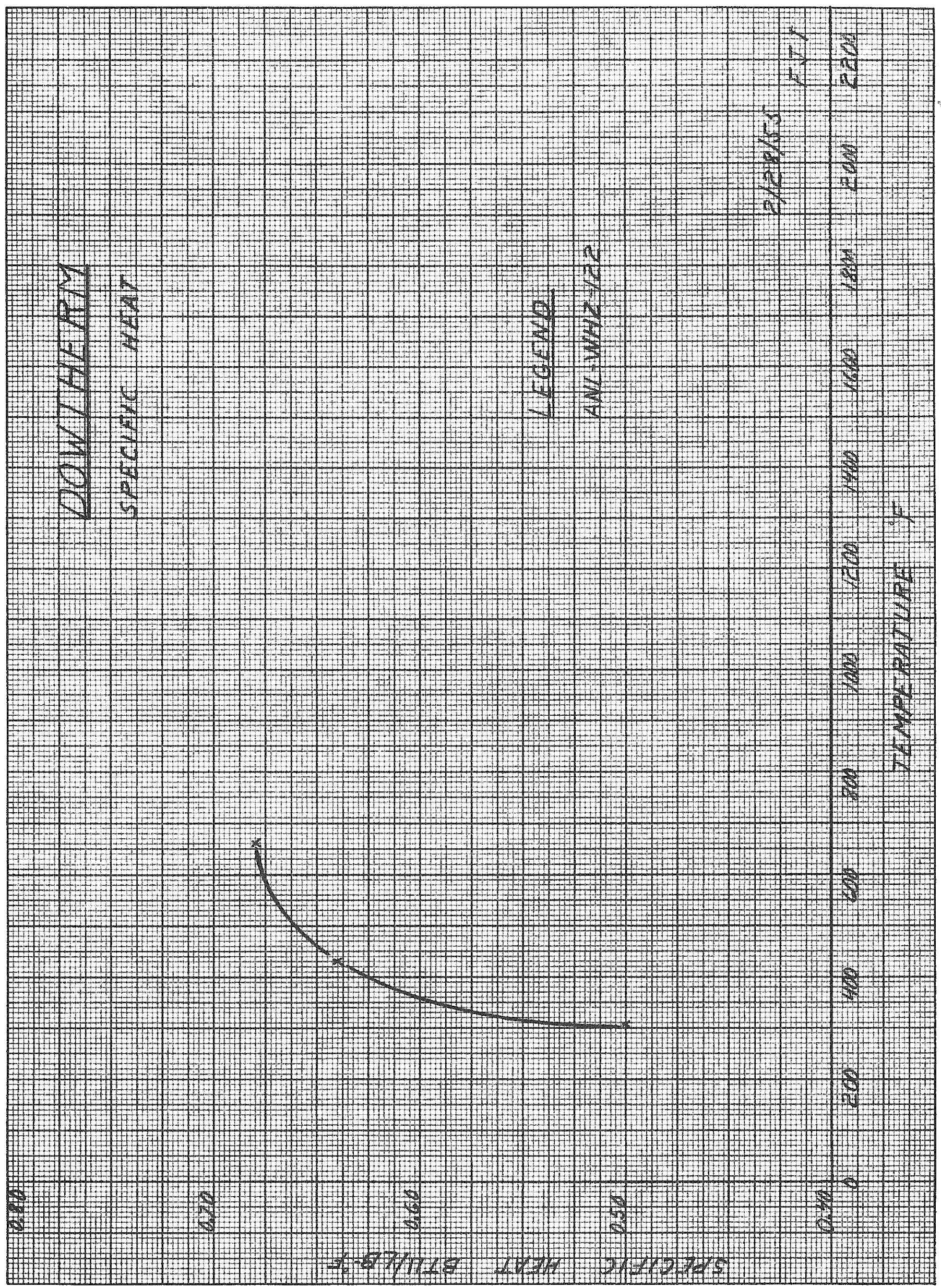




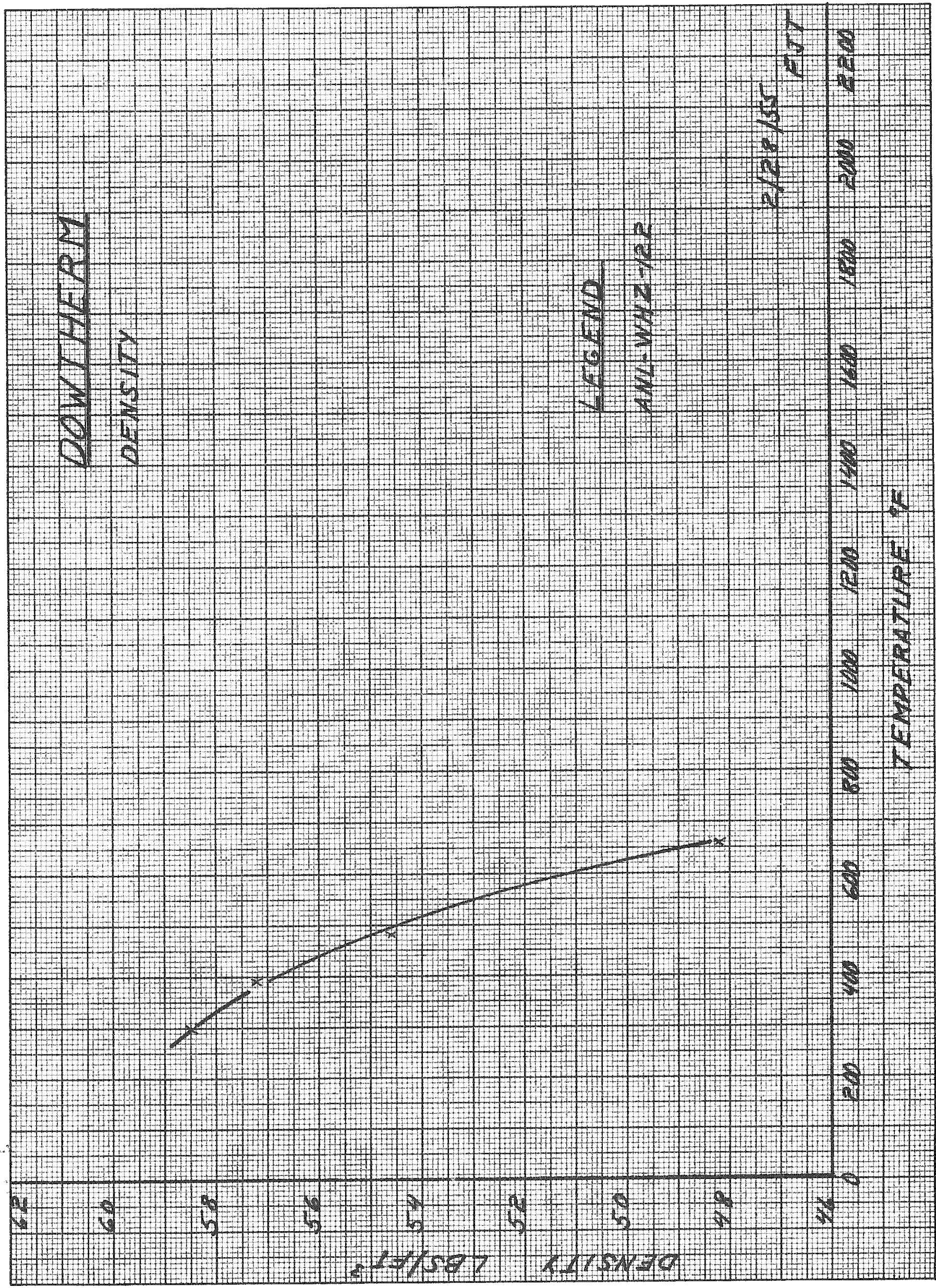




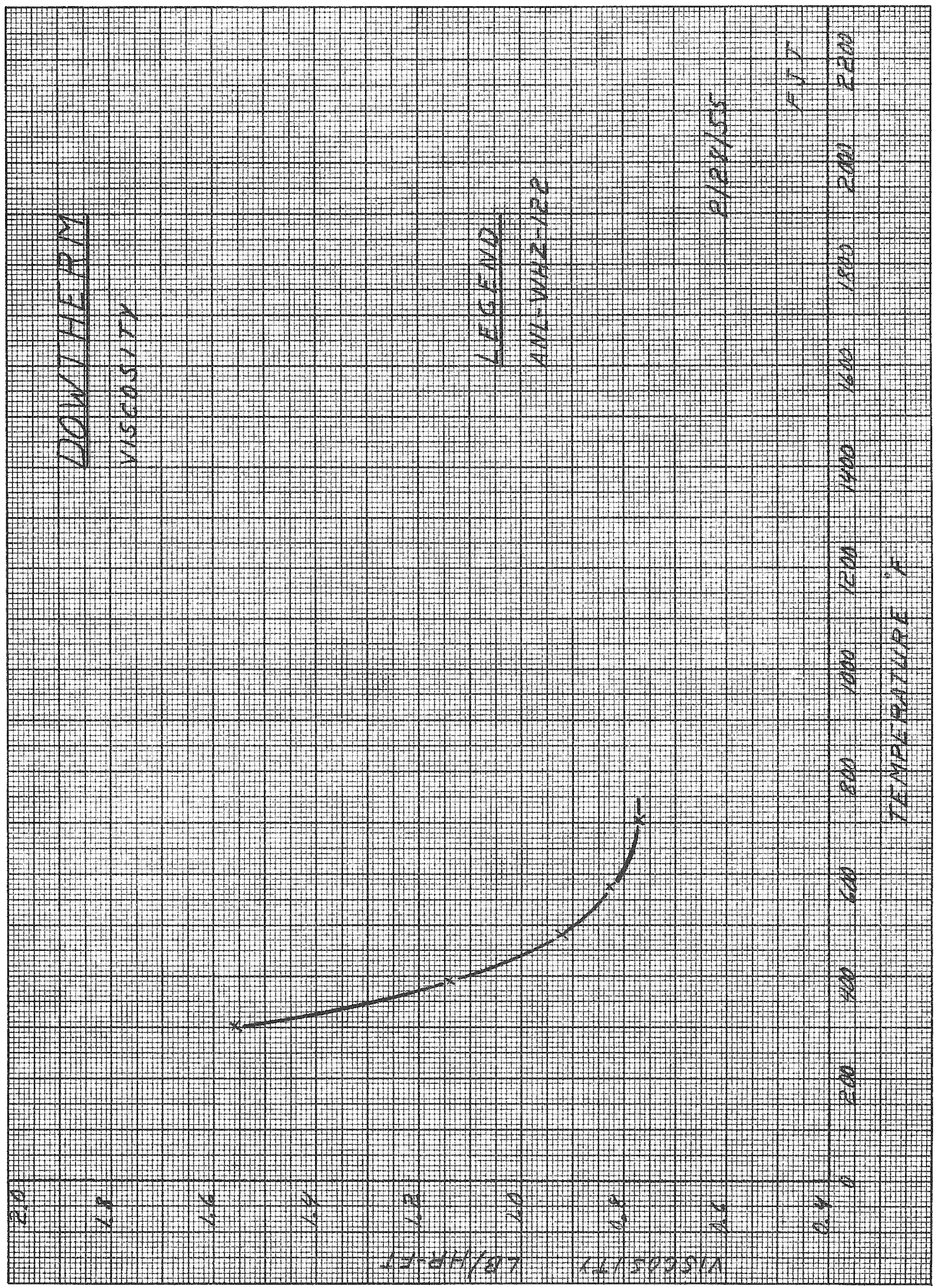




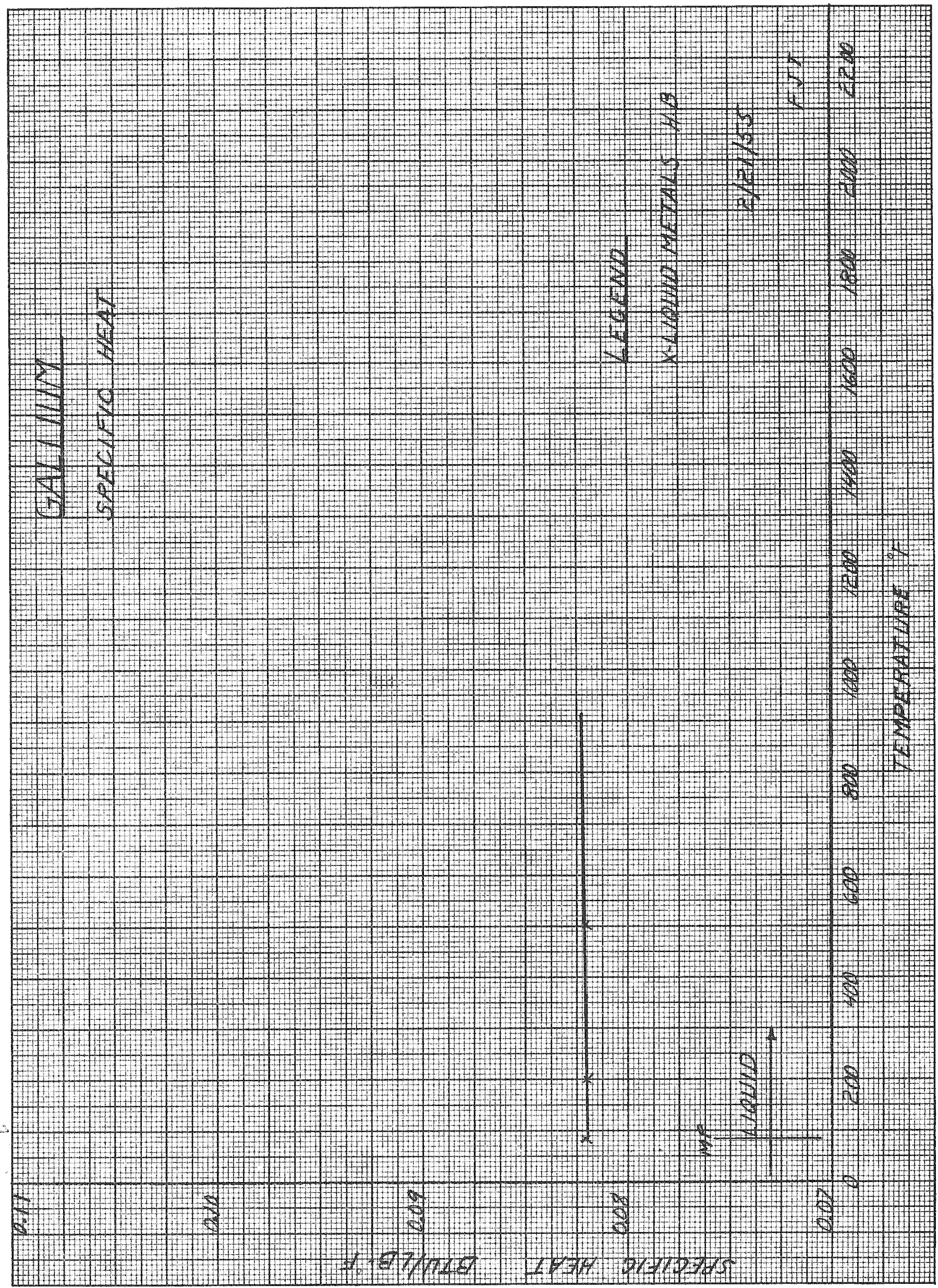




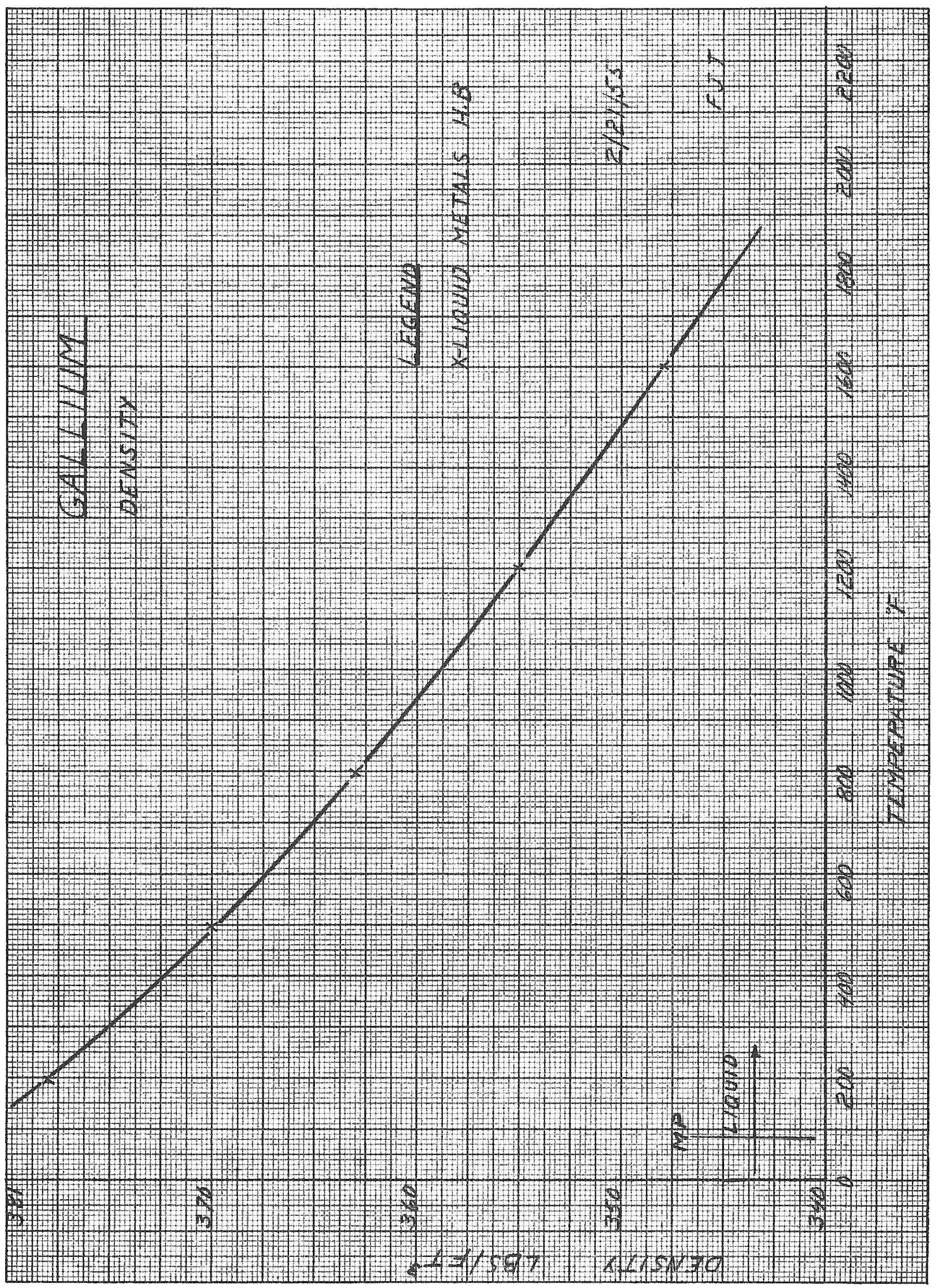




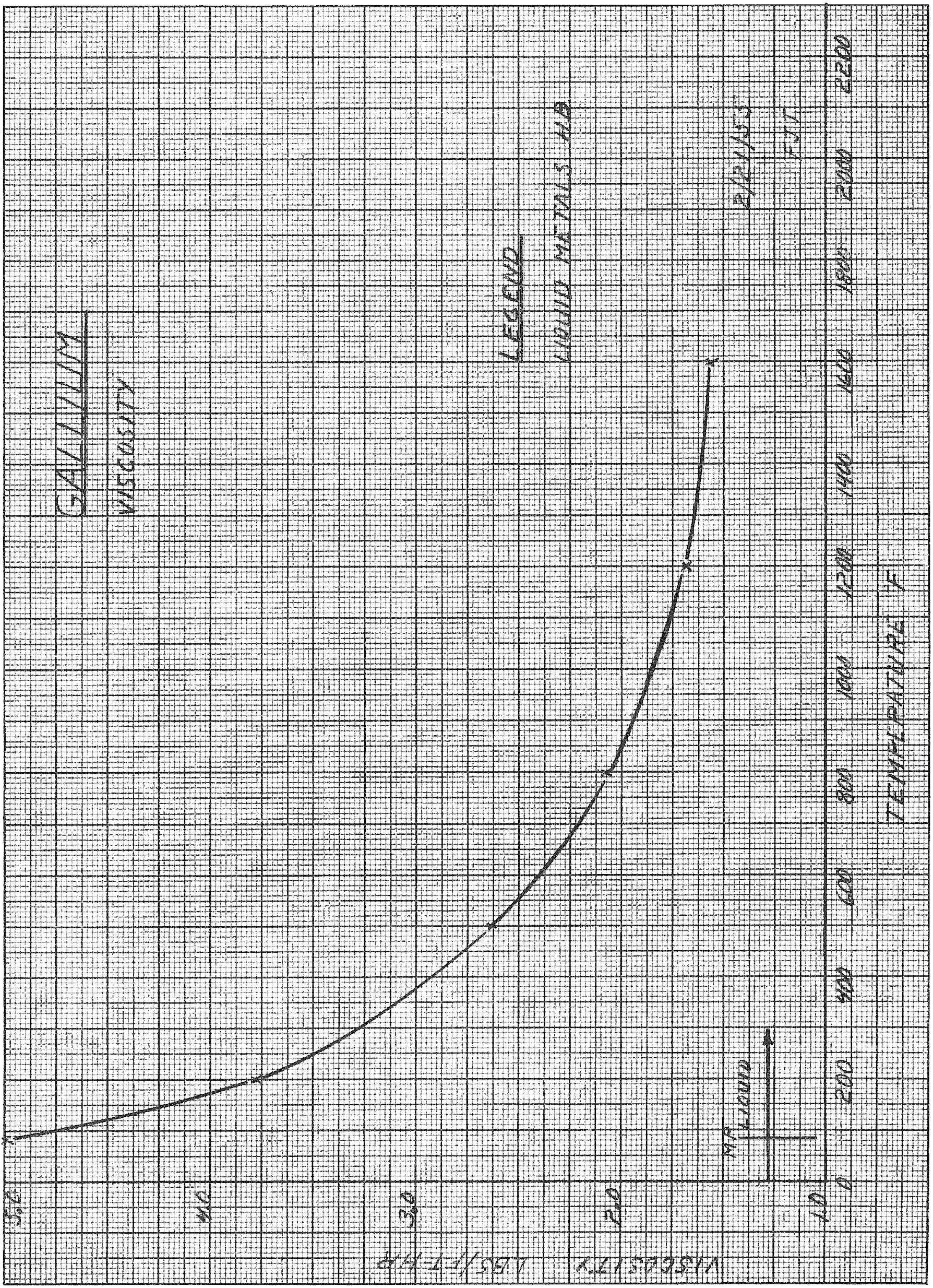




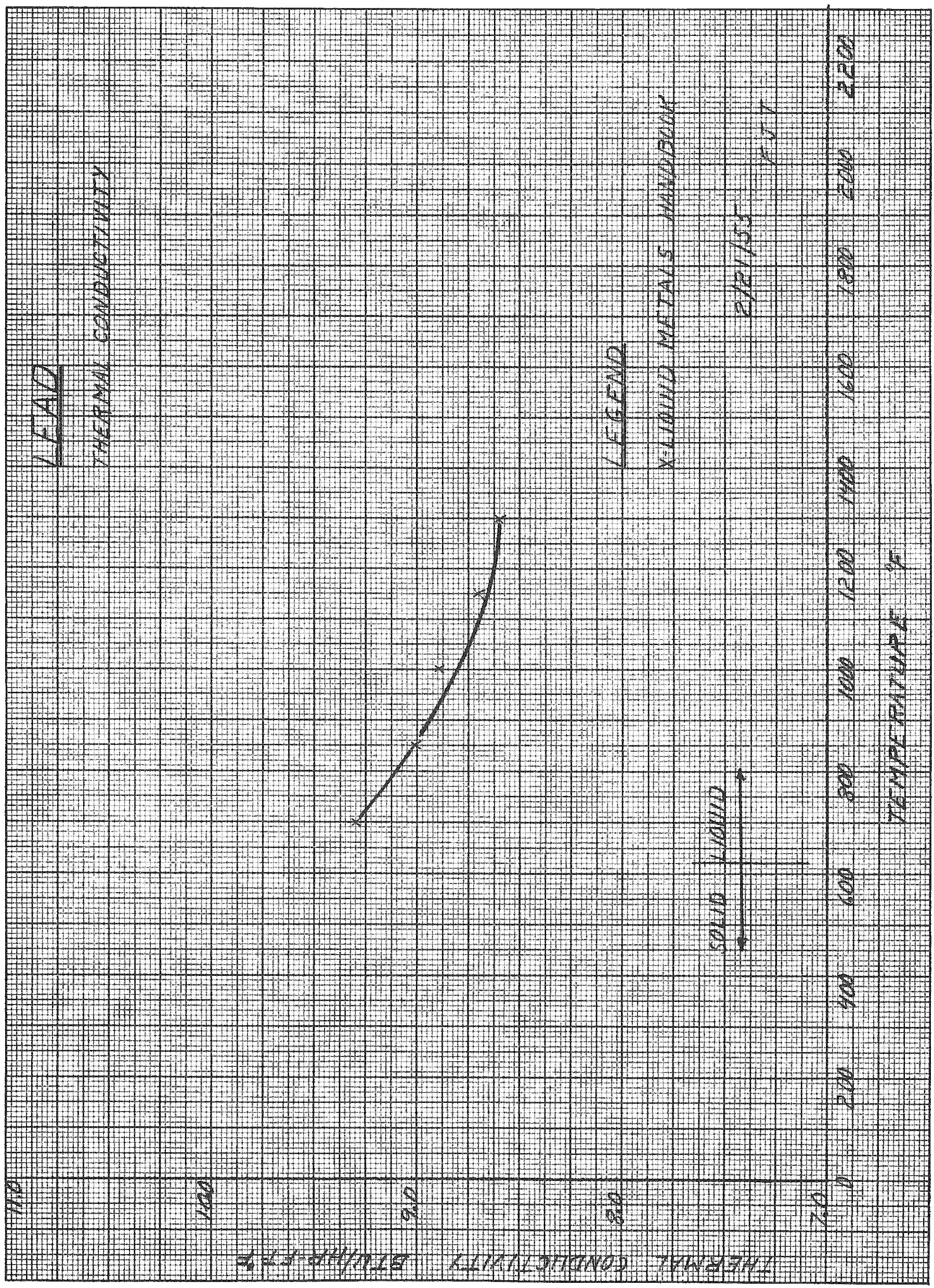




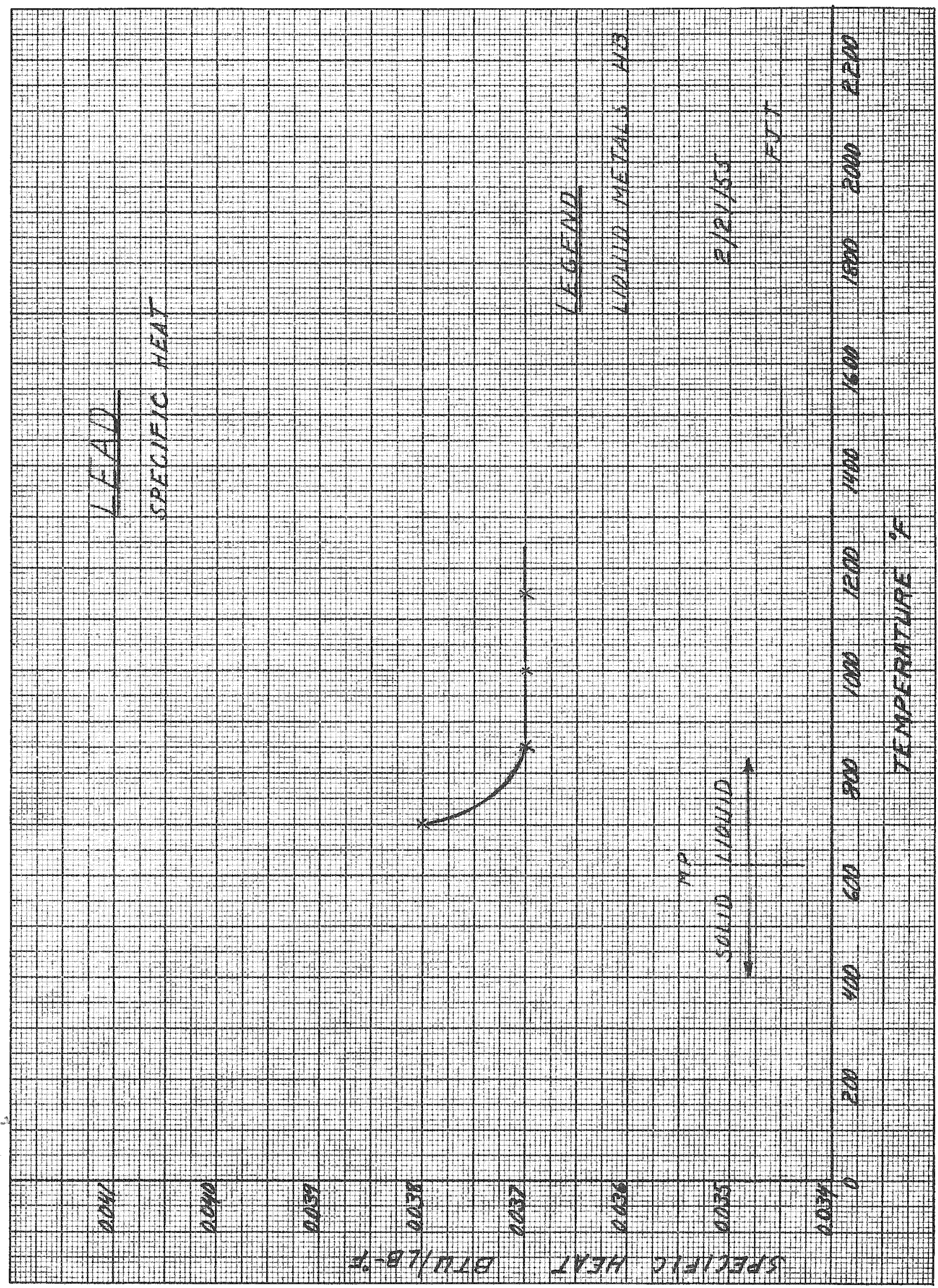




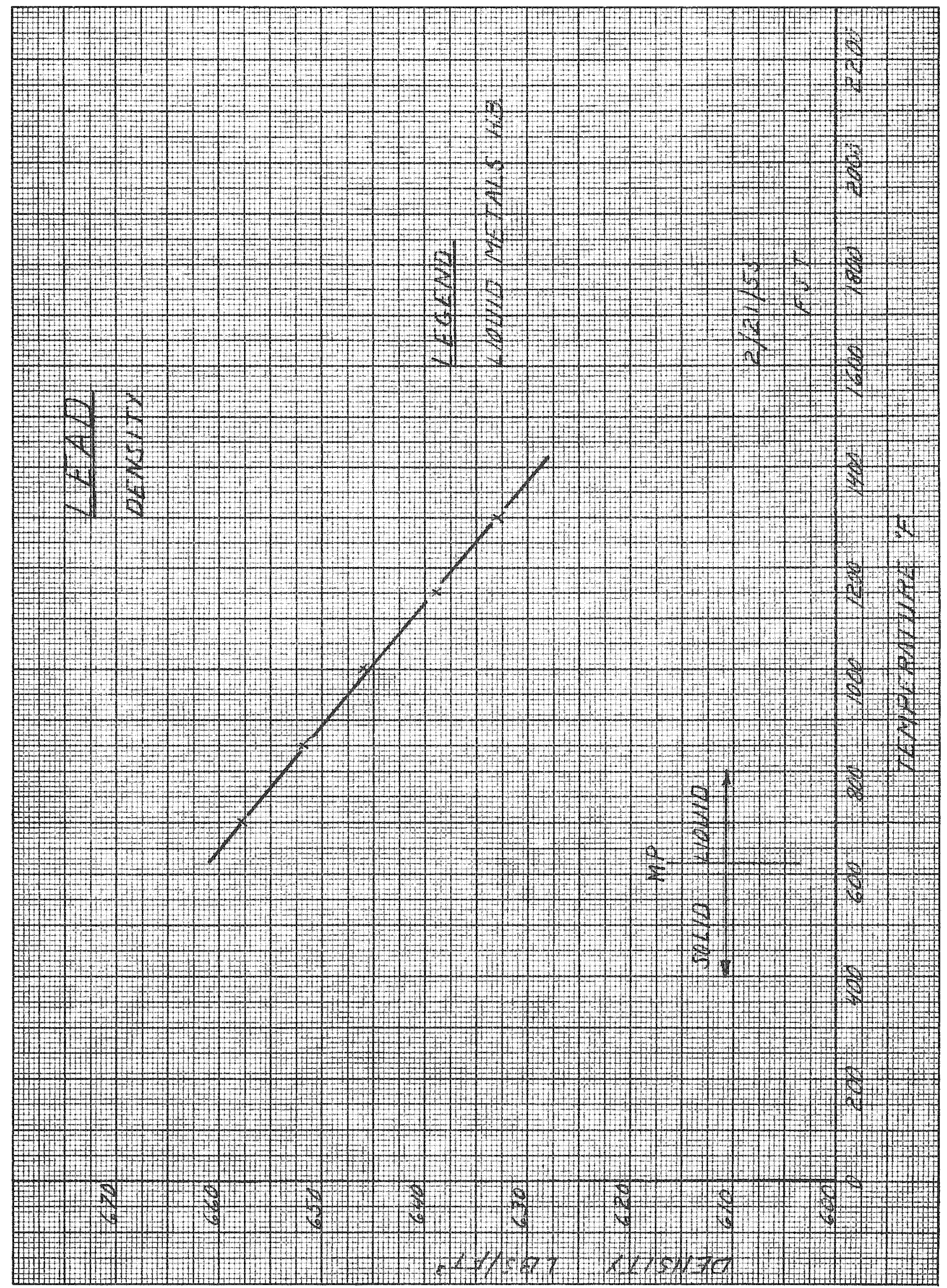




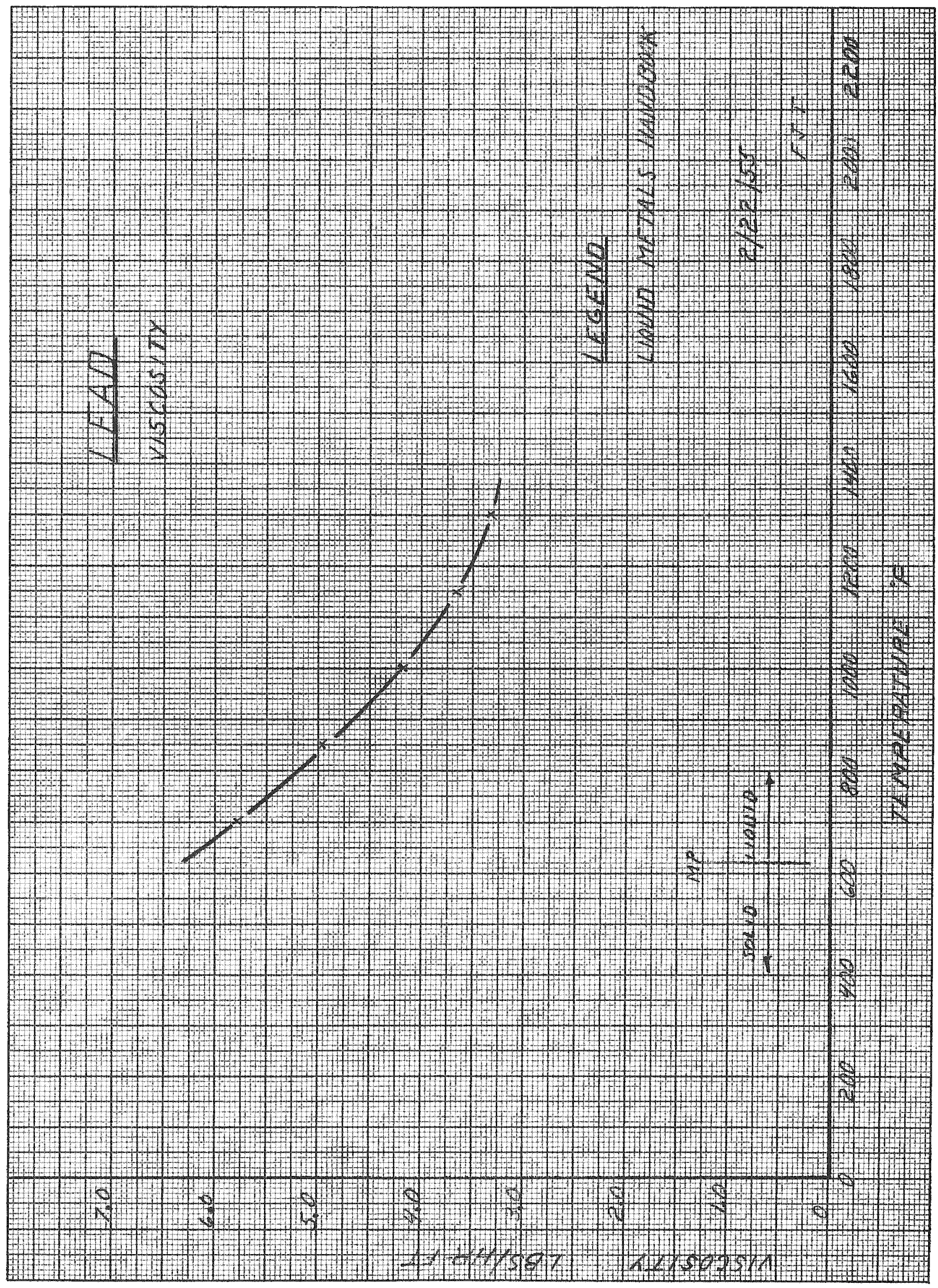




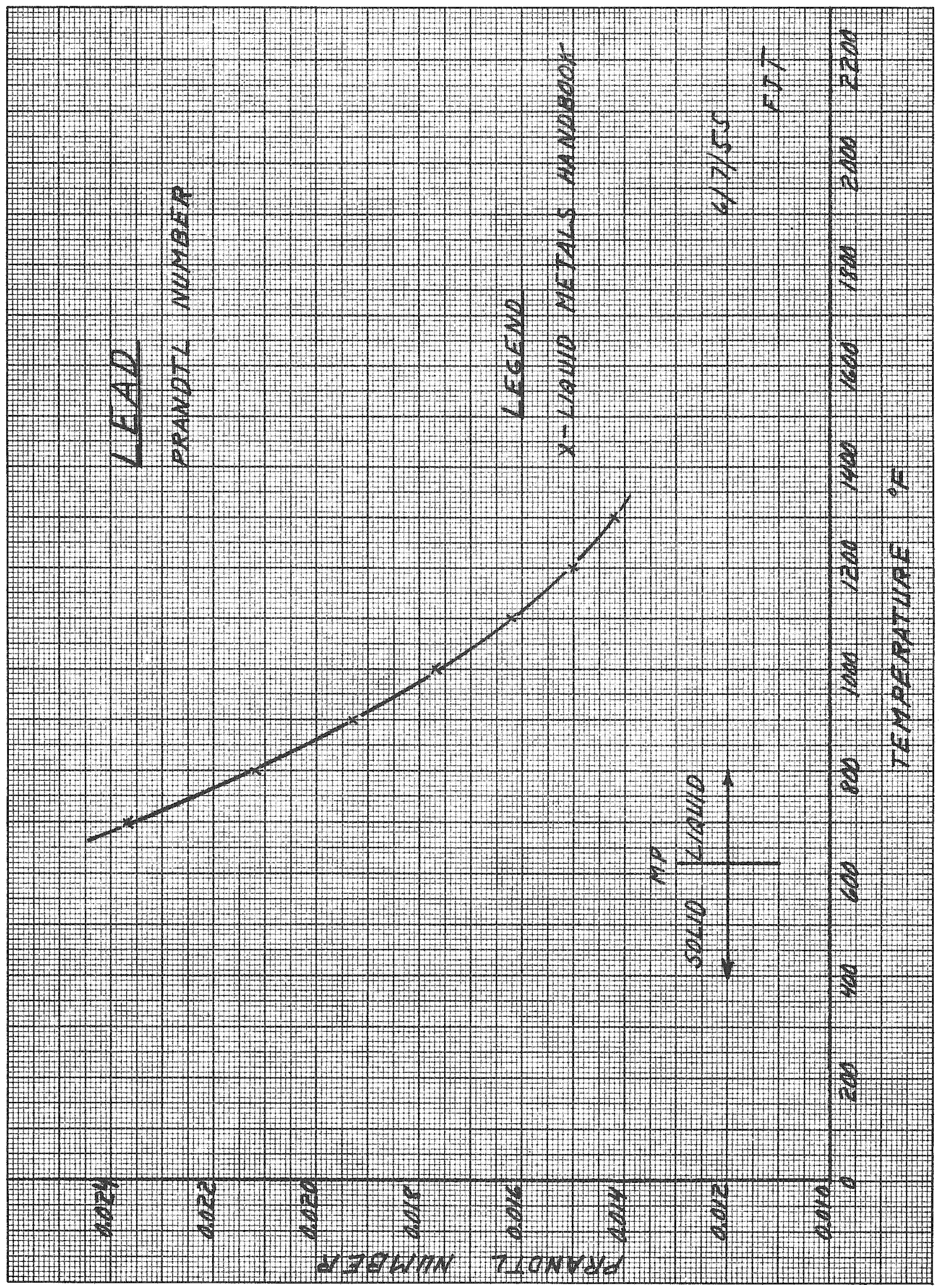




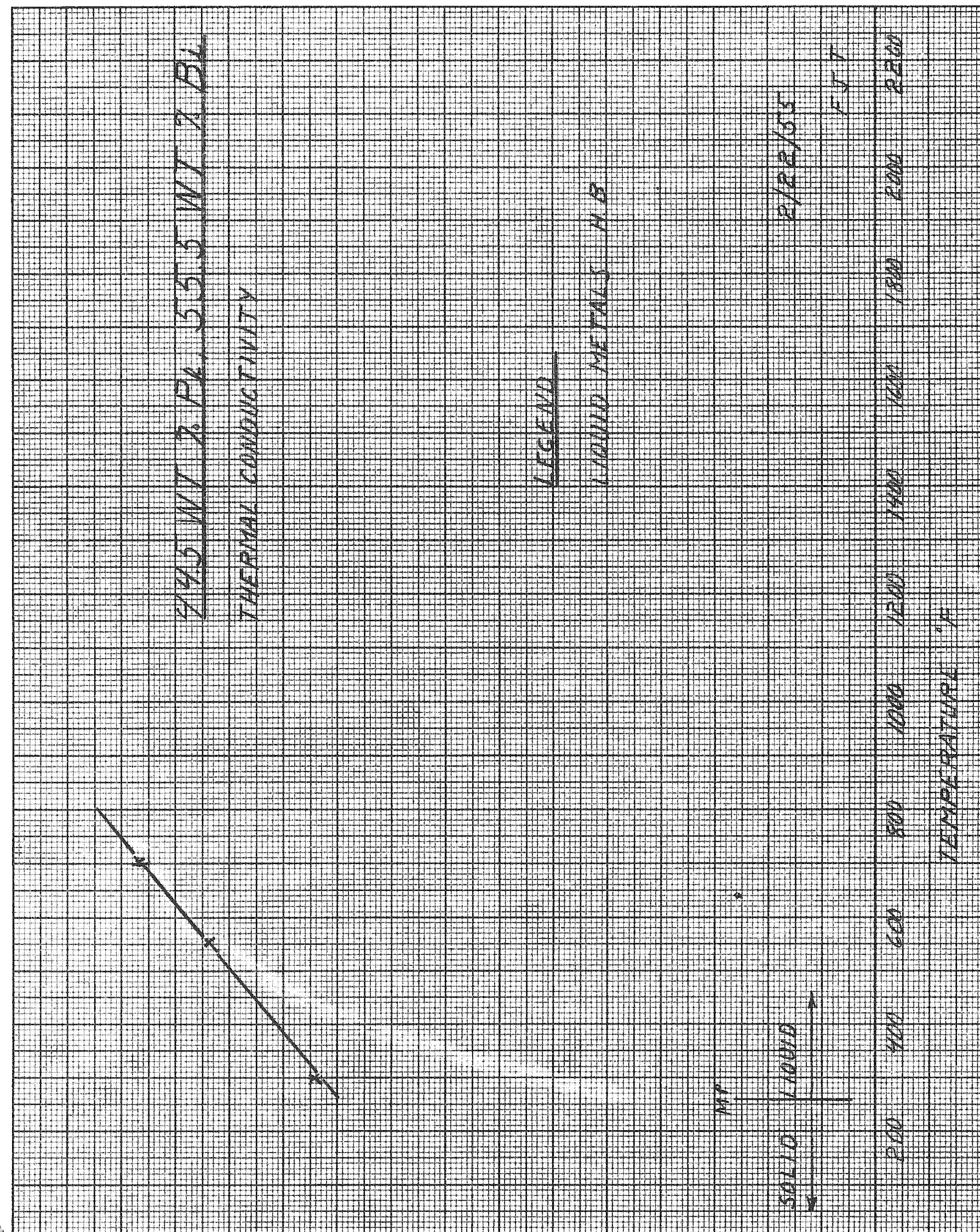

[1. 1.

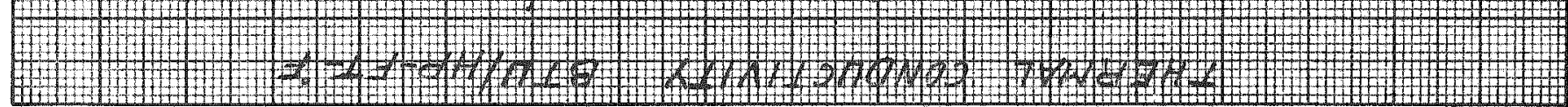




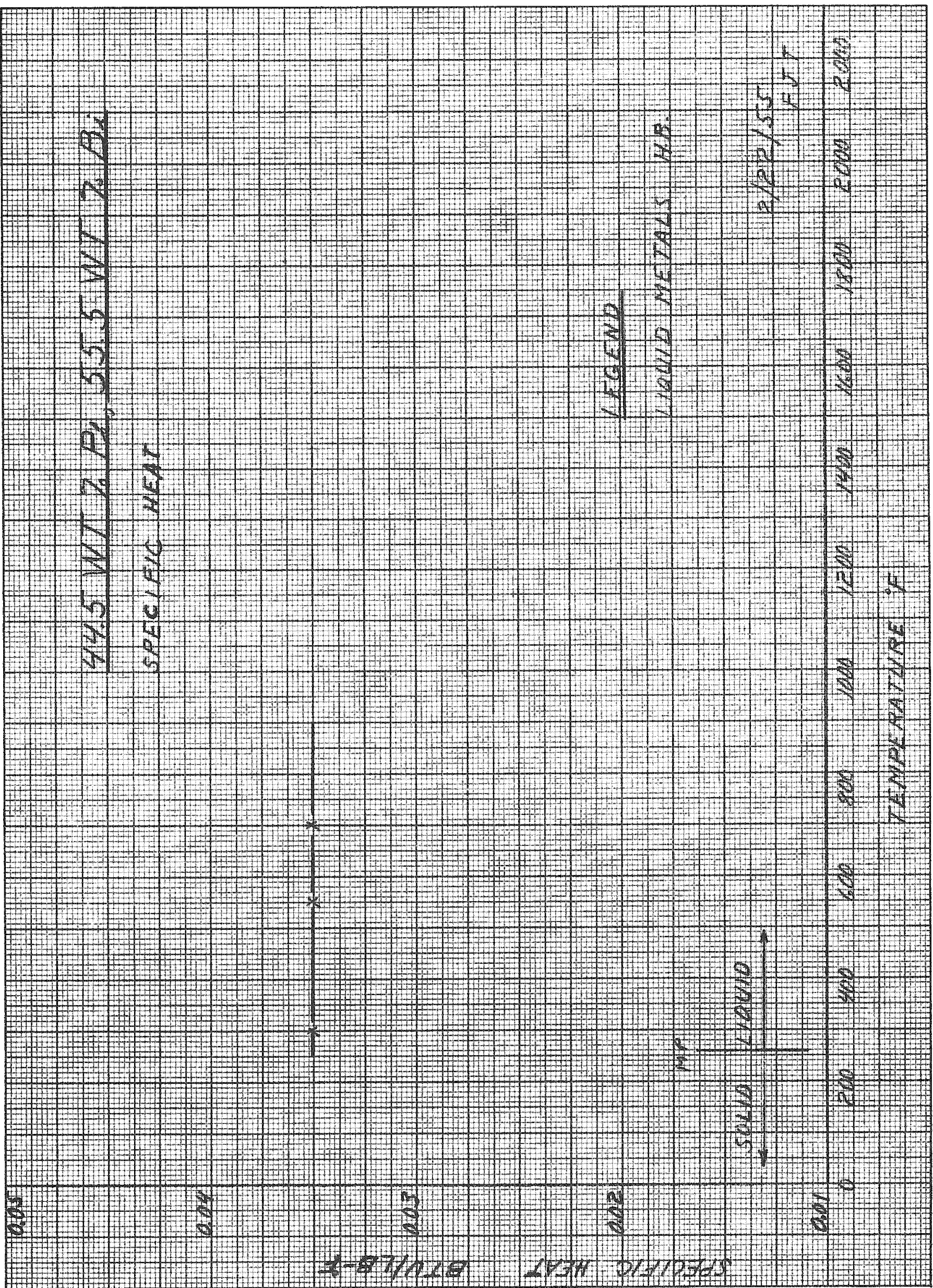




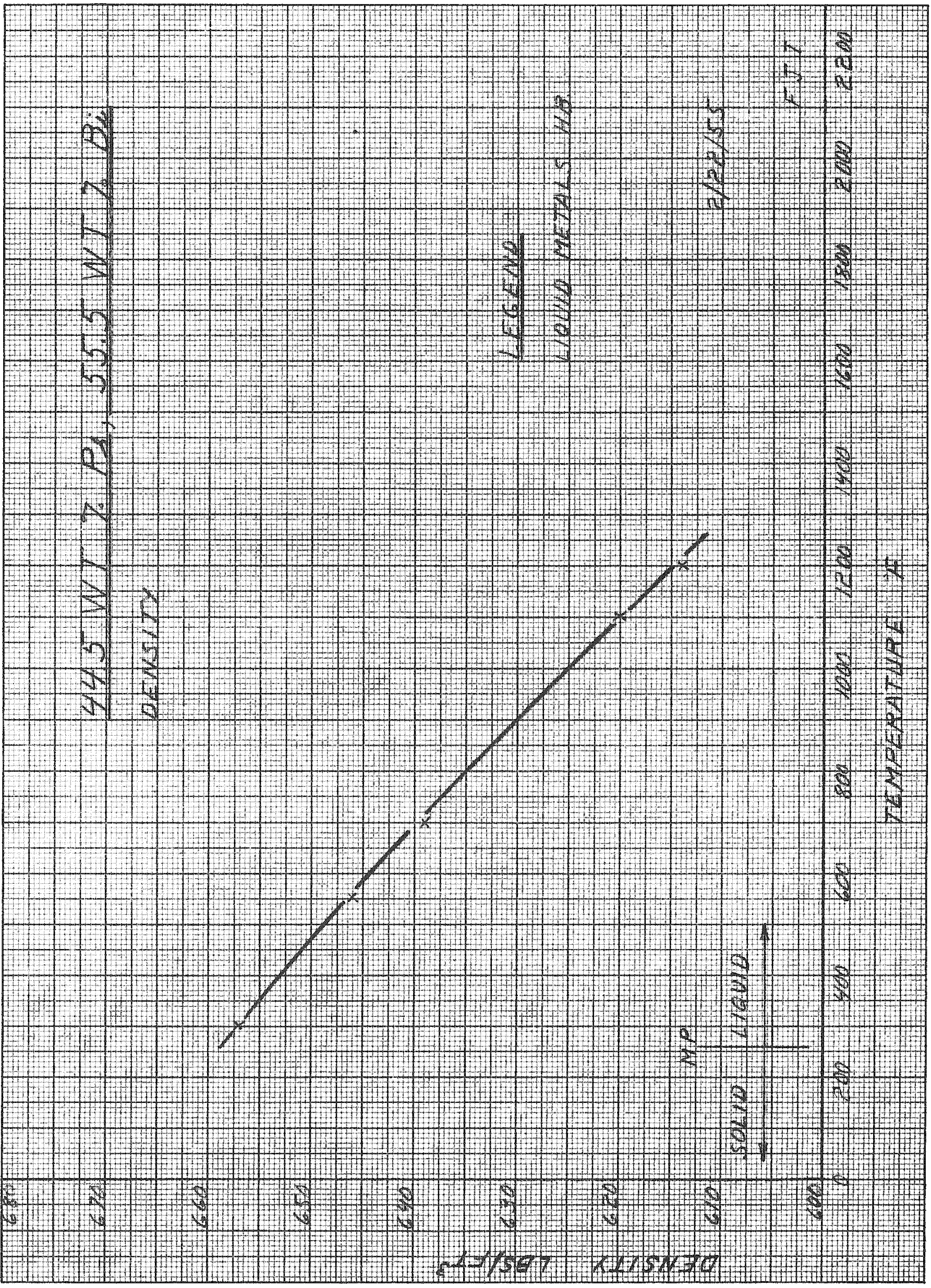




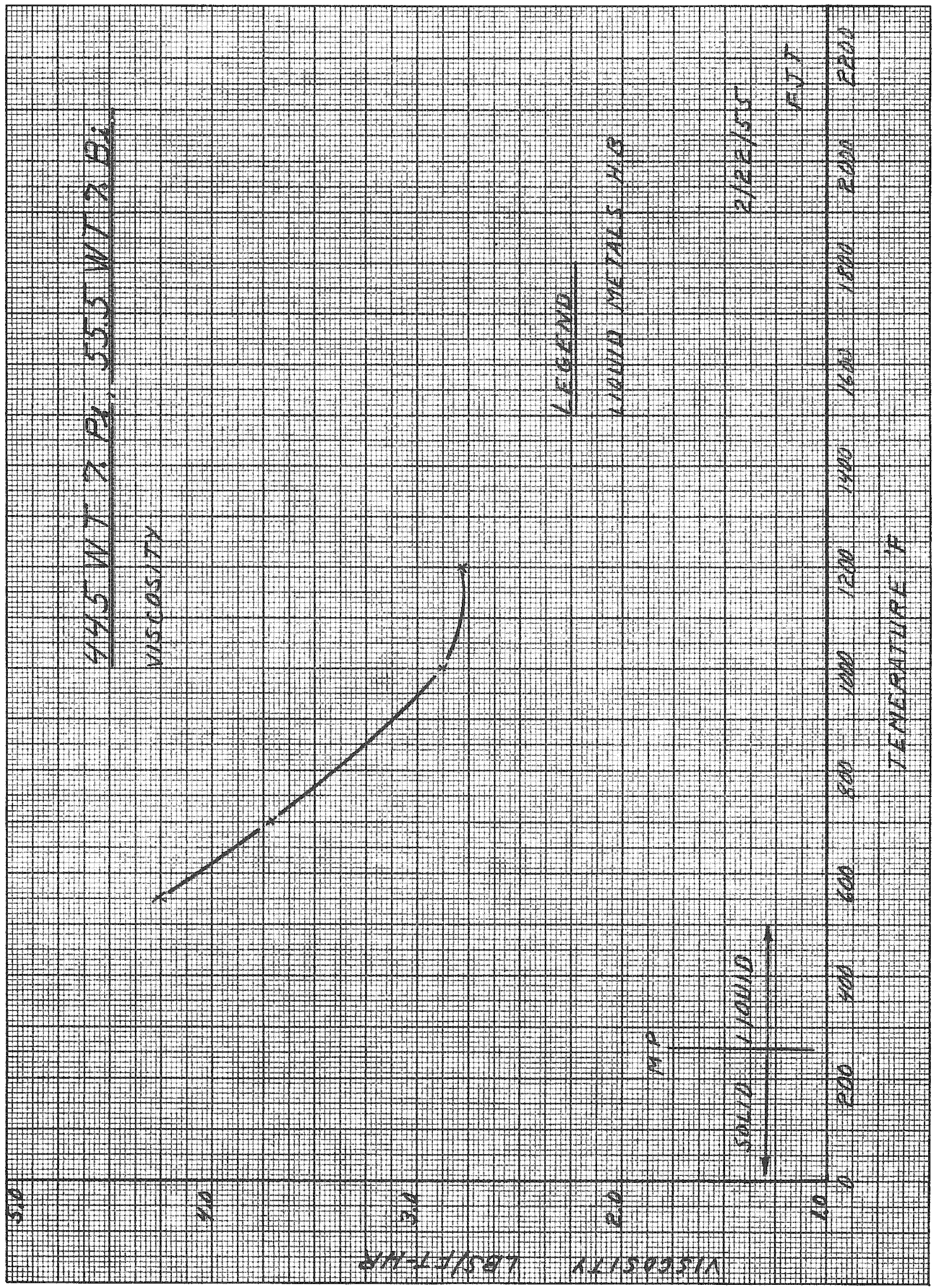




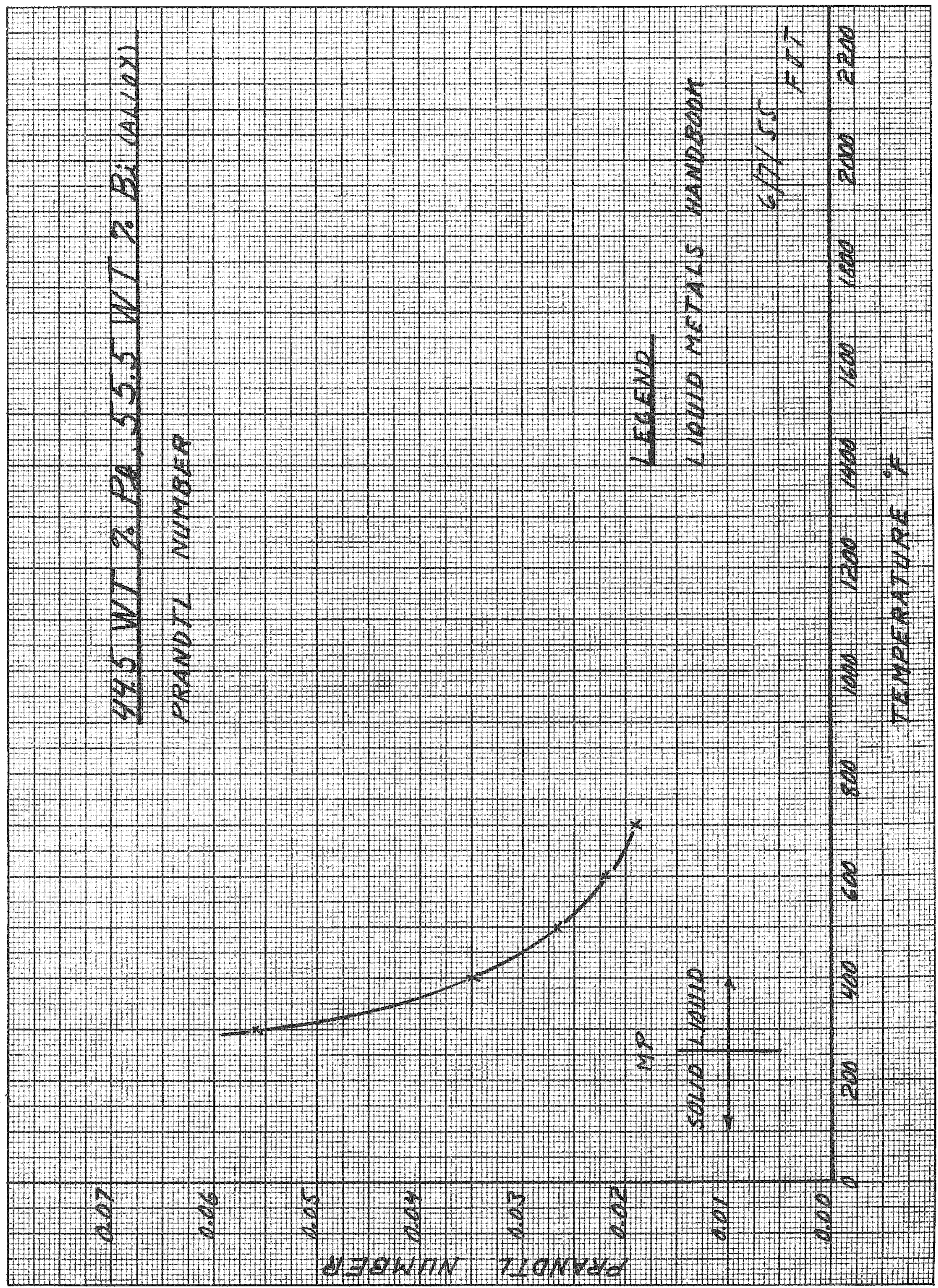




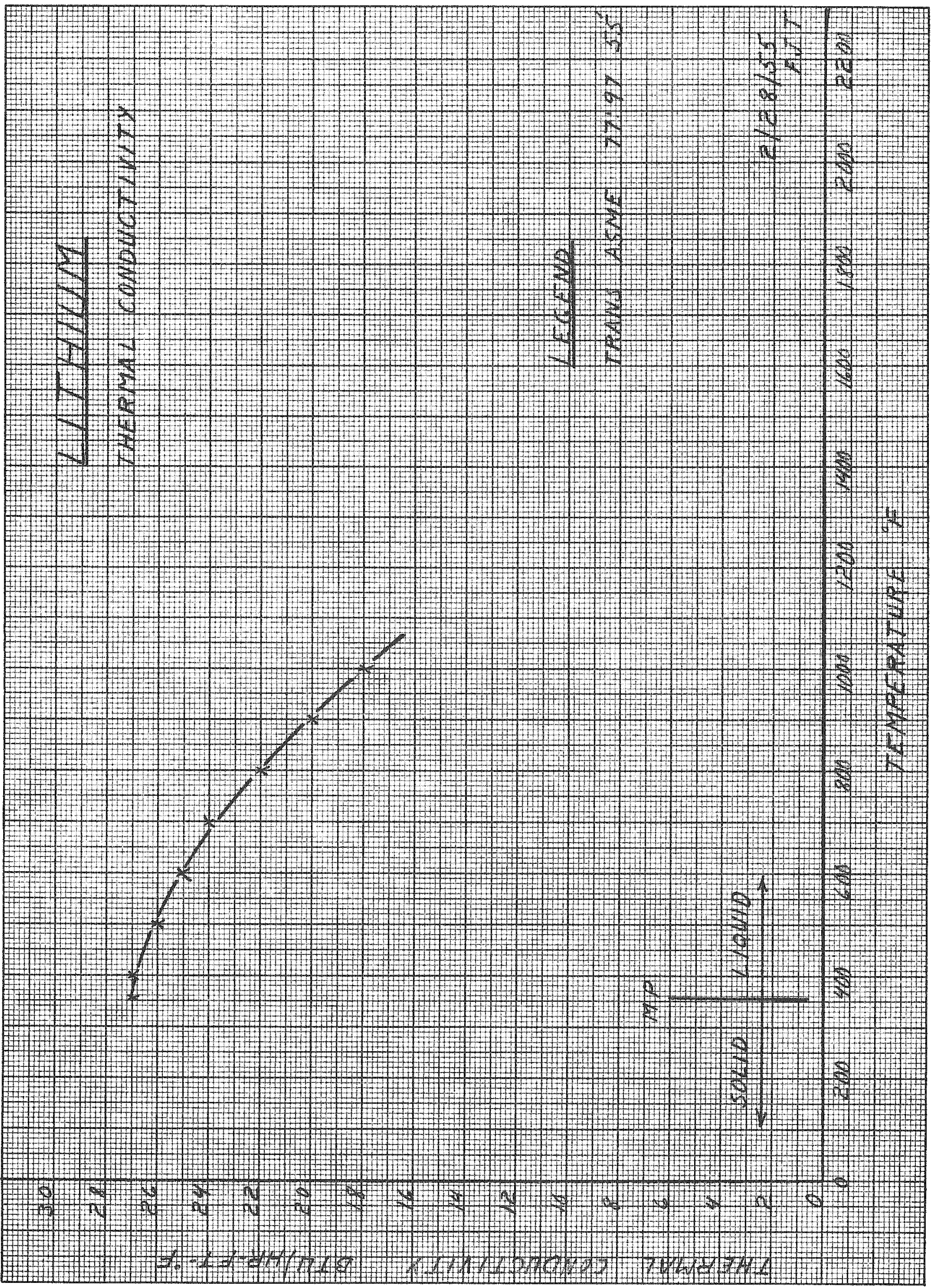




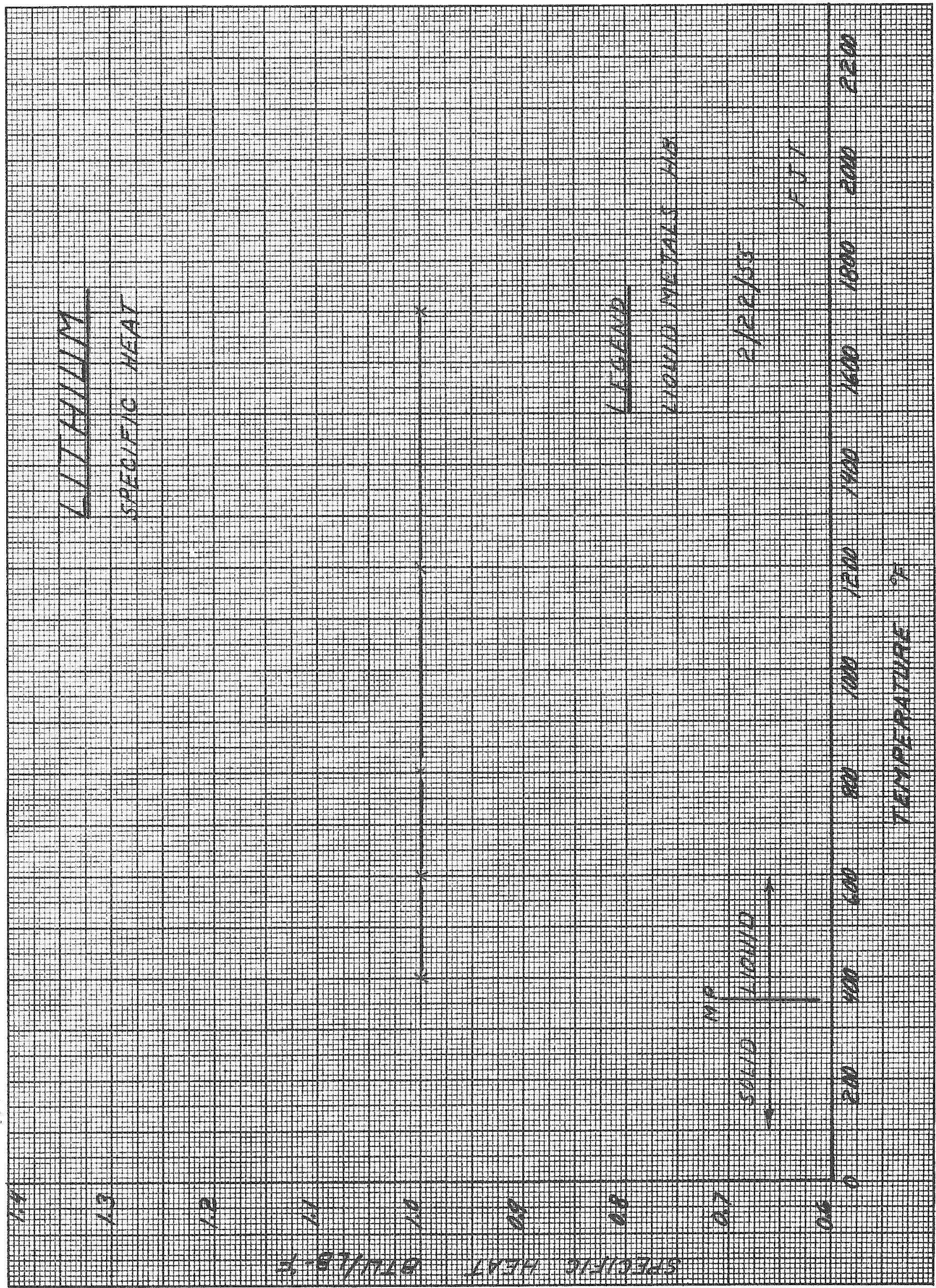


-
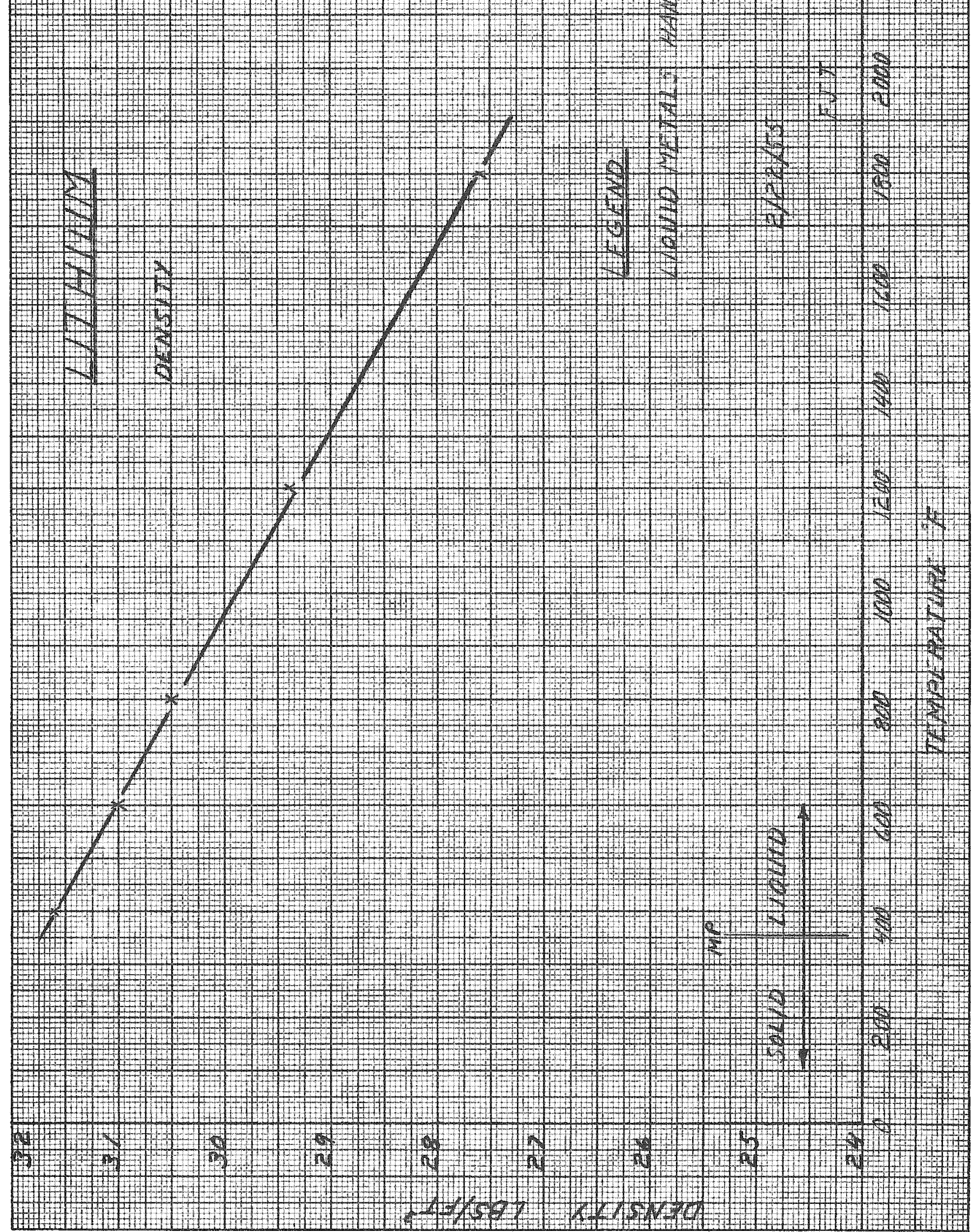


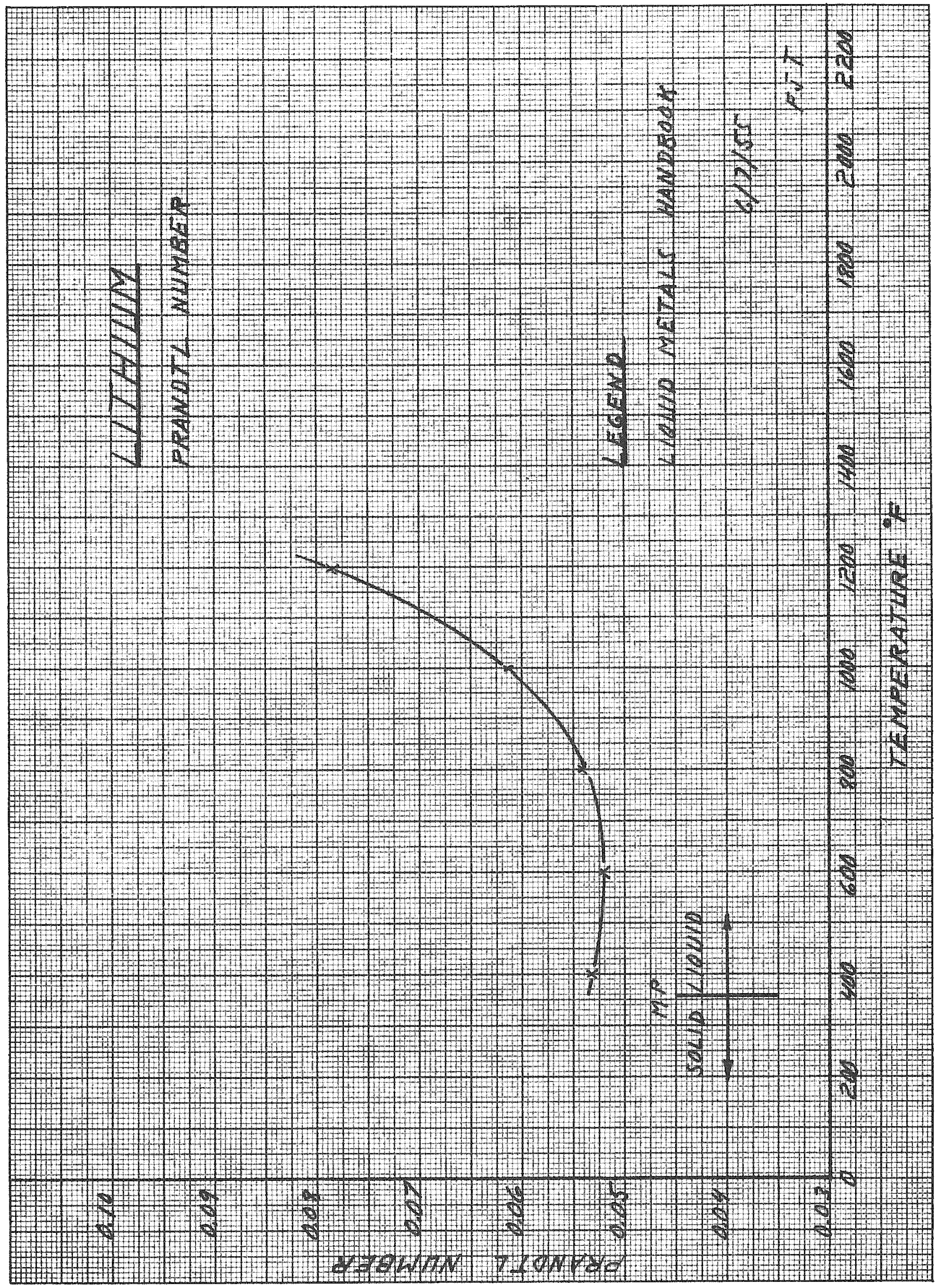




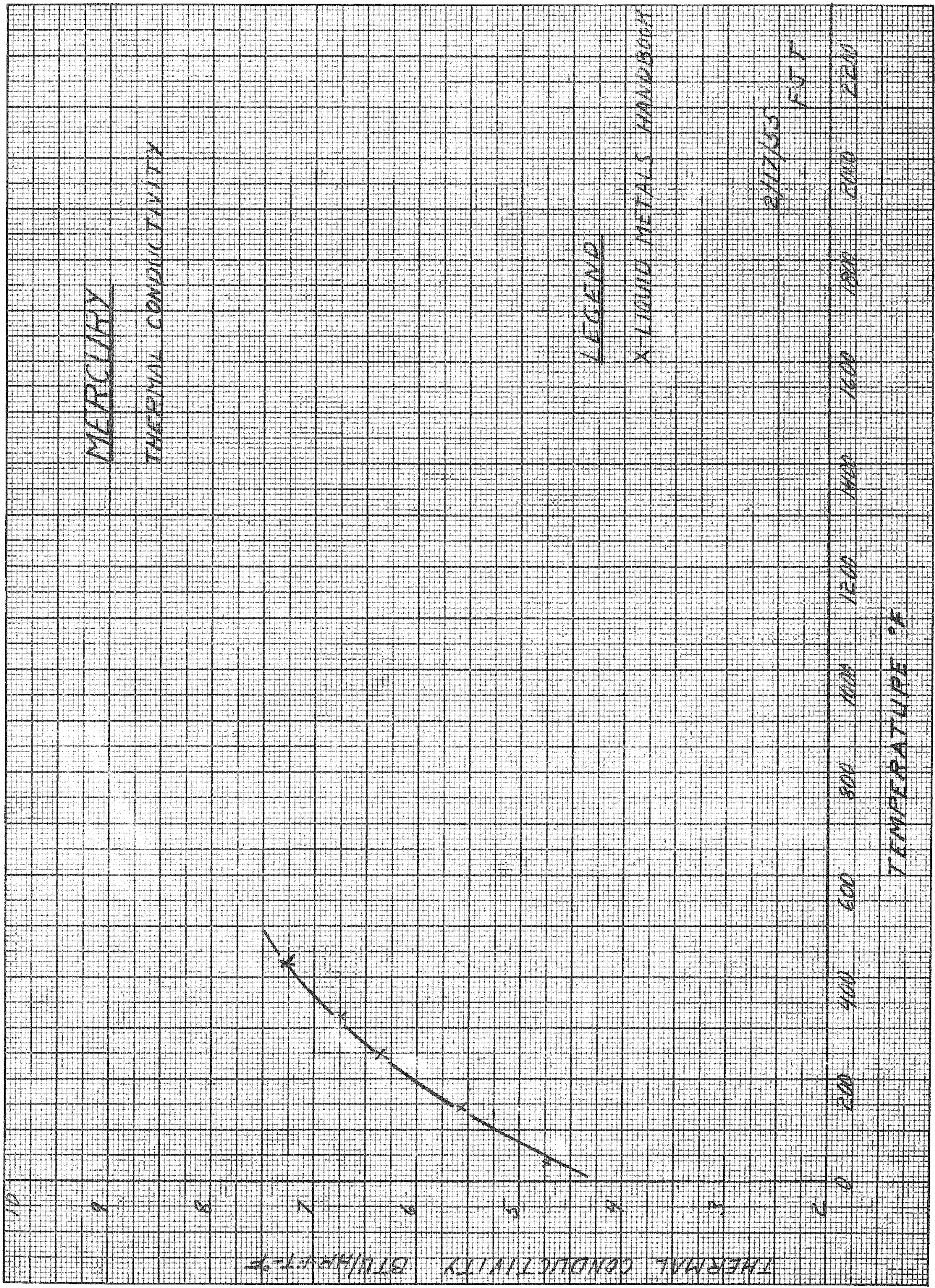




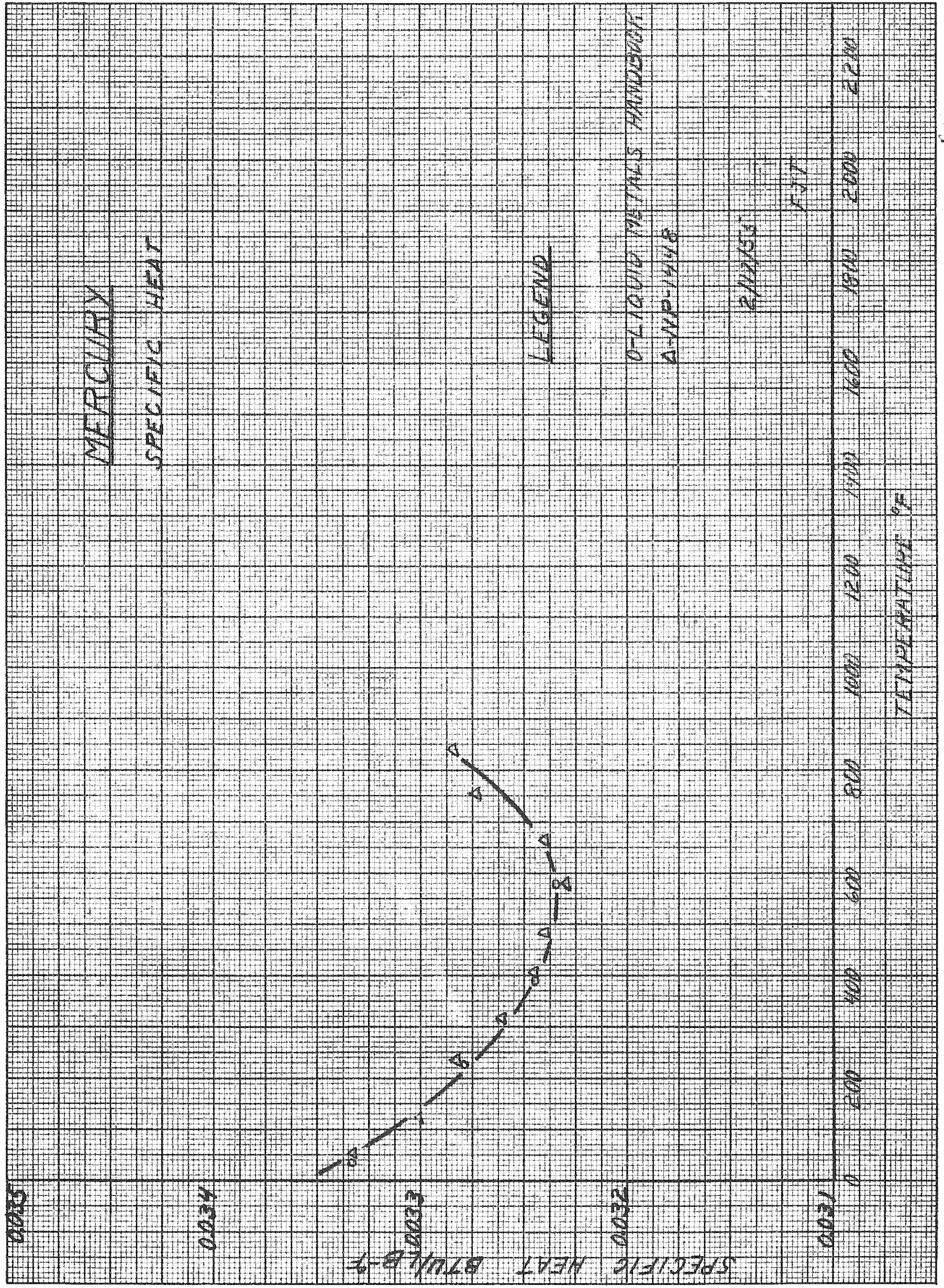




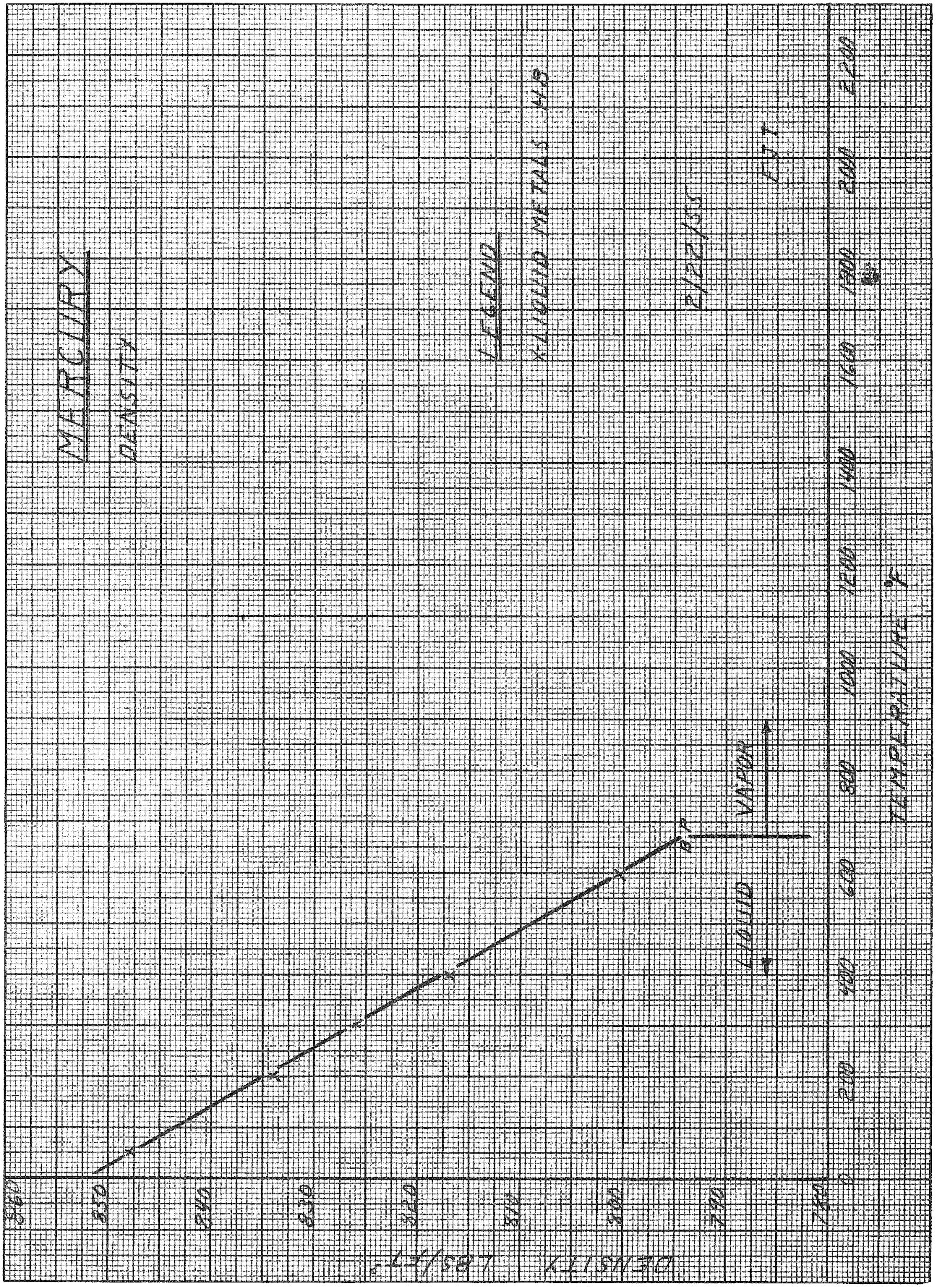




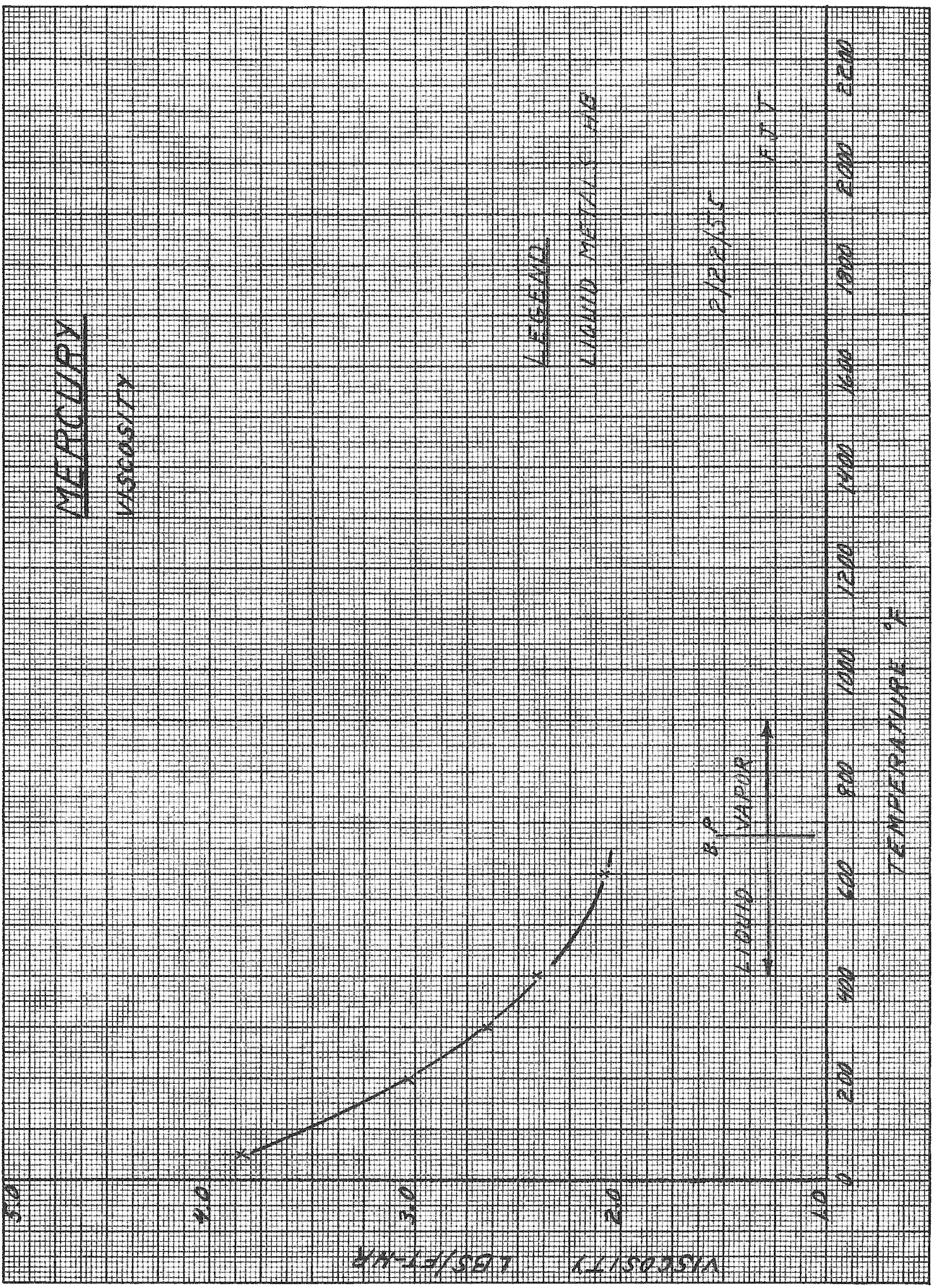




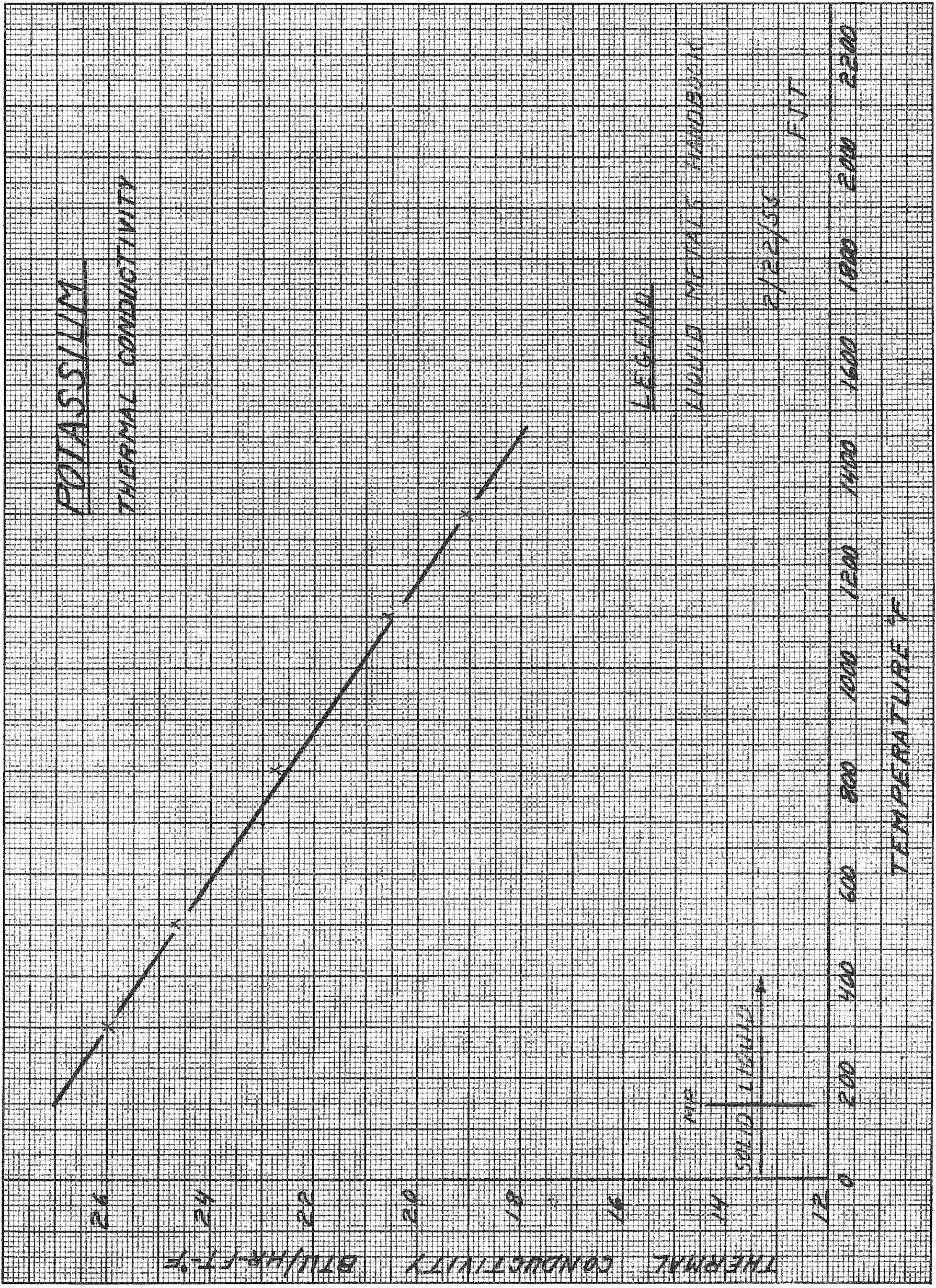




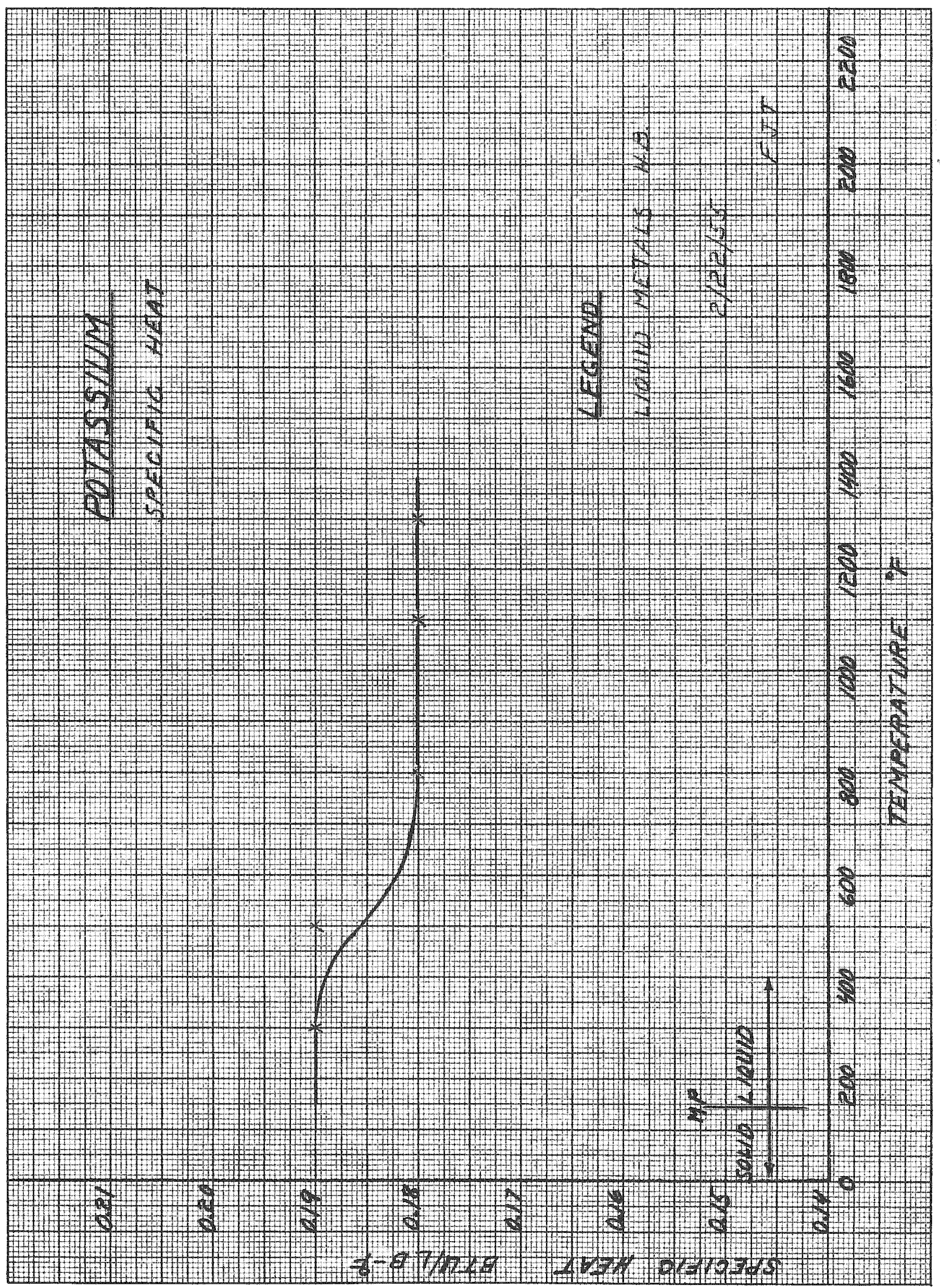




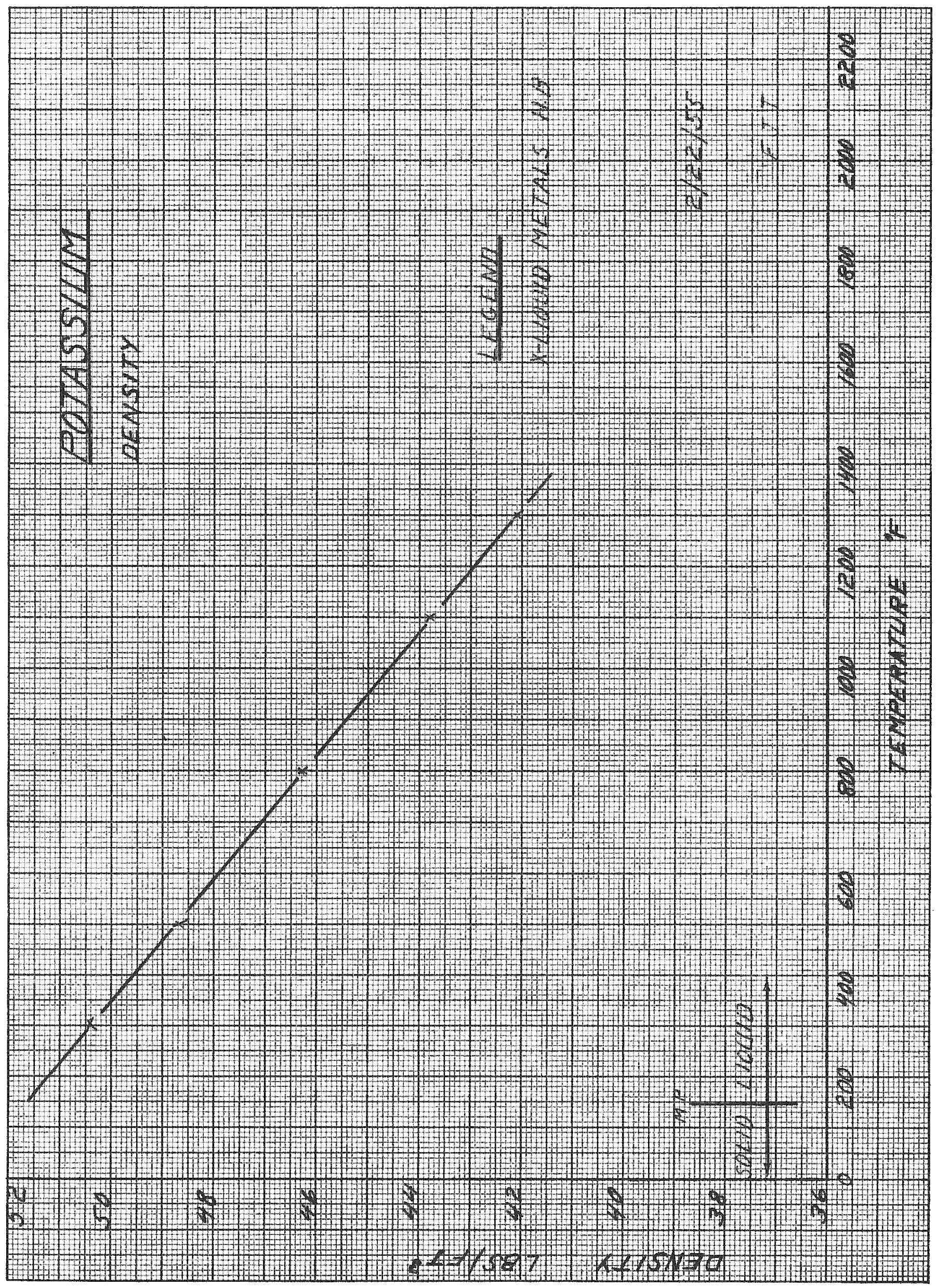




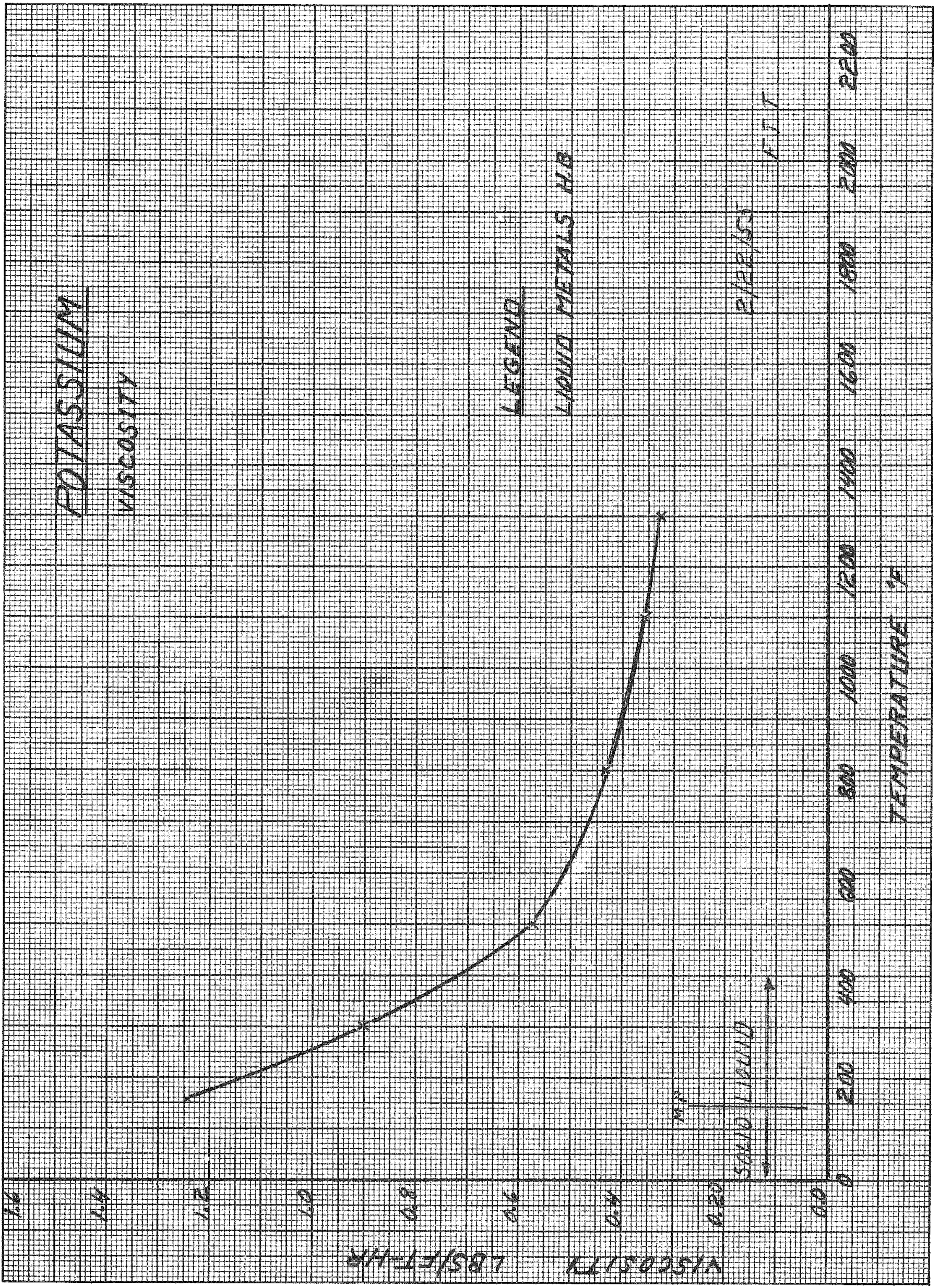




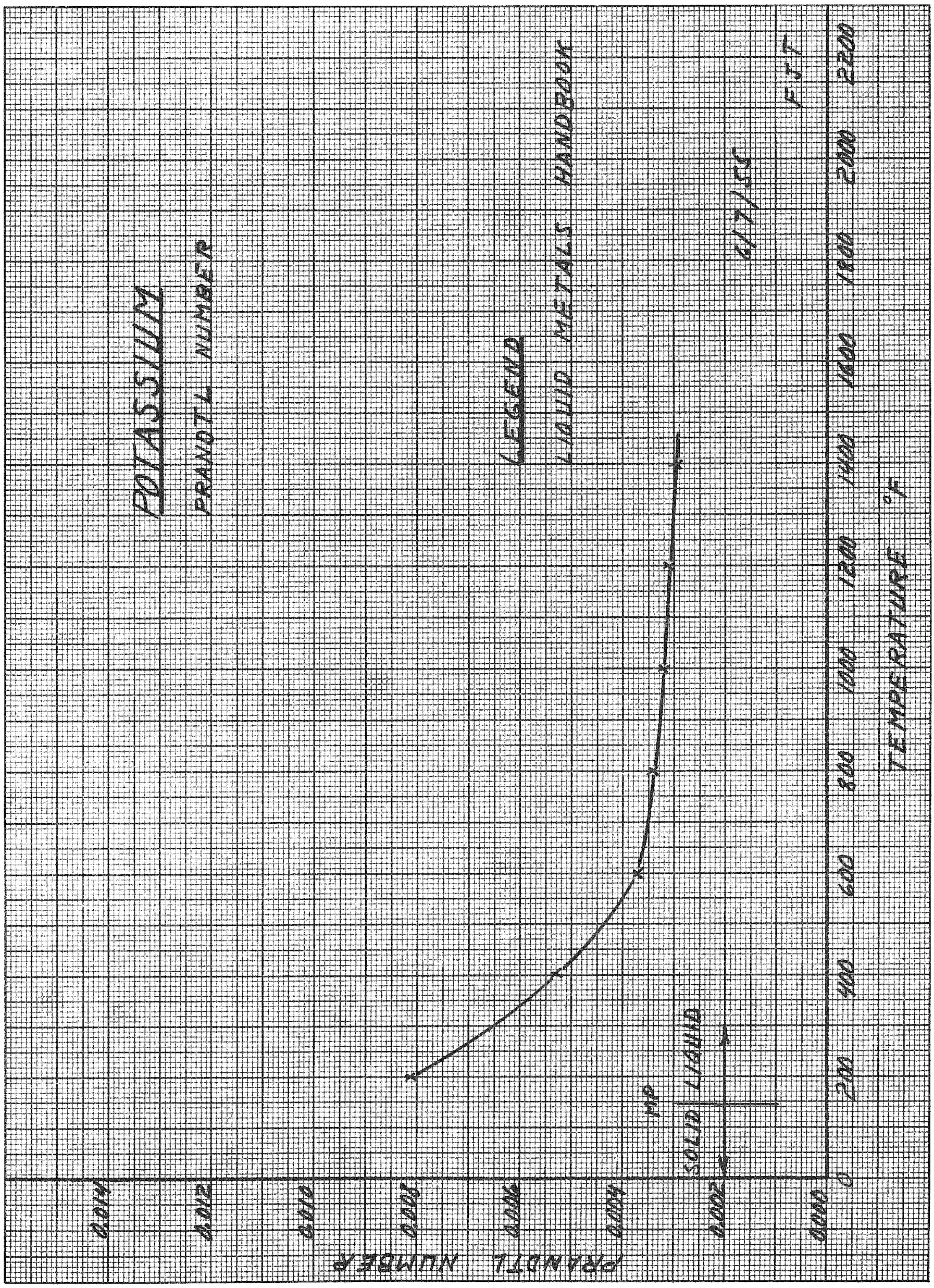




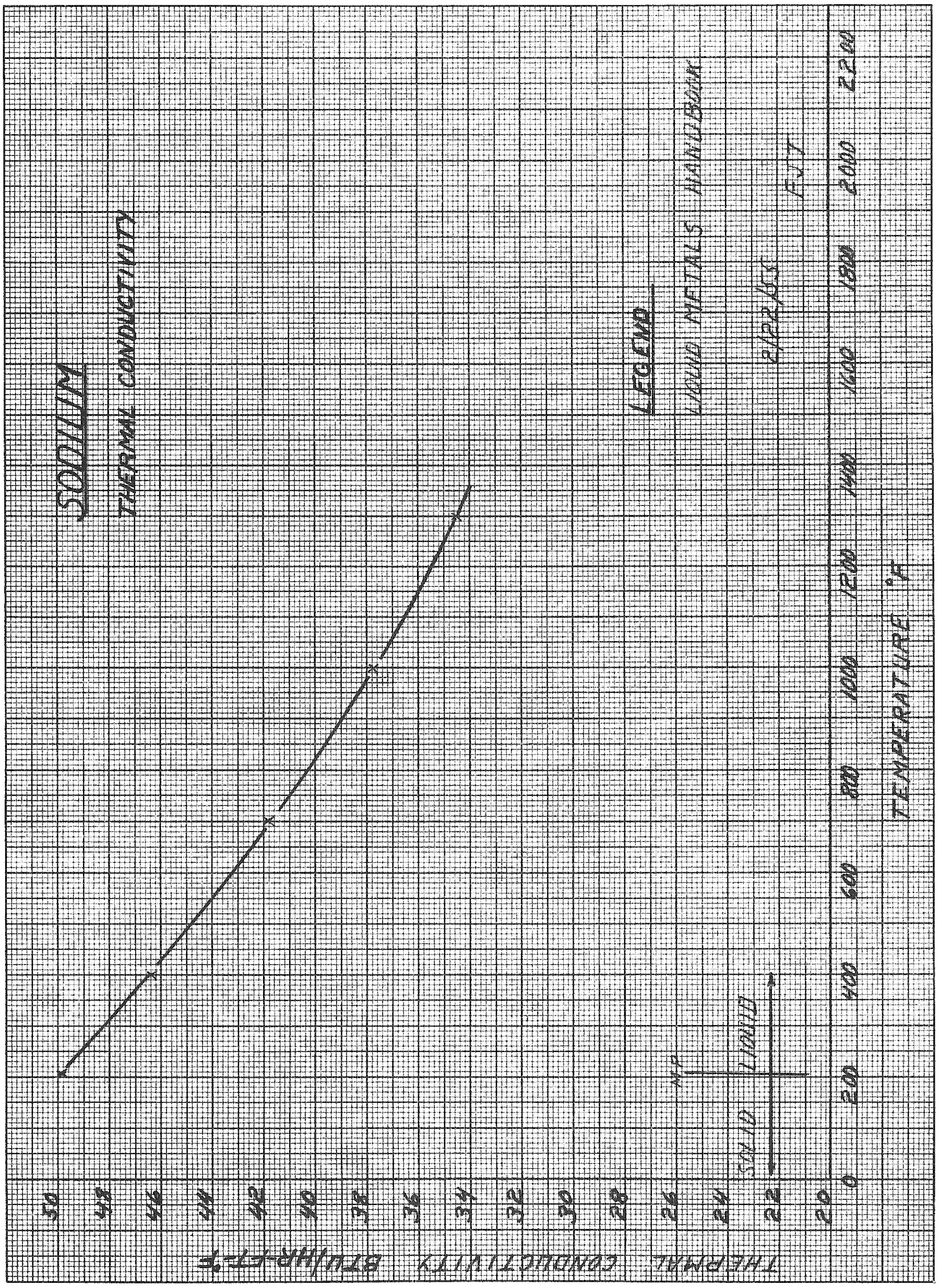




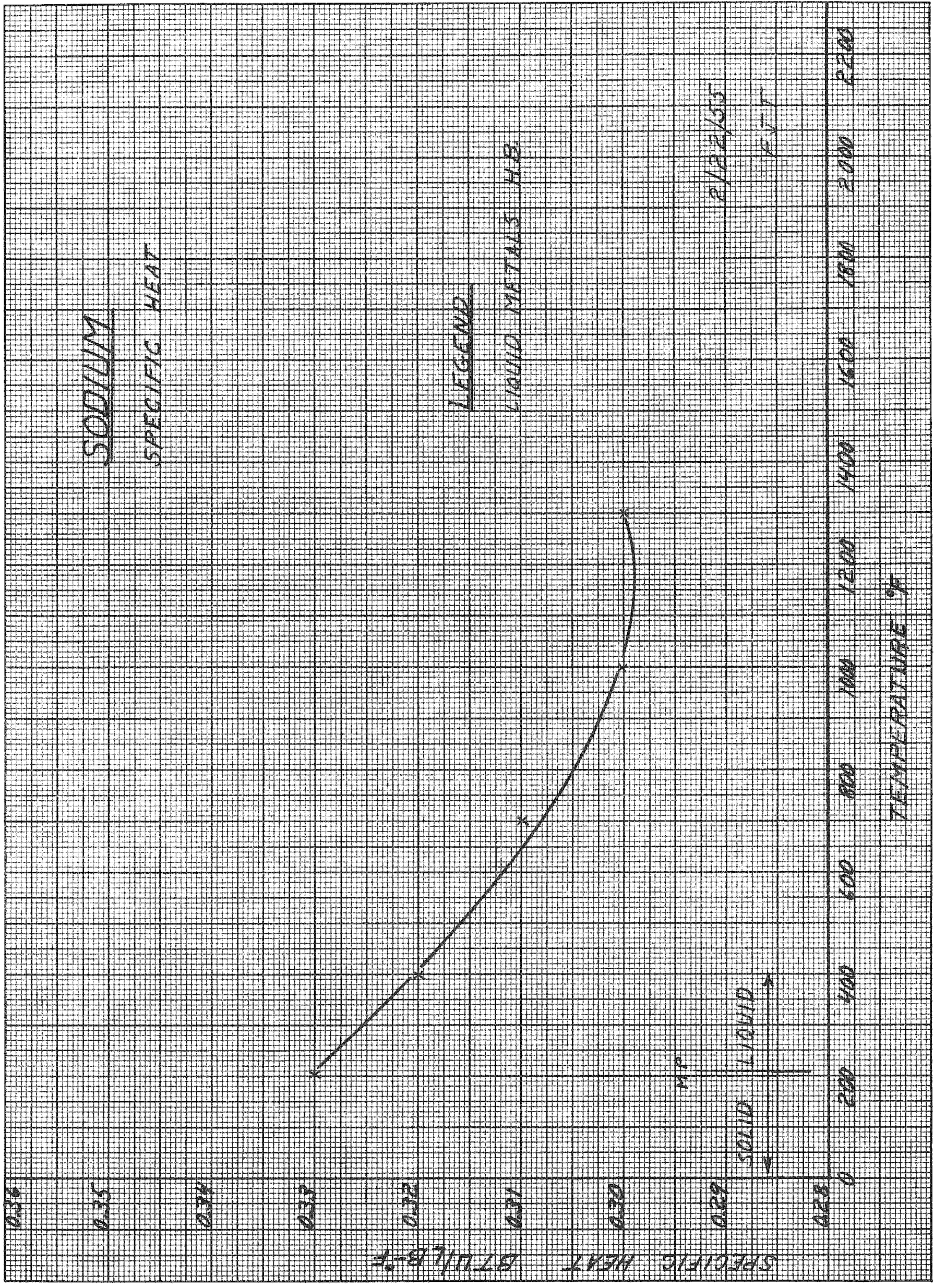




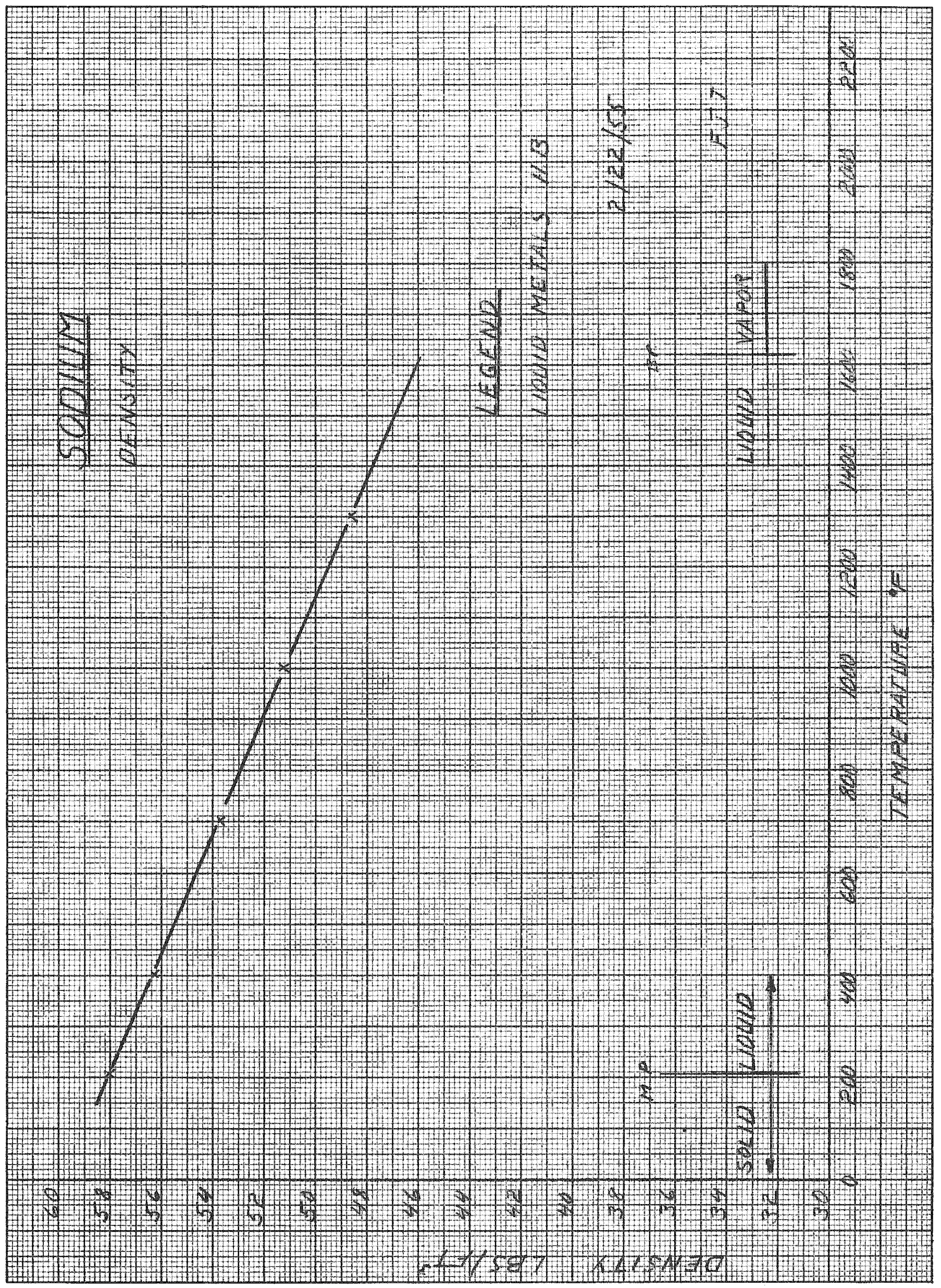




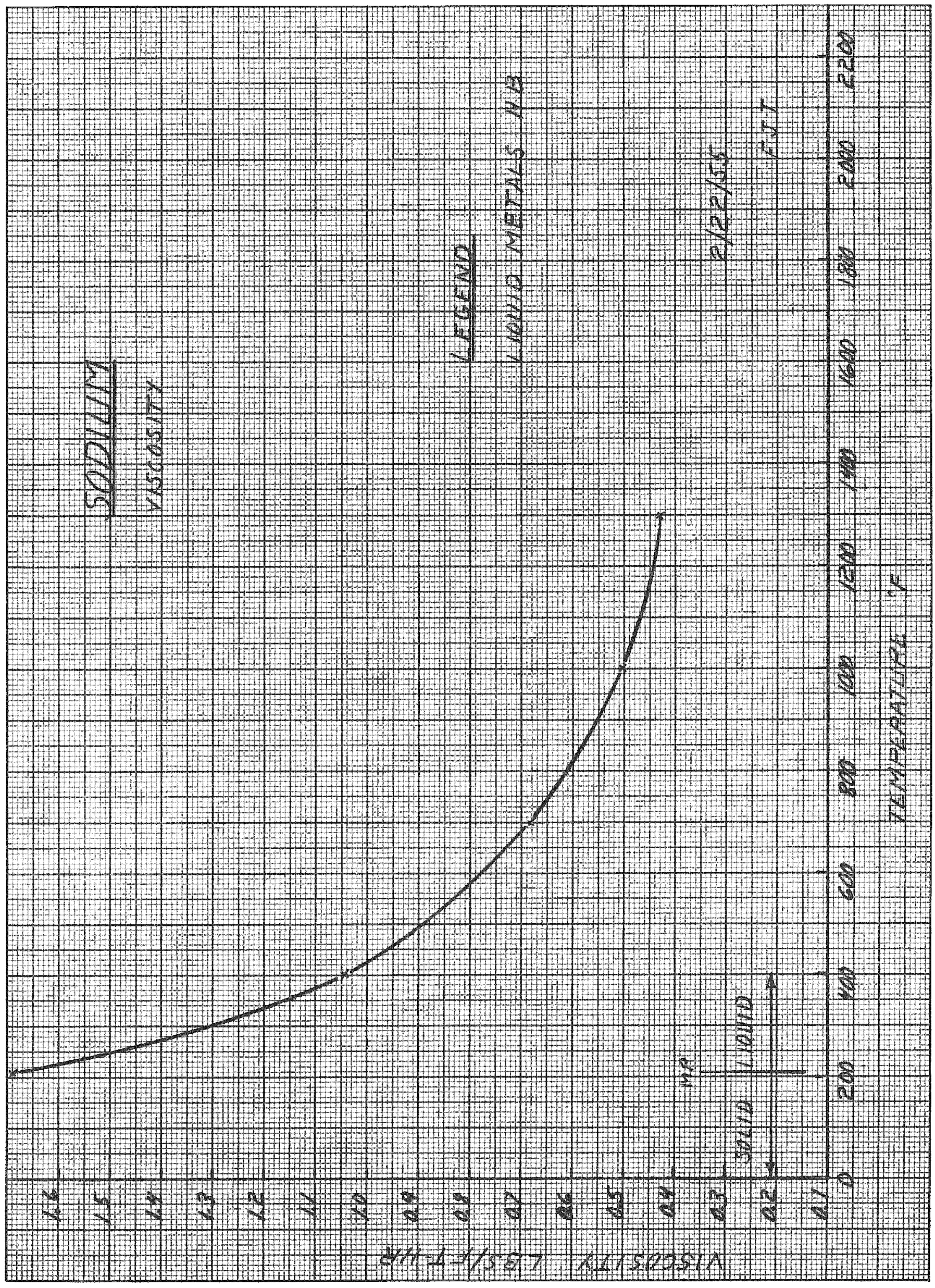




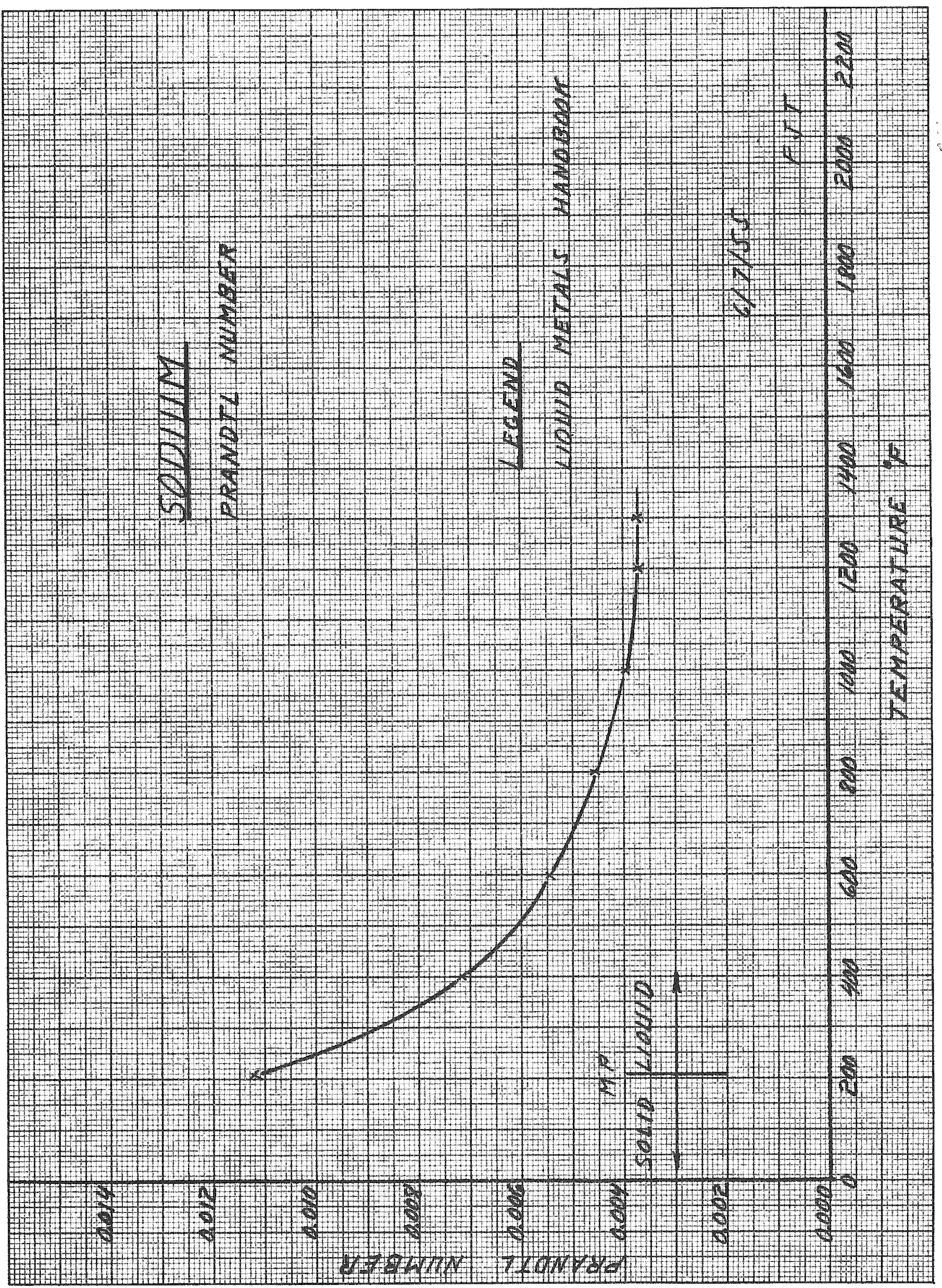




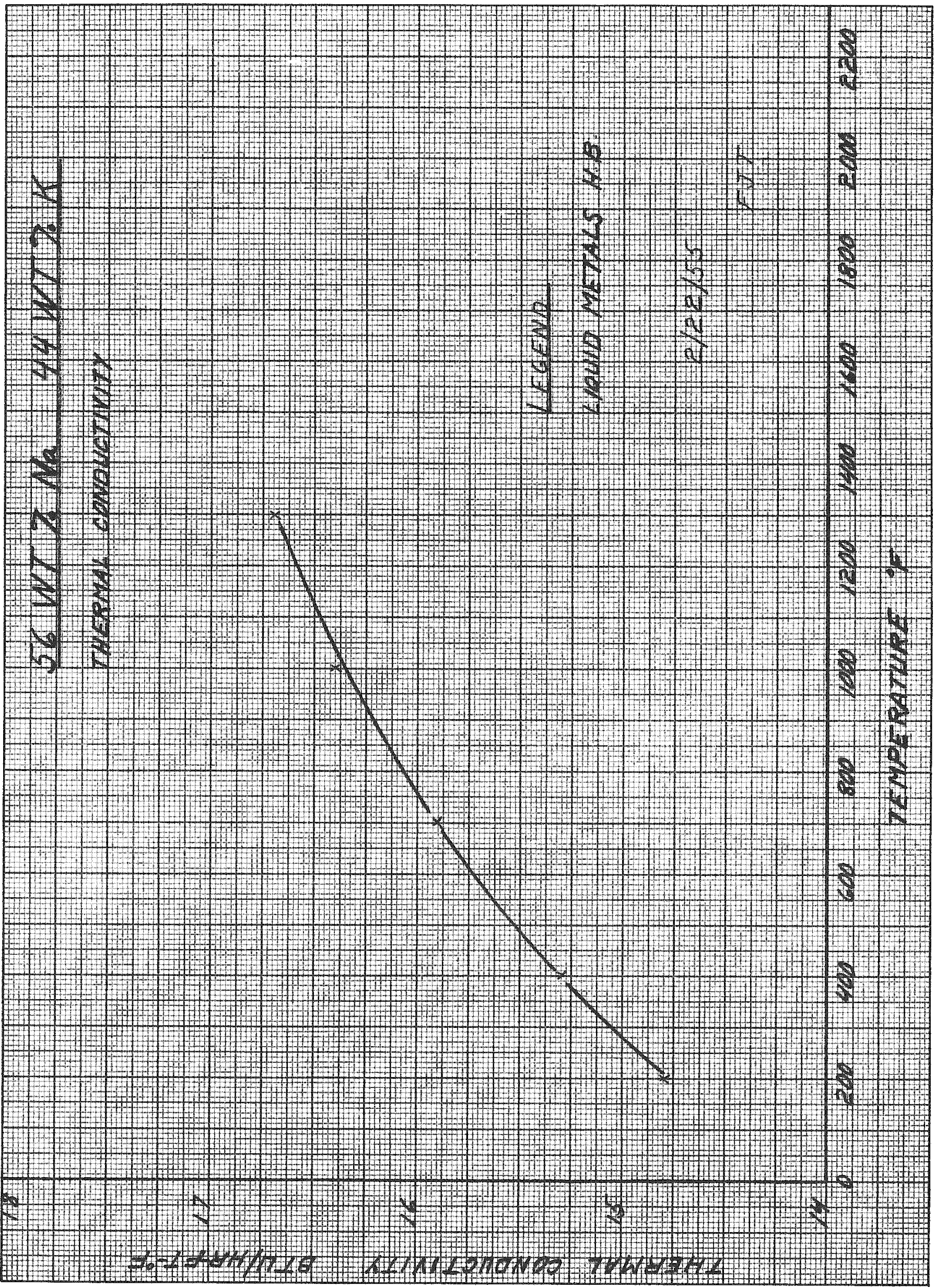




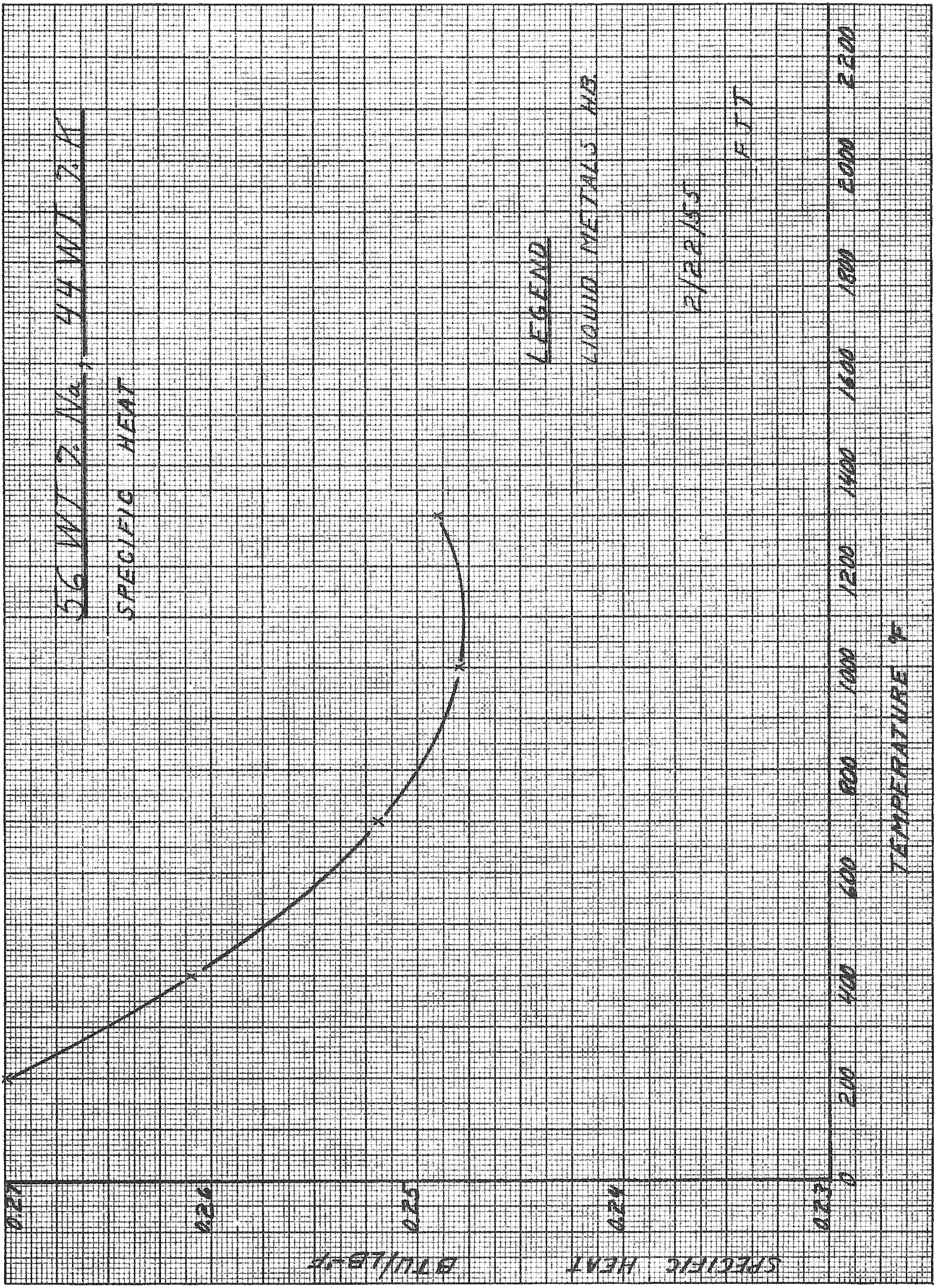




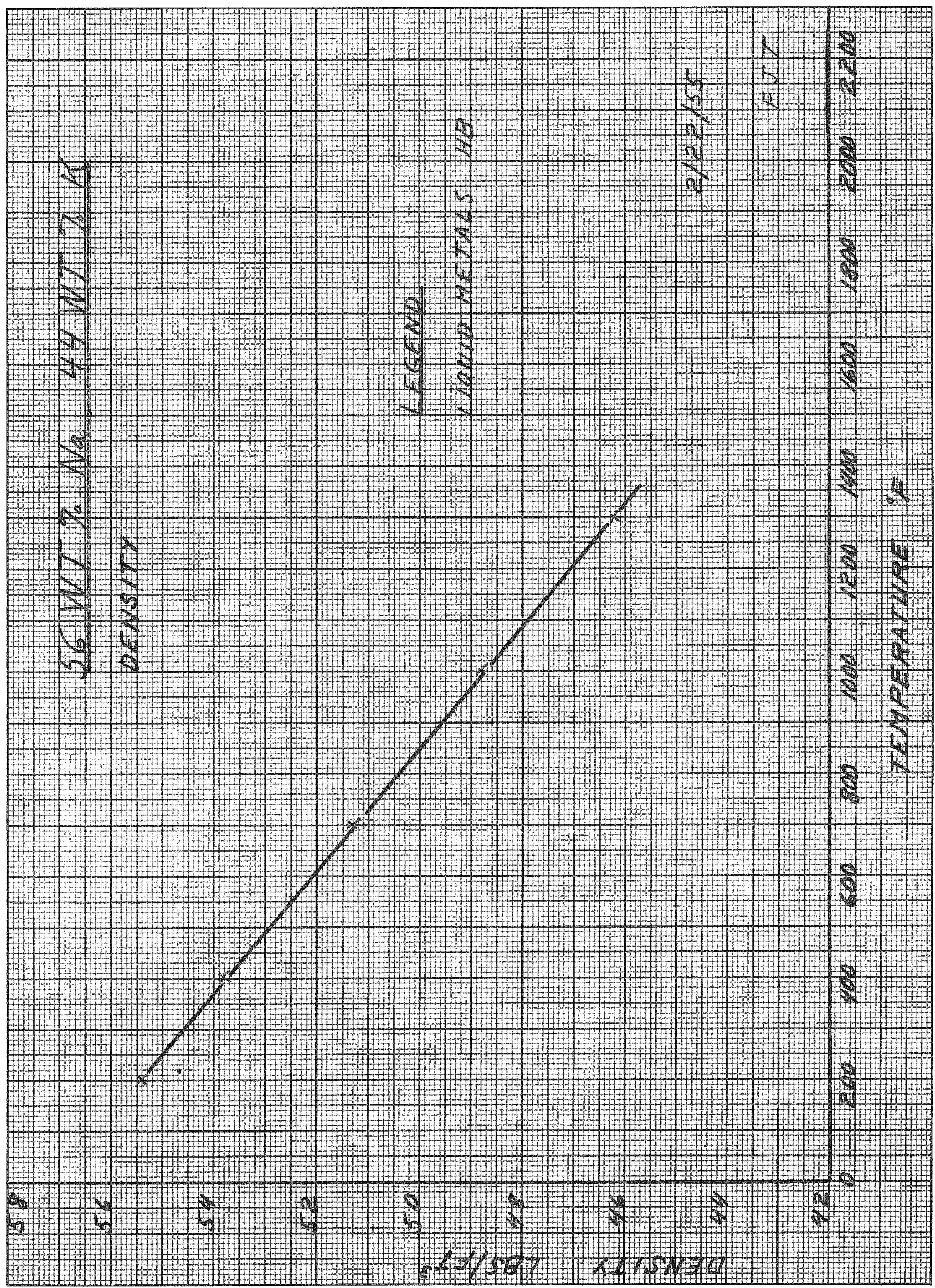




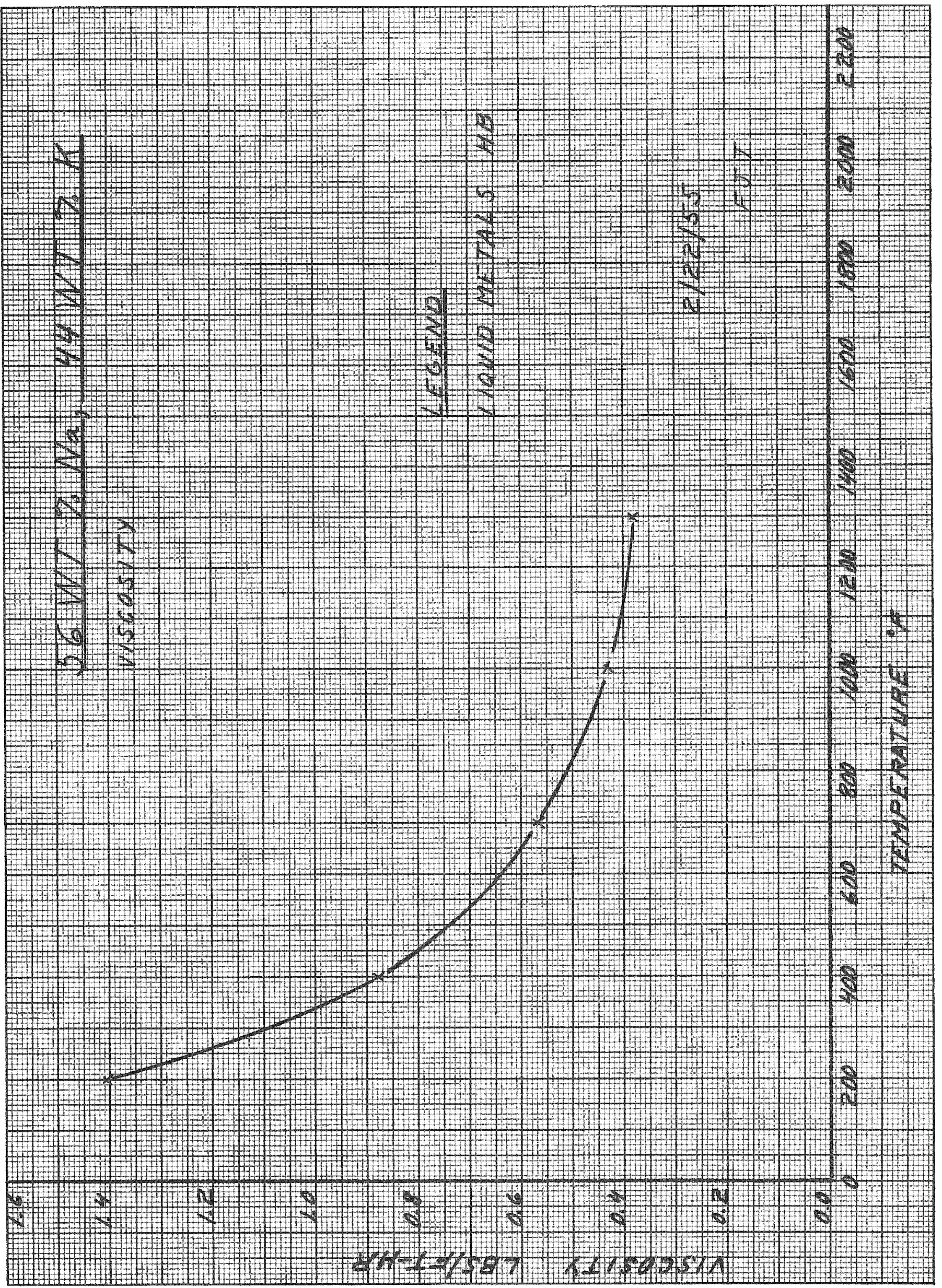




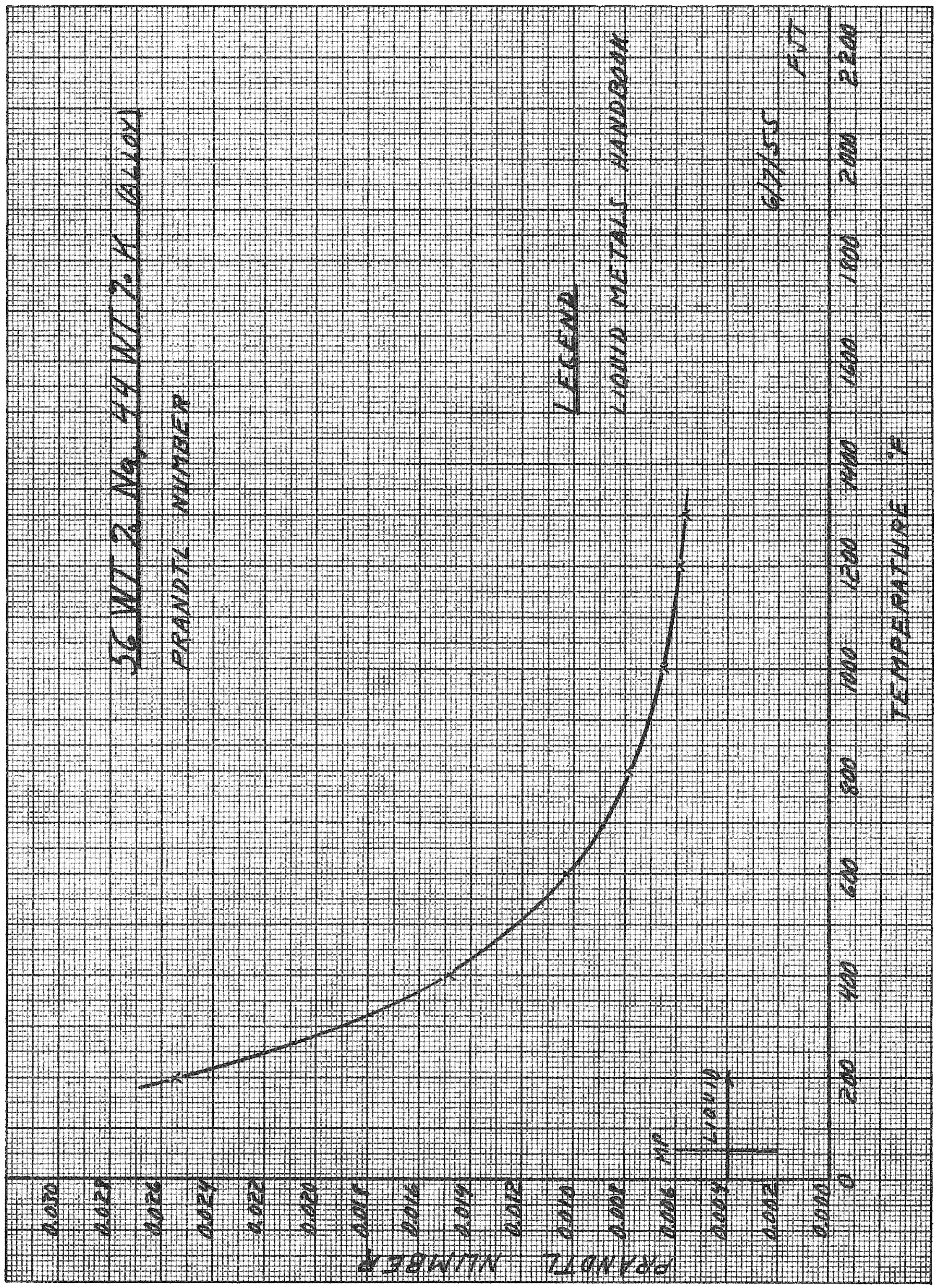




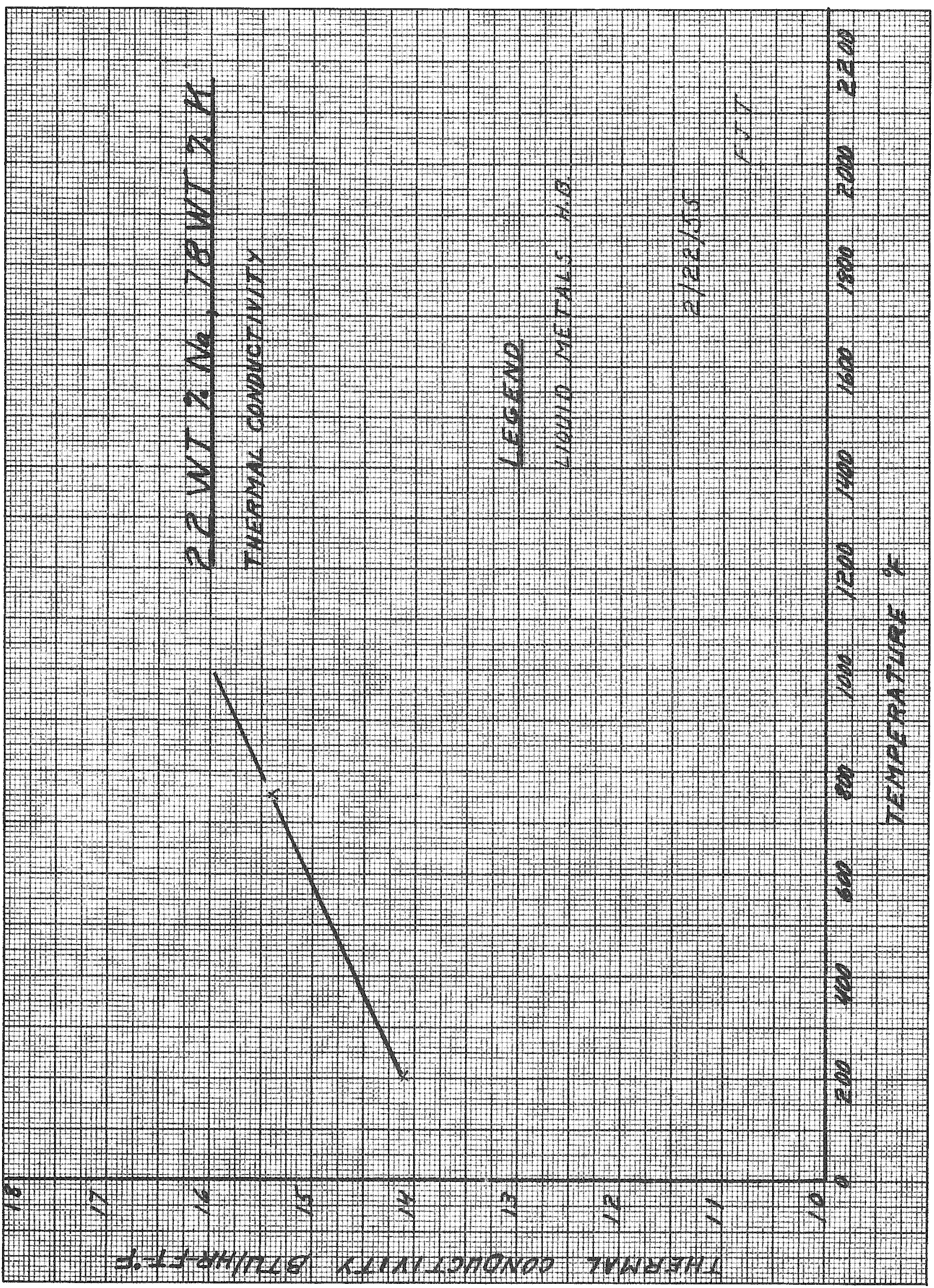




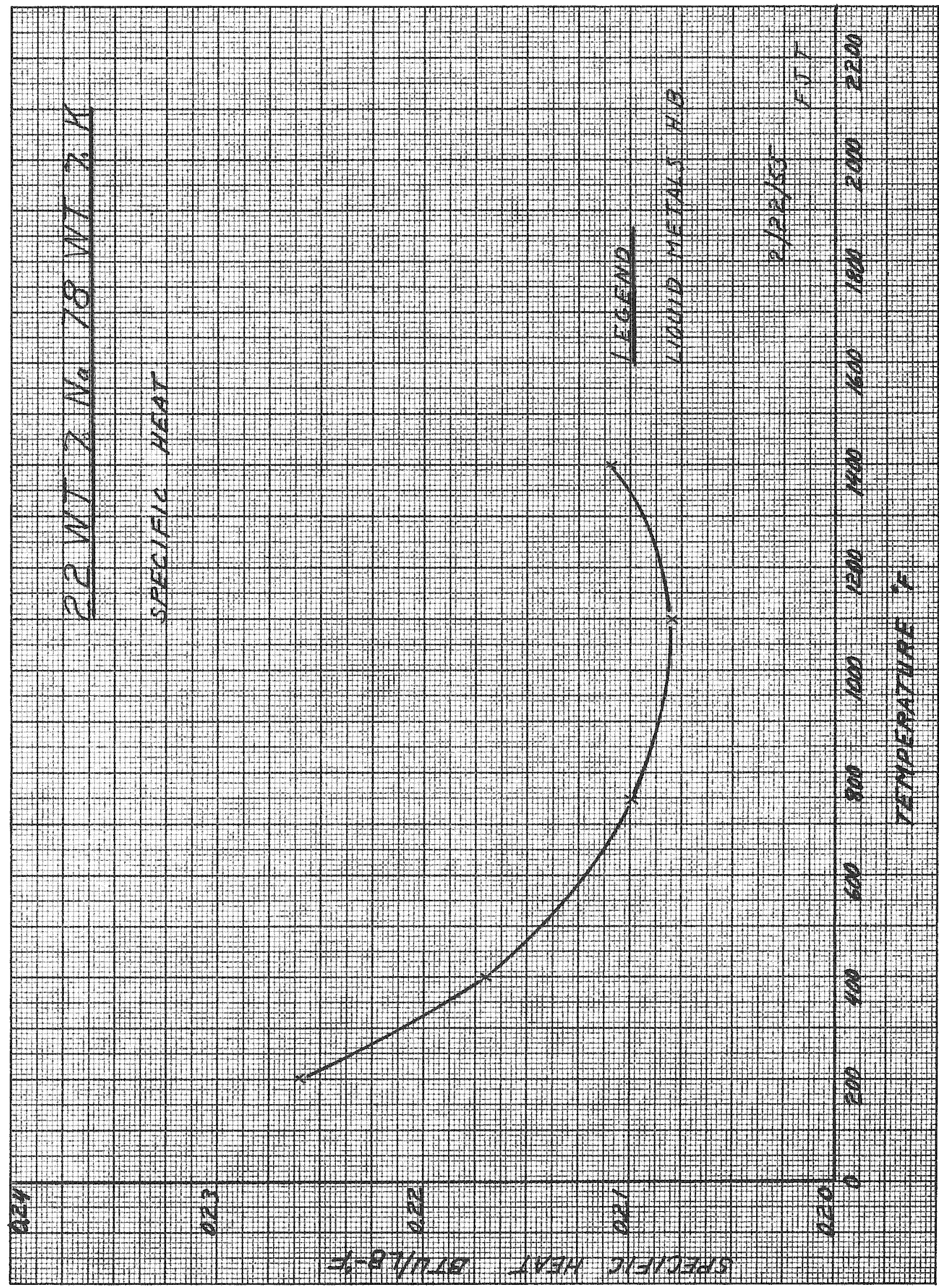




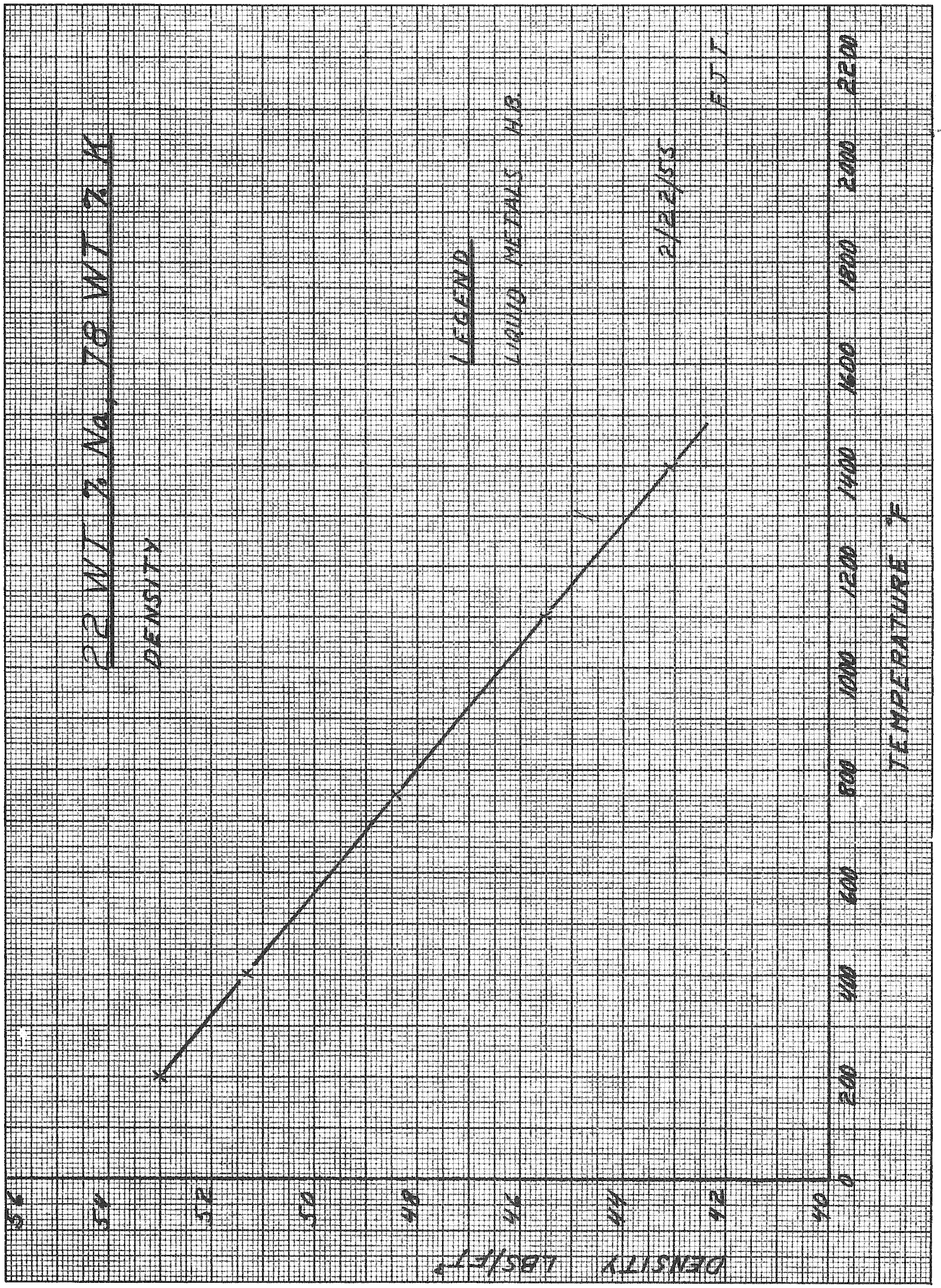




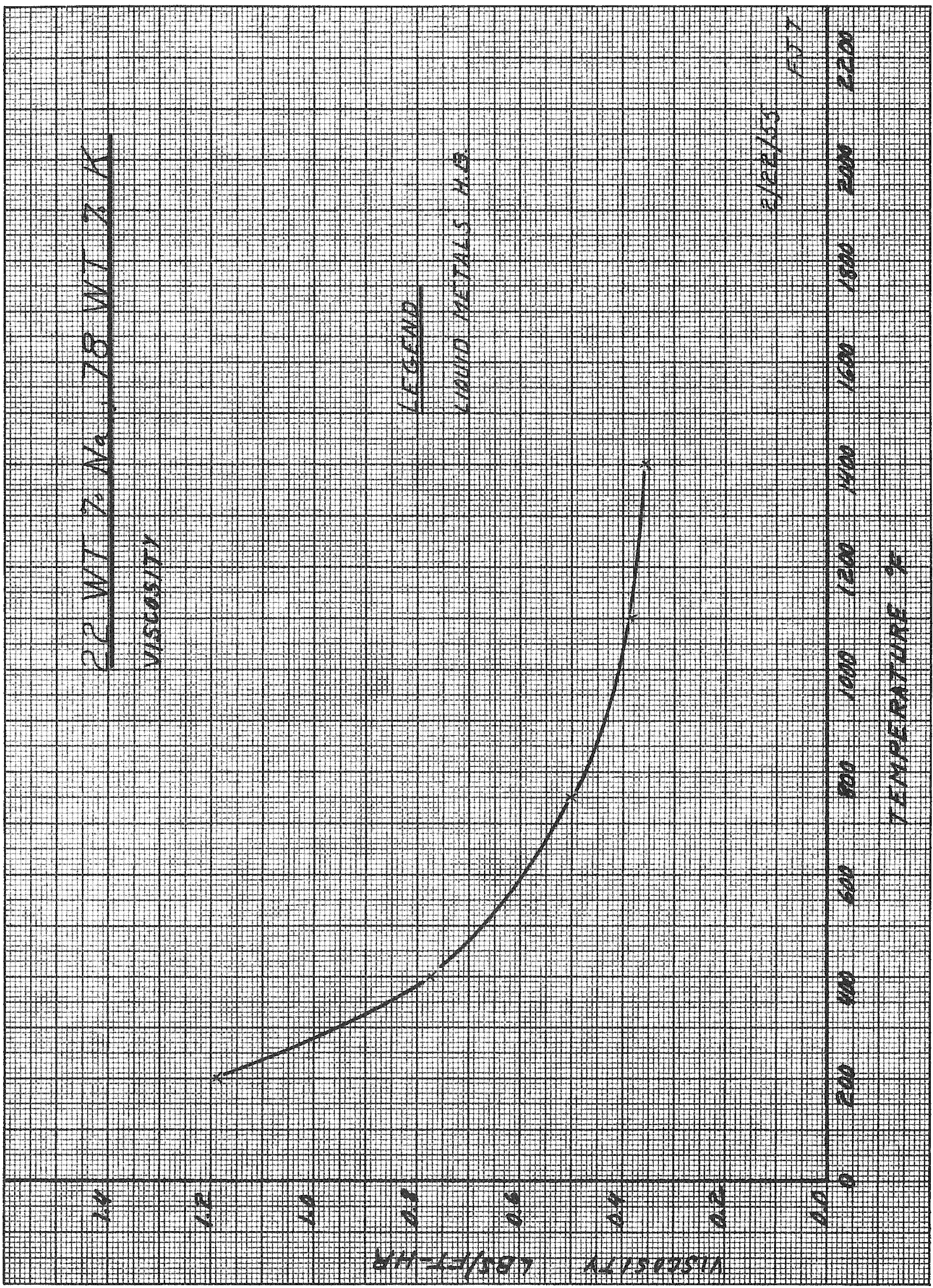




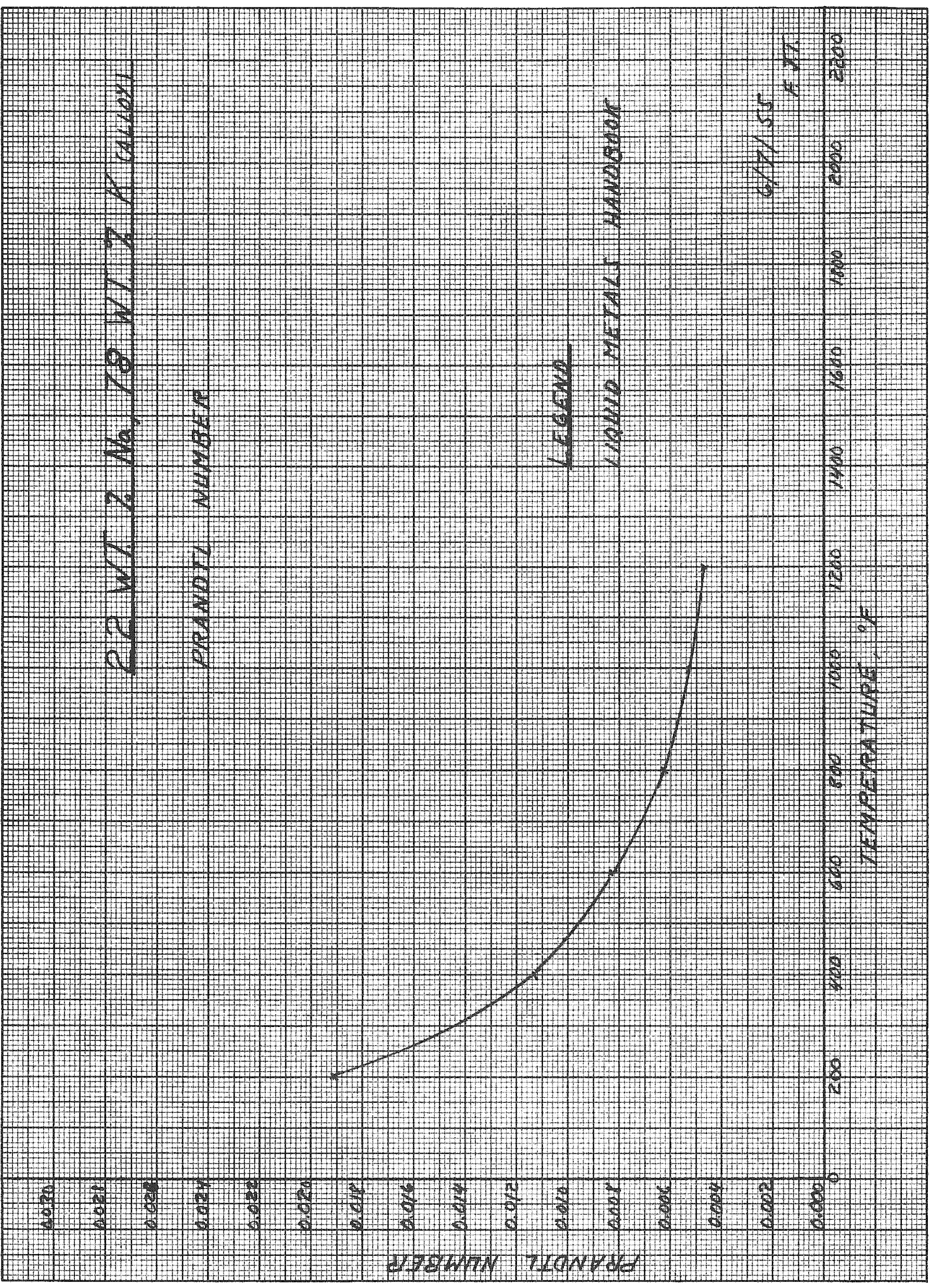




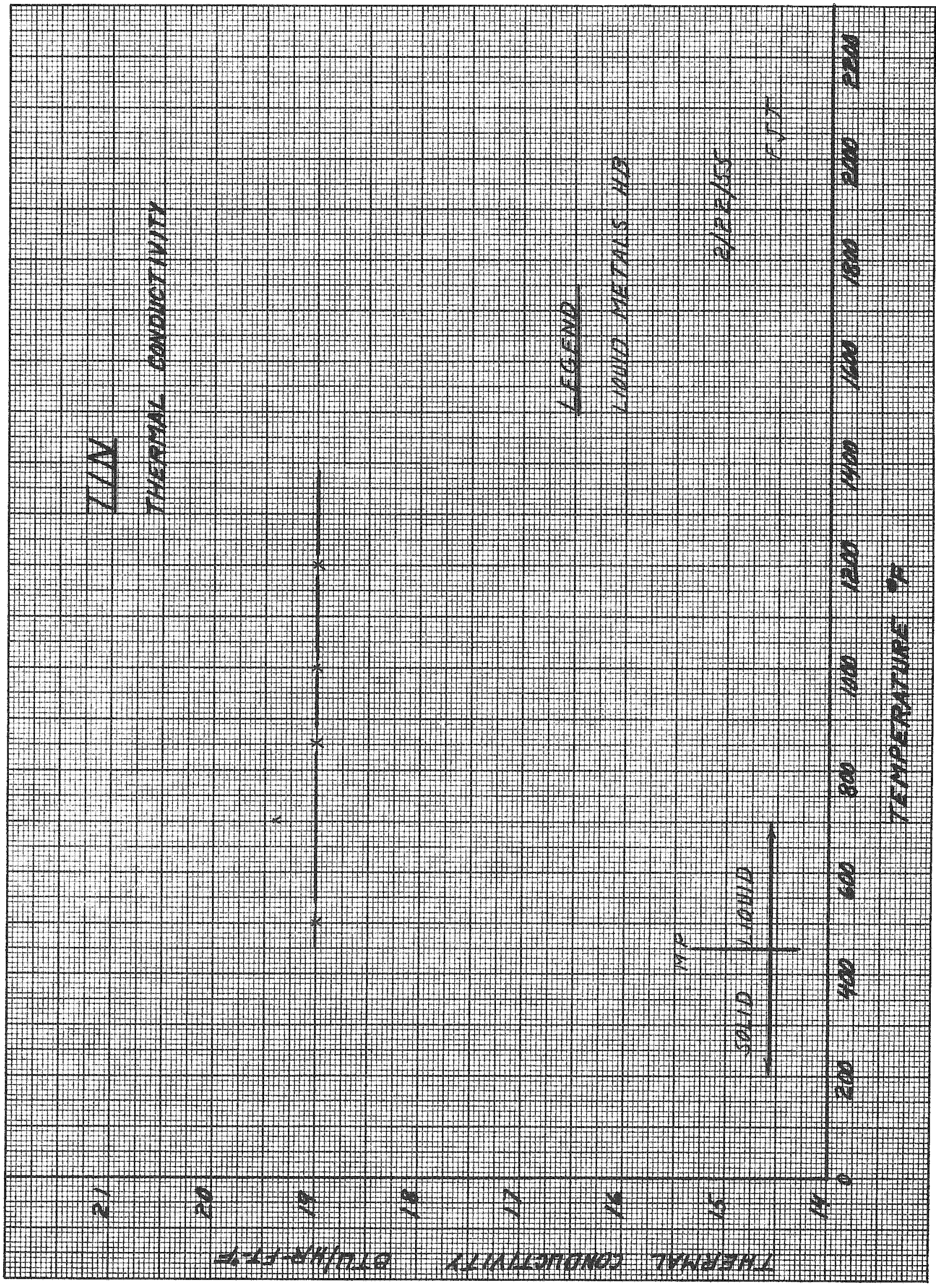



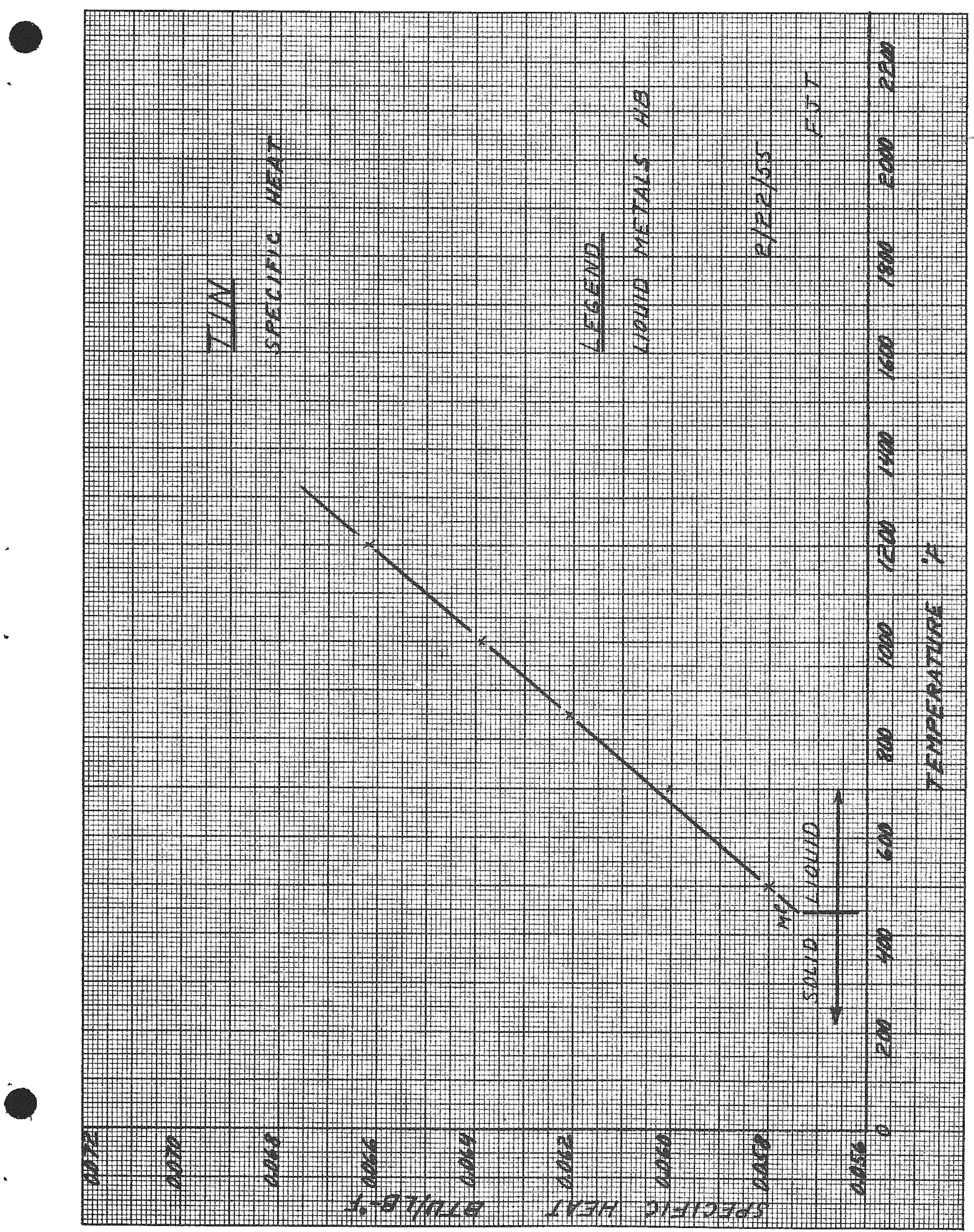


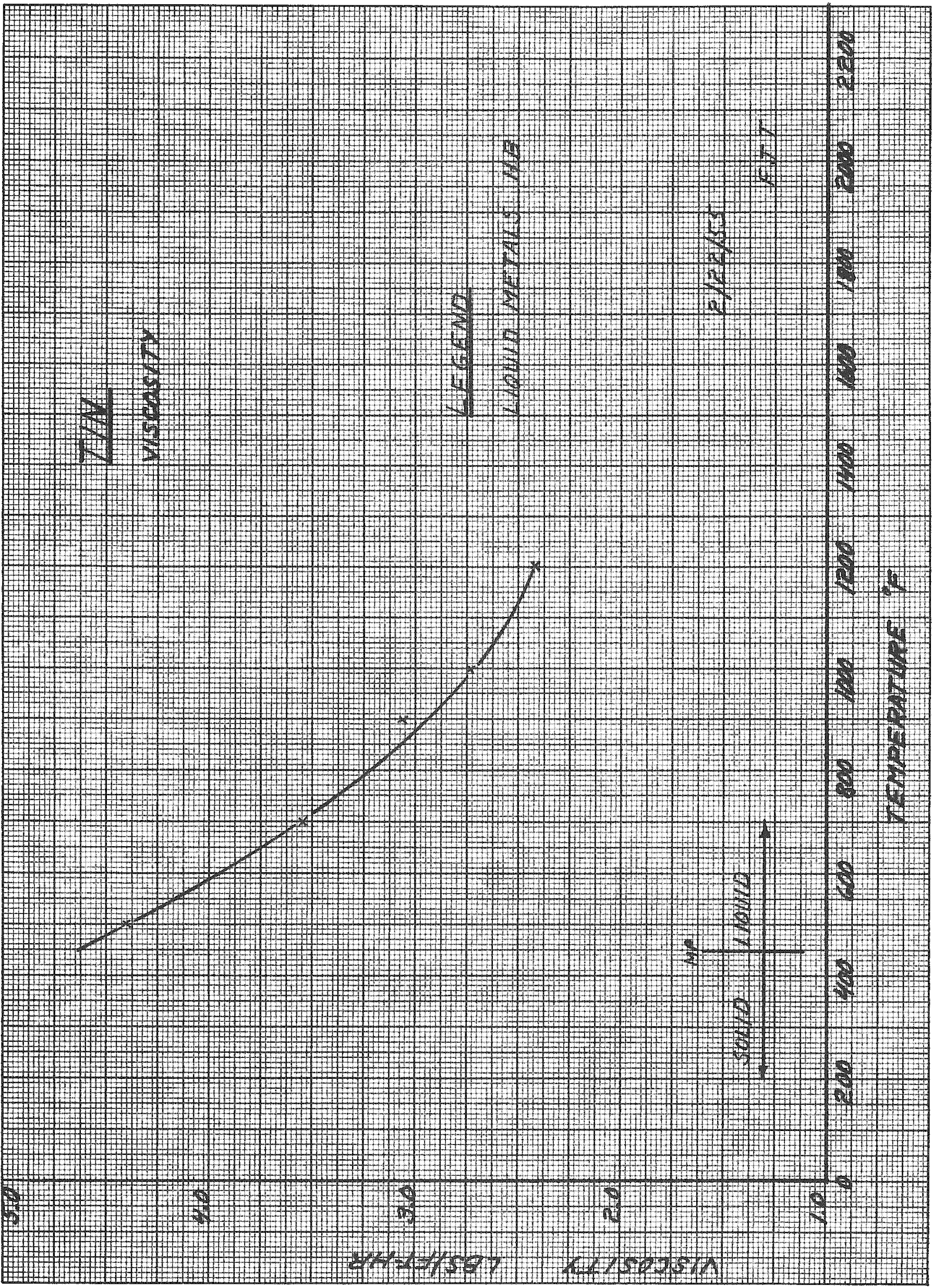




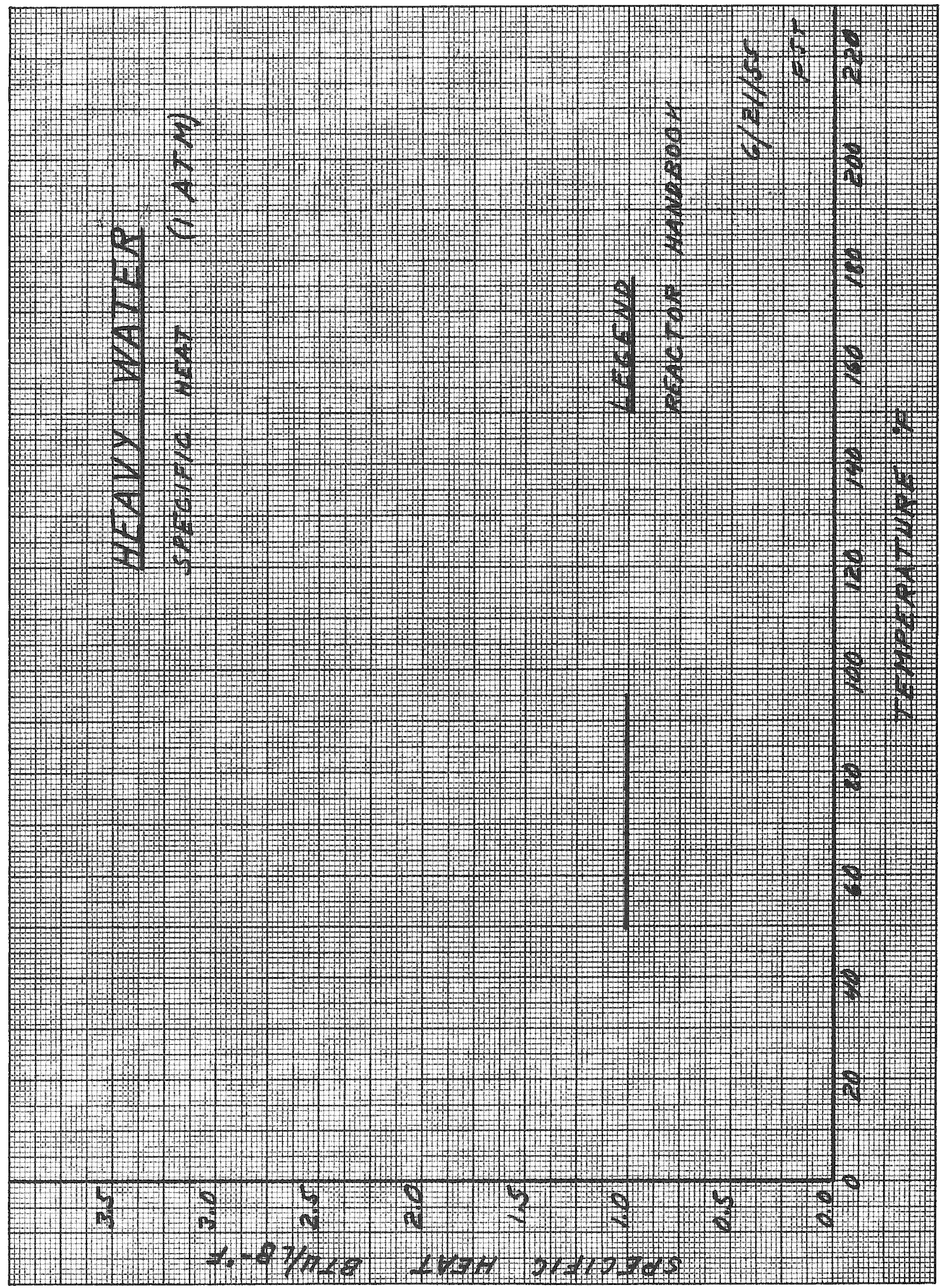




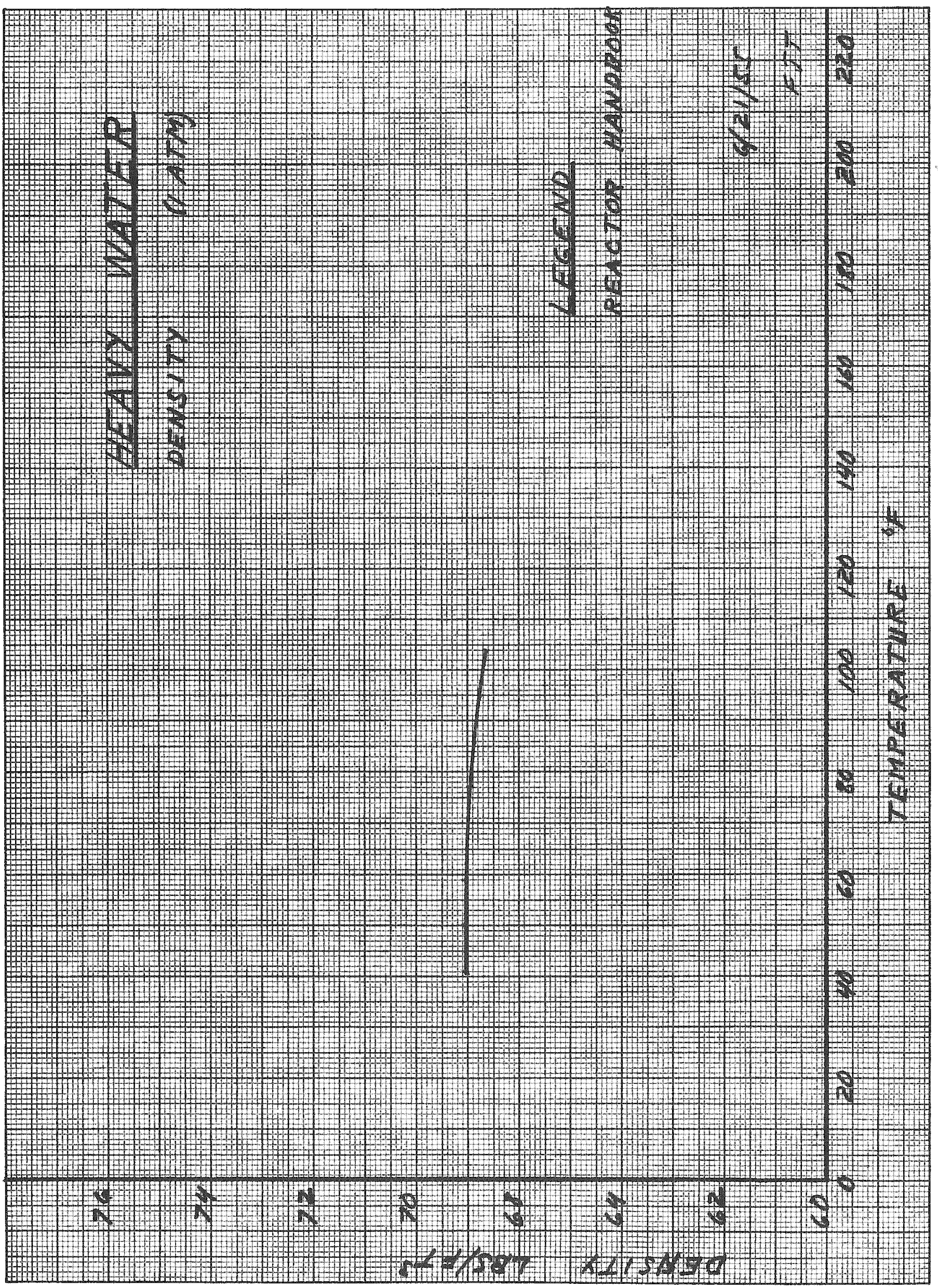




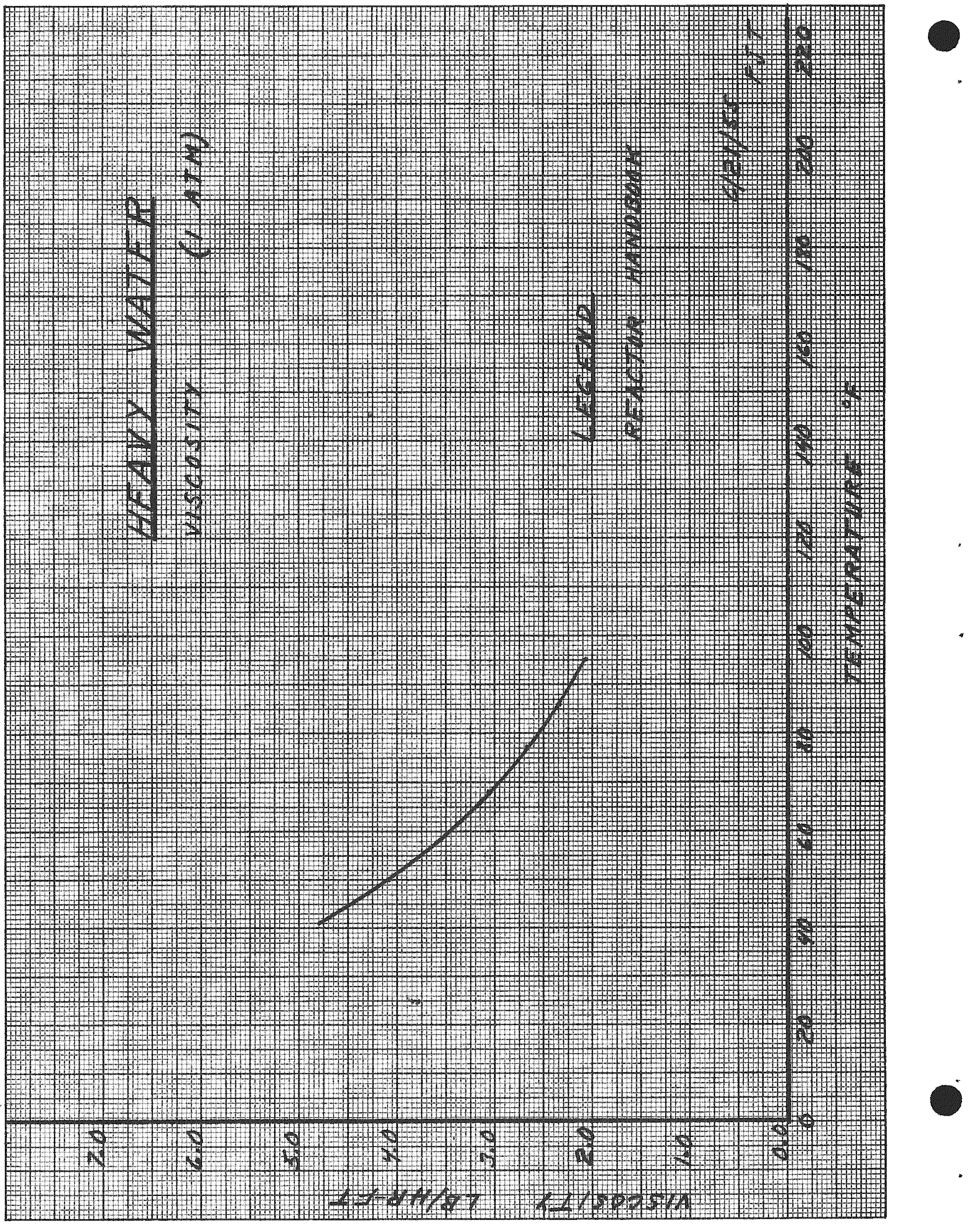




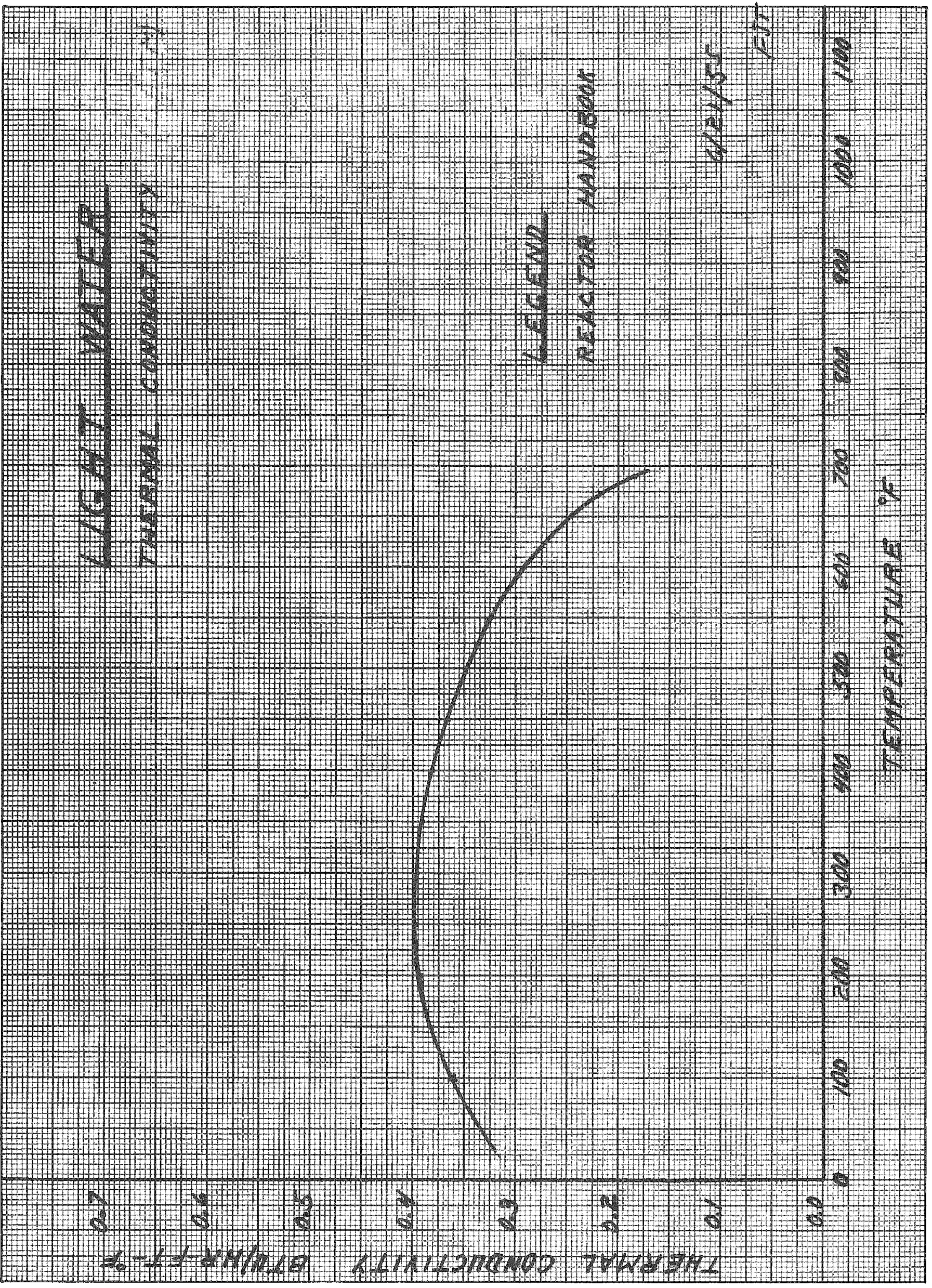




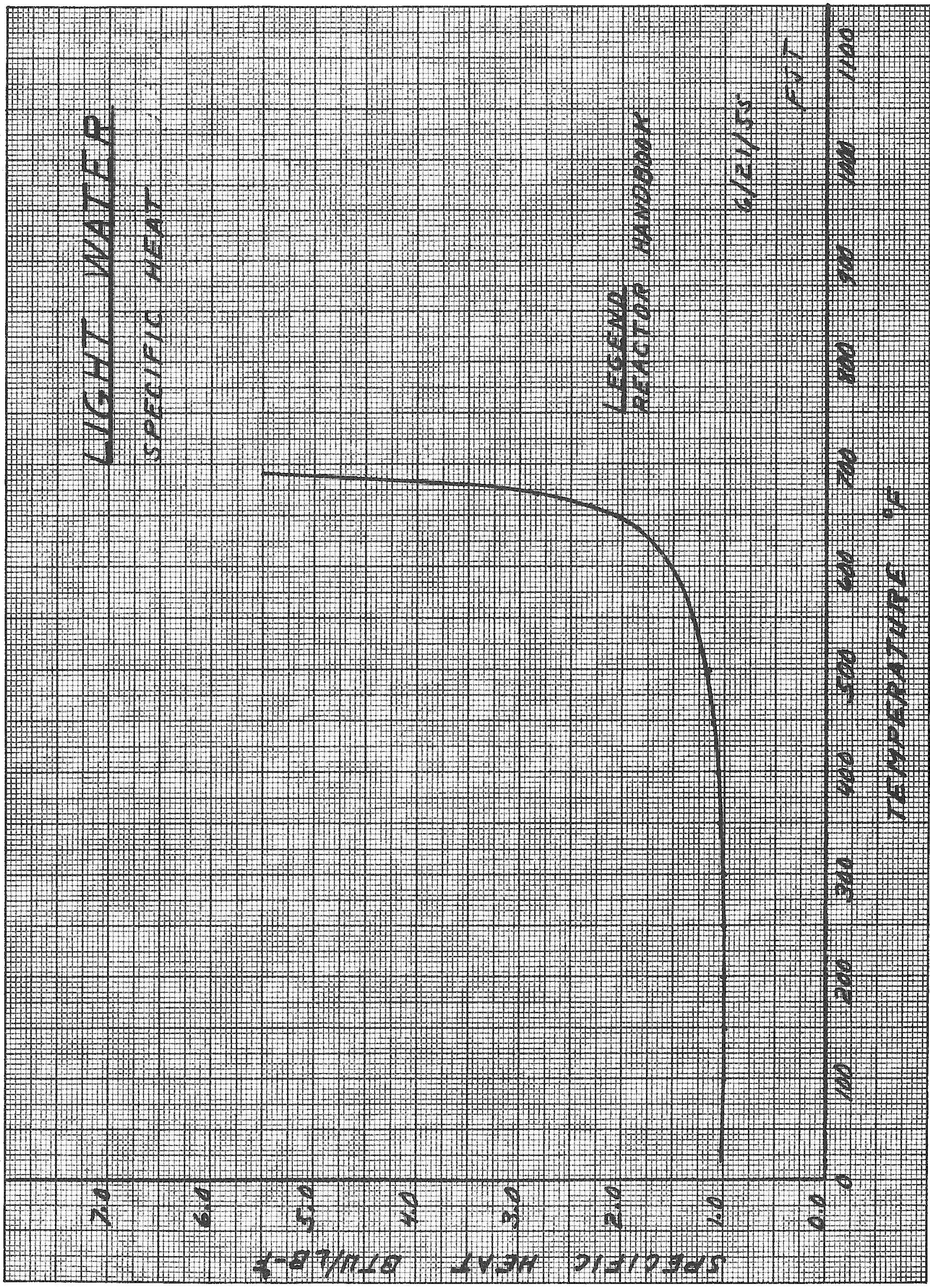




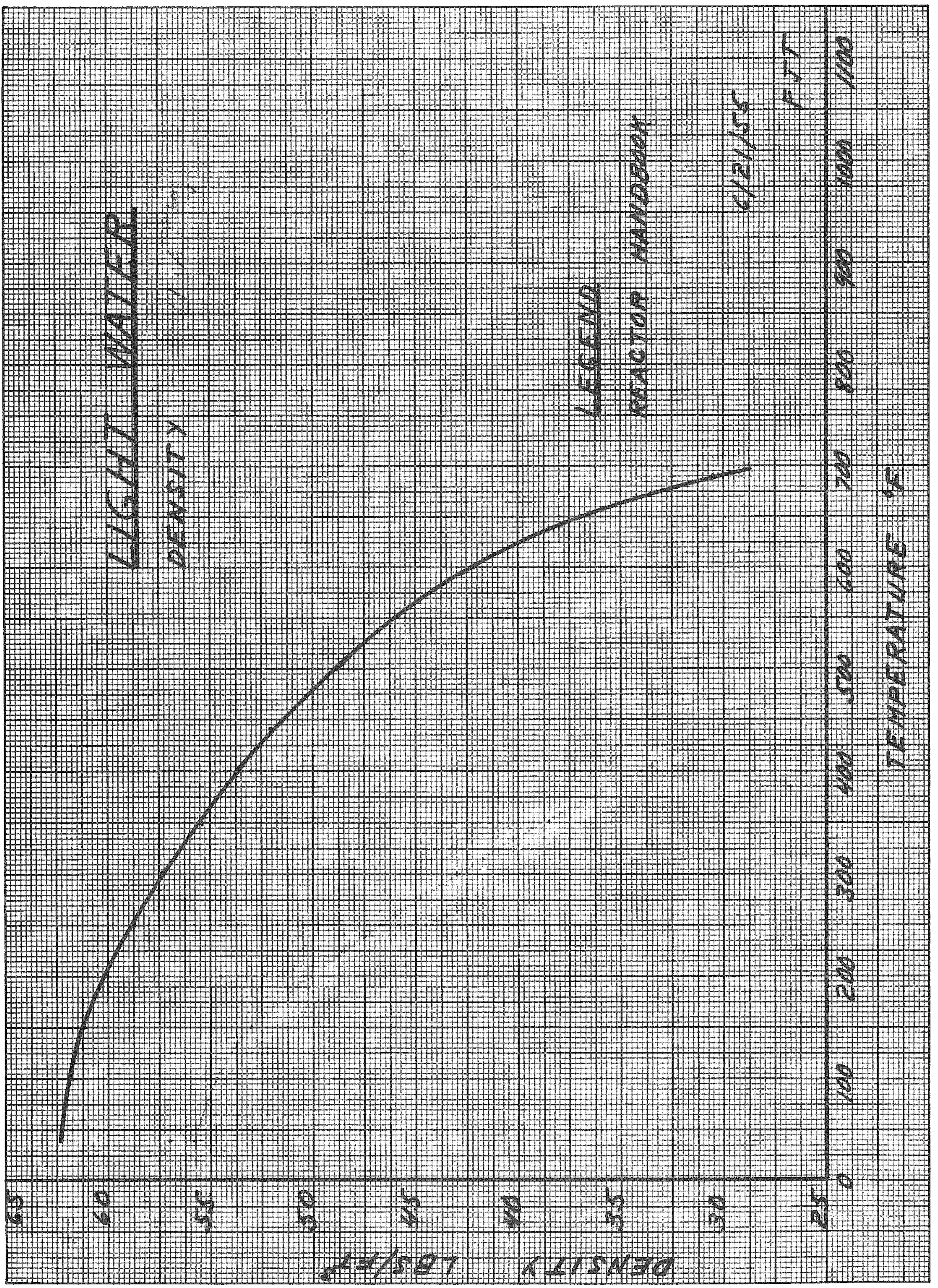




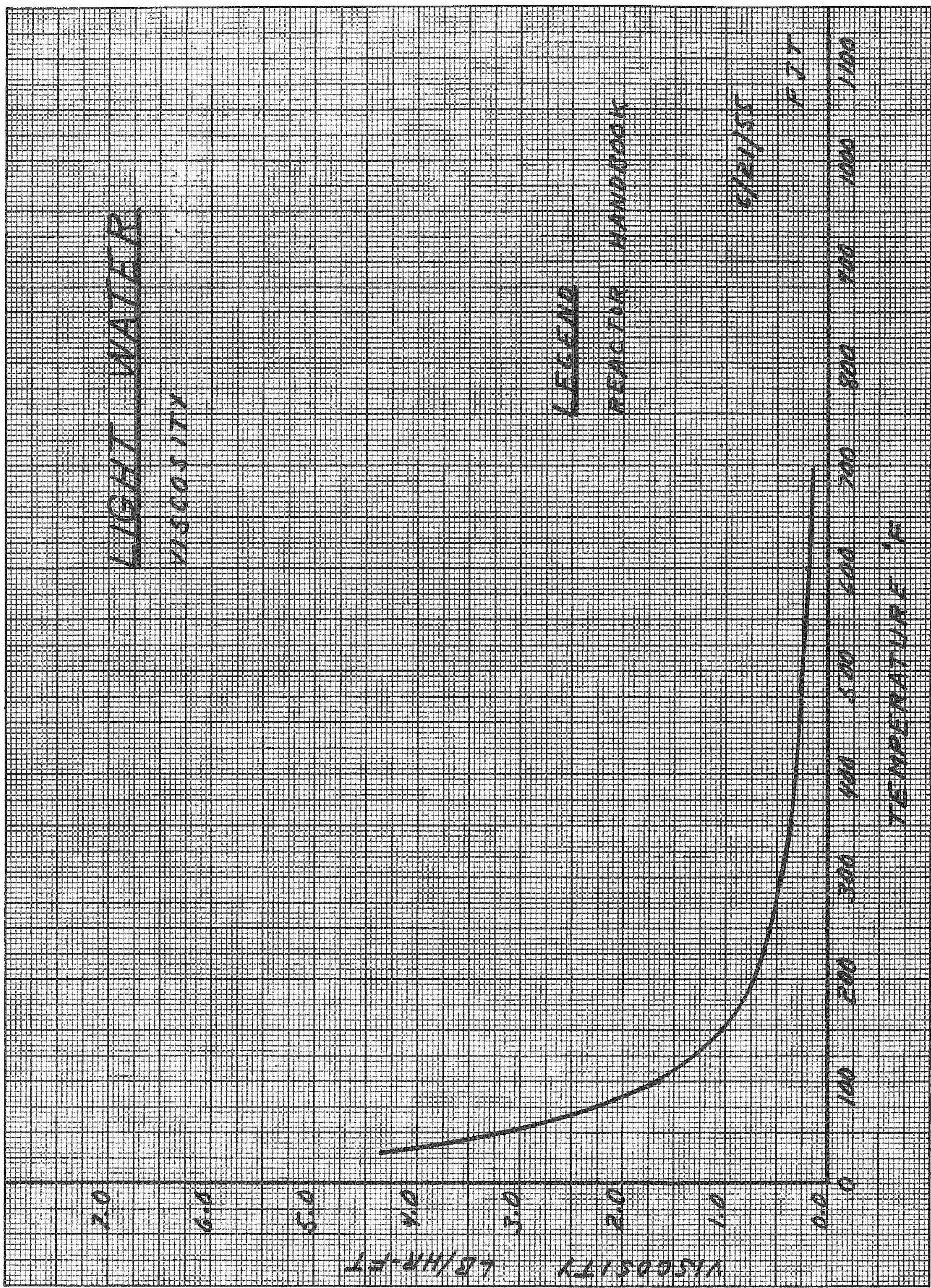




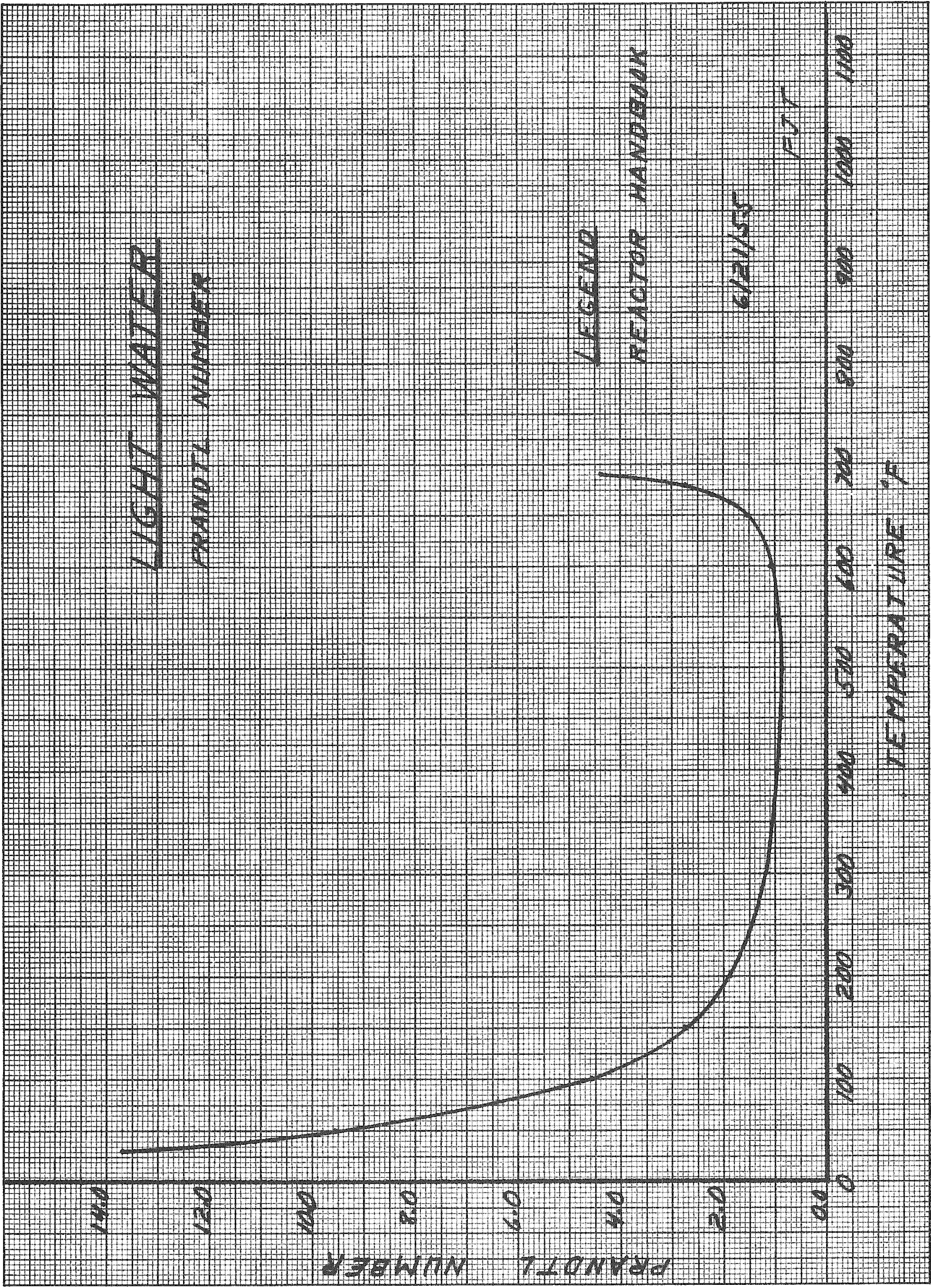




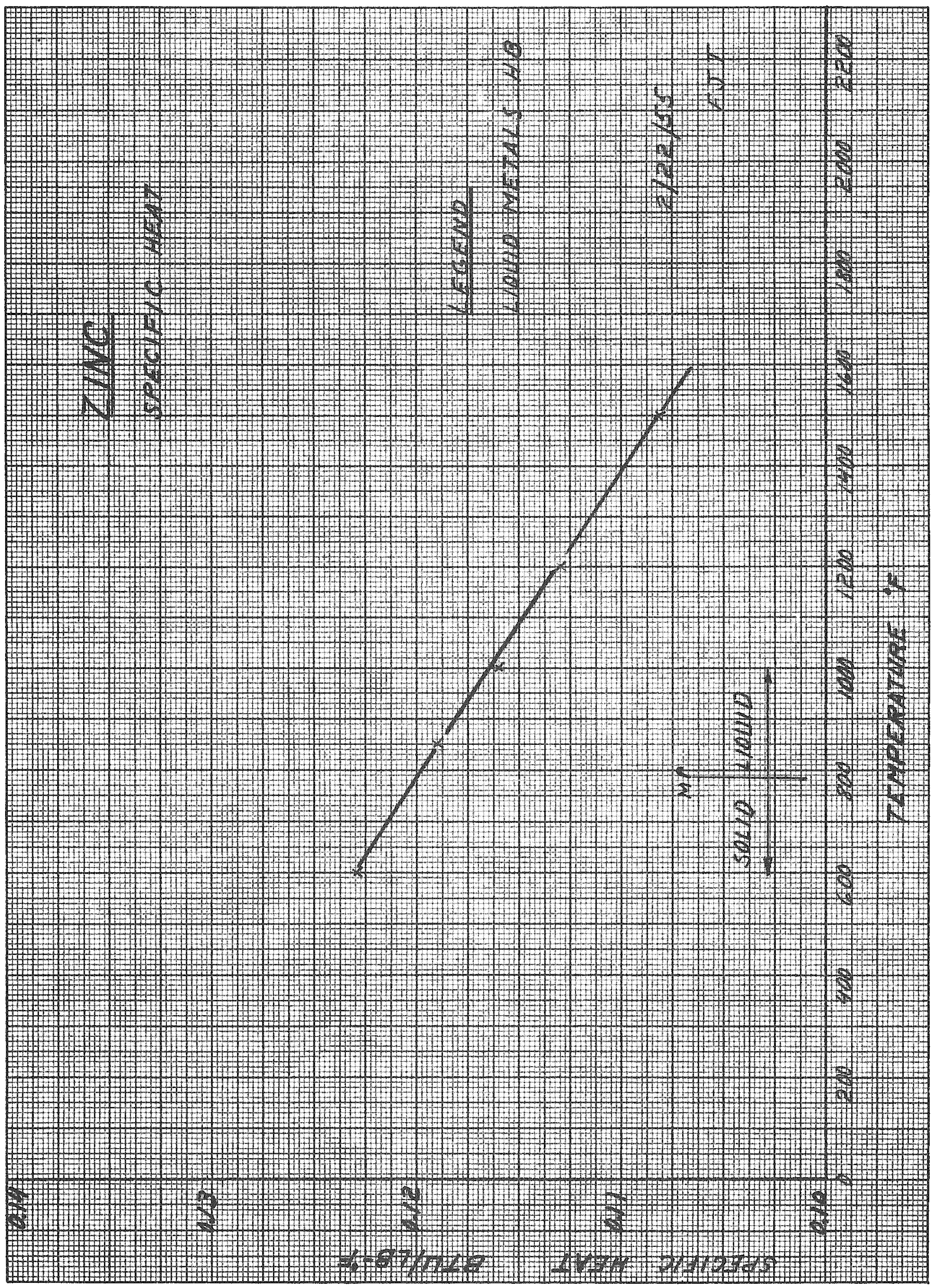




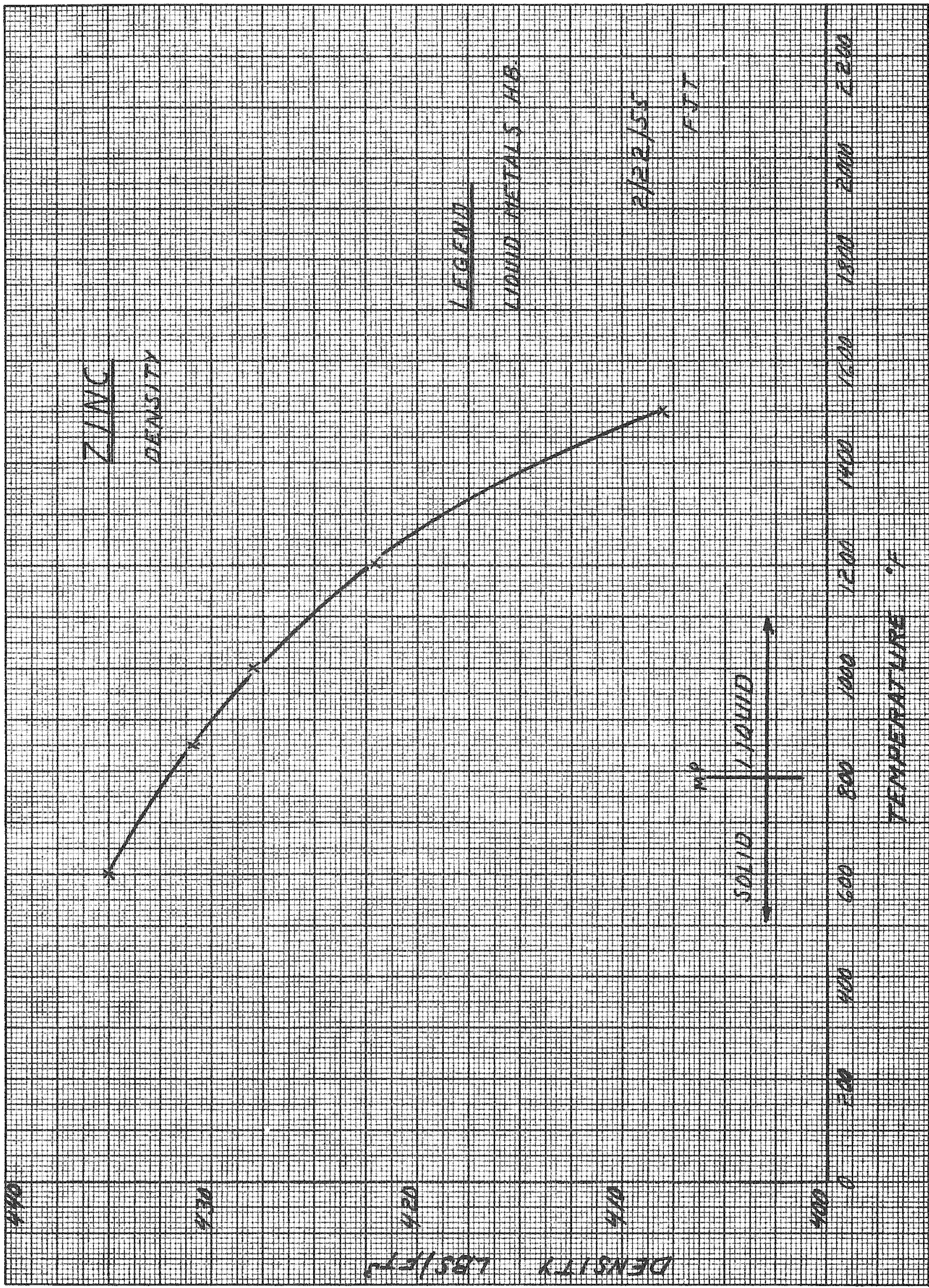




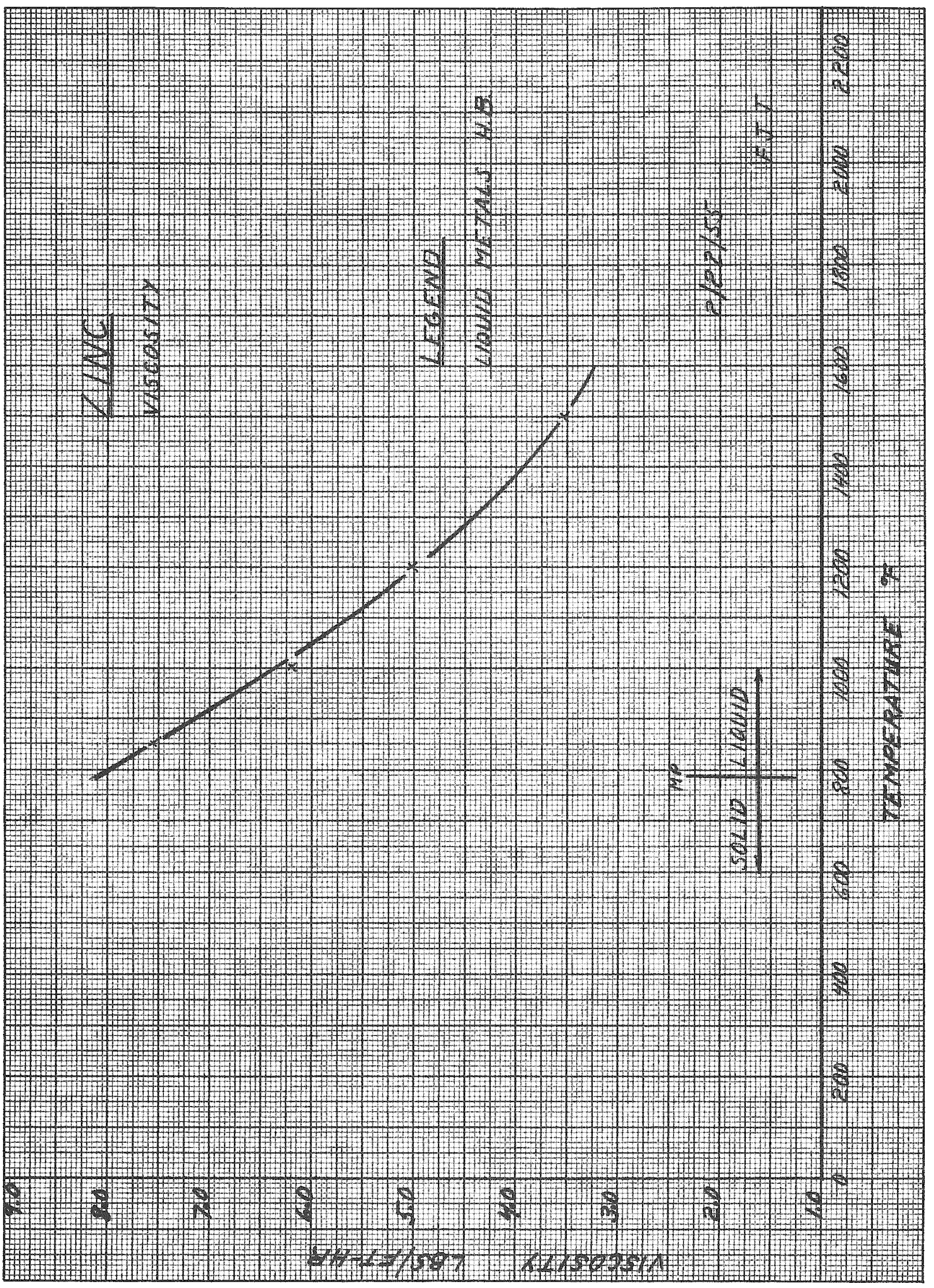




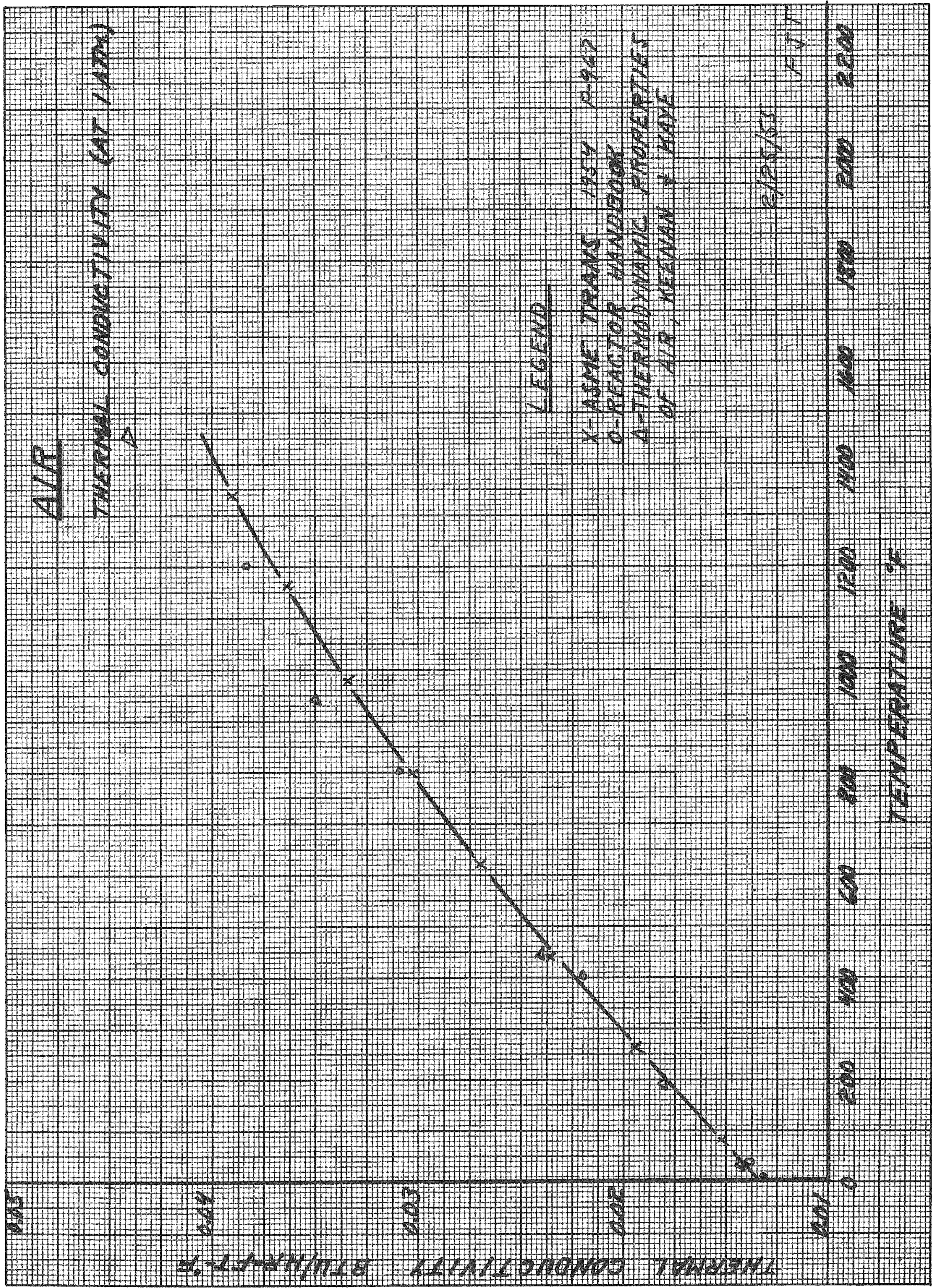




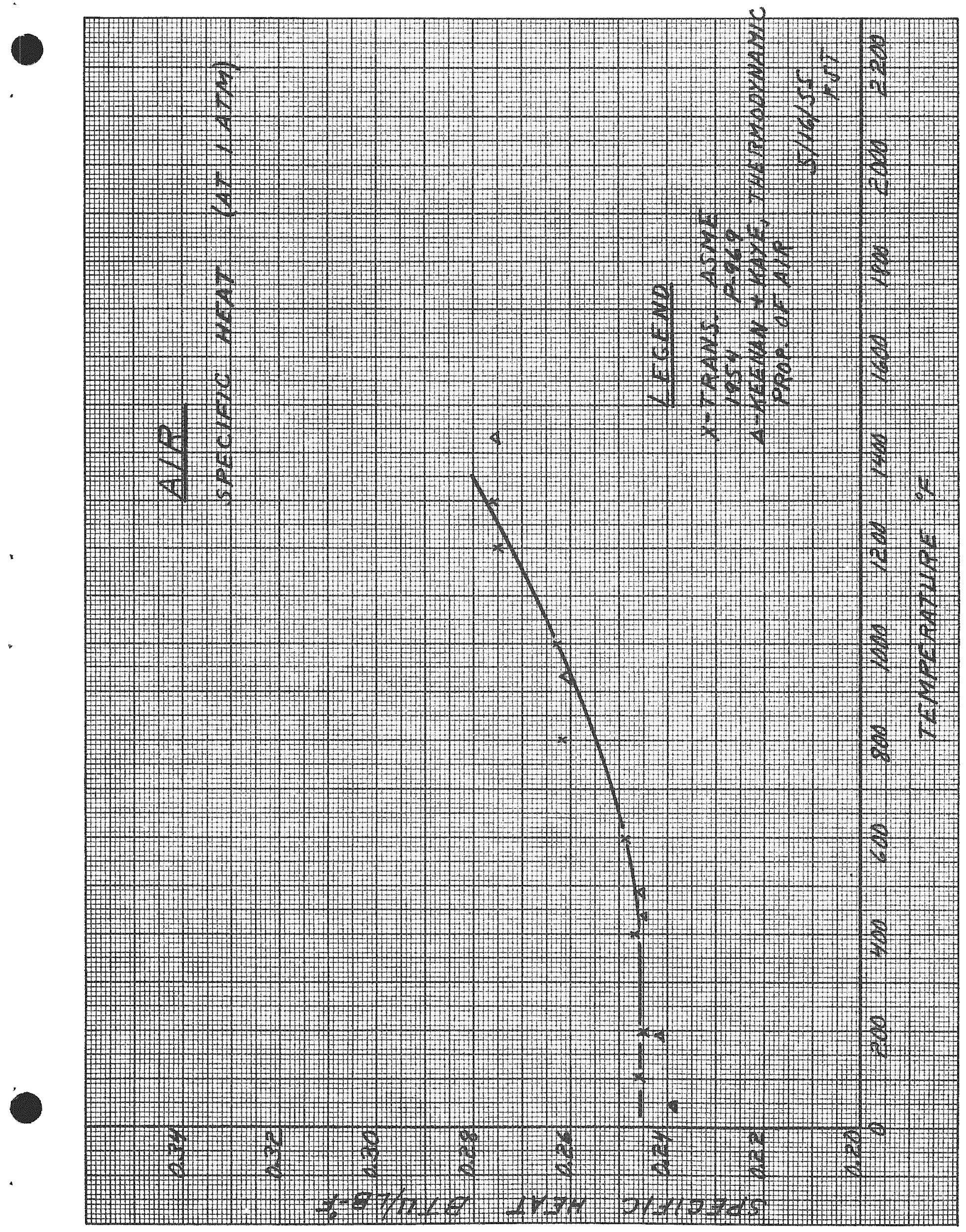




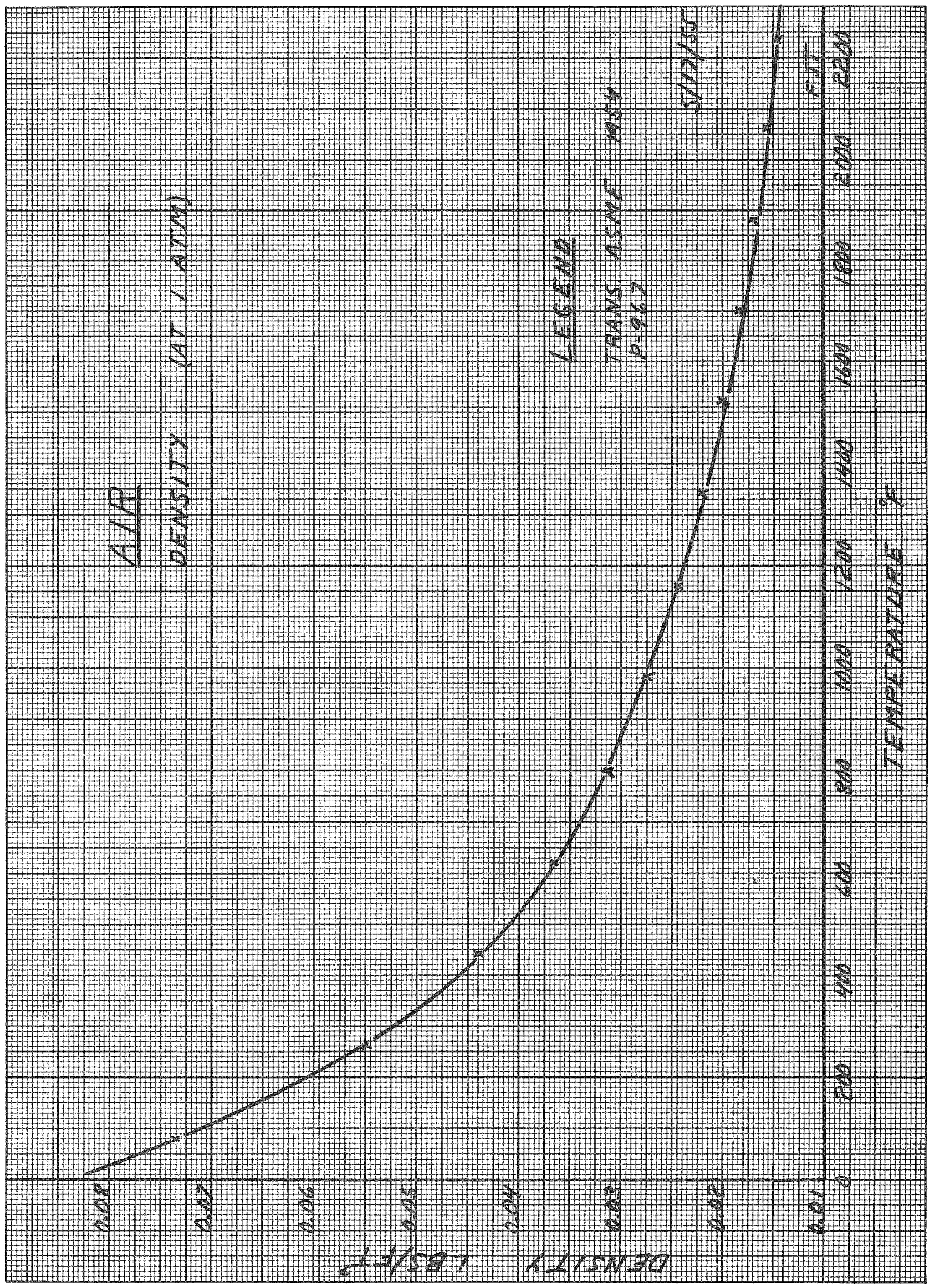




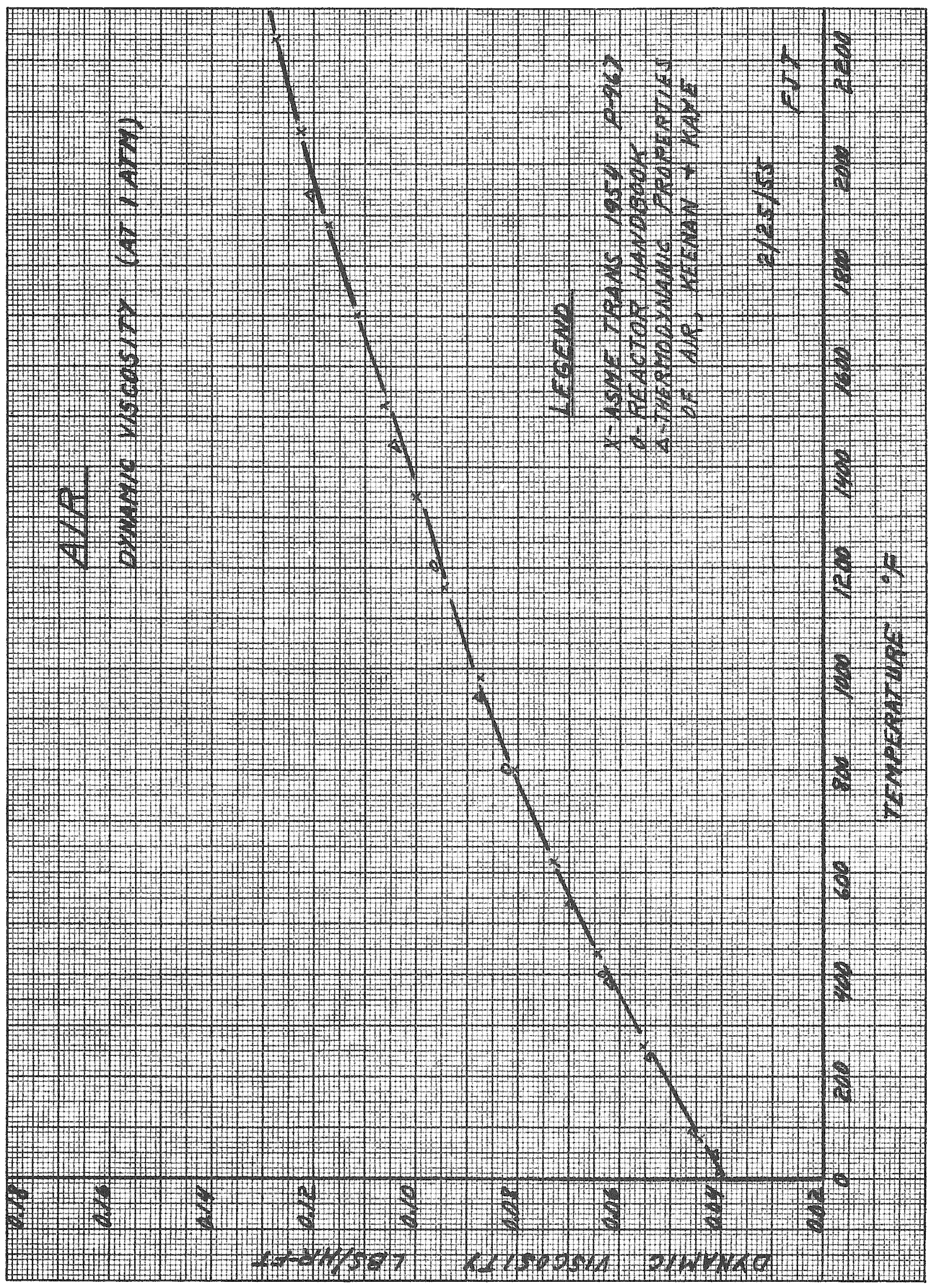




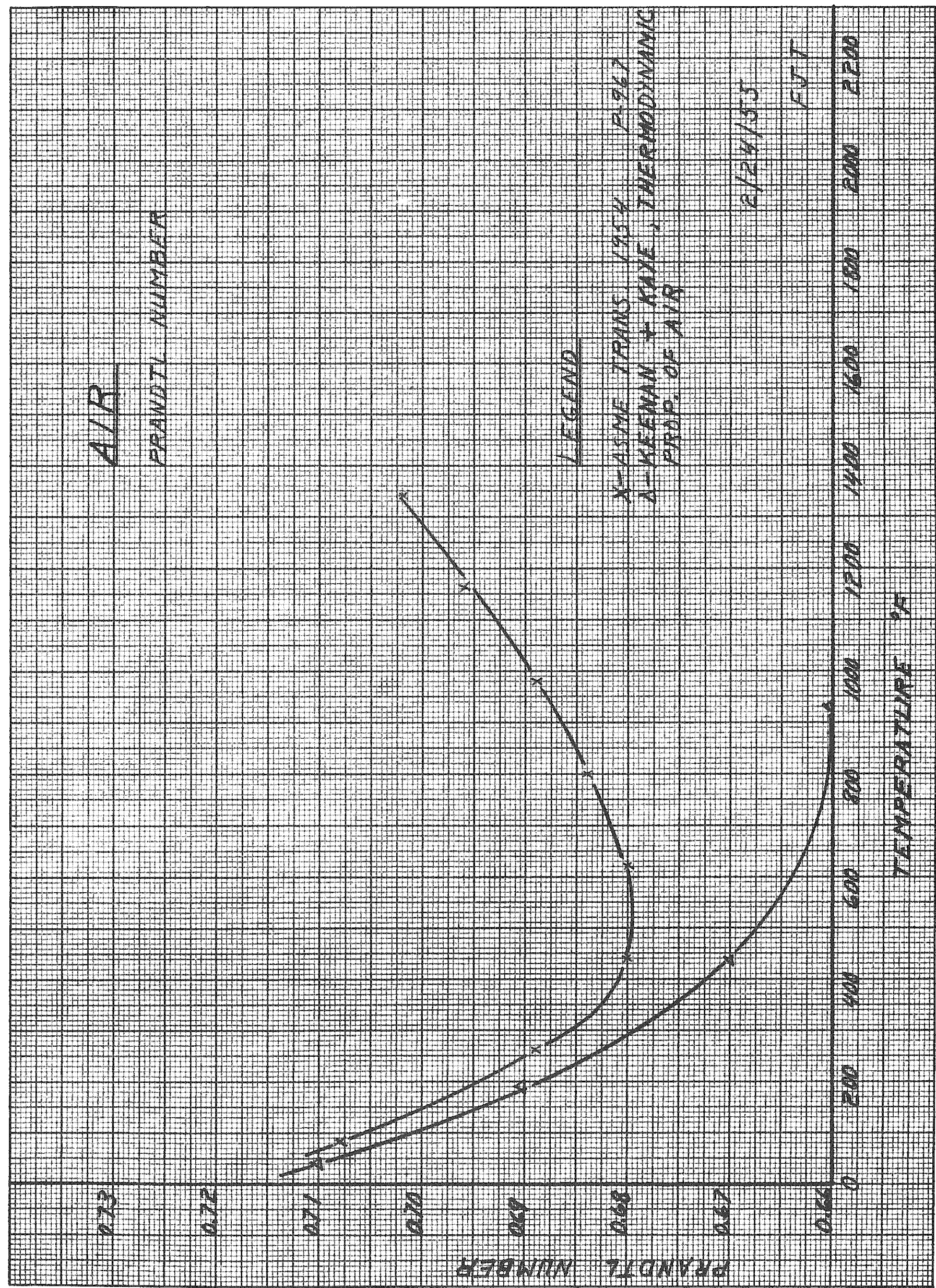




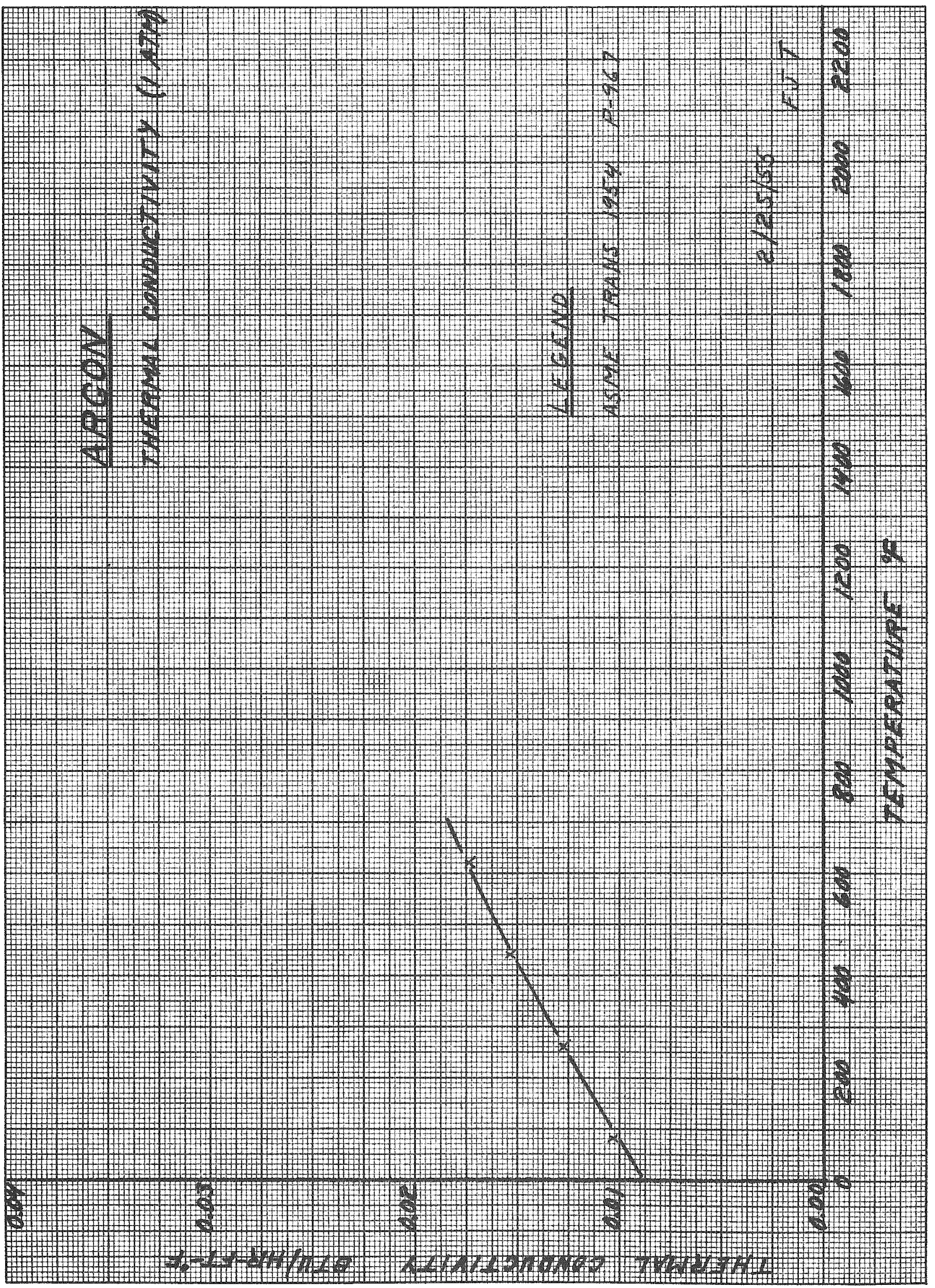




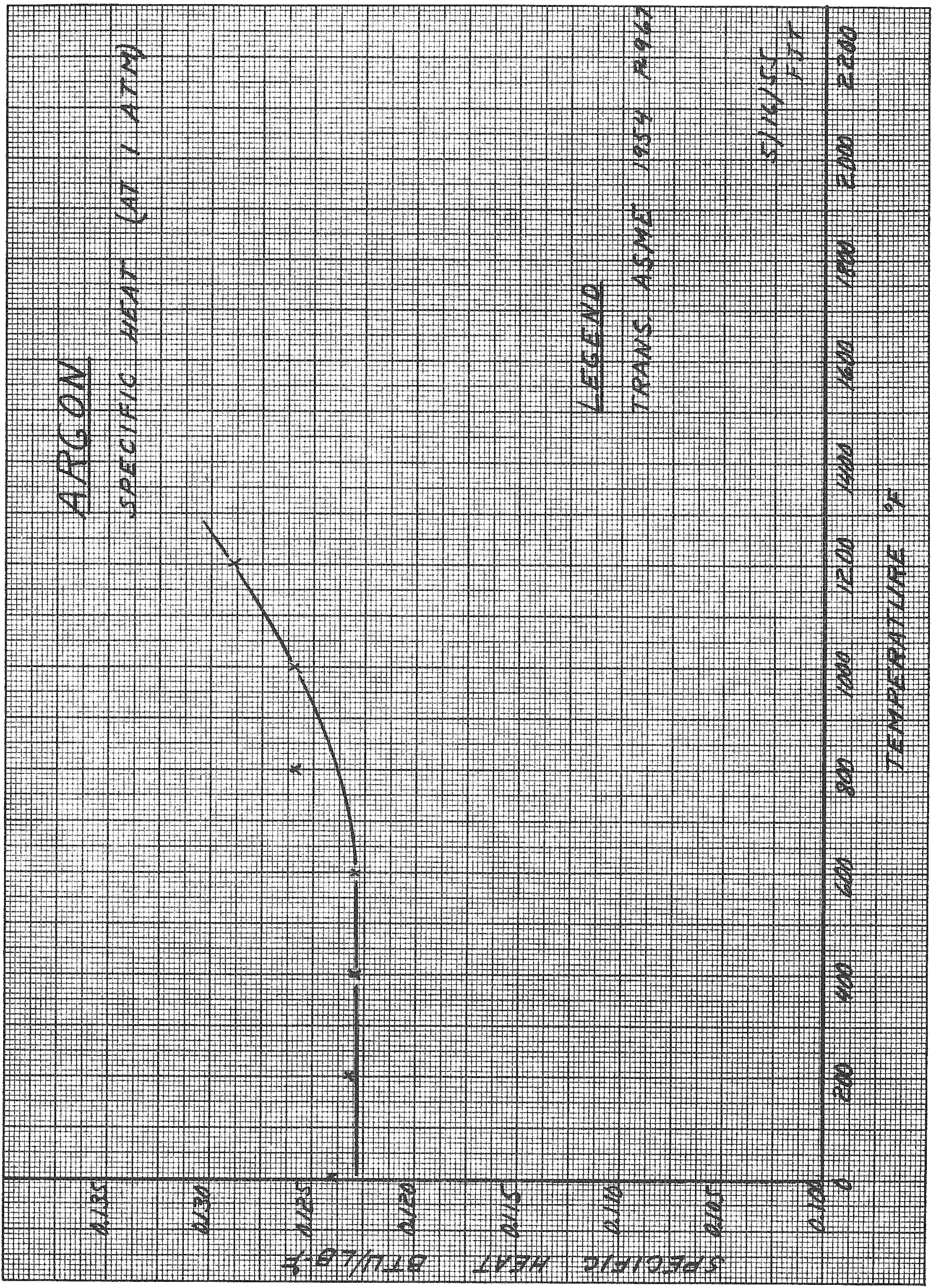




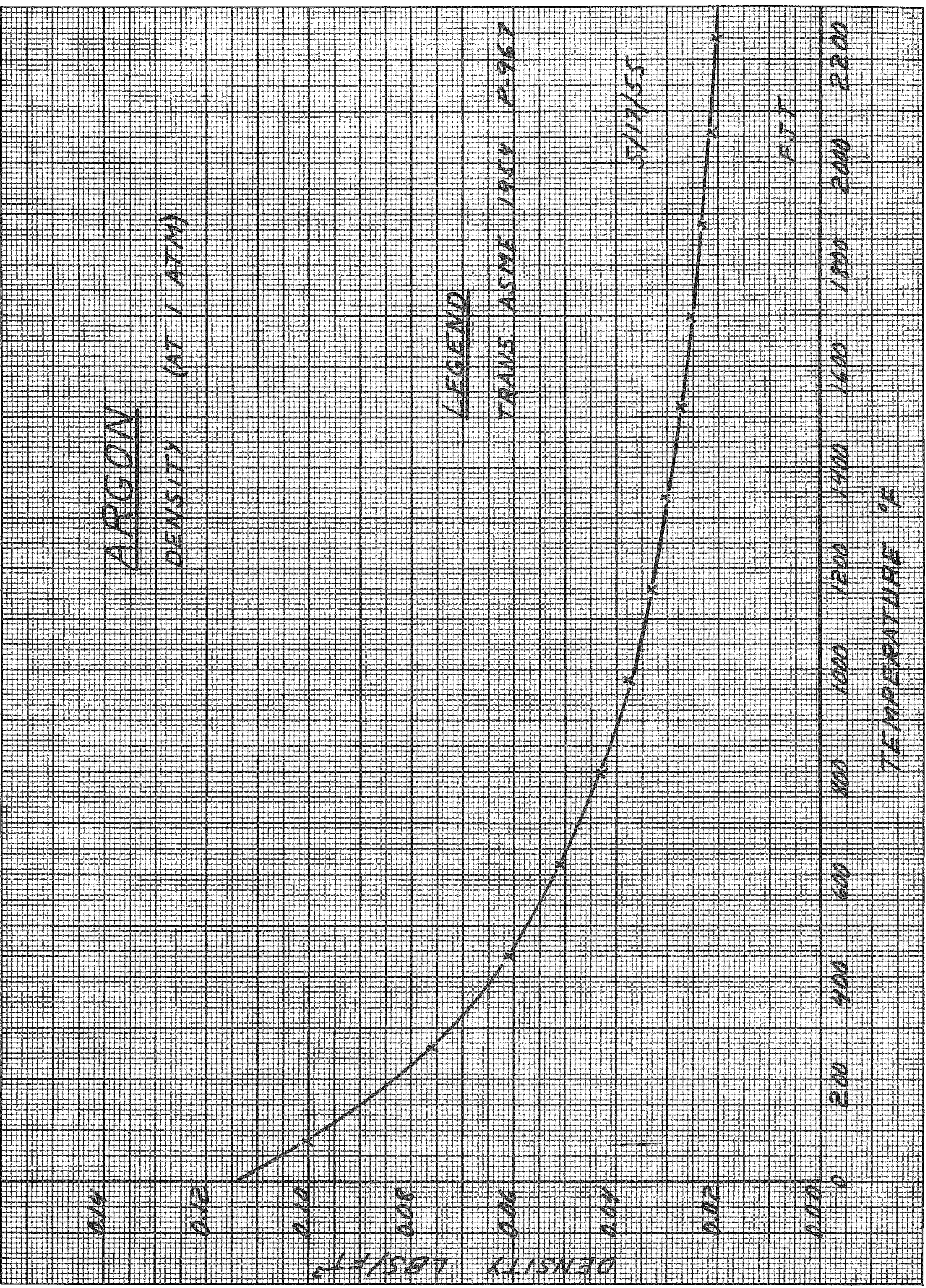




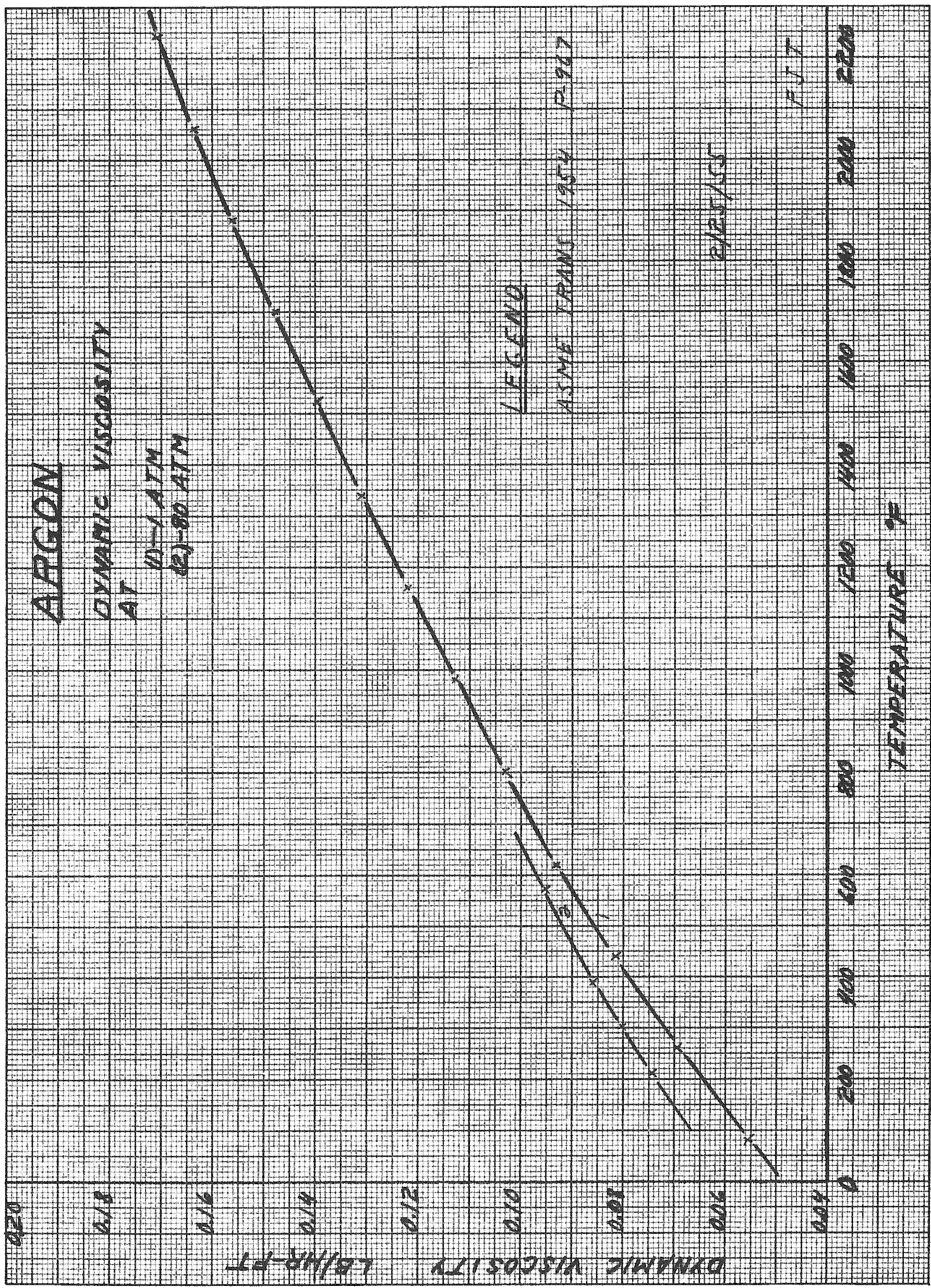




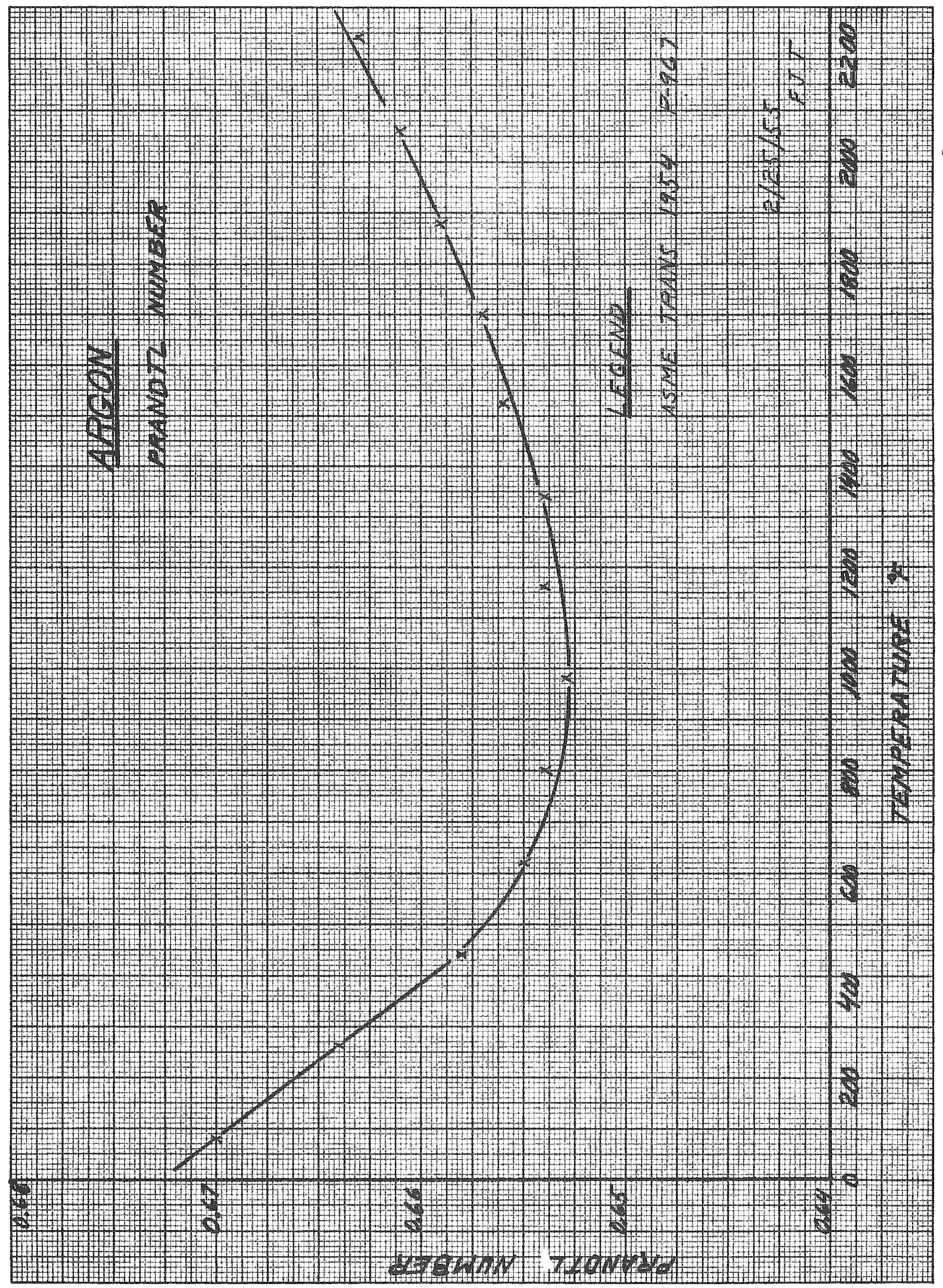




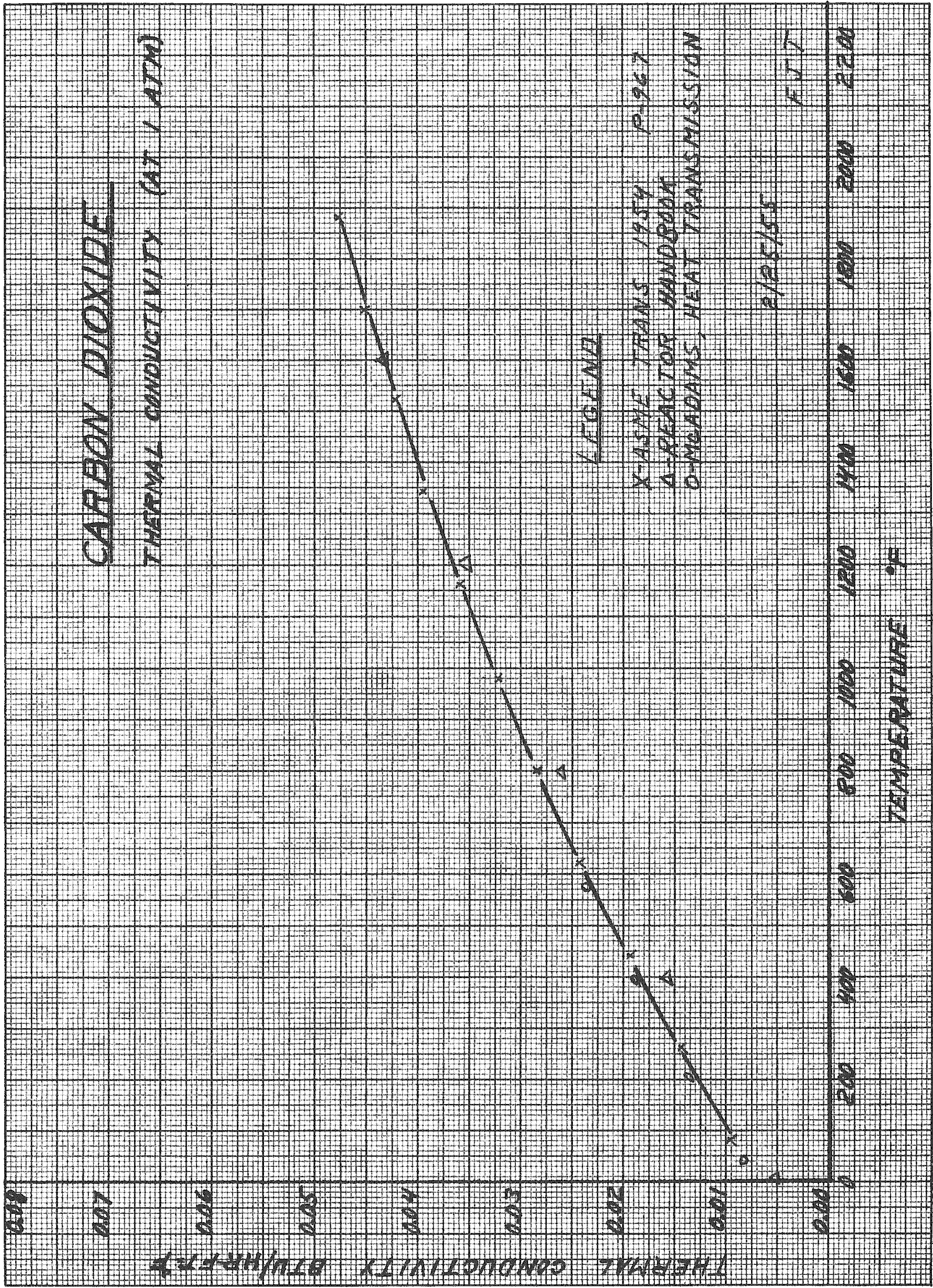




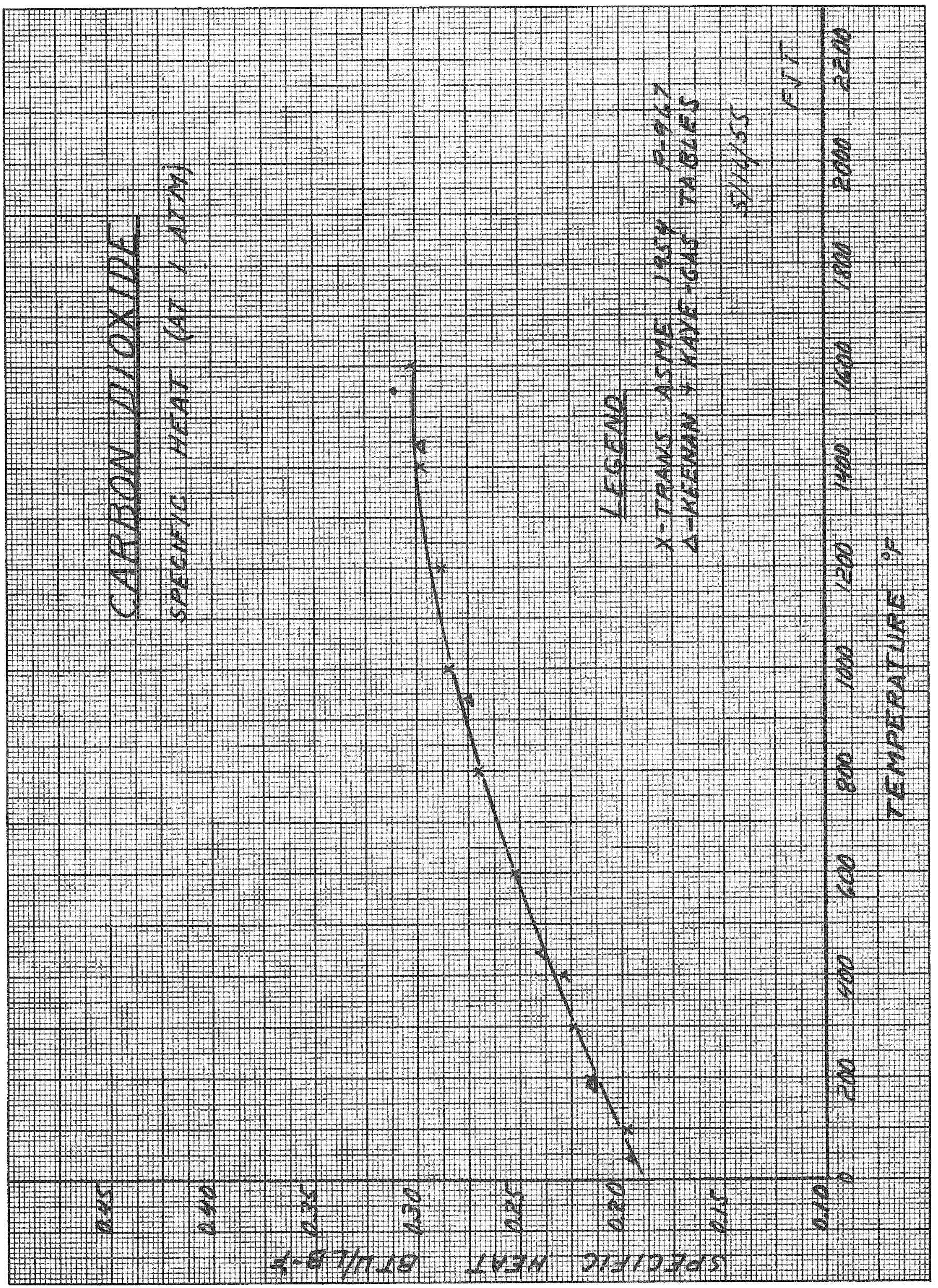




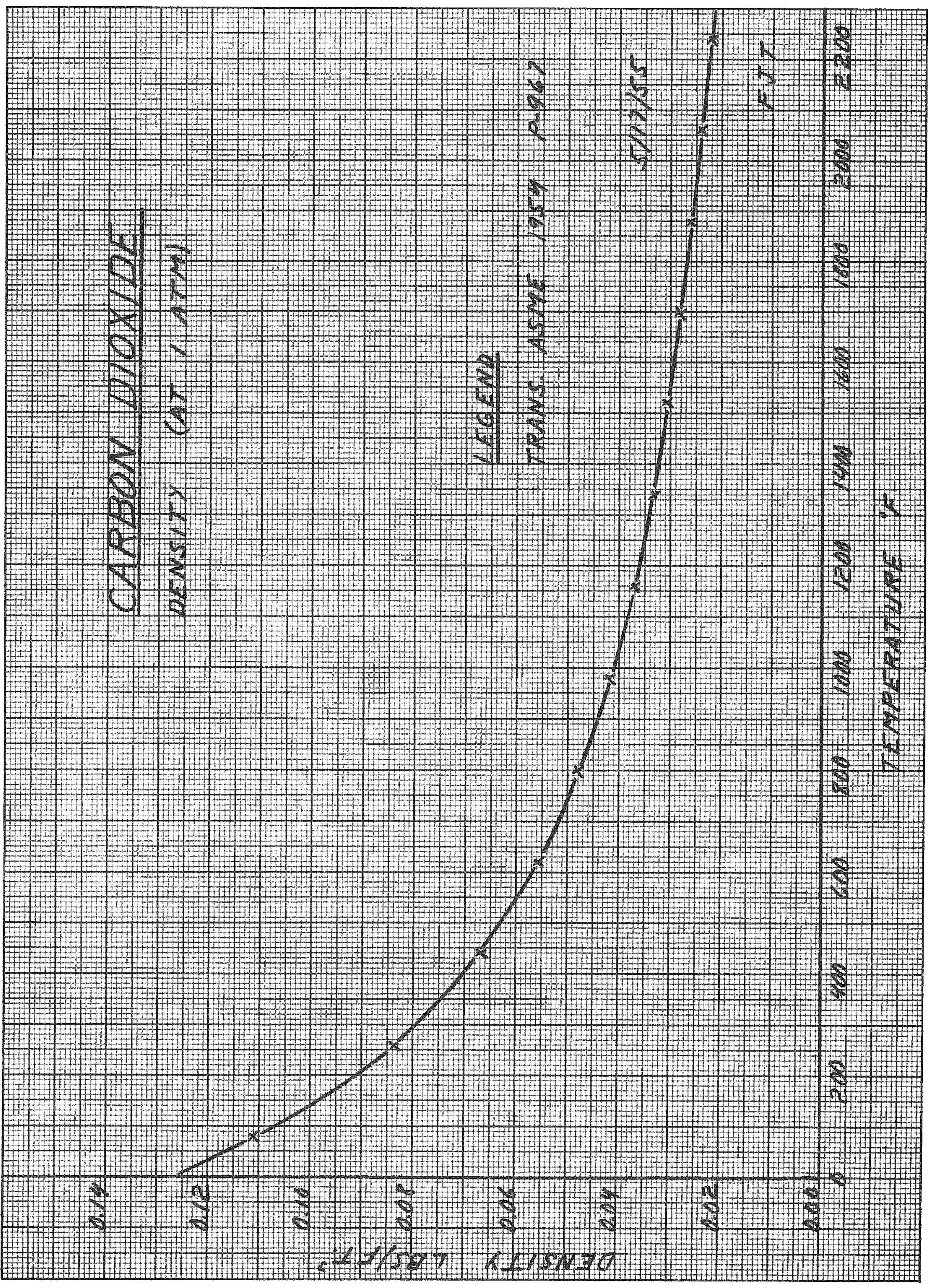




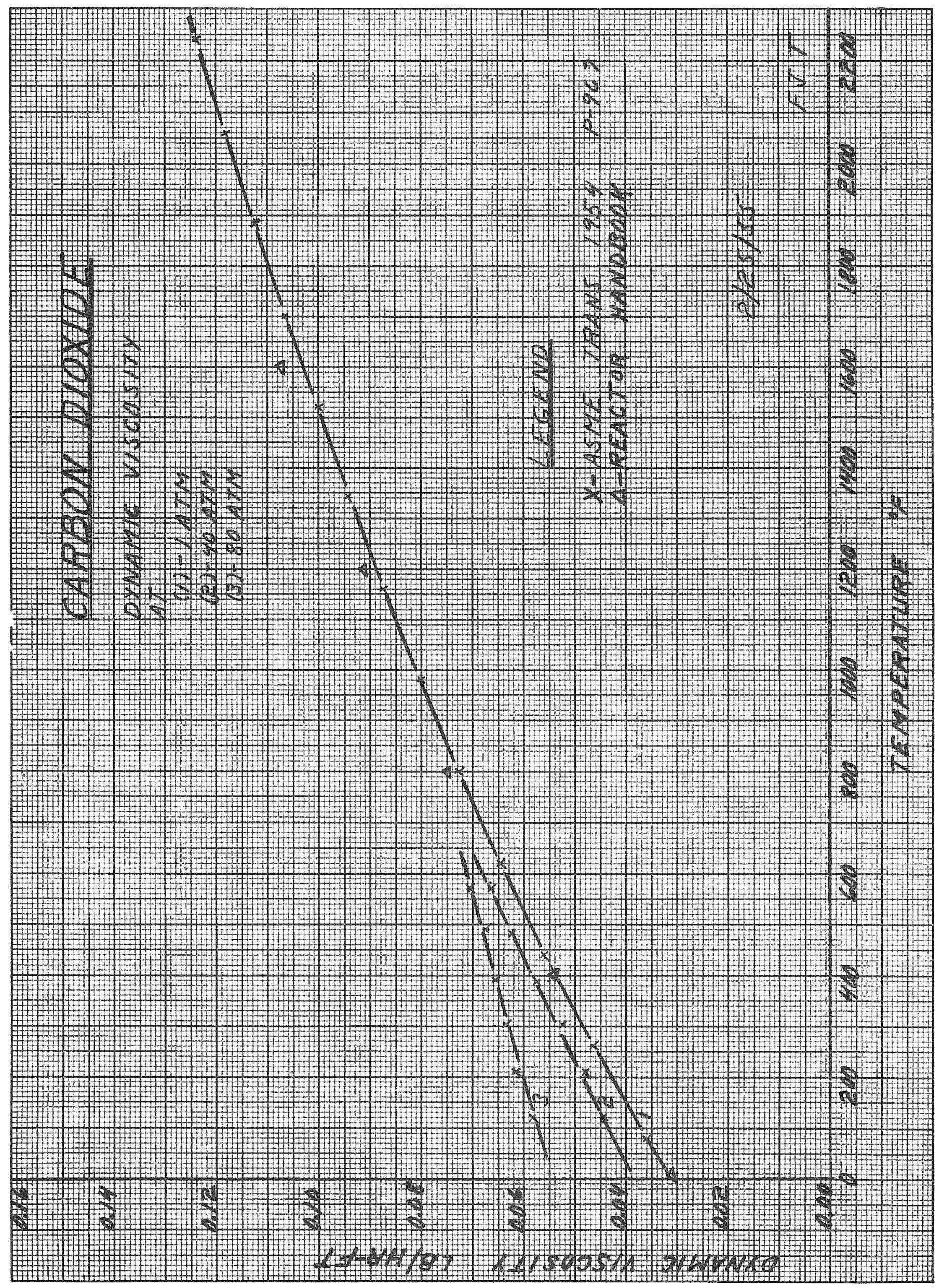




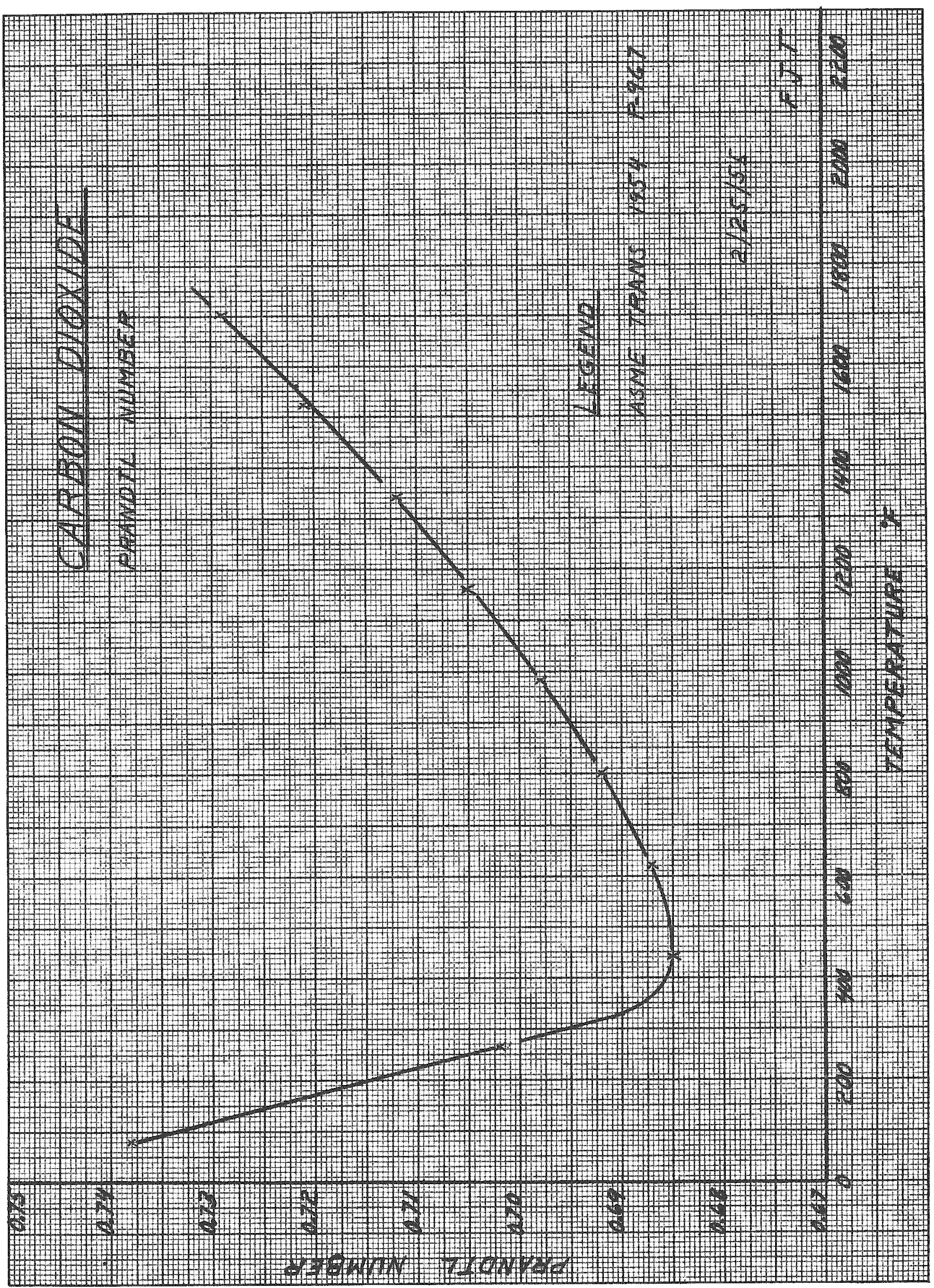




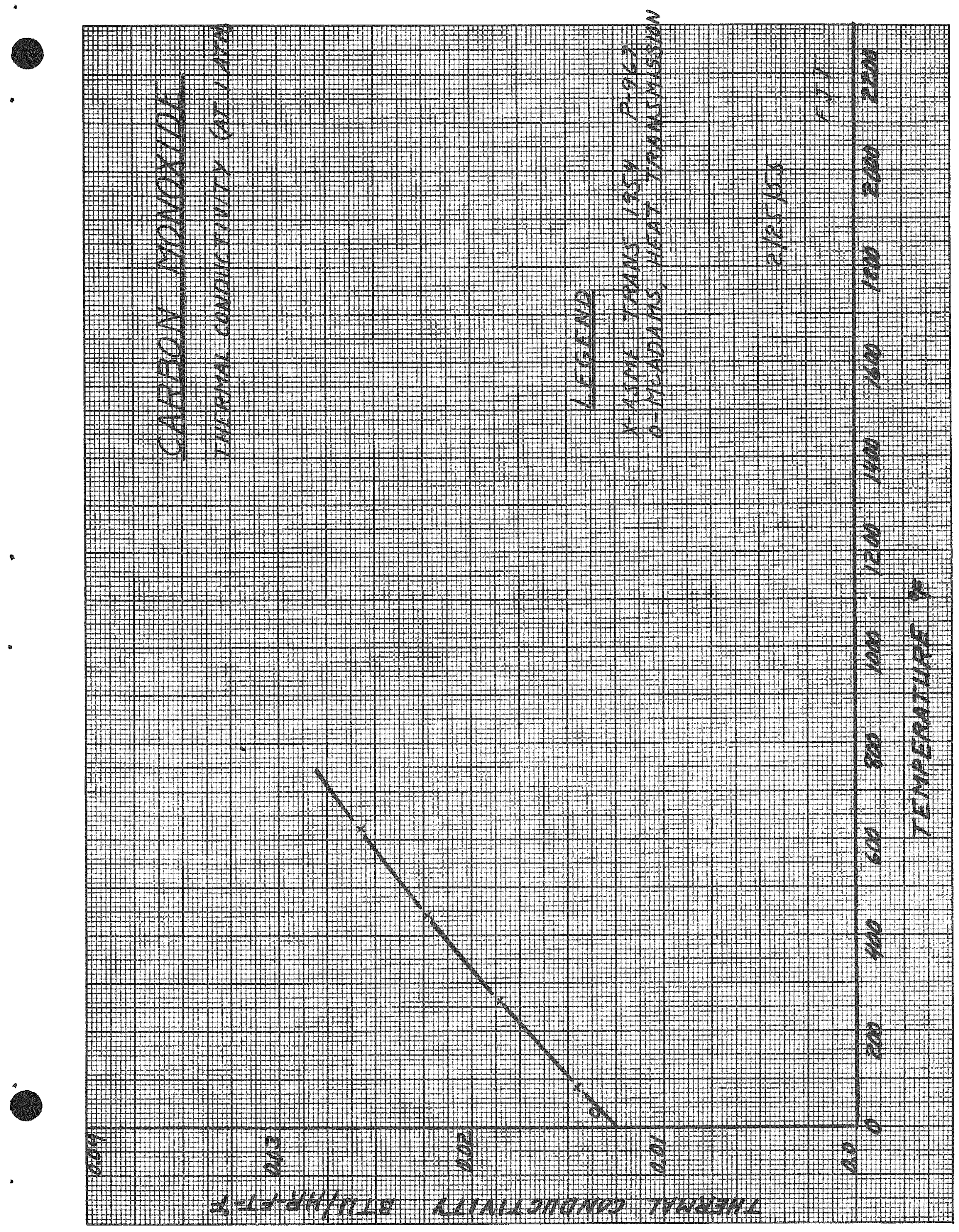




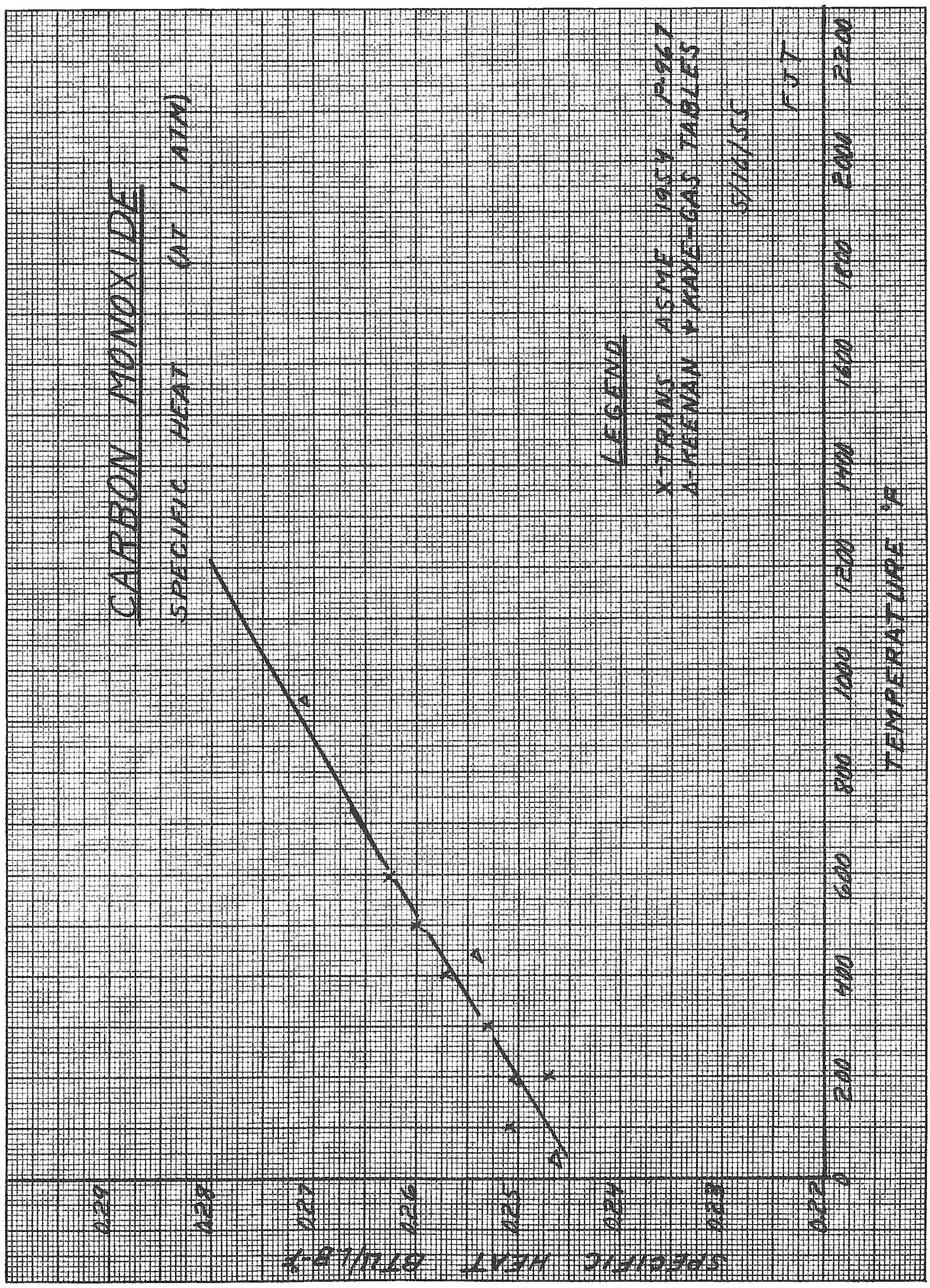




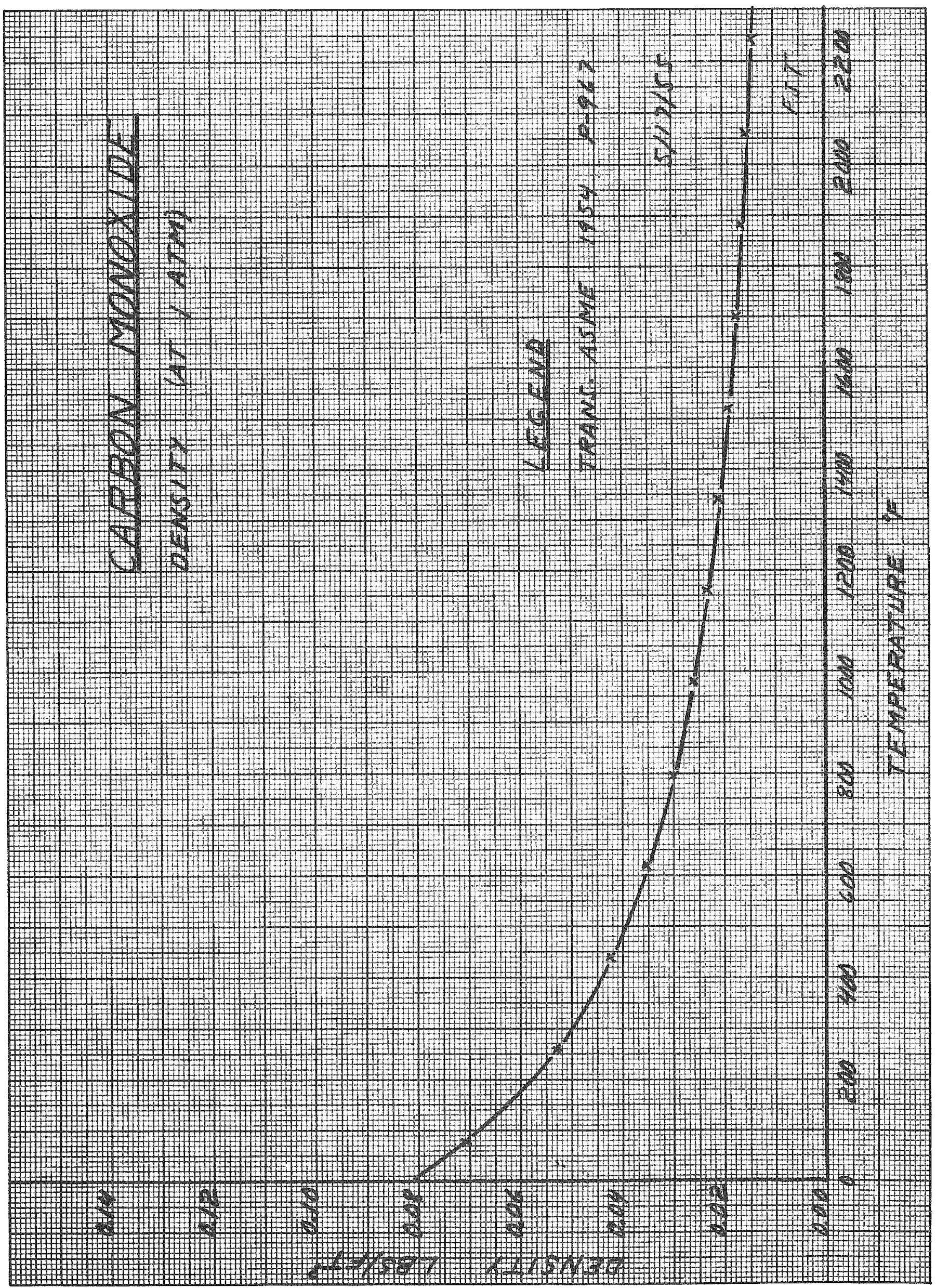




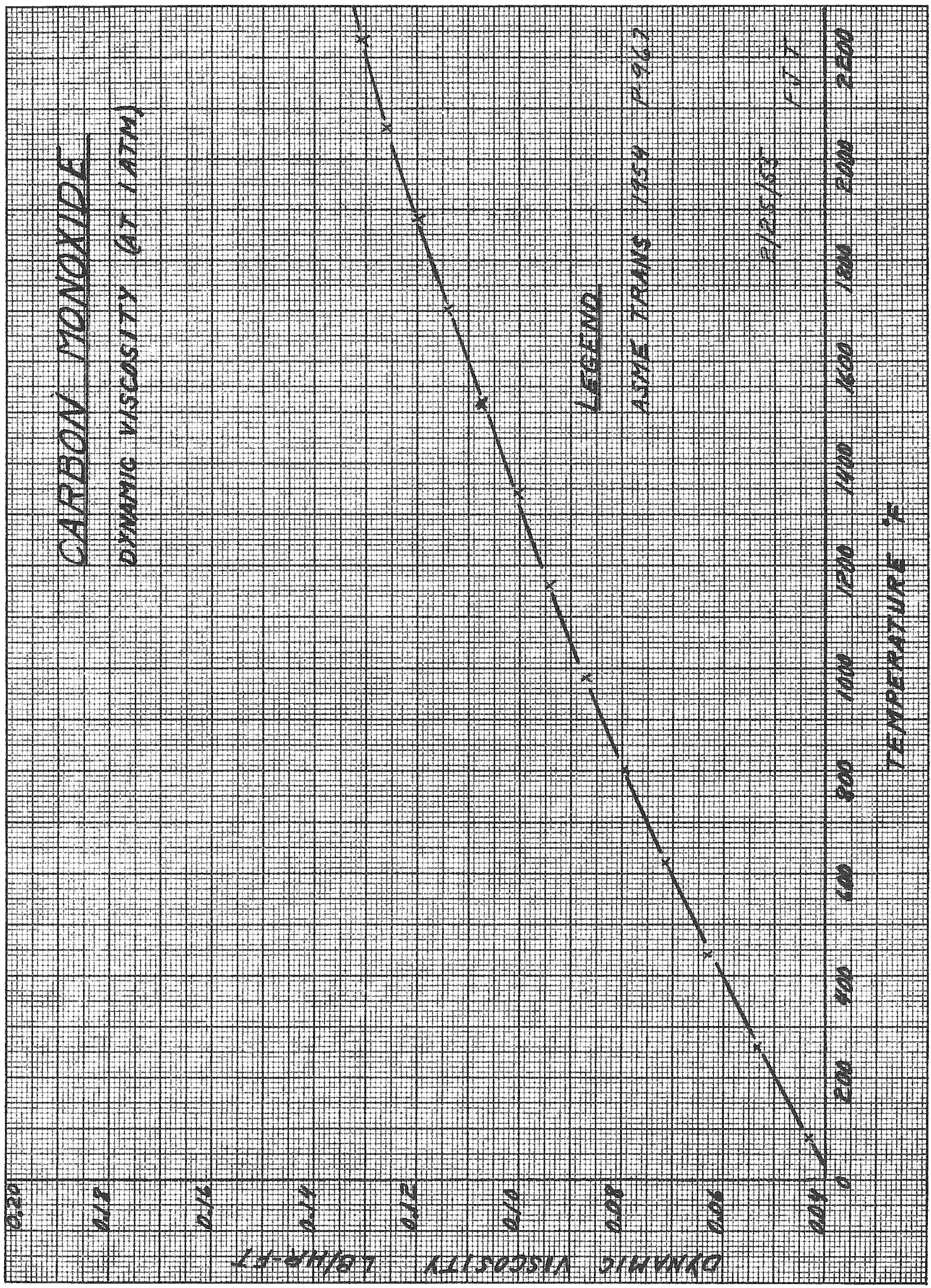




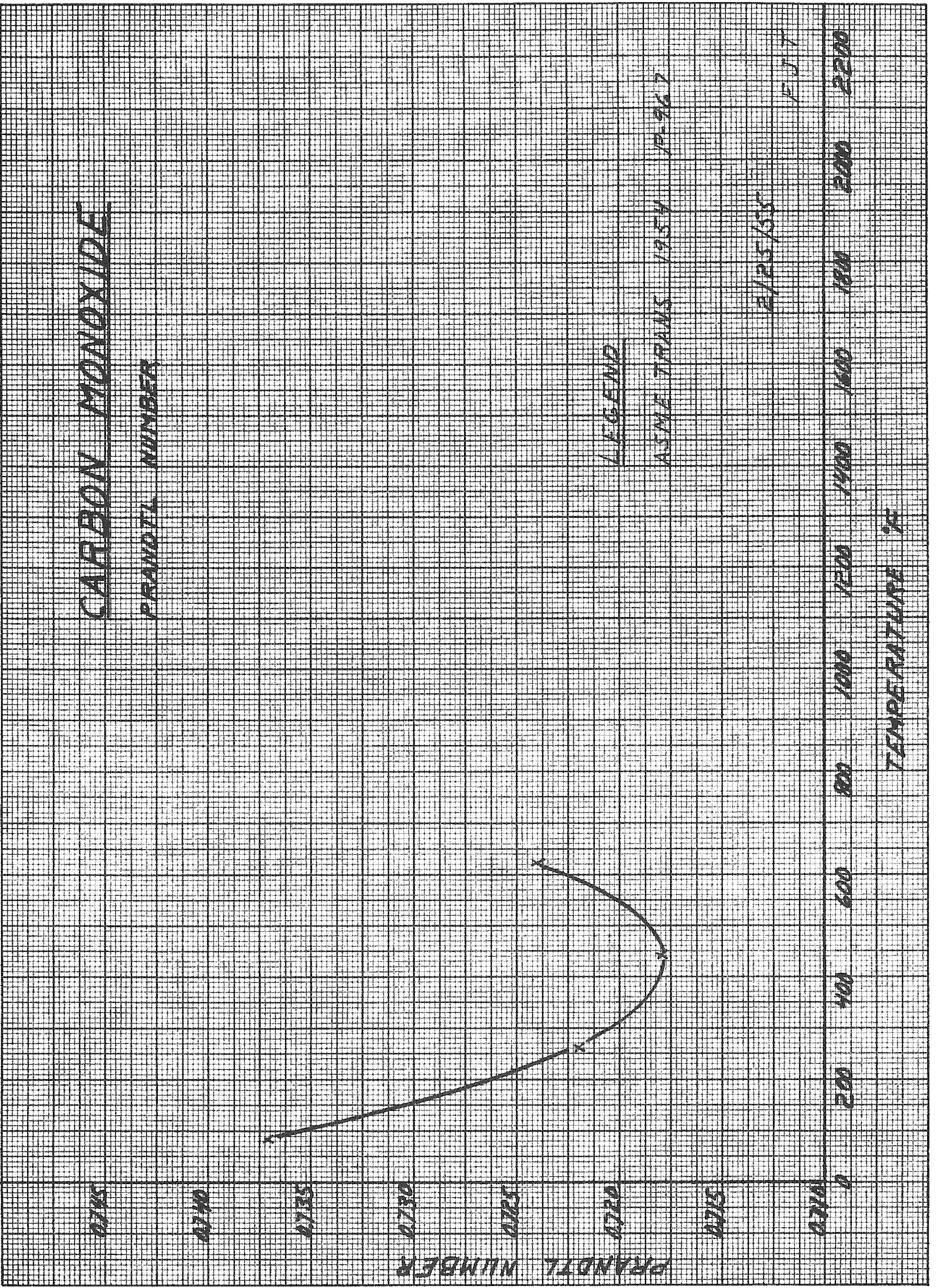




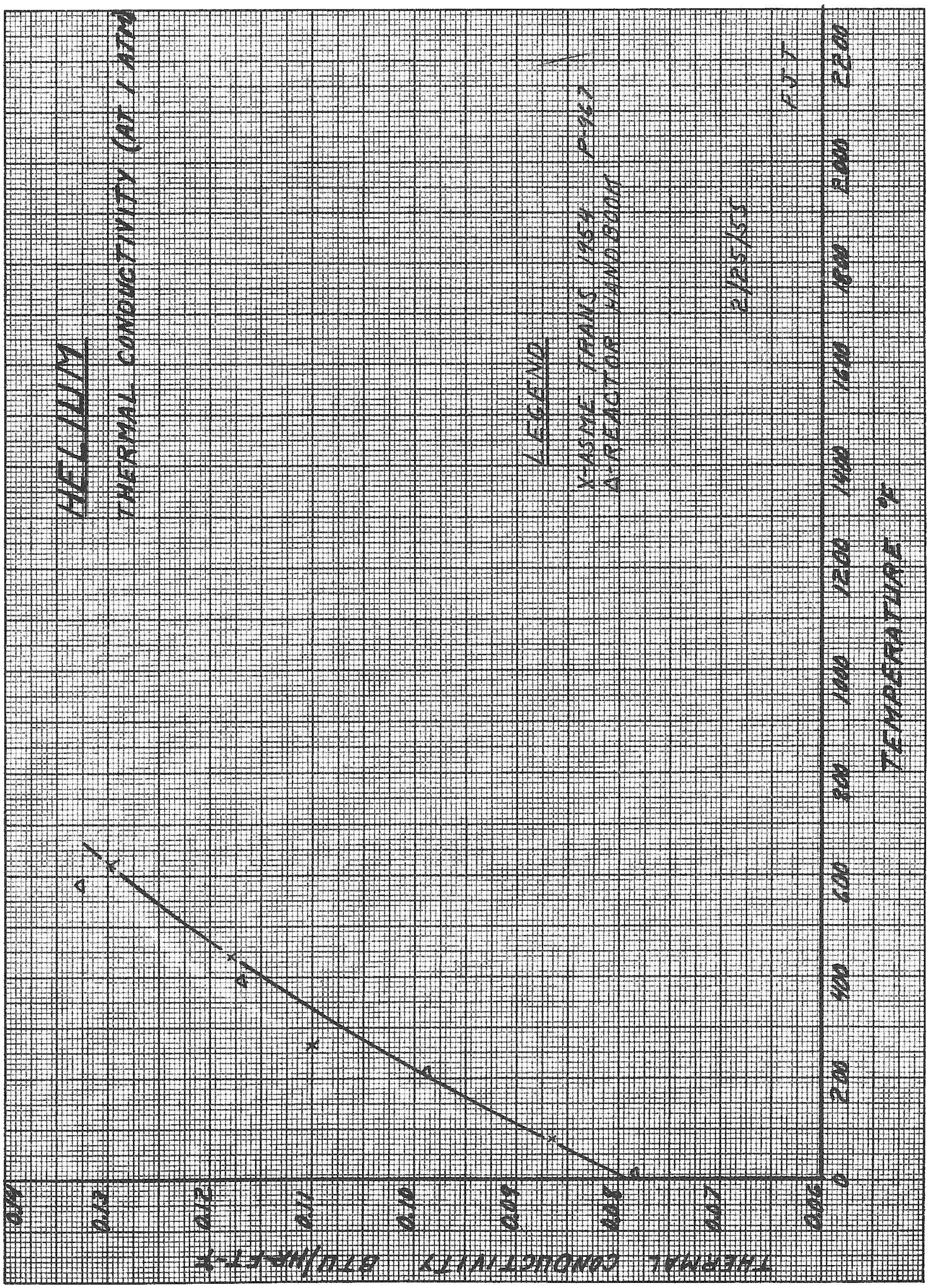




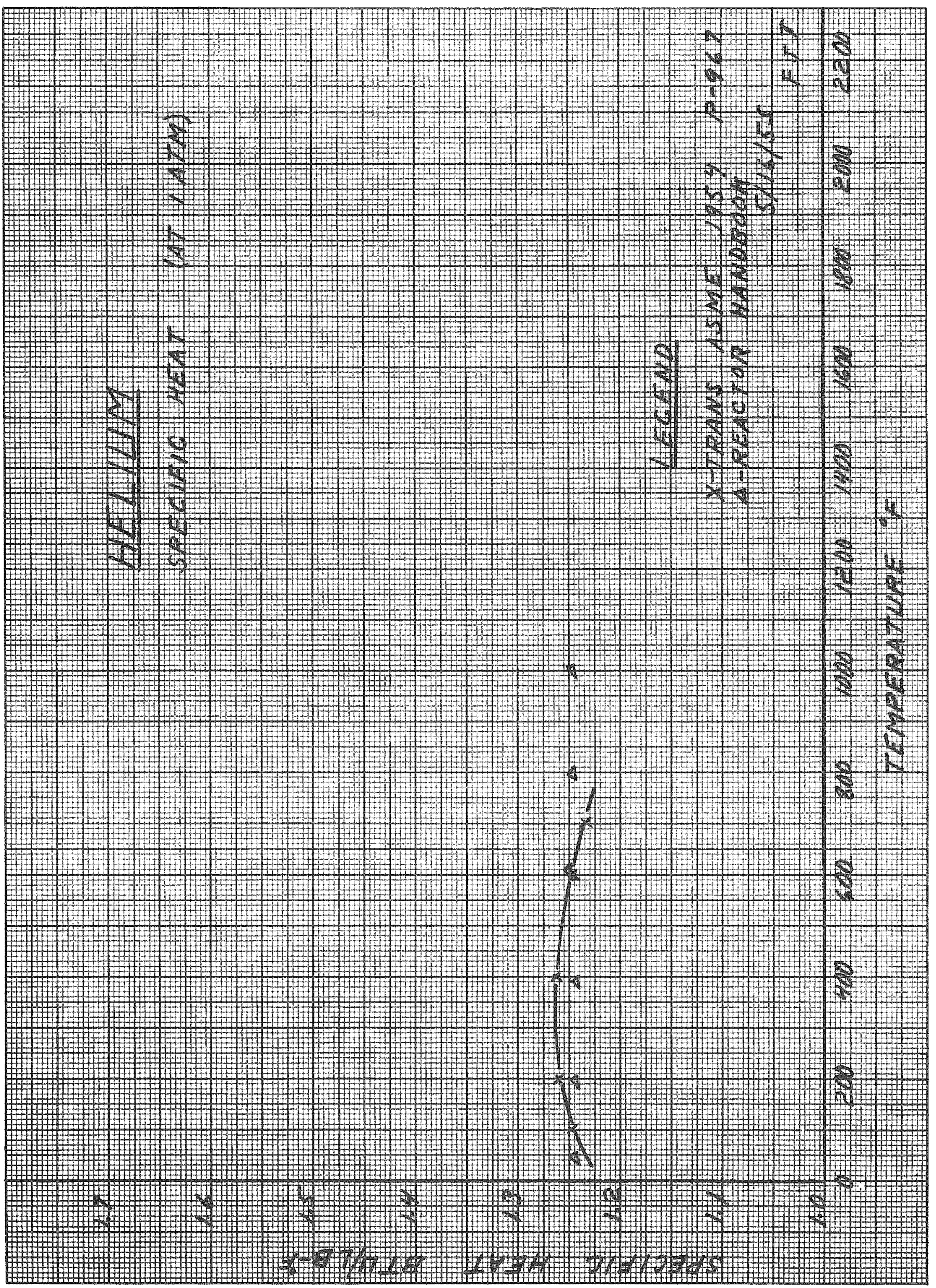




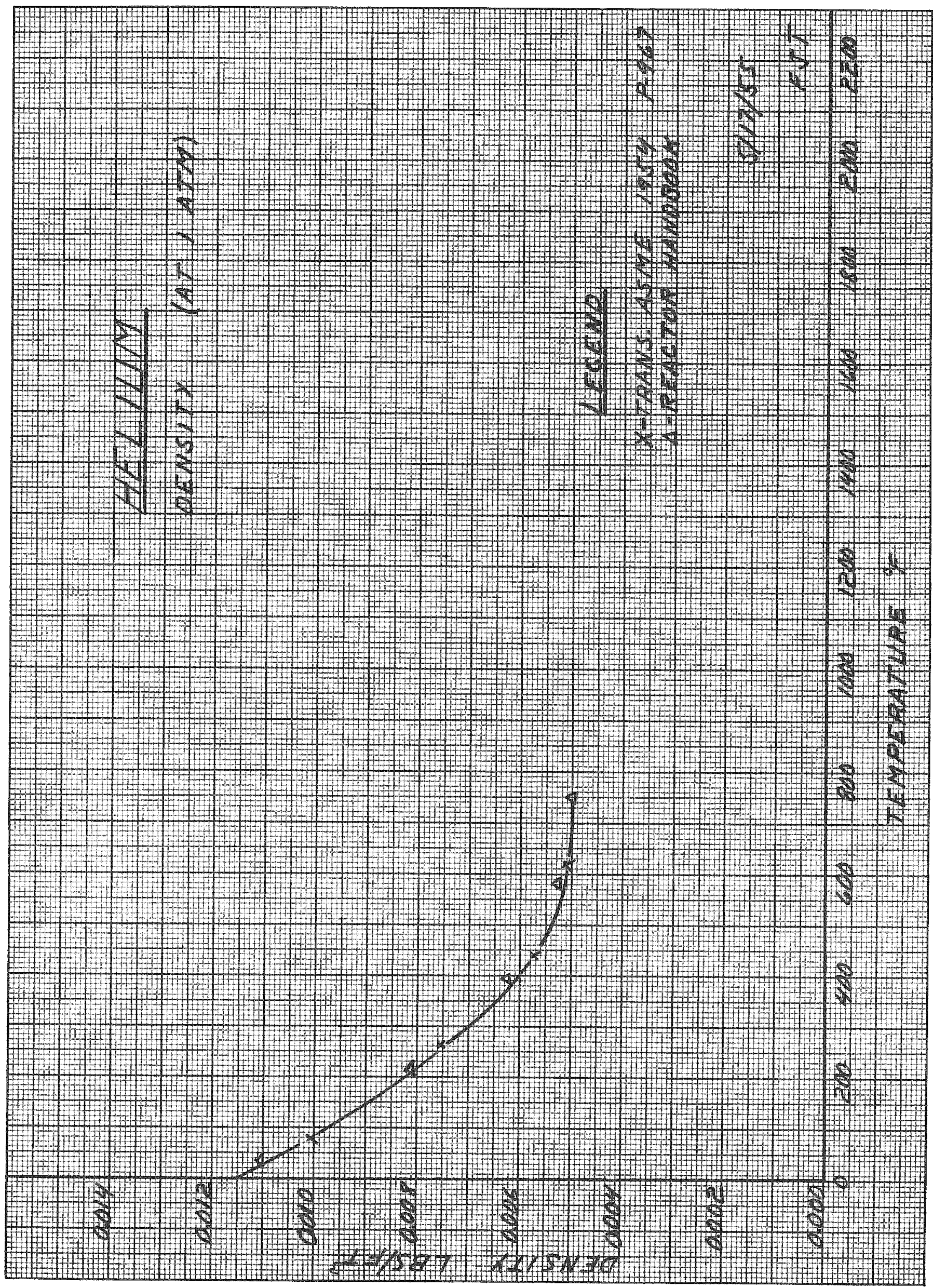




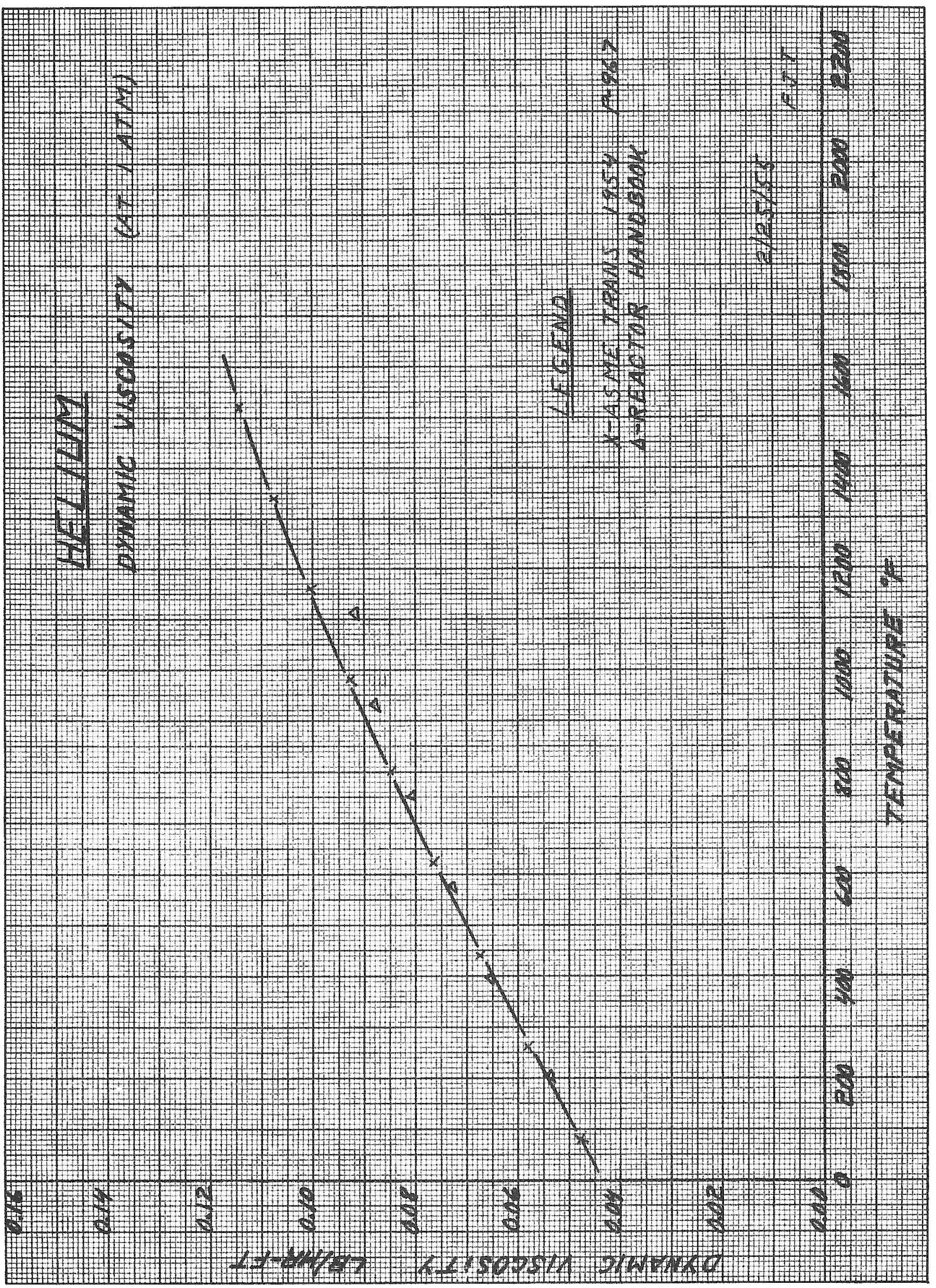




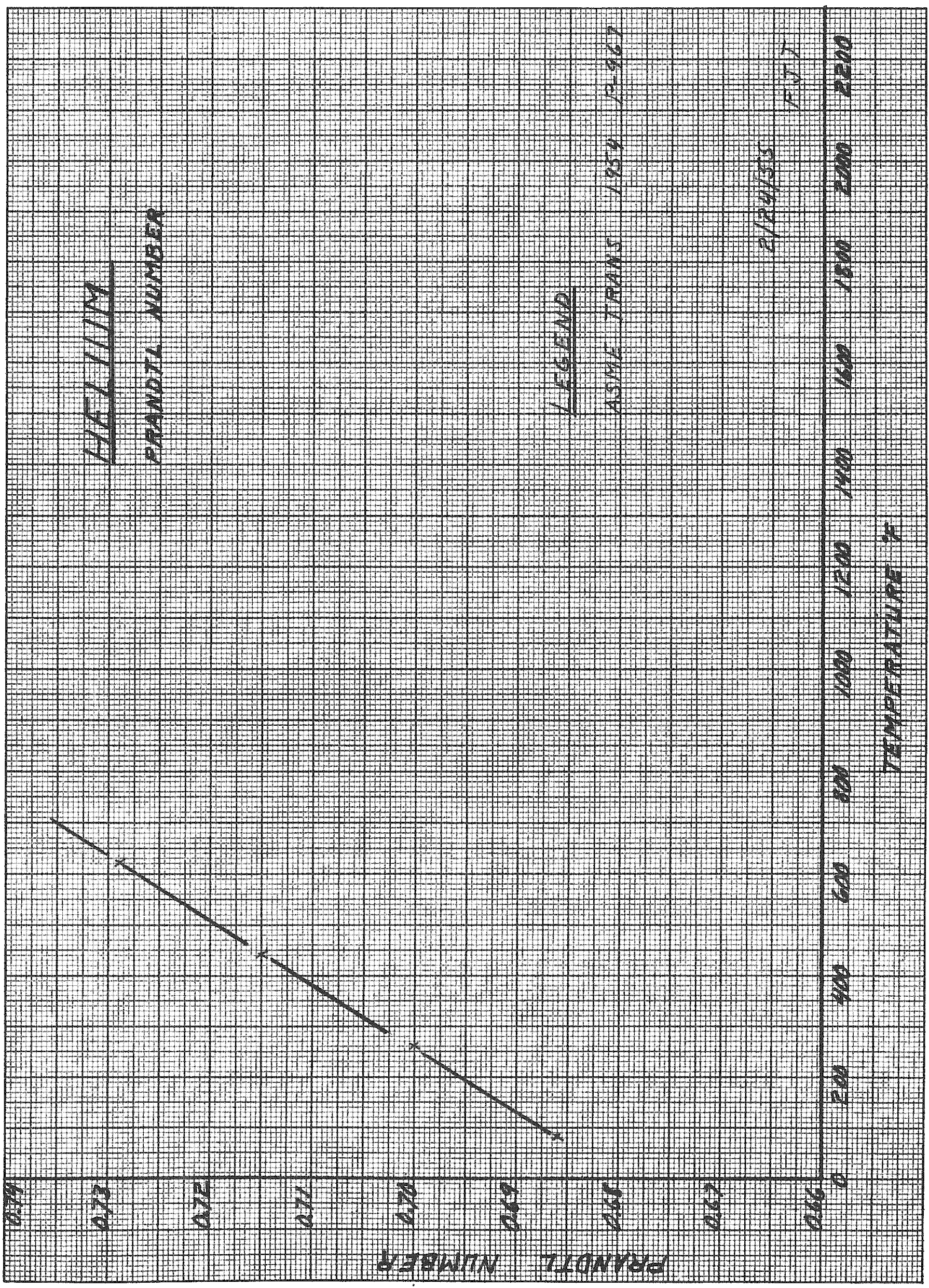




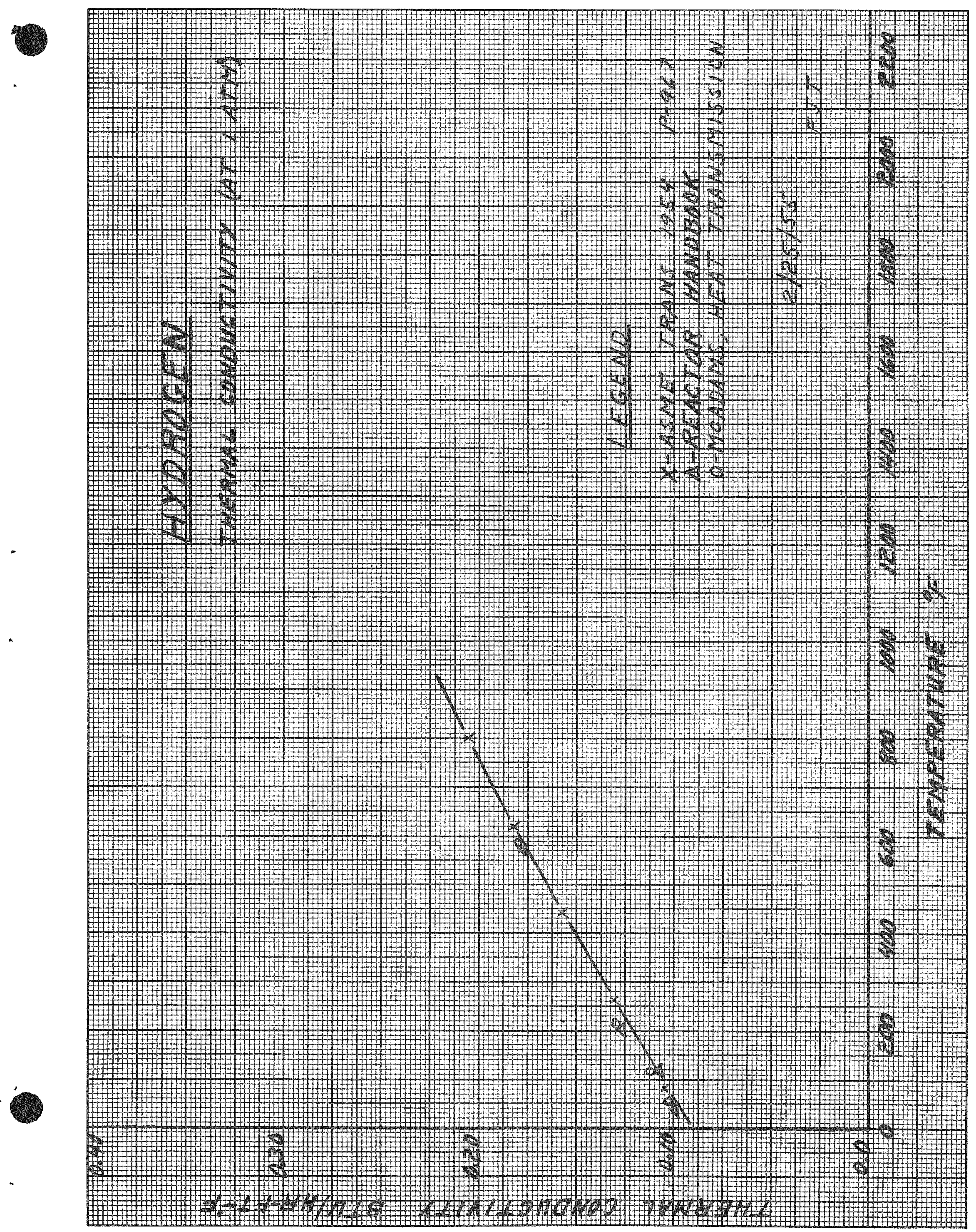




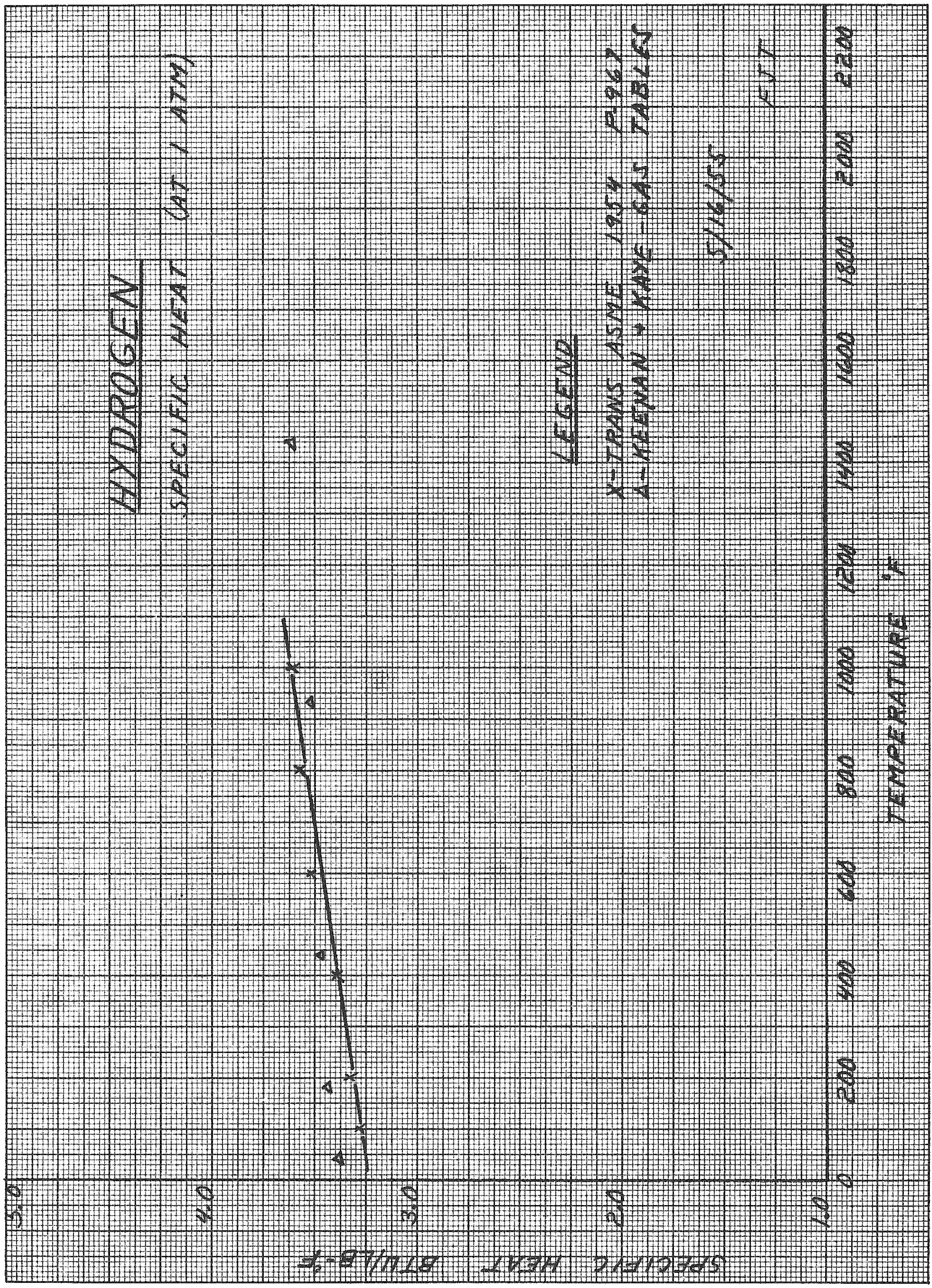




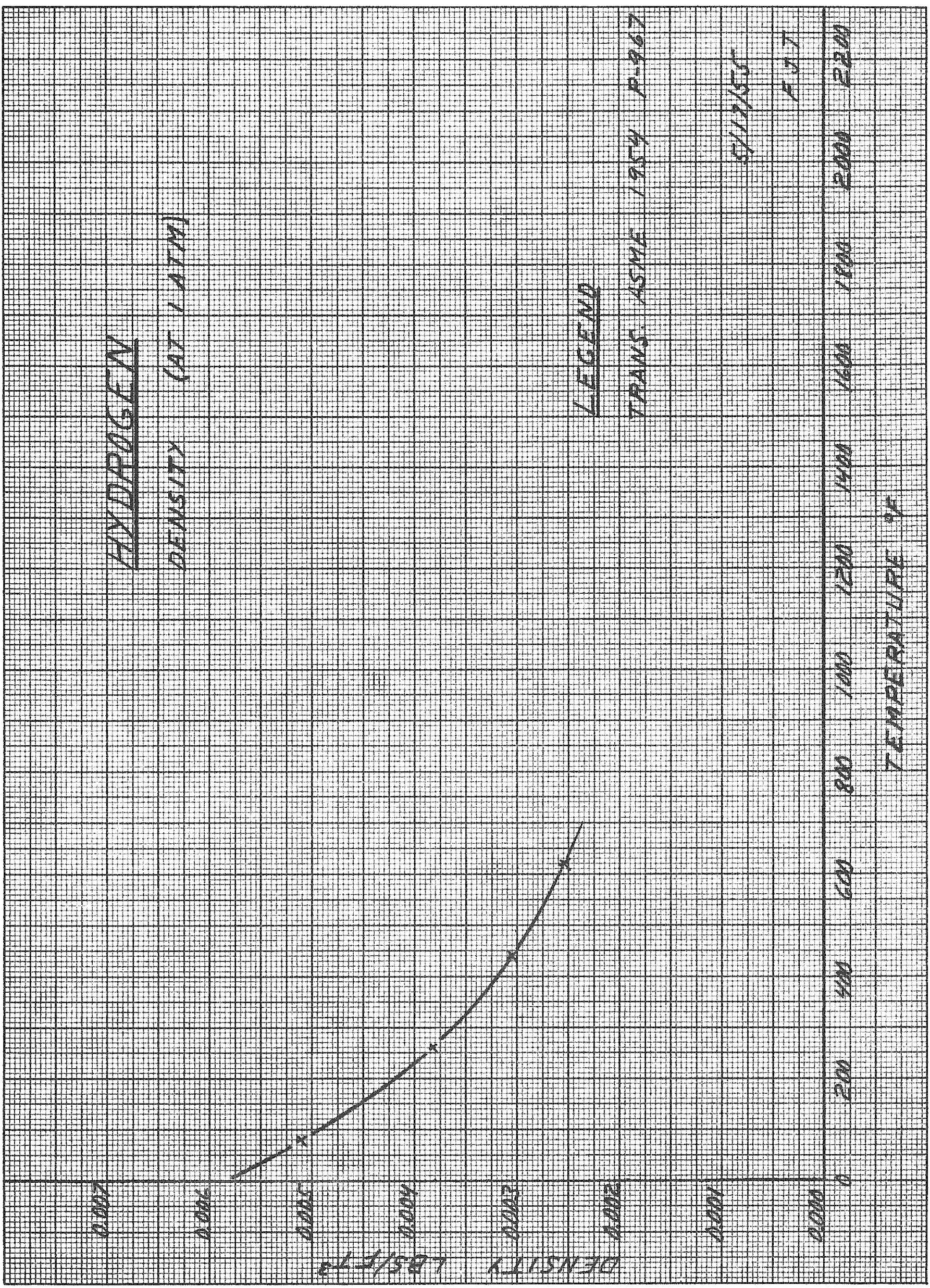




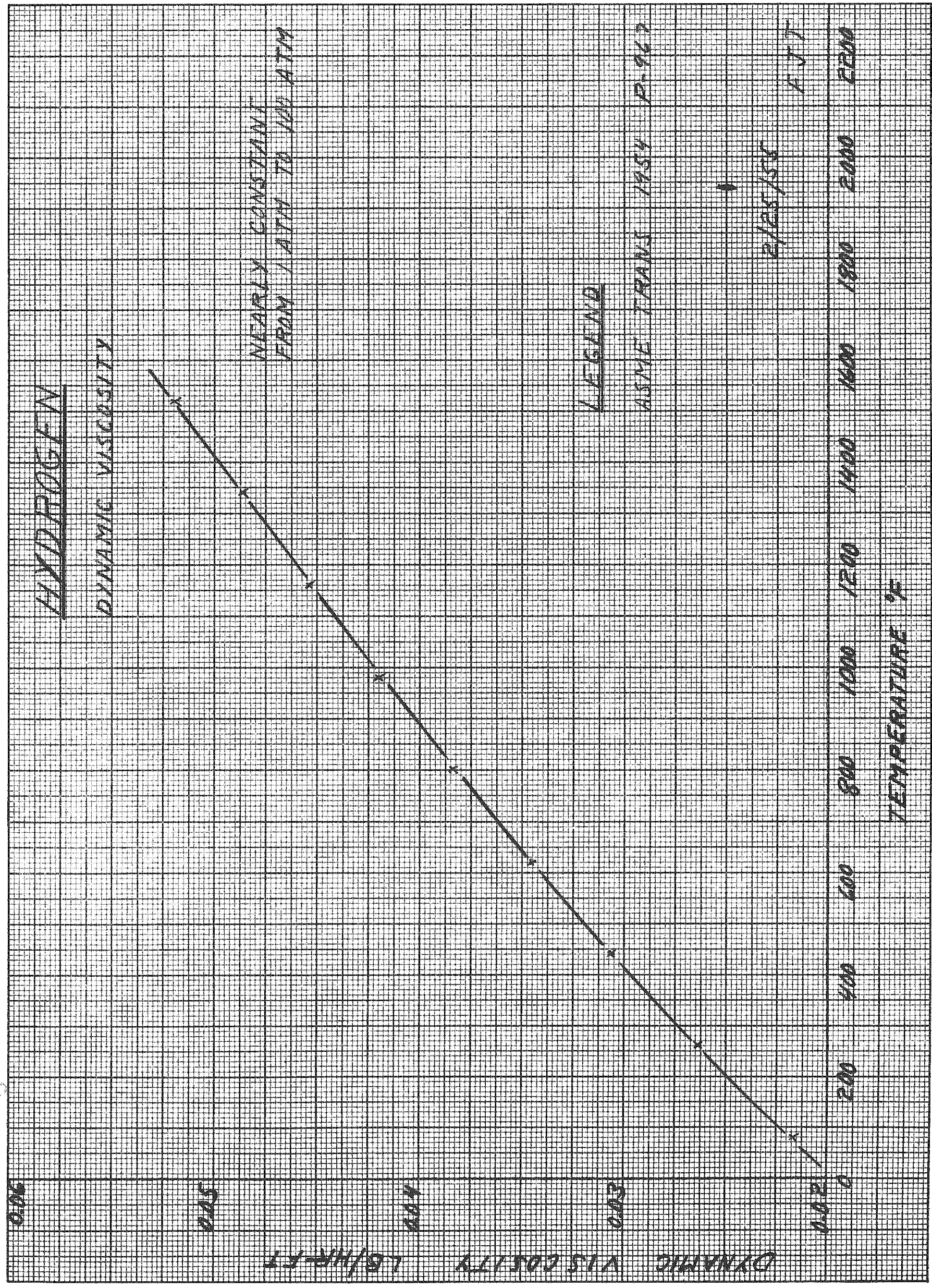




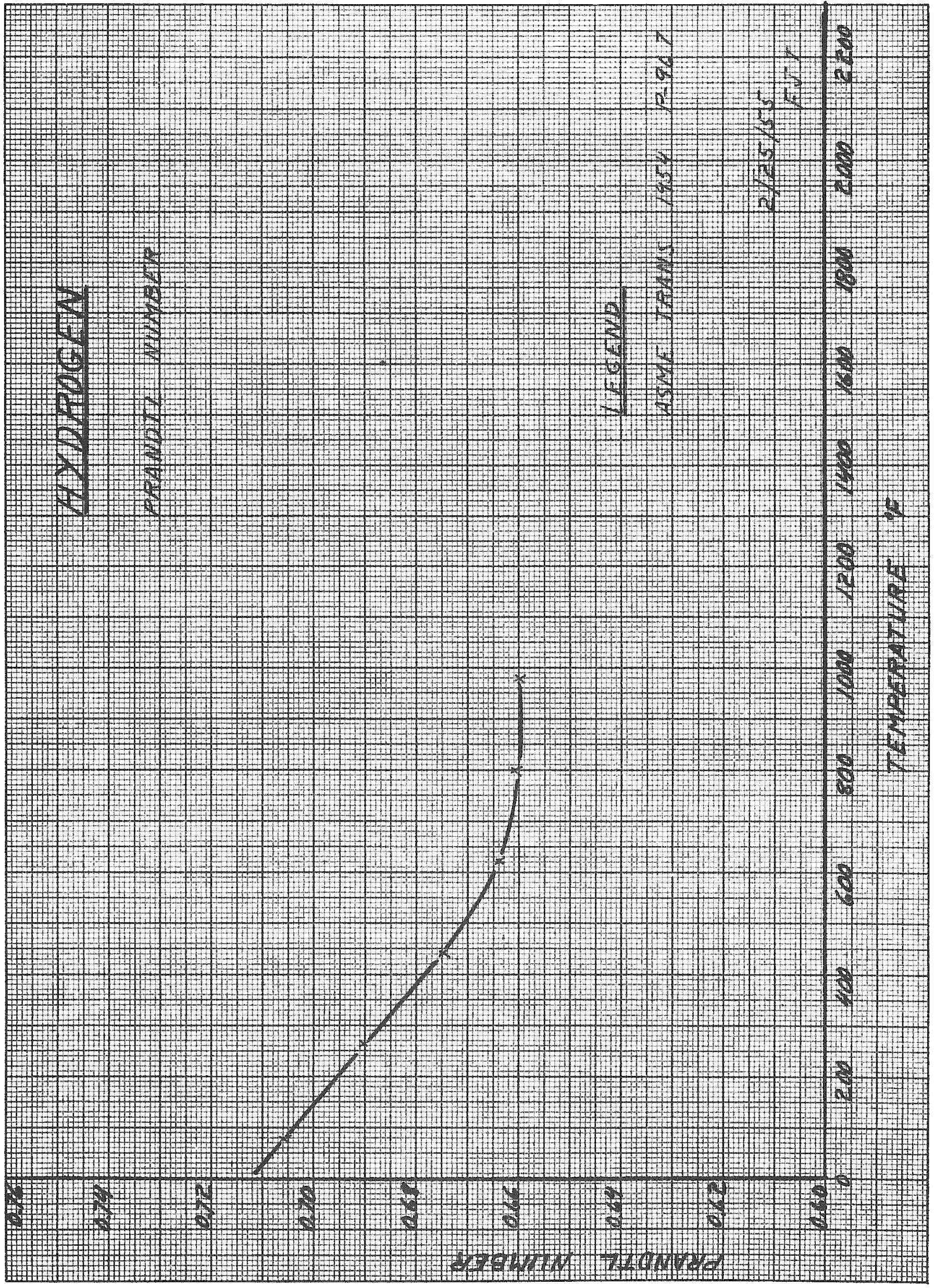




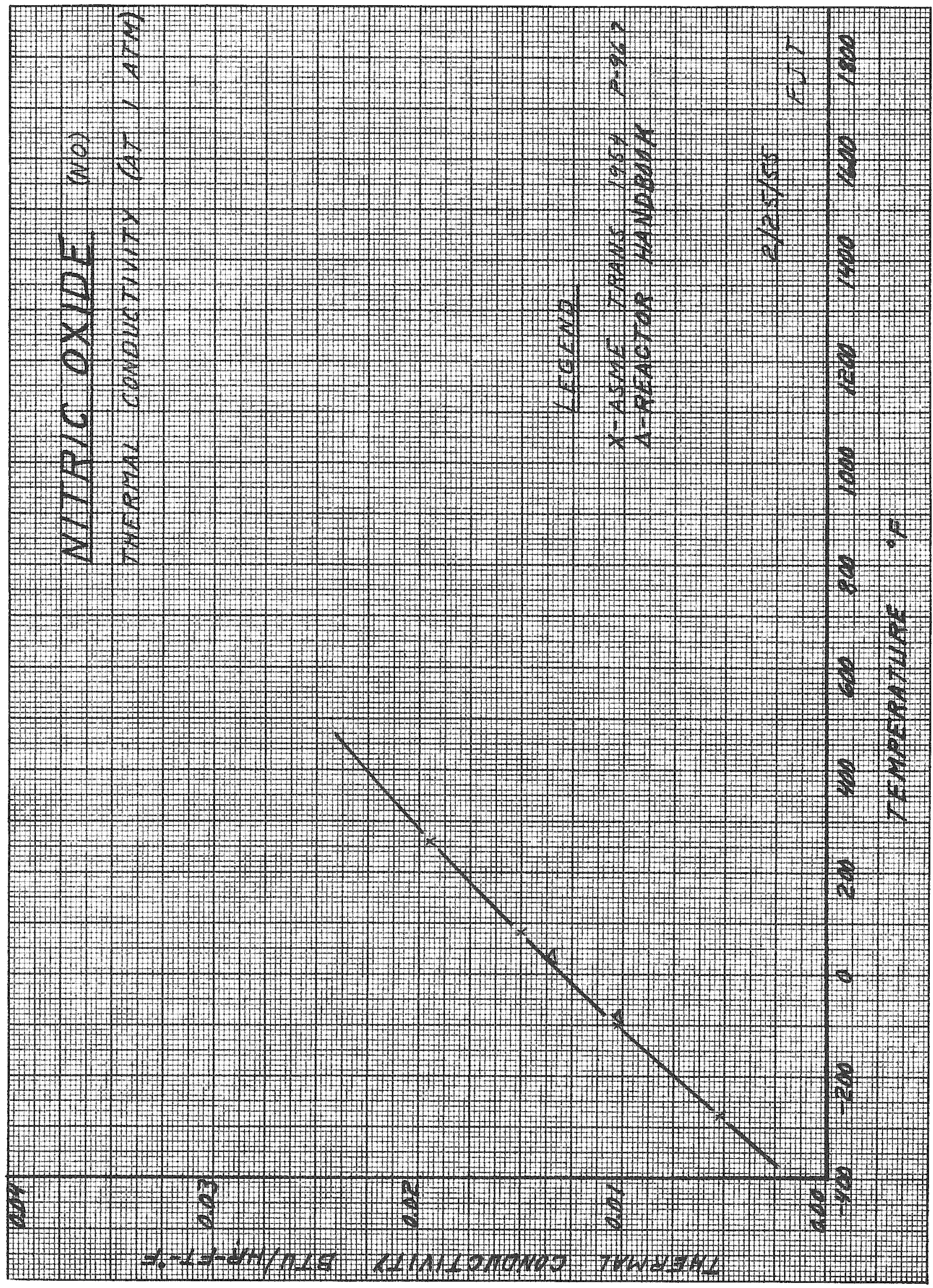




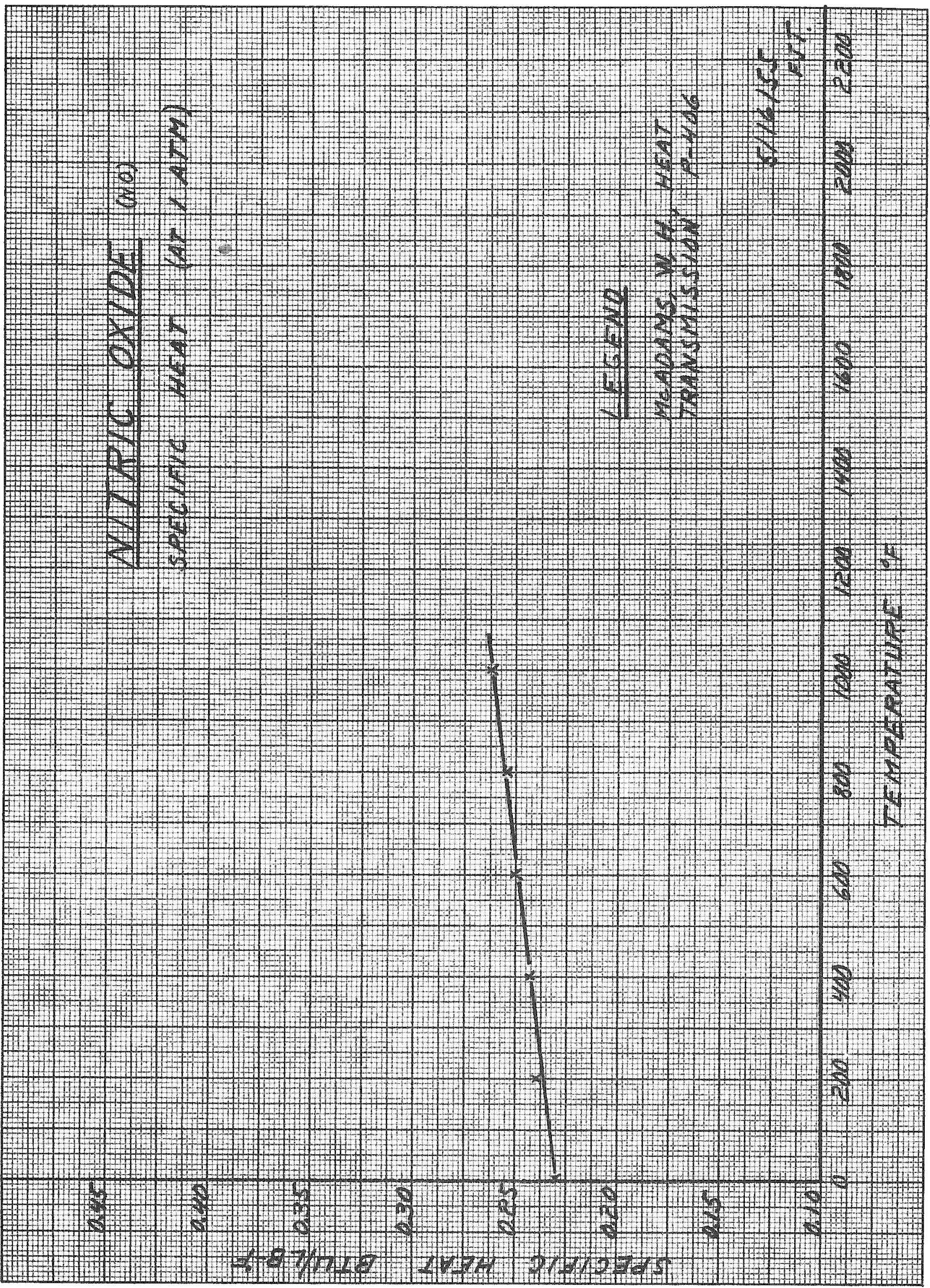




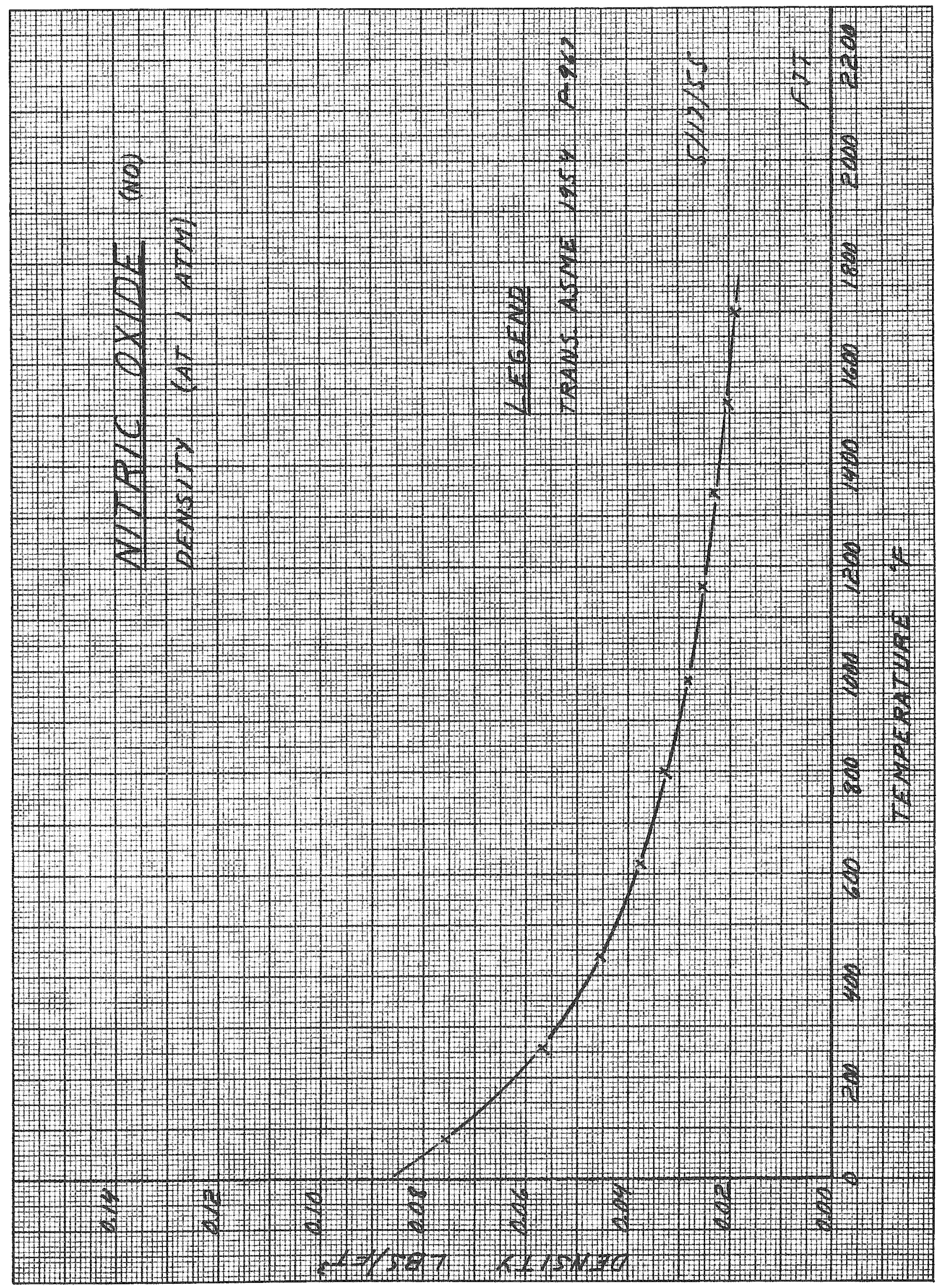




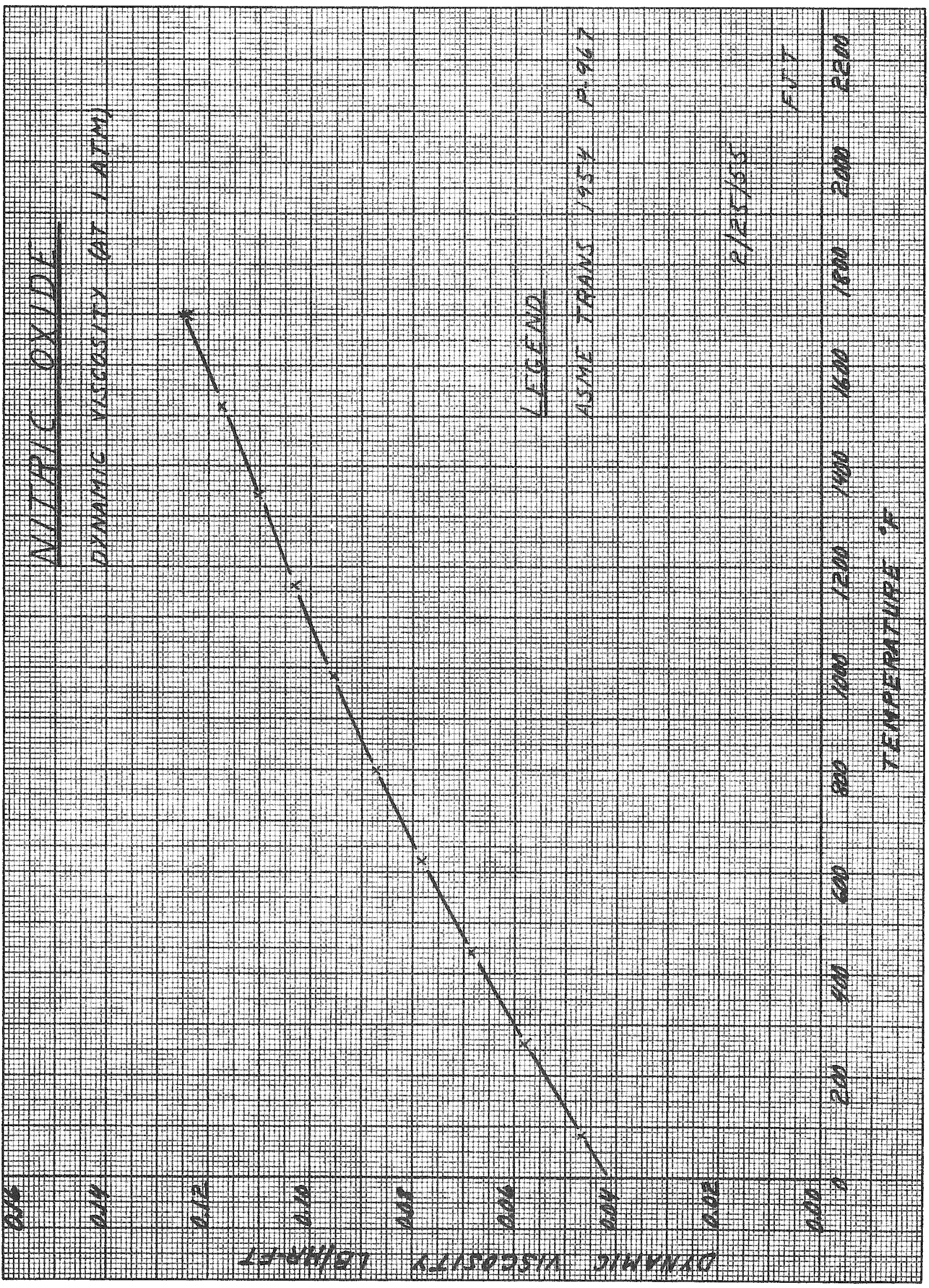




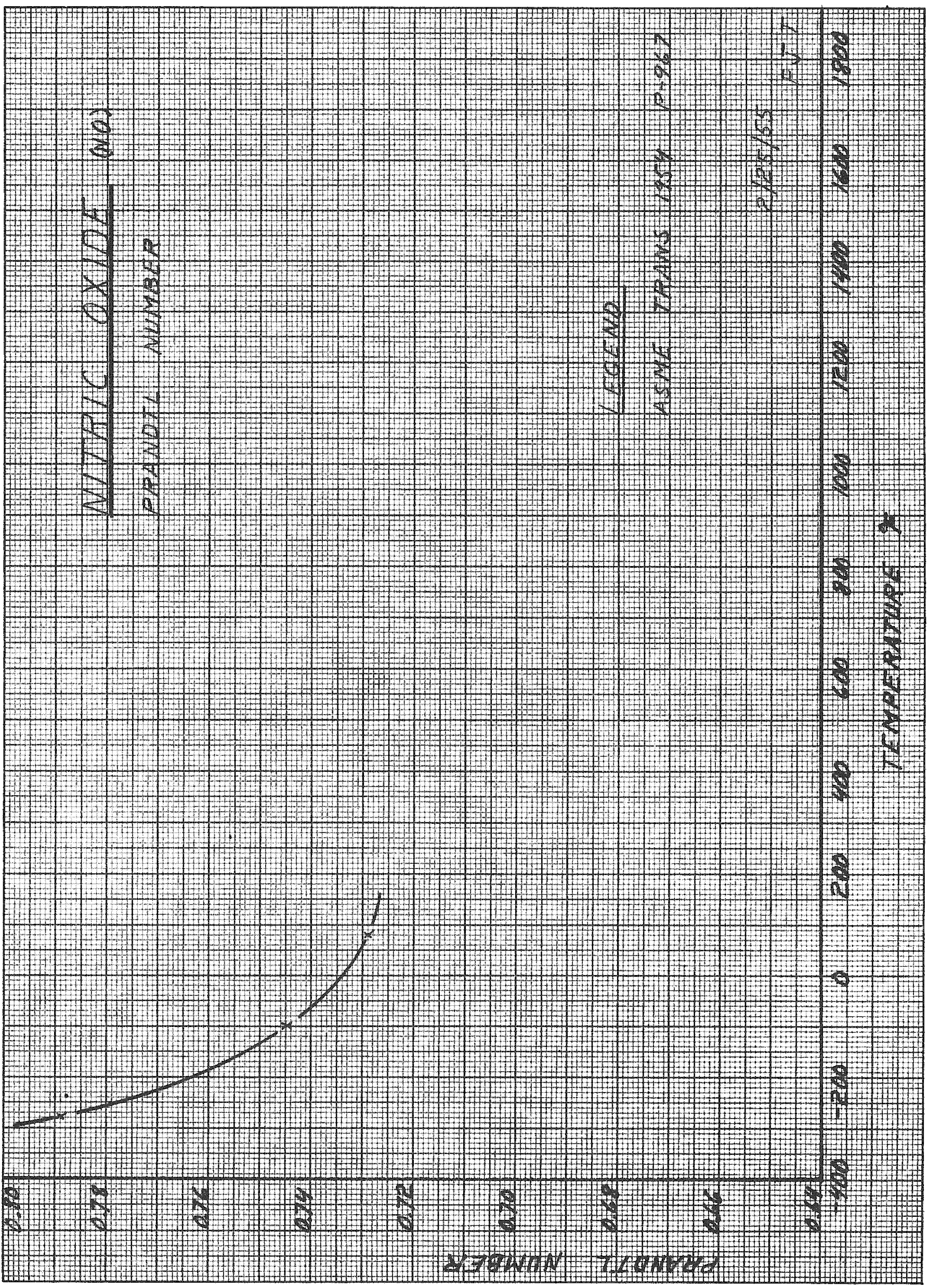



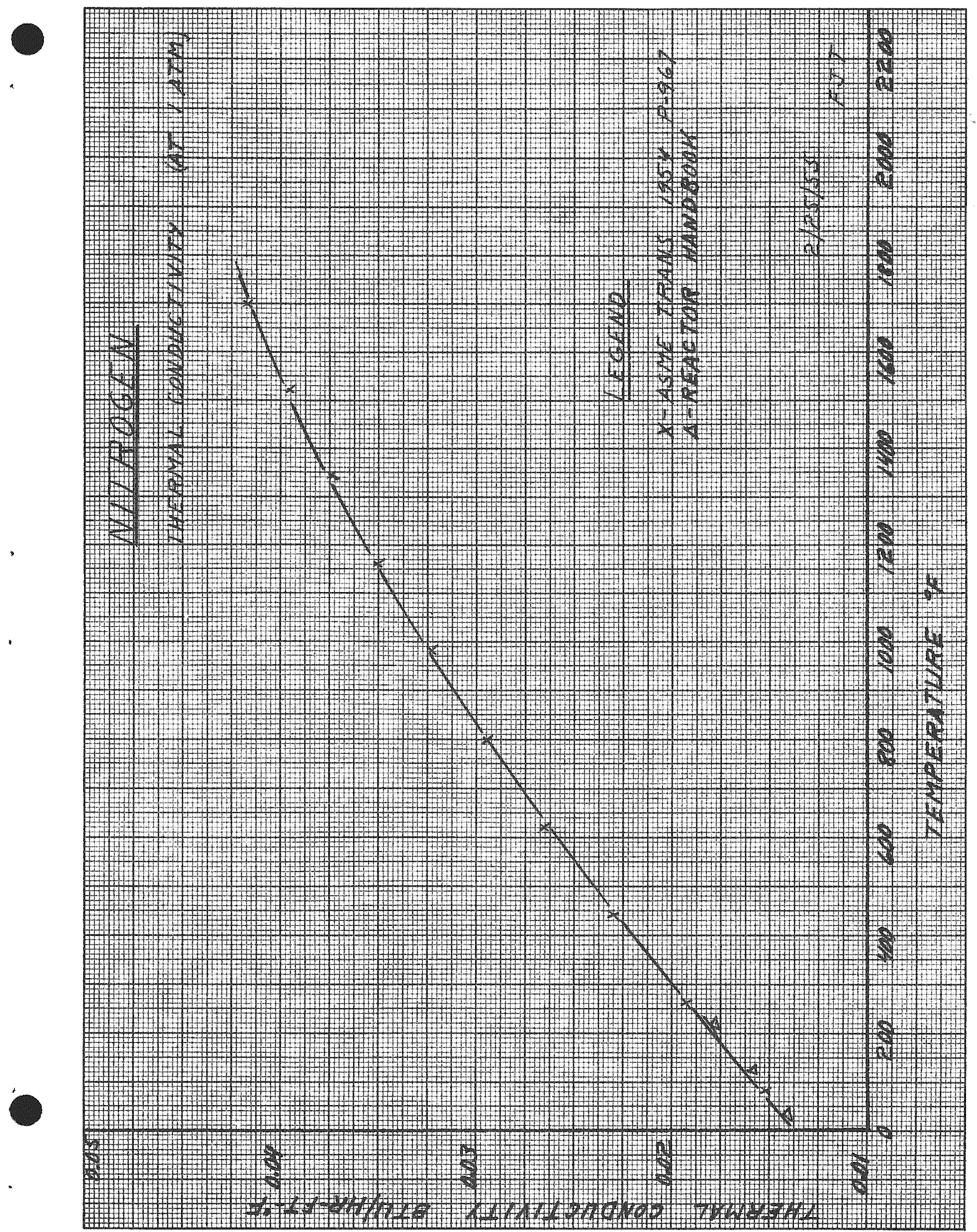


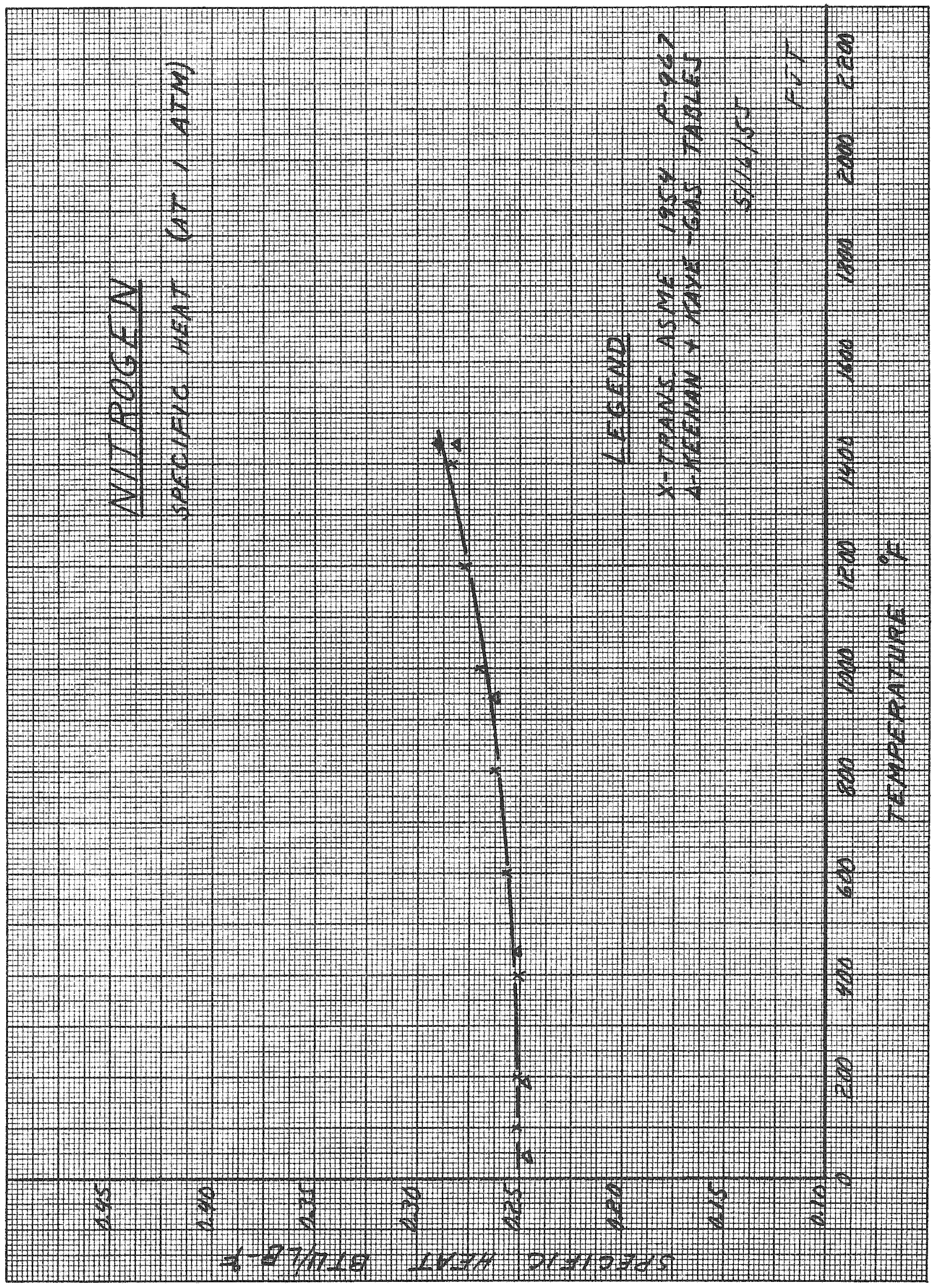




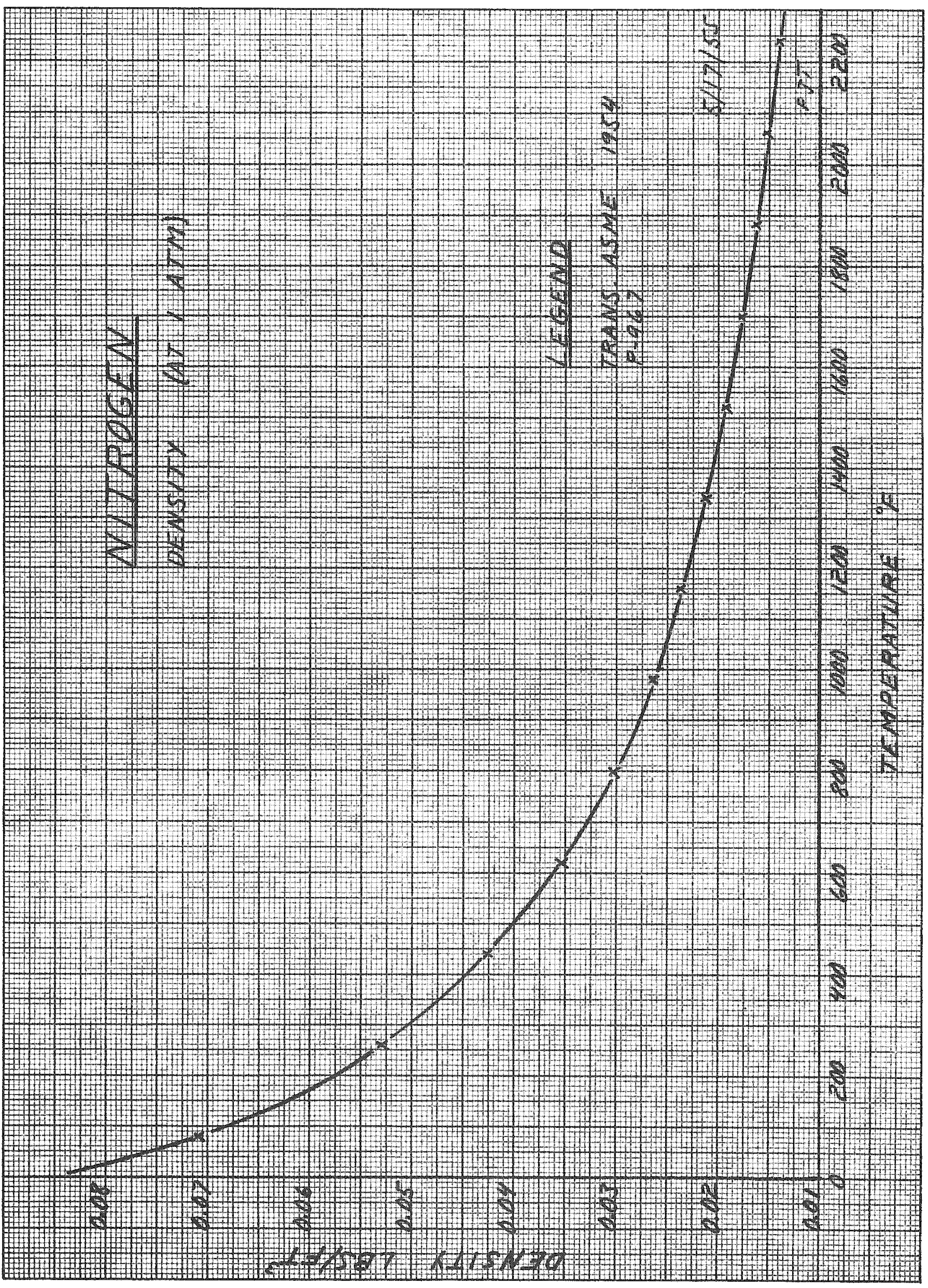




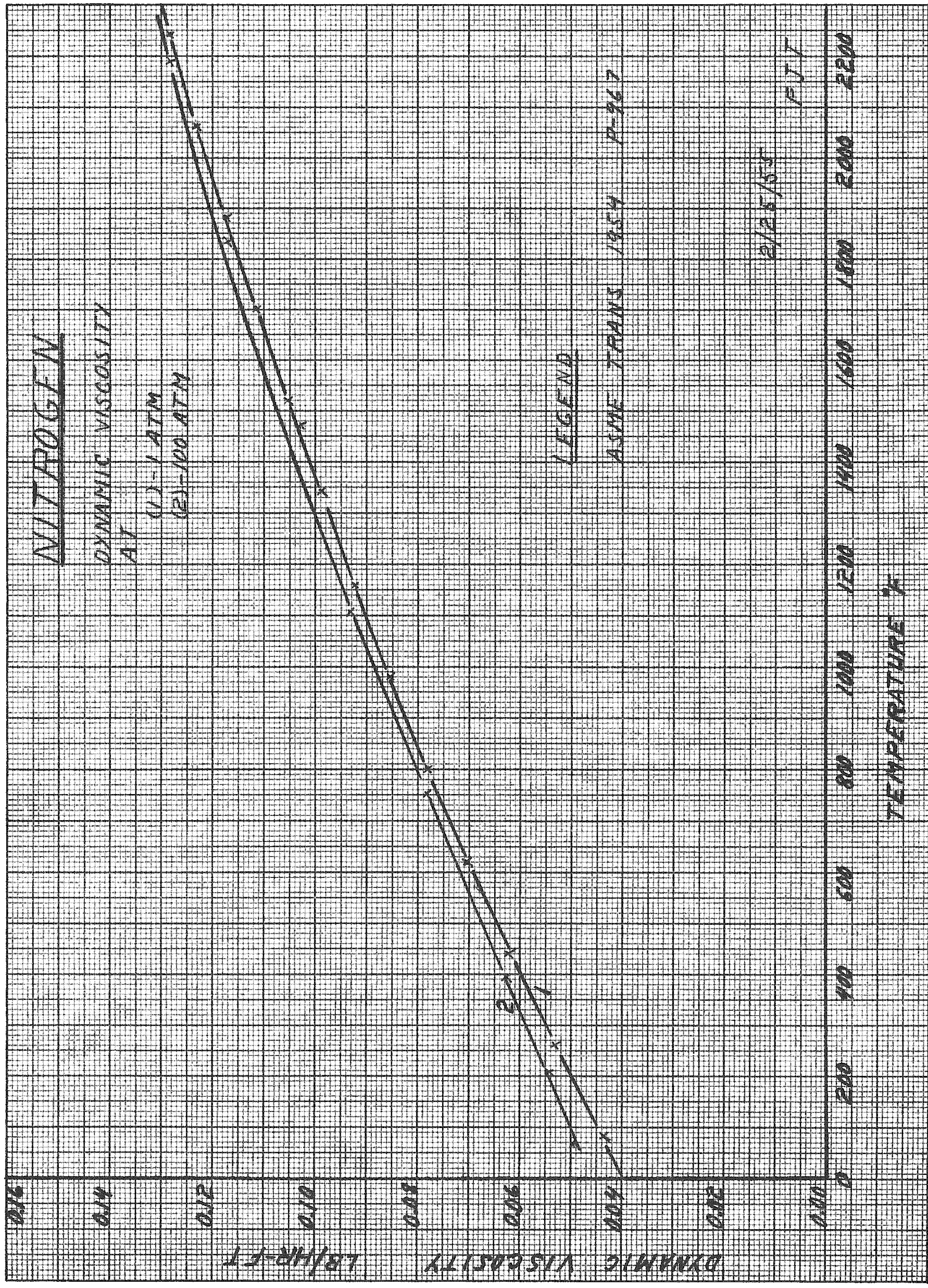




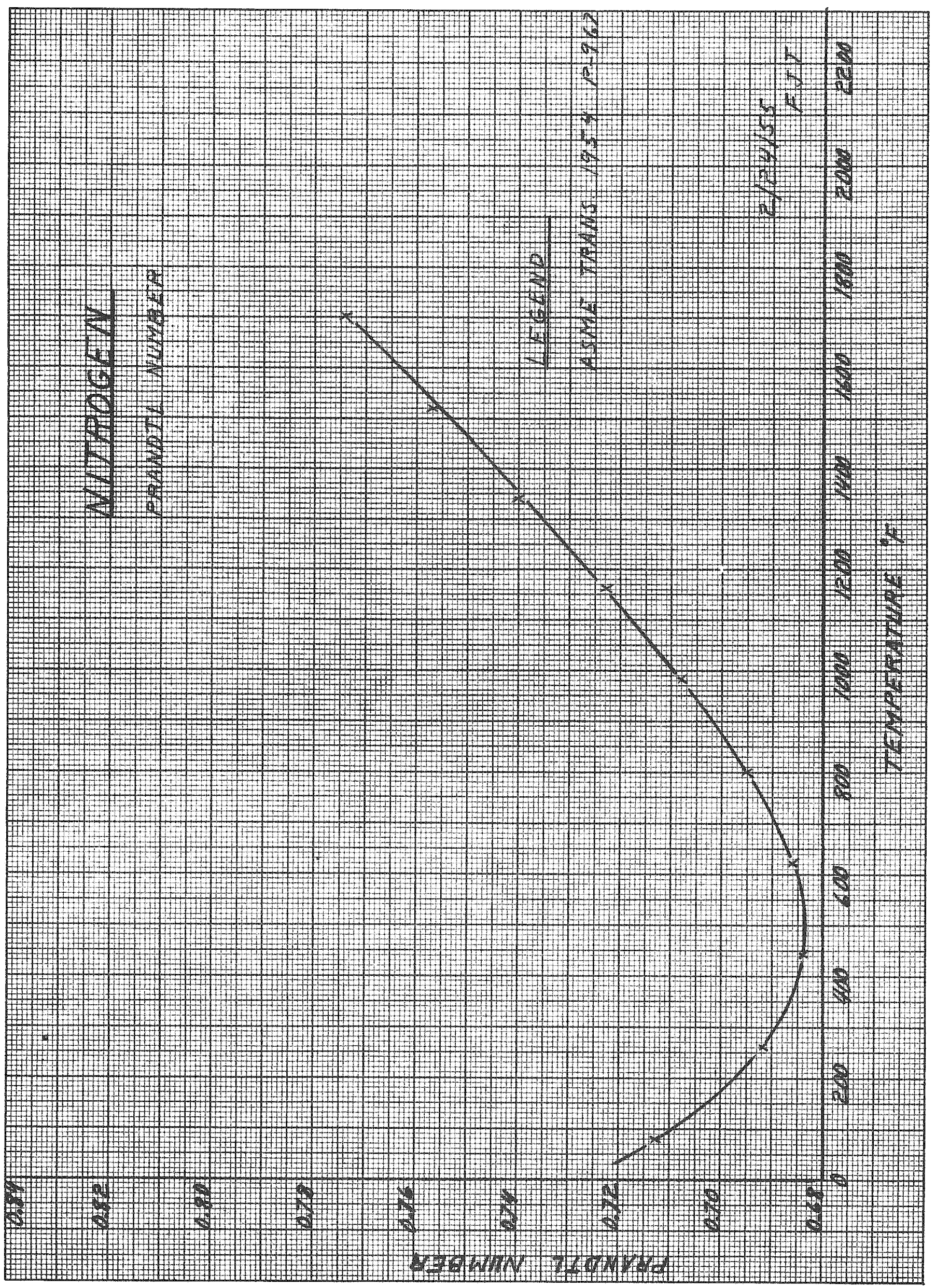




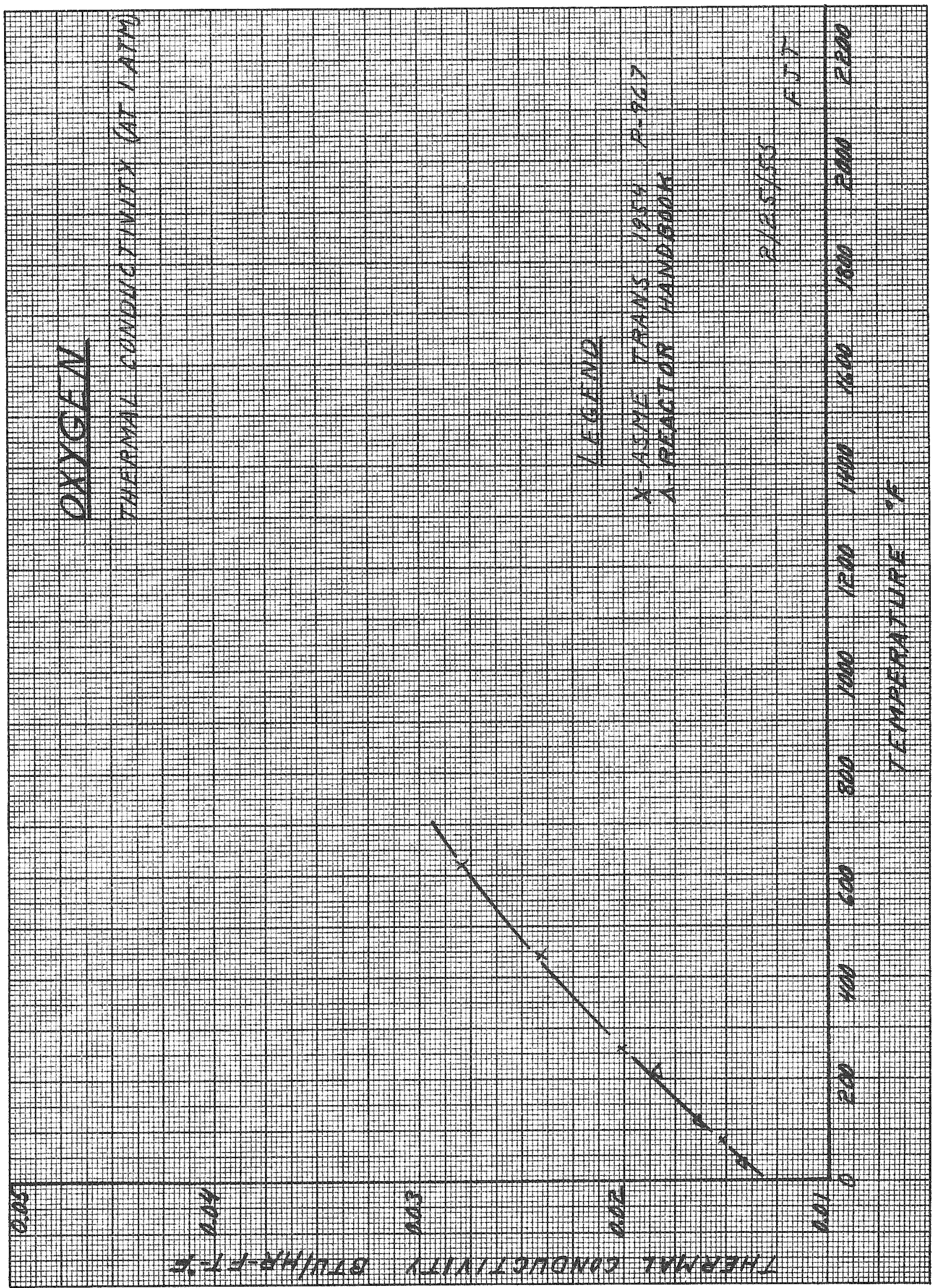




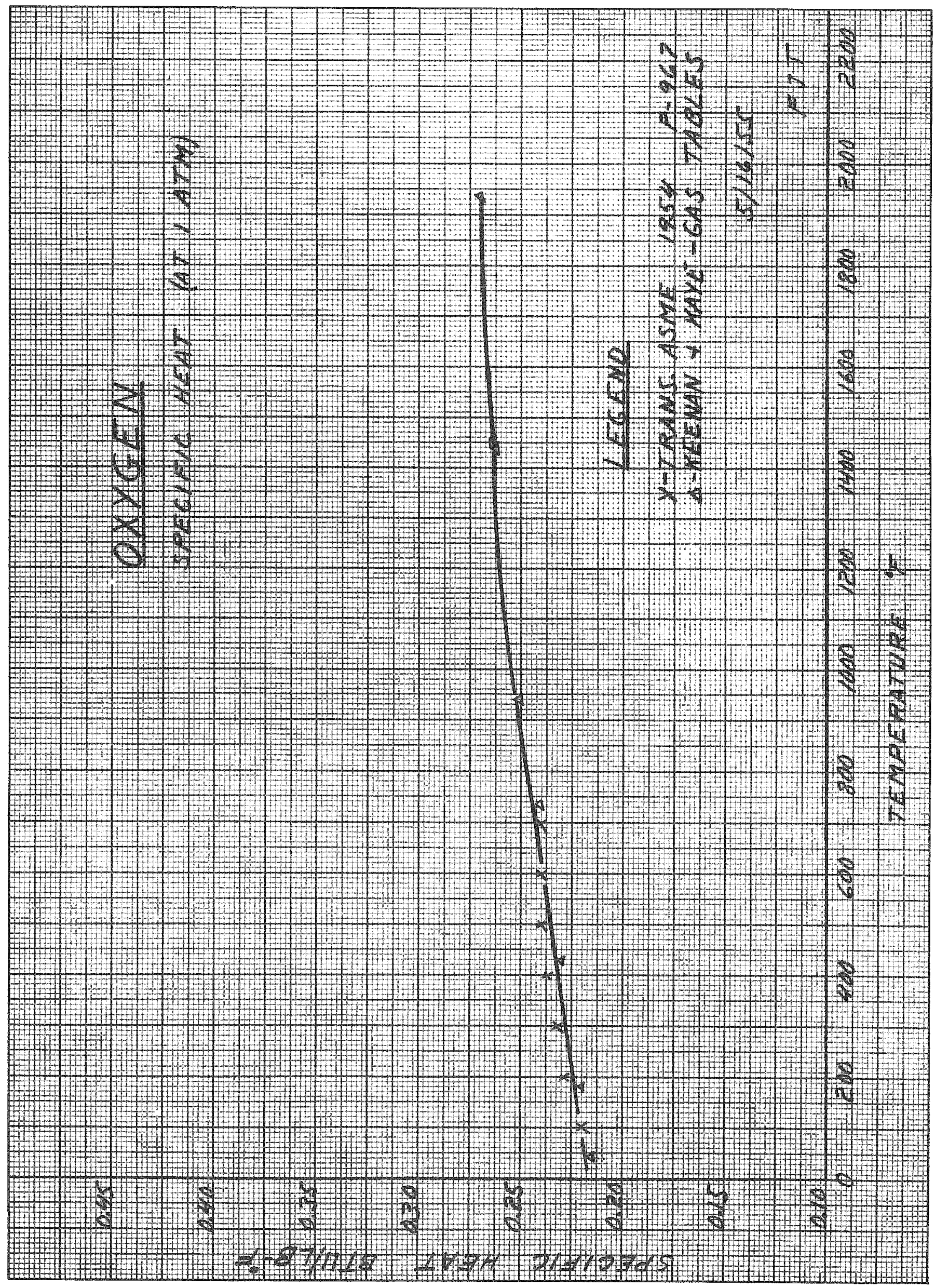




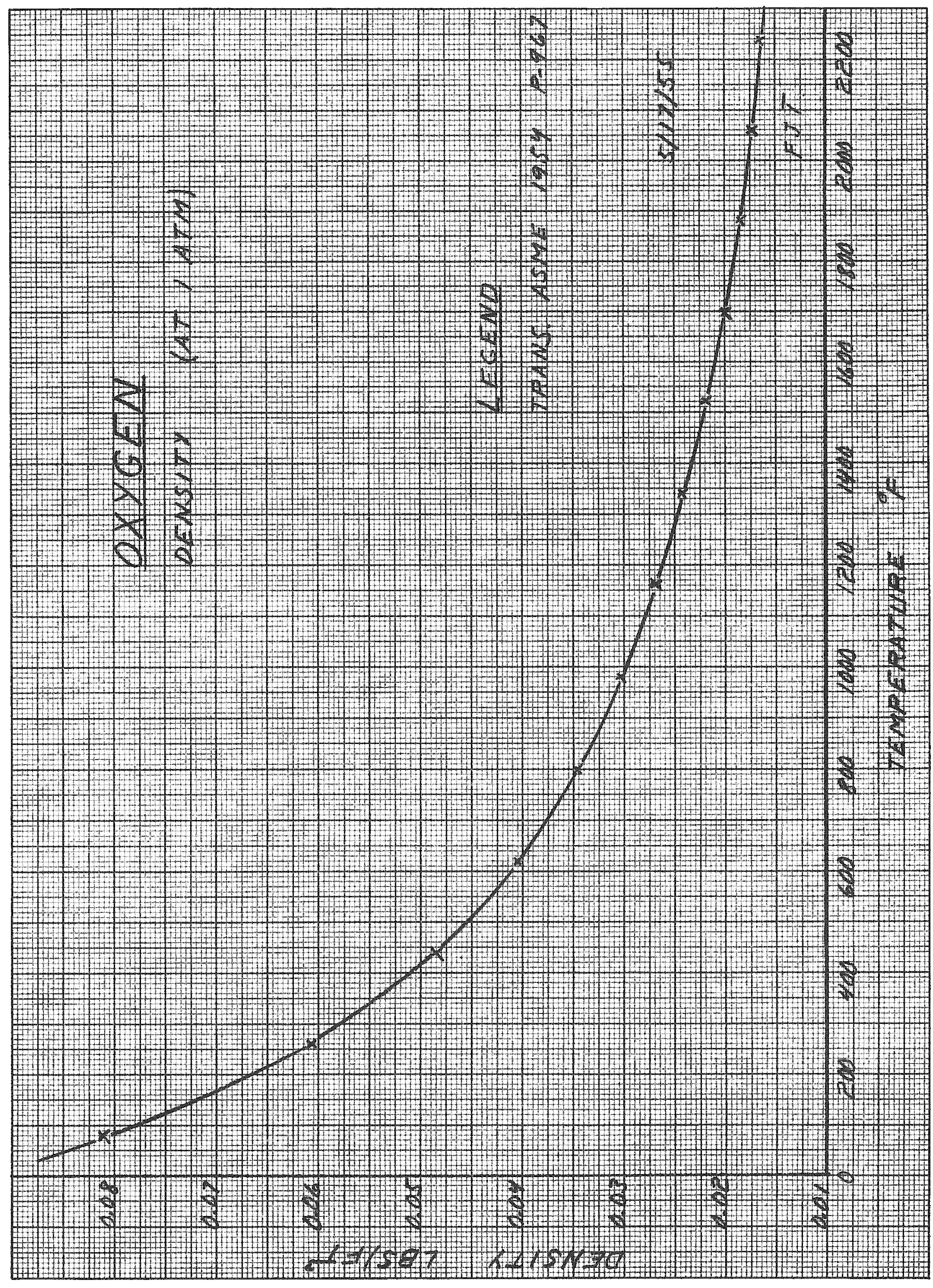




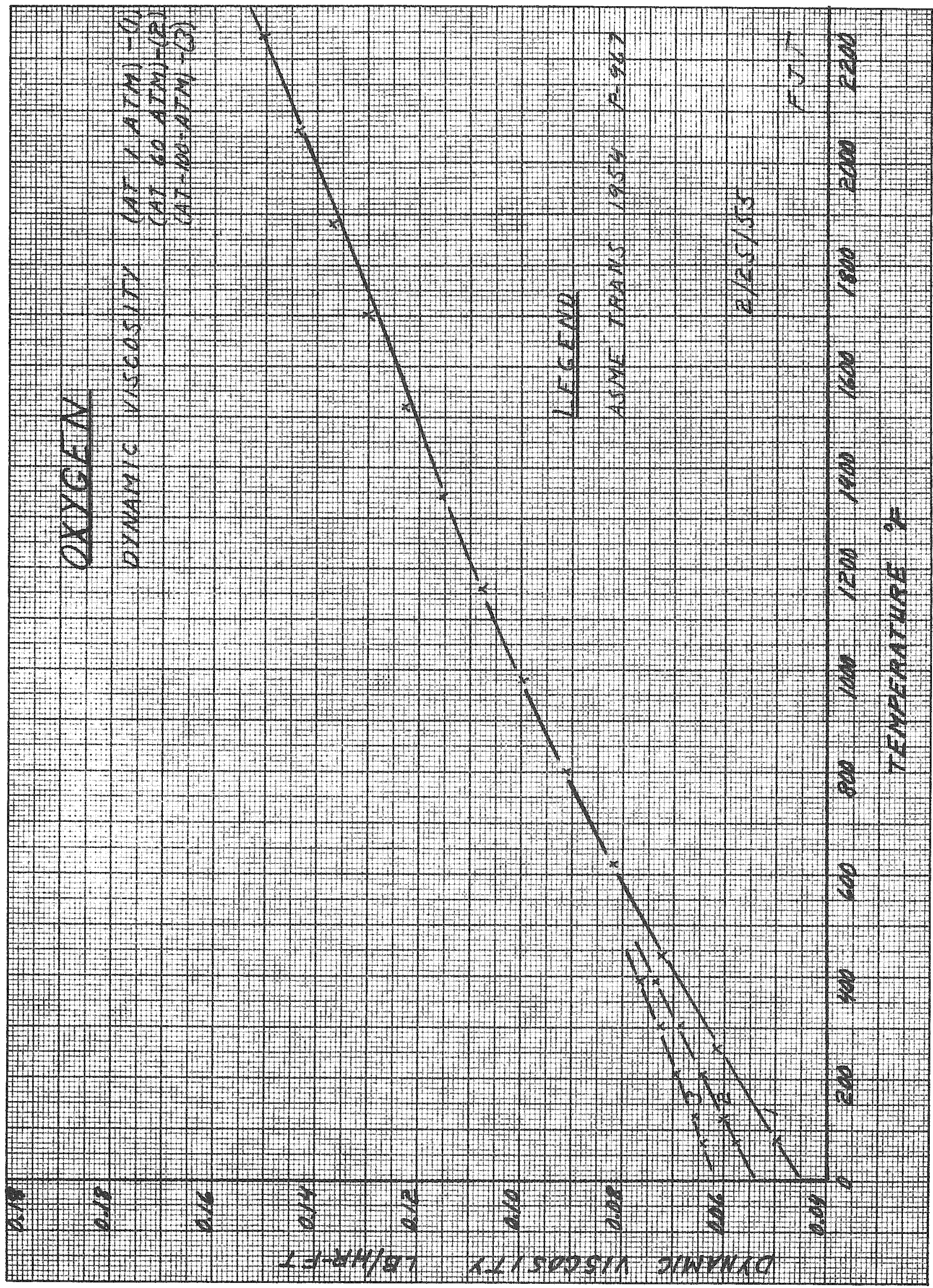




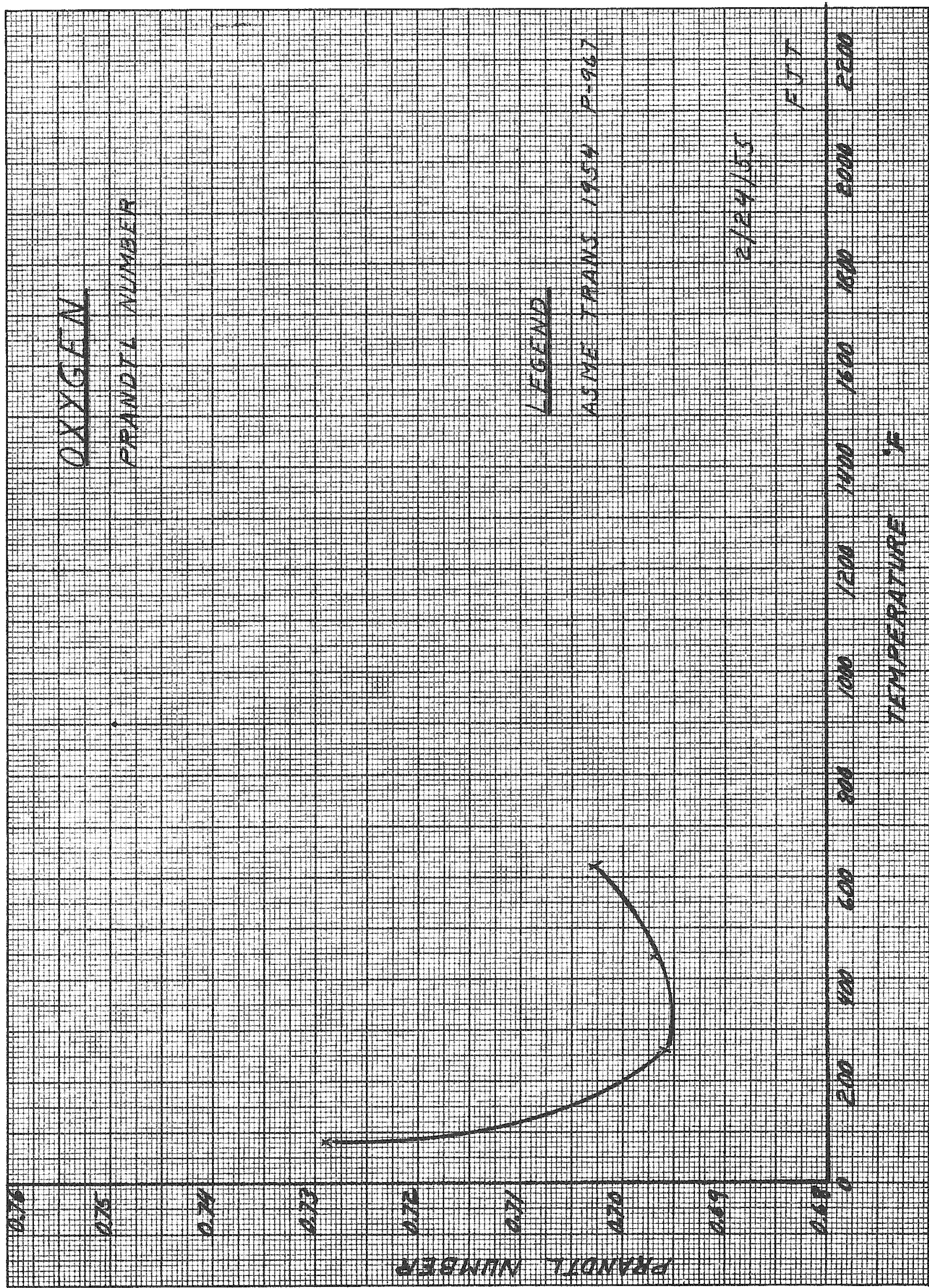




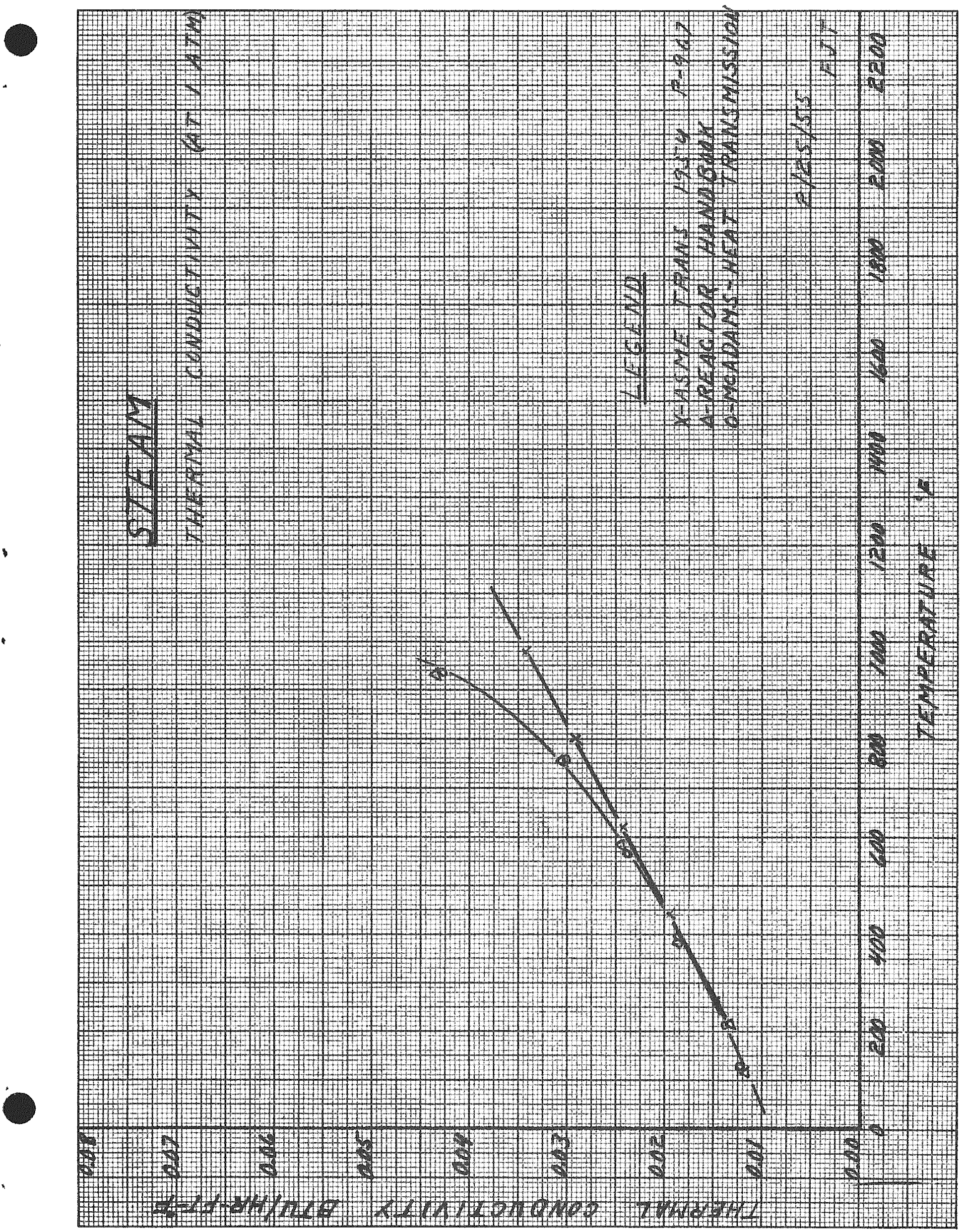




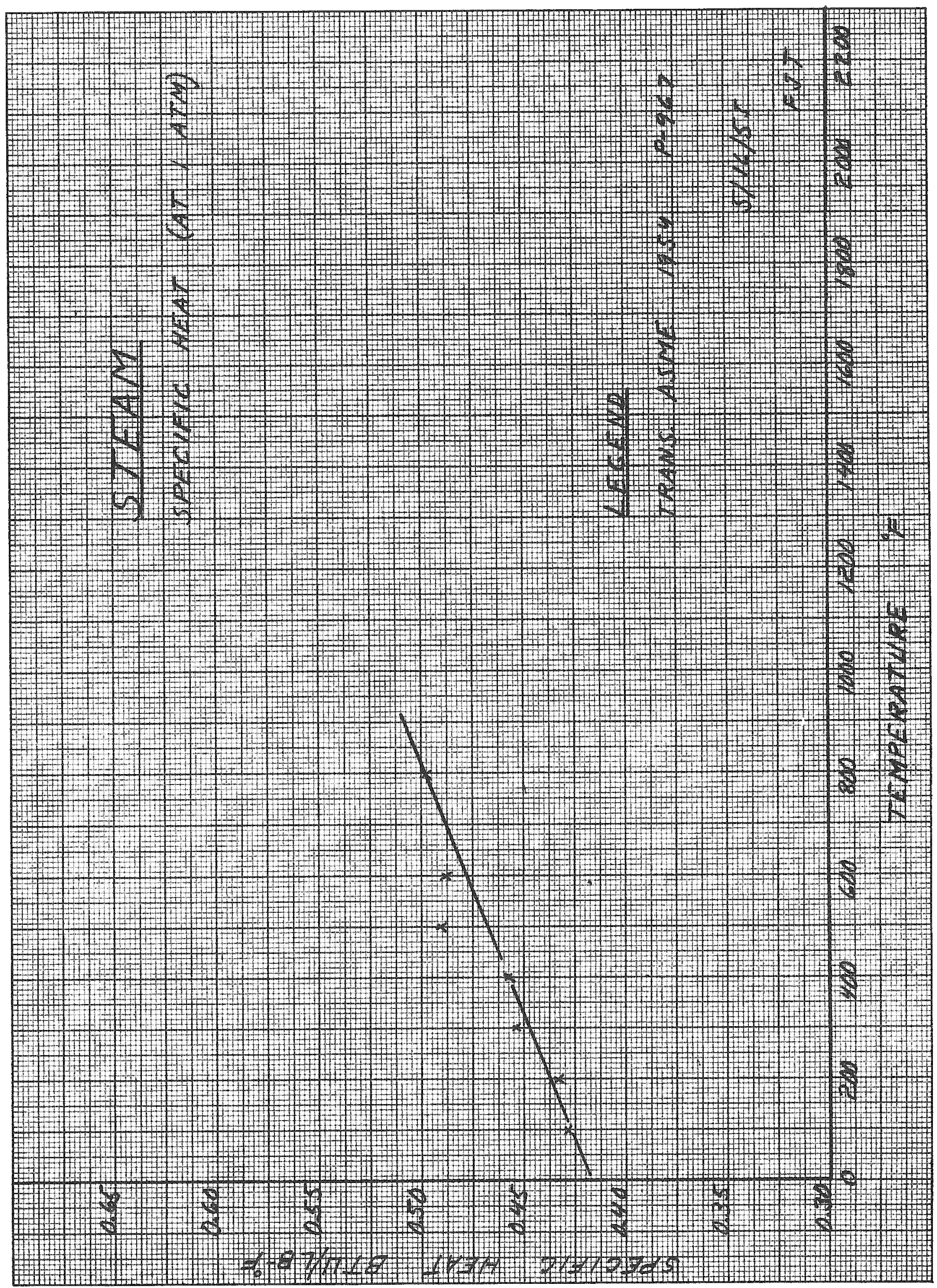




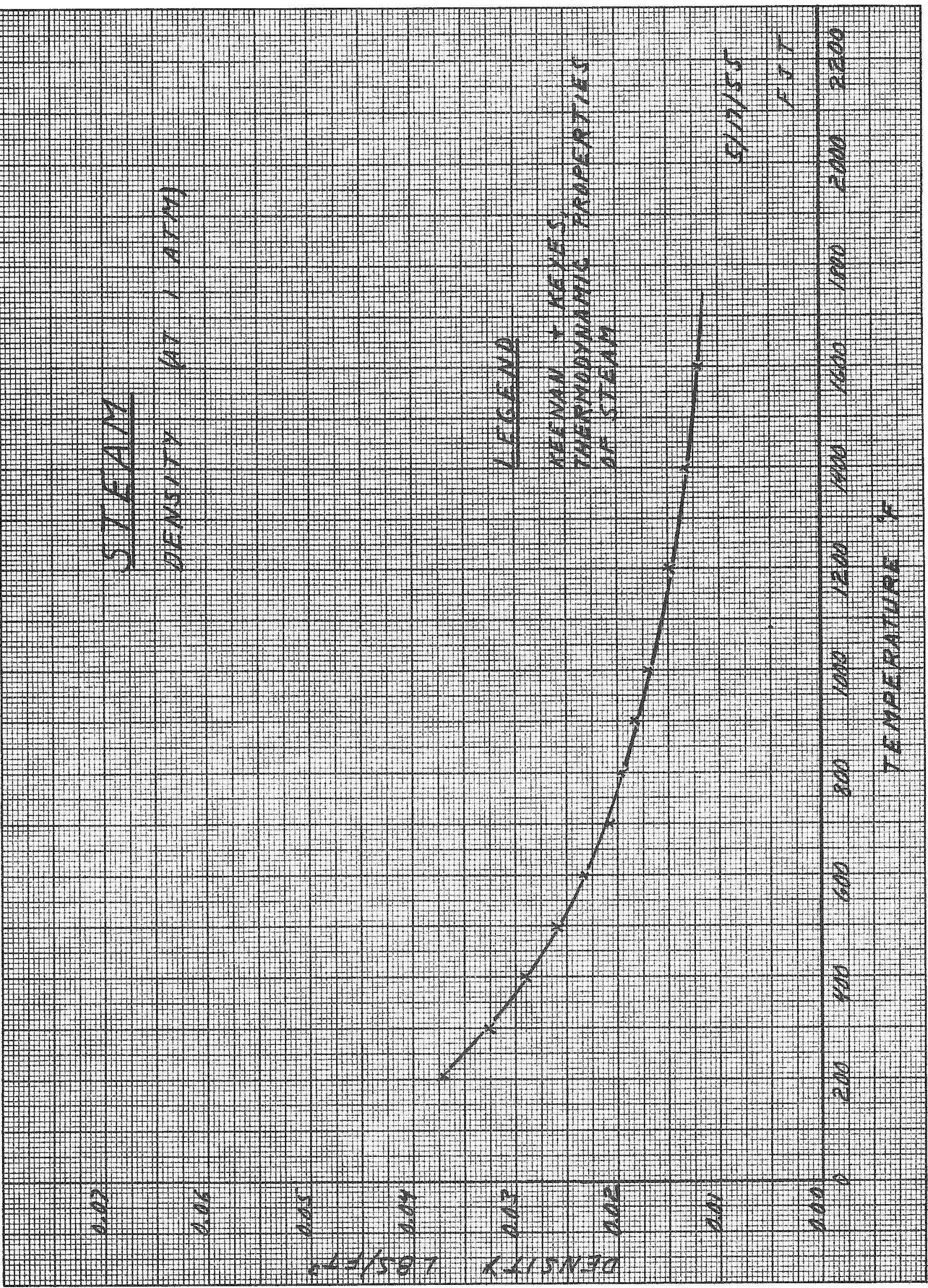




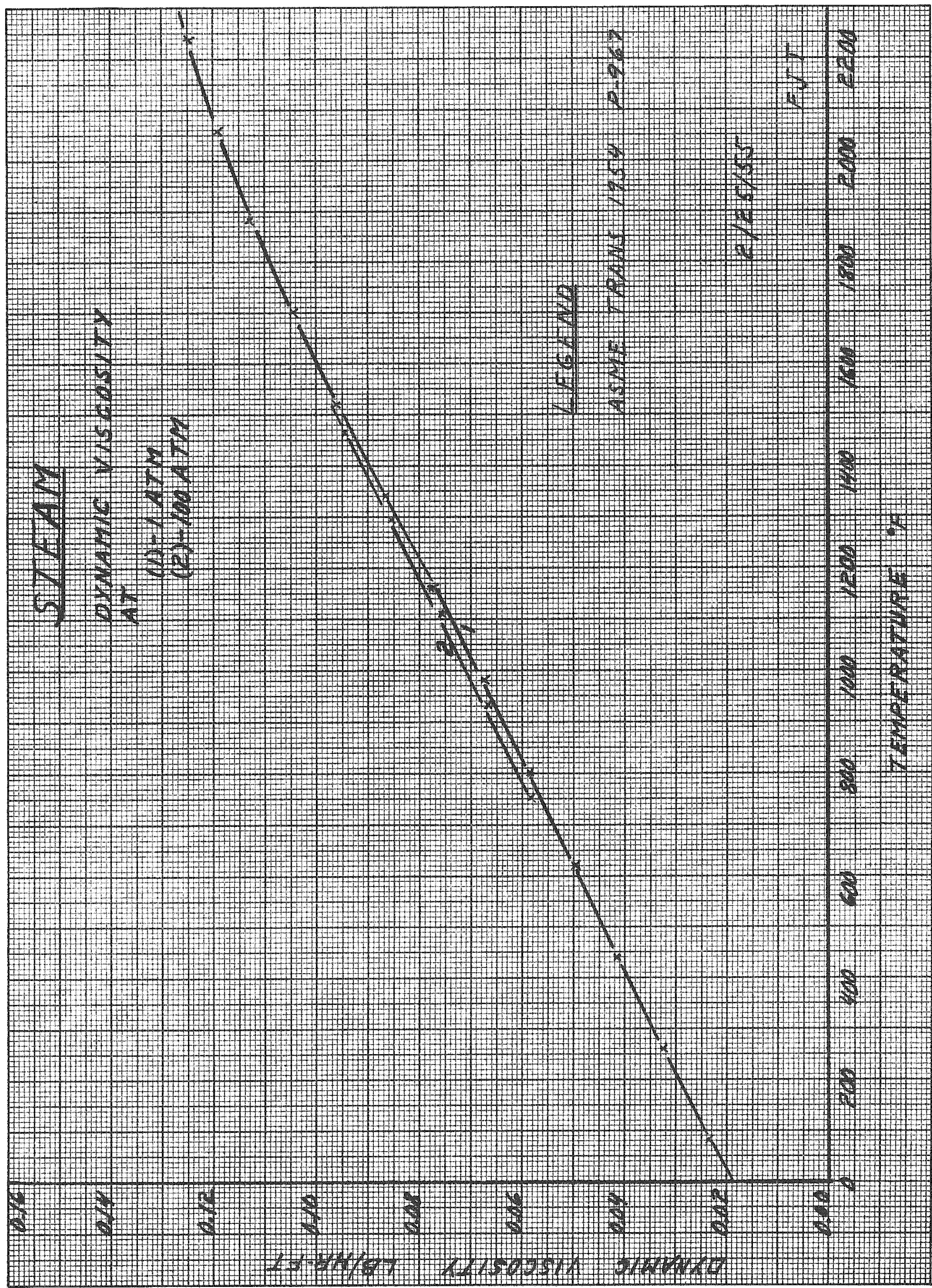




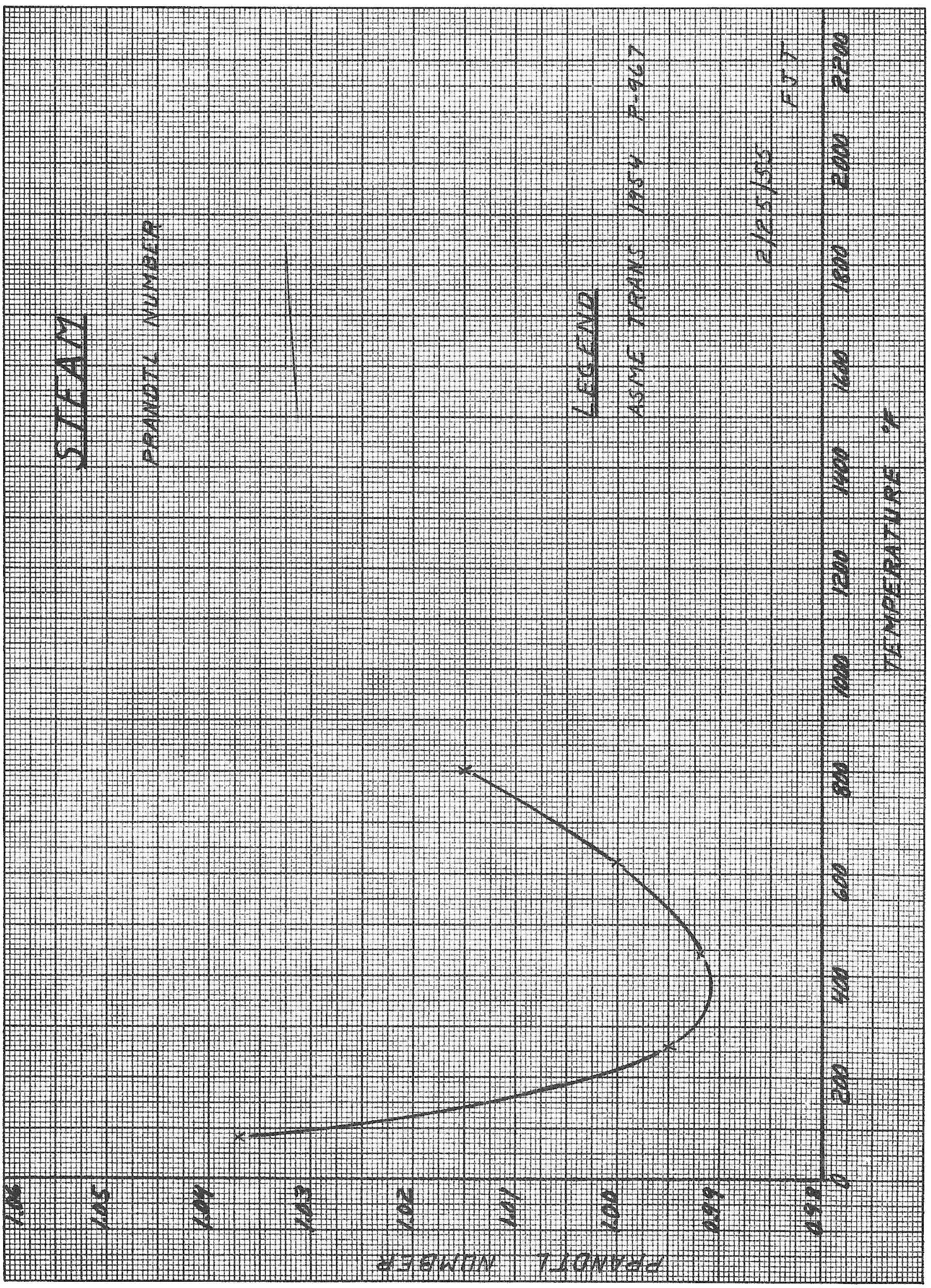




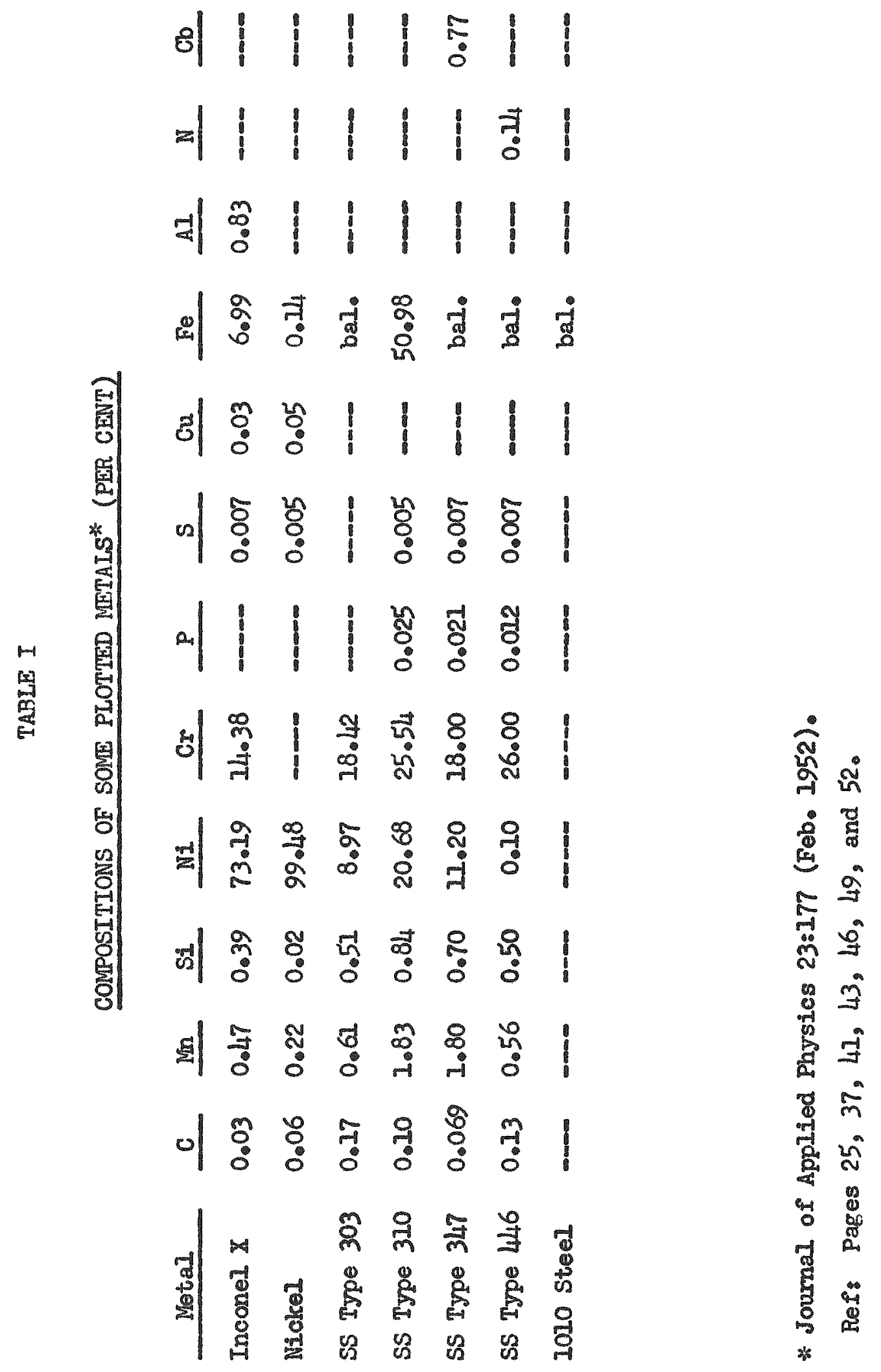


TabIe II

\section{SINGLE POINT VALUES}

\begin{tabular}{|c|c|c|c|}
\hline Solid & $\mathrm{k}(\mathrm{Btu} / \mathrm{hr}-\mathrm{rt}=\mathrm{F})$ & $C_{p}(B+u / I b-F)$ & $P\left(1 \mathrm{bs} / \mathrm{ft} t^{3}\right)$ \\
\hline Alumel & - & 0.13 & 537 \\
\hline Alumina & - & 0.23 & 248 \\
\hline Aluminam Alloy 2S & 128 & 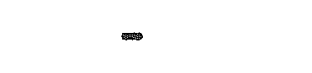 & 169 \\
\hline Alluminum AlIoy $24 \mathrm{~S}-\mathrm{TH}$ & $\infty$ & 0.23 & - \\
\hline Aluminum Al1oy 75S-T6 & 70 & 0.23 & - \\
\hline Beryllia & - & - & 188 \\
\hline Boron Carbide & - & 0.437 & 156 \\
\hline Cadmi um & - & - & 540 \\
\hline Cadmium-Silver Alloy & - & $\mathrm{x}$ & $\mathrm{X}$ \\
\hline Concrete, High Density & - & 0.266 & 269 \\
\hline Inconel & $\infty$ & 0.109 & - \\
\hline Inconel "X" & - & 0.109 & - \\
\hline Iron, Amco & $\Rightarrow$ & - & 487 \\
\hline Iron, Cast & - & 0.13 & 446 \\
\hline Iron, Pure & - & 0.11 & 490 \\
\hline Lead & - & - & 708 \\
\hline Magnesium AIIoy AN-1/4-29 & - & 0.25 & 111 \\
\hline Magnesium Oxide & - & 0.285 & 224 \\
\hline Molybdemum & - & - & 643 \\
\hline Monel & - & - & 552 \\
\hline "K" Monel & $\infty$ & 0.127 & - \\
\hline
\end{tabular}


Table II (Con't)

SINGLE POINT VALUES

\begin{tabular}{|c|c|c|c|}
\hline Solids & $k\left(B t u / h r-r t^{\circ}-F\right)$ & $C_{D}\left(B+a / I b^{\circ}-E\right)$ & $P\left(1 b s / f t^{3}\right)$ \\
\hline Nichrome V & 7.9 & - & 515 \\
\hline NickeI & - & - & 556 \\
\hline "An Nickel & - & 0.13 & 554 \\
\hline Nicke1 OxIde & $\infty$ & 0.159 & 425 \\
\hline Nickel-Zirconium Alloy & - & $x$ & $x$ \\
\hline Spinel & - & 0.257 & 221 \\
\hline Stainless Steel 301 & - & 0.12 & - \\
\hline Stainless Steel 303 & - & 0.12 & 493 \\
\hline StainlessSteel 304 & - & 0.12 & 493 \\
\hline Stainless Steel 310 & - & 0.12 & 493 \\
\hline Stainless Steel 316 & - & 0.12 & - \\
\hline Stainless Steel 321 & - & - & 500 \\
\hline Stainless Steel 430 & - & 0.11 & 481 \\
\hline Stainless Steel 446 & - & - & 475 \\
\hline Steel, Mild & - & 0.112 & 487 \\
\hline Steel, Low Carbon & - & 0.115 & 488 \\
\hline Steo1, Tungsten & - & 0.215 & 498 \\
\hline Tantalum & - & 0.036 & 1036 \\
\hline Thorium Dioxide & - & 0.0548 & 605 \\
\hline Mi tanium & 8.7 & - & - \\
\hline Titaniun Dioxide & - & 0.179 & 266 \\
\hline
\end{tabular}


Table II (Con't)

SINGLE POINT VALUES

\begin{tabular}{|c|c|c|c|}
\hline Solids & $k\left(B \operatorname{ta} / h r-t^{\circ} \mathrm{F}\right)$ & $C_{p}(B+u / 1 b-i)$ & $P\left(10 s / f t^{3}\right)$ \\
\hline Tungsten & - & $\Rightarrow$ & 1200 \\
\hline Urani um & - & - & 1174 \\
\hline UraniumeChromium Alloy & - & $\mathrm{X}$ & $x$ \\
\hline Uranlum-Zi rconium Alloys & - & $\mathrm{X}$ & $\infty$ \\
\hline U-2r ALIoy (I rradiated) & $\infty$ & 0.0319 & 1161 \\
\hline Uranfum Dioxide & - & - & 680 \\
\hline Vanadium & - & - & 382 \\
\hline Zinc Oxide & - & 0.121 & 353 \\
\hline Zi rconium-Tantalum AIIoy & - & $x$ & $x$ \\
\hline 21xconium-Tn A110J & - & $x$ & $x$ \\
\hline zarcon & - & 0.132 & 285 \\
\hline Zirconium Dioxide & - & 0.16 & 381 \\
\hline
\end{tabular}

- Graph

X No data presented 


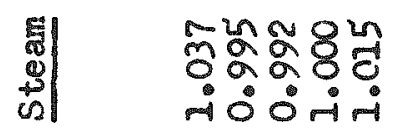

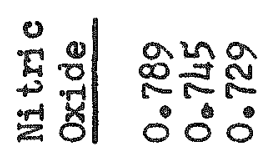

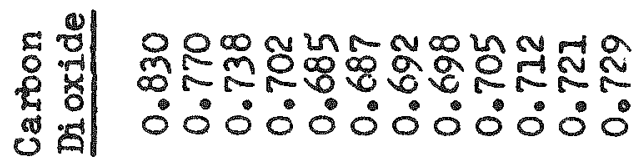

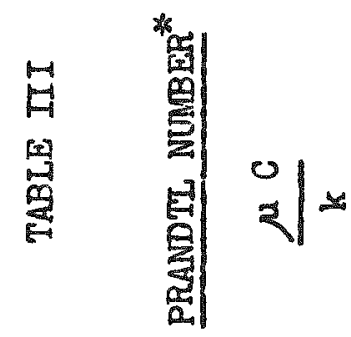

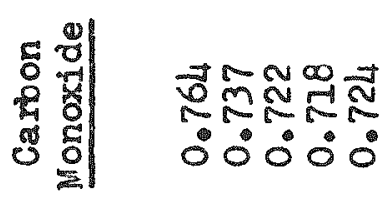

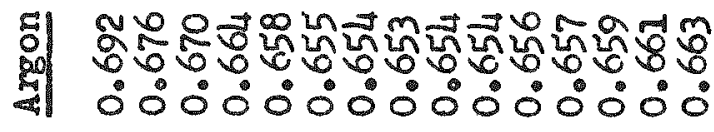

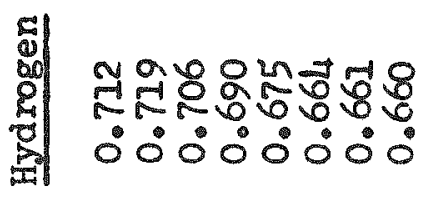

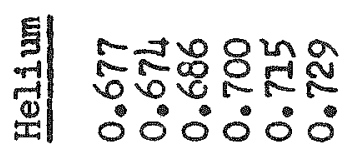

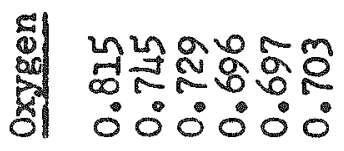

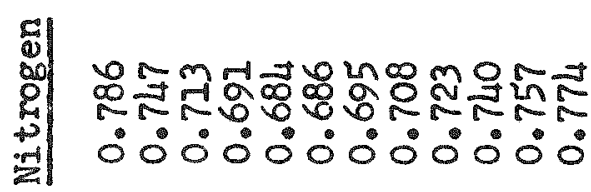

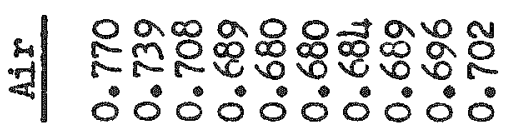

育叫 


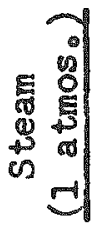

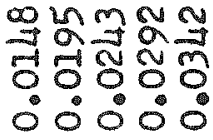

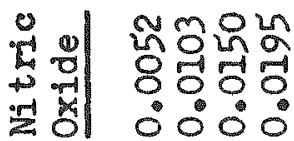

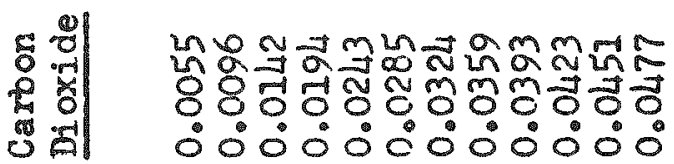

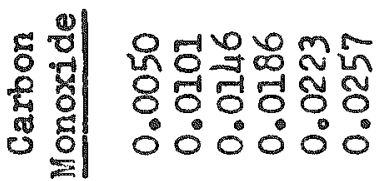

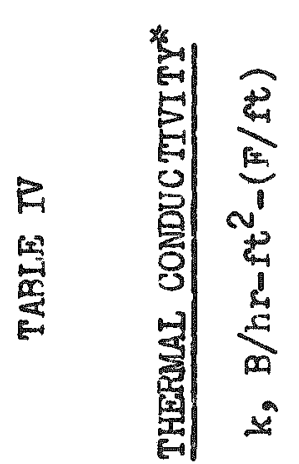

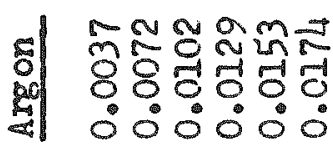

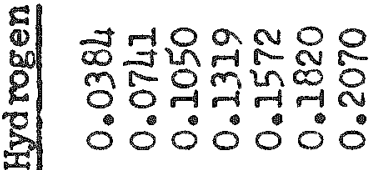

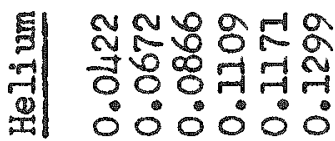

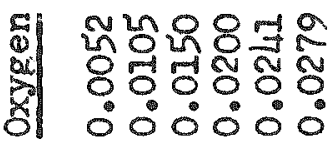

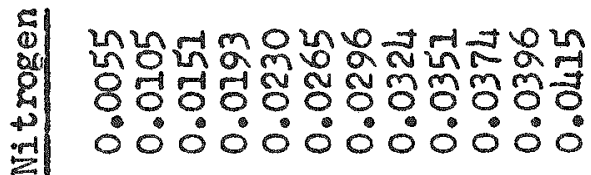

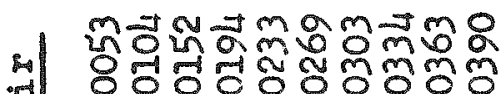

स 8.0 .000 .000

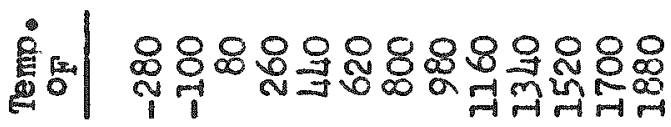




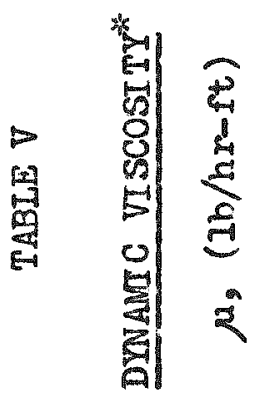

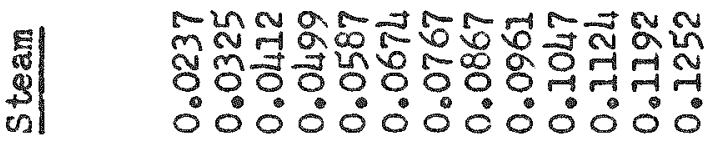

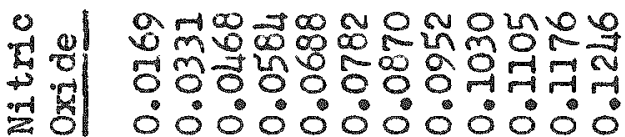

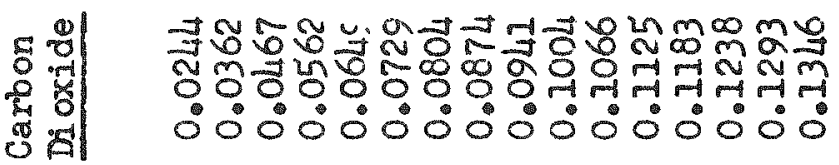

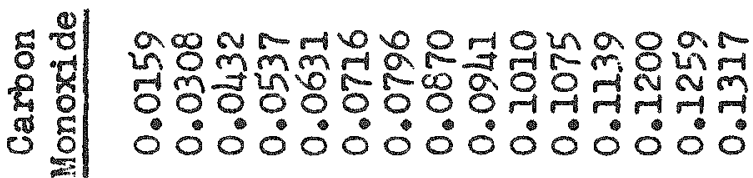

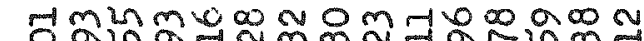

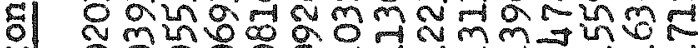

: $0.00007 \%$.

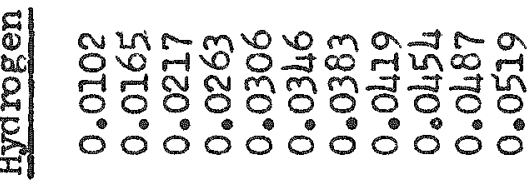

E $000 \times 6 \rightarrow \infty \sim 7 \infty 0$

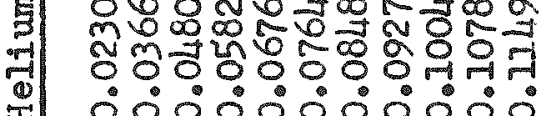

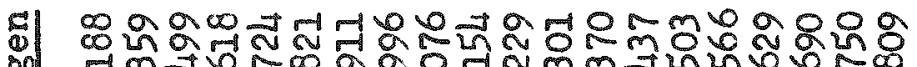

की

f| 00000000000000000000

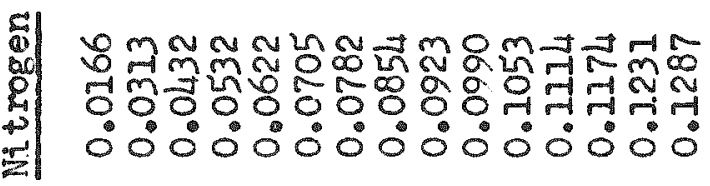

$\overrightarrow{\text { यू }}$

4)

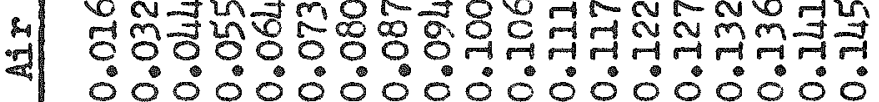

- 
TARLE VI

VISCOSITIES OF GASES UNDER PRESSURE - 1b/hr-It

MRCON

\begin{tabular}{|c|c|c|c|c|c|c|}
\hline $\begin{array}{c}\text { Temp. } \\
\mathrm{F} \\
\end{array}$ & $1 \mathrm{~atm}$ & $20 \mathrm{~atm}$ & $40 \mathrm{~atm}$ & $60 \mathrm{~atm}$ & $80 \mathrm{am}$ & $100 \mathrm{~atm}$ \\
\hline 77 & $-\infty+\infty$ & 0.0552 & 0.057 & 0.059 & 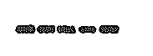 & \\
\hline 122 & 0.058 & 0.0595 & 0.061 & 0.063 & 0.066 & $-\infty$ \\
\hline 212 & 0.066 & 0.0665 & 0.067 & 0.069 & 0.072 & 0.074 \\
\hline 302 & 0.071 & 0.0723 & 0.074 & 0.075 & 0.077 & 0.080 \\
\hline 392 & 0.077 & 0.0784 & 0.080 & 0.081 & 0.083 & 0.086 \\
\hline 572 & 0.088 & 0.0883 & 0.089 & 0.091 & 0.092 & 0.095 \\
\hline
\end{tabular}

Ref: Trans. ASME 76:967 (1954) 
TABLE VII

VISCOSITIES OF GASES UNDER PRESSURE - Ib/hr-It

CARBON DIOXIDE

\begin{tabular}{|c|c|c|c|c|c|c|}
\hline Temp-s & 1 atm & 20 atm & $40 \mathrm{~atm}$ & 60 atm & $80 \mathrm{~atm}$ & 100 atm \\
\hline 122 & 0.039 & 0.0406 & 0.044 & 0.050 & 0.059 & \\
\hline 212 & 0.044 & 0.0452 & 0.048 & 0.052 & 0.062 & \\
\hline 302 & 0.050 & 0.0506 & 0.052 & 0.057 & 0.063 & \\
\hline 392 & 0.054 & 0.0559 & 0.058 & 0.061 & 0.065 & \\
\hline 482 & 0.059 & 0.0605 & 0.062 & 0.064 & 0.068 & \\
\hline 572 & 0.064 & 0.0649 & 0.066 & 0.068 & 0.070 & \\
\hline
\end{tabular}

Ref: Trans. ASME 76:967 (1954) 
TABLE VIII

VISCOSITIES OF GASES UNDER PRESSURE - lb/hr-It

HYDROGEN

\begin{tabular}{|c|c|c|c|c|c|c|}
\hline $\begin{array}{c}\text { Pemp } \\
\end{array}$ & 1 atm & $20 \mathrm{~atm}$ & 40 atm & $60 \mathrm{~atm}$ & $80 \mathrm{~atm}$ & 100 atm \\
\hline-238 & 0.012 & 0.012 & 0.012 & 0.012 & 0.012 & 0.013 \\
\hline-48 & 0.015 & 0.015 & 0.015 & 0.015 & 0.015 & 0.015 \\
\hline 32 & 0.020 & 0.020 & 0.020 & 0.020 & 0.021 & 0.021 \\
\hline 212 & 0.025 & 0.025 & 0.025 & 0.025 & 0.025 & 0.025 \\
\hline 392 & 0.029 & 0.029 & 0.029 & 0.030 & 0.030 & 0.030 \\
\hline 572 & 0.034 & 0.033 & 0.034 & 0.034 & 0.034 & 0.034 \\
\hline
\end{tabular}

Ref: Trans. ASME 76:967 (1954) 
TABLE IX

VISCOSITIES OF GASES UNDER PRESSURE - 1b/hr- It

NITROGEN

$\begin{array}{ccccccc}\begin{array}{ccccc}\text { Temp. } \\ \text { F }\end{array} & \text { I atm } & \text { 20 atm } & 40 \mathrm{~atm} & 60 \mathrm{~atm} & 80 \mathrm{~atm} & 100 \mathrm{~atm} \\ 68 & 0.042 & 0.043 & 0.044 & 0.046 & 0.078 & 0.050 \\ 122 & 0.046 & 0.046 & 0.047 & 0.048 & 0.049 & 0.050 \\ 212 & 0.054 & 0.051 & 0.051 & 0.052 & 0.053 & 0.054 \\ 392 & 0.060 & 0.060 & 0.061 & 0.061 & 0.062 & 0.062 \\ 752 & 0.076 & 0.076 & 0.077 & 0.077 & 0.077 & 0.078 \\ 1112 & 0.090 & 0.091 & 0.091 & 0.091 & 0.091 & 0.092 \\ 1472 & 0.104 & 0.104 & 0.104 & 0.104 & 0.104 & 0.102 \\ 1832 & 0.116 & 0.116 & 0.116 & 0.116 & 0.117 & 0.117 \\ 2192 & 0.127 & 0.127 & 0.127 & 0.129 & 0.128 & 0.128\end{array}$

Ref: Trans. ASME 76:967 (1954) 
TABLE X

VISCOSITIES OF GASES UNDER PRESSURE - 1b/hr-ft

ORYGEN

\begin{tabular}{|c|c|c|c|c|c|c|}
\hline $\begin{array}{c}\text { Temp } \\
\text { F }\end{array}$ & 1 atm & 20 atm & 40 atm & $60 \mathrm{~atm}$ & $80 \mathrm{~atm}$ & 100 atm \\
\hline 77 & 0.050 & 0.052 & 0.055 & 0.058 & 0.060 & 0.064 \\
\hline 122 & 0.053 & 0.055 & 0.057 & 0.060 & 0.063 & 0.066 \\
\hline 212 & 0.059 & 0.061 & 0.063 & 0.065 & 0.067 & 0.070 \\
\hline 302 & 0.065 & 0.066 & 0.067 & 0.069 & 0.071 & 0.0730 \\
\hline 392 & 0.070 & 0.071 & 0.072 & 0.074 & 0.075 & 0.077 \\
\hline
\end{tabular}

Ref: Trans. ASME 76:967 (1954) 
TABLE XI

VISCOSITIES OF GASES UNDER PRESSURE - Ib/hr=ft

STEMM

\begin{tabular}{|c|c|c|c|c|c|c|}
\hline $\begin{array}{l}\text { Temp } \\
\end{array}$ & 1 atm & $20 \mathrm{~atm}$ & $40 \mathrm{~atm}$ & 60 atm & $80 \mathrm{~atm}$ & $100 \mathrm{~atm}$ \\
\hline 77 & 0.0024 & & & & & \\
\hline 122 & 0.0257 & & & & & \\
\hline 212 & 0.0304 & & & & & \\
\hline 392 & 0.0389 & & & & & \\
\hline 572 & 0.0476 & 0.0479 & 0.0483 & 0.0489 & 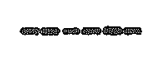 & $-\infty$ \\
\hline 752 & 0.0563 & 0.0566 & 0.0569 & 0.0572 & 0.0577 & 0.0583 \\
\hline 932 & 0.0651 & 0.0653 & 0.0655 & 0.0658 & 0.0662 & 0.0665 \\
\hline 1712 & 0.0739 & 0.0743 & 0.0745 & 0.0747 & 0.0750 & 0.0753 \\
\hline 1293 & 0.0891 & 0.0843 & 0.0844 & 0.0846 & 0.0848 & 0.0851 \\
\hline 1472 & 0.0937 & 0.0938 & 0.0940 & 0.0942 & 0.944 & 0.0945 \\
\hline 1652 & 0.1025 & & & & & \\
\hline 1832 & 0.1104 & & & & & \\
\hline 2012 & 0.1175 & & & & & \\
\hline 2192 & 0.1237 & & & & & \\
\hline
\end{tabular}

Ref: Trans. ASME 76:967 (1954) 
1. Powell, $R_{0}$ Io, and Blanpied, W. A, Thermal Conductivity of Metals and Alloys at Low Temperatures. NBS Circular 556, National Bureau of Standards (1954).

2. Pagerey, P. Ro, The Thermal Conductivity of Organic Ilquids. Preprint of paper contributed by Heat Transfer Division of the American Society of Mechanical Engineers for presentation at the Sem-Annual Meeting. Paper No. 54-SA-55, June 20, 1954 .

3. Sakiadis, B. C., and Coates, J., A Literature Survey of the Thermal Conductivity of Liquids. Engineering Experimental Station, Bulietin No. 34. College of Engineering, Louisiana State University (1954).

4. Iiquid Metals Handbook, edited by $R_{0} N$. Lyon, Departnent of the Navy and U.S. Atomic Enexgy Commission, NAVEXOS P-733, Chapter 2, June 1952.

5. Evans, J. E., Themal Conductivity of Fourteen Metals and Alloys Up to $1100^{\circ} \mathrm{F}$, Report NACA-PMI-E50LOT (1951).

6. The Reactor Handbook, Vol. 3, Materials, 1953.

7. Metals Handbook, Compiled by the American Society for Metals. 